Neuartige Pyrrol/Pyrazol-Bausteine für die Synthese von Hybrid-Makrozyklen, azyklischen Ligandsystemen und bimetallischen Komplexen
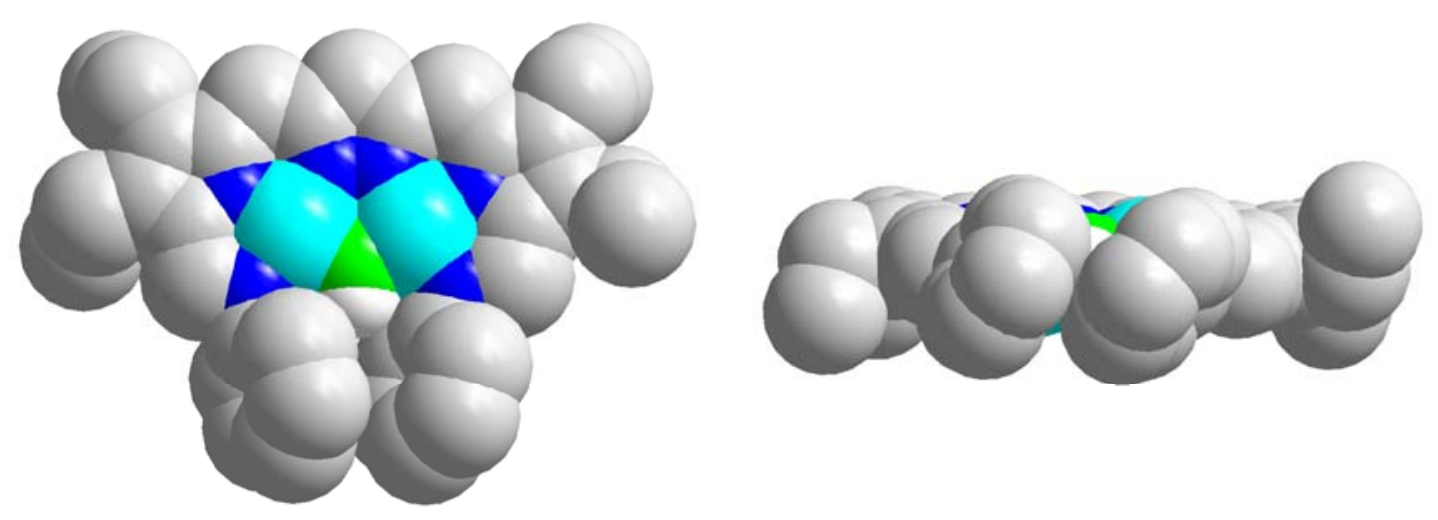

Stamatia Katsiaouni

Dissertation • Göttingen 2007 


\title{
Neuartige Pyrrol/Pyrazol-Bausteine für die Synthese von Hybrid-Makrozyklen, azyklischen Ligandsystemen und bimetallischen Komplexen
}

\author{
Dissertation \\ zur Erlangung des Doktorgrades \\ der Mathematisch-Naturwissenschaftlichen Fakultäten \\ der Georg-August-Universität zu Göttingen \\ vorgelegt von \\ Diplom-Chemikerin \\ Stamatia Katsiaouni \\ aus Serres \\ (Griechenland)
}

Göttingen 2007 
D7

Referent: $\quad$ Prof. Dr. Franc Meyer

Korreferent: Prof. Dr. Jörg Magull

Tag der mündlichen Prüfung: 01.11.2007 
Ich erkläre hiermit an Eides statt, dass ich diese Arbeit selbständig und ohne unerlaubte Hilfsmittel angefertigt habe. 
Die vorliegende Arbeit entstand im Zeitraum von Juni 2004 bis September 2007 unter Anleitung von Herrn Professor Dr. Franc Meyer am Anorganisch-Chemischen Institut der Georg-August-Universität Göttingen. 
„Erfahrung ist der Anfang aller Kunst und jedes Wissens“. Aristoteles (384-322 v. Chr; griech. Philosoph) 



\section{Inhaltsverzeichnis}

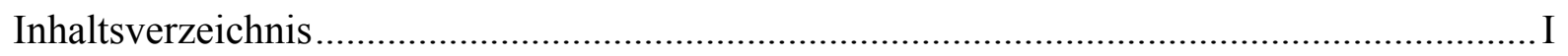

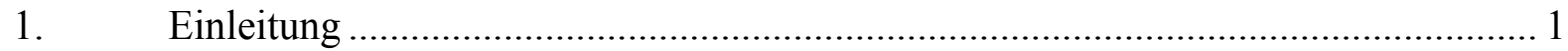

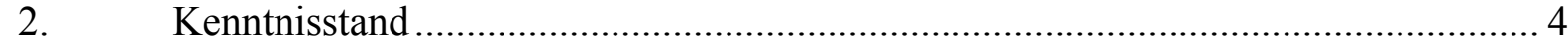

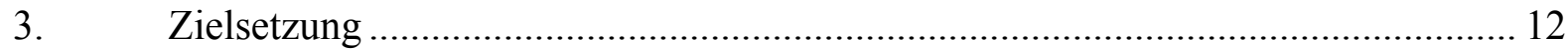

4. Synthese der Pyrrol/Pyrazol-basierten Bausteine ………………………………..... 14

5. Darstellung von neuartigen Makrozyklen ................................................................ 19

5.1 Darstellung von „Doppelporphyrinen“.......................................................... 19

5.2 Darstellung neuartiger Schiff-Base Makrozyklen .................................................. 33

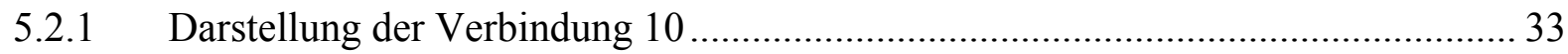

5.2.2 Darstellung neuartiger Pyrrol/Pyrazol-basierter Hybrid-Makrozyklen..................... 35

5.2.2.1 Strukturell charakterisierte Schiff-Base Makrozyklen .............................................. 38

5.2.2.2 UV/Vis-Untersuchungen der neuartigen Schiff-Base Makrozyklen.......................... 48

5.2.2.3 Untersuchung der spektroskopischen Sonde 2a ..................................................... 51

5.2.2.3.1 Titration von 2a mit Trifluoressigsäure.......................................................... 51

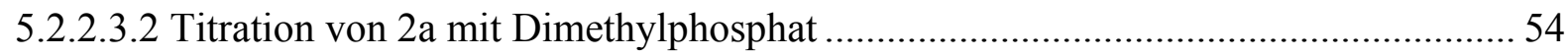

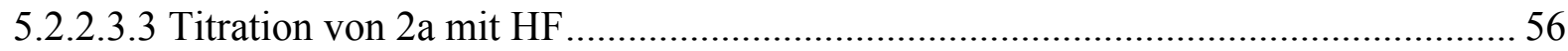

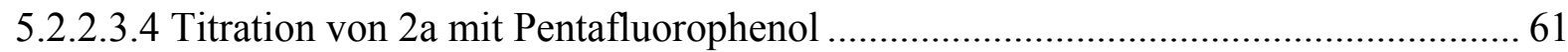

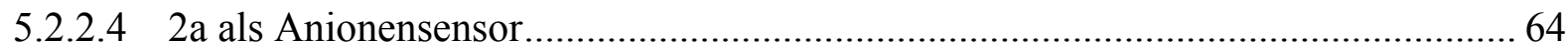

5.2.2.5 Untersuchung der Bindungseigenschaften von $2 \mathrm{~d}$ mittels UV/Vis-Spektroskopie . 68

5.2.2.6 Darstellung von Metallkomplexen der Schiff-Base Makrozyklen............................ 71

6. Neuartige Pyrrol/Pyrazole-basierte Diimin-Liganden ………………………….... 75

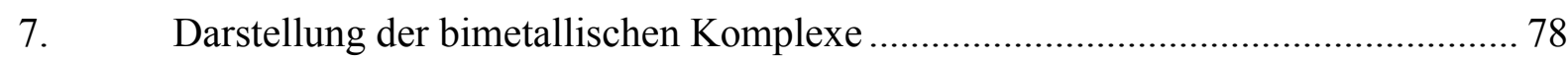

7.1 Bimetallische Kupfer(II)komplexe der Liganden $\mathrm{H}_{3} \mathrm{~L}^{1}, \mathrm{H}_{3} \mathrm{~L}^{3}, \mathrm{H}_{3} \mathrm{~L}^{4}$ und $\mathrm{H}_{3} \mathrm{~L}^{5}$ -

Strukturelle Charakterisierung, spektroskopische und magnetische Eigenschaften 78

7.2 Bimetallische Nickel(II)komplexe der Liganden $\mathrm{H}_{3} \mathrm{~L}^{1}, \mathrm{H}_{3} \mathrm{~L}^{3}$ und $\mathrm{H}_{3} \mathrm{~L}^{4}-$ Strukturelle

Charakterisierung und NMR-spektroskopische Untersuchungen ............................. 95

7.3 Bimetallischer Palladium(II)komplex mit den Liganden $\mathrm{H}_{3} \mathrm{~L}^{3}-$ Strukturelle

Charakterisierung und NMR-Untersuchungen...................................................... 102

7.4 Spektroskopische Untersuchungen der Metallkomplexe ……………………....... 107

7.4.1 IR-Spektren ausgewählter Metallkomplexe _......................................................... 107

7.4.2 Elektrochemische Untersuchungen ausgewählter bimetallischer Komplexe.......... 110 
7.4.3 UV/Vis-Spektren ausgewählter Metallkomplexe mit azyklischen Pyrrol/Pyrazolbasierten Diimin-Liganden.

7.5 Experimente zum Austausch des OH-Brückenliganden der Nickel(II)komplexe.. 115

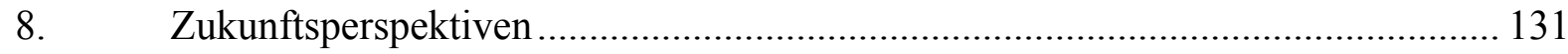

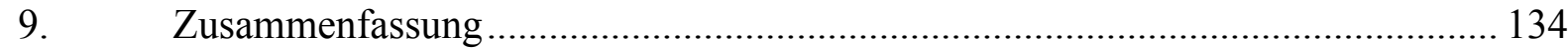

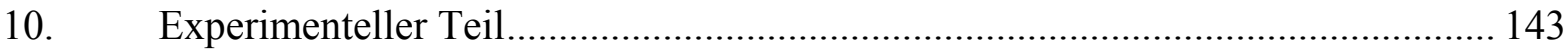

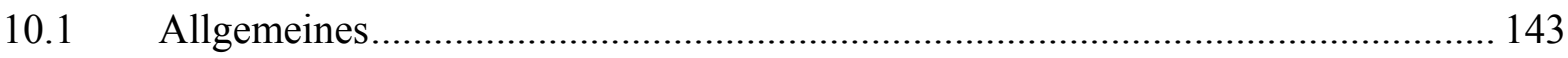

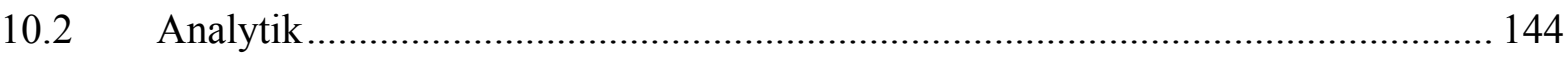

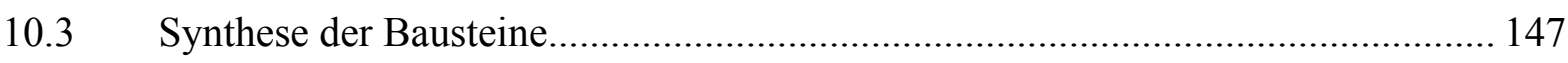

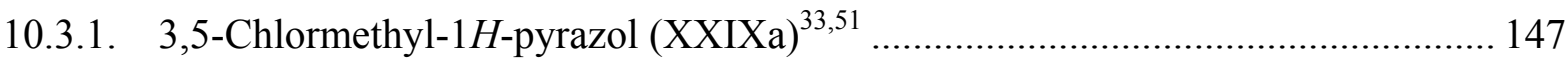

10.3.2. Synthese von 3,5-Bis-(chloromethyl-4-phenyl)-1H-pyrazol Hydrochlorid (4) ..... 148

10.3.3. Synthese von 3,5-Bis-(3,4-dimethyl-1H-pyrrol-2-yl-methyl)-1H-pyrazol (5a).... 150

10.3.4. Synthese von 3,5-Bis-(3,4-diethyl-1-formylpyrrol-2-ylmethyl)-1H-pyrazol (1a) . 151

10.3.5. Synthese von 3,5-Bis-(3,4-diethyl-1-hydroxy-2-yl-methyl)-1H-pyrazol (6) ........ 153

10.3.6. Synthese von 3,5-Bis-(3,4-dimethyl-1H-pyrrol-2-yl-methyl)-4-phenyl-1H-pyrazol $(5 b)$

10.3.7. Synthese von 3,5-Bis-(3,4-diethyl-1-formylpyrrol-2-yl-methyl)-4-phenyl-1H-

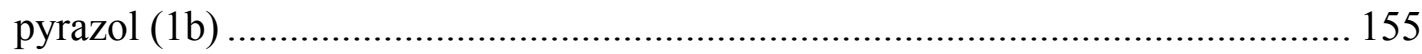

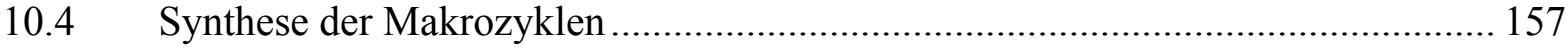

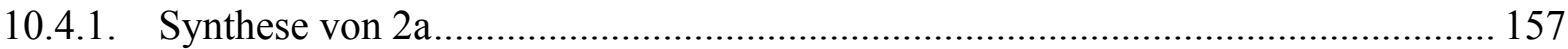

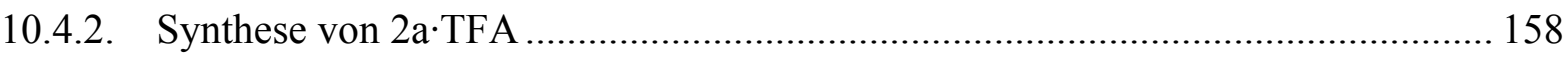

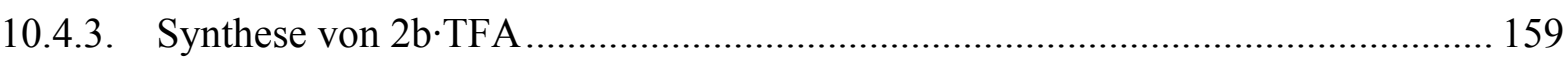

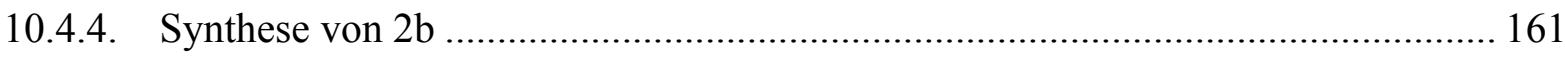

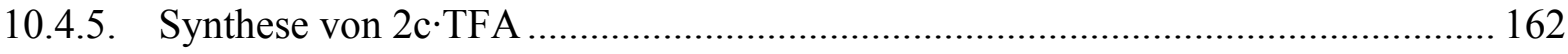

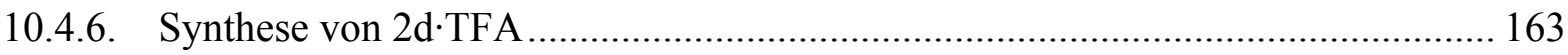

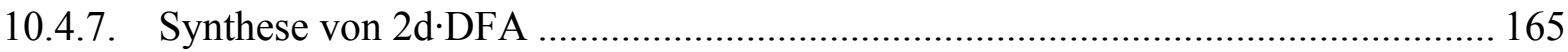

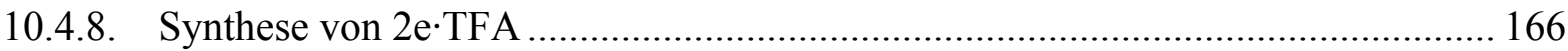

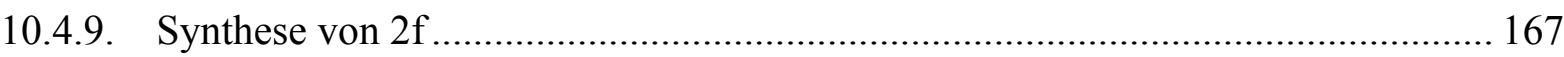

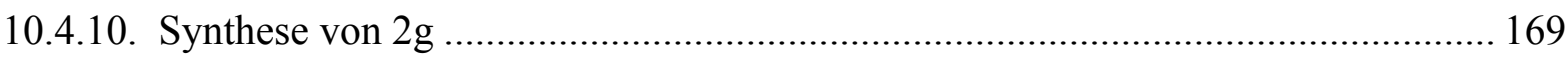

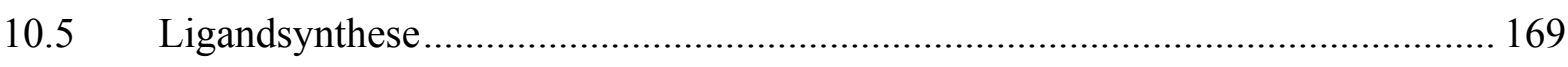

10.5.1. Synthese von 3,5-Bis-(2,3-dihydro-1H-perimidin)-1H-pyrazol (10).................... 169

10.5.2. Synthese von [5-(Morpholin-4-carbonyl)-2H-pyrazol-3-yl]-morpholin-4-yl-

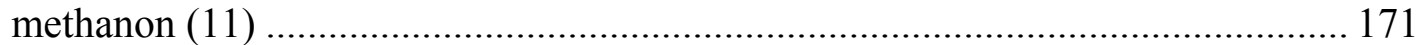

10.5.3. Synthese von 3,5-Bis-(1H-pyrrol-2-yl-methylen-amin)-1H-pyrazol (7) ............... 172 
10.5.4. Synthese von 3,5-Bis-(methyl-pyridin-2-yl-methylen-amin)-1H-pyrazol (8) ....... 173

10.5.5. Synthese von 3,5-Bis-(benzyliden-2-yl-methylenamin)-1H-pyrazol (9) ............... 174

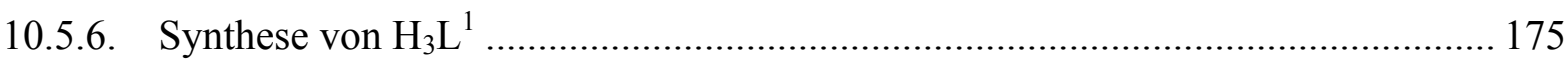

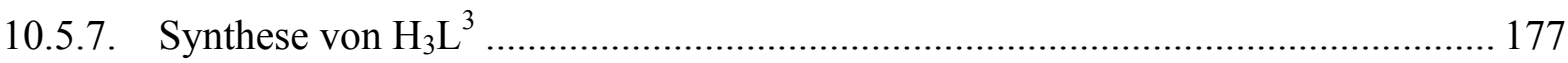

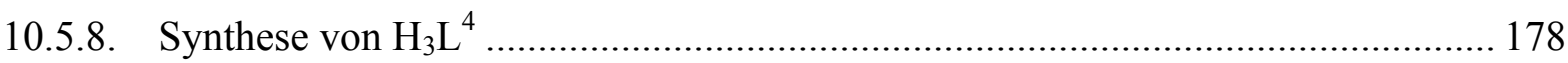

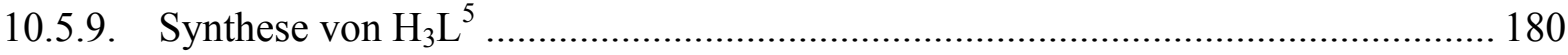

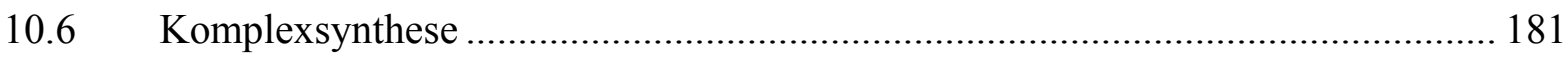

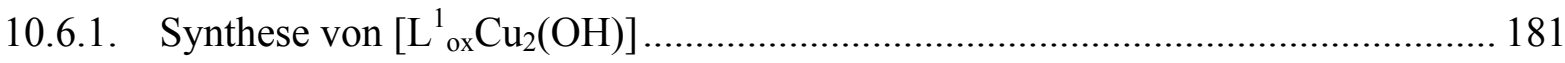

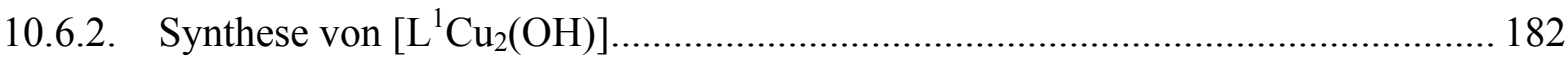

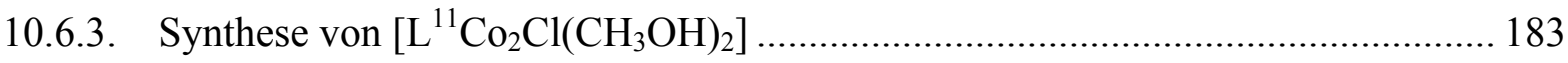

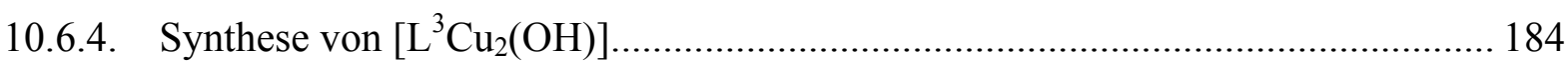

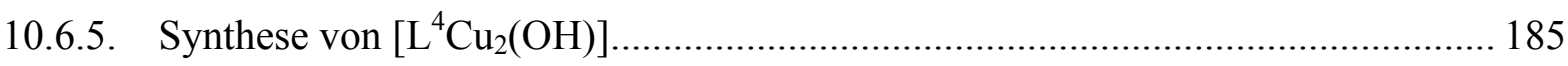

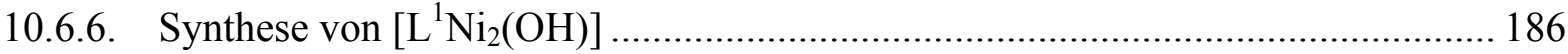

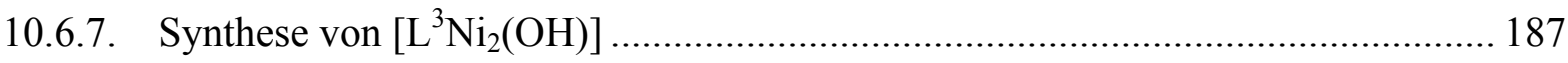

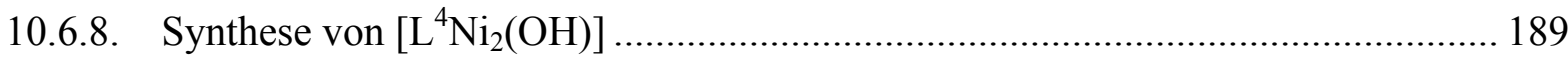

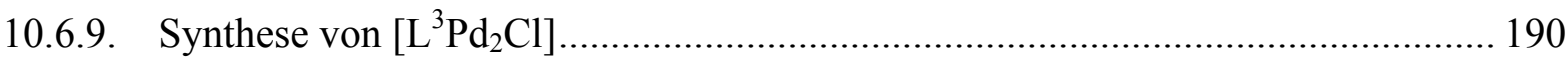

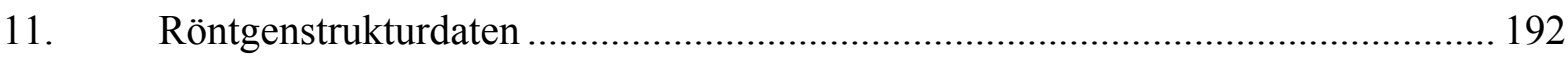

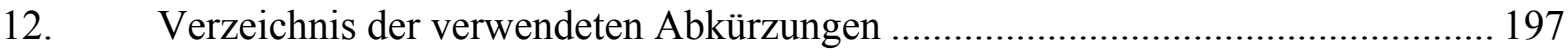

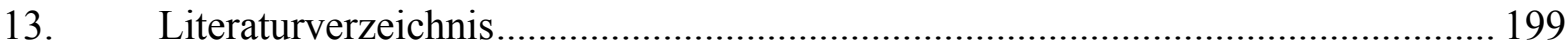





\section{Einleitung}

Porphyrine sind die meist untersuchten Tetrapyrrol-Makrozyklen. Durch ihre intensive Färbung werden sie auch als ,pigments of life“ bezeichnet. ${ }^{1}$ Porphyrine bzw. Metalloporphyrine sind an diversen biochemischen Synthesen beteiligt. Sie stellen die aktiven Zentren unterschiedlicher Enzyme dar und können somit unterschiedliche biochemische Reaktionen katalysieren. Durch den makrozyklischen Effekt besitzen sie eine recht hohe Stabilität und weisen durch ihren definierten Hohlraum im Inneren des Makrozyklus eine gewisse Starrheit auf. Der Makrozyklus-Hohlraum der Porphyrine bietet genügend Raum für unterschiedliche Metallionen, um eine Koordination zur ermöglichen. Ein bekanntes Beispiel sind hierbei die Cobalamine (z. B. Vitamin $\mathrm{B}_{12}$ ), welche Cobalt in einem teilkonjugierten Corrin-Ringgerüst beinhalten. Die Natur nutzt das Vitamin $\mathrm{B}_{12}$ Coenzym, um organische Spezies zu reduzieren und anschließend das H-Atom zu transferieren. Dabei sind zwei Einelektronen-Reduktionsschritte möglich.

Besonders bekannt sind die Eisenporphyrin-haltigen redoxaktiven Häm-Proteine, welche eine vielfältige Funktion in diversen Lebewesen beanspruchen. Hierzu gehören die Cytochrome ( $a$, $b, c, c 554$, bo etc.), welche als Elektronentransfer dienen. Sie können je nach Aufbau unterschiedliche Hämgruppierungen enthalten. ${ }^{2}$

Lebenswichtig für den Menschen und für viele andere Lebewesen sind die Häm- und Myoglobine. Ihre Funktion ist der Transport bzw. Speicherung von Sauerstoff $\left(\mathrm{O}_{2}\right)$ im Blut oder in den Muskeln. In Abbildung 1 ist das tetramere Protein des Oxy-Hämoglobins dargestellt. Durch die eisenhaltigen Porphyrinkomplexe können bei vollständiger Beladung vier Sauerstoffmoleküle $\left(\mathrm{O}_{2}\right)$ effizient gespeichert und anschließend transportiert werden (Abbildung 1 rechts).

Hierbei wird der Fe(II)-Komplex, welcher im Proteininneren von einem Imidazol-Ring des proximalen Histidins besetzt wird durch die Koordination des $\mathrm{O}_{2}$ Moleküls zu Fe(III) oxidiert (Postulierung nach Weiss 1964). ${ }^{3}$ Durch Reaktion und Weitergabe des Sauerstoffs kann das sechsfach koordinierte Fe(III)-Ion reversibel zum fünffach koordiniertem Fe(II)-Porphyrin reduziert werden.

Porphyrinhaltige Proteine dienen ebenfalls als Pigmente, die an der Lichtabsorption beteiligt sind. $\mathrm{Zu}$ den bekanntesten Pigmenten gehören neben den Chlorophyllen die Carotenoide, Phyceoethythrin und das Phycocyanin. Diese Pigmente können Licht bestimmter Wellenlänge absorbieren, was zu einer Ladungstrennung im Reaktionszentrum führt. 


\section{Einleitung}

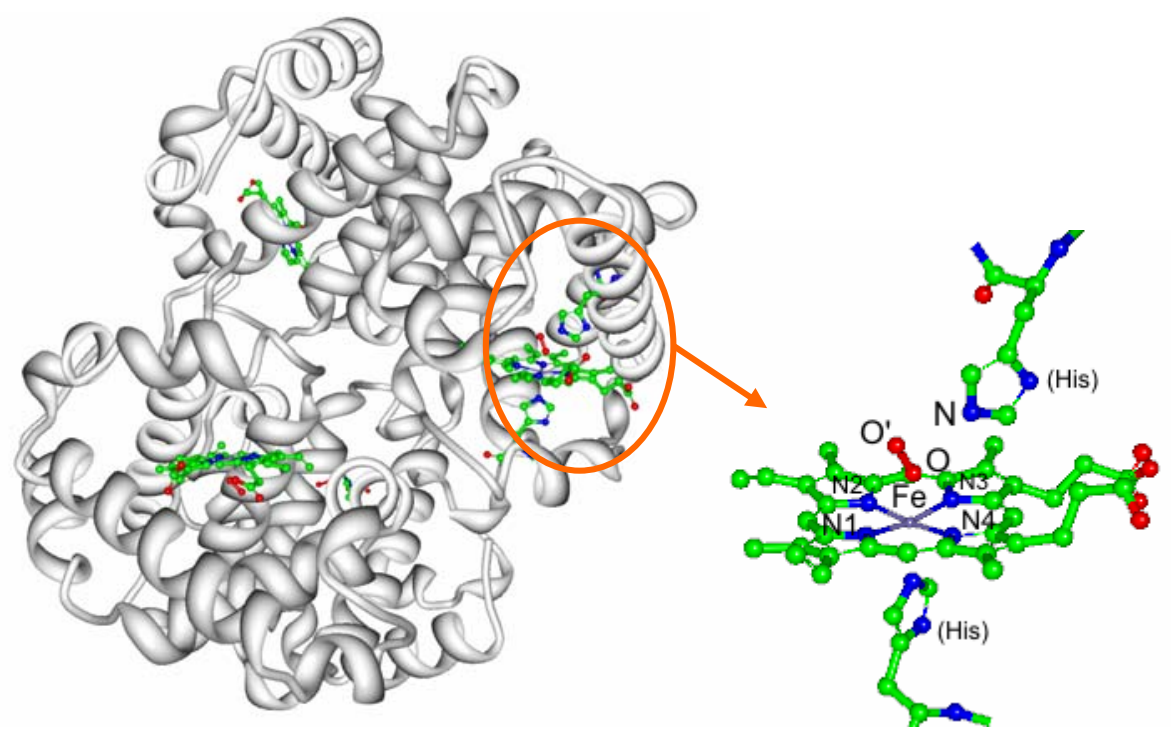

Abbildung 1: Proteinstruktur des Oxy-Hämoglobins isoliert aus dem Blut einer Graugans. Die Kristallstruktur (PID: 11717498) wurde aus der „Protein Data Bank“ entnommen und mit dem Programm „Protein Workshop 1.5 “ bearbeitet. ${ }^{4}$ Auf der linken Seite ist eine Vergrößerung des Metalloporphyrins in diesem Protein abgebildet.

Außerordentlich interessant sind die Chlorophylle, welche die Lichtreaktion der Photosynthese katalysieren. Chlorophylle sind üblicherweise $\mathrm{Mg}(\mathrm{II})-K o m p l e x e$, die ein teilhydriertes Porphyrin und einen ankondensierten Fünfring enthalten. In Abbildung 2 ist die Proteinstruktur (links) und das beinhaltete Mg(II)-Porphyrinkomplex des WSCP-Chl's (Water-Soluble Chlorophyll-binding Protein) (rechts) dargestellt.
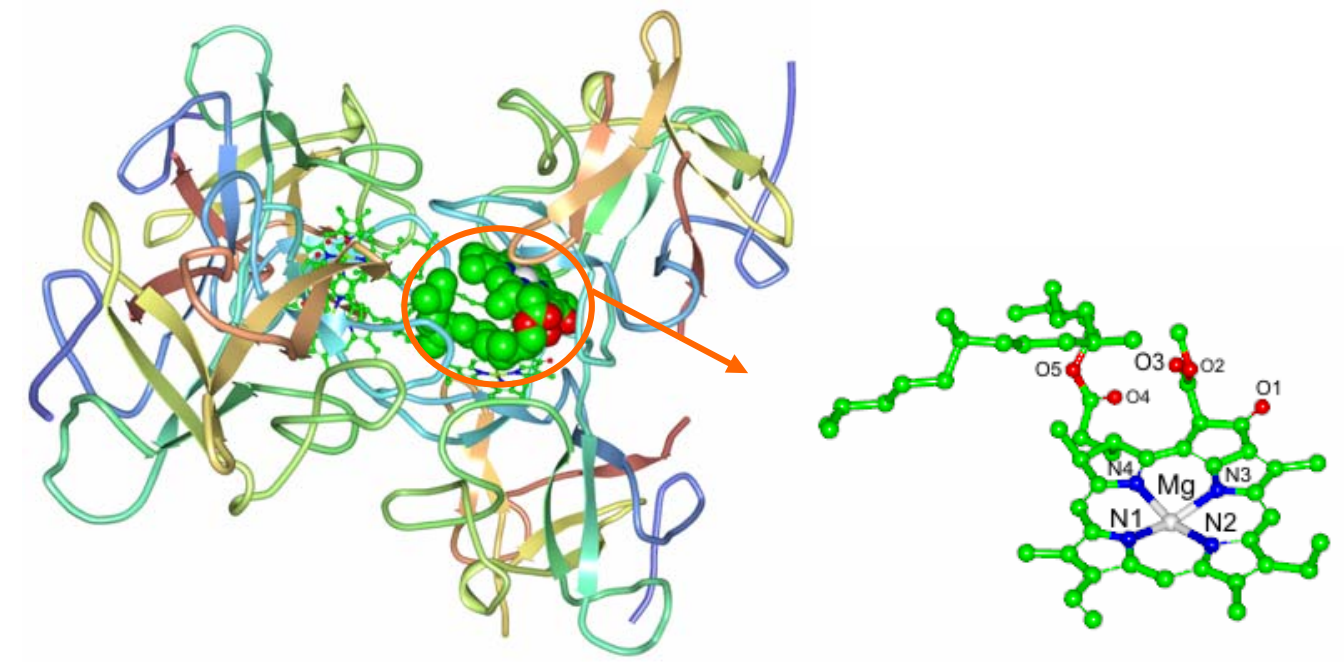

Abbildung 2: Tetramer Struktur des WSCP-Chl's (water-soluble chlorophyll-binding protein) Komplexes isoliert aus Kresse (Lepidium virginicum) (links). Das aktive Zentrum des Proteins besteht aus vier MgPorphyrin Komplexeinheiten. Zur besseren Übersicht wurde auf der rechten Seite dieser Abbildung das Metalloporphyrin vergrößert dargestellt. Die Kristallstruktur (PID: 17170107) wurde aus der „Protein Data Bank“ entnommen und mit dem Programm „Protein Workshop 1.5“ bearbeitet. $^{5}$ 
Das Substitutionsmuster der Porphyrine in den Chlorophyllen bestimmt hierbei die Lage der Absorptionsbanden. Als leichtes Atom ist $\mathrm{Mg}$ (II) redoxstabil und besitzt eine geringe SpinBahn-Kopplung. Dadurch wird ein mögliches ISC (Interssystem-Crossing) vom sehr kurzlebigen Singulett- zum langlebigen Triplettzustand verhindert. Diese Besonderheiten der Chlorophylle nutzt die Natur bei dem sehr komplexen Prozess der Photosynthese (PSI und PSII) aus, um aus Wasser und Kohlendioxid letztlich Disauerstoff und reduzierte Kohlenwasserstoffverbindungen zu erzeugen. ${ }^{6}$ Für die Bestimmung der dreidimensionalen Struktur eines photosynthetischen Reaktionszentrums bekamen drei Naturwissenschafter aus Deutschland (J. Deisenhofer, R. Huber, H. Michel) im Jahre 1988 den Nobelpreis für Chemie. Die Abhängigkeit von fossilen Brennstoffen und die stets ansteigende Produktion von Kohlendioxid in der Erdatmosphäre führen $\mathrm{zu}$ einem verstärkten wissenschaftlichen Bestreben nach einem Verständnis der Photosynthese. Die Erkenntnisse der sogenannten „Antennensysteme“ der Chlorophyllmoleküle haben in den letzten Jahren das Interesse an der Herstellung biomimetischer Solarzellen immens erhöht. ${ }^{7}$ Balaban et al. im Forschungszentrum Karlsruhe verwendeten Zinkporphyrine zur Darstellung und Entwicklung von Hybrid-Solarzellen mit selbstorganisierenden biomimetischen Antennensystemen. ${ }^{8}$

Durch die besonderen elektronischen und optischen Eigenschaften der Porphyrine bzw. Metalloporphyrine sind sie in der Anwendung ebenfalls seit Jahren stark verbreitet. Eine Reihe von Porphyrinderivaten werden seit den 70er Jahren in der photodynamischen Therapie (PDT) zur Behandlung von Hautkrebsvorstufen (aktinischen Keratosen) und bestimmten Formen von weissem Hautkrebs erfolgreich eingesetzt. $\mathrm{Zu}$ diesen Arzneimitteln gehören die Pharmaka PhotoDyn ${ }^{\circledR}$ 501, Photofrin ${ }^{\circledR}$ oder Termorfin Foscan ${ }^{\circledR}$. Die Porphyrine dienen dabei als Photosensibilisatoren. Beim Anleuchten der kranken Zellen mit einer definierten Wellenlänge kann mit Hilfe der Photosensibilisatoren, durch eine photodynamische Reaktion, Triplett Sauerstoff $\left({ }^{3} \mathrm{O}_{2}\right) \mathrm{zu}$ einem ,toxischen“ Singulett Sauerstoff $\left({ }^{1} \mathrm{O}_{2}\right)$ angeregt werden. Das für die Zellen toxische ${ }^{1} \mathrm{O}_{2}$ zerstört in nachfolgender Reaktion die kranken Zellen. ${ }^{9}$

Die besonderen vielfältigen optischen und elektronischen Eigenschaften der Porphyrine faszinieren weiterhin eine große Anzahl an Chemikern, Biologen und Materialwissenschaftlern. In den letzten Jahrzehnten wuchs das Interesse an modifizierten Porphyrogenen Verbindungen und an der Entwicklung einer neuen Klasse von Liganden, den „Expandierten Porphyrinen“. ${ }^{10}$ Eines der Ziele dieser Forschung ist die Expandierung (Vergrößerung) des Hohlraums im Makrozyklus durch die Erhöhung der Anzahl der Heterozyklen im System. Hierbei kann die Anzahl der Pyrroleinheiten erweitert bzw. weitere Heterozyklen wie das Furan oder Thiophene können intrigiert werden. ${ }^{11}$ Von besonderem 
Interesse sind neue Erkenntnisse zu den fundamentalen Eigenschaften der Aromazität bei den expandierten Systemen. Durch den größeren Hohlraum des Zyklus bietet sich ausreichend Platz für größere Metalle, wie z. B. Lanthanoide, Actinide oder weitere Übergangsmetalle. Je nach Größe können in eingen Fällen auch bimetallische Komplexe erfolgreich dargestellt werden. $^{12,13,14}$

In letzter Zeit stieg die Anwendung der expandierten Porphyrine im Bereich der AnionBindung und des Anionen-Transports. ${ }^{15,16,17}$ Durch Wasserstoffbrückenbindungen können dabei unterschiedliche Ionen selektiv nachgewiesen bzw. separiert werden.

In dieser Arbeit werden die Synthese von neuartigen Porphyrinogen-ähnlichen Makrozyklen, die neben Pyrrol auch Pyrazol als Heterozyklus enthalten und deren besondere spektroskopische Eigenschaften vorgestellt. Zudem werden verwandte offenkettige Pyrazol/Pyrrol-Hybridliganden für die Synthese binuklearer Komplexe genutzt.

\section{Kenntnisstand}

\section{I) Expandierte Porphyrine und Heteroporphyrine}

Robert Woodward präsentierte 1966 auf der "Aromaticity Conference“ in Sheffield, Großbritannien, erstmals einen Pentapyrrol-Makrozyklus. Dieser Makrozyklus wurde zufällig bei der Synthese von Vitamin $B_{12}$ entdeckt. Das war das erste expandierte Porphyrin, welches einige Jahre später Sapphyrin (I) ${ }^{18}$ genannt wurde (Abbildung 3). Seit der Erfindung des Sapphyrins hat sich das Gebiet der expandierten Porphyrine ab den 90er Jahren zunehmend entwickelt.
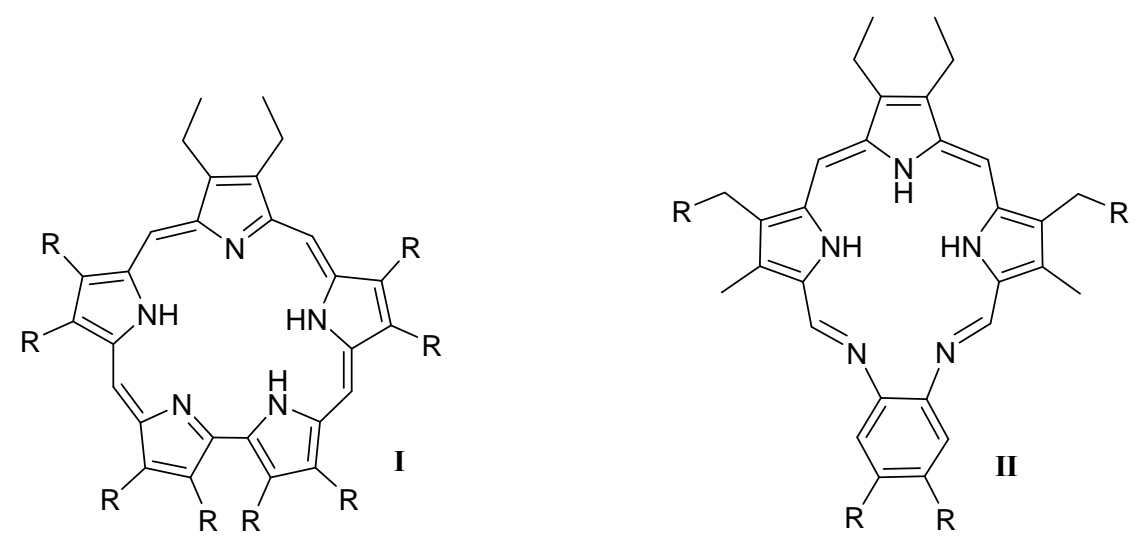

Abbildung 3: Struktur des Sapphyrins (I) ${ }^{18}$ und des Texaphyrins $(\text { II })^{19}$. 
Einen neuen Schwerpunkt in der Chemie der expandierten Porphyrin setzte einige Jahre später das von Sessler et al. synthetisierte Texaphyrin (II, Abbildung 3). ${ }^{19}$ Diese Klasse von expandierten Porphyrinen wurde über eine Schiff-Base Kondensation zwischen einem Diformyltripyrran und einem aromatischen 1,2-Diamin dargestellt. Das Texaphyrin war zu diesem Zeitpunkt das erste expandierte Porphyrin, welches eine Metallierungsreaktionen einging. Besonders gut untersucht wurden die Lanthanoid-Komplexe; diese werden heute als Adjuvanzien bei der Strahlungstherapie von Tumoren eingesetzt. ${ }^{20}$ Besonders bekannt wurde ein Texaphyrin-Lutetiumkomplex mit den Handelsnamen Lutrin bzw. Antrin, welcher als pontenzieller Photosensibilisator zur Behandlung von Krebs und kardiovaskulären Erkrankungen klinisch geprüft wird. ${ }^{21}$

Obwohl das Texaphyrin eines der meist untersuchten expandierten Porphyrine ist, gelang es erst 2001, 13 Jahre nach seiner Endeckung, das metallfreie Texaphyrin in Form eines monoprotoniertem $\mathrm{HPF}_{6}$-Adduktes zu isolieren. ${ }^{19 \mathrm{~b}}$

Das Ryburin (III) $)^{22}$ und das von Gossauer et al. synthetisierte Hexaphyrin (IV) ${ }^{23}$ gehören zu den ersten expandierten Porphyrinen mit mehr als fünf Pyrroleinheiten (Abbildung 4). Seitdem wurde über eine sehr große Zahl an Oligopyrrol-Makrozyklen berichtet.
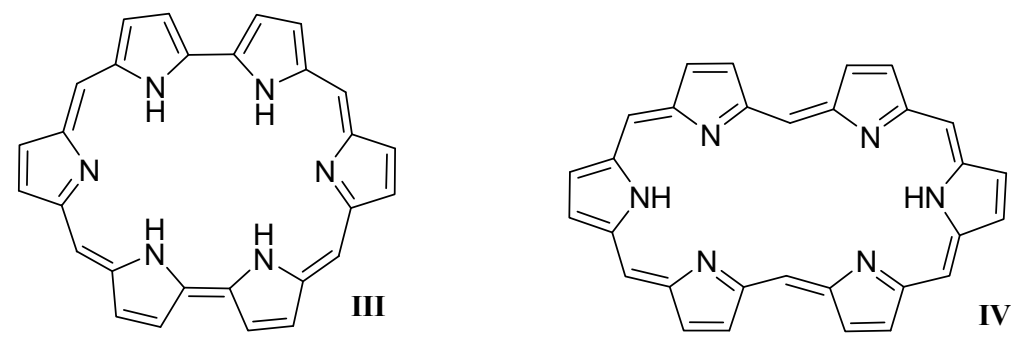

Abbildung 4: Struktur des Rybins (III) $)^{22}$ und des Hexaphyrins (IV) ${ }^{23}$.

Expandierte Porphyrine unterscheiden sich von den natürlich vorkommenden makrozyklischen Tetrapyrrolen durch einen größeren zentralen Ring mit mindestens 17 Atome. Die elektronische Struktur der expandierten Porphyrine im Vergleich zu den klassischen Porphyrinen ist dabei von großem Interesse. Hierbei wurde untersucht, ob die Hückel-Theorie mit der Definition für die Aromatizität $(4 n+2 \pi$-Elektronen) auch für expandierte Porphyrine oder Heteroporphyrine gilt. Sie eignen sich vor allem als Liganden zur Synthese von mono- und je nach Größe des Hohlraums auch von dinuklearen Metallkomplexen. 
Osuka et al. synthetisierten einige der ersten „N-confused“-Hexaphyrine (VI, VII) aus der Reaktion eines N-confused Tripyrrins (V) mit einem aromatischen Aldehyd (Abbildung 5). Bei der Reaktion konnten mehrere Makrozyklen mit unterschiedlichen Elektronenzuständen (26 bzw. $28 \pi$ Elektronen) isoliert werden. In späteren Arbeiten konnte gezeigt werden, dass diese N-confused-Hexaphyrine sich als Liganden zur Synthese von bimetallischen Komplexen eignen.<smiles>BrC(c1c[nH]c(C(Br)c2ccc[nH]2)c1)c1ccc[nH]1</smiles>

$\mathrm{ArCHO}$

$p$-TSA

p-Chloranil

$\mathbf{V}$

$\left(\mathrm{Ar}=\mathrm{C}_{6} \mathrm{~F}_{5}\right)$

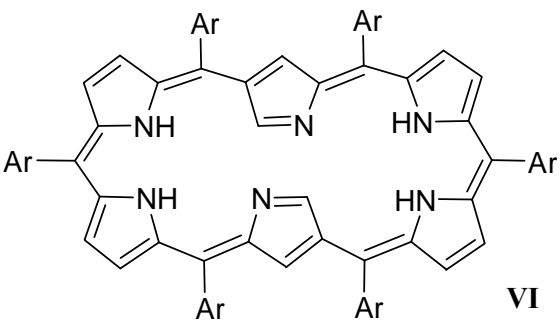

$[28 \pi]$

$+$

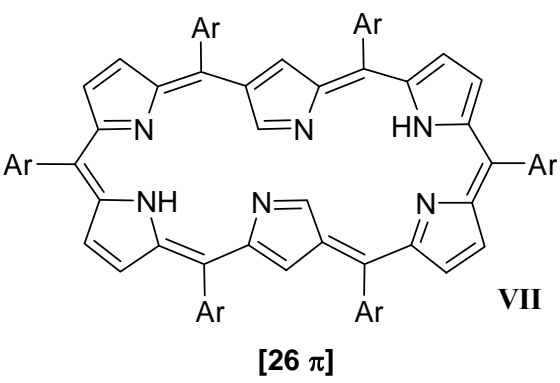

Abbildung 5: Synthese des „N-confused“--Hexaphyrins nach Furuta, Osuka et al. ${ }^{24}$

Eine Vielfalt an heterozyklischen Systemen wurde in porphyrinogenen Verbindungen untersucht, wie die Heterosmaragdyrine, Heterosapphyrine, oder N-konvertierte Heterosapphyrine. Diese Makrozyklen enthalten neben Pyrrol auch Furan-, Thiophen- oder andere heterozyklische Untereinheiten, die miteinander verknüpft sind (Abbildung 6). 


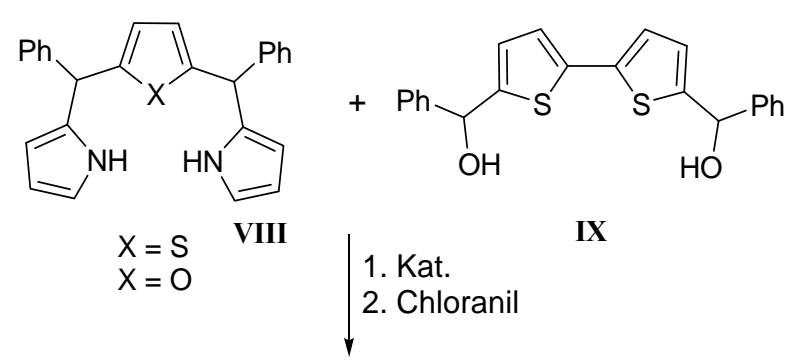

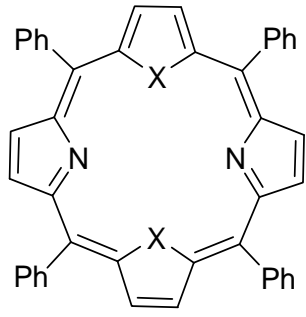

$\mathbf{X}$

Heteroporphyrin

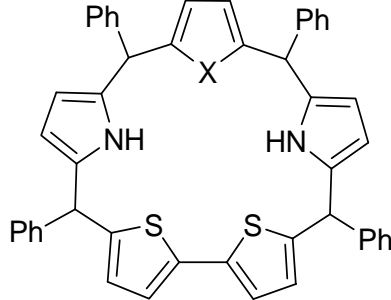

XI

Heterosapphyrin

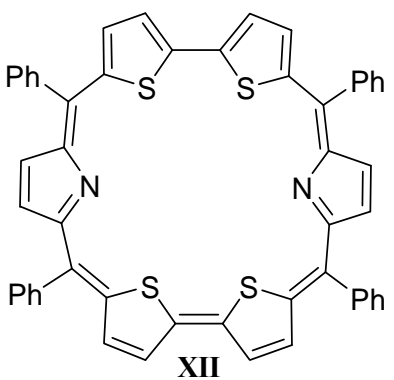

Heterorubyrin

Abbildung 6: Synthese von Heteroporphyrinen und weitere Analoga nach Chandrashekar et al. ${ }^{25}$

Chandrashekar et al. stellten die ersten Heterosapphyrine durch Kondensation eines Heterotripyrrans (VIII) mit dem Bithiophendiol (IX) und anschließende Oxidation dar. ${ }^{25}$ Neben dem Heterosapphyrin (XI) entstanden weitere Makrozyklen - das Heteroporphyrin (X) und Heterorubyrin (XII). Die Bildung dieser zwei Makrozyklen lässt sich durch partielle Hydrolyse und Rekombination der Ausgangsverbindungen erklären.

\section{II) Schiff-Base Makrozyklen}

Sessler et al. präsentierten die ersten Polypyrrol-Schiff-Base Lanthanoid-Komplexe, die erfolgreich in der photodynamischen Therapie eingesetzt wurden. ${ }^{26}$ Einige Jahre vor diesen außerordentlichen Entdeckungen stellten Mertes et al. die ersten „Akkordion“-Porphyrine dar. ${ }^{27}$ Sie entstanden in einer Kondensationsreaktion eines 5,5'-Diformyldipyrromethans mit Trimethylendiamine. Bowman-James et al. untersuchten die Akkordion Porphyrine als Hybridmodelle für Häm- und binukleare Monooxygenase. ${ }^{28}$ Love et al. synthetisierten einige Zeit später neuartige nicht-aromatische Schiff-Base Calixpyrrol-Makrozyklen (XIV) (Abbildung 7) und stellten damit bimetallische Palladiumkomplexen dar. ${ }^{29,30}$ 


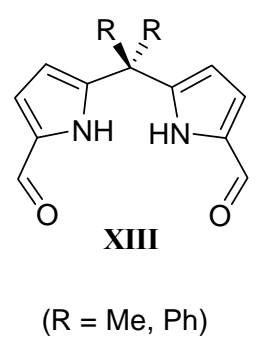

1. Diamin, $\left[\mathrm{H}^{+}\right]$

2. $\mathrm{NEt}_{3}$
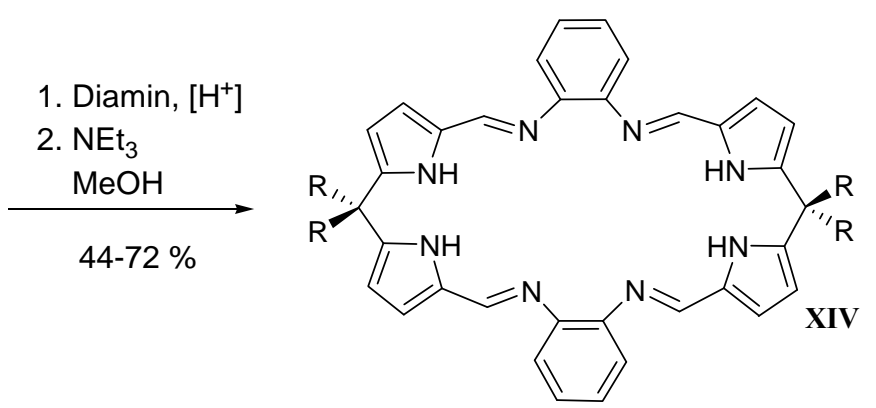

Abbildung 7: Synthese eines Schiff-Base Calixpyrrol-Makrozyklus nach Love et al. ${ }^{29,30}$

Das Interesse an Schiff-Base und Hetero-Schiff-Base Makrozyklen stieg immens in den letzten Jahren. So berichteten Won und Lee über die Synthese von pseudoaromatisch expandierten Porphyrin-Analoga. ${ }^{31}$ In diesen Arbeiten synthetisierten sie den ersten thiophenhaltigen Schiff-Base Makrozyklus aus dem Thiophen-Dialdehyd (XV) säurekatalytisch mit einem 1,2-Diamin (Abbildung 8). ${ }^{32}$ Sie nutzten als Erste ein Tetraaminobenzol, anstelle eines 1,2-Diamins, zur Synthese von heterobiszyklischen SchiffBase Makrozyklen (XVII).

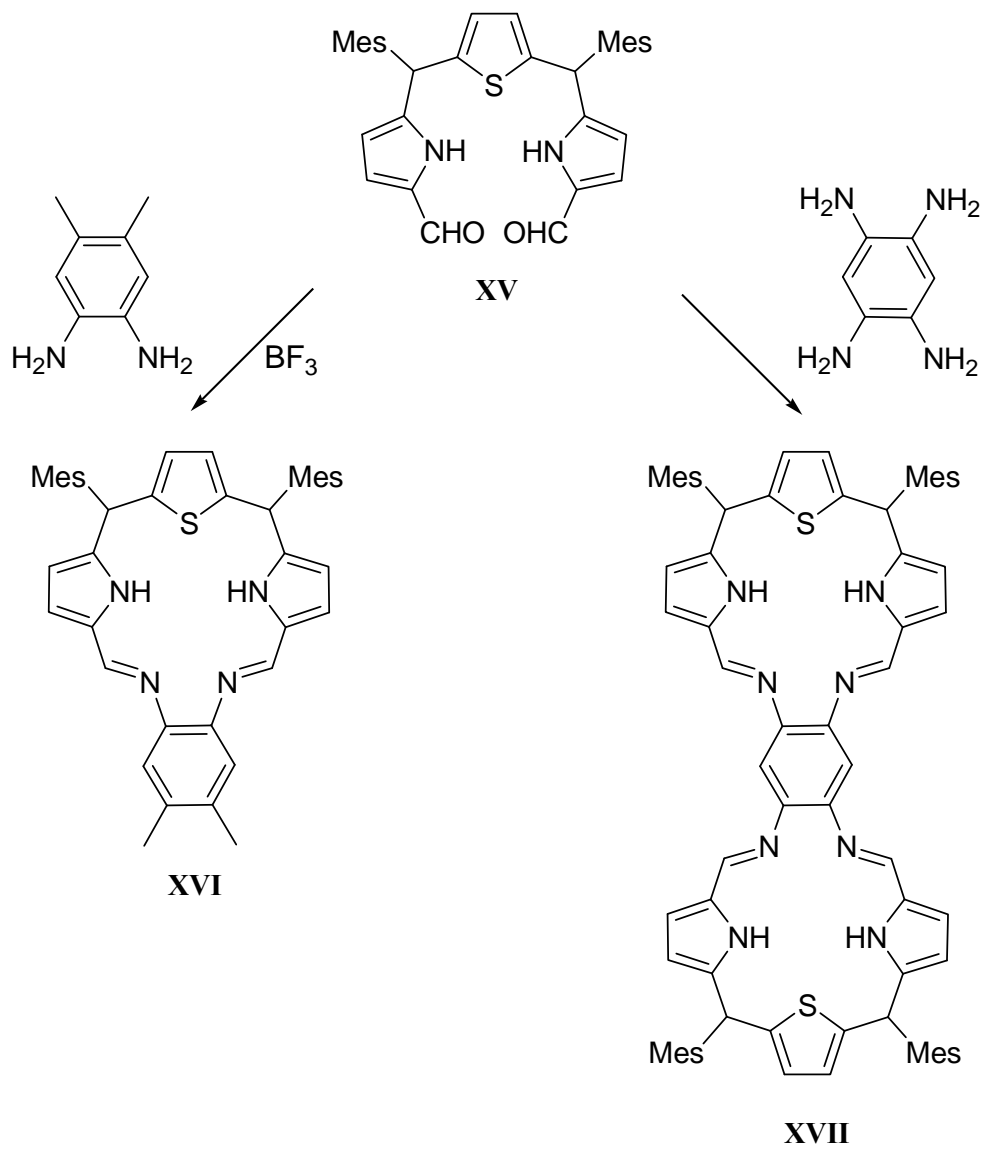

Abbildung 8: Synthese von thiophenhaltigen Schiff-Base Makrozyklen nach Won und Lee. ${ }^{32}$ 


\section{III) Makrozyklen auf der Basis 3,5-Disubstituierte Pyrazole}

3,5-Disubstituierte Pyrazolderivate werden erfolgreich als Brückenliganden bei der Darstellung von bimetallischen Komplexen eingesetzt. Bosnich et al. berichten in zwei Veröffentlichungen über die Synthese von bimetallischen Pyrazol-basierten Rhodium- und Iridium-Komplexen. ${ }^{33,34}$ Diese homobinuklearen Komplexe wurden bei oxidative Additionen und reduktiven Eliminierungsreaktion mit Acetylchlorid und Methyliodid untersucht. ${ }^{34}$

In unserer Arbeitsgruppe werden neuartige 3,5-disubstituierte Pyrazolliganden zur Darstellung von bimetallischen Komplexen entwickelt, die einer realistischen Modellierung von dinuklearen aktiven Zentren von Enzymen entsprechen. Hierbei werden präorganisierende Kompartimentliganden verwendet, in denen die beiden Koordinationseinheiten durch eine Pyrazolatbrücke verbunden sind. ${ }^{35}$ Durch das Anbringen von unterschiedlichen Seitenarmen an der 3,5-Position des Pyrazols kann der Metall-MetallAbstand der Komplexe variiert werden (Abbildung 9). ${ }^{35}$ Unterschiedliche zweikernige Pyrazolat-Metallkomplexe wurden hierzu synthetisiert und als Modellsysteme für biomimetische Oxidations- und Hydrolysereaktionen getestet. ${ }^{36,37}$

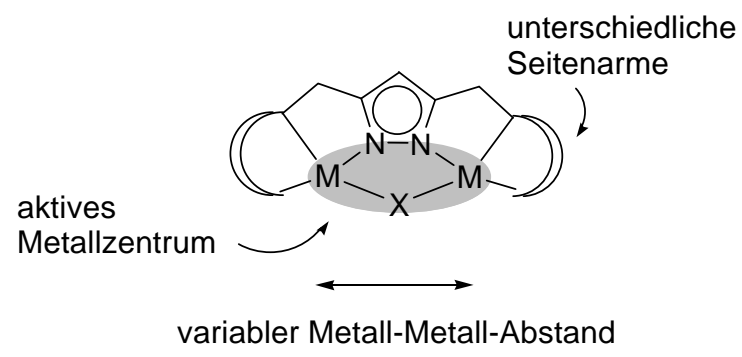

Abbildung 9: Schema eines bimetallischen Komplexes mit Pyrazol als Brückenligand.

Obwohl in der Literatur über zahlreiche Heteropolypyrrol-Makrozyklen berichtet wird, existiert nur eine geringe Zahl an Pyrazol-basierten Makrozyklen. E. Lind berichtet in seiner Dissertation im Jahre 1987 über eine Reihe von Pyrrol/Pyrrazol-haltigen Makrozyklen, die er durch Zyklokondensation von neuartigen Pyrrol/Pyrazol Bausteinen (XVIII und XIX) erhalten hatte. ${ }^{38}$ Allerdings wurde in dieser Arbeit eine Mischung aus vier unterschiedlichen Makrozyklen (XXa-d) erhalten, die sich nicht voneinander trennen ließen (Abbildung 10). Die Pyrazol-Einheiten in diesen Zyklen waren mit Benzylgruppen geschützt und konnten aus den entstandenen Makrozyklen nicht abgespalten werden. Somit konnte kein aromatisches System geschaffen werden. 
Navarro et al. veröffentlichten 1993 die ersten Polyamin-Makrozyklen von 3,5disubstituierten $1 H$-Pyrazolen. ${ }^{39}$ Einige dieser Makrozyklen werden in Abbildung 11 gezeigt (XXI - XXIII). In späteren Arbeiten konnten Navarro et al. zeigen, dass diese $1 H$-Pyrazol haltigen Makrozyklen sich als Rezeptoren zur Komplexierung von L-Glutamat bei physiologischen $\mathrm{pH}-$ Werten in Wasser eignen. ${ }^{40,41}$ Diese Makrozyklen sind zudem Liganden zur Synthese von doppelt verbrückten bimetallischen Komplexen. Navarro et al. synthetisierten Zn(II)-Komplexe, konnte aber die Existenz dieser Verbindungen nur massenspektrometrisch nachweisen. ${ }^{39,42}$

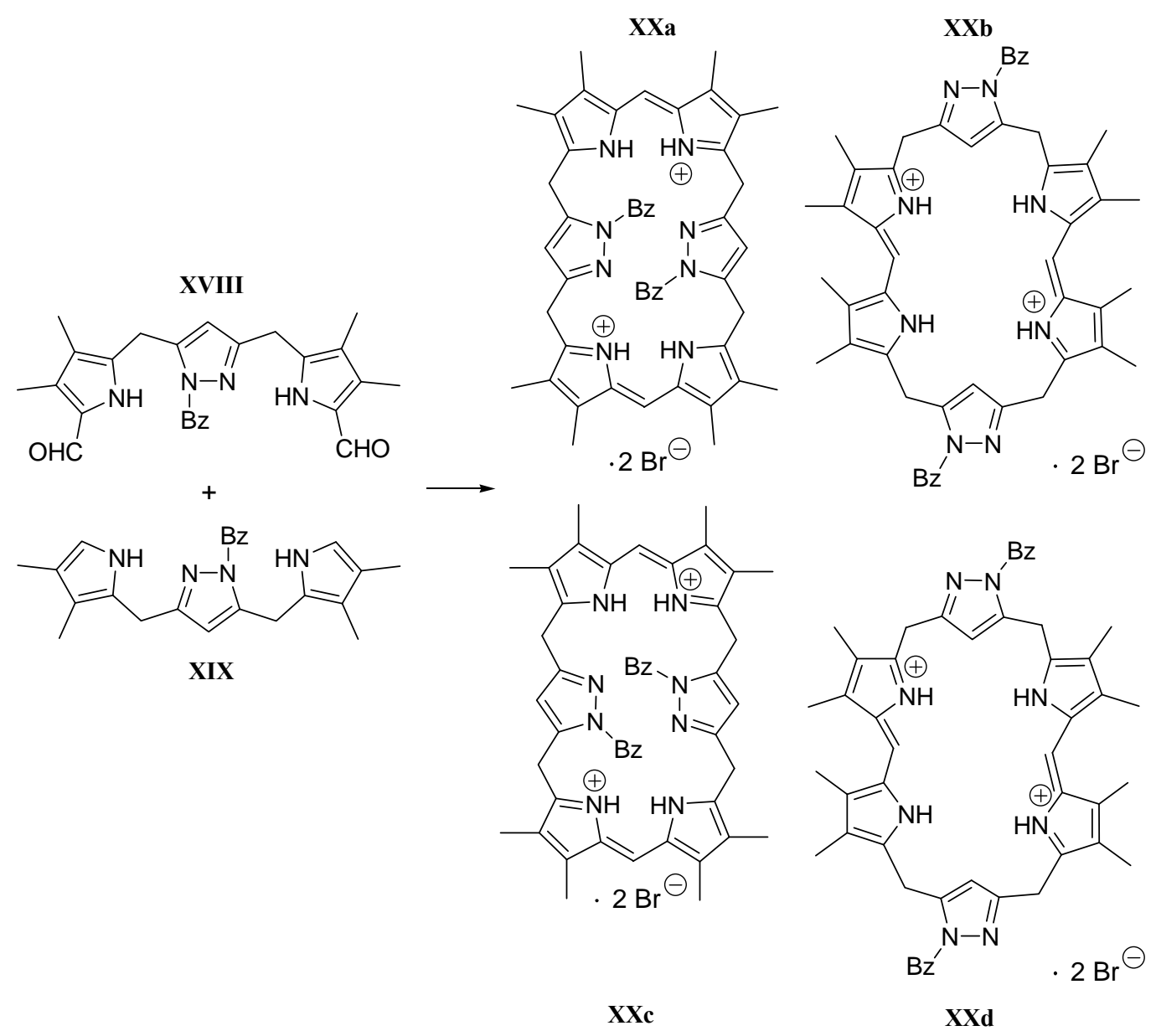

Abbildung 10: Syntheseweg zur Darstellung von Pyrrol/Pyrazol-basierten Makrozyklen nach Lind. ${ }^{38}$

Brooker et al. stellten jüngst einen ganz neuen Typ von Pyrazol-haltigen Schiff-Base Makrozyklen her. ${ }^{43}$ Die Synthese des metallfreien Makrozyklus war ihnen nicht möglich; stattdessen konnten sie in einer Templatsynthese aus 1,3-Diaminopropan, dem 3,5Pyrazoldicarbaldehyd ${ }^{41}$ und zwei Äquivalenten Kupferacetat den bimetallischen Komplex XXIV erfolgreich isolieren (Abbildung 12). 


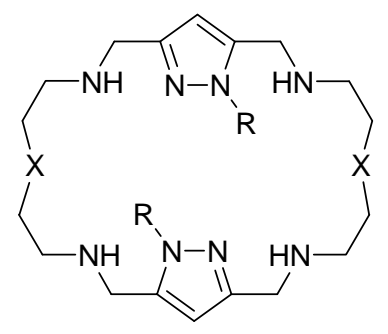

XXI

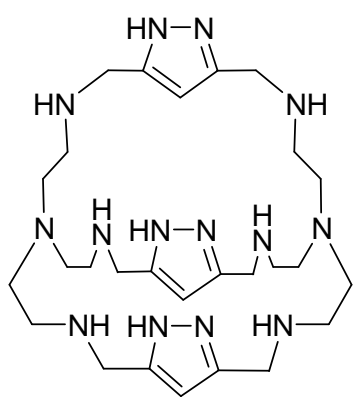

XXII

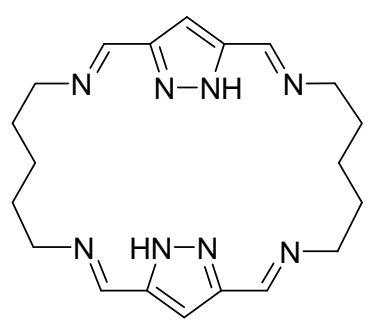

XXIII

$$
\mathrm{X}=\mathrm{O}, \mathrm{NH}
$$$$
\mathrm{R}=\mathrm{H}, \mathrm{Bn}
$$

Abbildung 11: Makrozyklen aus 3,5-disubstituiertem Pyrazol und Polyaminen nach Navarro et al. ${ }^{39,40,41}$

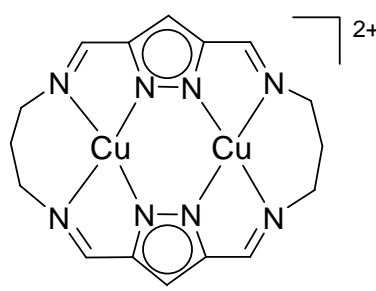

XXIV

Abbildung 12: Nach Brooker et al. synthetisierter bimetallischer Komplex aus einem Pyrazol-basiertem SchiffBase Makrozyklus.

Es ist bis heute der einzige röntgenographisch charakterisierte bimetallische Komplex aus dieser Klasse von erweiterten Schiff-Base Makrozyklen mit Pyrazol-Baustein.

Wie man aus den oben berichteten Ergebnissen entnehmen kann, ist die Anzahl der 3,5disubstituierten Pyrazol-Hybrid-Makrozyklen, auch aufgrund diverser synthetischer Schwierigkeiten, begrenzt. Dies macht die Erforschung und Entwicklung von neuartigen Pyrazol-basierten Makrozyklen besonders reizvoll und interessant. 


\section{Zielsetzung}

In der Literatur ist bis heute nur ein Artikel über die Darstellung von 3,5-Bis-(1H-pyrrol-2-ylmethyl)-1H-pyrazol-Derivaten bekannt. Lind berichtete in seiner Dissertation über die Synthese von solcher Bausteine zur Darstellung von Makrozyklen (Abbildung 10), die jedoch nicht in reiner Form isoliert werden konnten. ${ }^{38}$

Ziel dieser Arbeit ist die Darstellung von neuartigen 3,5-Bis-(1H-pyrrol-2-yl-methyl)- $1 H$ pyrazol-Bausteinen (1), wie sie in Abbildung 13 gezeigt sind. Der Pyrazol-Heterozyklus dient als Brückeneinheit in diesem System. Durch das Anbringen von unterschiedlichen Resten am Rückgrat der Pyrazol-4-Position bzw. an den 3,4-Positionen des Pyrrolringes kann eine Reihe neuartiger Bausteine entwickelt werden. Diese neuartigen Pyrrol/Pyrazol-Bausteine (1a und 1b) können vielfältig eingesetzt werden.

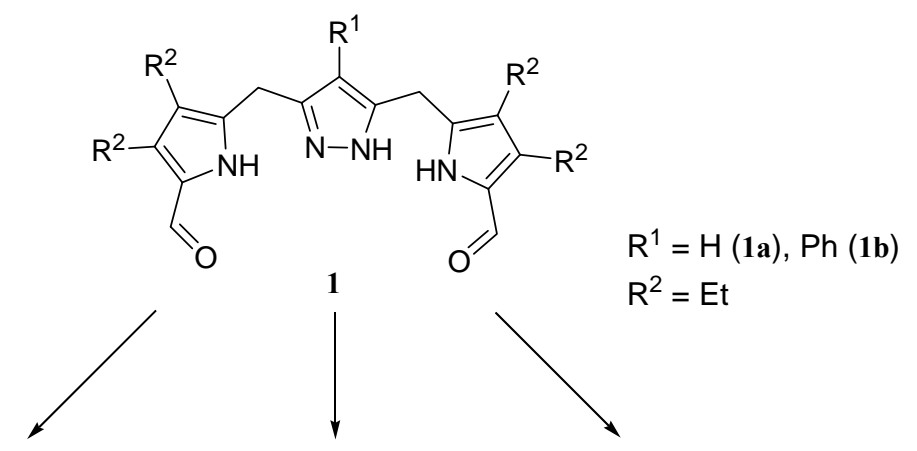

"Doppelporphyrine"

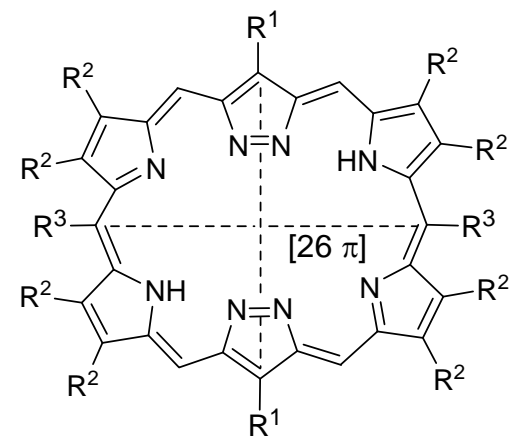

3
Schiff-Base Makrozyklen

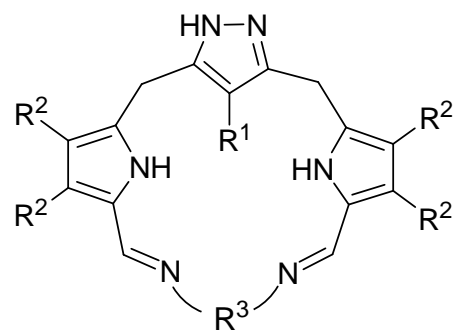

2

$\mathrm{R}^{1}=\mathrm{H}$

$\mathrm{R}^{2}=\mathrm{Et}$

$\mathrm{R}^{3}=$ aliphatische oder aromatische Diimine
Azyklische Diimin-Liganden<smiles>[R]N=Cc1[nH]c(Cc2n[nH]c(Cc3[nH]c(C=N[R])c([R])c3[R])c2[R])c([R])c1[R]</smiles>

$\mathbf{H}_{3} \mathbf{L}$

$\mathrm{R}^{1}=\mathrm{H}, \mathrm{Ph}$

$\mathrm{R}^{2}=\mathrm{Et}$

$\mathrm{R}^{3}=$ aromatische Amine

Abbildung 13: Baustein 3,5-Bis-(1H-pyrrol-2-yl-methyl)-1H-pyrazol (1) und die Darstellung von neuartigen Makrozyklen (3 und 2) bzw. azyklischen Diimin-Liganden $\left(\mathbf{H}_{\mathbf{3}} \mathbf{L}\right)$. 
Eines der Ziele ist die Synthese von neuartigen, expandierten Porphyrinen mit Pyrazoleinheiten im Ring; von uns sogenannten „Doppelporphyrinen“ (3). Das Gerüst dieses aromatischen Systems besteht, horizontal betrachtet, aus zwei Porphyrinfragmenten und vertikal aus zwei Pyrazolaten mit pyrrolhaltigen Seitenarmen an den 3,5-Positionen. Das Doppelporphyrin 3 besitzt $26 \pi$ Elektronen statt $18 \pi$ Elektronen wie die klassischen Porphyrine. Durch die Expandierung des Rings entstehen hierbei neue elektronische bzw. spektrale Merkmale und ungewöhnliche Komplexbildungseigenschaften.

Eine große Anzahl an Tripyrrin-haltigen Schiff-Base Makrozyklen und Analoga wurde in den letzten Jahren veröffentlicht. ${ }^{44}$ Dennoch wurde bis heute noch kein Pyrrol/Pyrazol-basierter Schiff-Base Makrozyklus dargestellt. Ein weiteres Ziel dieser Arbeit war die Synthese des ersten Bispyrrolpyrazol-basierten Schiff-Base Makrozyklus (2). Eine Reihe von neuartigen Schiff-Base Makrozyklen können durch säurekatalytische Kondensationsreaktion zwischen dem Dialdehyd 1a und diversen Diaminen synthetisiert werden. 2 besitzt eine flexible Kavität als Bindungstasche und weist besondere Wasserstoff-Brücken Motive auf. Diese Pyrrol/Pyrazol-Hybrid Makrozyklen können als mögliche spektroskopische Sonden eingesetzt werden.

3,5-Disubstituierte Pyrazolliganden werden erfolgreich zur Synthese von chelat-stabilisierten bimetallischen Komplexen eingesetzt. Die Bispyrrolpyrazol-basierten Bausteine (1a, 1b) sollten ebenfalls zur Synthese von neuartigen azyklischen Diimin-Liganden eingesetzt werden $\left(\mathbf{H}_{3} \mathbf{L}\right.$, Abbildung 13). Durch seinen Aufbau weist der Chelatligand $\mathbf{H}_{\mathbf{3}} \mathbf{L}$ zwei „Bindungstaschen“ auf, in welchen zwei Metallionen koordinativ gebunden werden können (Abbildung 14). Hierbei sollten die Bindungseigenschaften von $\mathbf{H}_{3} \mathbf{L}$ als Chelatligand und die spektroskopischen Eigenschaften der bimetallischen Komplexe untersucht werden, insbesondere Auswirkung sperriger Substituenten auf den Gast X in der Zange der zwei Metallionen.

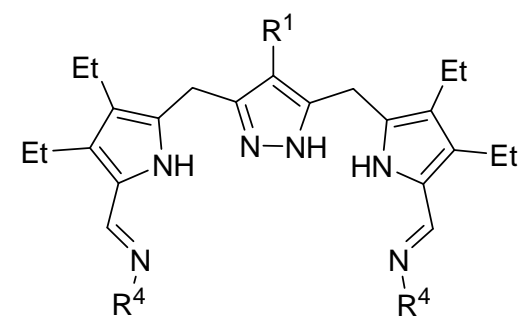

$\mathbf{H}_{3} \mathbf{L}$

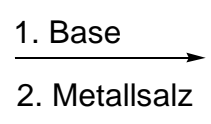

2. Metallsalz

Abbildung 14: Schematische Darstellung von neuartigen Pyrrol/Pyrazol-basierten bimetallischen Chelatkomplexen $\left[\mathbf{L}^{\mathbf{x}} \mathbf{M}_{\mathbf{2}} \mathbf{X}\right]$.

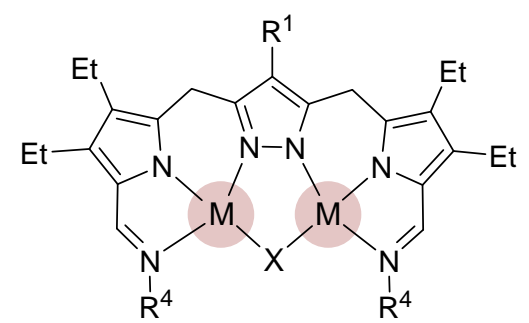

$\left[\mathbf{L}^{\mathrm{x}} \mathbf{M}_{2} \mathbf{X}\right]$ 


\section{Synthese der Pyrrol/Pyrazol-basierten Bausteine}

Die neuartigen Bausteine (1) wurden aus einer Pyrazol- und je zwei Pyrrolkomponenten dargestellt. Hierzu wurden eine Serie von neuen Bispyrrolpyrazolderivaten synthetisiert (Abbildung 15). Als Pyrazoledukte wurde in dieser Arbeit das 3,5-Bis-(chlormethyl)pyrazol Hydrochlorid (XXIX) und das 3,5-Bis-(chlormethyl)-4-phenyl-pyrazol Hydrochlorid (4) verwendet. Die Pyrrol-Ausgangsverbindungen bestanden aus dem käuflich erhältlichen Pyrrol und dem 3,4-Diethylpyrrol (XXXV).

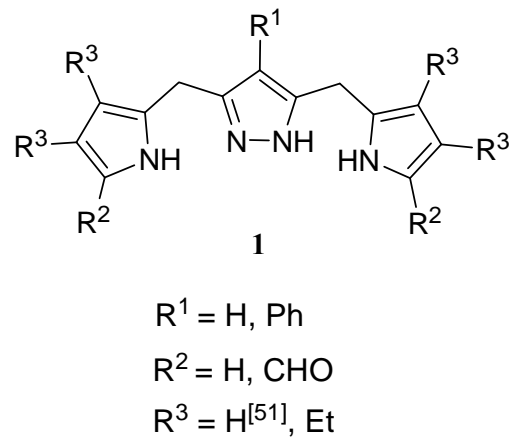

Abbildung 15: Pyrrol/Pyrazol-basierter Grundbaustein (1).

Zunächst wurde hier nach Literaturvorschrift das 3,5-Bis-(chlormethyl)pyrazol Hydrochlorid (XXIX) hergestellt. ${ }^{33}$ Hierzu wurde 3,5-Dimethylpyrazol (XXV) mit Kaliumpermanganat und $\mathrm{CO}_{2}$-Gas zum Monokaliumsalz der Pyrazol-3,5-dicarbonsäure (XXVI) oxidiert, welches anschließend säurekatalytisch mit Methanol verestert wurde.

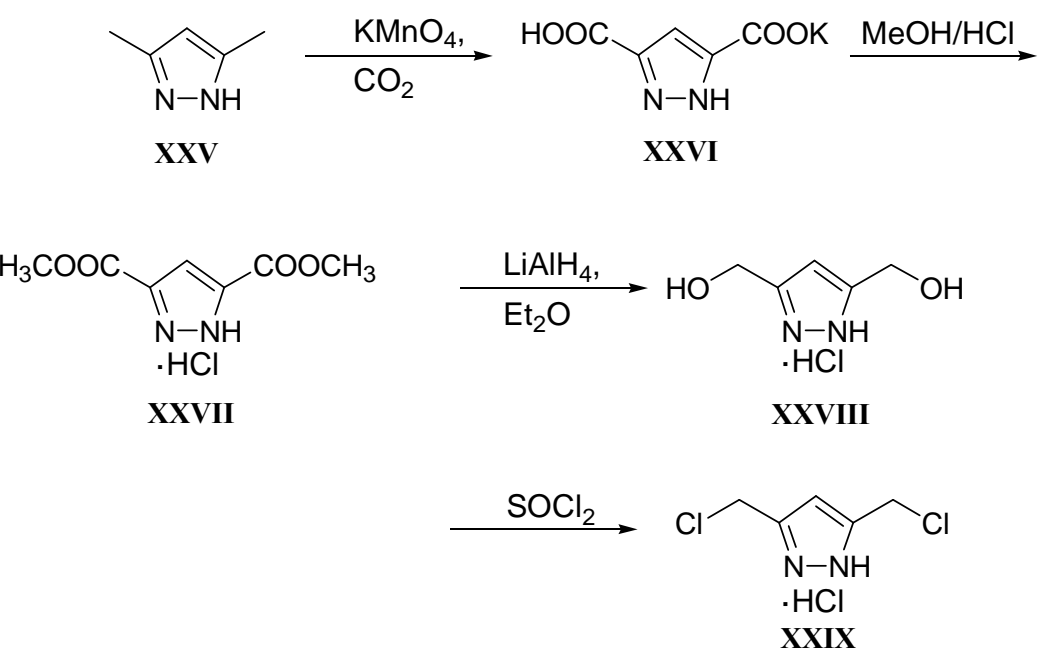

Abbildung 16: Synthese von 3,5-Bis-(chlormethyl)-1H-pyrazol Hydrochlorid. ${ }^{33}$ 
Der Ester (XXVII) wurde mit Lithiumaluminumhydrid zum Dialkohol (XXVIII) reduziert, welcher anschließend zur gewünschten Verbindung mit Thionylchlorid umgesetzt wurde (Abbildung 16).

Die zweite Pyrazol-Ausgangsverbindung, das 3,5-Bis(chlormethyl)-4-phenyl-pyrazol Hydrochlorid (4) wurde nach Literaturvorschrift hergestellt (Abbildung 17). ${ }^{45}$ Hierbei wurde Glycinmethylester Hydrochlorid (XXX) mit Natriumnitrit säurekatalytisch zum Diazoessigsäuremethylester (XXXI) umgesetzt. Durch Reaktion mit Zimtsäuremethylester bei $60-90{ }^{\circ} \mathrm{C}$ entsteht zunächst der Dimethylester (XXXII), ${ }^{45,46}$ welcher mit Brom zum 4Phenyl-1H-pyrazol-3,5-dicarboxydimethylester (XXXIII) oxidiert wird. Verbindung XXXIII wurde mit einem Überschuss an Lithiumaluminiumhydrid in Methanol zum Dialkohol (XXXIV) reduziert und anschließend mit Thionylchlorid chloriert. Einige dieser Stufen wurden optimiert und sind im experimentellen Teil detailliert beschrieben.

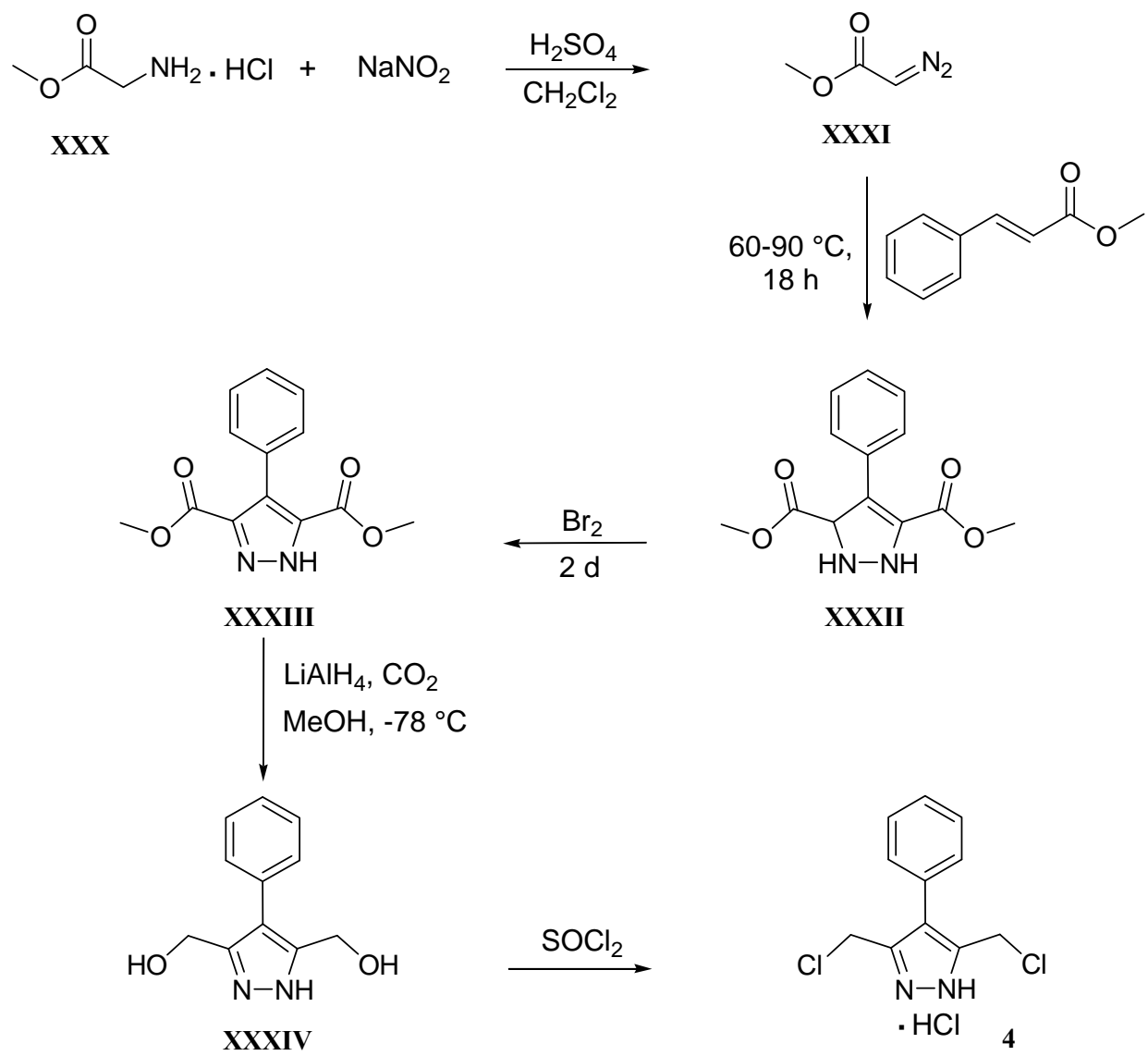

Abbildung 17: Syntheseweg zur Darstellung von 3,5-Bis-(chlormethyl)-4-phenyl-pyrazol Hydrochlorid (4).

Das 3,4-Diethylpyrrol (XXXV) wurde in einem aufwendigen mehrstufigen Syntheseweg ausgehend vom Isocyanoessigsäureethylester (XXXVI) ${ }^{47}$ und dem 4-Acetoxy-3-nitrohexan $(\mathbf{X X X V I I})^{48}$ dargestellt. Dazu wird Glycinethylester Hydrochlorid (XXXVIII) mit 
Methylformiat (XXXIX) und Triethylamin umgesetzt. Das isolierte Formylamin (XL) wird unter Inertatmosphäre bei $0{ }^{\circ} \mathrm{C}$ mit Phosphorylchlorid/Triethylamin versetzt (Abbildung 18). Der Isocyanoessigsäureethylester (XXXVI) ist auch bei tiefen Temperaturen und unter Inertatmosphäre nur begrenzt haltbar.

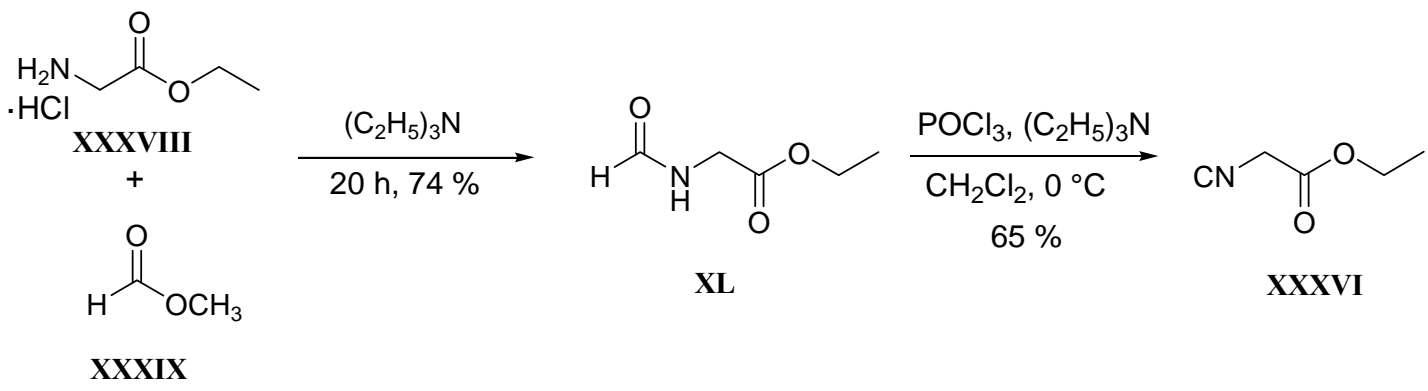

Abbildung 18: Syntheseweg von Isocyanoessigsäureethylester (XXXVI) ${ }^{47}$

Parallel wurde in einem weiteren Syntheseweg Propionaldehyd (XLI) in Reaktion mit Nitropropan (XLII) zum 4-Nitrohexan-3-ol (XLIII) und anschließender Versterung zu 4Acetoxy-3-nitrohexan (XXXVII) umgesetzt (Abbildung 19). ${ }^{48}$

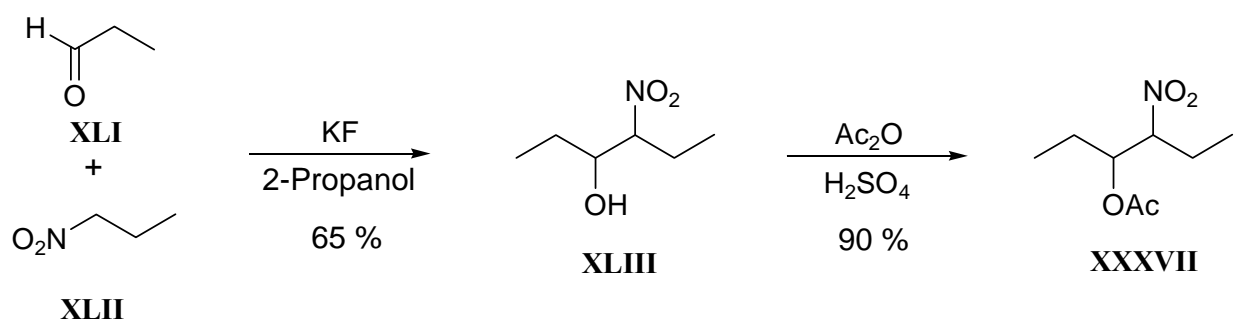

\footnotetext{
Abbildung 19: Syntheseweg von 4-Acetoxy-3-nitrohexan (XXXVII) ${ }^{48}$
}

In einer Zyklisierungsreaktion von XXXVI mit XXXVII unter basischen Bedingungen (Base: DBU = 1,8-Diazabicyclo-[5.4.0]undec-7-en) entsteht das 3,4-Diethyl-2-carboxylat (XLIV) und durch anschließende Decarboxylierung das 3,4-Diethylpyrrol (XXXV) (Abbildung 20). Die Pyrazol-Ausgangsverbindungen XXIX und 4 liegen beide als Hydrochloridsalze vor und müssen vor der Substitutionsreaktion mit den Pyrrolderivaten neutralisiert werden. 4 wird hierzu unter $\mathrm{N}_{2}$-Atm. in trockenem THF gelöst und bei $-78{ }^{\circ} \mathrm{C}$ mit einem Äquivalente $n$-BuLi neutralisiert. Parallel wird in einem weiteren Schlenkkolben ein Überschuss an 3,4Diethylpyrrol (3.5 - 4.0 eq.) in trockenem TFH gelöst mit $n$-BuLi bei $-78{ }^{\circ} \mathrm{C}$ und unter $\mathrm{N}_{2}$ Atm. deprotoniert. Nach 45 Min. wird das neutrale 4 langsam zum Pyrrolgemisch zugegeben (Abbildung 21). 
<smiles>[CH2+]CC(=O)OCC</smiles>

XXXVI<smiles>CCC(OC(C)=O)C(CC)[N+](=O)[O-]</smiles>

XXXVII

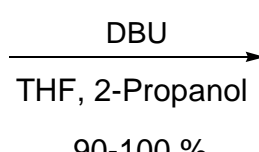

90-100\%<smiles>CCOC(=O)c1[nH]cc(CC)c1CC</smiles><smiles>CCc1c[nH]cc1CC</smiles>

XXXV

Abbildung 20: Syntheseweg von 3,4-Diethylpyrrol (XXXV) ${ }^{48}$

Das Reaktionsgemisch wurde $2 \mathrm{~h}$ bei $-78{ }^{\circ} \mathrm{C}$ gerührt und anschließend über Nacht auf Raumtemperatur erwärmt. Am nächsten Tag wurde das Reaktionsgemisch mit einer gesättigten $\mathrm{NH}_{4} \mathrm{Cl}$-Lösung gequencht. Die wässrige Phase wurde mit $3 \times 100 \mathrm{~mL} \mathrm{CH}_{2} \mathrm{Cl}_{2}$ extrahiert und die gesammelten organischen Phasen mit $\mathrm{Na}_{2} \mathrm{SO}_{4}$ bzw. $\mathrm{MgSO}_{4}$ getrocknet.

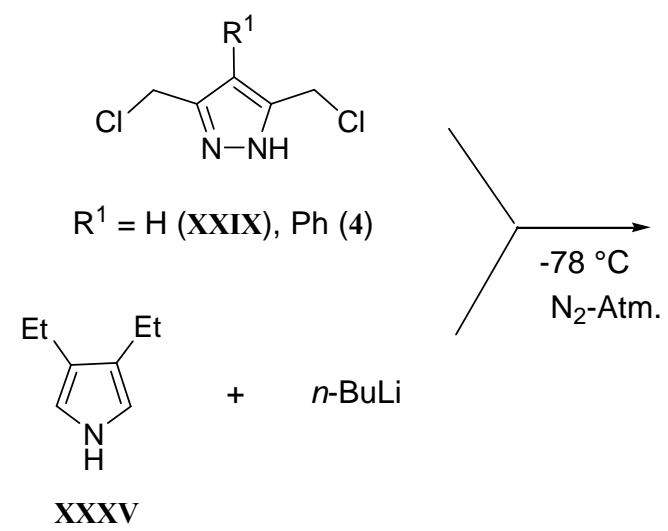

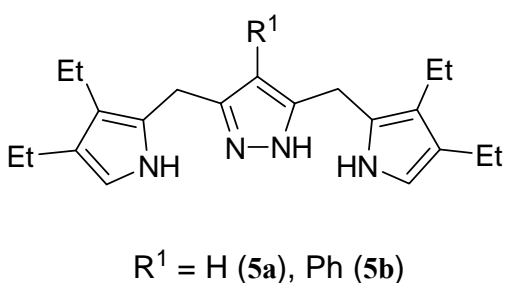

$$
\begin{array}{r}
\mathrm{R}^{1}=\mathrm{H}(\mathbf{5 a}), \mathrm{Ph}(\mathbf{5 b}) \\
\mid \begin{array}{l}
\mathrm{DMF}, \\
\mathrm{PhCOCl}, \\
2 \mathrm{~h}, 0^{\circ} \mathrm{C}
\end{array}
\end{array}
$$<smiles></smiles>

$$
\mathrm{R}^{1}=\mathrm{H}(\mathbf{1} \mathbf{a}), \mathrm{Ph}(\mathbf{1 b})
$$

Abbildung 21: Syntheseweg der Dialdehyde 1a bzw. 1b.

Das Lösungsmittel wurde im Vakuum entfernt und das braune ölige Rohprodukt wurden mittels Säulenchromatographie an Aluminiumoxid ([basisch, Aktivität I]) mit Dichlormethan/Methanol 40:1 als Laufmittel) gereinigt. Das Produkt 5b erhielt man als leicht 
bräunliches Öl welches sich leicht zersetzt. Da die Verbindung 5b hygroskopisch ist und leicht polymerisiert, wurde sie direkt für die nächste Reaktionsstufe eingesetzt.

Die Synthese des Pyrrol/Pyrazol-derivats 5a verläuft analog zu der oben beschriebenen Vorschrift. Im Vergleich zu 4 lässt sich das Hydrochlorid von XXIX leichter neutralisieren und kann außerdem als freie Base gelagert werden. XXIX wurde dafür in einer THF- oder Dichlormethan-Suspension mit einem leichten Überschuss an festem $\mathrm{Na}_{2} \mathrm{CO}_{3}$ versetzt und über Nacht stehen gelassen. Am nächsten Tag wurde das Reaktionsgemisch abfiltriert und der Rückstand mit THF oder Dichlormethan gewaschen. Im Filtrat befindet sich das neutrale XXIX, welches bei der Substitutionsreaktion mit den Pyrrolderivaten direkt eingesetzt werden kann. Die Reaktion mit neutralem XXIX und XXXV kann in diesem Fall auch in trockenem Dichlormethan statt THF durchgeführt werden.

Sowohl 5a als auch 5b polymerisieren zu braunen Ölen (aufgrund des Fehlens von Substituenten an der $\alpha$-Position des Pyrrols), was die Reinigung der Produkte erschwert. Deshalb empfiehlt es sich, diese Verbindungen direkt nach der Isolierung in der sogenannten Vilsmeier-Haack Formylierung umzusetzen. 5a bzw. 5b wird hierbei unter $\mathrm{N}_{2}$-Atm. und bei $0{ }^{\circ} \mathrm{C}$ in trockenem DMF gelöst und tropfenweise mit frisch destilliertem Benzolylchlorid (8 eq.) versetzt. Nach $2 \mathrm{~h}$ Rühren wurde das Reaktionsgemisch auf Raumtemperatur gebracht und 2-4 h bei gleicher Temperatur gerührt. Unter Eisbadkühlung wurde anschließend das Reaktionsgemisch mit einer 50\%igen-ethanolischen $\mathrm{Na}_{2} \mathrm{CO}_{3}$-Lösung neutralisiert. Das Reaktionsgemisch wurde mit $\mathrm{CH}_{2} \mathrm{Cl}_{2}$ extrahiert und die gesammelten organischen Phasen mit $\mathrm{Na}_{2} \mathrm{SO}_{4}$ getrocknet (Abbildung 21). Das Lösungsmittel wurde entfernt und das braune ölige Rohprodukt säulenchromatographisch an basischem Aluminiumoxid gereinigt. Die entstandenen Dialdehyde 1a bzw. 1b lassen sich etwas leichter isolieren als ihre Pyrrol/Pyrazol-Ausgangsverbindungen 5a bzw. 5b, wenn die Edukte in ausreichender Reinheit eingesetzt wurden. Bei Anwesenheit von diversen unerwünschten PyrrolNebenprodukten wird die Isolierung der Dialdehyde 1a bzw. 1b extrem erschwert und die Rohprodukte müssen mehrfach säulenchromatographisch gereinigt werden. Nach der säulenchromatographischen Reinigung sollten die erhaltenen Produkte aus Methanol/Essigsäureethylester umkristallisiert werden. 


\title{
5. Darstellung von neuartigen Makrozyklen
}

\subsection{Darstellung von „Doppelporphyrinen“}

Nach der Darstellung der Pyrrol/Pyrrazol-Bausteine wurden im nächsten Schritt neue expandierte Porphyrine synthetisiert. Diese neuartigen Makrozyklen wurden zunächst, ähnlich der klassischen Porphyrin-Synthese, in einer Kondensationsreaktion eines $\alpha$-unsubstituierten Pyrrolbausteines mit einem Aldehyd umgesetzt, oder über eine sogenannte MacDonaldMethode in Reaktion zwischen einem Diformyldipyrrol (XLIV) mit einem $\alpha$ diunsubstituierten Pyrrolbaustein (XLV) säurekatalytisch zu einem Porphyrin umgesetzt (Abbildung 22).

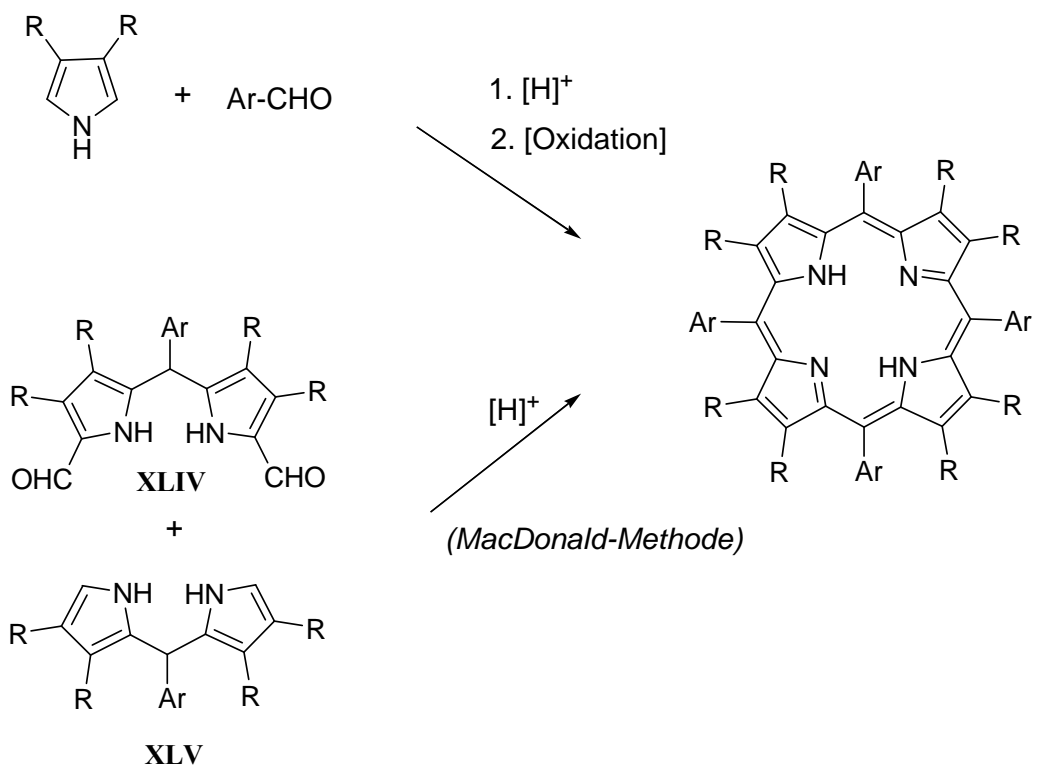

\begin{abstract}
Abbildung 22: Klassische Porphyrinsynthese aus einem Pyrrolderivat und einem Aldehyd ${ }^{49}$ (oben) und aus einem Dipyrrolmethan (XLV) nach der MacDonald-Methode ${ }^{50}$ (unten).
\end{abstract}

In dieser Arbeit wurden die Pyrrol/Pyrazol-Bausteine 5a und 5c in Reaktion mit Pentafluorbenzaldehyd bzw. Benzaldehyd verwendet (Abbildung 23). Pentafluorbenzaldehyd ist im Vergleich zum Benzaldehyd durch den fluorierten Aromaten aktiver und wird deshalb bei der Synthese von Porphyrinen bevorzugt verwendet. Verbindung 5c wurde im Rahmen von frühren Arbeiten analog zu 5a synthetisiert. ${ }^{51}$ Verbindung 5a ist durch die Substituenten an den 3,4-Positionen des Pyrrols stabiler als 5c und weniger empfindlich gegen 
unerwünschte Polymerisationsreaktionen. 5a bzw. 5c wurden in trockenen organischen Lösungsmitteln wie Dichlormethan oder Chloroform gelöst und unter $\mathrm{N}_{2}$-Atmospähre mit dem Aldehyd versetzt.

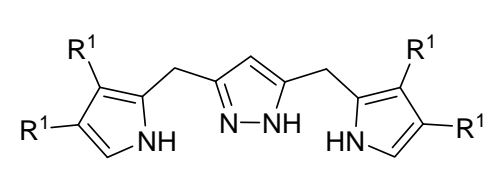

$\mathrm{R}^{1}=\mathrm{H}(\mathbf{5} \mathbf{c})$, Et $(\mathbf{5 a})$
1. $\mathrm{ArCHO}$

2. 0.1 - 10 eq $\left[H^{+}\right]$

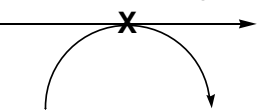

(l)

$\mathrm{R}_{2}=\mathrm{Cl}(\mathbf{X L V I}), \mathrm{CN}(\mathbf{X L V I I})$

$\mathrm{Ar}=\mathrm{Ph}, \mathrm{C}_{6} \mathrm{~F}_{5}$

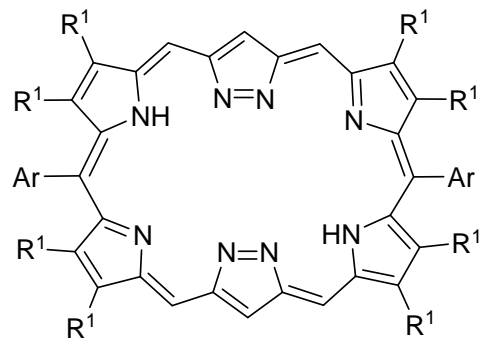

$\mathrm{R}^{1}=\mathrm{H}, \mathrm{Ar}=\mathrm{Ph}(\mathbf{3 a})$

$\mathrm{R}^{1}=\mathrm{H}, \mathrm{Ar}=\mathrm{C}_{6} \mathrm{~F}_{5}(\mathbf{3 b})$

$\mathrm{R}^{1}=\mathrm{Et}, \mathrm{Ar}=\mathrm{Ph}(\mathbf{3 c})$

$\mathrm{R}^{1}=\mathrm{Et}, \mathrm{Ar}=\mathrm{C}_{6} \mathrm{~F}_{5}(\mathbf{3 d})$

Abbildung 23: Syntheseweg zur Darstellung von Doppelphorphyrinen (3a-3d).

Benzaldehyd wurde mit einer Säure in Lösung aktiviert. Hierbei wurden unterschiedliche Säuren wie TFA, $p$-TSA bzw. $\mathrm{BF}_{3} \cdot \mathrm{OEt}_{2}(0.1$ bis 10 eq.) verwendet. Nach dieser Reaktion wurden die entstandenen Produkte mit einem Oxidationsmittel oxidiert. Hierbei wurde entweder Chloranil (XLVI) oder DDQ (XLVII) zum Reaktionsgemisch gegeben. XLVI und XLVII gehören zu den klassischen Oxidationsmitteln in der Porphyrinsynthese.

Obwohl die Reaktionsbedingungen vielfältig variiert wurden, konnte keine der Zielverbindungen (3a-3d) hergestellt werden. Bei der Reaktion von 5a mit Pentafluorbenzaldehyd konnten mit Hilfe der ESI(+)-Massenspektrometrie in einer Acetonitril-Lösung einige entstandene Spezies charakterisiert werden. Es wurden dabei zwei Hauptsignale beobachtet (Abbildung 24). Das erste Signal bei $730 \mathrm{~m} / \mathrm{z}$ könnte dem Produkt entsprechen, welches durch eine doppelte Substitutionsreaktion aus dem Pyrrol/Pyrazol-Baustein und je ein Molekül $\mathrm{C}_{6} \mathrm{~F}_{5} \mathrm{CHO}$ entsteht (3d'). 3d' die Summenformel $\left[\mathrm{C}_{35} \mathrm{H}_{31} \mathrm{~F}_{10} \mathrm{~N}_{4} \mathrm{O}_{2}\right]$, was einer molekularen Masse von $729 \mathrm{~g} \cdot \mathrm{mol}^{-1}$ entspricht. Bei Protonierung unter (ESI+)-Bedingungen würde man ein Signal für $[\mathrm{M}+\mathrm{H}]^{+}$bei $730 \mathrm{~m} / \mathrm{z}$ erwarten. 


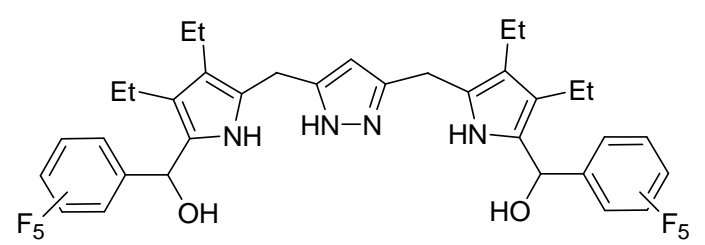

3d'

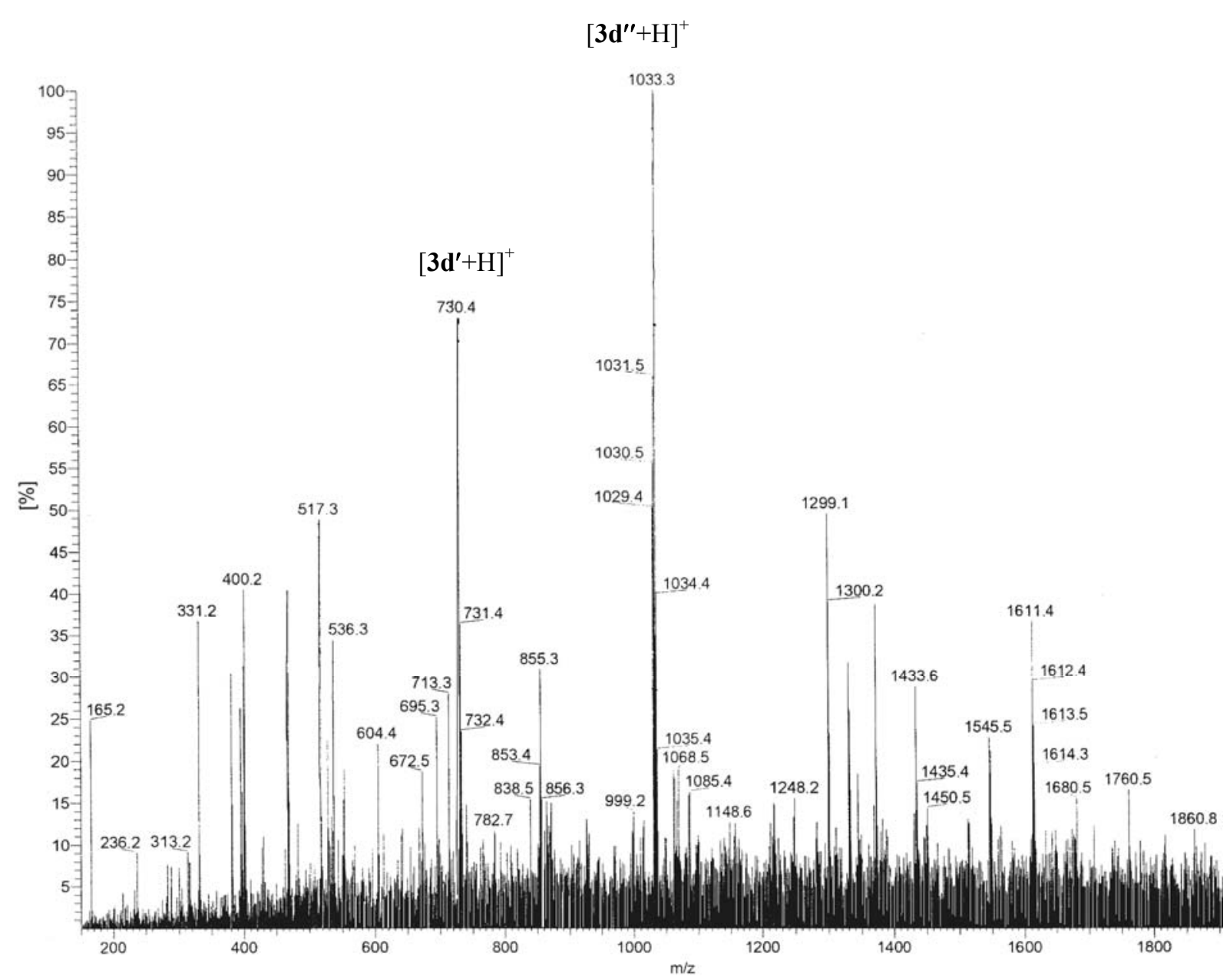

Abbildung 24: (ESI+)-Massenspektrum des Produkts aus der Reaktion von 5a mit Pentafluorbenzaldehyd aufgenommen in $\mathrm{CH}_{2} \mathrm{Cl}_{2} / \mathrm{MeCN}$.

Das Signal bei 1033 m/z kann dem nicht-oxidierten Makrozyklus (3d") zugeordnet werden, welcher eine molekulare Masse von $1032 \mathrm{~g} \cdot \mathrm{mol}^{-1}\left[\mathrm{C}_{56} \mathrm{H}_{58} \mathrm{~F}_{10} \mathrm{~N}_{8}\right]$ hat. Im Massenspektrum sind jedoch weitere Signale bei z.B. 1299.1 oder $1611.4 \mathrm{~m} / \mathrm{z}$ zu beobachten, welches als mögliche Bildung von Ketten gedeutet werden kann. Eine vollständige Oxidation zu einem aromatischen Makrozyklus (3d) konnte nie beobachtet werden. Durch frühere Arbeiten war bekannt, dass die Methylengruppen zwischen Pyrazol und Pyrrol durch klassische Oxidationsmittel nicht oxidierbar ist. ${ }^{51}$ Auch eine mögliche Oxidation an der meso-Position 
zwischen zwei Pyrrolen war nicht zu beobachten. Die Synthese der Spezies 3d', 3d" war jedoch nicht verlässlich reproduzierbar, und 3d' bzw. 3d" konnten auch nicht isoliert werden. Im nächsten Schritt wurden zwei weitere Syntheserouten untersucht, die zu einem Doppelporphyrin führen sollten (Abbildung 25). Hierbei wurde im Reaktionsweg A der neu synthetisierte Dialdehyd 1a mit 5a unter $\mathrm{N}_{2}$-Atmospähre säurekatalytisch mit einen Überschuss an Säure (TFA oder HBr) umgesetzt.

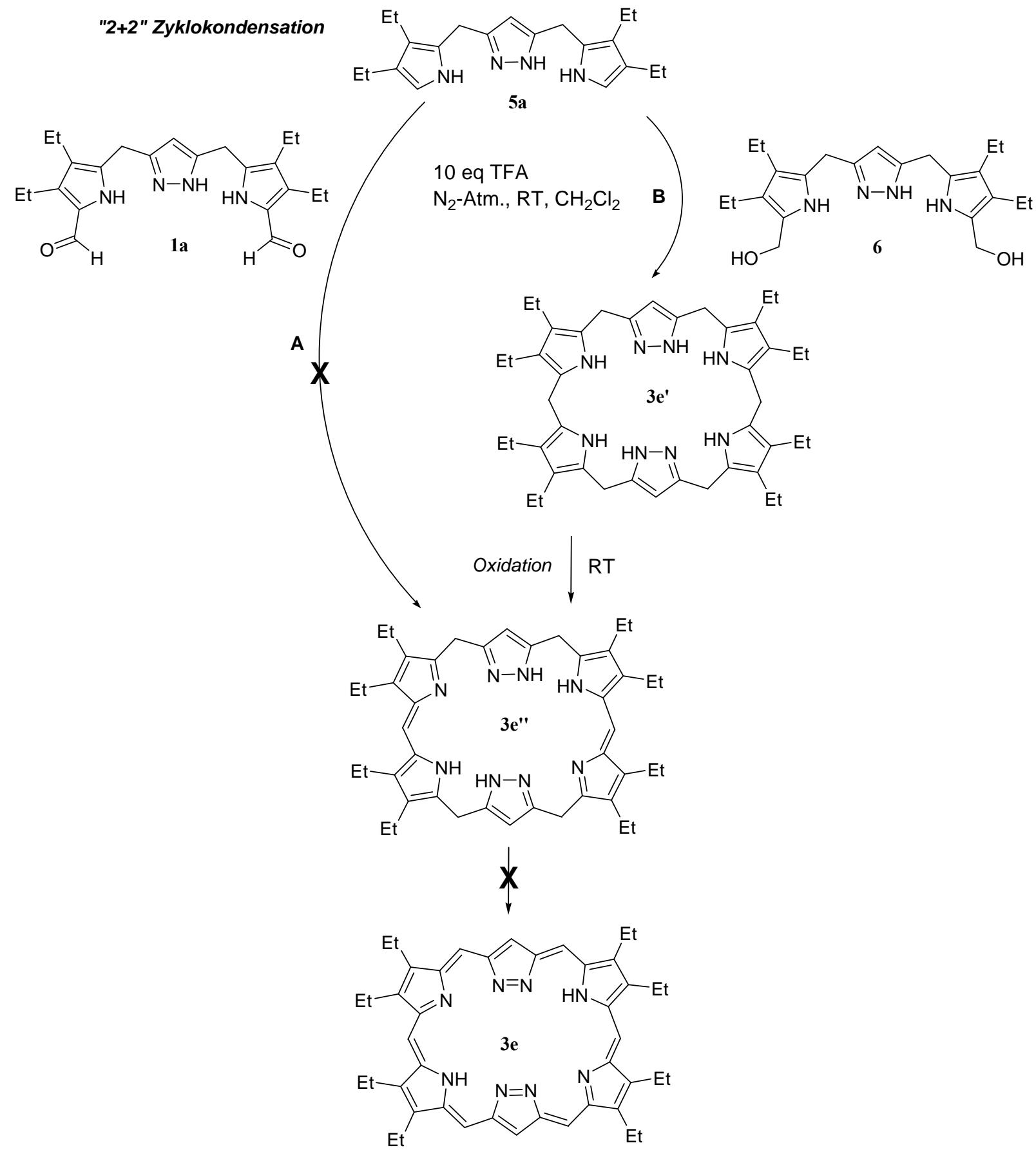

Abbildung 25: Syntheserouten A und B zur Darstellung eines Doppelporphyrins (3e). 


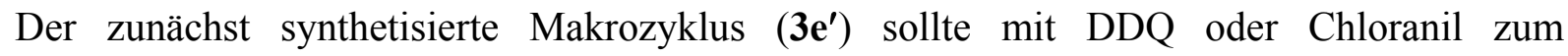
gewünschten aromatischen Doppelporphyrin (3e) führen. Der Reaktionsverlauf wurde mittels ${ }^{1}$ H-NMR-Spektroskopie verfolgt. Dazu wurden die Edukte 1a und 5a in $\mathrm{CDCl}_{3}$ gelöst und $20 \%$ TFA- $\mathrm{d}_{1}$ wurde dazugegeben. Hierbei konnten einige Änderungen der chemischen Verschiebungen, die durch die Protonierung von 1a mit TFA zustande kamen, beobachtet werden. Das Proton in $\mathrm{CH}^{\mathrm{pz}-4}$ verschiebt sich hierbei von 5.83 ppm ins Tieffeld bei 6.90 ppm und das CHO-Signal des Aldehydrests von 9.40 auf 9.00 ppm. Die $\mathrm{CH}_{2}$ - und $\mathrm{CH}_{3}$ - Gruppen der Pyrrol-Substituenten zeigen ebenfalls eine geringfügige Verschiebung. Für die Signale des $\alpha$-unsubstituierten Pyrroledukts 5a gab es keinerlei Veränderung, was darauf hinweist, dass es zu keiner Reaktion zwischen 5a und 1a gekommen ist. Durch dieses NMRExperiment konnte schliesslich demonstriert werden, dass das Dialdehyd 1a keine Reaktion mit 5a einging.

Lindsey et al. konnten bei einer analogen Porphyrinsynthese feststellen, dass es bei einer (2+2)-Zyklokondensation zwischen einem Pyrroldialdehyd und einem $\alpha$-unsubstituierten Dipyrromethanderivat zu keiner Reaktion kam. ${ }^{52}$ Sie lösten dieses Problem, indem sie die Dialdehyd-Derivate mit einem Überschuss an Natriumborhydrid zum Dialkohol reduzierten. Sie konnten in einer anschließenden säurekatalytischen Zyklokondensation die gewünschten Porphyrine erhalten.

Analog wurde im Syntheseweg B (Abbildung 25) der Dialdehyd 1a in Chlorofom/Methanol gelöst und mit einem Überschuss an Natriumborhydrid zum Dialkohol (6) reduziert. Über Dünnschichtchromatographie wurde hierbei die Reaktion verfolgt. Als das Edukt völlig reduziert vorlag wurde das Reaktionsgemisch mit Wasser aufgearbeitet und die Lösung mit Dichlormethan extrahiert. Die organische Phase wurde mit $\mathrm{MgSO}_{4}$ getrocknet und das Lösungsmittel im Vakuum entfernt. Der dabei entstandene Dialkohol 6 erwies sich als stark hygroskopisch und musste direkt in der nachfolgenden Zyklisierungsreaktion umgesetzt werden.

Der Dialkohol (6) wurde unter Inertatmosphäre in trockenem $\mathrm{CHCl}_{3}$ bzw. $\mathrm{CH}_{2} \mathrm{Cl}_{2}$ gelöst und ein Äquivalent 5a dazu gegeben. Das Reaktionsgemisch wurde mit 10 eq. TFA versetzt und mindestens $1 \mathrm{~h}$ bei Raumtemperatur gerührt. Anschließend wurde das entstandene Produkt mit einem Oxidationsmittel umgesetzt. Hierbei wurden unterschiedliche Oxidationsmittel ausprobiert, wie DDQ, Chloranil, Brom, $\mathrm{Pd} / \mathrm{C}, \mathrm{Ag}_{2} \mathrm{O}$ oder $o$-Iodylbenzoesäure $\left(\mathrm{IBX}^{53}\right)$. Die Reaktion wurde von Beginn an per UV/Vis-Spektroskopie und ESI-Massenspektrometrie verfolgt. Man kann beobachten, dass bei der Reaktion von 5a mit 6 nach Zugabe der Säure zuerst der Makrozyklus 3e' entsteht und relativ lange stabil in Lösung bleibt. Nach Zugabe 
des Oxidationsmittels kommt es zur Teiloxidation an den meso-Positionen und der Makrozyklus 3e" entsteht. Das UV/Vis-Spektrum von 3e" aus der Reaktionsmischung ist in Abbildung 26 (links) dargestellt.
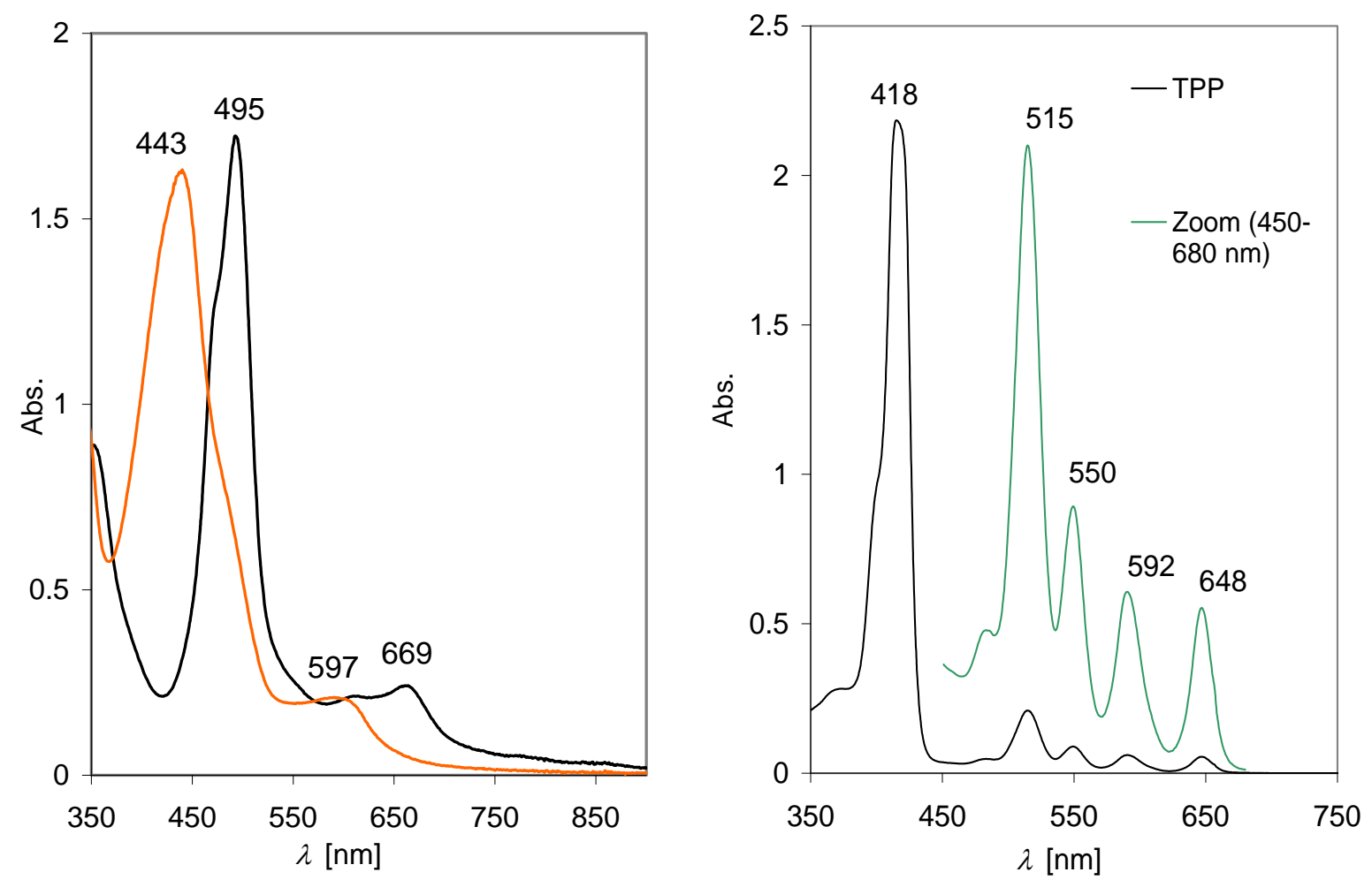

Abbildung 26: UV/Vis-Spektren von 3e" (schwarze Linie) und nach Deprotonierung mit $\mathrm{Et}_{3} \mathrm{~N}$ (rote Linie) (links). UV/Vis-Referenzspektrum von Tetraphenylporphyrin (TPP, schwarze Line) und Vergrößerung des Bereichs von 450-680 nm (grüne Linie) (rechts).

Es wird eine intensive Bande bei $495 \mathrm{~nm}$ und eine Schulter bei $669 \mathrm{~nm}$ beobachtet. Bei Addition einer Base (hier Triethylamin) kommt es zu einer hyperchromen Verschiebung der Banden (rote Linie). In Abbildung 26 (rechts) ist das UV/Vis-Spektrum vom Tetraphenylporphyrin als Referenzsubstanz angegeben. Porphyrine haben charakteristische Banden; sie bestehen aus einer intensiven Bande bei ca. $400 \mathrm{~nm}$, Sorret-Banden $\left(\pi \rightarrow \pi^{*}\right.$ Übergänge) genannt, und mehreren Q-Banden zwischen 450-700 nm. Bei expandierten Porphyrinen, wie z.B. den Hexaphyrinen, wird ebenfalls eine intensive Soret-Bande beobachtet und mehrere Q-Banden, die jedoch durch das expandierte Elektronensystem eine bathochrome Verschiebung gegenüber den klassischen Porphyrinen haben. ${ }^{54}$ Bei Addition einer Base (wie Triethylamin) gibt es bei den konjugierten aromatischen Porphyrinen keinerlei Änderung im UV/Vis-Spektrum, da die Basizität der Base nicht ausreicht um die zwei Protonen der Porphyrine zu entfernen. Da im UV/Vis-Spektrum von 3e" eine Verschiebung durch Zugabe an Base zu beobachten ist, kann daraus geschlossen werden, dass 
hierbei der entstandene Zyklus, wie bereits vermutet, kein aromatisches System besitzt. Beim UV/Vis-Spektrum von 3e" fehlen weiterhin Banden, die den Q-Banden beim Porphyrin entsprechen würden. Bei der Synthese von $3 \mathbf{e}^{\prime \prime}$ wird TFA verwendet, welches eine Protonierung am N-Atom des Pyrazols verursachen kann. Durch Addition von $\mathrm{Et}_{3} \mathrm{~N}$ zu der Lösung von 3e" kommt es zur Deprotonierung des N-Atoms im Pyrazol. Dies bestätig die Verschiebung der Banden im UV/Vis-Spektrum von 3e" nach Zugabe von Triethylamin.

Im Massenspektrum (ESI+) ist bei Beginn der Reaktion ein Signal bei $701 \mathrm{~m} / \mathrm{z}[\mathrm{M}+\mathrm{H}]^{+} \mathrm{zu}$ beobachten, welches dem Makrozyklus 3e' mit $700 \mathrm{~g} \cdot \mathrm{mol}^{-1}\left[\mathrm{C}_{44} \mathrm{H}_{60} \mathrm{~N}_{8}\right]$ zugeordnet werden kann (Abbildung 27).
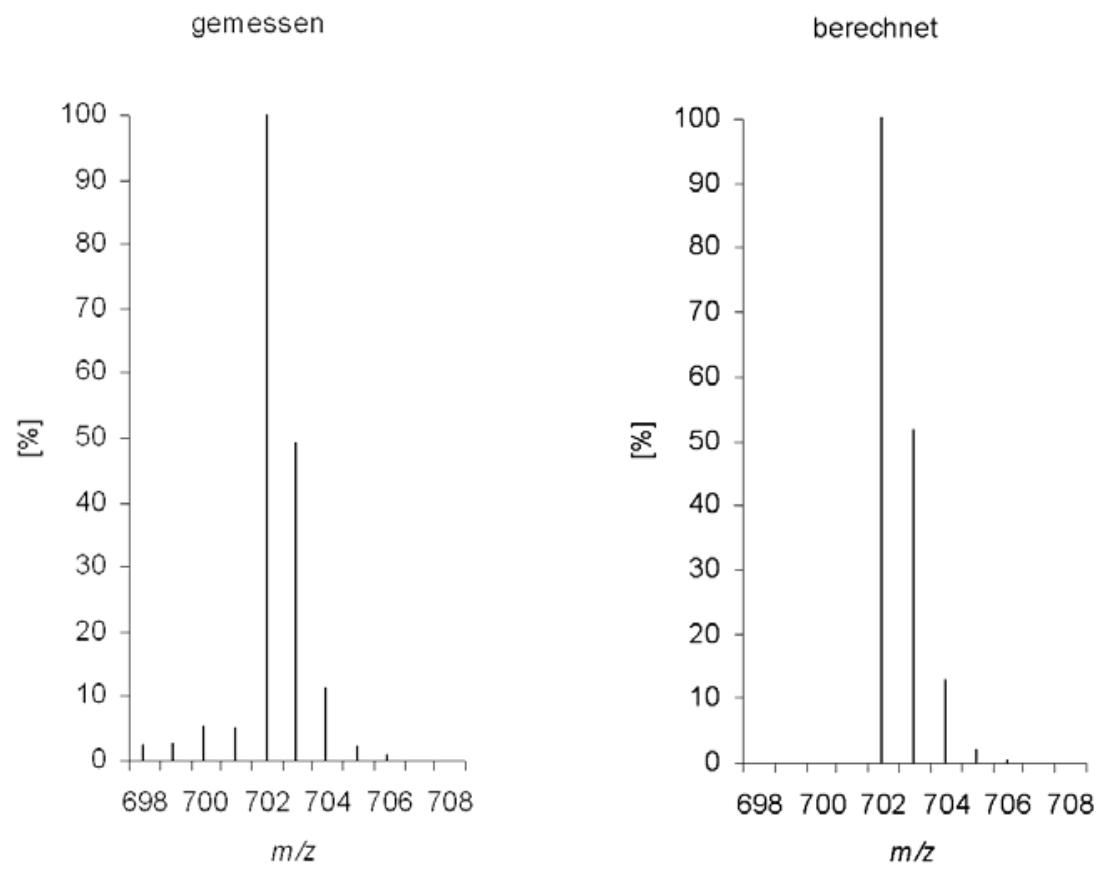

Abbildung 27: ESI-MS Spektrum von $3 \mathbf{e}^{\prime}$ gemessen in $\mathrm{CHCl}_{3} / \mathrm{MeCN}$ (links) und Simulation (rechts).

Durch langsame Oxidation an der Luft oder durch Zugabe eines (bereits erwähnten) Oxidationsmittels nimmt das Signal bei $701 \mathrm{~m} / \mathrm{z}$ an Intensität ab und eine neues Signal bei $697 \mathrm{~m} / \mathrm{z}$ für $[\mathrm{M}+\mathrm{H}]^{+}$entsteht (Abbildung 28). Dieses Signal kann den teiloxidierten Makrozyklus 3e" mit $696 \mathrm{~g} \cdot \mathrm{mol}^{-1}\left[\mathrm{C}_{44} \mathrm{H}_{56} \mathrm{~N}_{8}\right]$ zugeordnet werden. Ein weiteres benachbartes Signal bei $695 \mathrm{~m} / \mathrm{z}$ mit geringerer Intensität zeigt, dass womöglich eine weitere Oxidation dieses Zyklus stattfinden kann. Diese Spezies konnte nur in der Massenspektrometrie nachgewiesen werden.

Die Reaktionslösung des Syntheseweges B (Abbildung 25) wurde zudem mittels hochauflösender Massenspektrometrie (HR-MS(ESI+)) untersucht. Das gemessene 
Massenspektrum und eine Simulation sind in Abbildung 29 wiedergegeben. Auch dort ist das Signal bei $701.50127 \mathrm{~m} / \mathrm{z}$ für $[\mathrm{M}+\mathrm{H}]^{+}$zu finden. Der berechnete Wert $\mathrm{zu}$ dieser Zusammensetzung mit $701.50142 \mathrm{~m} / \mathrm{z}$ stimmt gut mit dem gemessenen überein und bestätigt erneut die Bildung des Makrozyklus 3e'.
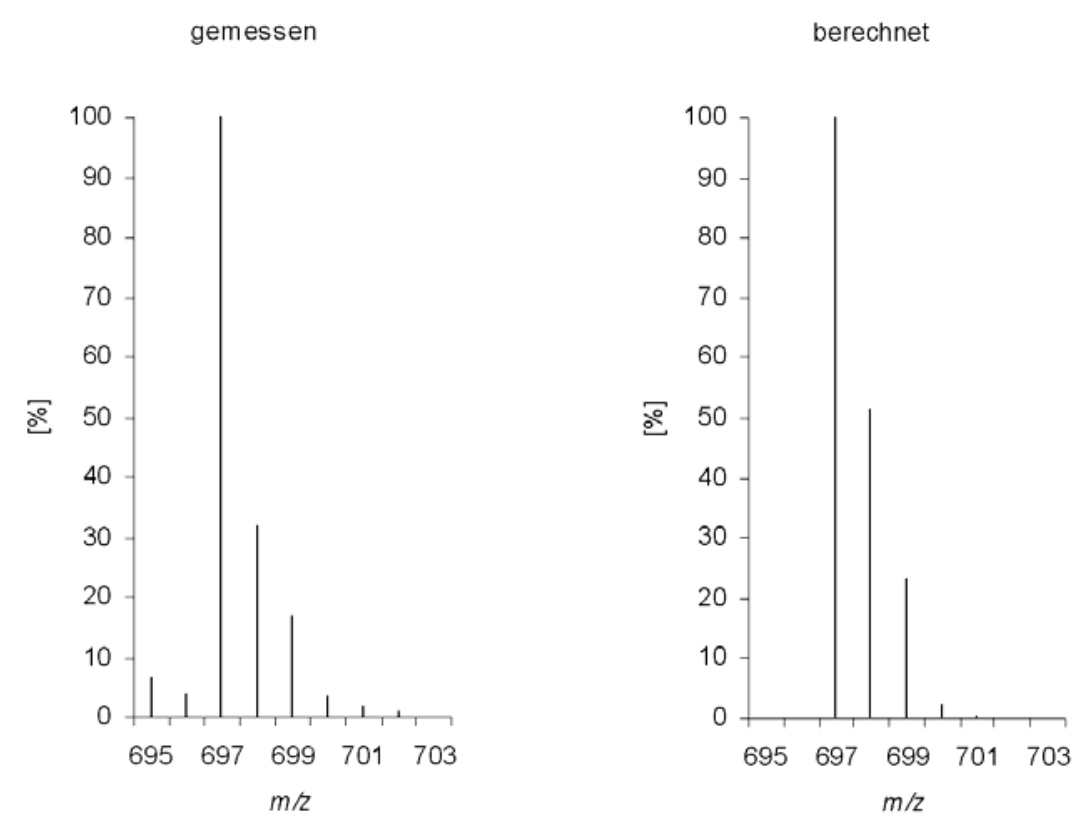

Abbildung 28: ESI-MS Spektrum von 3e" nach Oxidation mit Chloranil gemessen in $\mathrm{CHCl}_{3} / \mathrm{MeCN}$ (links) und Simulation (rechts).

Außerdem ist noch ein weiteres Signal bei $699.48654 \mathrm{~m} / \mathrm{z}$ zu beobachten, welches durch eine langsame Oxidation von $\mathbf{3} \mathbf{e}^{\prime}$ an der Luft $\mathrm{zu}[(\mathrm{M}-2 \mathrm{H})+\mathrm{H}]^{+}$entsprechen würde. Eine Simulation des Isotopenmusters für diese Zusammensetzung bestätigt diese Vermutung. Bei genauer Betrachtung des Signals bei $701.50127 \mathrm{~m} / \mathrm{z}$ sieht man, dass ein weiteres Signal bei $701.51965 \mathrm{~m} / \mathrm{z}$ aufzufinden ist, welches der Isotopenverteilung des ersten Signals bei $699 \mathrm{~m} / \mathrm{z}$ zugeordnet wird.

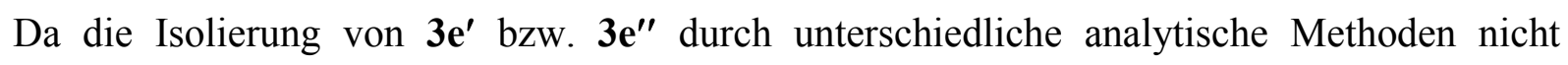
möglich war, wurde versucht, durch eine Templat-ähnliche Synthese direkt Metallkomplexe herzustellen. Dazu wurden unterschiedliche Metall(II)-Salze verwendet, wie $\mathrm{CdCl}_{2}$, $\mathrm{Zn}(\mathrm{AOc})_{2}, \mathrm{Ni}(\mathrm{OAc})_{2}, \mathrm{Cu}(\mathrm{OAc})_{2}$ oder $\mathrm{PdCl}_{2}$. Auch nach Zugabe dieser Metallsalze konnte die Entstehung eines Metallkomplexes jedoch nicht nachgewiesen werden.

In einem alternativen Ansatz kann Verbindung 5a analog der Synthese von expandierten Calixpyrrolen als Baustein genutzt werden. Sessler et al. synthetisierten eine neue Klasse von expandierten Calixpyrrolen aus dem (1H-pyrro-2-yl)-3,6-dimethyl-9H-carbazol in säurekatalytischer Reaktion mit Aceton bei Raumtemperatur. ${ }^{55}$ 

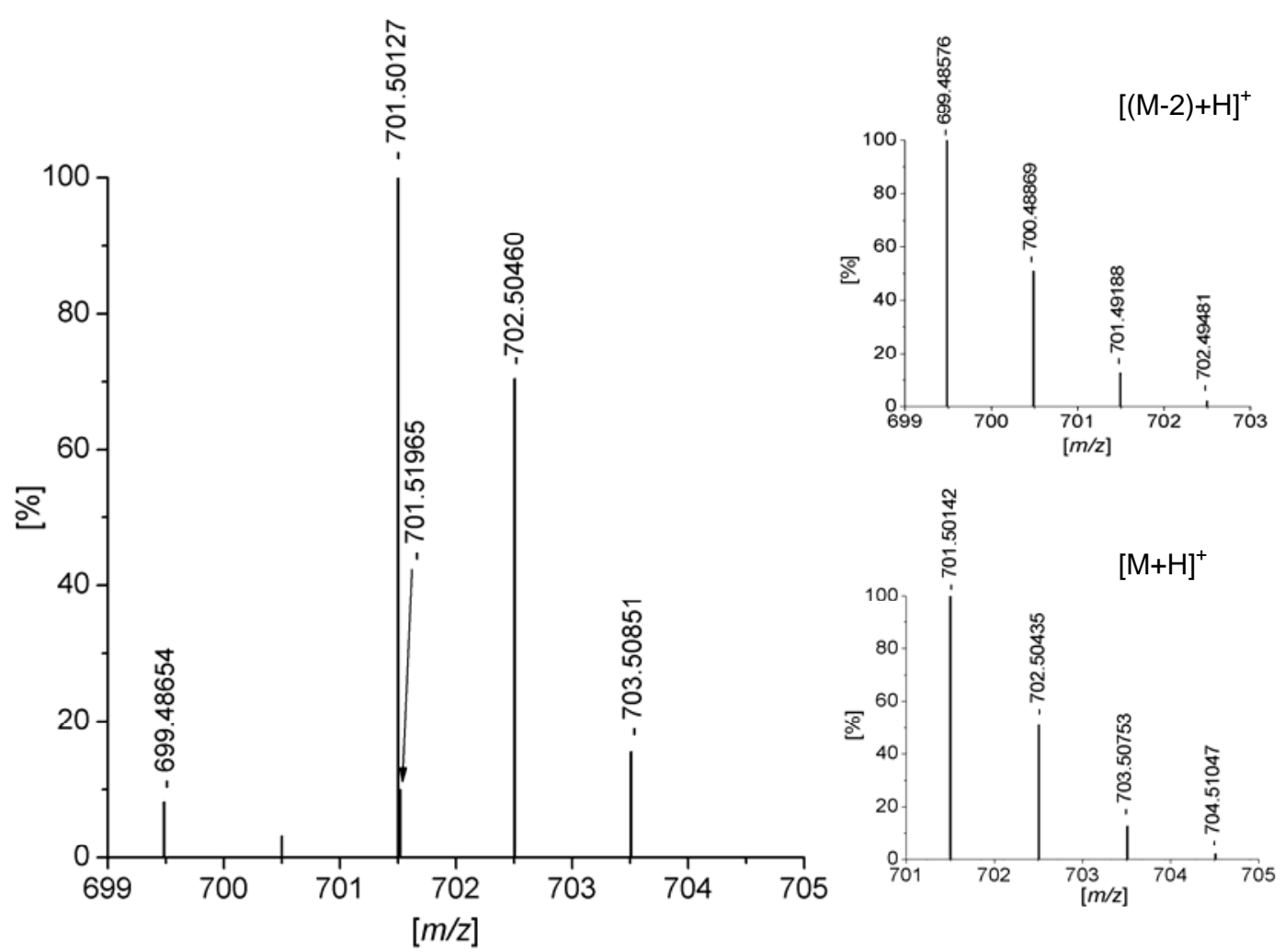

Abbildung 29: HR-MS (ESI + ) von $3 \mathbf{e}^{\prime}$ als $[\mathrm{M}+\mathrm{H}]^{+}$gemessen in $\mathrm{MeOH}$ (links) und Simulation der zwei Signale bei $699 \mathrm{~m} / \mathrm{z}[(\mathrm{M}-2 \mathrm{H})+\mathrm{H}]^{+}$und $701 \mathrm{~m} / \mathrm{z}[\mathrm{M}+\mathrm{H}]^{+}$(rechts).

Der neue Baustein (5a) wurde in einem entprechneden Experiment mit einem Überschuss Aceton unter Inertatmosphäre und unter Lichtausschluß umgesetzt (Abbildung 30). Hierbei wurden unterschiedliche Reaktionsbedingungen untersucht. Die Menge an Säure (TFA) wurde von 1 bis 5 eq. variiert, und die Reaktionszeit ( $1 \mathrm{~h}$ bis $2 \mathrm{~d}$ ) bzw. die Reaktionstemperatur (RT bis unter Rückfluss erhitzt) wurden ebenfalls varriert.<smiles>CCc1c[nH]c(Cc2cc(Cc3[nH]cc(CC)c3CC)[nH]n2)c1CC</smiles><smiles></smiles>

Abbildung 30: Syntheseweg zur Darstellung eines neuartigen expandierten Pyrazol-basiertem Callixpyrrol 3f. 
Die Reaktion wurde mittels DC und UV/Vis-Spektroskopie verfolgt. Die Reaktionslösung zeigt eine breite Bande mit schwacher Intensität bei $520 \mathrm{~nm}$ und ein weiteres schwächeres Signal bei $645 \mathrm{~nm}$. Bei der Zugabe von Triethylamin wurde die violett-rote Reaktionslösung rot-braun. Das UV/Vis-Spektrum ändert sich dementsprechend und eine sehr breite Bande bei $460 \mathrm{~nm}$ mit einer Schulter bei $535 \mathrm{~nm}$ entsteht. Weder die ${ }^{1} \mathrm{H}-\mathrm{NMR}$ - noch die ESI-Spektren zeigen charakteristische Signale für den erwarteten Makrozyklus (3f). Das ESI(-) Massenspektrum zeigt mehrere Signale, welche die Bildung von Ketten vermuten lassen. Unter diesen Reaktionsbedingungen konnte der Makrozyklus 3f bedauerlicherweise nicht dargestellt werden.

In einer weiteren alternativen Syntheseroute wurden andere, bereits zuvor bekannte Bausteine verwendet um durch eine säurenkatalytische Zyklokondensation das gewünschte „Doppelporphyrin“ (3a) zu erhalten (Abbildung 31). Dazu wurde 3,5-Bis(hydroxymethyl)pyrazol (XXVIII) als Hydrochlorid sowie als freie Base mit 5-Phenyldipyrromethan (XLVII) $^{56}$ in einer Zyklisierungsreaktion eingesetzt. Die Reaktion wurde säurekatalytisch mit einem Überschuss an TFA oder $p$-TSA unter Inertatmosphäre durchgeführt. Als Lösungsmittel wurde hierbei Dichlormethan oder auch zur besseren Löslichkeit des Pyrazoleduktes Acetonitril verwendet. Diese Reaktion wurde bei unterschiedlichen Reaktionsbedingungen durchgeführt.

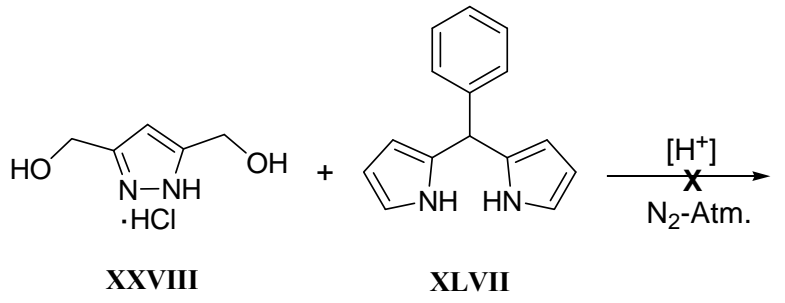

XXVIII

XLVII

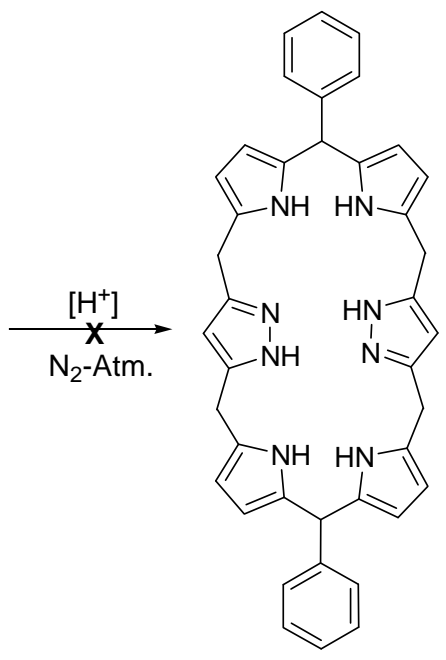

3a'

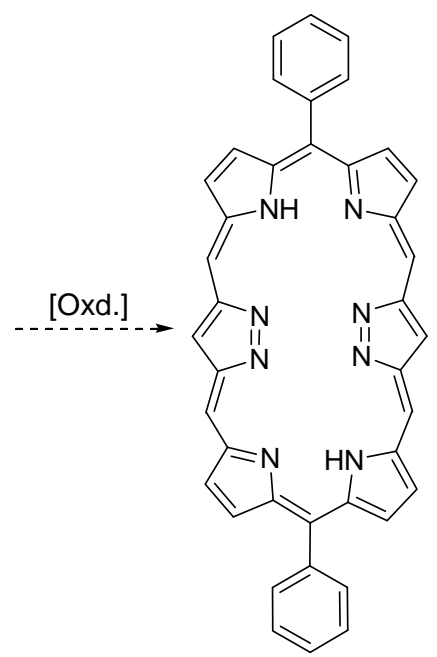

3a

Abbildung 31: Syntheseroute zur Darstellung des „Doppelporphyrins“ 3a.

Die Reaktionszeit wurde zwischen $1 \mathrm{~h}$ bis zu mehrere Tage variiert und die Temperatur von Raumtemperatur bis zur Siedetemperatur erhöht. Anschließend wurde das Reaktionsgemisch mit einem Oxidationsmittel wie DDQ oder Chloranil versetzt. Die Reaktion wurde mittels 
Dünnschichtchromatographie, UV/Vis-Spektroskopie und ESI-Massenspektrometrie verfolgt. Die UV/Vis-Spektren entsprachen dem oxidierten 5-Phenyl-dipyrromethan und die ESI-MS Spektren zeigten nur die Edukte bzw. die Entstehung des 5-Phenyl-4,6-dipyrrins. Dolphin et al. berichteten bereits in den 90er über die Oxidation der Dipyrromethanderivate in 4,6Dipyrrine. ${ }^{57}$ Durch mehrere analytische Messverfahren konnte gezeigt werden, dass das 3,5Bis-(hydroxymethyl)pyrazol (XXVIII) aufgrund seiner geringen chemischen Aktivität keine Reaktion mit dem Dipyrromethan (XLVII) einging.

Im nächsten Schritt sollte aus den Hydroxygruppen von XXVIII eine bessere Abgangsgruppe, wie das Tosylat dargestellt werden (Abbildung 32). Das Tosylat gehört zu einer der besten Abgangsgruppen und sollte bei der Substitutionsreaktion mit einem Dipyrroethanderivat eine Reaktion eingehen.

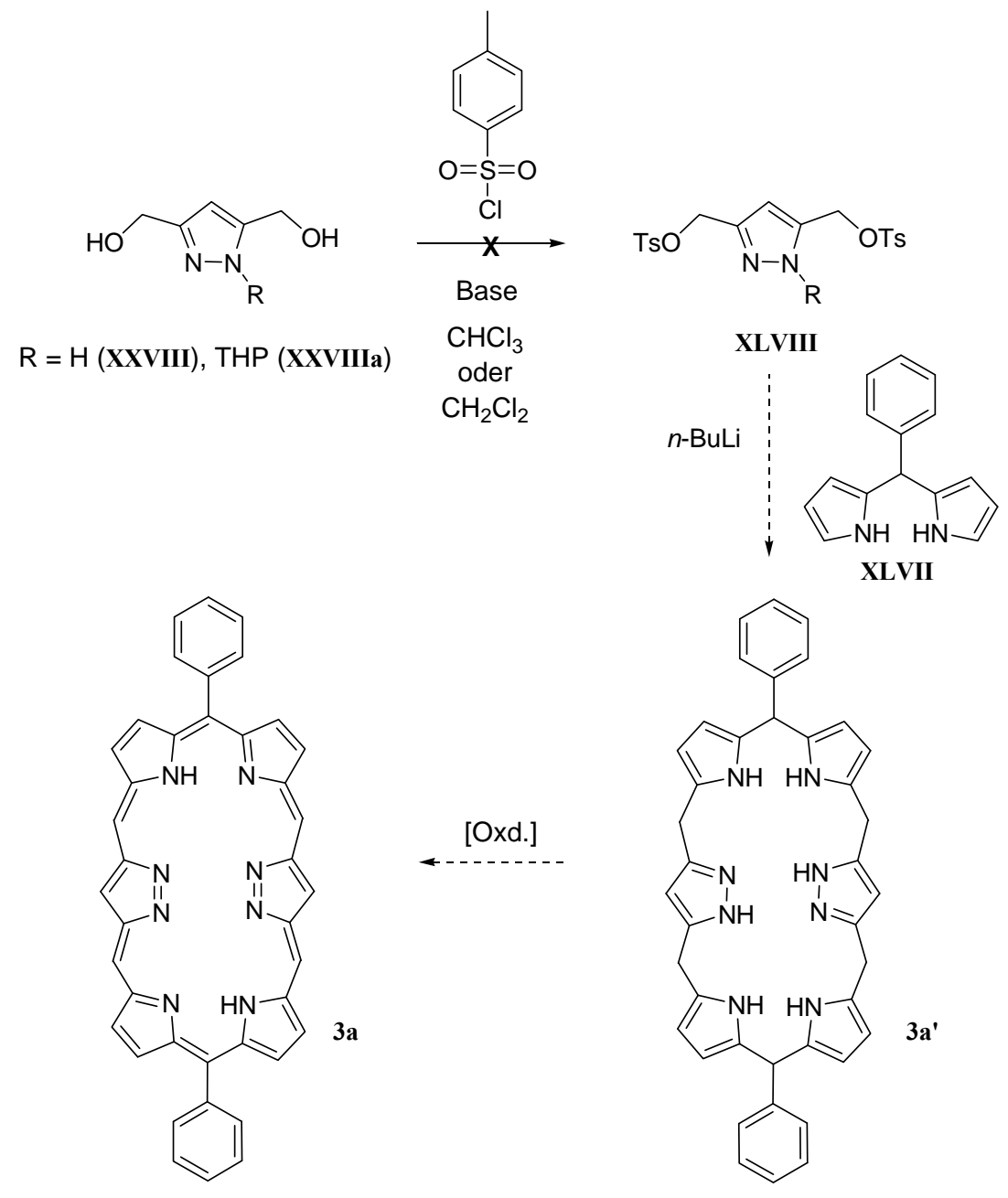

Abbildung 32: Syntheseweg zur Darstellung des Doppelporphyrins 3a.

Zunächst wurde das 3,5-Bis-(hydroxymethyl)-pyrazol in basischer Reaktion mit Triethylamin und Tosylchlorid umgesetzt. Da das gewünschte Produkt nicht entstand, wurden anstelle von Triethylamin auch Pyridin oder auch Dimethylaminopyridin (DMAP) zur Aktivierung des 
Dialkohols XXVIII eingesetzt und bis zur mehreren Tagen unter Rückfluss erhitzt. Der Reaktionsverlauf wurde mittels DC verfolgt. Diese Reaktionen führten jedoch nur zu uncharakterisierten Produkten. Der Dialkohol XXVIII ist eine in organischen Lösungsmitteln sehr schwerlösliche Substanz und wurde im nächsten Schritt durch den THP-geschützten Dialkohol XXVIIIa ersetzt. Durch die bessere Löslichkeit von XXVIIIa erhoffte man sich eine erfolgreiche Tosylierungsreaktion. Auch hier musste zuerst die Reaktion optimiert werden. Eine Erhöhung der Reaktionstemperatur wurde durch den Wechsel zu Toluol als Lösungsmittel (unter Rückfluss) und DMAP als Base erreicht.

Während der Reaktion entstand eine weiße Suspension. Das Reaktionsgemisch wurde bis zu drei Tagen unter Rückfluss erhitzt und anschließend mit einer $0.1 \mathrm{M}$ HCl-Lösung neutralisiert. Die DC-Kontrolle ergab keine Hinweise auf die Bildung neuer Produkte. In den ${ }^{1}$ H-NMR-Spektren konnten nur die eingesetzten Edukte beobachtet werden. Im ESI-MS oder auch im EI-MS konnten nur Signale gefunden werden, die eine geringere Masse (m/z) hatten als das gewünschte Produkt (XLVIII).

Die bis dahin synthetisierten Makrozyklen führten aus unterschiedlichen Gründen nicht zur Zielverbindung (3a-3e). Deshalb wurden weitere Pyrazol-basierte Bausteine synthetisiert, die anschließend in einer Zyklokondensationsreaktion eingesetzt wurden. Eine der Ausgangsverbindung war das 3,5-Bis-(aminomethyl)pyrazol Hydrochlorid (L). Dieses Pyrazolderivat wurde in einem zweistufigen Syntheseweg aus dem 3,5-Bis(chlormethyl)pyrazol Hydrochlorid (XXIX) nach Literaturvorschrift hergestellt. ${ }^{58}$

Anschließend wurde $\mathbf{L}$ mit 2 eq. aq. NaOH-Lösung neutralisiert und ein aromatischer Aldehyd wurde nach wenigen Minuten zugegeben. Hierbei wurden Benzaldehyd, 2Pyridincarbaldehyd und 1H-Pyrrol-2-carbaldehyd verwendet. Für die weiteren Untersuchungen war in dieser Arbeit nur Verbindung 7 von Interesse. Die anderen zwei Verbindungen (8 und 9) wurden zum Vergleich der Stabilität solcher Diimin-Verbindungen synthetisiert und werden im Experimentellen Teil detailliert beschrieben. Die Ausbeute von 79 ist relativ hoch, und die Produkte lassen sich durch Umkristallisation reinigen. Im Gegensatz zu 5a bzw. 5b ist die $\alpha$-unsubstituierte Pyrrolverbindung 7 unter Normalbedingungen stabil und muss nicht unter Inertatmosphäre gelagert werden.

Die Ausgangsverbindung 7 wurde im nächsten Schritt zur Synthese von „Doppelporphyrin“Analoga (3g und 3h) eingesetzt. 7 wurde hierbei unter $\mathrm{N}_{2}$-Atmospähre in trockenem Methanol gelöst und ein Äquivalent Benzaldehyd bzw. Pentafluorbenzaldehyd wurde dazugegeben (Abbildung 34). 


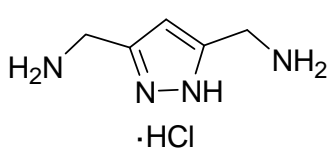

$\mathbf{L}$
1. 2 eq. $\mathrm{NaOH}$

2. $\stackrel{\mathrm{H}}{\mathrm{H}}_{\mathrm{H}}$<smiles>[R]C=NCc1cc(CN=C[R])[nH]n1</smiles><smiles>[R]</smiles><smiles>CCc1ccc[nH]1</smiles>

7<smiles>CC(=O)c1ccccn1</smiles>

8<smiles>Cc1ccccc1</smiles>

Abbildung 33: Synthese von Pyrazol-basierten Diimino-Derivaten (7-9).

Bei der Reaktion von 7 in $\mathrm{CH}_{2} \mathrm{Cl}_{2} /$ Methanol mit Benzaldehyd konnte man erst nach dreitägigem Erhitzen unter Rückfluss eine Reaktion der beiden Ausgangsverbindungen erreichen. Das Reaktionsgemisch wurde mit einem Überschuss TFA aktiviert.
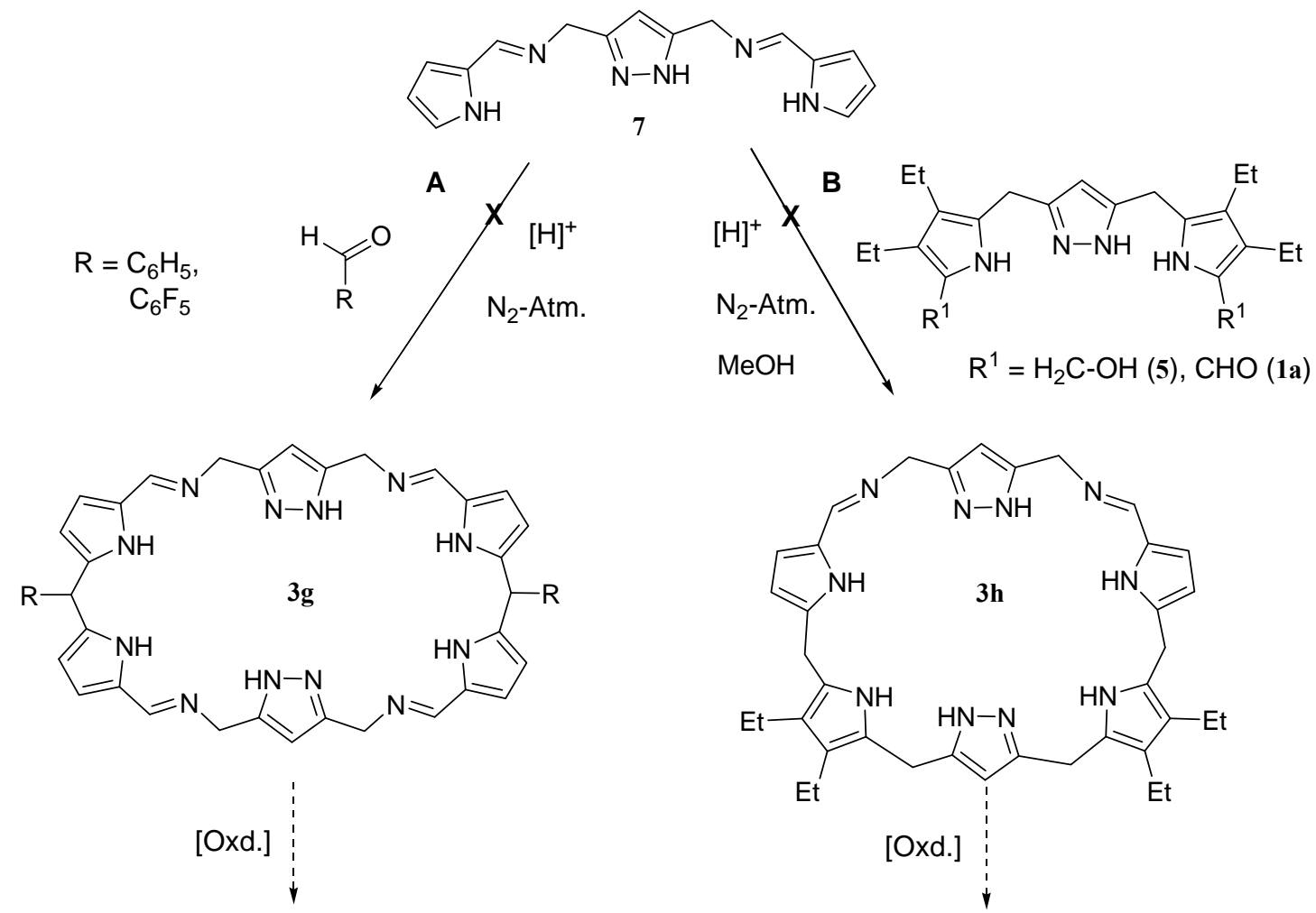

Abbildung 34: Syntheserouten A und B zur Darstellung von unterschiedlichen „Doppelporphyrin“-Analoga (3g bzw. 3h).

Einige der entstandenen Produkte konnten nur mittels ESI-Massenspektrometrie charakterisiert werden. Im Massenspektrum werden unter anderem zwei Signale bei $409 \mathrm{~m} / \mathrm{z}$ und bei $803 \mathrm{~m} / \mathrm{z}$ beobachtet. Die Ausgangsverbindungen reagieren miteinander vermutlich nicht $\mathrm{zu}$ einem Makrozyklus, sondern $\mathrm{zu}$ offenkettigen mono- und disubstituierten Pyrrolderivaten, wie in Abbildung 35 vorgeschlagen wird. 


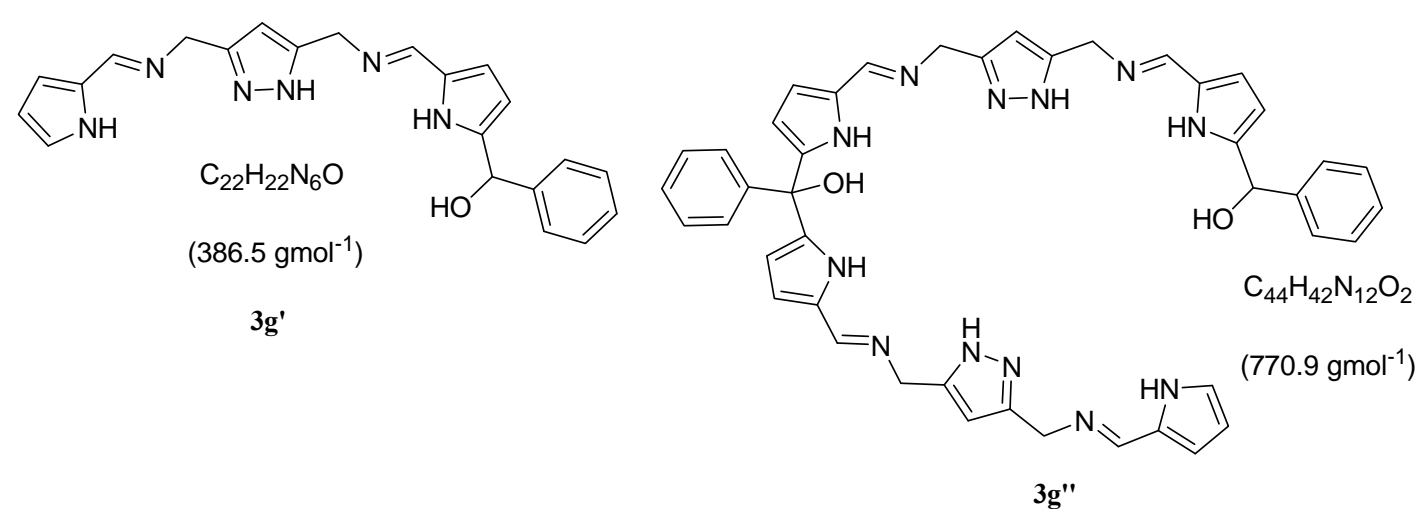

Abbildung 35: Mögliche Produkte (3g' und $\mathbf{3} \mathbf{g}^{\prime \prime}$ ) bei der der Reaktion von 7 mit Benzaldehyd (Weg A).

Wenn Verbindung $\mathbf{3} \mathbf{g}^{\prime}$ als Methanol-Addukt ionisiert wird entspricht dies der detektierten Masse von $409 \mathrm{~m} / \mathrm{z}$. Bei einer Ionisation von $\mathbf{3} \mathbf{g}^{\prime \prime}$ mit einem $\mathrm{Na}^{+}$-Ion würde man ein Signal bei $803 \mathrm{~m} / \mathrm{z}$ erwarten. Die Verbindungen $\mathbf{3} \mathbf{g}^{\prime}$ und $\mathbf{3} \mathbf{g}^{\prime \prime}$ scheinen relativ luftempfindlich zu sein und konnten nicht isoliert werden. 7 reagiert auch mit dem reaktiveren Pentafluorbenzaldehyd nicht zum erwarteten Makrozyklus 3g.

Beim Reaktionsweg B (Abbildung 34) wurden als Komponenten der Dialdehyd 1a bzw. der Dialkohol 6 verwendet. Der Dialdehyd 1a ging unter den getesteten Bedingungen keine Reaktion mit 7 ein. Deshalb wurde in einer weiteren Reaktion der Dialkohol 6 als reaktionsfreudigerer Partner eingesetzt. Die Reaktion wurde mit Hilfe der ESIMassenspektrometrie verfolgt. Hierbei kann beobachtet werden, dass bei dieser Reaktion eine Reihe an offenkettigen unerwünschten Produkten entstehen. Die Bildung eines Doppelporphyrins konnte in beiden Fälle nicht beobachtet werden. 


\subsection{Darstellung neuartiger Schiff-Base Makrozyklen}

Im Rahmen dieser Arbeit wurde eine Reihe neuartiger Pyrrol/Pyrazol-basierter Schiff-Base Makrozyklen dargestellt und untersucht. In dem kommenden Kapitel werden diese Verbindungen vorgestellt und ihre spektroskopischen Eigenschaften diskutiert.

\subsubsection{Darstellung der Verbindung 10}

Navarro et al. berichteten über die Synthese und Protonierungseigenschaften von 3,5disubstituierten Pyrazol-basierten Schiff-Base Makrozyklen (Abbildung 11). ${ }^{40,41}$ Inspiriert durch diese Arbeit und die Veröffentlichung von Love et al. ${ }^{29}$ über Akkordeon-artige SchiffBase Makrozyklen (Abbildung 7) wurden im Rahmen dieser Dissertation Schiff-Base Makrozyklen eines neuen Typs dargestellt.

Wie bei den Systemen von Navaro et al. sollte auch hier zunächst der 1H-Pyrazol-3,5dicarbaldehyd (LI) in einer Kondensationsreaktion mit einem Amin säurekatalytisch zu einem Makrozyklus (3i) mit zwei 3,5-substituierten Pyrazolringen und vier Iminfuktionen umgesetzt werden (Abbildung 36). $\mathrm{Zu}$ diesem Zweck wurde hier nach Literaturvorschrift der $1 \mathrm{H}$ Pyrazol-3,5-dicarbaldehyd (LI) durch Oxidation des Dialkohols (XXVIII, Abbildung 32) mit einem Überschuss an aktiviertem Manganoxid dargestellt. ${ }^{59,60}$ Der Dialdehyd LI wurde daraufhin in einer methanolischen Suspension mit 1,8-Diaminonaphtalin LII unter $\mathrm{N}_{2}$-Atm. unter Rückfluss erhitzt. Zur Aktivierung von LI wurden 2 eq. TFA oder $p$-TSA verwendet.

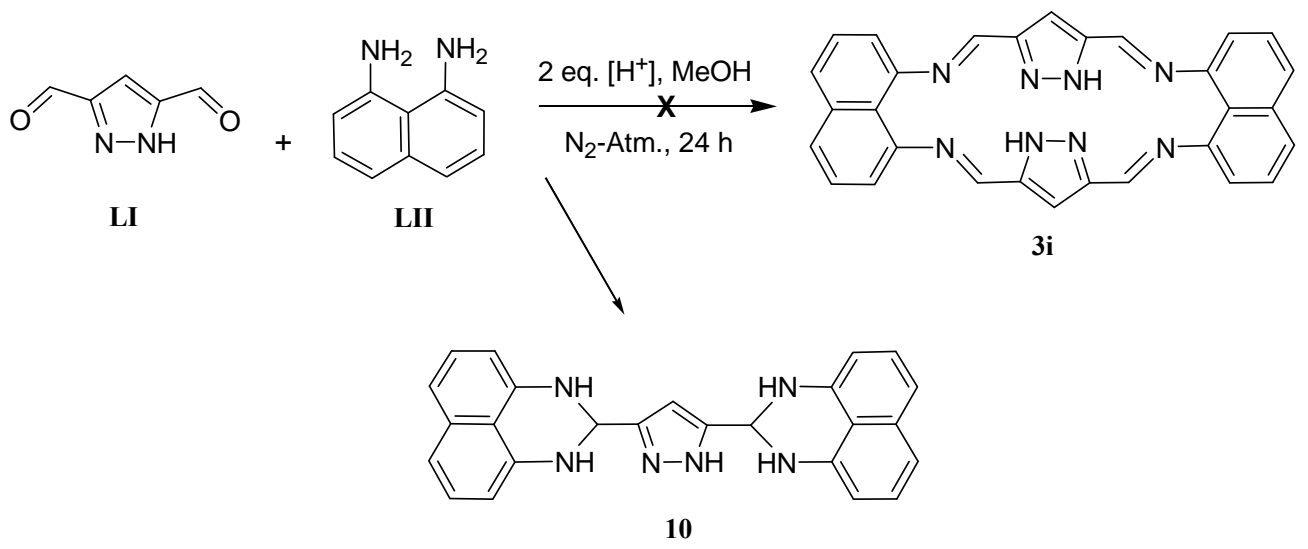

Abbildung 36: Synthese des 3,5-Bis-(2,3-dihydro-1H-perimidin)-1H-pyrazols (10). 
Statt des erwarteten Makrozyklus 3i entstand jedoch das 3,5-Bis-(2,3-dihydro-1H-perimidin)$1 H$-pyrazol (10), eine in diversen organischen Lösungsmittel äußerst schwerlösliche Verbindung. Aus der Literatur ist bereits bekannt, dass 1,8-Diaminopyridin in Reaktion mit Aldehyden Perimidinderivate bildet. ${ }^{61}$ Spektroskopische Untersuchungen, unter anderem IRund ${ }^{13} \mathrm{C}$-NMR-Spektroskopie, bestätigen eindeutig die Entstehung von 10. Die chemische Verschiebung der CH-Gruppe an der 3,5-Position des Pyrazols liegen im ${ }^{1} \mathrm{H}-\mathrm{NMR}$-Spektrum bei $5.50 \mathrm{ppm}$ und im ${ }^{13} \mathrm{C}$-NMR bei $60.3 \mathrm{ppm}$.

Verbindung 10 wurde in einer Reihe von Komplexierungsreaktionen als potentieller Ligand eingesetzt. $\mathrm{Zu}$ diesem Zweck wurde 10 mit 3 eq. Base deprotoniert ( $\mathrm{KO} t \mathrm{Bu}$ oder $\left.\mathrm{Et}_{3} \mathrm{~N}\right)$ und mit 2 eq. Metallsalz versetzt. Dabei wurden unterschiedliche Salze, wie $\mathrm{FeCl}_{3}, \mathrm{CuCl}_{2}$, $\mathrm{PdCl}_{2}(\mathrm{COD}), \mathrm{Ni}(\mathrm{OAc})_{2}$ oder $\mathrm{CrCl}_{3} \cdot 3 \mathrm{THF}$ verwendet. Die entstandenen Metallkomplexe erwiesen sich jedoch als extrem schwerlöslich, so dass die Umkristallisation und Charakterisierung der Komplexe sehr erschwert wurde. Massenspektrometrisch (ESI-MS) konnte im Falle von 10 mit M(II)-Ionen ermittel werden, dass der Komplexe des Typs $\mathrm{L}_{2} \mathrm{M}_{2} \mathrm{X}_{2}\left(\mathrm{X}=\mathrm{Cl}^{-}\right)$entstanden. 


\subsubsection{Darstellung neuartiger Pyrrol/Pyrazol-basierter Hybrid-Makrozyklen}

Aus dem synthetisierten Dialdehyd 1a konnte in Reaktion mit unterschiedlichen Diaminen eine Reihe von Schiff-Base Makrozyklen synthetisiert werden. 1a wurde in Inertatmospshäre unter Erwärmung in Methanol gelöst und mit einem Überschuss Trifluoressigsäure aktiviert. Nach Zugabe des Diamins wurde das Reaktionsgemisch 1-2 Tage unter Rückfluss erhitzt (Abbildung 37). Nach dem Entfernen des Lösungsmittels im Vakuum wurde das Rohprodukt säulenchromatographisch bzw. durch Umkristallisation aufgereinigt. Die Charakterisierung der hier präsentierten Makrozyklen ist im Experimentellen Teil detailliert beschrieben.<smiles>CCCc1[nH]c(C=O)c(CC)c1CC</smiles><smiles>CCc1c(C=O)[nH]c(Cc2ccn[nH]2)c1CC</smiles>

$1 \mathbf{a}$

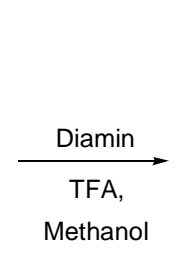

2

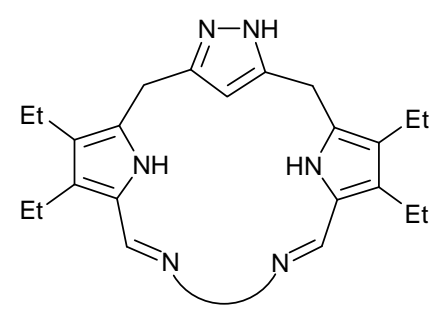

2

Diamine:

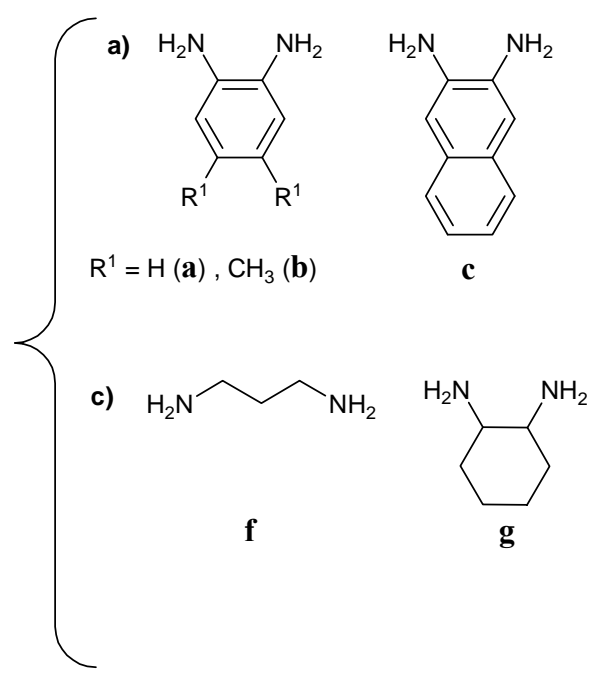

b)

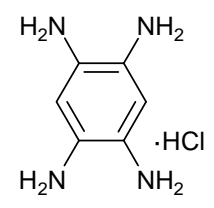

d

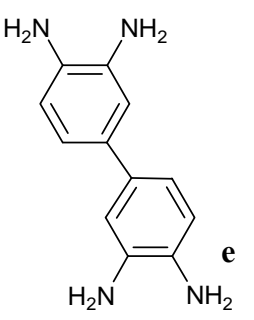

d)

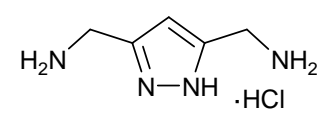

L

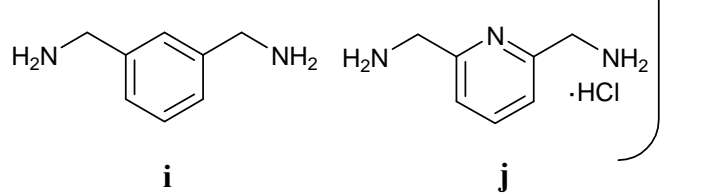

Abbildung 37: Synthese der neuartigen Schiff-Basen Makrozyklen.

Abbildung 38 zeigt alle im Rahmen dieser Arbeit neu synthetisierten Schiff-Base Makrozyklen. Aufgrund der säurekatalytischen Reaktion mit TFA liegen die HybridMakrozyklen nach der Synthese zunächst als TFA-Addukte vor. Durch Neutralisation mit einer Base oder mittels Filtration durch basisches Aluminiumoxid, werden die Makrozyklen als freie Schiff-Basen erhalten. 


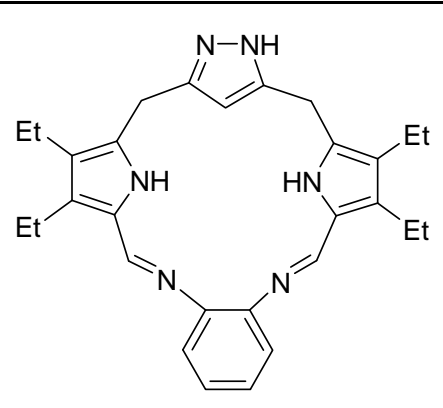

2a
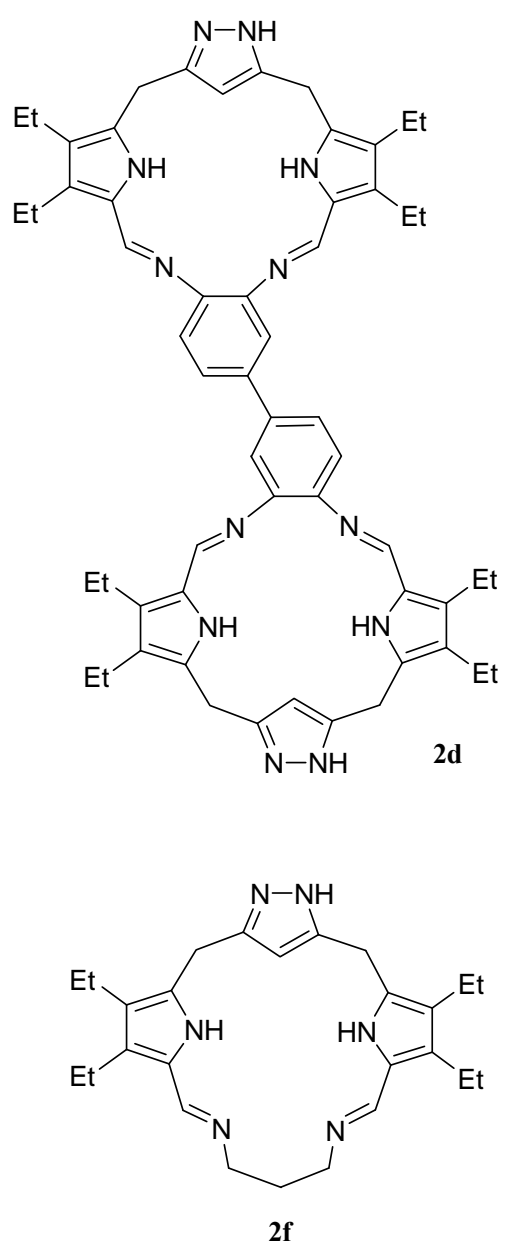

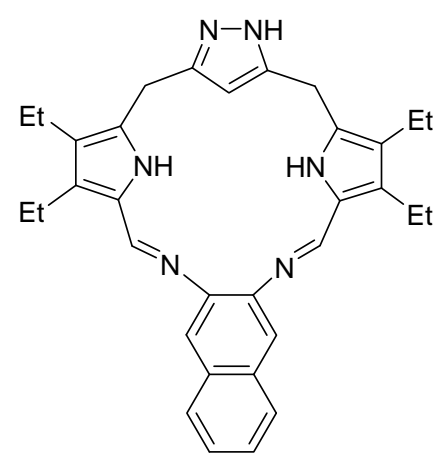

2c

2b

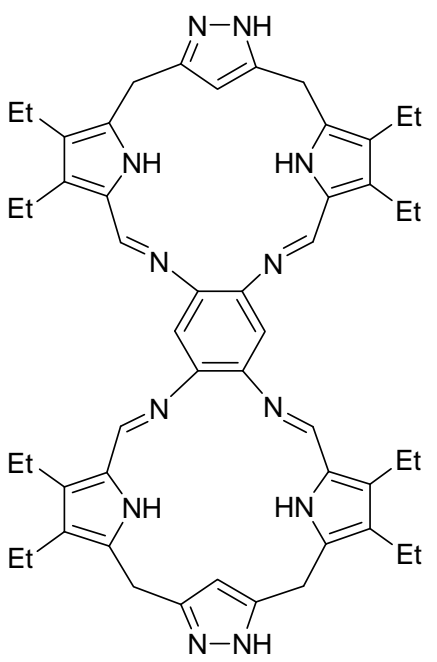

$2 \mathbf{e}$

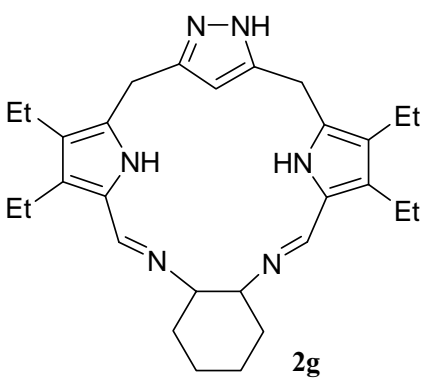

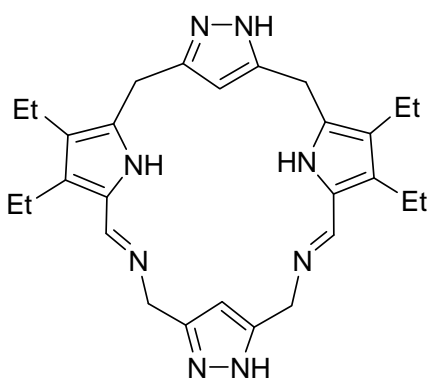

2L

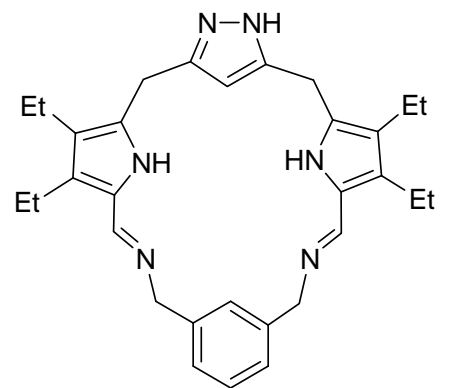

$2 i$

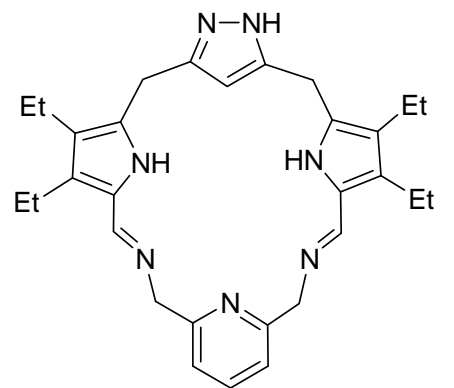

$2 \mathrm{j}$

Abbildung 38: Im Rahmen dieser Arbeit neu synthetisierte Pyrrol/Pyrazol-basierte Schiff-Base Makrozyklen. 
Es hat sich gezeigt, dass je sperriger das substituiere Amin gewählt wird, desto schwerer löslich werden die resultierenden Hybrid-Makrozyklen. Die freie Base 2a weist von allen diesen Makrozyklen die beste Löslichkeit in unpolaren organischen Lösungsmitteln auf. Von 2a zu 2c nimmt die Löslichkeit der Verbindungen jedoch drastisch ab. Die ürsprünglichen TFA-Addukte lösen sich hingegen in DMSO.

Die Schiff-Base Makrozyklen 2d und 2e wurden ausgehend von den Tetraaminen 3,3'Diaminobenzidin bzw. 1,2,4,5-Tetraaminobenzol Tetrahydrochlorid synthetisiert. Die resultierenden Bismakrozyklen (2d und 2e) verfügen durch ihren Aufbau über zwei Taschen potentiellen Wasserstoffbrücken-Bindungsstellen. Die resultierenden biszyklischen Pyrrol/Pyrazol-basierten Schiff-Base Makrozyklen erwiesen sich als schwerlöslich in diversen organischen Lösungsmitteln. Die Aufreinigung von 2e war aufgrund der vorliegenden Tetrahydrochlorid-Form nicht möglich. Die Charakterisierung von 2e erfolgte mit Hilfe der HR-Massenspektrometrie (ESI+). Das beobachtete Signal bei $855.52896 \mathrm{~m} / \mathrm{z}$ für die monoprotonierte Spezies $[\mathrm{M}+\mathrm{H}]^{+}$mit der Summenformel $\mathrm{C}_{52} \mathrm{H}_{63} \mathrm{~N}_{12}$ stimmt gut mit dem berechneten Wert $(855.52932 \mathrm{~m} / \mathrm{z})$ überein.

Neben aromatischen Di- bzw. Tetraaminen wurden in dieser Arbeit auch aliphatische Amine, wie das Diaminopropan (f) und das 1,2-Diaminozyklohexan (g) verwendet. Die Makrozyklen 2f und $\mathbf{2 g}$ tragen entsprechend eine aliphatische Brücke zwischen den Iminfunktionen und weisen damit im Vergleich $\mathrm{zu}$ den aromatischen Chromophoren 2a-2d andere spektroskopischen Eigenschaften aufweisen.

Die Schiff-Base Makrozyklen 2f und 2g wurden in guter Ausbeute erhalten. Säulenchromatographisch konnte 2f nach mehrfacher Aufreinigung isoliert und durch unterschiedliche spektroskopische Methoden charakterisiert werden. 2g dagegen konnte nur mittels ESI-Massenspektrometrie als monoprotoniertes Addukt $[\mathrm{M}+\mathrm{H}]^{+}$bei $474 \mathrm{~m} / \mathrm{z}$ nachgewiesen werden. Säulechromatographisch war es nicht möglich den Makrozyklus 2g aufzureinigen, da die Verbindung auf der Oberfläche der Säule haftet bzw. durch polare Lösungsmittel mit den vorhandenen Verunreinigungen elviert wird.

Die nächste Gruppe neu synthetisierter Makrozyklen stellen die Verbindungen $2 \mathbf{L}, \mathbf{2} \mathbf{i}$ und $\mathbf{2 j}$ dar. Die drei verwendeten Amine sind im Vergleich zu den verwendeten 1,2-Diaminen durch zwei Methylenbrücken erweitert. Durch Verwendung der Amine 3,5-Bis(aminomethyl)pyrazol Dihydrochlorid (L), 2,6-Bis-(aminomethyl)benzol (i), und 2,6-Bis(aminomethyl)pyridin (j) bilden die entsprechenden Verbindungen $\mathbf{2} \mathbf{L}, \mathbf{2} \mathbf{i}$ und $\mathbf{2 j}$ größere Makrozyklen aus und bieten somit mehr Platz für mögliche Metallionen bzw. spezifische Anionen. Das 2,6-Bis-(aminomethyl)pyridin (j) wurde in einem mehrstufigen Syntheseweg 
aus Pyridin-2,6-dicarbonsäure dargestellt. ${ }^{62,63,64,65}$ Die Makrozyklen wurden analog zu den bereits beschriebenen Schiff-Base Hybrid-Makrozyklen synthetisiert. Aufgrund der höheren Flexibilität dieser Spezies gestaltete sich die Isolierung der Makrozyklen $2 \mathbf{L}, \mathbf{2 i}$, und $\mathbf{2 j}$ als unerwartet schwierig. Weder zahlreiche Umkristallisationen noch säulenchromatographische Auftrennungen führten zu reinen Produkten. 2L, 2i und $\mathbf{2 j}$ konnten massenspektrometrisch $(\mathrm{ESI}+)$ nachgewiesen werden, wurden jedoch im Rahmen dieser Arbeit nicht weiter untersucht.

\subsubsection{Strukturell charakterisierte Schiff-Base Makrozyklen}

Mehrere der neu synthetisierten Schiff-Base Makrozyklen konnten röntgenographisch charakterisiert werden. Die Molekülstruktur der freien Base 2a ist in Abbildung 39 gezeigt. 2a kristallisiert in der Raumgruppe $P 2 / c$ mit vier Molekülen in der monoklinen Elementarzelle. Einige ausgewählte Abstände und Winkel von 2a sind in Tabelle 1 aufgelistet. Die Imin-Phenyleinheit des Makrozyklus ist nahezu planar. Die Pyrazoleinheit ist entgegen der Makrozyklus-Kavität gerichtet. Der Pyrazolring steht mit einem Winkel von $79^{\circ}$ zur planaren Imin-Phenyleinheit. Über dem Hohlraum des Makrozyklus 2a befindet sich ein Ethanolmolekül, welches Wasserstoffbrückenbindungen zu der Verbindung ausbildet. Das Ethanol dient dabei interessanterweise sowohl als Wasserstoffbrücken-Donor als auch als -Akzeptor. In Tabelle 2 sind die wichtigsten Bindungslängen und Abstände der Wasserstoffbrückenbindungen aufgelistet. Das Sauerstoffatom (O1) dient als Wasserstoffbrückenbindungs-Akzeptor zu den Pyrrol-NH Protonen (H3 bzw. H6) und das Ethanol O-H10 als Wasserstoffbrücken-Donor zu den Imin-N Atomen (N4 bzw. N5). Die Abstände von Donor zu Akzeptor betragen ca. 2.80 Å. Ein weiteres Ethanolmolekül bildet Wasserstoffbrückenbindungen mit der freistehenden Pyrazoleinheit des Makrozyklus aus. Durch Ausbildung von Wasserstoffbrückenbindungen zu zwei weiteren Ethanolmolekülen in der Elementarzelle kommt es zur Bildung einer dimeren Einheit von 2a (Abbildung 39). 

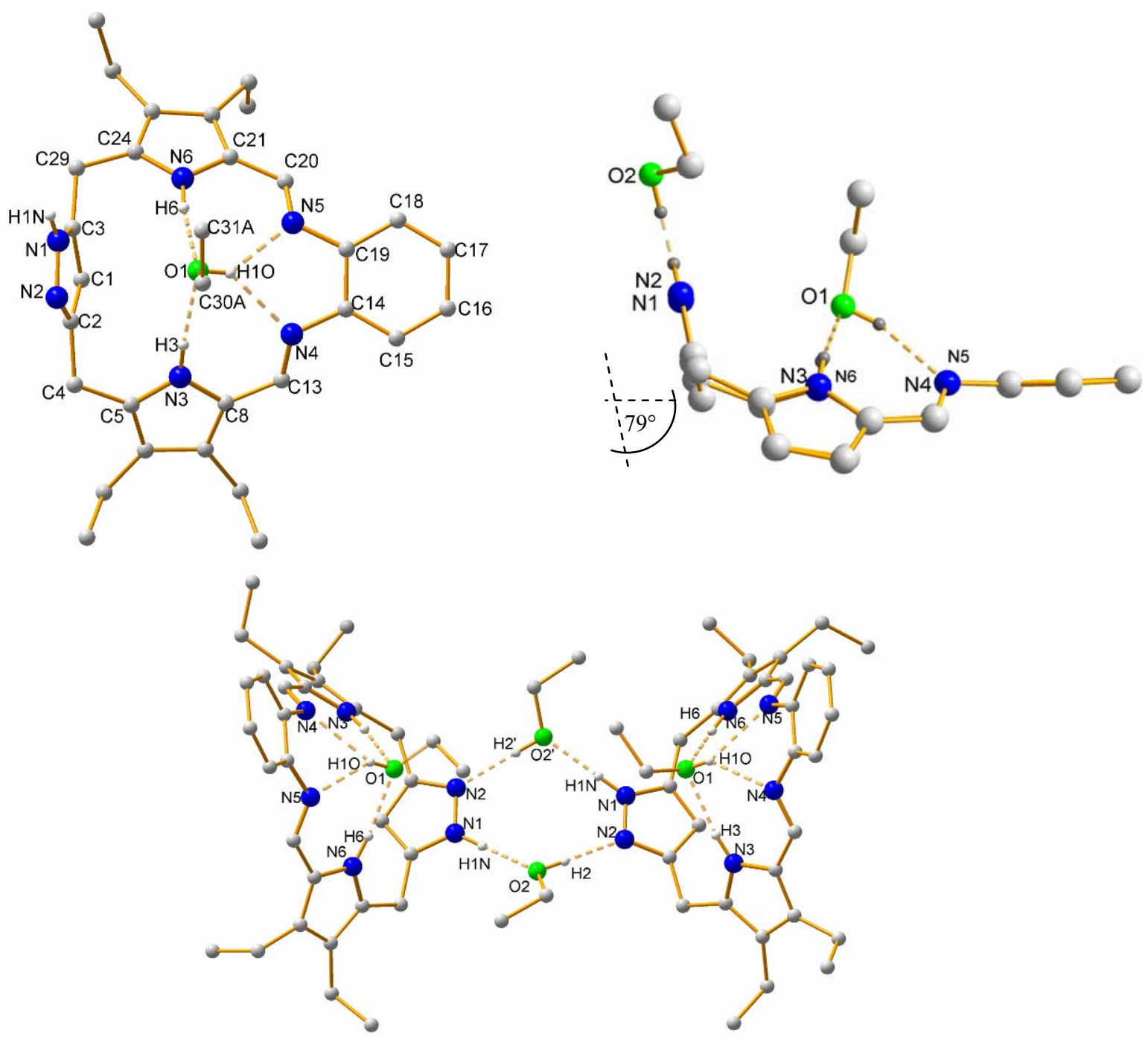

Abbildung 39: Molekülstruktur des neutralen Schiff-Base Makrozyklus [2a·EtOH] (oben links), Seitenansicht (oben rechts) und Dimerstruktur verbrückt über Wasserstoffbrückenbindungen (unten).

Tabelle 1: Ausgewählte Abstände und Winkel von [2a·EtOH].

Abstände $[\AA]$

\begin{tabular}{ll|ll}
\hline $\mathrm{N}(4)-\mathrm{C}(13)$ & $1.283(3)$ & $\mathrm{C}(3)-\mathrm{C}(29)$ & $1.507(3)$ \\
$\mathrm{N}(4)-\mathrm{C}(14)$ & $1.407(3)$ & $\mathrm{C}(4)-\mathrm{C}(5)$ & $1.501(3)$ \\
$\mathrm{N}(5)-\mathrm{C}(19)$ & $1.416(3)$ & $\mathrm{C}(8)-\mathrm{C}(13)$ & $1.429(3)$ \\
$\mathrm{N}(5)-\mathrm{C}(20)$ & $1.278(3)$ & $\mathrm{C}(20)-\mathrm{C}(21)$ & $1.437(3)$ \\
$\mathrm{C}(2)-\mathrm{C}(4)$ & $1.503(3)$ & $\mathrm{C}(24)-\mathrm{C}(29)$ & $1.505(3)$ \\
\hline \multicolumn{3}{|c|}{ Winkel $\left[{ }^{\circ}\right]$} & $120.81(18)$ \\
\hline \multicolumn{2}{|c|}{} & $\mathrm{C}(20)-\mathrm{N}(5)-\mathrm{C}(19)$ & $113.43(18)$ \\
\hline
\end{tabular}


Tabelle 2: Ausgewählte Abstände $(\AA)$ und Winkel $\left(^{\circ}\right)$ der Wasserstoffbrückenbindungen von $[\mathbf{2 a} \cdot \mathbf{E t O H}](\mathrm{D}$ : Donor, A: Akzeptor).

\begin{tabular}{lllll}
\hline $\mathrm{D}-\mathrm{H} \cdots \mathrm{A}$ & $\mathrm{d}(\mathrm{D}-\mathrm{H})$ & $\mathrm{d}\left(\mathrm{H}^{\cdots} \mathrm{A}\right)$ & $\mathrm{d}(\mathrm{D} \cdots \mathrm{A})$ & $<(\mathrm{DHA})$ \\
\hline $\mathrm{N}(1)-\mathrm{H}(1 \mathrm{~N}) \cdots \mathrm{O}\left(2^{\prime}\right) 1$ & $0.92(3)$ & $1.86(3)$ & $2.783(2)$ & $179(2)$ \\
$\mathrm{O}(1)-\mathrm{H}(1 \mathrm{O}) \cdots \mathrm{N}(5)$ & $0.82(3)$ & $2.25(3)$ & $2.934(2)$ & $141(3)$ \\
$\mathrm{O}(1)-\mathrm{H}(1 \mathrm{O}) \cdots \mathrm{N}(4)$ & $0.82(3)$ & $2.27(3)$ & $2.962(2)$ & $143(3)$ \\
$\mathrm{O}(2)-\mathrm{H}(2) \cdots \mathrm{N}(2)$ & $0.87(3)$ & $1.91(3)$ & $2.778(2)$ & $179(3)$ \\
$\mathrm{N}(3)-\mathrm{H}(3) \cdots \mathrm{O}(1)$ & $0.92(3)$ & $1.99(3)$ & $2.906(2)$ & $172(3)$ \\
$\mathrm{N}(6)-\mathrm{H}(6) \cdots \mathrm{O}(1)$ & $0.94(3)$ & $1.99(3)$ & $2.920(2)$ & $171(2)$ \\
\end{tabular}

Wird das Rohprodukt von 2a nach der Darstellung direkt und ohne basische säulenchromatographische Reinigung aus Dichlormethan umkristallisiert, werden Kristalle von 2a als protoniertes TFA-Addukt erhalten. In Abbildung 40 wird die Molekülstruktur von [2a·TFA] gezeigt. Die Verbindung kristallisiert in der Raumgruppe $P \overline{1}$ mit zwei Molekülen in der triklinen Zelle. In Tabelle 3 sind die wichtigsten Bindungsabstände und Winkel von [2a·TFA] aufgelistet. Über der Makrozyklus-Kavität ist ein deprotoniertes TFA-Molekül fixiert, welches zwei Wasserstoffbrückenbindungen mit den Pyrrol-NH Protonen (H6 bzw. H3) ausbildet. Eine dritte Wasserstoffbrücke existiert zwischen $\mathrm{O} 1$ und dem protonierten

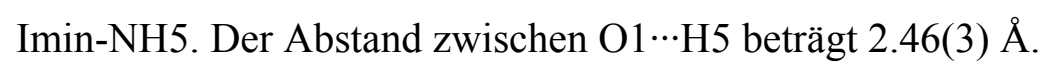
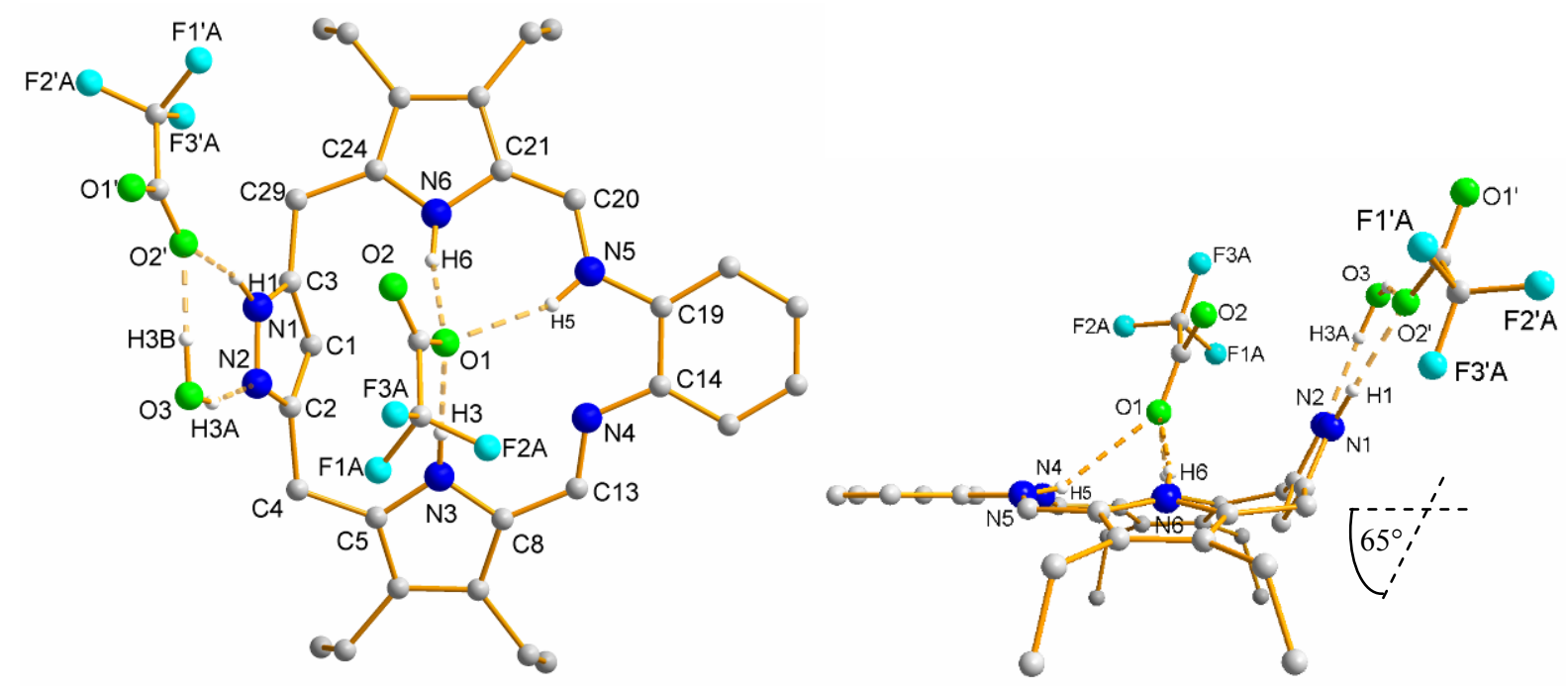

Abbildung 40: Molekülstruktur des protonierten Schiff-Base Makrozyklus [2a·TFA] (links) und Seitenansicht (rechts). 
Tabelle 3: Ausgewählte Abstände und Winkel von [2a·TFA].

\begin{tabular}{ll|ll}
\multicolumn{5}{c}{ Abstände $[\AA]$} \\
$\mathrm{N}(4)-\mathrm{C}(13)$ & $1.290(3)$ & $\mathrm{C}(3)-\mathrm{C}(29)$ & $1.491(4)$ \\
$\mathrm{N}(4)-\mathrm{C}(14)$ & $1.412(3)$ & $\mathrm{C}(4)-\mathrm{C}(5)$ & $1.505(4)$ \\
$\mathrm{N}(5)-\mathrm{C}(19)$ & $1.416(3)$ & $\mathrm{C}(8)-\mathrm{C}(13)$ & $1.425(4)$ \\
$\mathrm{N}(5)-\mathrm{C}(20)$ & $1.319(3)$ & $\mathrm{C}(20)-\mathrm{C}(21)$ & $1.392(4)$ \\
$\mathrm{C}(2)-\mathrm{C}(4)$ & $1.498(4)$ & $\mathrm{C}(24)-\mathrm{C}(29)$ & $1.500(4)$ \\
\hline \multicolumn{3}{|c|}{$\mathrm{Winkel} \mathrm{[{ } ^ { \circ } ]}$} & $121.6(2)$ \\
\hline $\mathrm{C}(2)-\mathrm{C}(4)-\mathrm{C}(5)$ & $\mathrm{C}(13)-\mathrm{N}(4)-\mathrm{C}(14)$ & $127.8(2)$ \\
$\mathrm{C}(3)-\mathrm{C}(29)-\mathrm{C}(24)$ & $115.7(2)$ & $\mathrm{C}(20)-\mathrm{N}(5)-\mathrm{C}(19)$ & \\
\hline
\end{tabular}

Ausgewählte Winkel und Bindungsabstände der Wasserstoffbrückenbindungen sind in Tabelle 4 wiedergegeben. Es zeigt sich, dass die Wasserstoffbrückenbindungen, ausgehend von O1 des TFA-Moleküls, ungleich sind (Tabelle 4). Der kürzeste Abstand (D‥A) wurde bei N6-H6…O1 mit $2.82 \AA$ gefunden. Die zweite Wasserstoffbrückenbindung, welche aus den N3H3-Pyrrrol-Atomen zum O1 des TFA-Moleküls ausgebildet wird ist etwas länger (2.93 $)$. Die längste Wasserstoffbrückenbindung, welche vom O1 des TFA-Moleküls ausgebildet wird, wurde mit $3.24 \AA$ zu der protoniertem N5H5-Iminposition gefunden.

Die protonierte Imin-Bindung (N4-C20) ist mit $1.32 \AA$ länger als die unprotonierte N4-C13 Bindung mit $1.29 \AA$. Die Bindungslängen der Imine zeigen, dass nur eine Seite des Makrozyklus protoniert wurde.

Tabelle 4: Ausgewählte Abstände $(\AA)$ und Winkel $\left(^{\circ}\right)$ der Wasserstoffbrückenbindungen von $[\mathbf{2 a} \cdot \mathbf{T F A}]$. (D: Donor, A: Akzeptor).

\begin{tabular}{lllll}
\hline $\mathrm{D}-\mathrm{H} \cdots \mathrm{A}$ & $\mathrm{d}(\mathrm{D}-\mathrm{H})$ & $\mathrm{d}(\mathrm{H} \cdots \mathrm{A})$ & $\mathrm{d}(\mathrm{D} \cdots \mathrm{A})$ & $<(\mathrm{DHA})$ \\
\hline $\mathrm{N}(1)-\mathrm{H}(1) \cdots \mathrm{O}\left(2^{\prime}\right)$ & $0.94(4)$ & $1.92(4)$ & $2.838(3)$ & $164(3)$ \\
$\mathrm{N}(3)-\mathrm{H}(3) \cdots \mathrm{O}(1)$ & $0.87(3)$ & $2.07(4)$ & $2.931(3)$ & $170(3)$ \\
$\mathrm{N}(5)-\mathrm{H}(5) \cdots \mathrm{O}(1)$ & $0.90(3)$ & $2.46(3)$ & $3.242(3)$ & $145(2)$ \\
$\mathrm{N}(5)-\mathrm{H}(5) \cdots \mathrm{N}(4)$ & $0.90(3)$ & $2.11(3)$ & $2.605(3)$ & $113(2)$ \\
$\mathrm{N}(6)-\mathrm{H}(6) \cdots \mathrm{O}(1)$ & $0.96(4)$ & $1.88(4)$ & $2.824(3)$ & $166(3)$ \\
$\mathrm{O}(3)-\mathrm{H}(3 \mathrm{~A}) \cdots \mathrm{N}(2)$ & $0.95(5)$ & $1.99(5)$ & $2.912(3)$ & $164(4)$ \\
$\mathrm{O}(3)-\mathrm{H}(3 \mathrm{~B}) \cdots \mathrm{O}\left(2^{\prime}\right)$ & $1.01(5)$ & $1.78(5)$ & $2.749(3)$ & $161(4)$ \\
\hline
\end{tabular}


Beim Vergleich der Molekülstrukturen von [2a·EtOH] und [2a·TFA], wird festgestellt, dass sich die Konformation des Makrozyklus nach der Protonierung ändert. Durch die Protonierung erhält die Pyrrol-Imin-Einheit eine planarisierte Konformation. Der Pyrazolring steht mit einem Winkel von $65^{\circ}$ zur planaren Imin-Phenyleinheit. Diese Konformationsänderung wird in den folgenden Kapiteln mittels unterschiedlicher spektroskopischer Methoden untersucht.

In dieser Arbeit ist es gelungen auch weitere Schiff-Base Makrozyklen erfolgreich zu kristallisieren. Nach Synthese von 2b wurde der Rückstand der Reaktion in $\mathrm{MeOH} / \mathrm{Et}_{2} \mathrm{O}$ bei $5{ }^{\circ} \mathrm{C}$ umkristallisiert. Das Produkt [2b-TFA] wurde in Form kupfer-roter Kristalle erhalten. [2b·TFA] kristallisiert analog zu [2a·TFA] in der Raumgruppe $P \overline{1}$ mit zwei Molekülen in der triklinen Zelle (Abbildung 41). Auch hier ist ein TFA-Molekül über der Makrozyklus-Kavität fixiert und bildet Wasserstoffbrückenbindungen zu den Pyrrol-NH (H3 bzw. H6) und zur protonierten Imin-Position (NH4) aus. Obwohl TFA bei der Makrozyklussynthese im starken Überschuss eingesetzt wird, wird die Verbindung nur an einer Imin-Position protoniert. Die N4-C13-Bindung ist auch hier mit $1.32 \AA$ entprechend länger als die unprotonierte IminBindung N5-C22 mit 1.28 Å. Ausgewählte Abstände und Winkel von [2b·TFA] und den ausgebildeten Wasserstoffbrückenbindungen sind in Tabelle 5 bzw. Tabelle 6 zusammengefasst. Entsprechend der Molekülstruktur von [2a·TFA] befinden sich in der Elementarzelle weitere TFA- bzw. Wassermoleküle, die durch Wasserstoffbrückenbindungen zu der Ausbildung dimerer [2b·TFA] Einheiten führen (Abbildung 41 unten). Der Pyrazolring steht in diesen Einheit mit einem Winkel von $68^{\circ}$ zur planaren Imin-Phenyleinheit. Die Änderung dieses Winkels in den Makrozyklen weist darauf hin, dass das System über eine gewisse Flexibilität verfügt und relativ frei um einen bestimmten Winkel rotieren kann.

Durch Neutralisation von [2b·TFA] und anschließende Umkristallisation aus einer gesättigten $\mathrm{MeOH} / \mathrm{CH}_{2} \mathrm{Cl}_{2} / \mathrm{Et}_{3} \mathrm{~N}$-Lösung unter langsamem Abdampfen der Lösungsmittel konnten nach einigen Tagen gelbe Kristalle von der freien Base 2b erhalten werden. Aufgrund der mangelhaften Qualität der Kristalle konnte die Molekülstruktur der neutralen Verbindung 2b nicht vollständig aufgeklärt werden. Dennoch konnte aus den gemessenen Daten entnommen werden, dass es sich hierbei um die freie Base handelt und analog zu 2a ein MethanolMolekül über dem Hohlraum des Makrozyklus platziert ist. 

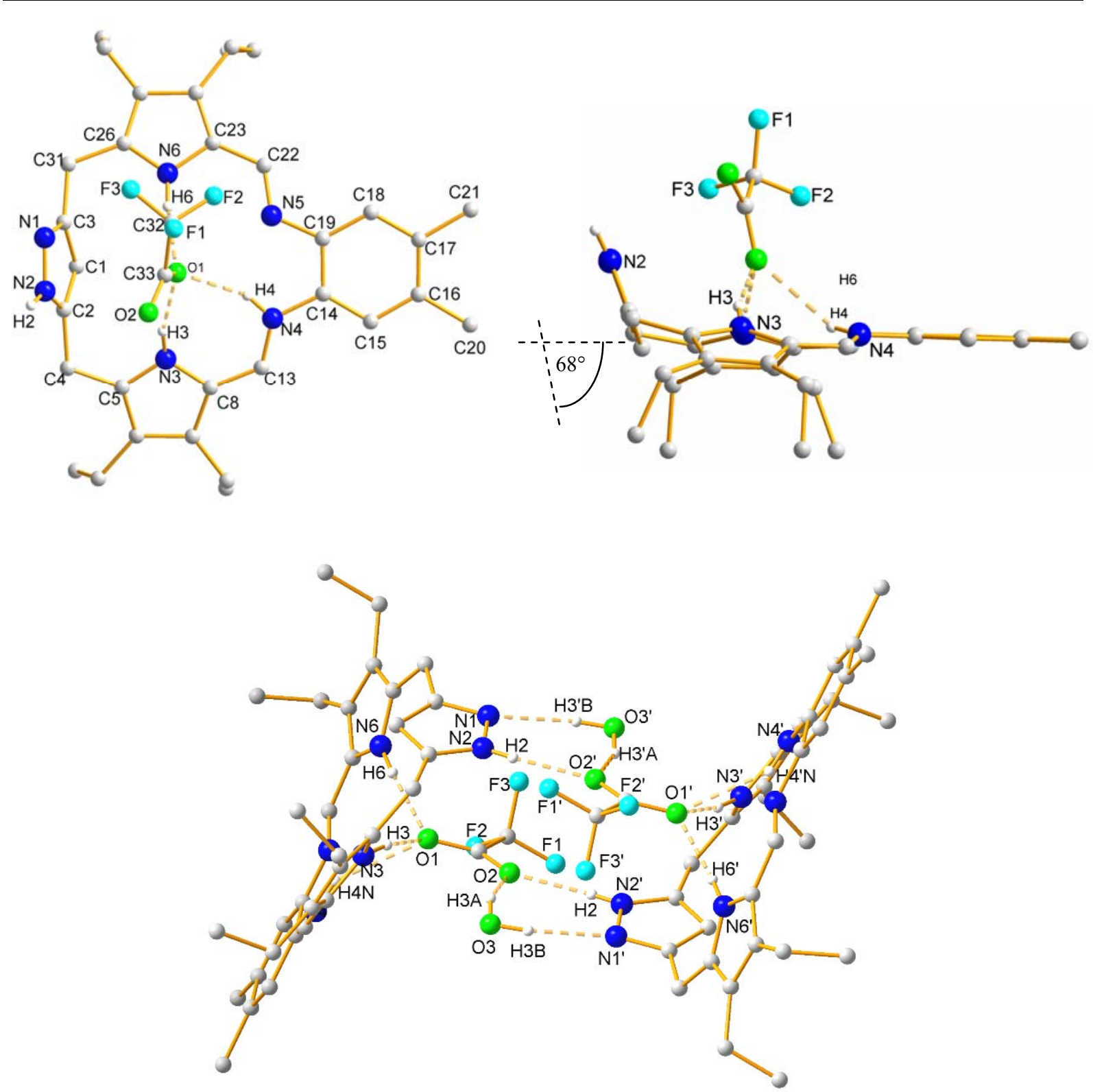

Abbildung 41: Molekülstruktur von [2b-TFA] (oben links) und Seitenansicht (oben rechts). Wasserstoffbrückenbindungen der dimeren [2b-TFA] Einheit zu je einem Wasser- und einem TFA-Molekül (unten).

Tabelle 5: Ausgewählte Abstände und Winkel von [2b·TFA].

Abstände $[\AA]$

\begin{tabular}{ll|ll}
\hline $\mathrm{N}(4)-\mathrm{C}(13)$ & $1.320(4)$ & $\mathrm{C}(3)-\mathrm{C}(31)$ & $1.497(5)$ \\
$\mathrm{N}(4)-\mathrm{C}(14)$ & $1.418(4)$ & $\mathrm{C}(4)-\mathrm{C}(5)$ & $1.498(5)$ \\
$\mathrm{N}(5)-\mathrm{C}(19)$ & $1.408(4)$ & $\mathrm{C}(8)-\mathrm{C}(13)$ & $1.388(5)$ \\
$\mathrm{N}(5)-\mathrm{C}(22)$ & $1.284(4)$ & $\mathrm{C}(22)-\mathrm{C}(23)$ & $1.417(4)$ \\
$\mathrm{C}(2)-\mathrm{C}(4)$ & $1.502(5)$ & $\mathrm{C}(26)-\mathrm{C}(31)$ & $1.512(5)$ \\
\hline
\end{tabular}




\begin{tabular}{ll|ll}
\hline \multicolumn{4}{c}{ Winkel [ $\left.{ }^{\circ}\right]$} \\
\hline $\mathrm{C}(3)-\mathrm{C}(31)-\mathrm{C}(26)$ & $115.3(3)$ & $\mathrm{C}(13)-\mathrm{N}(4)-\mathrm{C}(14)$ & $127.7(3)$ \\
$\mathrm{C}(5)-\mathrm{C}(4)-\mathrm{C}(2)$ & $114.9(3)$ & $\mathrm{C}(22)-\mathrm{N}(5)-\mathrm{C}(19)$ & $122.2(3)$ \\
\hline
\end{tabular}

Tabelle 6: Ausgewählte Abstände $(\AA)$ und Winkel $\left({ }^{\circ}\right)$ der Wasserstoffbrückenbindungen von [2b·TFA] (D: Donor, A: Akzeptor).

\begin{tabular}{lcccc}
\hline $\mathrm{D}-\mathrm{H} \cdots \mathrm{A}$ & $\mathrm{d}(\mathrm{D}-\mathrm{H})$ & $\mathrm{d}(\mathrm{H} \cdots \mathrm{A})$ & $\mathrm{d}(\mathrm{D} \cdots \mathrm{A})$ & $<(\mathrm{DHA})$ \\
\hline $\mathrm{N}(2)-\mathrm{H}(2 \mathrm{~N}) \cdots \mathrm{O}\left(2^{\prime}\right)$ & $0.82(5)$ & $2.07(5)$ & $2.878(4)$ & $167(5)$ \\
$\mathrm{N}(3)-\mathrm{H}(3 \mathrm{~N}) \cdots \mathrm{O}(1)$ & $0.88(4)$ & $1.93(5)$ & $2.789(4)$ & $165(4)$ \\
$\mathrm{N}(4)-\mathrm{H}(4 \mathrm{~N}) \cdots \mathrm{O}(1)$ & $0.88(4)$ & $2.40(4)$ & $3.176(4)$ & $146(3)$ \\
$\mathrm{N}(6)-\mathrm{H}(6 \mathrm{~N}) \cdots \mathrm{O}(1)$ & $0.91(4)$ & $2.05(4)$ & $2.950(4)$ & $168(3)$ \\
$\mathrm{O}(3)-\mathrm{H}(3 \mathrm{~A}) \cdots \mathrm{O}(2)$ & $0.93(2)$ & $1.88(3)$ & $2.752(4)$ & $155(6)$ \\
$\mathrm{O}(3)-\mathrm{H}(3 \mathrm{~B}) \cdots \mathrm{N}\left(1^{\prime}\right)$ & $0.93(2)$ & $2.20(3)$ & $3.056(4)$ & $154(5)$ \\
\hline
\end{tabular}

Durch langsames Abdampfen einer gesättigten $\mathrm{MeOH} / \mathrm{CH}_{2} \mathrm{Cl}_{2}$ Lösung konnten nach einigen Tagen Einkristalle von 2c erhalten und röntgenographisch analysiert werden. Der Schiff-Base Makrozyklus 2c kristallisiert wie die anderen Makrozyklen als TFA-Addukt in der der Raumgruppe $P^{\overline{1}}$ mit zwei Molekülen in der triklinen Zelle. Die Festkörperstruktur von [2c·TFA] ist in Abbildung 42 gezeigt. Ausgewählte Abstände bzw. Winkel werden in Tabelle 7 aufgelistet.

In diesem System wird ebenfalls eine Monoprotonierung an einer der Imin-Positionen (N4H4) erfasst. Die protonierte Imin-Bindung N4-C13 hat eine Länge von 1.32(6) $\AA$ und die entsprechende unprotonierte Imin-Bindung N5-C24 eine Länge von 1.31(6) Å. Erneut befindet sich ein deprotoniertes TFA-Molekül über dem Makrozyklus-Hohlraum und bildet Wasserstoffbrückenbindungen zu den Pyrrol-NH Protronen (NH3 bzw. NH6) aus. 

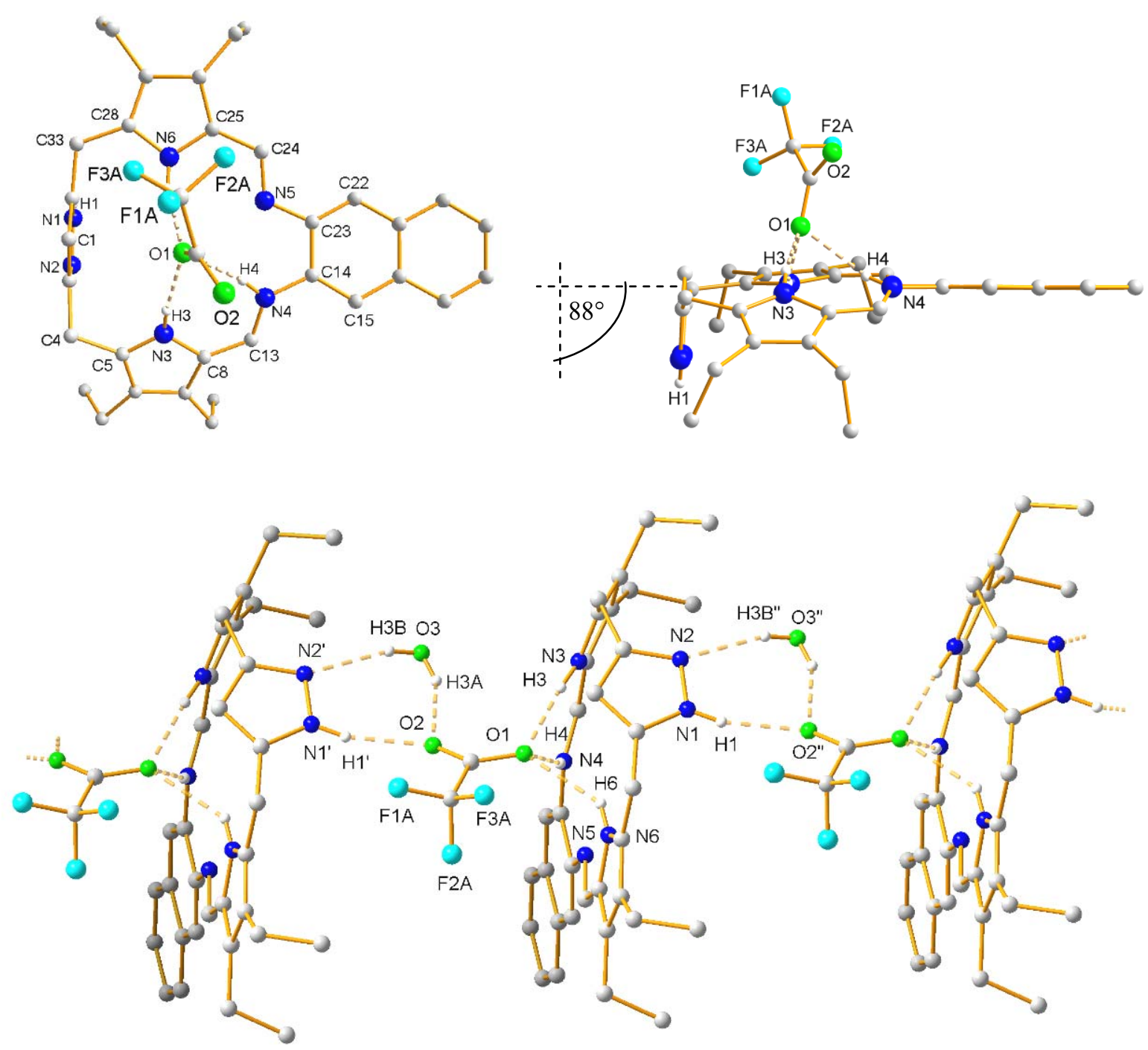

Abbildung 42: Molekülstruktur von [2c·TFA] (oben links) und Seitenansicht (oben rechts). Ausbildung von TFA und Wasser verbrückter Ketten aus 2c durch Wasserstoffbrückenbindungen (unten).

Die Abstände und Winkel der Wasserstoffbrückenbindungen in der Elementarzelle von [2c-TFA] werden in Tabelle 8 aufgeführt. Wie bei den anderen TFA-Addukten sind auch hier die Abstände der Wasserstoffbrückenbindungen des deprotonierten TFA O1-Atoms zu den drei H-Donoren ungleich groß. Durch Wasser- und TFA-Moleküle in der Elementarzelle kommt es zur Ausbildung von Ketten (Abbildung 42 unten).

Die N-Atome des Pyrazols befinden sich auch in diesem Molekül außerhalb der Kavität und der Pyrazolring steht insgesamt mit einem Winkel von $88^{\circ}$ zur planaren Imin-Naphtyleinheit. 
Tabelle 7: Ausgewählte Abstände und Winkel von [2c·TFA].

\begin{tabular}{|c|c|c|c|}
\hline \multicolumn{4}{|c|}{ Abstände $[\AA]$} \\
\hline $\mathrm{N}(4)-\mathrm{C}(13)$ & $1.321(6)$ & $C(3)-C(4)$ & $1.499(6)$ \\
\hline $\mathrm{N}(4)-\mathrm{C}(14)$ & $1.423(6)$ & $C(4)-C(5)$ & $1.510(7)$ \\
\hline $\mathrm{N}(5)-\mathrm{C}(23)$ & $1.419(6)$ & $\mathrm{C}(8)-\mathrm{C}(13)$ & $1.396(7)$ \\
\hline $\mathrm{N}(5)-\mathrm{C}(24)$ & $1.307(6)$ & $C(24)-C(25)$ & $1.436(6)$ \\
\hline $\mathrm{C}(2)-\mathrm{C}(33)$ & $1.490(7)$ & $\mathrm{C}(28)-\mathrm{C}(33)$ & $1.503(7)$ \\
\hline \multicolumn{4}{|c|}{ Winkel $\left[^{\circ}\right]$} \\
\hline$C(3)-C(4)-C(5)$ & $110.7(4)$ & $C(13)-N(4)-C(14)$ & $126.4(4)$ \\
\hline $\mathrm{C}(2)-\mathrm{C}(33)-\mathrm{C}(28)$ & $114.4(4)$ & $\mathrm{C}(24)-\mathrm{N}(5)-\mathrm{C}(23)$ & $119.6(4)$ \\
\hline
\end{tabular}

Tabelle 8: Ausgewählte Abstände $(\AA \AA)$ und Winkel $\left(^{\circ}\right)$ der Wasserstoffbrückenbindungen von [2c·TFA]. (D: Donor, A: Akzeptor).

\begin{tabular}{lllll}
\hline $\mathrm{D}-\mathrm{H} \cdots \mathrm{A}$ & $\mathrm{d}(\mathrm{D}-\mathrm{H})$ & $\mathrm{d}(\mathrm{H} \cdots \mathrm{A})$ & $\mathrm{d}(\mathrm{D} \cdots \mathrm{A})$ & $<(\mathrm{DHA})$ \\
\hline $\mathrm{N}(1)-\mathrm{H}(1) \cdots \mathrm{O}\left(2^{\prime}\right)$ & $0.854(10)$ & $2.07(2)$ & $2.871(4)$ & $156(5)$ \\
$\mathrm{N}(3)-\mathrm{H}(3) \cdots \mathrm{O}(1)$ & $0.853(10)$ & $1.888(13)$ & $2.738(6)$ & $175(6)$ \\
$\mathrm{N}(4)-\mathrm{H}(4) \cdots \mathrm{O}(1)$ & $0.854(10)$ & $2.21(2)$ & $3.010(5)$ & $156(5)$ \\
$\mathrm{N}(4)-\mathrm{H}(4) \cdots \mathrm{N}(5)$ & $0.854(10)$ & $2.23(6)$ & $2.633(6)$ & $109(4)$ \\
$\mathrm{N}(6)-\mathrm{H}(6) \cdots \mathrm{O}(1)$ & $0.849(10)$ & $2.24(3)$ & $2.999(5)$ & $148(5)$ \\
$\mathrm{O}(3)-\mathrm{H}(3 \mathrm{~B}) \cdots \mathrm{N}\left(2^{\prime}\right)$ & $0.823(10)$ & $2.21(2)$ & $3.002(5)$ & $162(5)$ \\
$\mathrm{O}(3)-\mathrm{H}(3 \mathrm{~A}) \cdots \mathrm{O}(2)$ & $0.822(10)$ & $2.09(3)$ & $2.840(5)$ & $152(4)$ \\
\hline
\end{tabular}

Abschließend werden die röntgenographisch charakterisierten Pyrrol/Pyrazol HybridMakrozyklen in einer Übersichtstabelle (Tabelle 9) miteinander verglichen. Die Besonderheit in diesen Systemen stellt die Monoprotonierung der Imin-Position dar. In den röntgenographisch charakterisierten TFA-Addukten von 2a-2c kann eindeutig die protonierte $\mathrm{C}=\mathrm{N}-\mathrm{H}-$ Position ermittelt werden. Wie aus der Tabelle $9 \mathrm{zu}$ entnehmen ist, verlängert sich die $\mathrm{C}=\mathrm{N}-$ Iminbindung nur geringfügig nach der Protonierung mit TFA. Solche ProtonierungsAddukte sind nicht nur in azyklischen Systemen ${ }^{66}$, sondern auch in verwandte Schiff-Basen Makrozyklen bekannt. ${ }^{29,67,68}$ Ähnlich wie in dieser Arbeit sind die Bindungsabstände der protonierten Imin-Gruppen in literaturbekannten Schiff-Base-Derivaten zwischen 1.28 - 
$1.32 \AA$ lang. Die Bindungslängen der nicht protonierten Imin-Gruppen in den gleichen Systemen sind analog zu den hier ermittelten Imin-Abständen. ${ }^{29,66-68}$

Tabelle 9: Übersichtstabelle der Bindungslängen der Imin-Positionen bzw. Winkel zwischen dem Pyrazolring und der planaren Imin-Phenyleinheit der röntgenographisch charakterisierten Schiff-Base Makrozyklen.

\begin{tabular}{|c|c|c|c|}
\hline Verbindung & $\begin{array}{c}\operatorname{Imin} 1 \\
[\AA]]\end{array}$ & $\begin{array}{c}\operatorname{Imin} 2 \\
{[\AA]}\end{array}$ & $\begin{array}{l}\text { Winkel zwischen } \\
\text { Pyrazolring und der } \\
\text { planaren Imin- } \\
\left.\text { Phenyleinheit }{ }^{\circ}{ }^{\circ}\right]\end{array}$ \\
\hline 2a.EtOH & $\begin{array}{c}\mathrm{N}(4)-\mathrm{C}(13) \\
1.283(3)\end{array}$ & $\begin{array}{c}\mathrm{N}(5)-\mathrm{C}(20) \\
1.278(3)\end{array}$ & 79 \\
\hline 2a.TFA & $\begin{array}{c}\mathrm{N}(4)-\mathrm{C}(13) \\
1.290(3)\end{array}$ & $\begin{array}{c}\mathrm{N}(5)-\mathrm{C}(20) \\
1.319(3)\end{array}$ & 65 \\
\hline $2 \mathrm{~b} \cdot \mathrm{TFA}$ & $\begin{array}{c}\mathrm{N}(4)-\mathrm{C}(13) \\
1.320(4)\end{array}$ & $\begin{array}{c}\mathrm{N}(5)-\mathrm{C}(22) \\
1.284(4)\end{array}$ & 68 \\
\hline 2c.TFA & $\begin{array}{c}\mathrm{N}(4)-\mathrm{C}(13) \\
1.321(6)\end{array}$ & $\begin{array}{c}\mathrm{N}(5)-\mathrm{C}(24) \\
1.307(6)\end{array}$ & 88 \\
\hline
\end{tabular}

Aus den Daten der röntgenographisch charakterisierten Schiff-Base Makrozyklen kann entnommen werden, dass das Pyrazolring im System zwischen den Methylenbrücken rotieren kann. Die Änderung dieses Winkels $\left(65-88^{\circ}\right)$ in den Schiff-Base Makrozyklen weist darauf hin, dass der Pyrazolring über eine gewisse Flexibilität verfügt und relativ frei um einen bestimmten Winkel rotieren kann.

Die Molekülstrukturen der TFA-Addukte (2a-2c) zeigen, dass eins der O-Atome des Trifluoracetates Wasserstoffbrücken zu dem protonierten Imin bzw. zu den Pyrrol-NH ausbilden kann. Die Abstände der Wasserstoffbrückenbindungen $(\mathrm{d}(\mathrm{H} \cdots \mathrm{A}))$ zum Anion haben eine Länge von 1.89-2.46 ppm und stimmen gut mit dem in der Literatur gefundene Werte überein. ${ }^{67}$ 


\subsubsection{UV/Vis-Untersuchungen der neuartigen Schiff-Base Makrozyklen}

Die freien Basen der dargestellten Makrozyklen zeigen eine gelbe Farbe, während die TFAAddukte der entsprechenden Zyklen eine rote bis rot-braune Färbung aufweisen. Diese interessante Eigenschaft der nicht aromatischen Makrozyklen wurde mit Hilfe der UV/VisSpektroskopie untersucht.

$$
\mathrm{Mz}+\mathrm{HX} \rightleftharpoons \mathrm{MzH}^{+}+\mathrm{X}^{-}
$$

Wie die allgemeine Reaktionsgleichung oben zeigt, konnten die freien Schiff-Base Makrozyklen (Mz) nach Zugabe von Säuren unterschiedlicher Stärke $[\mathrm{pK} a=0.5$ bis 6.0] (TFA, $\mathrm{HCl}, \mathrm{H}_{2} \mathrm{SO}_{4}$, Dimethylphosphat, Zitronensäure, 3-Hydoxypropionsäure, Glykolsäure, Pentafluorophenol ect.) protoniert werden. Hierbei schlug die gelbe Farbe der Lösung in rot um:

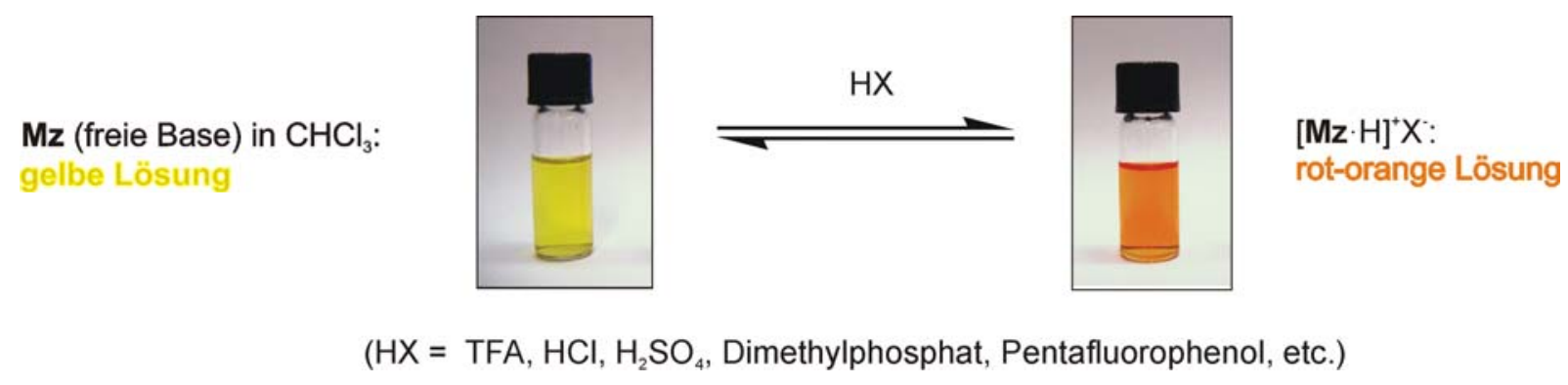

In Abbildung 43 sind die UV/Vis-Spektren sowohl der freien Basen von 2a, 2b, 2c, 2f, 2d und 2e als auch jene der mit TFA protonierten Spezies (gemessen in Chloroform) gezeigt. Alle Makrozyklen weisen eine scharfe Bande zwischen 312-366 nm auf, gefolgt von einer langwelligen Schulter mit schwächerer Intensität. 2f hat als einziger hier gezeigter Makrozyklus einen aliphatischen Imin-Teil. Im UV/Vis-Spektrum zeigt $2 \mathbf{f}$ eine Bande bei $312 \mathrm{~nm}$ und eine Schulter bei $351 \mathrm{~nm}$. Diese Banden sind auf $\pi \rightarrow \pi^{*}$ Übergange der

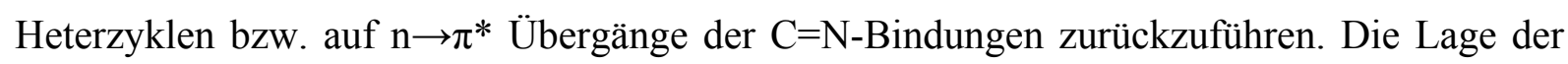
scharfen Bande ist vom Aufbau der Imin-Phenyl-Einheit abhängig. Je ausgeweiteter das aromatische System des Imin-Phenyl-Chromophors ist, desto stärker ist die bathochrome Verschiebung der Absorption. Tabelle 10 zeigt eine Übersicht der Absorptionen der hier untersuchten Makrozyklen und ihrer entsprechenden Extinktionskoeffizienten $(\varepsilon)$. Die zwei biszyklischen Systeme 2d und 2e haben aufgrund des Vorliegens zweier Chromophore erwartungsgemäß den höchsten Extinktionskoeffizienten. 


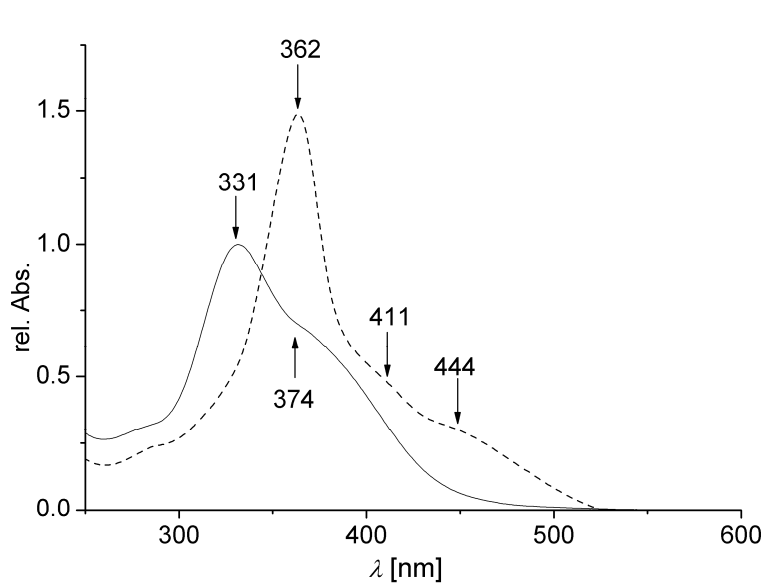

2a

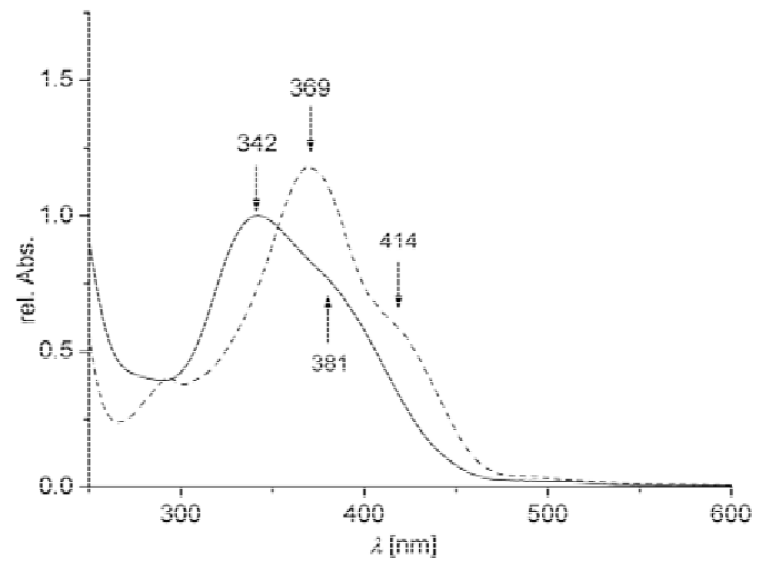

2c

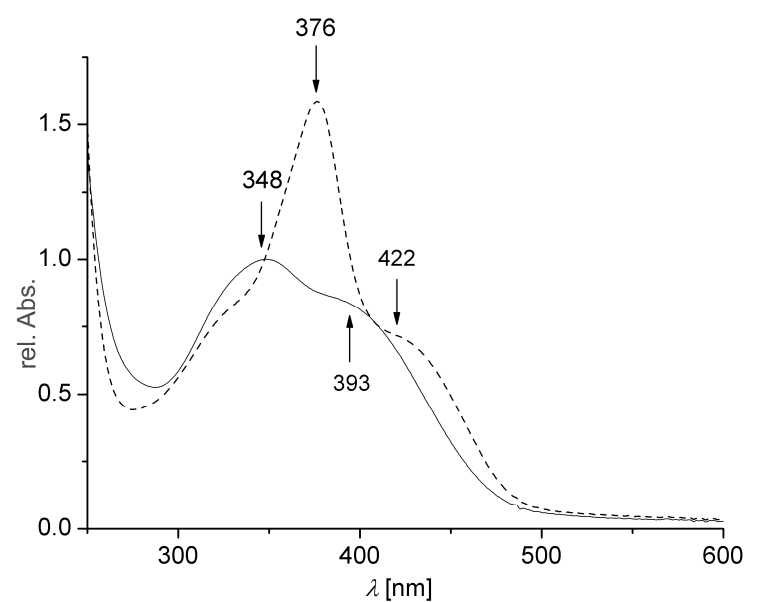

$2 d$

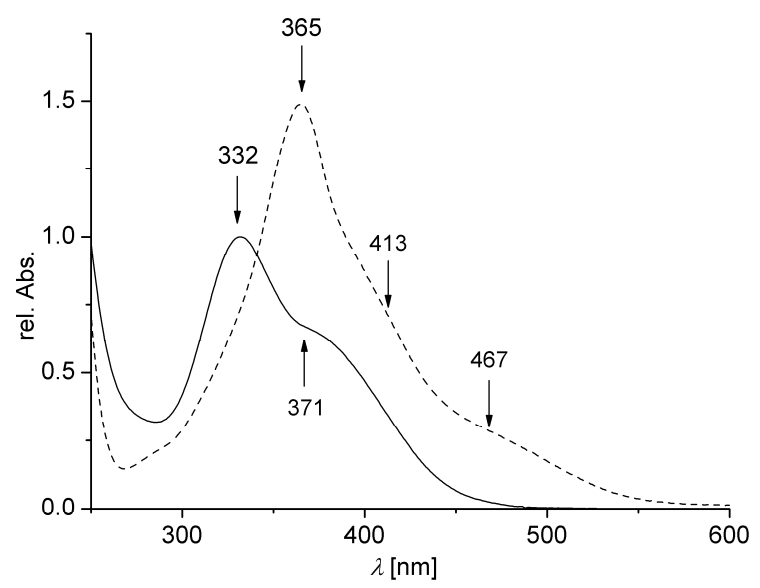

$\mathbf{2 b}$

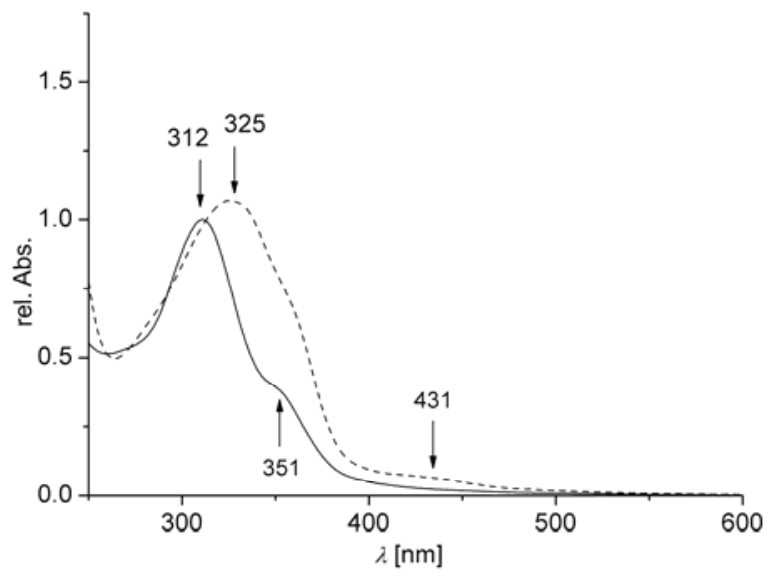

2f

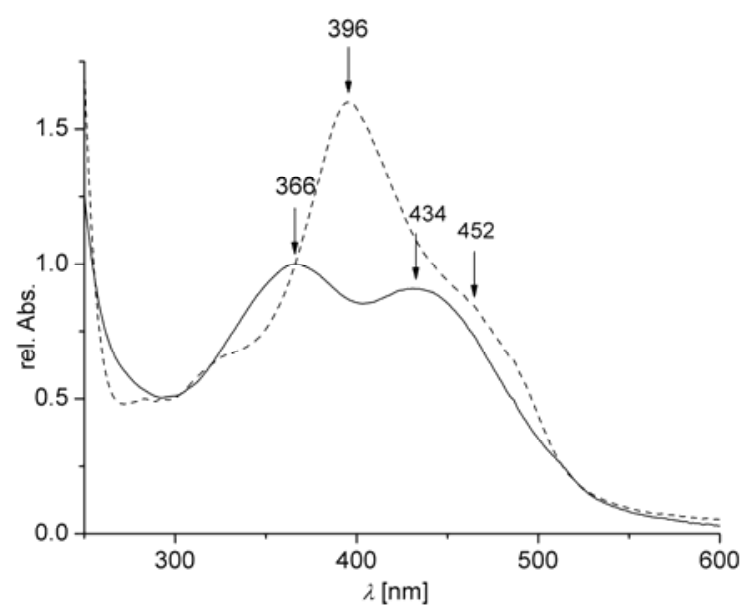

2e

Abbildung 43: Ausgewählte UV/Vis-Spektren der Schiff-Base Makrozyklen in $\mathrm{CHCl}_{3}$ (2a-2c aromaticher IminRest; 2 f aliphatischer Imin-Rest; 2d und 2e biszyklische Verbindungen). Deprotonierter Zyklus mit $0.01 \% \mathrm{Et}_{3} \mathrm{~N}$ (durchgezogene Linie) und protonierter Zyklus mit einem Überschuss an TFA (gestrichelte Linie). 
Tabelle 10: Absorptionen der protonierten bzw. deprotonierten Schiff-Base Makrozyklen in Chloroform $\left(^{*}\right.$ zur besseren Löslichkeit wurde dieser Makrozyklus mit Methanol versetzt).

\begin{tabular}{|c|c|c|}
\hline \multirow{2}{*}{ Schiff-Base } & $\begin{array}{c}\text { UV/Vis-Banden in } \mathrm{nm} \\
\text { Deprotonatiert mit } 0.01 \% \mathrm{Et}_{3} \mathrm{~N} \\
{\left[\varepsilon \times 10^{4} \mathrm{M}^{-1} \mathrm{~cm}^{-1}\right]}\end{array}$ & $\begin{array}{c}\text { UV/Vis-Banden in nm } \\
\text { Protoniert mit einem TFA Überschuss } \\
{\left[\varepsilon \times 10^{4} \mathrm{M}^{-1} \mathrm{~cm}^{-1}\right]}\end{array}$ \\
\hline 2a & $331[2.37], 374(\mathrm{sh})$ & $362[3.58], 411(\mathrm{sh}), 444(\mathrm{sh})$ \\
\hline 2b & $332[2.06], 371(\mathrm{sh})$ & $365[2.88], 413(\mathrm{sh}), 467(\mathrm{sh})$ \\
\hline 2c & $342[2.44], 381(\mathrm{sh})$ & $369[2.87], 414(\mathrm{sh})$ \\
\hline 2f & $312[1.56], 351(\mathrm{sh})$ & $325[1.70], 431(\mathrm{sh})$ \\
\hline 2e* & $366[3.06], 434[2.79]$ & $396[5.02], 452(\mathrm{sh})$ \\
\hline 2d & $348[2.39], 393$ & $376[3.79], 422(\mathrm{sh})$ \\
\hline
\end{tabular}

Nach Protonierung der Makrozyklen mit TFA können bathochrome, als auch hyperchromen Verschiebungen beobachtet werden. Die UV/Vis-Untersuchungen der Schiff-Base Makrozyklen haben gezeigt, dass die neu dargestellten Verbindungen durch die reversible Protonierungsreaktion an einer der Imin-Positionen als Säure-Base-Indikatoren fungieren können. Die besonderen Eigenschaften der Makrozyklen als spektroskopische Sonden werden in den nächsten Kapiteln ausführlich diskutiert. 


\subsubsection{Untersuchung der spektroskopischen Sonde 2a}

Der Schiff-Base Makrozyklus 2a unterscheidet sich von allen anderen neu synthetisierten Makrozyklen durch seine gute Löslichkeit in relativ unpolaren Lösungsmitteln, wie Dichlormethan bzw. Chloroform. In diesem Kapitel werden die spektroskopischen Untersuchungen der Protonierungsreaktion von 2a mit Hilfe der UV/Vis- bzw. NMRSpektroskopie beschrieben und diskutiert.

\subsection{Titration von 2a mit Trifluoressigsäure}

Zunächst sollte die Protonierung von 2a mit einer starken Säure $\left(\mathrm{pK}_{\mathrm{a}}(\mathrm{TFA})=0.52\right.$ gemessen in Wasser $)^{69}$ aufgeklärt werden. Hierzu wurde $\mathbf{2 a}$ in Chloroform $\left(4.2 \times 10^{-5} \mathrm{M}\right)$ mit einer Standard TFA-Lösung titriert. Der Titrationsvorgang wurde mittels UV/Vis-Spektroskopie verfolgt. In Abbildung 44 ist die spektrophotometrische Titration von 2a gezeigt. Der isosbestische Punkt ist bei $344 \mathrm{~nm}$ zu beobachten und weist zusammen mit dem Verlauf der Messkurven auf eine einheitliche Reaktion hin.

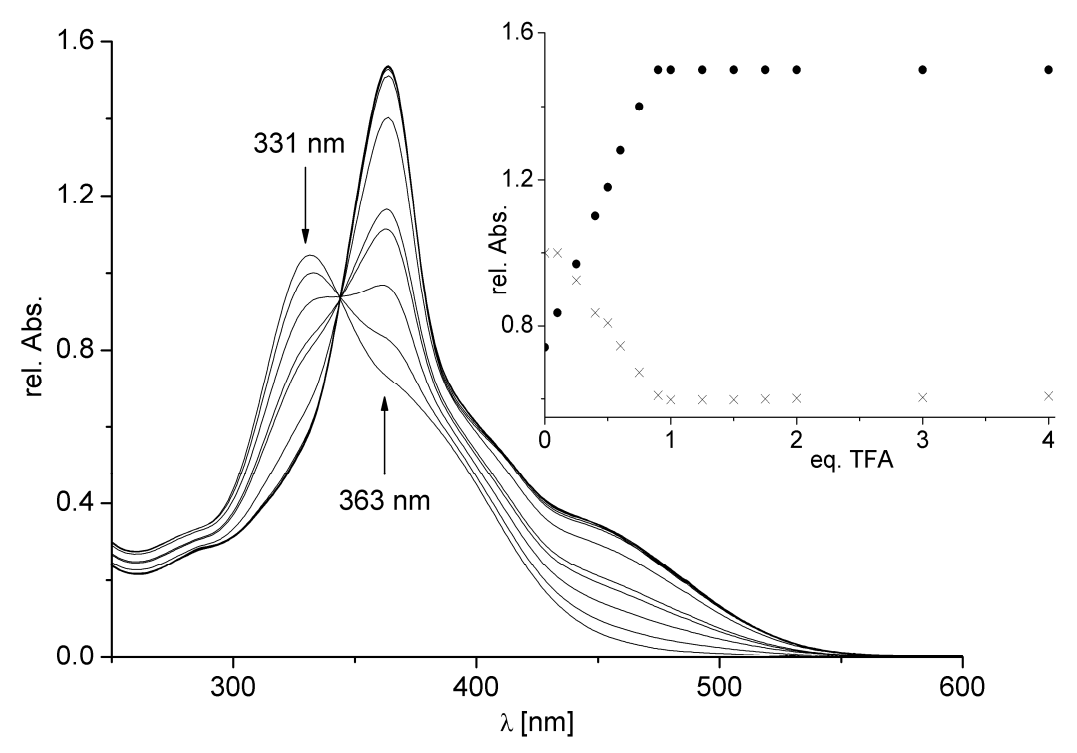

Abbildung 44: UV/Vis-spektrophotometrische Titration von $2 \mathbf{a}\left(4.2 \times 10^{-5} \mathrm{M}\right)$ mit einer Standard TFA-Lösung $\left(0.01 \mathrm{M}\right.$ in $\left.\mathrm{CHCl}_{3}\right)$. Ein isosbestischer Punkt bei $344 \mathrm{~nm}$ wird beobachtet. Oben rechts ist das Titrationsprofil bei $331 \mathrm{~nm}(\times)$ und $362 \mathrm{~nm}(\bullet)$ gegen TFA Äquivalente dargestellt.

Dem Titrationsprofil kann entnommen werden, dass die Bande bei $331 \mathrm{~nm}$ während der Addition von TFA kontinuierlich abnimmt und eine weitere Bande bei $362 \mathrm{~nm}$ an Intensität 
zunimmt. Nach der Titration von 1 eq. TFA ist das System gesättigt und es werden keine weiteren Änderungen mehr beobachtet. Zusammen mit den Ergebnissen der Röntgenstrukturanalyse untermauern diese Messungen die postulierte Monoprotonierung. Der Protonierungsvorgang von 2a mit TFA wurde auch mittels der ${ }^{1} \mathrm{H}-\mathrm{NMR}-$ Spektroskopie untersucht. Zu diesem Zweck wurde 2a in $\mathrm{CDCl}_{3}$ gelöst und bei Raumtemperatur mit 0.1 bis 4.0 eq. TFA titriert. Die Änderungen der chemischen Verschiebungen in den ${ }^{1} \mathrm{H}-\mathrm{NMR}-$ Spektren sind in Abbildung 45 gezeigt.

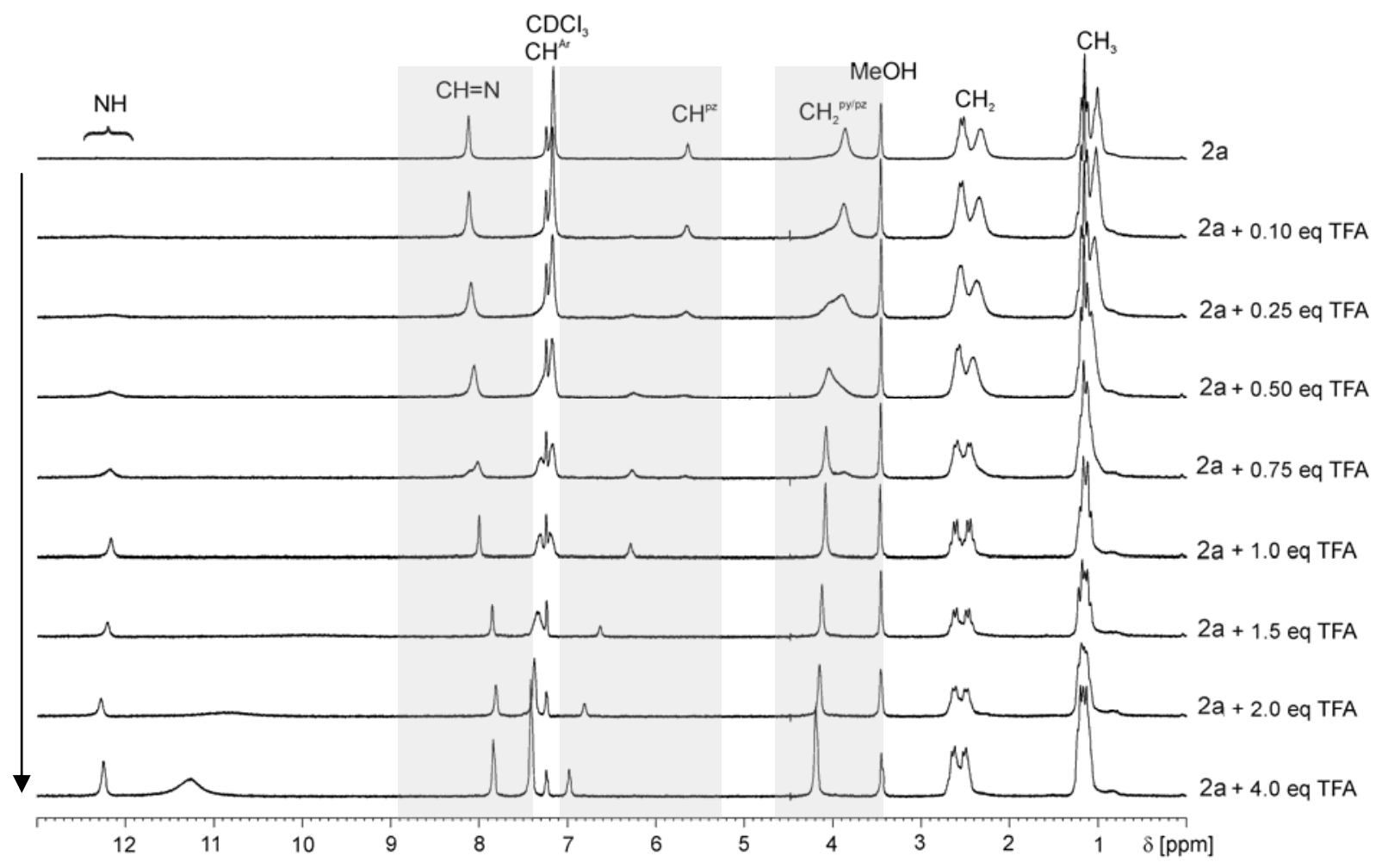

Abbildung 45: ${ }^{1} \mathrm{H}-\mathrm{NMR}-S p e k t r e n$ des Titrationsverlauf von 2a $\left(3.6 \times 10^{-2} \mathrm{M}\right)$ in $\mathrm{CDCl}_{3}$ mit $0-4$ eq. einer Standard TFA-Lösung (0.4 M in $\mathrm{CDCl}_{3}$ ). Spektren wurden auf einem $200 \mathrm{MHz}$ NMR-Messgerät aufgenommen.

Vor Beginn der Titration zeigen die $\mathrm{CH}^{\mathrm{pz}}$-Gruppen (5.67 ppm), $\mathrm{CH}_{2}{ }^{\mathrm{py} / \mathrm{pz}}$-Gruppen (3.88 ppm) und die Ethylgruppen (1.01-2.27 ppm) des Makrozyklus (2a) relativ breite Signale. Schon nach der Zugabe von 0.1 eq. TFA entsteht eine kleine Schulter beim Signal der $\mathrm{CH}_{2}{ }^{\mathrm{py} / \mathrm{pz}}-$ Gruppe. Nach weiterer Addition von TFA wird diese Schulter zu einem neuen Signal bei 4.20 ppm. Jedoch sind während der Titration noch weitere Änderungen zu beobachten. Nach einem halben Äquivalent TFA sind zwei breite Signale für die $\mathrm{CH}^{\mathrm{pz}}$-Gruppen bei 5.67 und $6.21 \mathrm{ppm} z u$ beobachten, welche auf zwei unterschiedliche Spezies in der Reaktionslösung 
hinweisen. Nach einem Äquivalent Säure wird nur noch ein einziges Signal beobachtet, welches sich bei einem TFA Überschuss geringfügig ins Tieffeld verschiebt. Diese Tieffeldverschiebung kann möglicherweise auf die Ausbildung externer Wasserstoffbrückenbindungen des Pyrazols in 4-Position $\left(\mathrm{CH}^{\mathrm{pz}}\right)$ mit einem weiteren TFAMolekül in der Lösung zurückgeführt. Durch Überschuss an TFA kommt es außerdem zur Protonierung des N-Atoms im Pyrazol. Dies führt zur Änderung des elektronischen Zustands im Heterozyklus und folglich zur Änderung der chemischen Verschiebung der $\mathrm{CH}^{\mathrm{pz}}$-Gruppe. Bei der Protonierung des Imins verändert sich seine chemische Umgebung. Während der Titration wird eine Verbreiterung des Signals für die $\mathrm{CH}=\mathrm{N}-$ Gruppe beobachtet. Nach vollständiger Protonierung bei 1.0 eq. Säure findet keine nennenswerte chemische Verschiebung mehr statt. Die dennoch beobachtete Änderung der chemischen Verschiebung in den $\mathrm{CH}^{\mathrm{pz}}$ - und $\mathrm{CH}^{\mathrm{Ar}}$-Signalen ist auf die Konformationsänderung durch externe Wasserstoffbrückenbindungen zum freien TFA zurückzuführen. Eine Veränderung ist ebenfalls an den aromatischen $\mathrm{CH}^{\mathrm{Ar}}$-Protonen zu erkennen. Vor Beginn der Reaktion wird ein Signal bei 7.17 ppm für die $\mathrm{CH}^{\mathrm{Ar}}$-Gruppe beobachtet. Nach der Zugabe von 1.0 eq. TFA entstehen zwei zu einander symmetrische Multipletts mit gleicher Intensität. Die spricht für ein symmetrisches System im aromatischen Phenylrest, welches durch schnelle Tautomerisation des Protons zwischen den zwei C=N-Imin-Positionen verursacht wird.

Die chemische Verschiebung dieser Signale wandelt sich jedoch bei einem Überschuss TFA $\mathrm{zu}$ einem Singulett bei $7.55 \mathrm{ppm}$ um. Dies zeigt, dass die Ausbildung der Wasserstoffbrückenbindungen im System nicht nur die chemische Umgebung des Imins bzw. des Pyrazols beeinflusst, sondern die des gesamten Makrozyklus.

Die drei NH-Protonen von 2a tauschen am Anfang der Titration so schnell aus, dass sie nicht detektiert werden können. Während der Titration entsteht ein neues breites Signal bei ca. 12.3 ppm, welches den vorhandenen NH-Protonen zugeordnet wird. Nach der Zugabe von vier Äquivalenten Säure ist schließlich zudem das COOH-Signal des TFA bei 11.4 ppm zu erkennen.

Schiff-Basen haben eine charakteristische Imin-Valenzschwingung im Bereich von $1600 \mathrm{~cm}^{-1}$. Die Imin-Streckschwingung der freien Base (2a) wird bei $1612 \mathrm{~cm}^{-1}$ beobachtet (Abbildung 46 links). Die protonierte Spezies [2a·TFA] zeigt im IR-Spektrum mehre Banden im Bereich 1643-1690 cm-1 (Abbildung 46 rechts). Dieser Befund bestätigt die Annahme der schnellen Tautomerisation des Protons zwischen den zwei $\mathrm{C}=\mathrm{N}$-Imin-Positionen, da mehr als nur eine Imin-Bande detektiert wird. Die Verschiebung der Bande zu höheren Schwingungsfrequenzen zeigt, dass die Imin-Bindung gestärkt wird. 


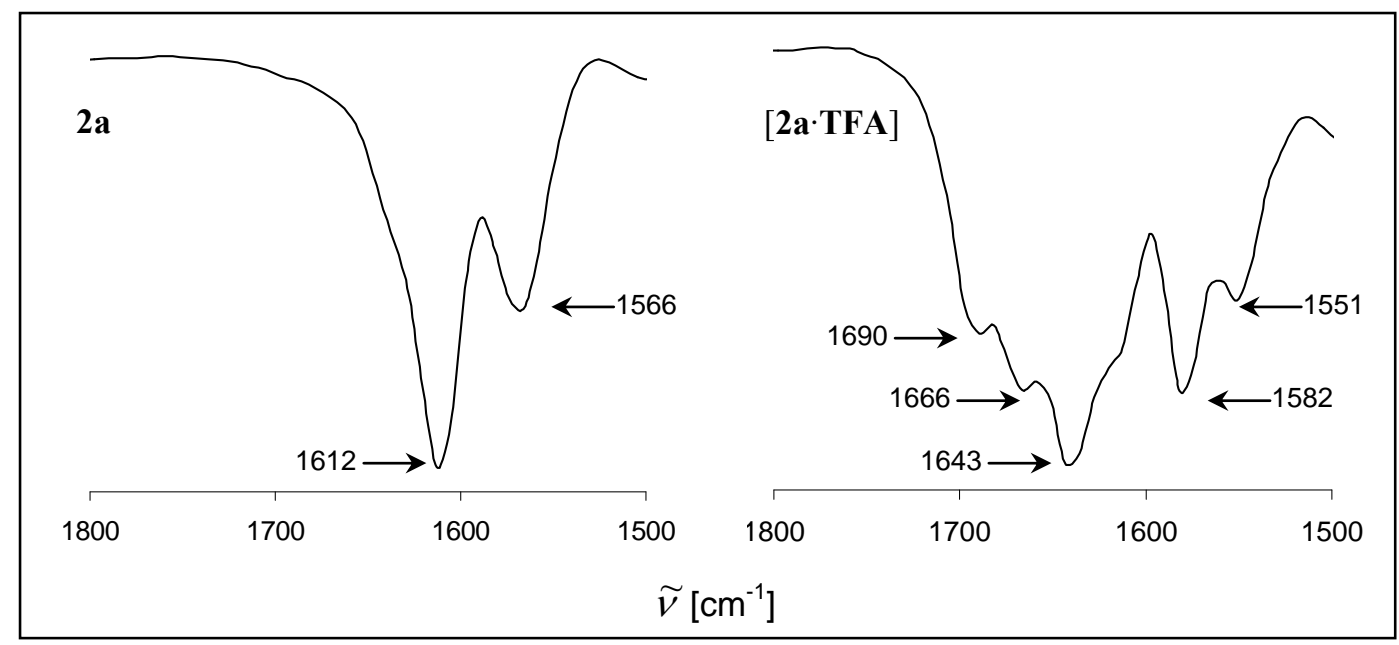

Abbildung 46: IR-Spektren von 2a und [2a·TFA] gemessen in $\mathrm{KBr}$.

\subsection{Titration von 2a mit Dimethylphosphat}

Als weitere starke Säure wurde das Dimethylphosphat $(\mathrm{DMP})\left((\mathrm{OH}) \mathrm{P}(\mathrm{OMe})_{2}(=\mathrm{O})\right)\left(\mathrm{pK}_{\mathrm{a}}=\right.$ 0.47 in $\mathrm{H}_{2} \mathrm{O}$ bzw. 1.88 in Ethanol/ $\left.\mathrm{H}_{2} \mathrm{O}\right)^{70}$ gewählt. Analog zur Titration mit TFA wurde 2a in Chloroform $\left(4.2 \times 10^{-5} \mathrm{M}\right)$ mit einer frisch angesetzten 0.01 M DMP Lösung in Chloroform titriert. Die UV/Vis-spektrophotometrische Titration von 2a mit DMP ist in Abbildung 47 gezeigt. Während der Titration nimmt die Bande bei $331 \mathrm{~nm}$ ab und eine neue Bande bei $363 \mathrm{~nm}$ wächst im bathochromen Bereich. Hierbei wird entsprechend der Titration mit TFA ein hyperchromer Effekt der Bande bei $363 \mathrm{~nm}$ beobachtet.

Wie das Titrationsprofil in Abbildung 47 rechts zeigt, ist das System nach einem Äquivalent DMP gesättigt. Ab diesen Titrationspunkt werden keine weitere Änderung im UV/VisSpektrum mehr beobachtet. Ein isosbestischer Punkt ist bei $344 \mathrm{~nm}$ analog der TFA-Titration zu erkennen.

Der Protonierungsvorgang wurde ebenfalls mit Hilfe der NMR-Spektroskopie $\left({ }^{1} \mathrm{H}\right.$ und $\left.{ }^{31} \mathrm{P}\right)$ verfolgt. Die Titration von $2 \mathrm{a}\left(3.57 \times 10^{-2} \mathrm{M}\right)$ mit DMP $\left(0.4 \mathrm{M}\right.$ Standard-Lösung in $\left.\mathrm{CDCl}_{3}\right)$ wurde in der ${ }^{1} \mathrm{H}$ - bzw. ${ }^{31} \mathrm{P}-\mathrm{NMR}$-Spektroskopie bei Raumtemperatur untersucht. In Abbildung 48 werden die ${ }^{1}$ H-NMR-Spektren des Titrationsverlaufes gezeigt (ausgewählter Bereich von 3.0-13.0 ppm). Für DMP werden drei Signale beobachtet. Ein Dublett bei 3.72-3.78 ppm (kommt durch die ${ }^{1} \mathrm{H}_{-}{ }^{31} \mathrm{P}-\mathrm{K}$ opplung zu Stande) repräsentieren die jeweiligen $\mathrm{OCH}_{3}-$ Gruppen und ein breites Signal bei 9.30 ppm wird der OH-Gruppe des DMP zugeordnet. 


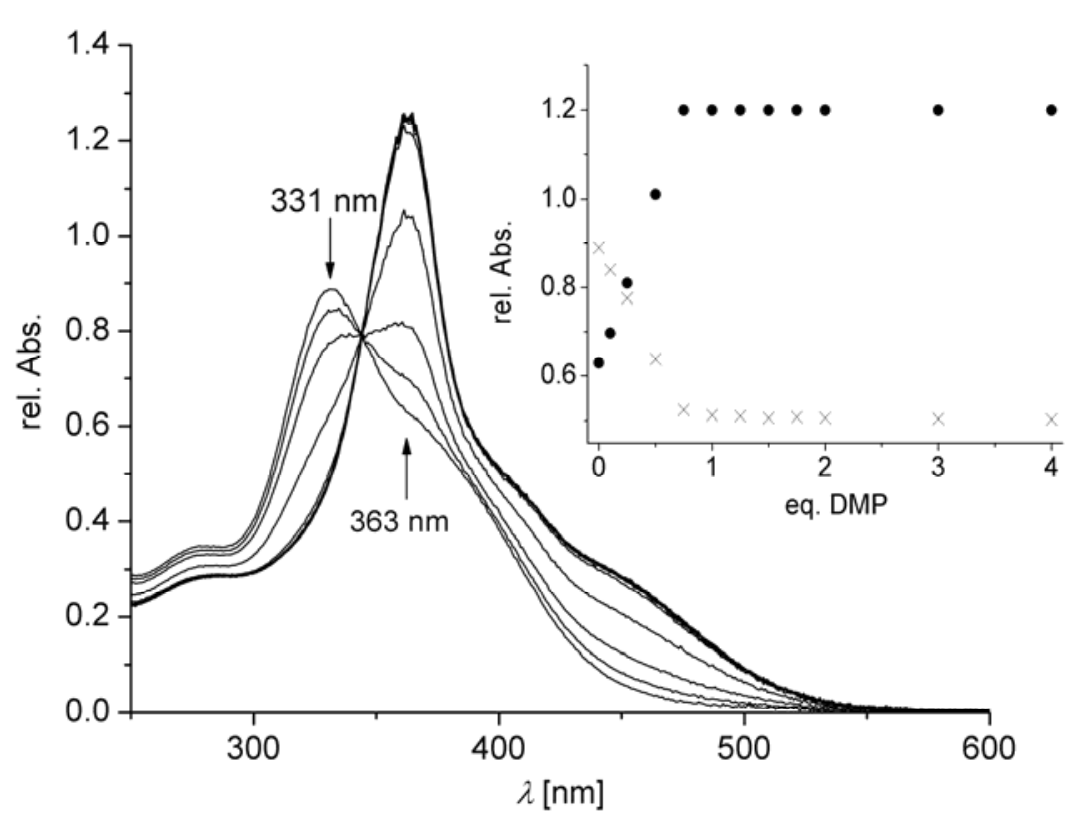

Abbildung 47: UV/Vis-spektrophotometrische Titration von 2a $\left(4.2 \times 10^{-5} \mathrm{M}\right)$ mit einer Standard Lösung von Dimethylphosphat $\left(0.01 \mathrm{M}_{\text {in }} \mathrm{CHCl}_{3}\right)$. Ein isosbestischer Punkt bei $344 \mathrm{~nm}$ wird beobachtet. Oben rechts ist das Titrationsprofil bei $331 \mathrm{~nm}(\times)$ und $363 \mathrm{~nm}(\bullet)$ gegen die Äquivalente an DMP dargestellt.

Während der DMP-Zugabe werden Änderungen der chemischen Verschiebungen bei fünf Signalen von 2a beobachtet. Die $\mathrm{CH}_{2}{ }^{\mathrm{py} / \mathrm{pz}}$-Gruppen sind vor Beginn der Reaktion bei 3.88 ppm zu beobachten. Nach 0.25 eq. DMP breitet sich dieses Signal aus und bildet bei weiterer DMP Zugabe ein scharfes Signal bei $4.12 \mathrm{ppm}$. Dies kann durch die Konformationsänderung, beeinflusst durch die mögliche Drehung der Pyrazol-Einheit bzw. die gewonnene Starrheit der $\mathrm{CH}_{2}{ }^{\mathrm{py} / \mathrm{pz}}$-Gruppen begründet werden.

Vor Beginn der Reaktion erscheint das Proton der $\mathrm{CH}^{\mathrm{pz}}$-Gruppe bei $5.67 \mathrm{ppm}$. Das Signal breitet sich bei der Zugabe von 0.10-0.50 eq. DMP zunächst, aufgrund von schnellem Protonen-Austausch, aus. Ab 0.75 eq DMP kann das Singulett der $\mathrm{CH}^{\mathrm{pz}}$-Gruppe bei $6.20 \mathrm{ppm}$ detektiert werden. Bei einem DMP-Überschuss (3.0-4.0 eq.) findet eine erneute TieffeldVerschiebung um $\Delta \delta=0.53 \mathrm{ppm}$ statt. Durch Überschuss an DMP kommt es außerdem zur Protonierung des N-Atoms im Pyrazol. Dies führt zur Änderung des elektronischen Zustands im Heterozyklus und folglich zur Änderung der chemischen Verschiebung der $\mathrm{CH}^{\mathrm{pz}}-G r u p p e$. Dieser Effekt wurde bei der Titration von 2a mit TFA ebenfalls beobachtet. Die restlichen Änderungen der Spektren sind analog der Titration mit TFA.

Das Reaktionsgeschehen wurde mittels ${ }^{13}$ P-NMR-Spektroskopie parallel verfolgt. Für DMP ist zu Beginn der Titration ein Singulett bei 3.57 ppm zu sehen. 


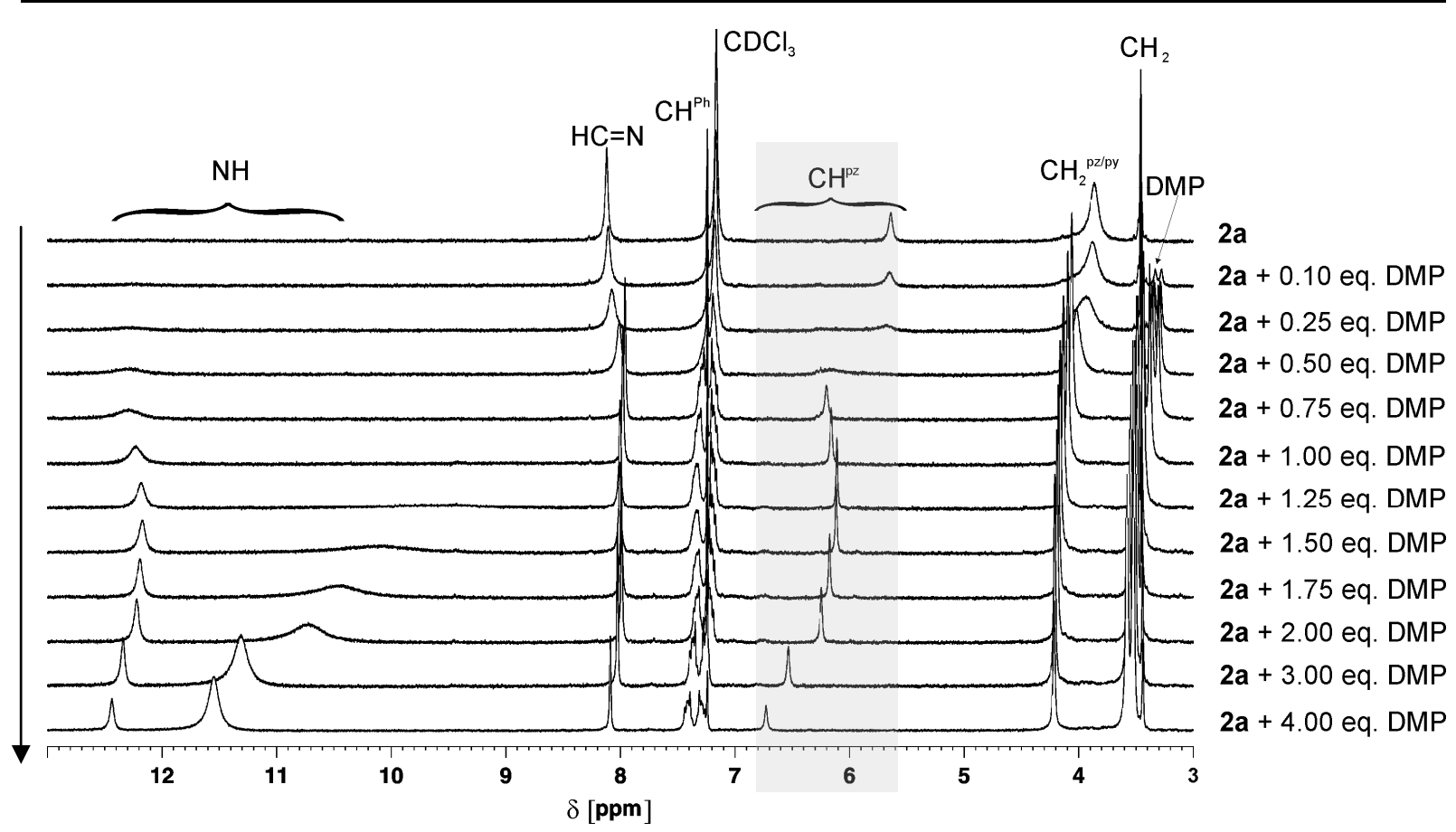

Abbildung 48: ${ }^{1} \mathrm{H}-\mathrm{NMR}-\mathrm{Titration}$ von 2a $\left(3.57 \times 10^{-2} \mathrm{M}\right)$ mit einer Standard DMP-Lösung $\left(0.4 \mathrm{M}\right.$ in $\mathrm{CDCl}_{3}$ gemessen bei $200 \mathrm{MHz}$ ).

Nach der Zugabe von 0.1 eq. zu 2a kommt es zur einen geringen Verschiebung des Signals zu $\delta=2.13 \mathrm{ppm}$. Nach Beendigung der Reaktion hat sich dieses Signal zu $1.20 \mathrm{ppm}$ verschoben. Da die chemische Verschiebung des ${ }^{31}$ P-Signals aus DMP relativ klein ist, können in Bezug auf den Protonierungsprozess keine konkreten Rückschlüsse gezogen werden.

\subsection{Titration von 2 a mit HF}

Der Schiff-Base Makrozyklus 2a wurde im nächsten Schritt mit der relativ schwachen Säure

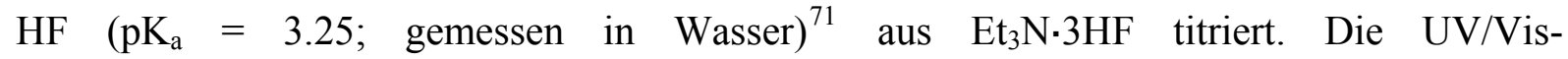
spektrophotometrische Titration von $2 \mathbf{a}\left(4.2 \times 10^{-5} \mathrm{M}\right)$ mit einer Standard-Lösung von $\mathrm{Et}_{3} \mathrm{~N} \cdot 3 \mathrm{HF}\left(2.1 \times 10^{-3} \mathrm{M}\right.$ in Chloroform) ist in Abbildung 49 gezeigt. Die bathochrome Verschiebung der Bande bei $331 \mathrm{~nm}$ zu einer neuen Bande bei $363 \mathrm{~nm}$ mit einem isosbestischen Punkt bei $344 \mathrm{~nm}$ ist analog zu den gezeigten Titrationen mit starken Säuren wie TFA. Anders als bei den starken Säuren, reicht hier die Zugabe von 1.0 eq. HF nicht aus, um die vollständige Monoprotonierung zu beobachten. Das Titrationsprofil in Abbildung 49 zeigt, dass nach 1.0 eq. HF weniger als die Hälfe von 2a protoniert vorliegt. Erst die Zugabe von 5.0 eq. HF führt zur vollständigen Monoprotonierung der Imin-Position. 


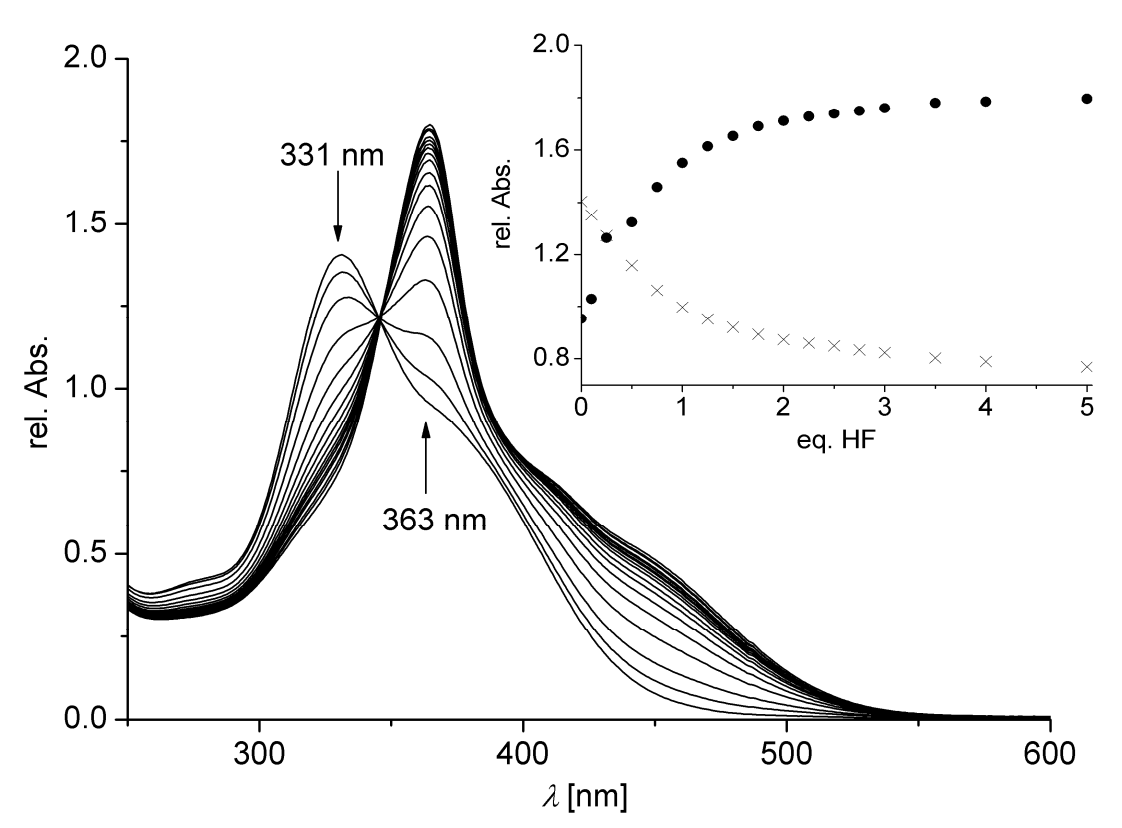

Abbildung 49: UV/Vis-spektrophotometrische Titration von 2a $\left(4.2 \times 10^{-5} \mathrm{M}\right)$ mit einer Standard-Lösung von $\mathrm{Et}_{3} \mathrm{~N} \cdot 3 \mathrm{HF}\left(2.1 \times 10^{-3} \mathrm{M}\right.$ in $\left.\mathrm{CHCl}_{3}\right)$. Ein isosbestischer Punkt bei $344 \mathrm{~nm}$ wird beobachtet. Oben rechts ist das Titrationsprofil bei $331 \mathrm{~nm}(\times)$ und $363 \mathrm{~nm}(\bullet)$ gegen die Äquivalente an HF dargestellt.

Der Titrationsverlauf von 2a mit HF wurde mit Hilfe der ${ }^{1} \mathrm{H}$ - und ${ }^{19} \mathrm{~F}-\mathrm{NMR}-$ Spektroskopie verfolgt. Die Änderungen im ${ }^{1} \mathrm{H}-\mathrm{NMR}-$ Spektrum sind analog $\mathrm{zu}$ den restlichen bereits gezeigten ${ }^{1} \mathrm{H}$-NMR-Titrationsverläufen. In den ${ }^{19} \mathrm{~F}-\mathrm{NMR}$-Spektren sind bis zur Zugabe von 1.25 eq. HF aufgrund der möglichen Austauschprozesse keine Fluor-Signale zu erkennen (Abbildung 50). Ab 1.5 eq. HF zu 2a ist ein schwaches Signal bei 32.5 ppm zu erkennen. Bei weiterer Zugabe wird ein zweites breites Signal bei $14.0 \mathrm{ppm}$ detektiert, welches sich während weiterer Zugabe von HF bis zu 7.5 ppm ins Hochfeld verschiebt. Die ${ }^{19}$ F-NMRSpektren entsprechen nach einem hohen HF Überschuss dem der freien Säure.

Wird 2a mit 1.5 eq. HF bei $500 \mathrm{MHz}$ statt $200 \mathrm{MHz}$ gemessen, so wird durch die Einwirkung des stärkeren Feldes ein Signal im ${ }^{19}$ F-NMR-Spektrum bei 75.5 ppm detektiert. Tieftemperatur-Messungen dieser Probe bis $214 \mathrm{~K}$ zeigen, dass ab $263 \mathrm{~K}$ ein weiteres Signal mit geringer Intensität bei 34.4 ppm vorliegt. Diese Ergebnisse legen nahe, dass das HFSystem äußerst komplex ist. Die ${ }^{19}$ F-Signale könnten sowohl aus dem freien HF, als auch aus den $\mathrm{F}^{-}$-Anionen stammen, die in der Lösung parallel vorliegen. Sowohl das freie HF, als auch die $\mathrm{F}^{-}$-Anionen sind in der Lage Wasserstoffbrückenbindungen zu $2 \mathbf{a}$ zu bilden. Dies erklärt die große Anzahl der Fluor-Signale im ${ }^{19}$ F-NMR-Spektrum. 


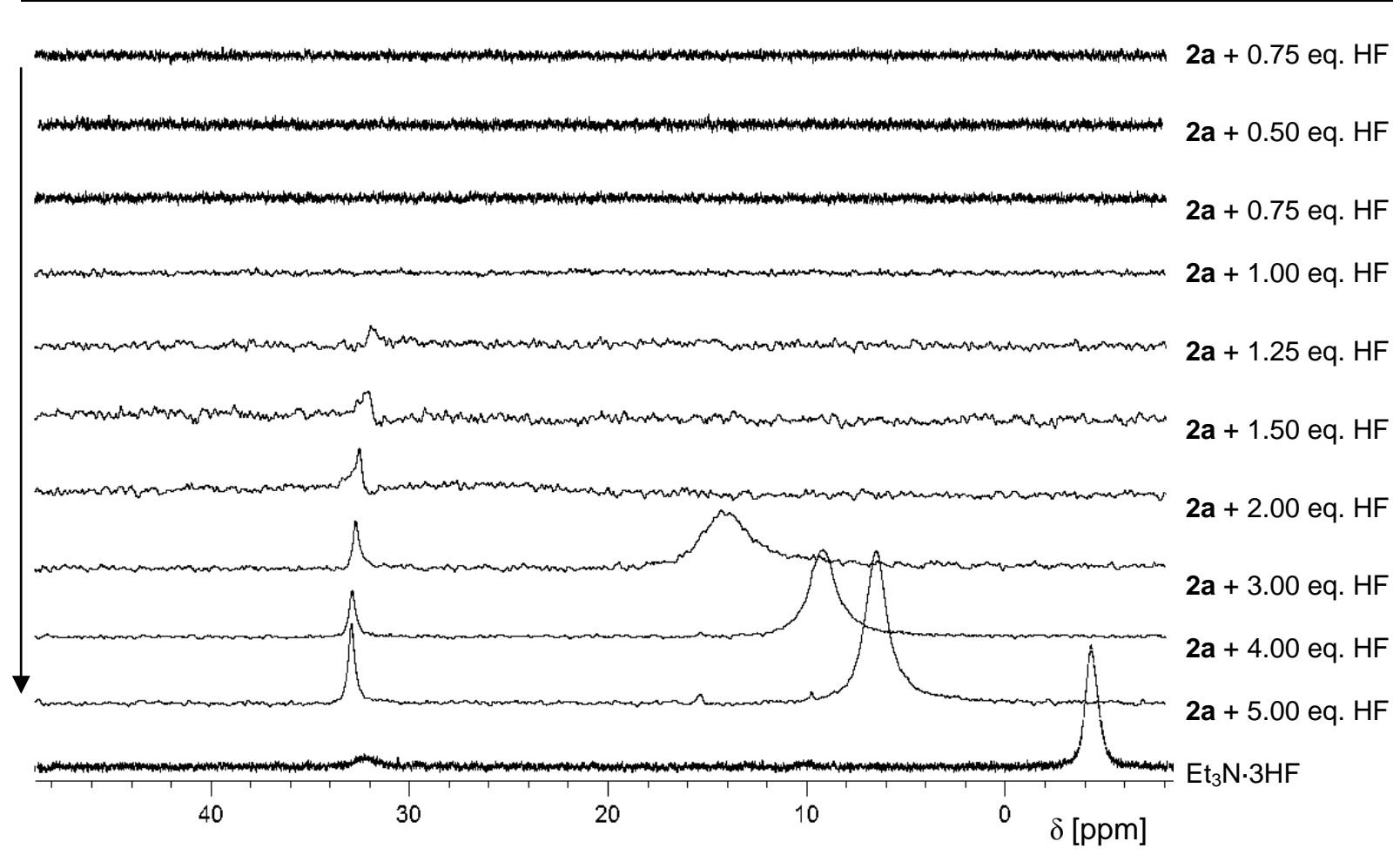

Abbildung 50: ${ }^{19} \mathrm{~F}-\mathrm{NMR}-\mathrm{Spektren}$ von $\mathbf{2 a}\left(4.37 \times 10^{-2} \mathrm{M}\right)$ titriert mit $0.25-5.0$ eq. $\mathrm{Et}_{3} \mathrm{~N} \cdot 3 \mathrm{HF}$ in $\mathrm{CDCl}_{3}\left(\mathrm{Standard}_{\text {: }}\right.$ $\mathrm{C}_{6} \mathrm{~F}_{6}$; Spektren wurde bei $188 \mathrm{MHz}$ aufgenommen).

Die mit 1.5 eq. HF titrierte 2a-Lösung, in der dass Fluor-Signal bei 32.5 ppm im ${ }^{19}$ F-NMRSpektrum erstmals beobachtet werden konnte, wurde zusätzlich mittels der ${ }^{1} \mathrm{H}-\mathrm{NMR}$ Spektroskopie bei $500 \mathrm{MHz}$ gemessen.

In Abbildung 51 ist das ${ }^{1} \mathrm{H}-\mathrm{NMR}-$ Spektrum von 2a mit 1.5 eq HF (A) und zum Vergleich reines 2a (B) und gezeigt. Spektrum B deutet darauf hin, dass der Makrozyklus 2a bereits nach der Zugabe von 1.5 eq. HF vollständig protoniert vorliegt. Die bereits diskutierten UV/Vis-Messungen konnten zeigen, dass der Makrozyklus 2a erst nach 5.0 eq. HF vollständig protoniert ist. Die vermeintlich vollständige Protonierung des Makrozyklus im ${ }^{1} \mathrm{H}-$ NMR-Spektrum kommt vermutlich durch die höhere Konzentration der Probe $\left(4.37 \times 10^{-2} \mathrm{M}\right)$ und entsprechende HF-Konzentration zu Stande. 


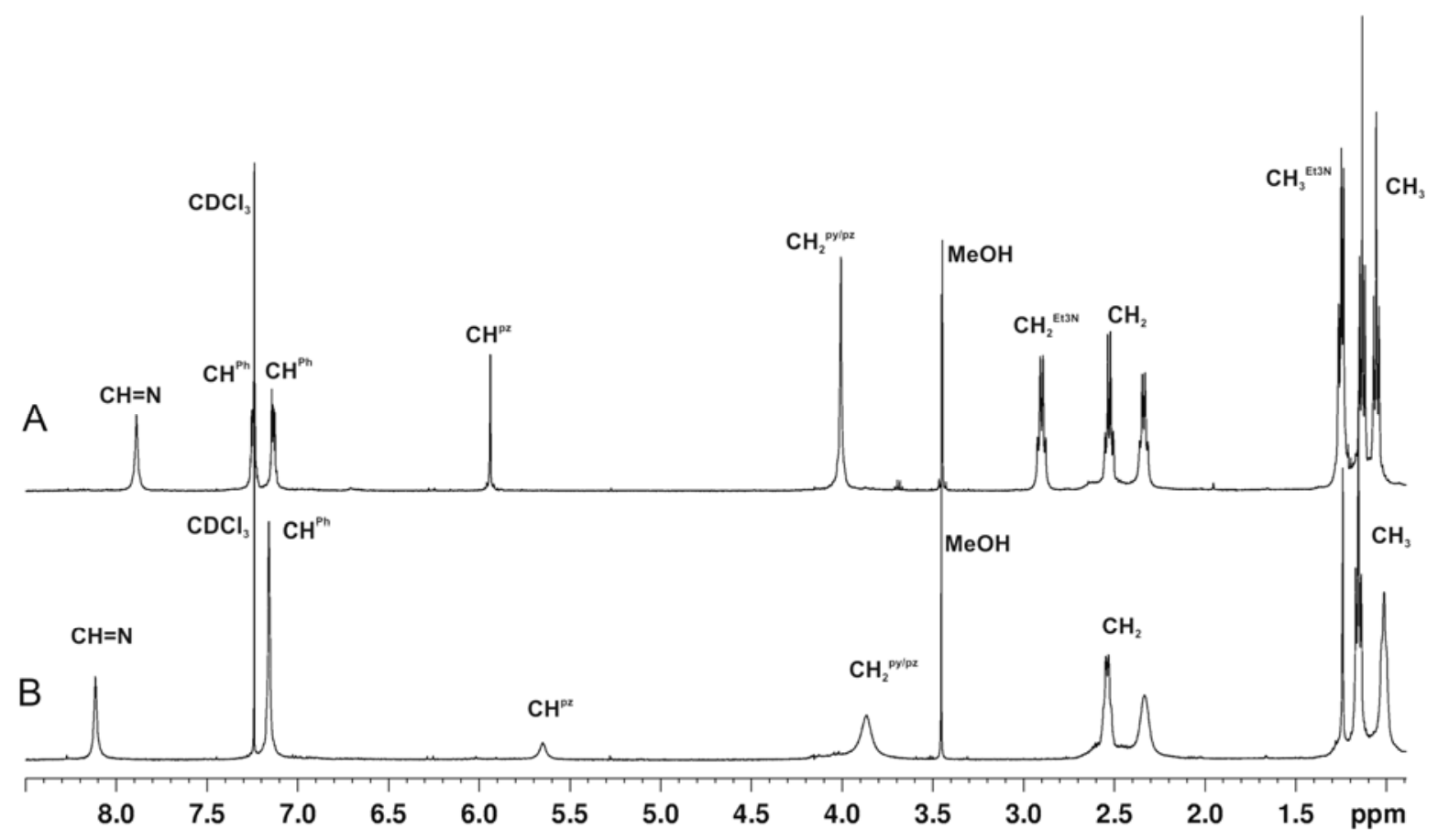

Abbildung 51: ${ }^{1} \mathrm{H}-\mathrm{NMR}-$ Spektrum von 2a $\left(4.37 \times 10^{-2} \mathrm{M}\right)$ titriert mit 1.5 eq. HF gemessen bei $500 \mathrm{MHz}$ (A) und Vergleichsspektrum 2a in $\mathrm{CDCl}_{3}$, beide gemessen bei $300 \mathrm{~K}$ (B).

Durch schnellen Austausch der Protonen bzw. der Fluor-Atomen könnten die Signale ebenfalls als einfache Siguletts statt der erwarteten Multiples erscheinen. Die gleiche Probe $(\mathbf{2} \mathbf{a}+1.5$ eq. HF) wurde erneut bei tiefen Temperaturen gemessen, um weitere Spezies in der Probe identifizieren zu können. In Abbildung 52 ist das ${ }^{1} \mathrm{H}-\mathrm{NMR}-$ Spektrum von 2a mit 1.5 eq. HF bzw. 2a bei $223 \mathrm{~K}$ gezeigt. Bei dieser Temperatur können die Signale der NHGruppen des protonierten 2a ab 12 ppm mit einem Intensitätsverhältnis von 1:1.7:1.6 (12.8, 14.6 bzw. $15.3 \mathrm{ppm}$ ) beobachtet werden.

Die Signale der $\mathrm{CH}=\mathrm{N}-$ Gruppe für 2a mit 1.5 eq. HF (Abbildung 52, A) werden in zwei Signale bei 7.59 bzw. 8.09 ppm mit einer Intensität von 1:1.2 aufgespalten. Die Aufspaltung der $\mathrm{CH}=\mathrm{N}-$ Gruppe lässt auf eine unterschiedliche chemische Umgebung schließen.

Bei tiefen Temperaturen ist es somit möglich die protonierte und nicht protonierte IminPosition von $2 \mathbf{a}$ zu unterscheiden. Das Proton der $\mathrm{CH}^{\mathrm{pz}}$-Einheit spaltet in keine weiteren Signale auf und bleibt mit einer Intensität 1 unverändert. Ein breites Signal bei 4.98 ppm mit einer Intensität von 1 deutet auf HF in der Lösung hin. Außer der Aufspaltung der IminGruppen werden weitere Aufspaltungen bei den $\mathrm{CH}_{2}$-Gruppen von $\mathrm{CH}_{2}{ }^{\mathrm{py} / \mathrm{pz}}$ (3.39-3.66, 4.13 ppm mit 1:3) bzw. der Pyrrol-Ethylgruppen $\left(\mathrm{CH}_{2} ; 2.14,2.39-2.52\right.$ ppm mit der Intensität 2:6) beobachtet. 


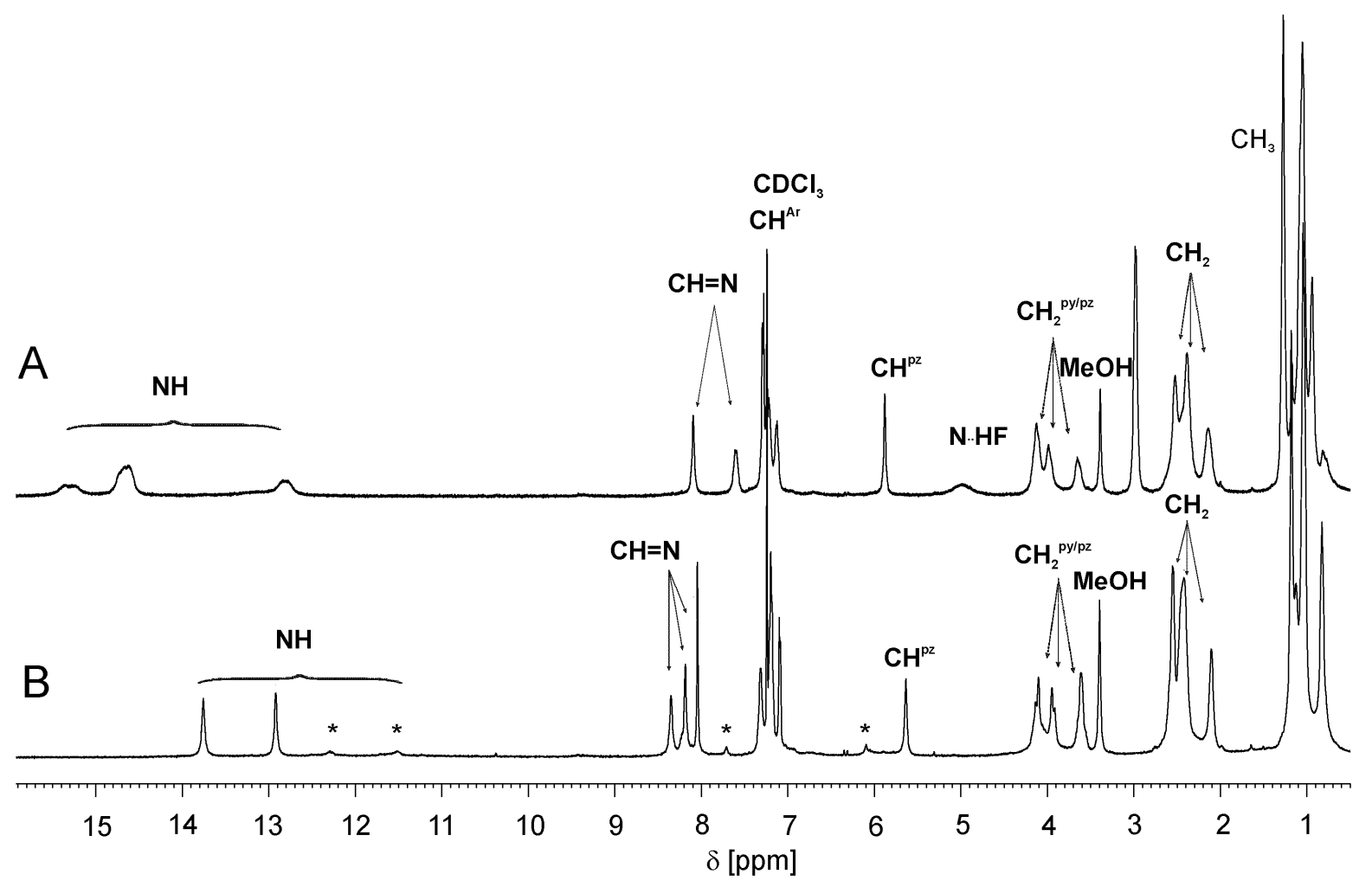

Abbildung 52: ${ }^{1} \mathrm{H}-\mathrm{NMR}-\mathrm{Spektrum}$ von 2a mit 1.5 eq. $\mathrm{HF}$ (A) und 2a (B) in $\mathrm{CDCl}_{3}$, beide gemessen bei $223 \mathrm{~K}$ bei $500 \mathrm{MHz}$.

Da sich die Gesamtintensität der Signale nicht ändert, erfolgt die Aufspaltung der Signale bei 2a mit 1.5 eq. HF (223 K) aufgrund unterschiedlicher Konformationen des Makrozyklus bedingt durch die Protonierung von 2 a.

Ein Vergleich der ${ }^{1}$ H-NMR-Spektren des freien Makrozyklus 2a mit der protonierten [2aH] ${ }^{+}$Spezies bei $223 \mathrm{~K}$ (Abbildung 52, B) zeigt, dass auch hier analog zu $[\mathbf{2} \mathbf{a H}]^{+}$eine Aufspaltung der $\mathrm{CH}_{2}{ }^{\mathrm{py} / \mathrm{pz}}$ - und Pyrrol-Ethylgruppen $\left(\mathrm{CH}_{2}\right)$ mit gleicher chemischer Verschiebung und deckungsgleicher Intensität stattfindet. Die $\mathrm{CH}^{\mathrm{pz}}$-Gruppe zeigt ebenfalls ein Singulett, allerdings bei $5.64 \mathrm{ppm}$. Interessanterweise kommt es auch bei der unprotonierten 2a-Spezies zur Aufspaltung der Imin-Gruppen. Hierbei werden jedoch drei Signale bei 8.04, 8.19 und $8.34 \mathrm{ppm}$ mit der Intensität von 1:0.9:0.7 beobachtet. Dieser Befund zeigt, dass die Struktur von 2a eine gewisse Flexibilität besitzt, und somit unterschiedliche Konformationen einnehmen kann, die bei tiefer Temperaturen auf der NMR-Zeitskala offenbar einigefroren sind.

Im ${ }^{1}$ H-NMR-Spektrum von 2a werden die zwei Signale bei 12.9 bzw. 13.8 ppm mit einer 1:1 Intensität den NH-Protonen zugeordnet (Abbildung 52, B). Die unterschiedlichen chemischen Verschiebungen der NH-Protonen von 2a und $[\mathbf{2 a H}]^{+}$belegen die Existenz der protonierten 
$[\mathbf{2 a H}]^{+}$Spezies. Die NH-Protonen des Makrozyklus bilden Wasserstoffbrückenbindungen zu HF bzw. den $\mathrm{F}^{-}$-Anionen in der Lösung. Dies hat zur Folge, dass die NH-Protonen, wie in $[\mathbf{2} \mathbf{a H}]^{+}$beobachtet, ins Tieffeld verschoben werden.

Im ${ }^{1}$ H-NMR-Spektrum von 2a werden noch weitere Signale mit schwacher Intensität beobachtet (Abbildung 52, siehe Kennzeichung in B). Diese Signale haben die gleiche chemische Verschiebung wie analoge Signale der protonierten Spezies in $[\mathbf{2} \mathbf{a H}]^{+}$(Spektrum A). Diese experimentelle Beobachtung zeigt, dass vermutlich Spuren von Säure in $2 \mathbf{a}$ eine geringe Protonierung verursachen. Säurespuren können aus der Synthese von 2a mit TFA stammen, oder durch Spuren von $\mathrm{HCl}$ in deuteriertem Chloroform.

Die Ergebnisse der ${ }^{1} \mathrm{H}-\mathrm{NMR}-S p e k t r o s k o p i e ~ v o n ~ 2 a ~ b z w . ~[2 \mathbf{a H}]^{+}$zeigen, dass der Schiff-Base Makrozyklus als Protonen-Sonde fungieren kann und selbst in der Lage ist geringe Säurespuren zu detektieren.

\subsection{Titration von 2a mit Pentafluorophenol}

Der Makrozyklus 2a wurde in einer weiteren Reaktion mit Pentafluorophenol $\left(\mathrm{PFP}, \mathrm{pK}_{\mathrm{a}}=\right.$ 4.10) ${ }^{72}$ protoniert. Mit Phenolen als Säure konnte keine Reaktion beobachtet werden.

Eine Lösung von $\mathbf{2 a}\left(4.2 \times 10^{-5} \mathrm{M}\right.$ in $\left.\mathrm{CHCl}_{3}\right)$ wurde mit einer frisch zubereiteten Pentafluorophenol-Lösung (0.01 M in Chloroform) stufenweise titriert. Die Titration wurde zunächst mit Hilfe der UV/Vis-Spektroskopie verfolgt (Abbildung 53). Die Verschiebung der Banden ist analog zu denen der bereits beschriebenen Säure-Base Reaktionen mit 2a. Das Titrationsprofil zeigt, dass der Protonierungsprozeß entsprechend der Titration mit einer schwachen Säure verläuft. Es wird ein Überschuss an PFP benötigt (mind. 12 eq.), um eine vollständige Monoprotonierung des Makrozyklus zu erreichen. Das aufgenommene Titrationsprofil spiegelt dabei die Stärke der verwendeten Säure wider.

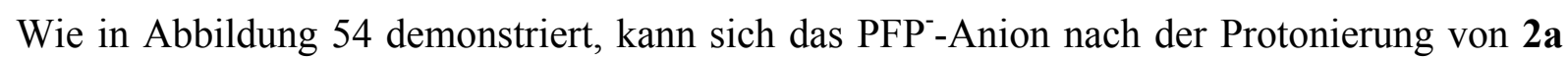
über der Makrozyklus-Kavität fixieren und Wasserstoffbrückenbindungen $\mathrm{zu}$ den $\mathrm{NH}-$ Gruppen ausbilden. 


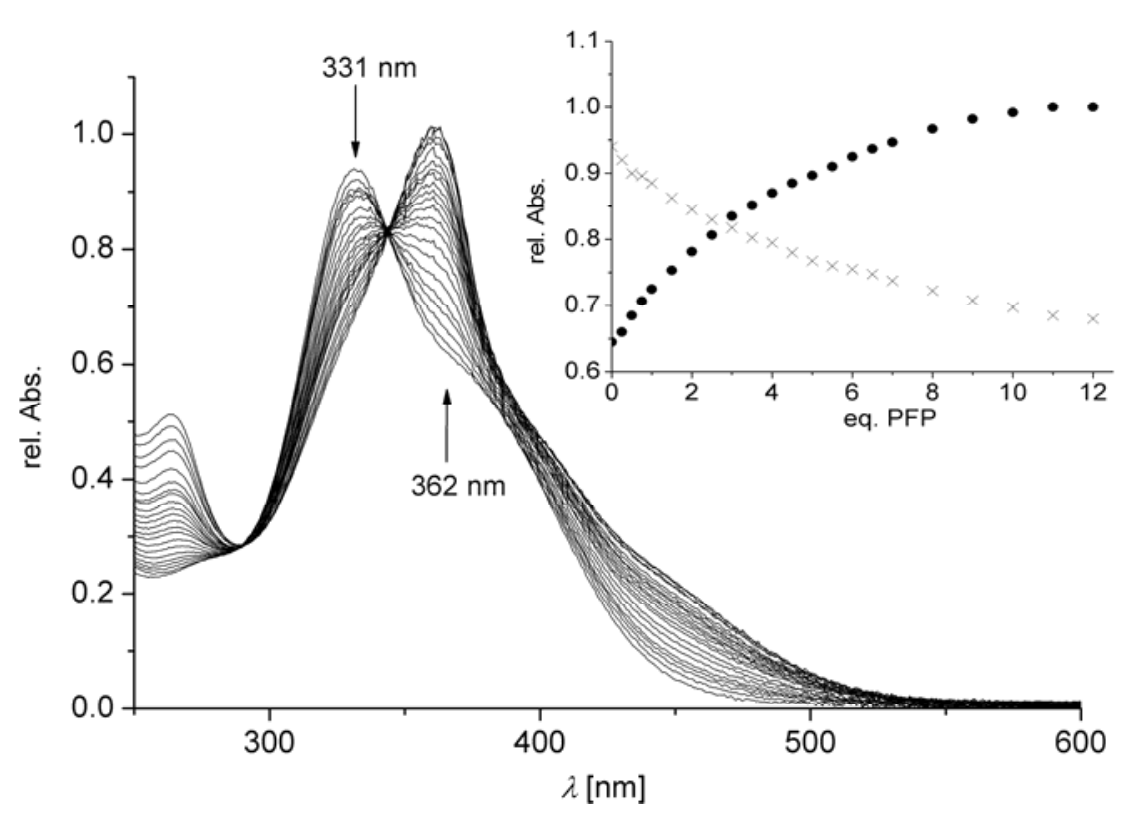

Abbildung 53: UV/Vis-spektrophotometrische Titration von 2a $\left(4.2 \times 10^{-5} \mathrm{M}\right)$ mit einer Standard-Lösung von Pentafluorophenol $\left(0.01 \mathrm{M}\right.$ in $\left.\mathrm{CHCl}_{3}\right)$. Ein isosbestischer Punkt bei $344 \mathrm{~nm}$ wird beobachtet. Oben rechts ist das Titrationsprofil bei $331 \mathrm{~nm}(\times)$ und $363 \mathrm{~nm}(\bullet)$ aufgetragen gegen die Äquivalente an PFP dargestellt.

Die ${ }^{1}$ H-NMR-Titration verlief analog zu den bereits diskutierten NMR-Titrationen von 2a mit anderen Säuren. Der Titrationsverlauf wurde zusätzlich mittels der ${ }^{19}$ F-NMR-Spektroskopie parallel verfolgt. In Abbildung 55 sind die Ergebnisse der Titration von 2a mit bis zu 12 eq. PFP dargestellt.<smiles>Oc1c(F)c(F)c(F)c(F)c1F</smiles>

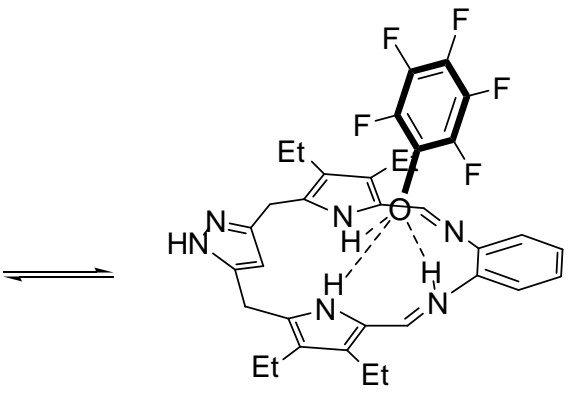

$[2 \mathrm{aH}]^{+} \cdot\left(-\mathrm{O}-\mathrm{C}_{6} \mathrm{~F}_{5}\right)$

Abbildung 54: Reaktionsschema der Monoprotonierung von 2a mit PFP.

Das PFP (0.05 M in $\mathrm{CDCl}_{3}$ ) zeigt vor Beginn der Reaktion drei Fluor-Signale bei -6.8, -1.8 bzw. -1.7 ppm für die F-Atome der ortho-, meta- und para-Position. Bei der Reaktion von 2a mit PFP verschieben sich diese Fluor-Signale bereits bei einer Zugabe von 0.5 eq. PFP stark ins Hochfeld. Besonders signifikant verschiebt sich das ${ }^{19} \mathrm{~F}$-Signal in der para-Position des deprotoniertem PFP (-22.1 ppm). 
a)

a)

h

PFP

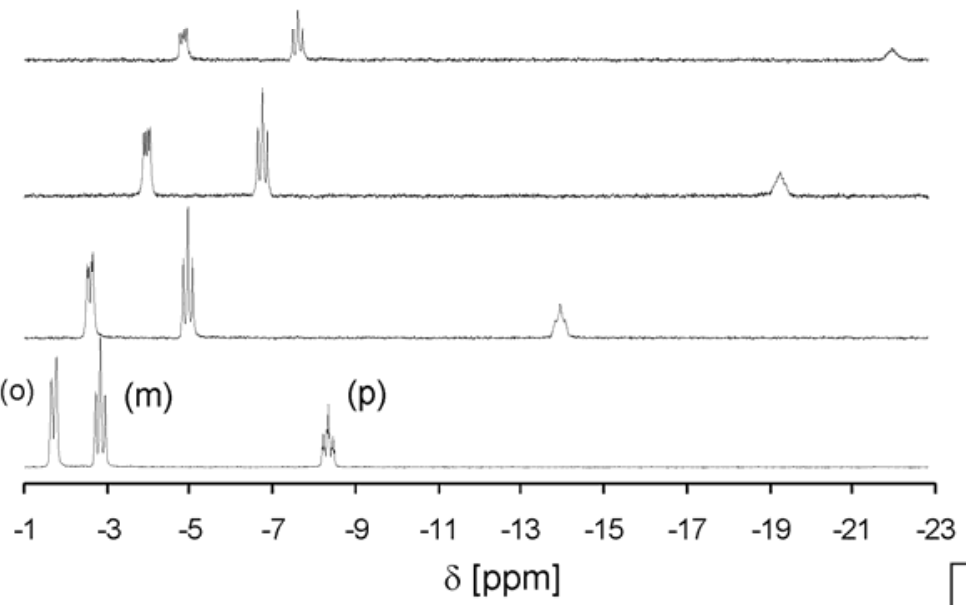

$2 a+0.5$ PFP

$2 a+1.0$ PFP

$2 a+2.0$ PFP

$2 a+12$ PFP

b)

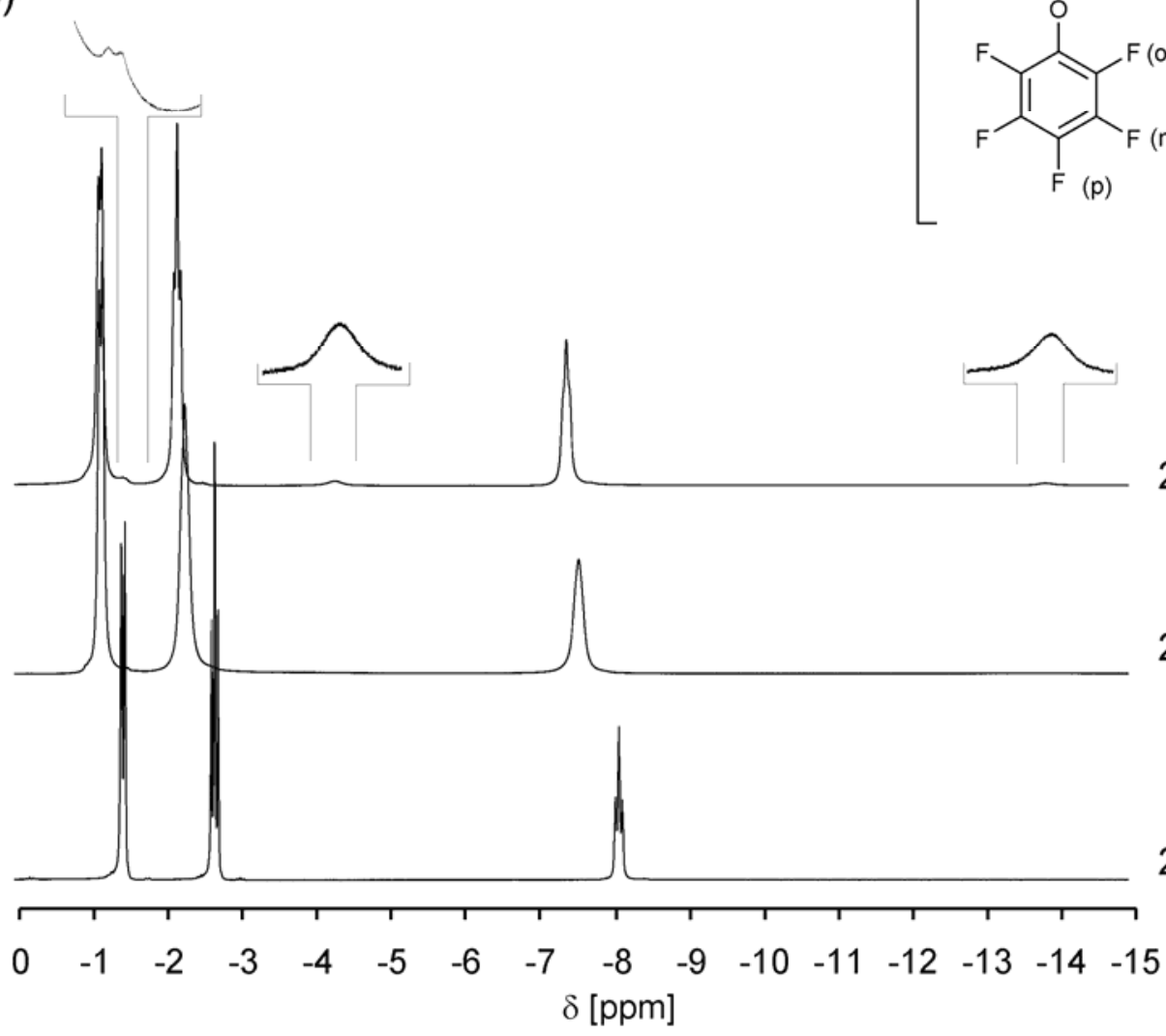

$218 \mathrm{~K}$

$233 \mathrm{~K}$

297 K

Abbildung 55: a) ${ }^{19}$ F-NMR Titration von 2a $\left(3.6 \times 10^{-2} \mathrm{M}\right)\left(\right.$ mit $\mathrm{C}_{6} \mathrm{~F}_{6}$ als interner Standard) mit einer StandardLösung von PFP (0.05 $\mathrm{M}$ in $\left.\mathrm{CDCl}_{3}\right)$ gemessen bei $188 \mathrm{MHz}$. b) ${ }^{19}$ F-NMR Tieftemperatur Messungen von 2a mit 12 eq. PFP gemessen bei $470 \mathrm{MHz}$.

Abbildung 56 zeigt, die Entstehung des Pentapfluorphenolats nach Protonierung von 2a. Die Grenzstruktur des $\mathrm{PFP}^{-}$-Anions verdeutlicht, dass nach der Protonierung das F-Atom an der 
para-Position elekronenreicher ist, und das entsprechende Signal somit ins Hochfeld verschoben wird.

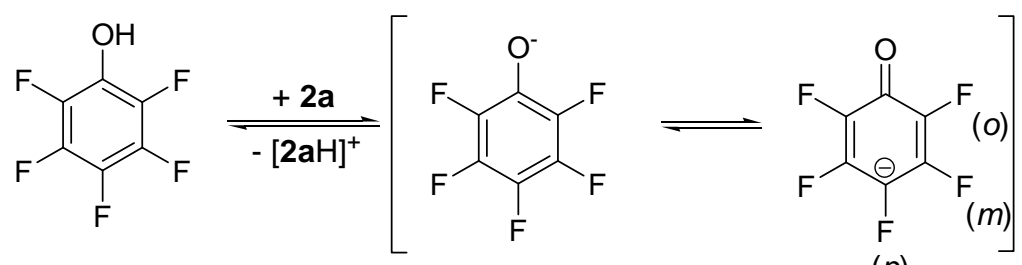

$(p)$

Abbildung 56: Deprotonierung des Pentafluorophenols mit 2a und Grenzstrukturen des PFP-Anions.

Am Beginn der Titration ( 0.5 eq. PFP) liegt das Substrat fast vollständig deprotoniert vor. Durch weitere PFP Zugabe zum Makrozyklus während der Titration verschieben sich die drei ${ }^{19}$ F-Signale zum ursprünglichen Spektrum des Pentafluorophenols hin zurück ins Tieffeld.

Bei weiter Addition an PFP zu 2a liegt jedoch sowohl das unprotonierte als auch protoniert Pentafluorophenol vor. Da der Austausch dieser zwei Spezies offenbar extrem schnell ist, werden keine separaten Signale für die jeweiligen Fluor-Atome beobachtet.

Eine Probe von 2a vesetzt mit 12 eq. PFP wurde bei tiefen Temperaturen in der ${ }^{19}$ F-NMRSpektroskopie untersucht. Beim Abkühlen der Probe wurden bei $218 \mathrm{~K}$ drei weitere F-Signale bei -13.9, -4.3 bzw. -1.4 ppm detektiert (Abbildung 55, b). Diese Signale werden dem Pentafluorphenolat in der Lösung zugeordnet.

Die in diesem Kapitel diskutierten Ergebnisse konnten zeigen, dass der Schiff-Base Makrozyklus 2a durch das saure Pentafluorophenol protoniert werden kann. Die Protonierungsreaktion kann sowohl mittels der UV/Vis- als auch der ${ }^{1} \mathrm{H}$ - und ${ }^{19} \mathrm{~F}-\mathrm{NMR}$ Spektroskopie verfolgt werden.

\subsubsection{2a als Anionensensor}

Anionenrezeptoren sind sowohl in chemischen als auch in einer Reihe biologischer Systeme von großer Bedeutung. Entsprechend wurde in den letzen Jahren eine große Anzahl neuer Anionenrezeptoren entwickelt. $\mathrm{Zu}$ den bekanntesten Anionenrezeptoren gehören die mesoOctamethylporphyrinoge, auch Calix[4]pyrrole genannt (Abbildung 57 links). ${ }^{73}$ 

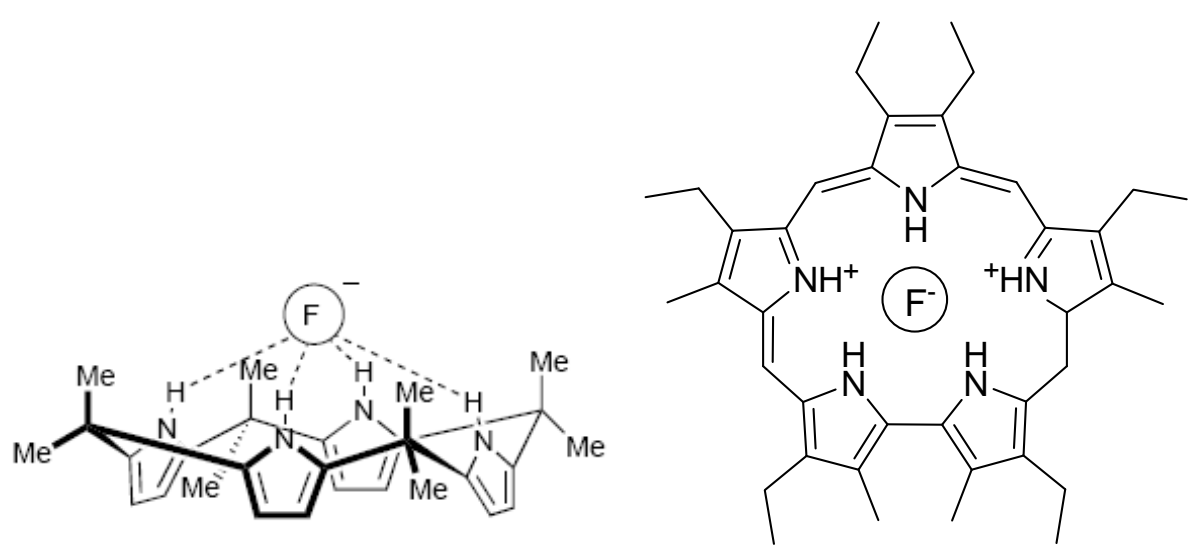

Abbildung 57: Fluor-Komplex mit dem nicht-aromatischen Octamethylcalix[4]pyrrol ${ }^{74}$ (links) und zweifach protoniertes Sapphyrin-Fluoro-Komplex ${ }^{75}$ (rechts).

Diese nicht-aromatischen Makrozyklen können durch ihre Pyrrol-NH-Gruppen selektiv Wasserstoffbrückenbindungen zu benachbarten Anionen ausbilden. Je nach Art des Anions können mit Hilfe der UV/Vis- bzw. NMR-Spektroskopie detaillierte Bindungsstudien durchgeführt werden. Auch die zweifach protonierten Sapphyrine können als Anionenrezeptoren fungieren (Abbildung 57 rechts).

Die in dieser Arbeit neu synthetisierten Makrozyklen haben strukturelle Ähnlichkeit zu den Calix[4]pyrrolen und zu den Sapphyrinen. Der Schiff-Base Makrozyklus 2a ist ebenfalls ein nicht konjugierter Makrozyklus und besitzt im Pyrazol- bzw. Pyrrolring NH-Gruppen, die als Anionen-Bindungsstellen fungieren könnten.

Es wurden daher eine Reihe unterschiedlicher Anionen zum Makrozyklus 2a gegeben und die Lösungen mit Hilfe der UV/Vis-Spektroskopie untersucht. Es konnten jedoch keine signifikanten Änderungen im UV/Vis-Spektrum von 2a detektiert werden. Die oben beschriebenen Änderungen der UV/Vis-Banden von 2a und den restlichen Makrozyklen werden aufgrund der Protonierung der Imin-Position beobachtet. Ein Anion ist hingegen lediglich in der Lage Wasserstoffbrückenbindungen zu den Pyrrol-NH bzw. Pyrrazol-NHGruppen auszubilden. Der elektronische Zustand des Chromophors 2a wird durch die Anionenbindung offenbar nicht beeinflusst, oder die Anionen-Affinität ist nur sehr gering.

Anionenrezeptor-Systeme können durch unterschiedliche Faktoren beeinflusst werden. Hierzu zählen sowohl die Größe und Geometrie des ausgewählten Anions als auch die Wahl des Lösungsmittels, da polare Lösungsmittel starke Wasserstoffbrückenbindungen zu dem jeweiligen Anion ausbilden und somit das System beeinflussen können. 
Die Umsetzung von 2a mit dem $\mathrm{F}^{-}$-Anion aus Tetrabutylammoniumfluorid (TBAF) in DMSO zeigt im UV/Vis-Spektrum (Abbildung 58) lediglich eine geringe hyperchrome Verschiebung der Bande (Schulter bei $400 \mathrm{~nm}$ ).

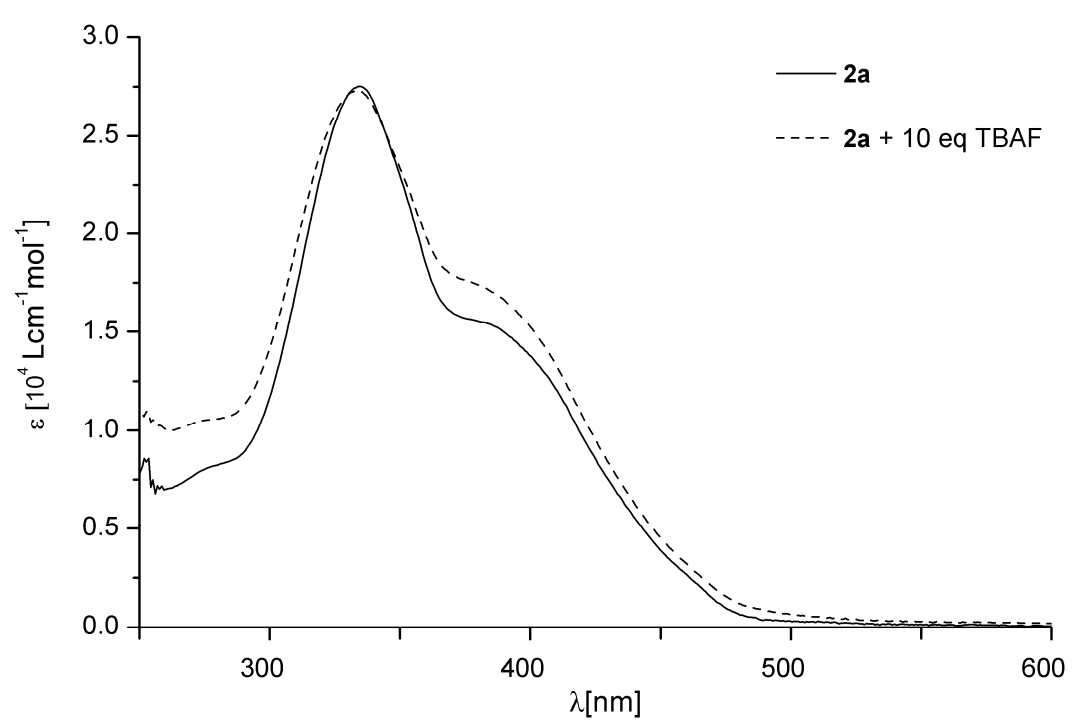

Abbildung 58: UV/Vis-Spektrum von 2a $\left(1.8 \cdot 10^{-4} \mathrm{M}\right.$ in DMSO) mit 10 eq. Fluorid aus einer Lösung von Tetrabutylammoniumfluorid (1.0 M in DMSO).

Das relativ kleine Fluorid-Ion kann sich über der Makrozyklus Kavität platzieren und zu den Pyrrol-NH-Gruppen Wasserstoffbrückenbindungen ausbilden. Aus der Röntgenstrukturanalyse von 2a ist bekannt, dass die NH-Gruppe des Pyrazolrings außerhalb der Makrozyklus-Kavität positioniert ist. Dies ermöglicht, wie Abbildung 59 zeigt, die Bildung einer weiteren Wasserstoffbrückenbindung mit einem zweiten Fluoridion.

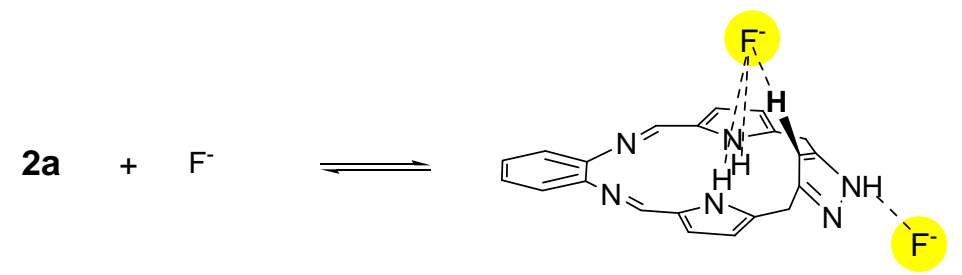

Abbildung 59: Mögliche Ausbildung von Wasserstoffbrückenbindungen zwischen 2a und Fluoridionen.

Dieses mögliche Anionenrezeptor-System wurde in der ${ }^{1} \mathrm{H}$ - bzw. ${ }^{19}$ F-NMR-Spektroskopie untersucht. Die NMR-Messungen wurden zuerst in $\mathrm{CDCl}_{3}$ durchgeführt, jedoch konnte aufgrund von schnellen Austauschreaktionen der Protonen bzw. Fluoratome keine signifikante Änderung in den ${ }^{1} \mathrm{H}$ - und ${ }^{19} \mathrm{~F}$-NMR-Spektren beobachtet werden. Die Titration 
wurde daher in DMSO- $\mathrm{d}_{6}$ durchgeführt. Die chemischen Verschiebungen des $\mathrm{CH}^{\mathrm{pz}}$-Protons während des Titrationsverlaufes sind in Abbildung 60 gezeigt.

Die NH-Protonen von 2a, die bei der Ausbildung von Wasserstoffbrückenbindungen zu den NH-Gruppen beeinflusst werden, konnten aufgrund schneller Austauschprozesse wiederum nicht detektiert werden. Bedingt durch den relativ hohen Schmelzpunkt von DMSO konnten keine Tieftemperatur Messungen durchgeführt werden.

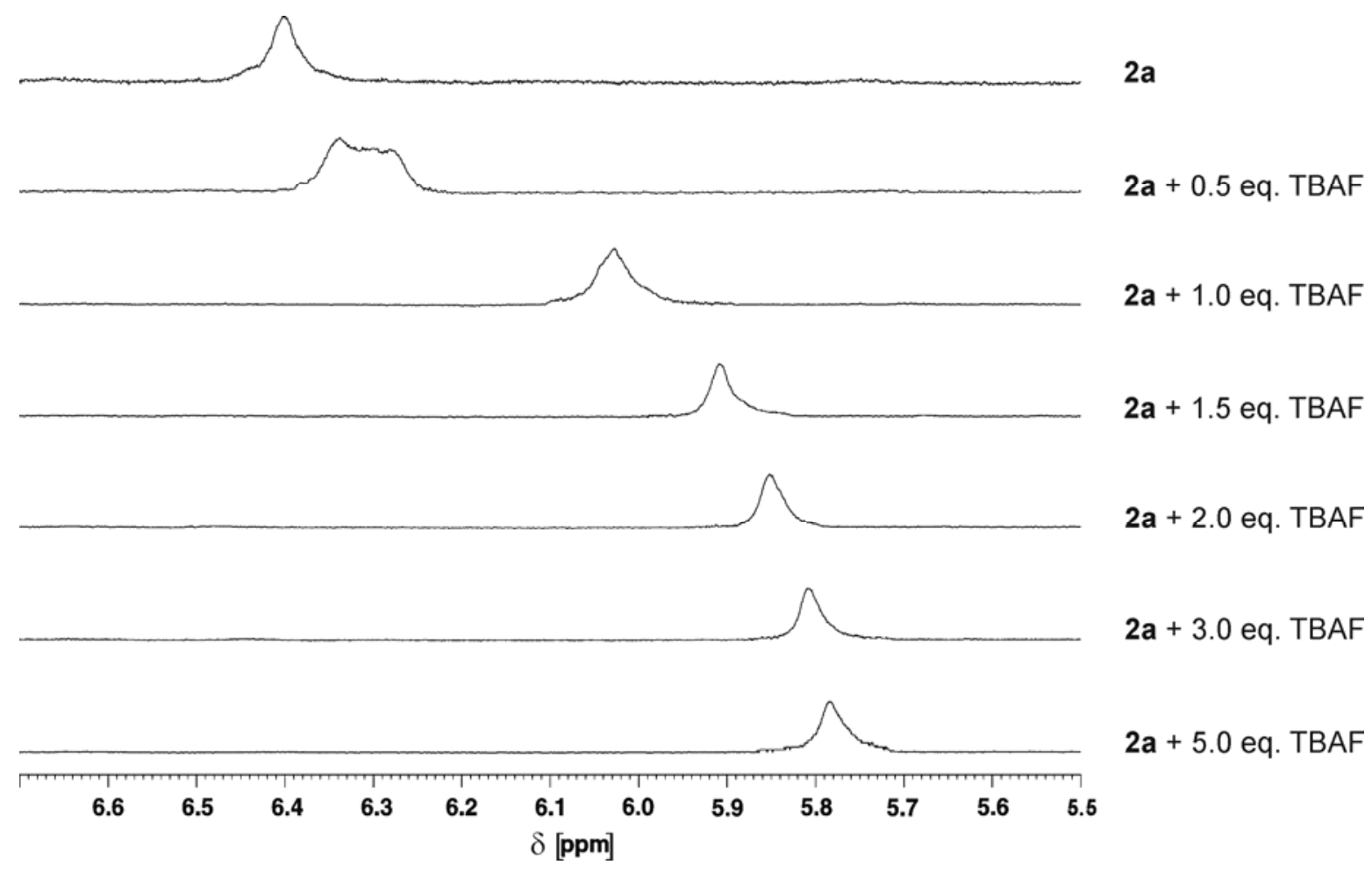

Abbildung 60: ${ }^{1} \mathrm{H}-\mathrm{NMR}$ Titration von 2a $\left(6.7710^{-2} \mathrm{M}\right)$ mit $\mathrm{F}^{-}$aus einer Standard $n$-Bu $\mathrm{H}_{4} \mathrm{NF}-\mathrm{Lösung}(1.02 \mathrm{M}$ in DMSO- $\left.\mathrm{d}_{6}\right)$. Das Signal bei $6.50 \mathrm{ppm}\left(\mathrm{CH}^{\mathrm{pz}}\right)$ zeigt eine deutliche Verschiebung bei der Zugabe von F-Ionen.

Während der Reaktion ist die Verschiebung der Lage des $\mathrm{CH}^{\mathrm{pz}}$-Protons von $6.50 \mathrm{ppm}$ auf $5.82 \mathrm{ppm}$ zu beobachten. Diese Verschiebung kann durch zwei Effekte versacht werden. Wie in Abbildung 59 bereits gezeigt, kann das $\mathrm{F}^{-}$-Anion externe Wasserstoffbrückenbindungen zu den Pyrazol-NH-Gruppen ausbilden. Eine weitere Möglichkeit ist die Bildung von Wasserstoffbrücken zwischen dem $\mathrm{CH}^{\mathrm{pz}}$-Proton und einem $\mathrm{F}^{-}$-Anion, welches über der Makrozyklus-Kavität fixiert ist. Diese zwei Wasserstoffbrückenbindungen beeinflussen die chemische Umgebung des Pyrazolringes und folglich die chemische Verschiebung des $\mathrm{CH}^{\mathrm{pz}}-$ Protons im ${ }^{1} \mathrm{H}-\mathrm{NMR}-$ Spektrum. 
Die Titration wurde parallel mittels der ${ }^{19} \mathrm{~F}-\mathrm{NMR}-$ Spektroskopie verfolgt. Hierbei sind während der Titration eine Reihe von F-Signalen zu erkennen, die jedoch nicht eindeutig zugeordnet werden konnten und im Rahmen dieser Arbeit nicht weiter untersucht wurden.

\subsubsection{Untersuchung der Bindungseigenschaften von 2d mittels UV/Vis-Spektroskopie}

Im Rahmen dieser Arbeit wurden die zwei biszyklischen Schiff-Base Makrozyklen 2d und 2e synthetisiert. Wie in den vorhergehenden Kapiteln diskutiert, können Schiff-Base Makrozyklen durch Protonierung an einer der Imin-Positionen ihren elektronischen Zustand ändern. Diese Protonierungsreaktionen können in der UV/Vis-Spektroskopie verfolgt werden.
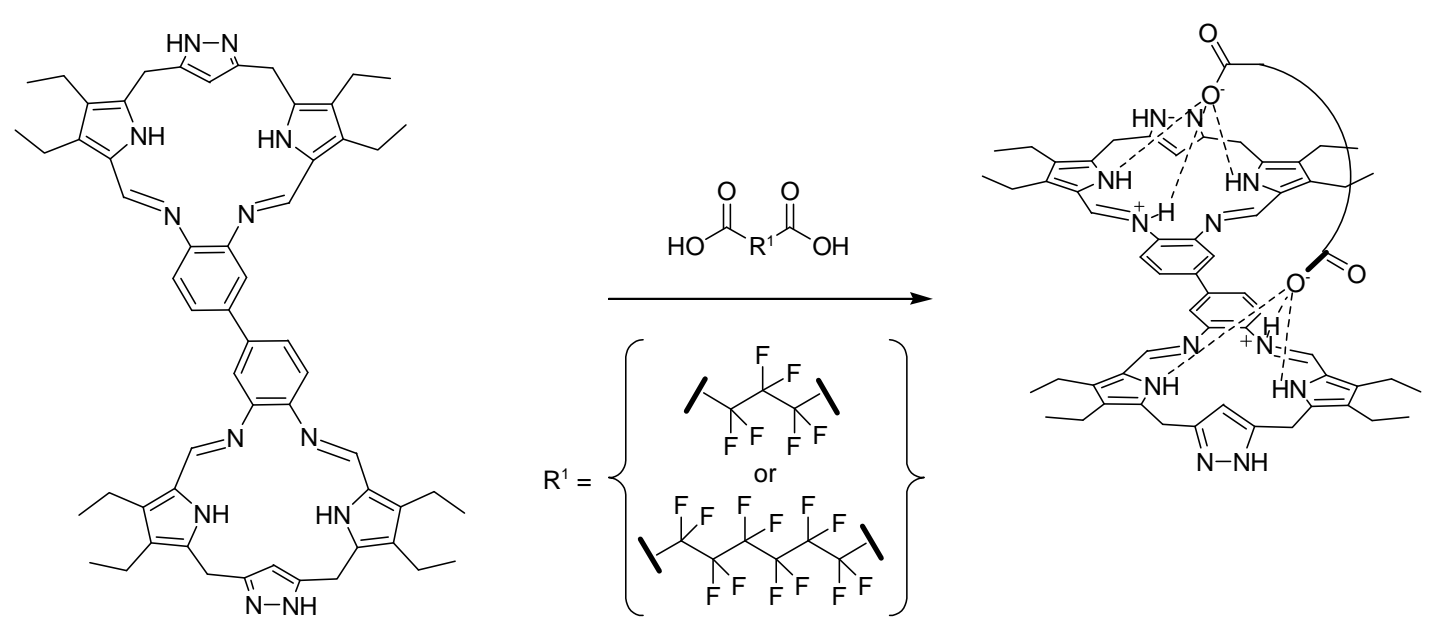

Abbildung 61: Schematische Darstellung der Protonierungs- bzw. Bindungseigenschaften von 2d mit unterschiedlich langen Fluordicarbonsäuren.

Da die Löslichkeit von 2e nur sehr gering ist, wurden die nachfolgenden Untersuchungen nur mit dem biszyklischen System 2d durchgeführt. Im Rahmen der Untersuchungen wurden zwei unterschiedlich lange Fluorocarbonsäuren ausgewählt, mit denen die Protonierungsbzw. Bindungseigenschaften der Säuren gegenüber 2d studiert werden sollten. Ausgewählt wurden die Dodekafluordiessigsäure (DFA) und die Hexafluorglutarsäure (HFGA) ( $\mathrm{pK}_{\mathrm{a}}=$ $0.07 \pm 0.10$ bzw. $\left.\mathrm{pK}_{\mathrm{a}}=0.08 \pm 0.10\right)^{76}$

Wie in Abbildung 62 gezeigt, bietet 2d durch seinen Aufbau die Möglichkeit zur Bildung von Wasserstoffbrückenbindungen. Nach Protonierung der Imin-Positionen kann sich das Säureanion dazu über dem Makrozyklus positionieren. 
Eine Chlorofom/Methanol-Lösung von 2d $\left(3.0 \times 10^{-5} \mathrm{M}\right)$ wurde stufenweise mit einer Dodecafluordiessigsäure Lösung in Acetonitril $\left(9.0 \times 10^{-3} \mathrm{M}\right)$ titriert. Die Intensität der Bande des Chromophors bei $352 \mathrm{~nm}$ nimmt im Laufe der Titration ab während eine neue intensivere Bande bei $380 \mathrm{~nm}$ entsteht. Analog zu der Säure-Base Reaktion von 2a wird ein isosbestischer Punkt bei $364 \mathrm{~nm}$ beobachtet. Dies lässt vermuten, dass auch hier die Protonierungsreaktion einheitlich verläuft.

In Abbildung 62 ist der Titrationsverlauf von 2d mit Dodecafluordiessigsäure und das Titrationsprofil der Reaktion gezeigt. Nach der Zugabe von einem Äquivalent Säure zum Makrozyklus werden keine weiteren signifikanten Änderungen im UV/Vis-Spektrum beobachtet.

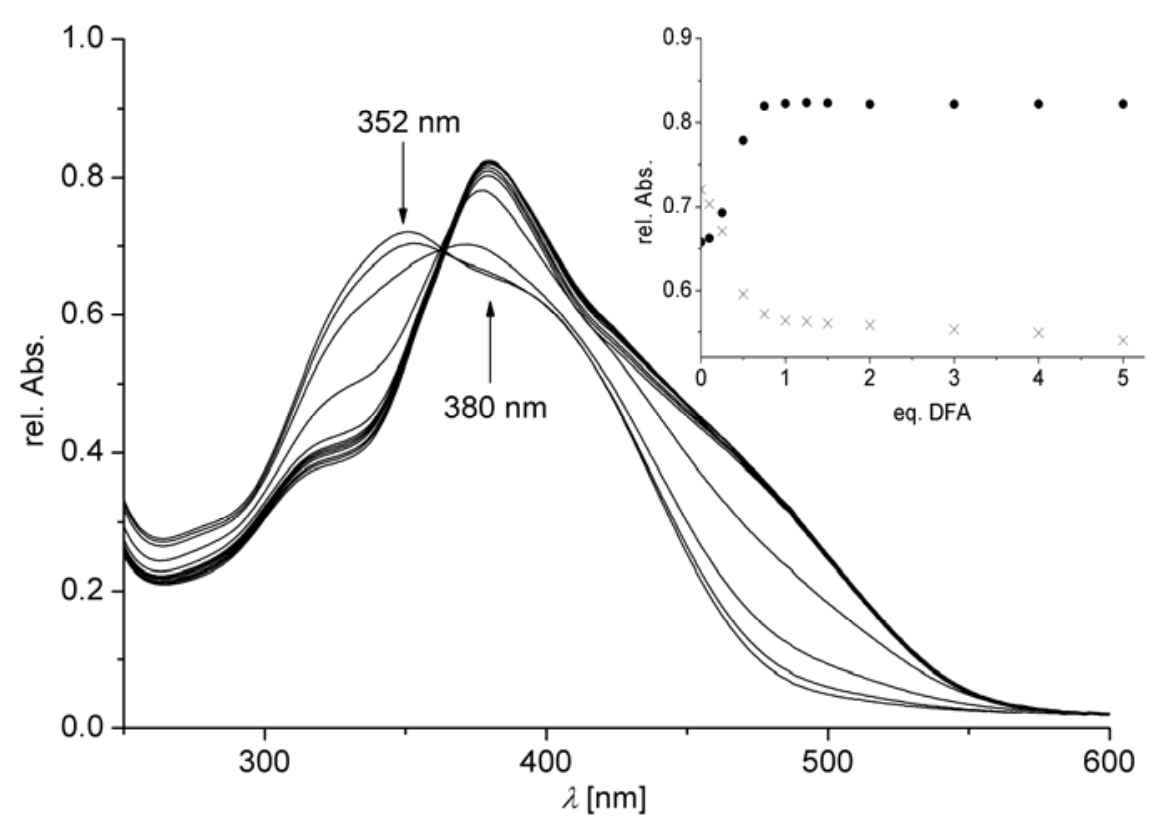

\begin{abstract}
Abbildung 62: UV/Vis-spektrophotometrische Titration von 2d (3.0×10-5 $\mathrm{M}$ in Chlorofom/Methanol 19:1) mit einer Standard-Lösung von Dodecafluordiessigsäure $\left(9.0 \times 10^{-3} \mathrm{M}\right.$ in Acetonitril). Oben rechts ist das Titrationsprofil mit relativer Absorption bei $352 \mathrm{~nm}(\times)$ und $379 \mathrm{~nm}(\bullet)$ in Form der relativen Absorption aufgetragen gegen die Äquivalente an DFA dargestellt.
\end{abstract}

Eine Imin-Position pro Makrozyklus-Kavität wird in 2d durch je ein Proton der Dicarbonsäure protoniert und ein 1:1 Komplex kann entstehen. Die Titration wurde analog mit der kürzeren Hexafluoroglutarsäure durchgeführt. Wie Abbildung 63 zeigt ist der Verlauf der Titration von 2d mit Hexafluorglutarsäure vergleichbar mit dem der Dodecafluordiessigsäure (Abbildung 62). 


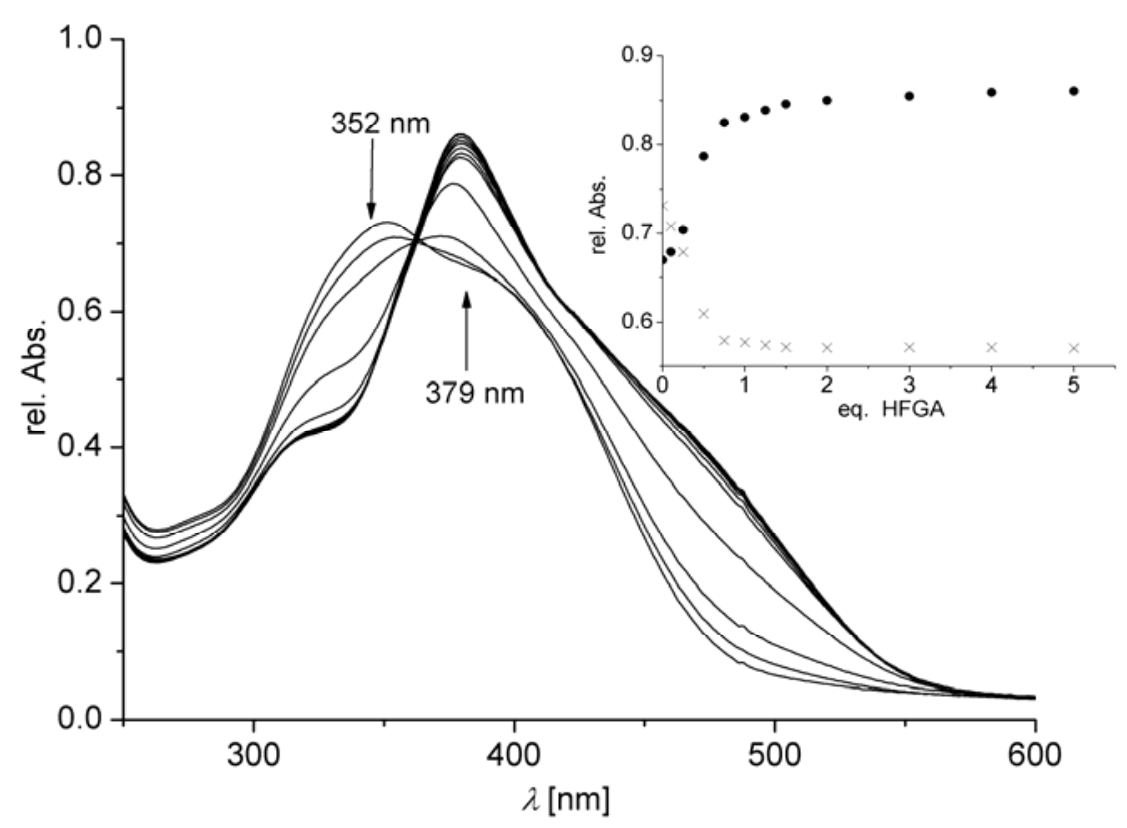

Abbildung 63: UV/Vis-spektrophotometrische Titration von $2 \mathbf{d}\left(3.0 \times 10^{-5} \mathrm{M}\right.$ in Chloroform) mit einer StandardLösung von Hexafluorglutarsäure $\left(9.0 \times 10^{-3} \mathrm{M}\right.$ in Acetonitril). Oben rechts ist das Titrationsprofil mit relativer Absorption bei $352 \mathrm{~nm}(\times)$ und $379 \mathrm{~nm}(\bullet)$ aufgetragen gegen die Äquivalente an HFGA dargestellt.

Das Titrationsprofil dieser Titration zeigt, dass das System nach der Zugabe von einem Äquivalent HFGA zu 2d nahezu vollständig reagiert hat. Bei weiterer Addition von HFGA ist eine sehr schwache hyperchrome Verschiebung der Bande bei $379 \mathrm{~nm}$ zu beobachten. Diese Verschiebung kommt durch den höheren $\mathrm{pK}_{\mathrm{a}}-$ Wert der Säure $\left(\mathrm{pK}_{\mathrm{a}}=0.08 \pm 0.10\right)$ zustande. Die zweite Dissoziationskonstante von HFGA ist kleiner als der erste, so dass 2d nach Zugabe eines Äquivalents HFGA unvollständig vorliegt. Es wird daher mehr als ein Äquivalent HFGA zur vollständigen Protonierung von 2d benötigt.

Vergleicht man das Titrationsprofil von $\mathbf{2 d}$ mit dem von 2a (Titration mit TFA) so stellt man fest, dass bei der Titration von 2d kein scharfer isosbestischer Punkt (bei $364 \mathrm{~nm}$ ) vorliegt. Diese Beobachtung läßt vermuten, dass der Protonierungsvorgang anders als bei 2a, nicht einheitlich, sondern über mehrere Prozesse abläuft. In Abbildung 64 sind zwei mögliche Protonierungszenarien von 2d dargestellt. Die Benzidinbrücke von 2d ist innerhalb ihrer Längsachse frei drehbar. Bei der Protonierung von 2d kann das Säureanion über Wasserstoffbrückenbindungen einen 1:1 Komplex mit dem Makrozyklus bilden. Durch die freie Drehbarkeit der Benzindinbrücken kann das Säureanion jedoch auch verdrängt werden. Wahrscheinlicher ist daher die Bildung von Ketten. Da der Makrozyklus von je zwei unterschiedlichen Säuremolekülen protoniert wird, verläuft die Reaktion vermutlich nicht einheitlich, sondern in mindestens zwei Stufen ab. 


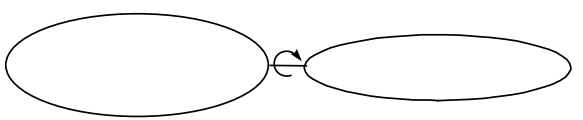

$$
\downarrow \stackrel{\mathrm{HO}_{\mathrm{R}^{1}}}{\mathrm{O}} \stackrel{\mathrm{OH}}{\mathrm{OH}}^{\mathrm{O}}
$$
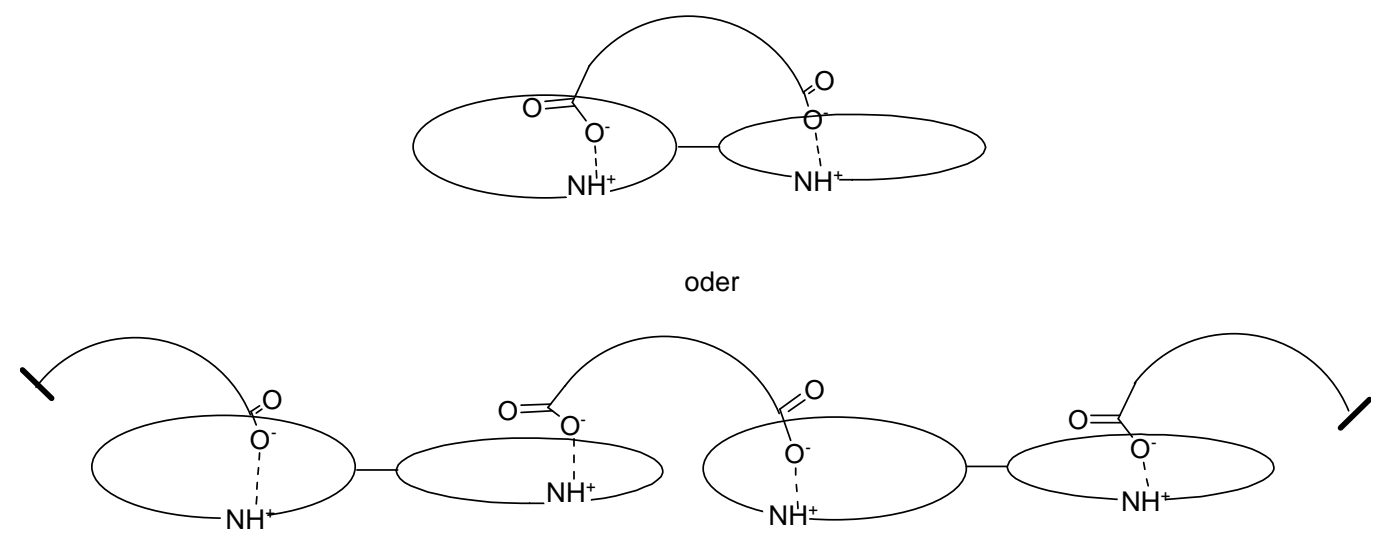

Abbildung 64: Schematische Darstellung möglicher Protonierungsprozesse von 2d mit einer Dicarbonsäure.

\subsubsection{Darstellung von Metallkomplexen der Schiff-Base Makrozyklen}

Die neuartigen Schiff-Base Makrozyklen (2a-2e) wurden als Chelatliganden bei der Komplexsynthese eingesetzt. Die Makrozyklen bieten prinzipiell sowohl an den IminPositionen als auch an den zwei Pyrrol-N und den zwei Pyrazol-N Atome mögliche Koordinationsstellen für die Metallkomplexierung an. So könnten, wie in Abbildung 65 gezeigt, quadratisch planare Metallkomplexe synthetisiert werden. Abhänging von der Beschaffenheit des eingesetzten Metalls könnte der Pyrazolring in die Koordinationssphäre eingebunden werden. Lanthanoide gehören $\mathrm{zu}$ der Metallgruppe, die eine Mehrfachkoordination erlauben.

Im Rahmen dieser Arbeit wurde eine Reihe unterschiedlicher Metallsalze $\left(\mathrm{Hg}(\mathrm{OAc})_{2}\right.$, $\mathrm{PdCl}_{2}(\mathrm{COD}), \mathrm{Cu}(\mathrm{OAc})_{2}, \mathrm{Ni}(\mathrm{OAc})_{2}, \mathrm{CrCl}_{3} \cdot \mathrm{THF}, \mathrm{Pb}\left(\mathrm{ClO}_{4}\right)_{2} \cdot 3 \mathrm{H}_{2} \mathrm{O}, \mathrm{ScCl}_{3}, \mathrm{Sm}\left(\mathrm{NO}_{3}\right)_{3} \cdot 3 \mathrm{H}_{2} \mathrm{O}$, $\mathrm{CdCl}_{2}, \mathrm{FeCl}_{3}, \mathrm{Ag}_{2}(\mathrm{OAc}), \mathrm{NdCl}_{3}$ in $\mathrm{TFH}$ etc.) zur Darstellung von Metallkomplexen eingesetzt. Die Komplexsynthese hat sich jedoch als viel anspruchsvoller erwiesen als erwartet. In manchen der Reaktionen konnte die Entstehung des Metallkomplexes in Lösung nachgewiesen werden. 


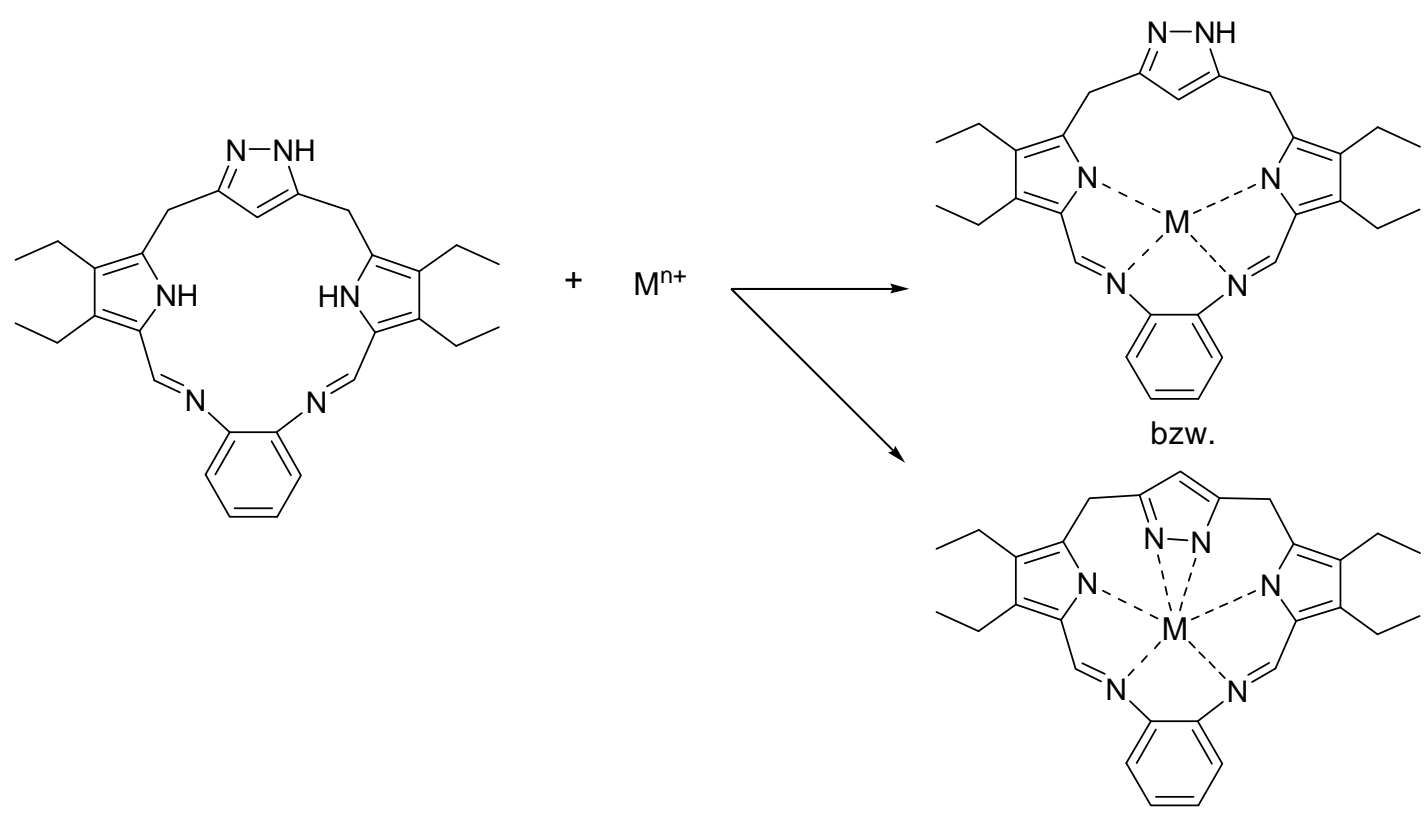

Abbildung 65: Allgemeine Metalllomplexsynthese mit 2a als Chelatligand.

Im Falle des Quecksilberacetats wurde der Makrozyklus 2a in einem Gemisch aus Chloroform/Acetonitril gelöst und anschließend mit $\mathrm{Hg}(\mathrm{OAc})_{2}$ versetzt. Das Reaktionsgemisch wurde einen Tag bei Raumtemperatur gerührt.

Die Reaktionslösung wurde mittels der ESI-Massenspektrometrie untersucht (Abbildung 66). Der freie Makrozyklus ist nach der Reaktion noch zu einem geringen Anteil nachweisbar (bei $\left.467 \mathrm{~m} / \mathrm{z}[\mathrm{M}+\mathrm{H}]^{+}\right)$. Im Massenspektrum werden drei relevante Signale detektiert, die einem Quecksilberkomplex mit 2a als Chelatligand zugeordnet werden können. Das Signal bei $667 \mathrm{~m} / \mathrm{z}$ entspricht der Zusammensetzung von [2aHg] als einfach protonierte Spezies $([\mathbf{2 a H g}]+\mathrm{H})^{+}$. Desweiteren existiert in der Lösung noch ein Komplex-Dimer $\left((2 \times[\mathbf{2 a H g}]+\mathrm{H})^{+}\right)$bei $1331 \mathrm{~m} / \mathrm{z}$ und ein Komplex-Trimer $\left((3 \times[\mathbf{2 a H g}]+\mathrm{H})^{+}\right)$bei $1994 \mathrm{~m} / \mathrm{z}$. Die Isotopenverteilung der gemessenen Signale entspricht der berechneten Verteilung. Der nach der Komplexsynthese gewonnene gelbe Feststoff von [2aHg] konnte nicht in einkristalliner Form für die Röntgenstrukturanalyse erhalten werden.

Weitere Metallkomplexe mit Pd(II)-, Ni(II)- und $\mathrm{Nd}(\mathrm{III})$-Salzen konnten in wenigen Fällen massenspektrometrisch nachgewiesen werden, jedoch gelang es nicht diese verläßlich zu reproduzieren. 


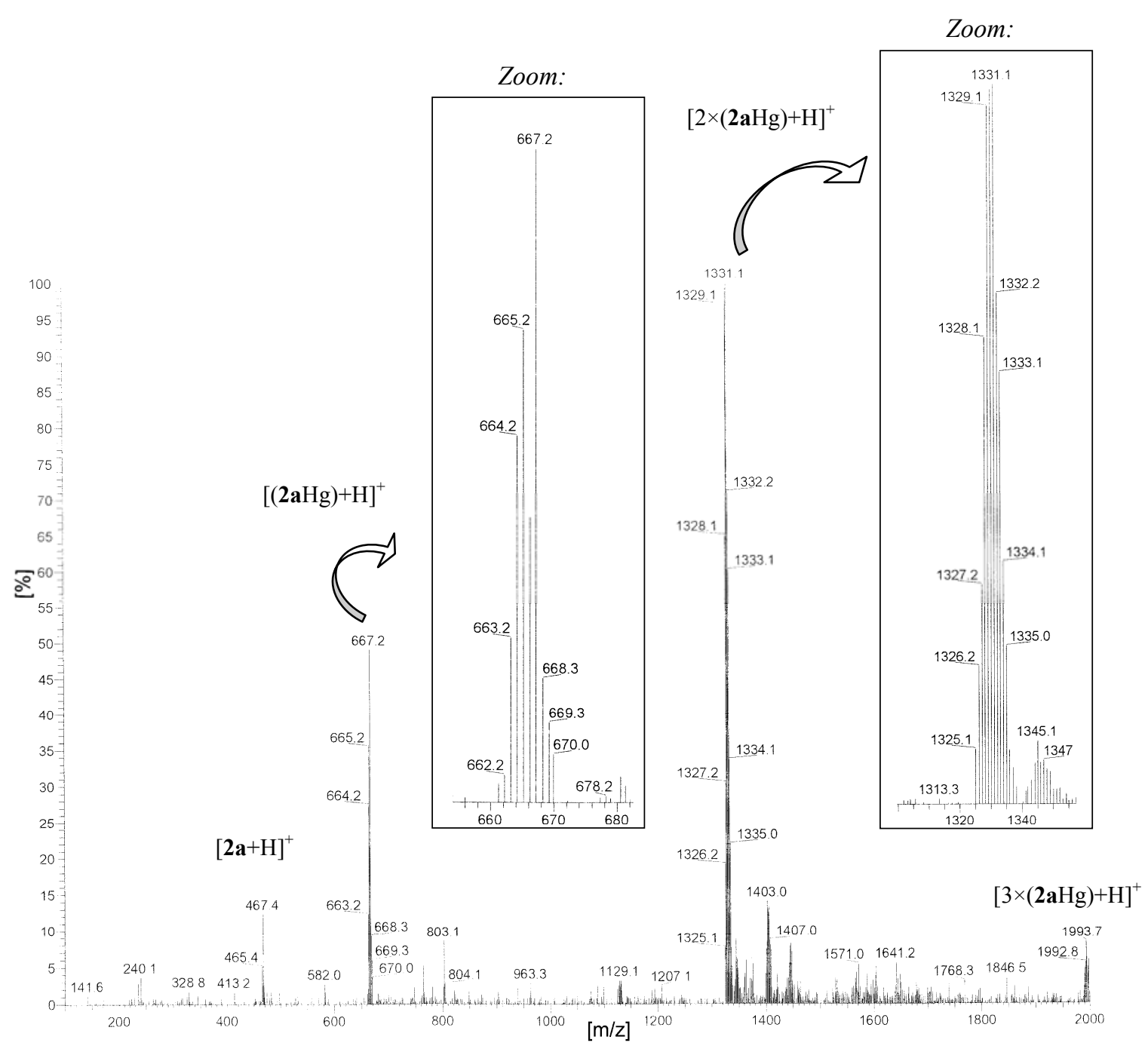

Abbildung 66: ESI(+)-Massenspektrum von [2aHg] in $\mathrm{MeCN} / \mathrm{CHCl}_{3}$.

Analog zu den Doppelporphyrinen wurde auch hier versucht einen konjugierten Makrozyklus aus den Schiff-Base Makrozyklen (2) zu synthetisieren. Allerdings ließen sich die $\mathrm{CH}_{2}{ }^{\mathrm{py} / \mathrm{pz}-}$ Brücken mit den in der Porphyrinchemie häufig verwendeten Oxidationsmittel DDQ und $p$ Chloranil nicht zur Aromatisierung der Pyrazolderivate bringen. Auch bei der Verwendung von anderen Oxidationsmitteln wie $\mathrm{BaMnO}_{4}{ }^{77}$ oder $\mathrm{MnO}_{2}$ konnte kein voll aromatisches System beobachtet werden. In den Arbeiten von Sessler et al. wurde berichtet, dass Texaphyrinogene durch die Anwesenheit einer Base bzw. eines Übergangmetalls (Cd(II), $\mathrm{Hg}(\mathrm{II}), \mathrm{La}(\mathrm{III})$ etc.) die aromatische Texaphyrine in Form eines Komplexes synthetisiert werden konnten. ${ }^{19}$ Analog $\mathrm{zu}$ diesen wissenschaftlichen Arbeiten wurden auch hier die Pyrrol/Pyrazol-basierten Schiff-Base Makrozyklen mit einer Base bzw. einem Übergangmetalls umgesetzt. Die Bildung eines konjugierten Systems konnte in keinem Fall beobachtet werden. Pyrazolderivate sind aufgrund der zwei elektronegativen N-Atome 
weniger reaktiver bei einem elektrophilen Angriff und bei einer Oxidationsreaktion, als die Pyrrolderivate. $^{78}$

Die Spacefilling-Modelle von 2a in Abbildung 67 zeigen, dass der Raum in der Makrozyklus Kavität relativ klein ist. Größere Metalle können sich entsprechend in die Kavität nicht hineinpassen. Dies würde begründen, weshalb es in dem meisten Fällen nicht zur Komplexbildung kommen konnte.
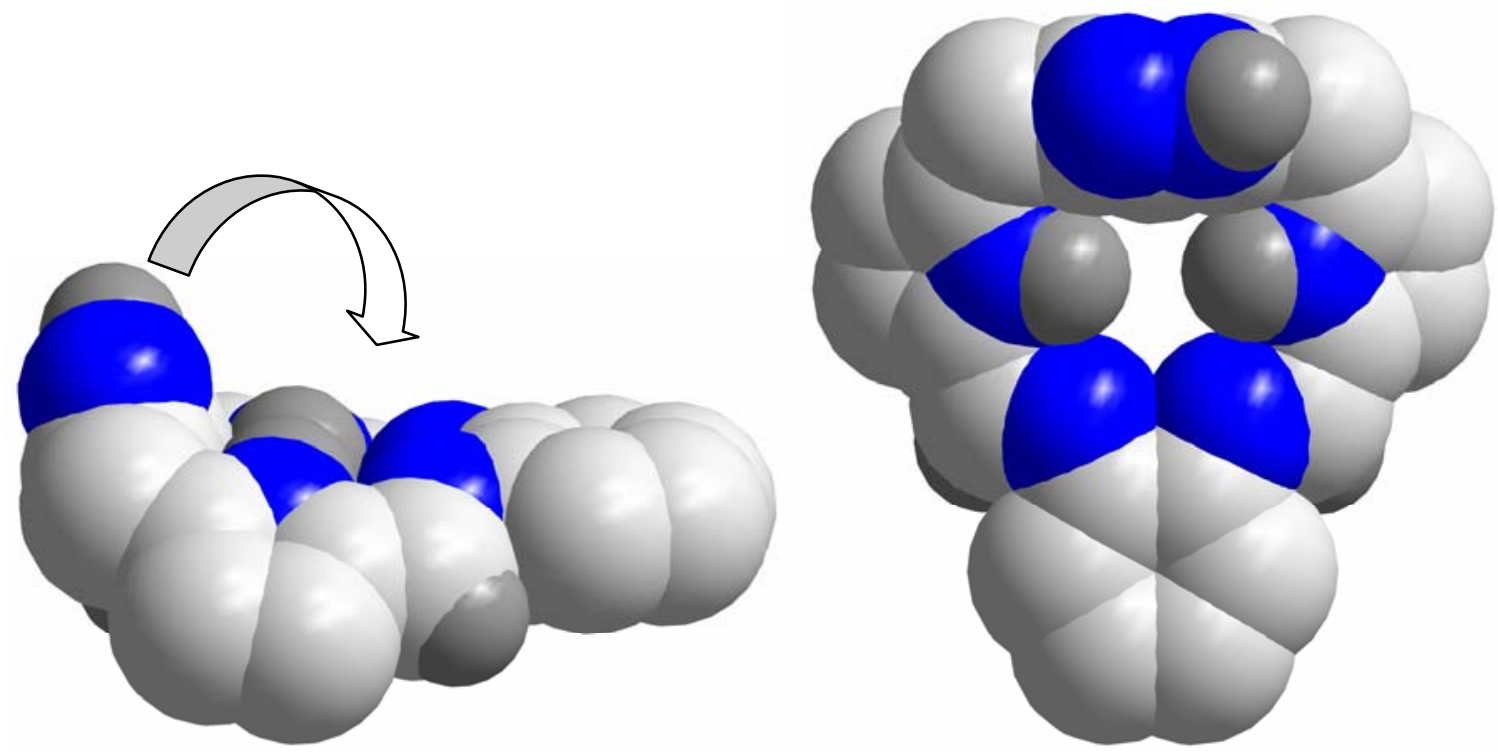

Abbildung 67: Spacefilling-Modelle von 2a.

Ein weiterer wichtiger Grund ist die Positionierung des Pyrazolrings. In den Molekülstrukturen der Pyrrol/Pyrazol-basierten Hybrid-Makrozyklen 2a, 2b bzw. 2c konnte bereits gezeigt werden, dass der Pyrazolring eine gewisse Flexibilität besitzt (Kapitel 5.2.2.1), der Pyrazolring zu einem gewissen Grad um die $\mathrm{CH}_{2}{ }^{\mathrm{py} / \mathrm{pz}}$-Brücken rotieren kann. Ein schwach koordiniertes Metallion könnte durch die Rotation des Pyrazol-Ringes somit über dem Makrozyklus Hohlraum verdrängt werden und der gebildete Komplex würde dissoziieren. 


\section{Neuartige Pyrrol/Pyrazole-basierte Diimin-Liganden}

\section{Einleitung}

Pyrroleinheiten gelten nicht nur als interessante Bausteine in der Porphyrinchemie, sondern auch als Liganden in einer Reihe azyklischer Systeme. ${ }^{79,80,81}$ Weber et al. synthetisierten schon in den sechziger Jahren tetradentate Diimin-Pyrrol Liganden des Typs LIII (Abbildung 68) durch Reaktion von Pyrrol-2-carbaldehyd mit einem Diamin. ${ }^{82}$ Mit Hilfe dieser Liganden konnten diverse quadratisch-planare Metallkomplexe ( $\mathrm{M}=\mathrm{Pd}(\mathrm{II}), \mathrm{Ni}(\mathrm{II}), \mathrm{Cu}(\mathrm{II})$ und $\mathrm{Zn}(\mathrm{II})$ ) synthetisiert werden, um ihre spektroskopischen bzw. magnetischen Eigenschaften zu untersuchen. $^{83}$

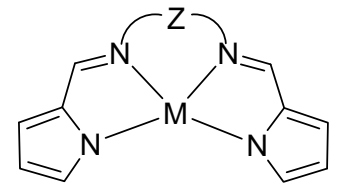

LIII

$Z=$ aromatischer oder aliphatischer Spacer

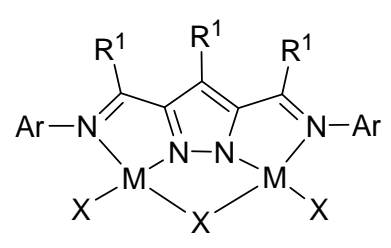

LIV

$\mathrm{R}^{1}=\mathrm{H}, \mathrm{CH}_{3}$

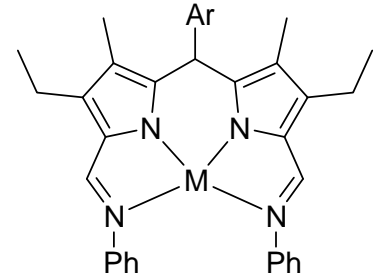

LV

Abbildung 68: Literaturbekannte Pyrrol- bzw. Pyrrazol-basierte Diimin-Liganden und Komplexe.

Die zunehmende Forschungstätigkeit auf dem Gebiet bimetallischer Komplexe hat zur Entwicklung einer großen Anzahl von Pyrazolatverbindungen als Brückenliganden geführt. 3,5-Pyrazol-basierte $\alpha$-Diimin Palladium(II)- und Nickel(II)-Komplexe (LIV, Abbildung 68) wurden bereits in der Arbeitsgruppe Meyer dargestellt. ${ }^{84,85}$ Diese neuartigen zweikernigen Metallkomplexe eignen sich als Präkatalysatoren in der Ethylenpolymerisation. ${ }^{84}$ Durch die Einführung unterschiedlicher sterisch anspruchsvoller Gruppen am Rückgrat $\left(\mathrm{R}^{1}\right)$ und an den Iminsubstituenten der Liganden (Aryl-Substituenten) kann die Aktivität der Katalysatoren gezielt modifiziert werden.

Katayev et al. veröffentlichten kürzlich eine interessante Arbeit über die Synthese und Reaktivität von neuartigen Ni-, Pd-, bzw. Pt-Diiminodipyrromethan-Komplexen (LV, Abbildung 68). ${ }^{86}$ Diese Komplexe sind in der Lage Sauerstoff Metall-abhängig zu aktivieren und folglich eine Autoxidation des Liganden auszulösen.

In Anlehnung an diese Studien wurden in der vorliegenden Arbeit neuartige 3,5disubstituierte Pyrrol/Pyrazol-basierte Diimin-Liganden synthetisiert, die als Liganden zur 
Darstellung bimetallischer Komplexe des Typs $\mathbf{L M}_{2} \mathbf{X}$ eingesetzt wurden (Abbildung 69). Derzeit sind in der Literatur keine Beispiele von 3,5-disubstituierten Pyrrol/Pyrazol-basierten Diimin-Liganden bekannt. Die Synthese dieser Liganden wird erstmals im Rahmen dieser Arbeit beschrieben. Das Pyrazolat dient bei den Verbindungen als verbrückende Einheit. Insgesamt bietet der Chelatligand am Pyrazol-, an den zwei Pyrrol-Einheiten und an den Imin-Positionen mehrere N-Donoratome zur Koordination von zwei Metallen an. Durch die Wahl von unterschiedlichen Resten $\left(\mathrm{R}^{2}\right)$ mit verschiedenem sterischen Anspruch an der IminPosition kann die individuelle Koordinationsumgebung der Metalle modifiziert werden. Die spektroskopischen und magnetischen Eigenschaften der resultierenden Metallkomplexe wurden eingehend untersucht.

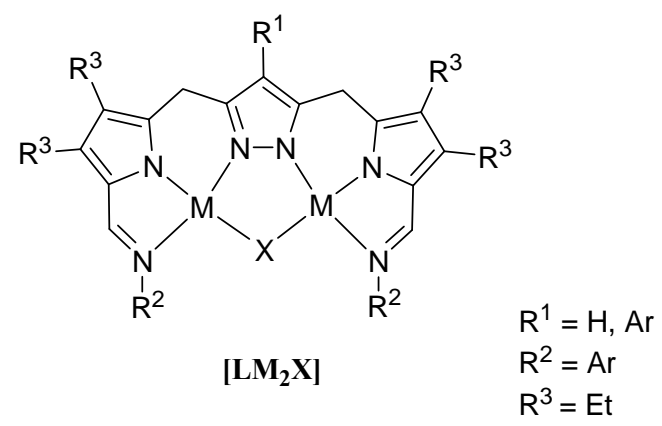

Abbildung 69: Generelle Struktur der Zielverbindungen des Typs [ $\left.\mathbf{L M}_{2} \mathbf{X}\right]$.

Als Bausteine zur Darstellung der 3,5-disubstituierten Pyrrol/Pyrazol-basierten DiiminLiganden $\left(\mathbf{H}_{3} \mathbf{L}\right)$ wurden die Dialdehyde 1a und $\mathbf{1 b}$ verwendet. Das jeweilige Edukt wurde unter $\mathrm{N}_{2}$-Atmosphäre bei $50{ }^{\circ} \mathrm{C}$ in trockenem Methanol gelöst und mit einem Überschuss einer Serie von ausgewählten Aminen versetzt (Abbildung 70).

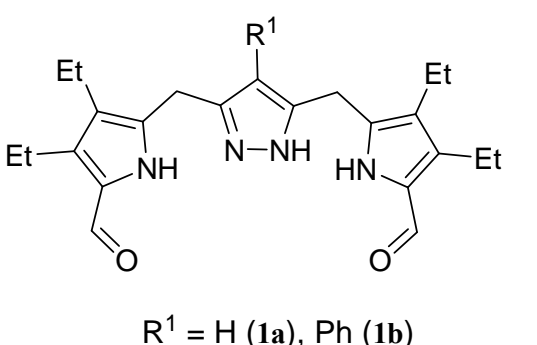

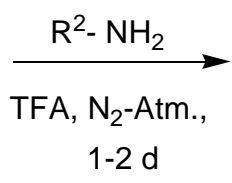

$R^{1}=H(1 a), P h(1 b)$

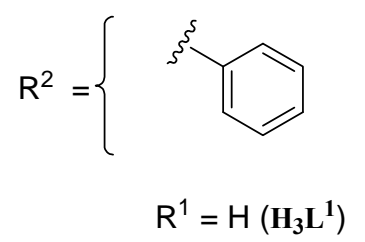

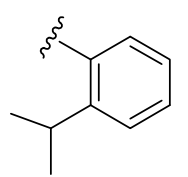

$\mathrm{R}^{1}=\mathrm{H}\left(\mathbf{H}_{3} \mathbf{L}^{2}\right)$
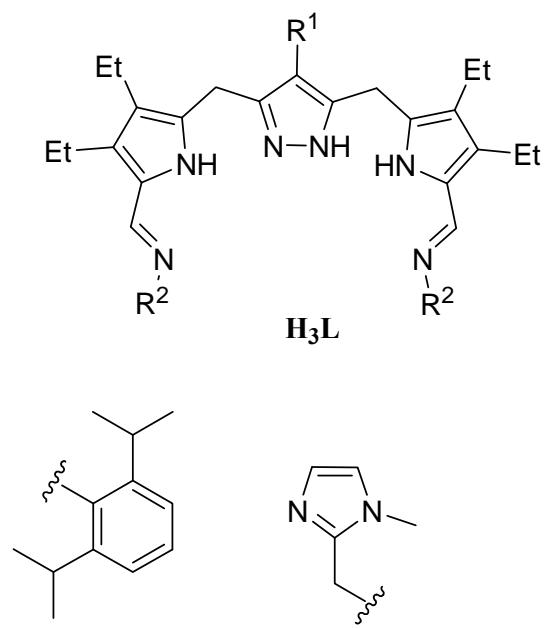

$R^{1}=H\left(H_{3} L^{3}\right) ; \quad R^{1}=H\left(H_{3} L^{5}\right)$

$\mathrm{R}^{1}=\mathrm{Ph}\left(\mathrm{H}_{3} \mathbf{L}^{4}\right)$

Abbildung 70: Synthese der 3,5-Pyrazol/Pyrrol-basierten Diimin Liganden. 
Nach einigen Minuten wurde ein Überschuss an Trifluoressigsäure zum Reaktionsgemisch gegeben und die Lösung ein bis zwei Tage unter Rückfluss erhitzt. Der Syntheseverlauf hat gezeigt, dass je höher der Überschuss des eingesetzten Amins ist (2-6 eq.), desto höher ist die Ausbeute des gewünschten Liganden.

Alle neuen synthetisierten Liganden konnten säulenchromatographisch aufgereinigt werden. Jedoch mussten bei der Reaktion entstandene Rohprodukte oft mehrfach säulenchromatographisch gereinigt und anschließend umkristallisiert werden, um eine für die Metallkomplexierung ausreichende Reinheit zu besitzen. In Abbildung 70 sind die Liganden $\mathbf{H}_{3} \mathbf{L}^{1}, \mathbf{H}_{3} \mathbf{L}^{2}, \mathbf{H}_{3} \mathbf{L}^{3}, \mathbf{H}_{3} \mathbf{L}^{4}$ und $\mathbf{H}_{3} \mathbf{L}^{5}$ dargestellt die im Rahmen dieser Arbeit synthetisiert werden konnten.

Einzig die Verbindung $\mathbf{H}_{3} \mathbf{L}^{2}$ konnte zwar synthetisiert, aber bis heute nicht isoliert werden. Dieser Ligand wurde mit der ESI(+/-)-Massenspektrometrie nachgewiesen. Das ESI(+)Massenspektrum zeigt ein einziges Signal bei $629.6 \mathrm{~m} / \mathrm{z}(100 \%)[\mathrm{M}+\mathrm{H}]^{+}$, welches der molekularen Zusammensetzung von $\mathbf{H}_{3} \mathbf{L}^{2}$ mit $628.9 \mathrm{~g} \cdot \mathrm{mol}^{-1}\left[\mathrm{C}_{41} \mathrm{H}_{52} \mathrm{~N}_{6}\right]$ entspricht. Die anderen Liganden konnten durch Anwendung mehrerer analytischer Methoden vollständig charakterisiert werden. Eine detaillierte Beschreibung findet sich im Experimentellen Teil (Kapitel 10.5) dieser Arbeit.

In den spektroskopischen Untersuchungen der dargestellten Liganden sind die Imin-Gruppen besonders charakteristisch. Mittels IR-Spektroskopie wurden die $\mathrm{C}=\mathrm{N}-\mathrm{Streckschwingungen}$ der Liganden bei 1615-1620 $\mathrm{cm}^{-1}$ detektiert (Kapitel 7.4.1). Im ${ }^{1} \mathrm{H}-\mathrm{NMR}-$ Spektrum werden die charakteristischen Imin-Signale zwischen 7.85-8.10 ppm beobachtet. 


\section{Darstellung der bimetallischen Komplexe}

Die dargestellten Liganden $\mathbf{H}_{3} \mathbf{L}^{1}, \mathbf{H}_{3} \mathbf{L}^{3}, \mathbf{H}_{3} \mathbf{L}^{4}$ und $\mathbf{H}_{3} \mathbf{L}^{5}$ wurden im nächsten Schritt in der Synthese von Metallkomplexen eingesetzt. $\mathrm{Zu}$ diesem Zweck wurde der jeweilige Ligand in einem organischen Lösungsmittel gelöst und mit Triethylamin umgesetzt. Anschließend wurde das Metallsalz (Pd(II)-, Pt(II)-, Ni(II)-, $\mathrm{Co}(\mathrm{II})-$ und $\mathrm{Cu}(\mathrm{II})-\mathrm{Salze}$ ) hinzugefügt und das Reaktionsgemisch 1-4 Tage gerührt.

Hierbei entstanden neutrale bimetallische Komplexe, in denen die Metallionen quadratisch planar koordiniert sind. Metallionen wie Fe(III) oder $\mathrm{Zn}$ (II), die eine tetraedrische bzw. quadratisch pyramidale Koordination bevorzugen, bilden mit diesen Liganden keine Koordinationsverbindungen. Unter Verwendung von Pt-Salzen, wie dem $\mathrm{K}_{2} \mathrm{PtCl}_{4}$, kommt es zur Komplexbildung mit den Liganden $\mathbf{H}_{3} \mathbf{L}^{\mathbf{1}}$ und $\mathbf{H}_{3} \mathbf{L}^{\mathbf{3}}$, jedoch konnten die isolierten Verbindungen aufgrund ihrer extrem geringen Löslichkeit in diversen Lösemitteln nicht untersucht werden.

\subsection{Bimetallische Kupfer(II)komplexe der Liganden $\mathrm{H}_{3} L^{1}, \mathrm{H}_{3} \mathrm{~L}^{3}, \mathrm{H}_{3} \mathrm{~L}^{4}$ und $\mathrm{H}_{3} \mathrm{~L}^{5}-$ Strukturelle Charakterisierung, spektroskopische und magnetische Eigenschaften}

Zur Darstellung des Kupferkomplexes von $\mathbf{H}_{3} \mathbf{L}^{1}$ wurde der Ligand mit 2.1 eq. Kupferacetat umgesetzt (Abbildung 71). Der entstandene Komplex wurde mittels der ESI(+)Massenspektrometrie charakterisiert. Für den Komplex $\left[\mathbf{L}^{1} \mathbf{C u}_{\mathbf{2}}(\mathbf{O H})\right]$ mit der Molmasse von $684 \mathrm{~g} \cdot \mathrm{mol}^{-1}$ würde bei Protonierung in der ESI(+)-Massenspektrometrie ein Signal bei $685 \mathrm{~m} / \mathrm{z}$ für $[\mathrm{M}+\mathrm{H}]^{+}$zu erwarten sein. Hierbei konnte jedoch ein Signal bei $683 \mathrm{~m} / \mathrm{z}(100 \%)$ für $[\mathrm{M}+\mathrm{H}]^{+}$beobachtet werden, welches einer Masse der Summenformnel $\left[\mathrm{C}_{36} \mathrm{H}_{36} \mathrm{~N}_{6} \mathrm{Cu}_{2} \mathrm{O}\right]$ entspricht.

Durch die Elektronenionisation kommt es wahrscheinlich zu einer Oxidation des Komplexes, so dass die Verbindung zwei Protonen verliert und daher die beobachtete Massendifferenz auftritt. In Abbildung 72 wird der mögliche Oxidationsweg während der Ionisation aufgezeigt. Es ist allgemein bekannt, dass sich Pyrrol- bzw. Polypyrrolderivate relativ leicht oxidieren lassen. Durch die Abgabe von zwei Protonen bzw. zwei Elektronen werden die Methylengruppen des Liganden anscheinend oxidiert. Dadurch wird die effektive 
Ladungsdichte an den Kupferatomen so stark verändert, dass es zu einer Änderung der formalen Oxidationsstufe kommt.
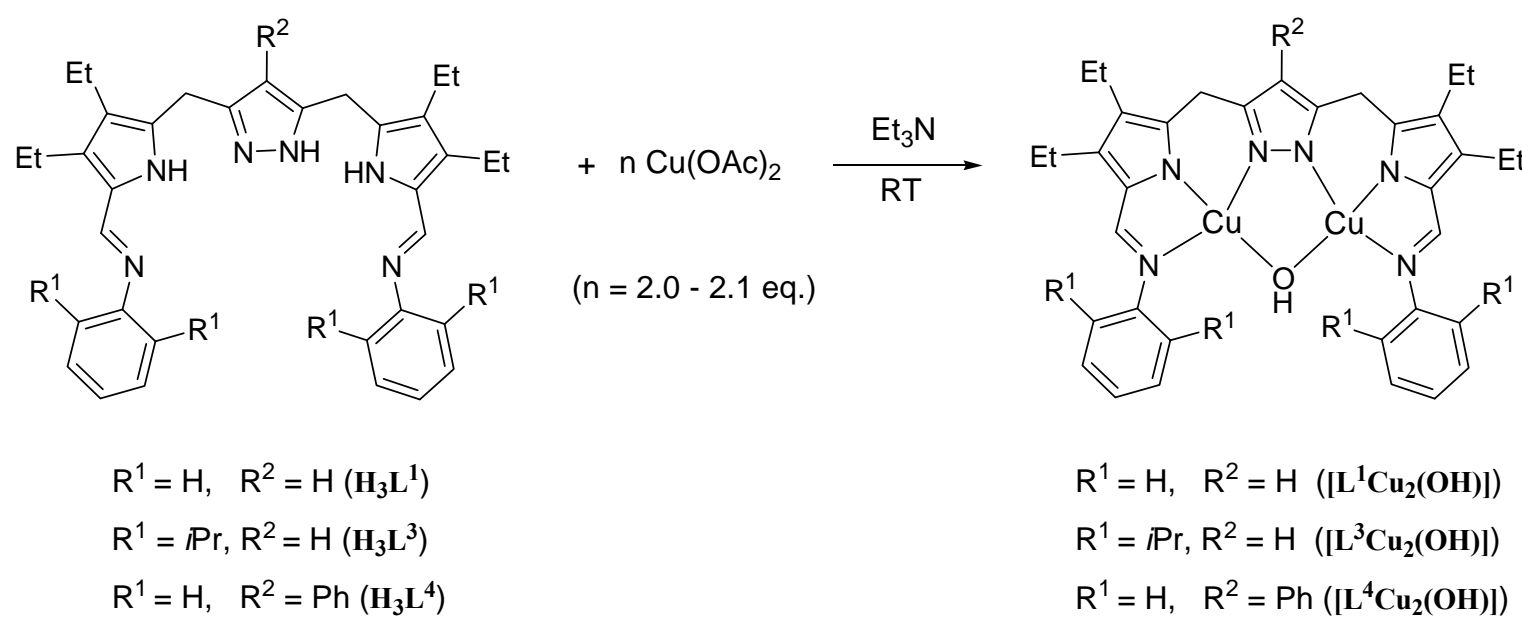

Abbildung 71: Synthese der bimetallischen Komplexe $\left[\mathbf{L}^{1} \mathbf{C u}_{2}(\mathbf{O H})\right],\left[\mathbf{L}^{3} \mathbf{C u}_{2}(\mathbf{O H})\right]$ und $\left[\mathbf{L}^{4} \mathbf{C u}_{2}(\mathbf{O H})\right]$.

In diesem Fall ist die Zuordnung der Oxidationsstufen im Liganden und in den Kupferatomen nicht mehr eindeutig. So können zwei mögliche Grenzstrukturen für die neutrale Spezies mit der Molmasse $682 \mathrm{~g} \cdot \mathrm{mol}^{-1}$ formuliert werden.
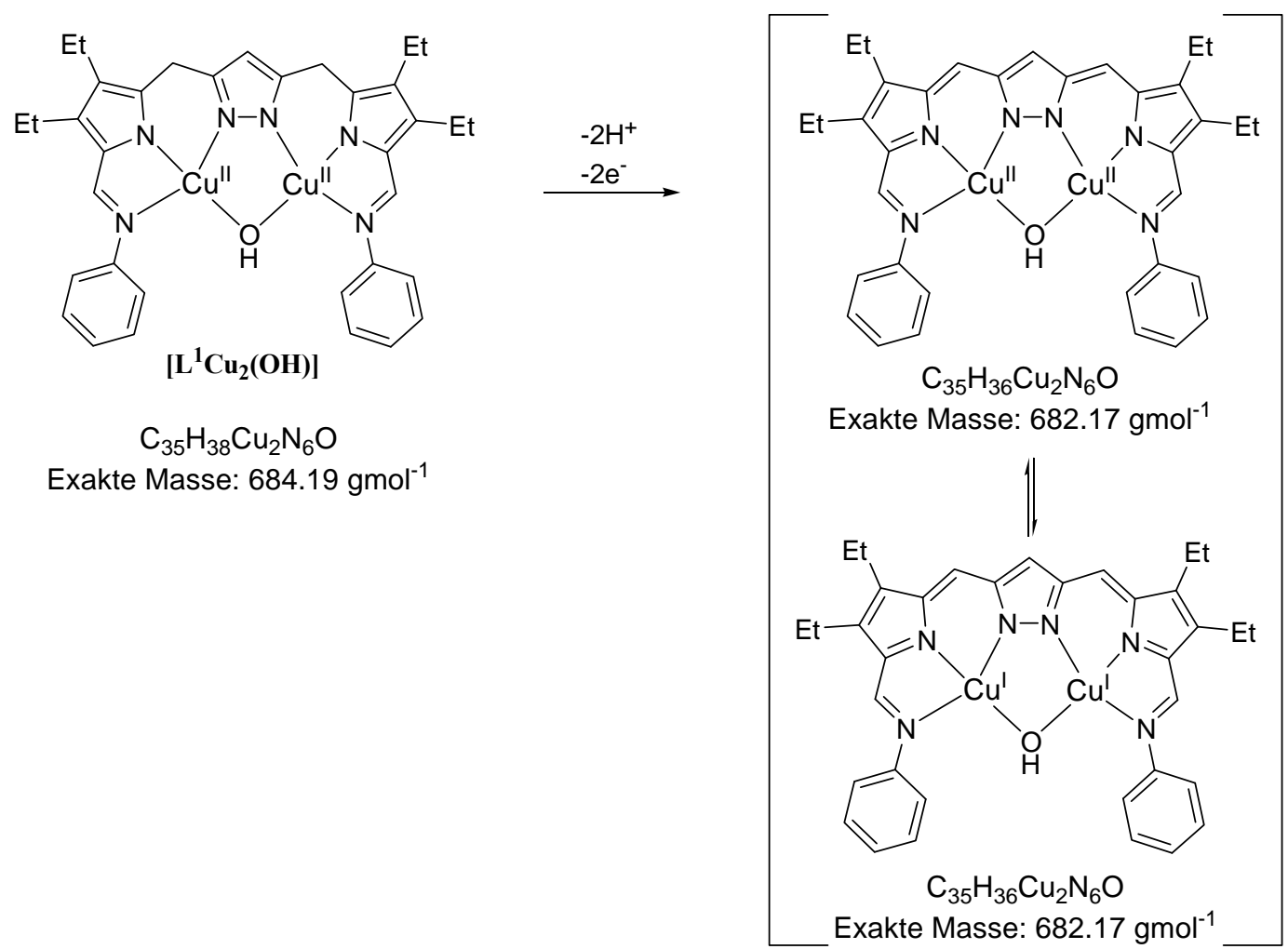

Abbildung 72: Postulierte Oxidationsreaktion des Metallkomplexes $\left[\mathbf{L}^{1} \mathbf{C u}_{2}(\mathbf{O H})\right]$ während der Elektrospray Ionisation. 
Dies ist somit ein typischer Fall eines „,non-innocent“ Liganden. Dieses Phänomen wurde ebenfalls bei den weiteren in diesem Kapitel beschriebenen Metallkomplexen beobachtet.

Durch langsames Verdampfen der verwendeten Lösungsmittel konnte nach mehrmaligem Umkristallisieren ein Einkristall zur röntgenographischen Charakterisierung erhalten werden. Der Komplex kristallisiert in der Raumgruppe $C 2 / c$ mit vier Molekülen in der monoklinen Zelle. Beide $\mathrm{Cu}-$ Ionen sind quadratisch planar koordiniert und weisen einen relativ kleinen $\mathrm{Cu}^{\cdots} \cdot \mathrm{Cu}$ Abstand von 3.35(7) $\AA$ auf (Abbildung 73). Zwischen den Kupferionen befindet sich eine $\mu$-OH Gruppe. Der $\mathrm{Cu} \cdots \mathrm{Cu}$ Abstand des bimetallischen Kupfer(II)komplexes stimmt mit literaturbekannten Werten für $\mu$-Hydroxo-Kupferkomplexe überein. ${ }^{87,88}$ Ausgewählte Bindungsabstände und Winkel sind in Tabelle 11 aufgeführt.

$$
\begin{aligned}
& \text { spontane Oxidation an den C3-bzw. C3'-Atomen zu } \\
& \text { einer Carbonyl-Funktion }
\end{aligned}
$$

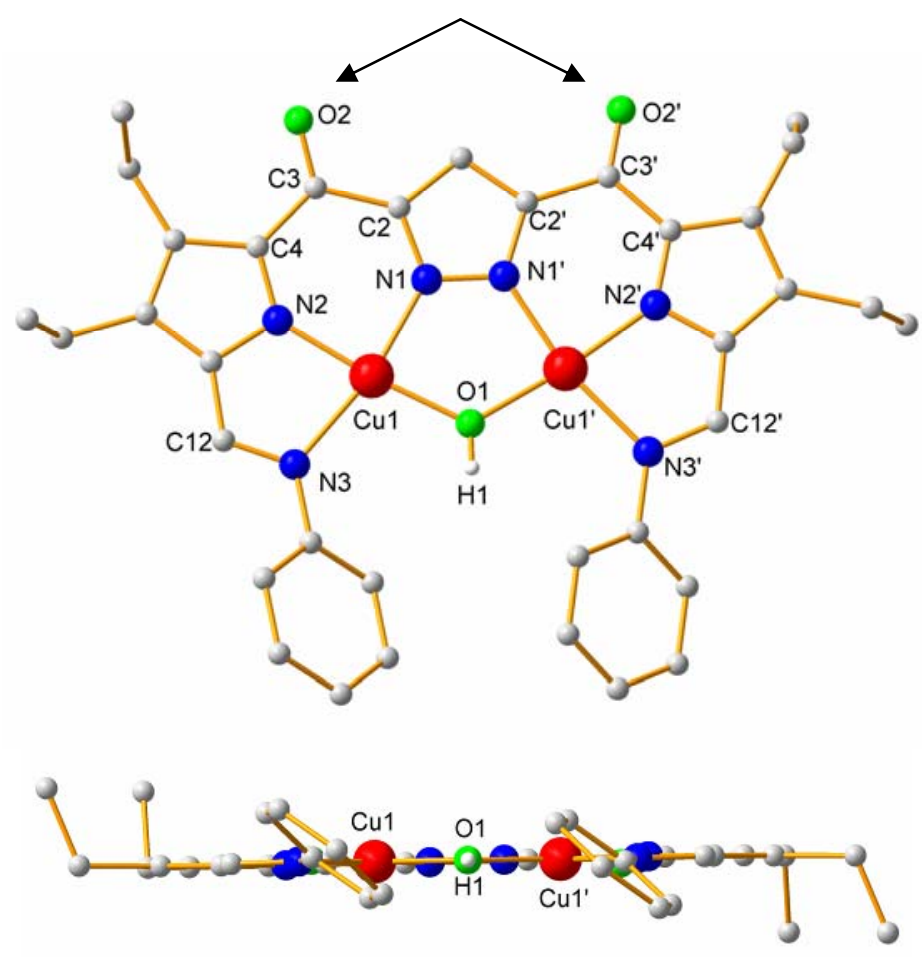

Abbildung 73: Molekülstruktur von $\left[\mathbf{L}^{1}{ }_{\text {ox }} \mathbf{C u}_{2}(\boldsymbol{\mu}-\mathbf{O H})\right]$ (Vorderansicht - oben) und Seitenansicht (unten).

Obwohl der Komplex unter Verwendung von Kupferacetat synthetisiert wurde, ist keine Acetat-Brücke zwischen den Metallionen zu beobachten, da das starre Liganden-System nicht genügend Raum zur Verfügung stellt. Die $\mathrm{CH}_{2}$-Gruppen an den 3,5-Positionen des Pyrazols (C3 bzw. C3') wurden bei Kristallisation des Komplexes an der Luft langsam zu Carbonylfunktionen ([ $\left.\left.\mathbf{L}^{\mathbf{1}}{ }_{\mathbf{o x}} \mathbf{C} \mathbf{u}_{\mathbf{2}}(\boldsymbol{\mu}-\mathbf{O H})\right]\right)$ oxidiert (Abbildung 73$)$. 
Tabelle 11: Ausgewählte Abstände und Winkel von $\left[\mathbf{L}^{1}{ }_{\text {ox }} \mathbf{C u} \mathbf{u}_{2}(\boldsymbol{\mu}-\mathbf{O H})\right]$.

\begin{tabular}{|c|c|c|c|}
\hline \multicolumn{4}{|c|}{ Abstände $[\AA]$} \\
\hline $\mathrm{Cu}(1)-\mathrm{Cu}\left(1^{\prime}\right)$ & $3.3483(7)$ & $\mathrm{Cu}(1)-\mathrm{N}(3)$ & $2.059(2)$ \\
\hline $\mathrm{Cu}(1)-\mathrm{O}(1)$ & $1.9063(15)$ & $\mathrm{N}(3)-\mathrm{C}(12)$ & $1.306(4)$ \\
\hline $\mathrm{Cu}\left(1^{\prime}\right)-\mathrm{O}(1)$ & $1.9063(15)$ & $C(2)-C(3)$ & $1.486(4)$ \\
\hline $\mathrm{Cu}(1)-\mathrm{N}(1)$ & $1.959(2)$ & $\mathrm{C}(3)-\mathrm{C}(4)$ & $1.437(5)$ \\
\hline $\mathrm{Cu}(1)-\mathrm{N}(2)$ & $1.913(2)$ & & \\
\hline \multicolumn{4}{|c|}{ Winkel $\left[^{\circ}\right]$} \\
\hline $\mathrm{N}(1)-\mathrm{Cu}(1)-\mathrm{N}(3)$ & $168.78(9)$ & $\mathrm{O}(1)-\mathrm{Cu}(1)-\mathrm{N}(2)$ & $174.68(11)$ \\
\hline $\mathrm{N}(2)-\mathrm{Cu}(1)-\mathrm{N}(1)$ & $87.05(9)$ & $\mathrm{O}(1)-\mathrm{Cu}(1)-\mathrm{N}(3)$ & $103.49(11)$ \\
\hline $\mathrm{N}(2)-\mathrm{Cu}(1)-\mathrm{N}(3)$ & $81.73(10)$ & $\mathrm{Cu}\left(1^{\prime}\right)-\mathrm{O}(1)-\mathrm{Cu}(1)$ & $122.85(16)$ \\
\hline $\mathrm{O}(1)-\mathrm{Cu}(1)-\mathrm{N}(1)$ & $87.73(10)$ & $C(2)-C(3)-C(4)$ & $120.2(3)$ \\
\hline
\end{tabular}

Die gleichen Reaktionen wurden ebenfalls unter Luftausschluss (Arbeiten in der Glovebox) durchgeführt und mit dem bereits diskutierten Komplex verglichen. Die Reaktion wurde unter den gleichen Bedingung mit einem Überschuss an Kupferacetat (2.1 eq) bzw. mit 2.0 eq. Kupferacetat durchgeführt.

Die Ergebnisse zeigen in allen Fällen die Entstehung von $\left[\mathbf{L}^{1} \mathbf{C} \mathbf{u}_{2}(\mathbf{O H})\right]$, statt des oxidierten Komplexes [ $\mathbf{L}^{1}{ }_{\text {ox }} \mathbf{C} \mathbf{u}_{2}(\mathbf{O H})$ ]. Eine Oxygenierung an den 3,5-Positionen des Pyrazols wurde lediglich im Fall der röntgenographisch charakterisierten Verbindung $\left[\mathbf{L}^{1}{ }_{\text {ox }} \mathbf{C u}_{\mathbf{2}}(\mathbf{O H})\right]$ beobachtet, konnte jedoch nicht reproduziert werden. In früheren Arbeiten und in einer Reihe von Oxidationsexperimenten mit Pyrrol/Pyrazol-basierten Schiff-Base Makrozyklen wurde bereits versucht die $\mathrm{CH}_{2}{ }^{\mathrm{py} / \mathrm{pz}}$-Gruppen der 3,5-Position des Pyrazols gezielt zu oxidieren. Die Oxygenierung in $\left[\mathbf{L}^{\mathbf{1}}{ }_{\mathbf{~} x} \mathbf{C u}_{\mathbf{2}}(\mathbf{O H})\right]$ ist jedoch anders, als die in dem ESI-MS gefundene Massendifferenz (Abbildung 72), die durch eine Oxidation (Abgabe von H-Atomen) verursacht wird. Die Oxygeniering der C3 bzw. C3' zu einer Carbonyl-Funktion in $\left[\mathbf{L}^{1}{ }_{\text {ox }} \mathbf{C u}_{2}(\mathbf{O H})\right]$ erinnert an den von Katayev et al. gefundene Autoxidation von Pyrrolbasierten Diiminliganden mit Sauerstoff in Komplexen mit Übergansmetalle. ${ }^{86}$

Durch eine neue Syntheseroute sollten daher die oxidierten Liganden $\mathbf{H}_{3} \mathbf{L}^{\mathbf{1}}$ ox und $\mathbf{H}_{\mathbf{3}} \mathbf{L}^{\mathbf{1 a}}{ }_{\text {ox }}$ dargestellt werden. Speziell $\mathbf{H}_{\mathbf{3}} \mathbf{L}^{\mathbf{1 a}}{ }_{\text {ox }}$ (Abbildung 74) ist sehr interessant, da die Carbonylgruppen an der 3,5-Position des Pyrazols die Möglichkeit zu einer strukturellen Erweiterung der Liganden bieten. An dieser Stelle können weitere Substituenten angebracht und so das Ligandengerüst gezielt modifiziert werden. 
Zur Darstellung des $\mathbf{H}_{\mathbf{3}} \mathbf{L}^{\mathbf{1 a}}$ ox wurde $\mathbf{L V I} \mathbf{I}^{43,89}$ in trockenem 1,2-Dichlorethan suspendiert und mit einem Überschuss Morpholin versetzt (Route 1, Abbildung 74). Im nächsten Schritt sollte das Pyrrol in einer Vielmeier-Haack ähnlichen Reaktion substituiert werden.<smiles>O=C(Cl)c1cc(C(=O)Cl)[nH]n1</smiles>

LVI

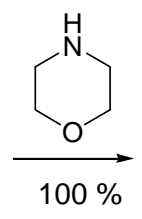

Route 1<smiles>O=C(c1cc(C(=O)N2CCOCC2)[nH]n1)N1CCOCC1</smiles>

11<smiles>CC(C)(C)c1ccc[nH]1</smiles>

$(*)$<smiles>O=C(c1cc(C(=O)c2ccc[nH]2)[nH]n1)c1ccc[nH]1</smiles>

12

$\mathrm{PhCOCl}$

DMF

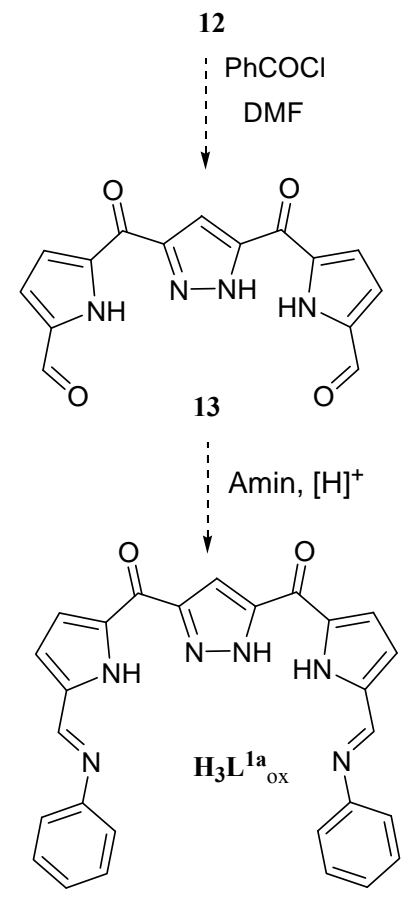

Amin, $[H]^{+}$

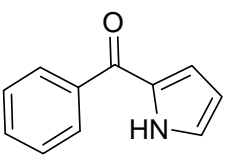

Abbildung 74: Syntheserouten zur Darstellung von $\mathbf{H}_{3} \mathbf{L}^{\mathbf{1 a}}{ }_{\text {ox }}$ aus LVI ${ }^{*}$ Reaktionsbedingungen für diese Synthese werden in Tabelle 12 aufgelistet).

Hierbei wurden unterschiedlichen Reaktionsbedingungen getestet. Die experimentellen Details der Sytheseroute 1 (Abbildung 74, *) sind in Tabelle 12 wiedergegeben. Verbindung 11 wurde zunächst mit $\mathrm{POCl}_{3}$ oder Benzoylchlorid zu einer Vilsmeier-Haack aktiven Spezies umgewandelt und anschliessend mit Pyrrol umgesetzt. Hierbei wurde sowohl die Menge an Pyrrol als auch die Reaktionszeit und das verwendete Lösungsmittel variiert. Zur Aufarbeitung wurde $\mathrm{Na}_{2} \mathrm{CO}_{3}$ verwendet. Es zeigt sich, dass mit Benzoylchlorid als Reagenz statt der hier gewünschten Zwischenstuffe (12) das Phenyl-(1H-pyrrol-2-yl)keton entsteht (Tabelle 12, Nr. 2). Auch der Einsatz des Grignard-Reagenzes EtMgCl zur Aktivierung des Pyrrols (Tabelle 12, Nr. 3) hat nicht zum gewünschten Produkt geführt. 
Tabelle 12: Auflistung der unterschiedlichen Reaktionsbedingungen zur Darstellung von Verbindung 12 in Syntheseroute 1 (Abbildung 74).

\begin{tabular}{|c|c|c|c|}
\hline $\mathrm{Nr}$. & Reagenzien & Reaktionsbedingungen & Ergebnis \\
\hline 1 & $\begin{array}{l}\text { 1) } 11-15 \text { eq. } \mathrm{POCl}_{3} \\
\text { 2) 2-4 eq. Pyrrol } \\
\text { 3) } \mathrm{Na}_{2} \mathrm{CO}_{3}\end{array}$ & $\begin{array}{l}3.5 \mathrm{~h} \text { bis } 2 \mathrm{~d} \text { unter Rückfluss in 1,2-Dichlorethan } \\
\text { oder in Chlorbenzol unter } \mathrm{N}_{2} \text {-Atm. erhitzt }\end{array}$ & $-/-$ \\
\hline 2 & $\begin{array}{l}\text { 1) } 20 \text { eq. Ph-COCl, } \mathrm{N}_{2} \text { - atm. } \\
\text { 2) Pyrrol, 1,2- Dichlorethan } \\
\text { 3) wäß. } \mathrm{Na}_{2} \mathrm{CO}_{3}\end{array}$ & $\begin{array}{l}\text { zu 1.) } 4 \text { h } 140{ }^{\circ} \mathrm{C} \text {, anschließend } 1 \mathrm{~d} R \mathrm{RT} \text {; } \\
\text { zu 2.) unter Rückfluss erhitzt } 6 \mathrm{~h} \text {; } \\
\text { zu 3.) unter Rückfluss erhitzt } 3 \mathrm{~h}\end{array}$ & $\begin{array}{l}\text { Nebenprodukt: } \\
\text { Phenyl-(1H- } \\
\text { pyrrol-2-yl)- } \\
\text { keton }\end{array}$ \\
\hline 3 & $\begin{array}{l}\text { 1) } \mathrm{Pyrrol}, \mathrm{EtMgCl} \\
\text { 2) } \mathrm{NH}_{4} \mathrm{Cl}\end{array}$ & $\begin{array}{l}\text { zu 1.) }-20^{\circ} \mathrm{C}, \mathrm{N}_{2} \text {-Atm., THF } \\
\text { anschließend } 3 \mathrm{~h} \text { bei RT }\end{array}$ & $-/-$ \\
\hline
\end{tabular}

Schließlich wurde ein neuer Syntheseweg durchgeführt, der ausgehend von LVI in einer Reaktion mit Pyrrolylmagnesiumbromid zu Verbindung 12 führen sollte (Syntheseroute 2, Abbildung 74). Hierzu wurde LVI in trockenem THF gelöst und auf $-45{ }^{\circ} \mathrm{C}$ gekühlt. Parallel wurde bei gleicher Temperatur das Pyrrol mit EtMgBr aktiviert und anschließend zu der THF-Lösung gegeben. Die Reaktionsmischung wurde $1 \mathrm{~h}$ bei $-45^{\circ} \mathrm{C}$ gerührt und danach auf Raumtemperatur erwärmt. Das Reaktionsgemisch wurde mit einer gesättigten Ammoniumchlorid-Lösung aufgearbeitet, die wässrige Phase abgetrennt und mit Dichlormethan mehrfach extrahiert. Die organische Phase wurde über $\mathrm{MgSO}_{4}$ getrocknet und das Lösungsmittel im Vakuum entfernt. Das entstandene braun-schwarze Rohprodukt wurde mittels EI-MS untersucht. Detektiert werden konnte ein Signal $[\mathrm{M}]^{+}$mit hoher Intensität bei $254 \mathrm{~m} / \mathrm{z}$, welches Verbindung 12 mit $254.1 \mathrm{~g} \cdot \mathrm{mol}^{-1} \quad\left(\mathrm{C}_{13} \mathrm{H}_{10} \mathrm{~N}_{4} \mathrm{O}_{2}\right)$ entspricht. Säulenchromatographisch ließ sich das stark verunreinigte Rohprodukt nicht aufreinigen. Zudem entsteht Verbindung 12 in nur sehr kleinen Ausbeuten.

Wie weiterführende Untersuchungen gezeigt haben, eignet sich die neu synthetisierte Vorstufe von 12, Verbindung 11, als Brückenligand zur Darstellung von bimetallischen Komplexen. $\mathbf{H L}^{\mathbf{1 1}}$ wurde dazu in einer Mischung aus Methanol/Ethanol gelöst und mit einigen Tropfen Triethylamin versetzt. Es wurde ein Metallsalz (Kupferacetat bzw. Cobaltacetat Tetrahydrat) zugegeben und über Nacht bei Raumtemperatur gerührt. Das eingeengte Reaktionsgemisch wurde mit Diethylether überschichtet, woraufhin nach wenigen 
Tagen grüne Kupferkomplexe bzw. lila-rote Kristalle erhalten werden konnten. Massenspektrometrische Untersuchungen (ESI) konnten in beiden Fällen zeigen, dass bimetallische Komplexe des Typs $\mathrm{L}_{2} \mathrm{M}_{2} \mathrm{X}_{4}$ entstehen (Abbildung 75).

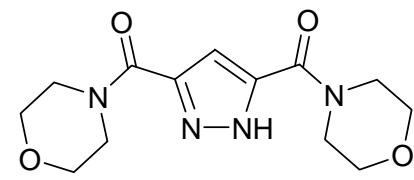

11

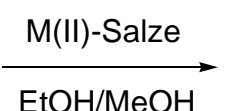

$\mathrm{M}=\mathrm{Co}, \mathrm{Cu}$ $\mathrm{X}=\mathrm{Cl}, \mathrm{MeOH}$

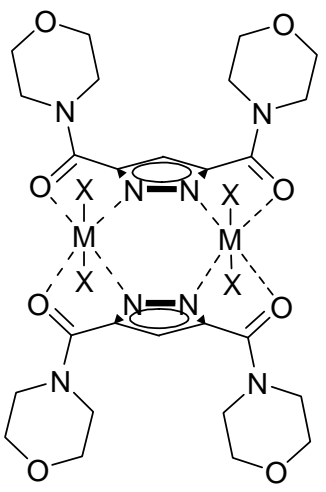

$\left[\mathrm{L}^{11} \mathbf{M}_{2} \mathrm{Cl}_{2}(\mathrm{MeOH})_{2}\right]$

Abbildung 75: Synthese von bimetallischen Komplexen des Typs $\mathrm{L}_{2} \mathrm{M}_{2} \mathrm{X}_{4}$ mit dem Liganden 11 (bzw. $\mathbf{H L}^{11}$ ).

Im Falle des Cobaltkomplexes zeigten die erhaltenen Kristalle eine ausreichende Qualität für die Röntgenstrukturanalyse. Der Komplex kristallisiert in der Raumgruppe $C 2 / c$ mit vier Molekülen in der monoklinen Zelle. Die Festkörperstruktur des Komplexes bestätigt die Bildung eines Komplexes des Typs $\mathrm{L}_{2} \mathrm{M}_{2} \mathrm{X}_{4}$ (Abbildung 76), in übereinstimmung mit den ESI-MS Ergebnissen.

Die zwei Cobaltionen haben eine oktaedrische Koordinationssphäre mit je einem Methanol und einem Chloridion in den axialen Positionen. Die äquatoriale Ebene wird aus jeweils einem N-Atom des Pyrazols und dem O-Atom der Carbonylgruppe eines Ligandmoleküls ausgebildet. Das N-Atom der Morpholineinheit ist an der Koordination nicht beteiligt. Der Co $\cdots$ Co Abstand beträgt $4.04 \AA$ und liegt somit im erwarteten Bereich. ${ }^{90,91}$ Ausgewählte Bildungswinkel und Abstände sind in Tabelle 13 aufgelistet.

Die O-Atome der Morpholineinheit ermöglichen die Ausbildung von externen Wasserstoffbrückenbindungen. Somit liegen in der Elementarzelle Komplex-Ketten vor, die durch Wasserstoffbrückenbindungen von O3 bzw. O3' zum benachbarten Methanol-Molekül gebildet werden. Da der Komplex Dimere des Typs $\mathrm{L}_{2} \mathrm{M}_{2} \mathrm{X}_{4}$ bildet, ist es nicht in der Lage weitere Substate an sich $\mathrm{zu}$ binden und als Katalysator $\mathrm{zu}$ fungieren. Daher wurde der Komplex im Rahmen dieser Arbeit nicht näher untersucht.

Analog zu $\mathbf{H}_{3} \mathbf{L}^{\mathbf{1}}$ konnten erfolgreich weitere Kupfer(II)komplexe mit $\mathbf{H}_{\mathbf{3}} \mathbf{L}^{\mathbf{3}}$ synthetisiert werden. Bei langsamem Abdampfen des Lösungsmittels aus der Mutterlauge entstanden 
braune kubische Kristalle. Die Festkörperstruktur des Kupferkomplexes $\left[\mathbf{L}^{3} \mathbf{C u}_{\mathbf{2}}(\mathbf{O H})\right]$ zeigt ähnlich wie bei $\left[\mathbf{L}^{1}{ }_{\text {ox }} \mathbf{C u}_{2}(\mathbf{O H})\right]$ erneut den Einbau eines $\mu$-OH als Brückenliganden zwischen den zwei Kupferatomen (Abbildung 77). Der Komplex kristallisiert in der Raumgruppe $P \overline{1}$ mit zwei Molekülen in der triklinen Zelle.

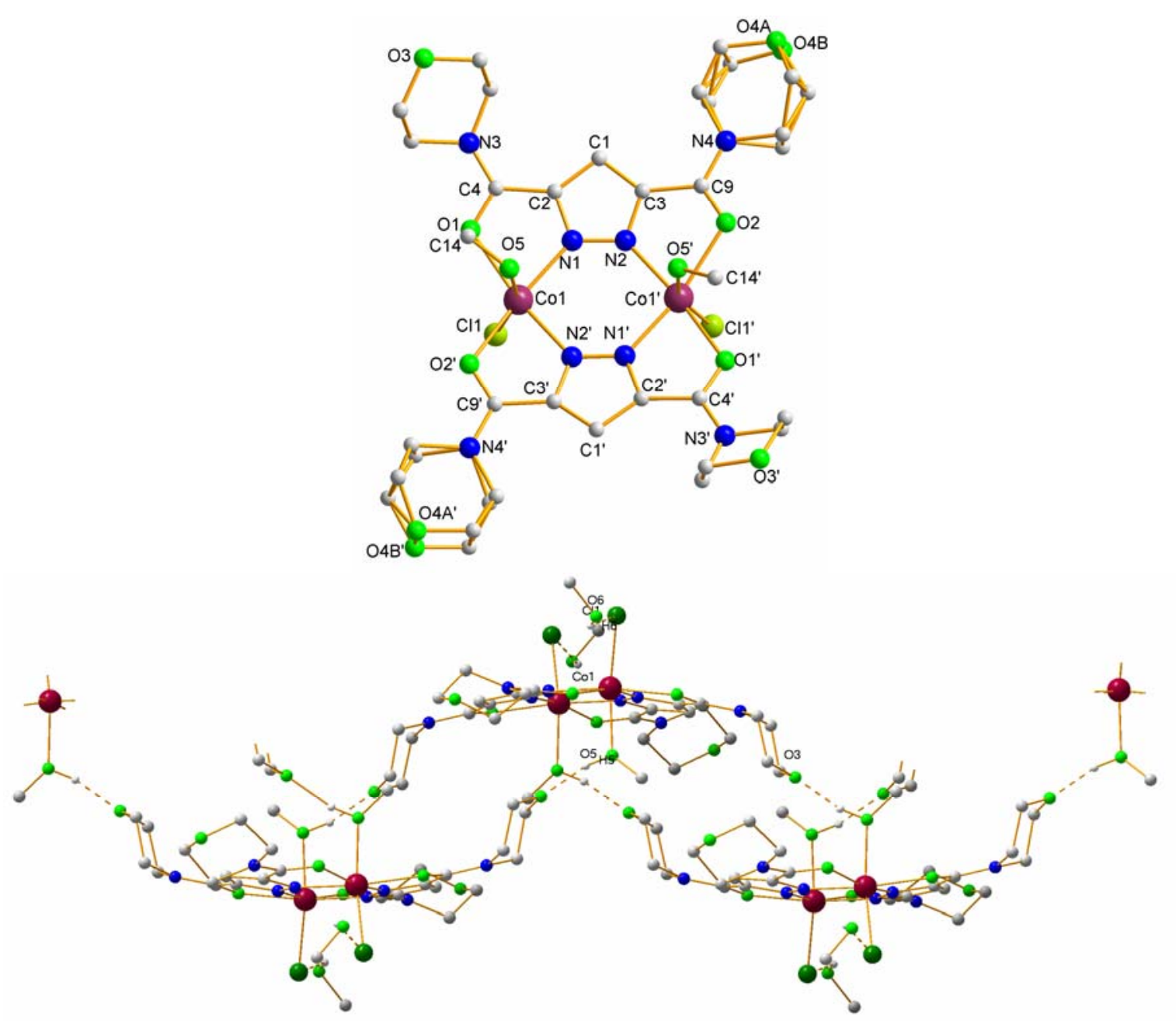

Abbildung 76: Festkörperstruktur von $\left[\mathbf{L}^{11} \mathbf{C o}_{2} \mathbf{C l}_{2}(\mathbf{M e O H})_{2}\right]$ (oben) und Bildung von Ketten in der Elementarzelle (unten).

Tabelle 13: Ausgewählte Abstände und Winkel von $\left[\mathbf{L}^{11} \mathbf{C o}_{2} \mathbf{C l}_{\mathbf{2}}(\mathbf{M e O H})_{2}\right]$.

Abstände $[\AA]$

\begin{tabular}{ll|ll}
\hline $\operatorname{Co}(1)-\operatorname{Co}\left(2^{\prime}\right)$ & $4.036(1)$ & $\operatorname{Co}(1)-\mathrm{O}(5)$ & $2.156(3)$ \\
$\operatorname{Co}(1)-\mathrm{N}(1)$ & $2.055(3)$ & $\operatorname{Co}\left(1^{\prime}\right)-\mathrm{O}(2)$ & $2.239(3)$ \\
$\operatorname{Co}(1)-\mathrm{N}\left(2^{\prime}\right)$ & $2.059(3)$ & $\operatorname{Co}(1)-\mathrm{O}\left(2^{\prime}\right)$ & $2.239(3)$ \\
$\operatorname{Co}\left(1^{\prime}\right)-\mathrm{N}(2)$ & $2.059(3)$ & $\operatorname{Co}(1)-\mathrm{Cl}(1)$ & $2.3462(11)$ \\
$\operatorname{Co}(1)-\mathrm{O}(1)$ & $2.180(3)$ & & \\
\hline
\end{tabular}




\begin{tabular}{lr|cc}
\hline \multicolumn{4}{c}{ Winkel $\left[^{\circ}\right]$} \\
\hline $\mathrm{N}(1)-\mathrm{Co}(1)-\mathrm{N}\left(2^{\prime}\right)$ & $97.97(12)$ & $\mathrm{O}(5)-\mathrm{Co}(1)-\mathrm{O}\left(2^{\prime}\right)$ & $79.00(11)$ \\
$\mathrm{N}(1)-\mathrm{Co}(1)-\mathrm{O}(5)$ & $90.02(12)$ & $\mathrm{O}(1)-\mathrm{Co}(1)-\mathrm{O}\left(2^{\prime}\right)$ & $111.64(10)$ \\
$\mathrm{N}\left(2^{\prime}\right)-\mathrm{Co}(1)-\mathrm{O}(5)$ & $92.14(13)$ & $\mathrm{N}(1)-\mathrm{Co}(1)-\mathrm{Cl}(1)$ & $104.01(9)$ \\
$\mathrm{N}(1)-\mathrm{Co}(1)-\mathrm{O}(1)$ & $74.54(11)$ & $\mathrm{N}\left(2^{\prime}\right)-\mathrm{Co}(1)-\mathrm{Cl}(1)$ & $100.12(9)$ \\
$\mathrm{N}\left(2^{\prime}\right)-\mathrm{Co}(1)-\mathrm{O}(1)$ & $169.97(11)$ & $\mathrm{O}(5)-\mathrm{Co}(1)-\mathrm{Cl}(1)$ & $159.76(10)$ \\
$\mathrm{O}(5)-\mathrm{Co}(1)-\mathrm{O}(1)$ & $81.35(11)$ & $\mathrm{O}(1)-\mathrm{Co}(1)-\mathrm{Cl}(1)$ & $88.33(7)$ \\
$\mathrm{N}(1)-\mathrm{Co}(1)-\mathrm{O}\left(2^{\prime}\right)$ & $166.14(12)$ & $\mathrm{O}\left(2^{\prime}\right)-\mathrm{Co}(1)-\mathrm{Cl}(1)$ & $88.82(8)$ \\
$\mathrm{N}\left(2^{\prime}\right)-\mathrm{Co}(1)-\mathrm{O}\left(2^{\prime}\right)$ & $74.25(11)$ & & \\
\hline
\end{tabular}

Analog zu $\left[\mathbf{L}^{\mathbf{1}}{ }_{\mathbf{o x}} \mathbf{C u}_{\mathbf{2}}(\mathbf{O H})\right]$ werden auch hier beide Kupferzentren durch jeweils einen Pyrazol-N-Donor, ein N-Atom aus der Pyrroleinheit sowie ein Imin-N-Atom koordiniert. Beide Kupferatome besitzen eine quadratisch planare Koordinationssphäre.

In Tabelle 14 sind ausgewählte Abstände und Winkel von $\left[\mathbf{L}^{3} \mathbf{C u}_{\mathbf{2}}(\mathbf{O H})\right]$ wiedergegeben. Der $\mathrm{Cu} \cdots \mathrm{Cu}$ Abstand ist mit $3.31 \AA$ relativ kurz und vergleichbar mit den restlichen Kupferkomplexen dieser Arbeit. Beim Betrachten der Molekülstruktur des Komplexes zeigt sich, dass das Molekül im Gegensatz zum planaren $\left[\mathbf{L}^{\mathbf{1}}{ }_{\text {ox }} \mathbf{C u}_{\mathbf{2}}(\boldsymbol{\mu}-\mathbf{O H})\right]$ eine leichte Wölbung aufweist (Abbildung 77, unten). Beide Kupferatome befinden sich nicht exakt in der Ebene, der Pyrroleinheiten.
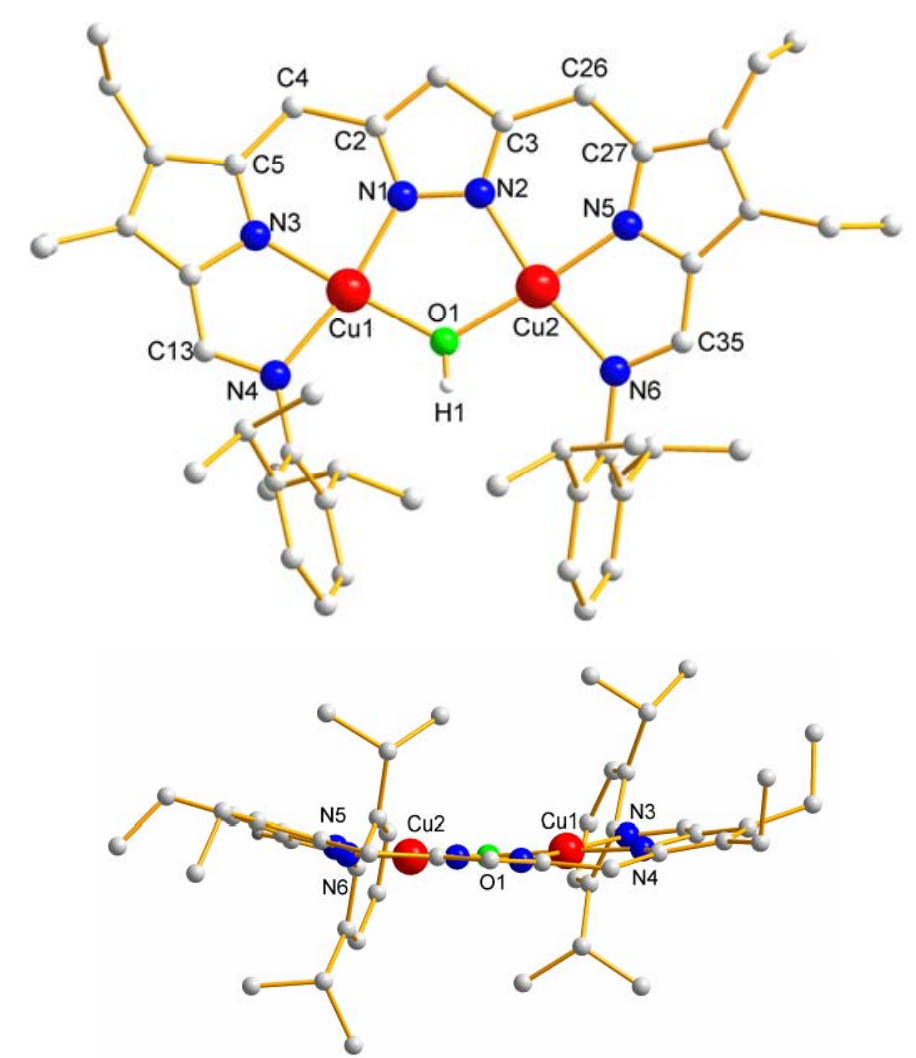

Abbildung 77: Molekülstruktur von $\left[\mathbf{L}^{3} \mathbf{C} \mathbf{u}_{2}(\mathbf{O H})\right]$ im Festkörper (oben) und Seitenansicht (unten). 
Tabelle 14: Ausgewählte Abstände und Winkel von $\left[\mathbf{L}^{3} \mathbf{C u}_{\mathbf{2}}(\boldsymbol{\mu - O H})\right]$.

\begin{tabular}{ll|ll}
\multicolumn{5}{c}{ Abstände $[\AA]$} & \\
\hline $\mathrm{Cu}(1)-\mathrm{Cu}(2)$ & $3.3108(3)$ & $\mathrm{Cu}(2)-\mathrm{N}(6)$ & $2.0409(15)$ \\
$\mathrm{Cu}(1)-\mathrm{N}(1)$ & $1.9522(14)$ & $\mathrm{Cu}(1)-\mathrm{O}(1)$ & $1.9101(13)$ \\
$\mathrm{Cu}(1)-\mathrm{N}(3)$ & $1.9010(15)$ & $\mathrm{Cu}(2)-\mathrm{O}(1)$ & $1.9056(13)$ \\
$\mathrm{Cu}(1)-\mathrm{N}(4)$ & $2.0327(14)$ & $\mathrm{N}(6)-\mathrm{C}(35)$ & $1.312(2)$ \\
$\mathrm{Cu}(2)-\mathrm{N}(2)$ & $1.9530(15)$ & $\mathrm{C}(3)-\mathrm{C}(26)$ & $1.510(2)$ \\
$\mathrm{Cu}(2)-\mathrm{N}(5)$ & $1.9006(15)$ & $\mathrm{C}(26)-\mathrm{C}(27)$ & $1.496(3)$ \\
\hline & & & $81.84(6)$ \\
& & $\mathrm{Ninkel}\left[{ }^{\circ}\right]$ & $89.50(6)$ \\
$\mathrm{N}(1)-\mathrm{Cu}(1)-\mathrm{N}(4)$ & $169.38(6)$ & $\mathrm{N}(5)-\mathrm{Cu}(2)-\mathrm{N}(6)$ & $100.78(6)$ \\
$\mathrm{N}(2)-\mathrm{Cu}(2)-\mathrm{N}(6)$ & $169.60(6)$ & $\mathrm{O}(1)-\mathrm{Cu}(1)-\mathrm{N}(1)$ & $89.65(6)$ \\
$\mathrm{N}(3)-\mathrm{Cu}(1)-\mathrm{N}(1)$ & $87.85(6)$ & $\mathrm{O}(1)-\mathrm{Cu}(2)-\mathrm{N}(2)$ & $117.16(15)$ \\
$\mathrm{N}(3)-\mathrm{Cu}(1)-\mathrm{N}(4)$ & $81.95(6)$ & $\mathrm{C}(27)-\mathrm{C}(26)-\mathrm{C}(3)$ & $100.68(6)$ \\
$\mathrm{N}(3)-\mathrm{Cu}(1)-\mathrm{O}(1)$ & $176.81(6)$ & $\mathrm{O}(1)-\mathrm{Cu}(2)-\mathrm{N}(6)$ & $120.38(7)$ \\
$\mathrm{N}(5)-\mathrm{Cu}(2)-\mathrm{O}(1)$ & $173.10(6)$ & $87.76(6)$ & \\
$\mathrm{N}(5)-\mathrm{Cu}(2)-\mathrm{N}(2)$ & &
\end{tabular}

Die Ebenen, die die jeweiligen Kupferatome in einem fünfgliedrigen Chelatring zu den NPyrrol und N-Imin (Cu2-N5-N6 zu Cu1-N3-N4) aufspannen haben einen Winkel von 18.2 zueinander. Bei der Komplexsynthese aus $\mathbf{H}_{\mathbf{3}} \mathbf{L}^{\mathbf{3}}$ und Kupferacetat konnte auch bei wiederholter Synthese an der 3,5-Position des Pyrazols keine Oxidation der $\mathrm{CH}_{2}$-Guppen zu Carbonylgruppen beobachtet werden.

Die magnetischen Eigenschaften des bimetallischen Komplexes $\left[\mathbf{L}^{3} \mathbf{C u}_{\mathbf{2}}(\mathbf{O H})\right]$ wurden mittels SQUID-Messung untersucht. Hierzu wurden die isolierten Kristalle zu einem Pulver verrieben und in eine Gel-Kapsel gefüllt. Die magnetische Suszeptibilität $\chi_{\mathrm{M}}$ von $\left[\mathbf{L}^{3} \mathbf{C} \mathbf{u}_{\mathbf{2}}(\mathbf{O H})\right]$ wurde bei $5000 \mathrm{G}$ und $2000 \mathrm{G}$, im Temperaturbereich zwischen $295 \mathrm{~K}$ und $2 \mathrm{~K}$ gemessen. Es konnte keine signifikante Feldabhängigkeit bei der Messung der Probe beobachtet werden. Die Temperaturabhängigkeit der magnetischen Suszeptibilität $\chi_{\mathrm{M}}$ und des Produkts $\chi_{\mathrm{M}} T$ für die Verbindung $\left[\mathbf{L}^{3} \mathbf{C u}_{\mathbf{2}}(\mathbf{O H})\right]$ bei $5000 \mathrm{G}$ ist in Abbildung 78 dargestellt. Der $\chi_{\mathrm{M}} T$-Wert von $0.25 \mathrm{~cm}^{3} \mathrm{Kmol}^{-1}\left(1.42 \mu_{\mathrm{B}}\right)$ bei $295 \mathrm{~K}$ ist deutlich niedriger als der erwartete „,spin-only“ Wert für zwei ungepaarte Kupfer(II)-Ionen von $0.79 \mathrm{~cm}^{3} \mathrm{Kmol}^{-1}$ für $g=2.05$. Diese Werte wurden nach den Gleichungen 1 und 2 mit $S_{A}=S_{B}=1 / 2$ berechnet. Der $\chi_{\mathrm{M}} T$-Wert des Kupferkomplexes nimmt mit sinkender Temperatur ab und tendiert schließlich nach Null. 


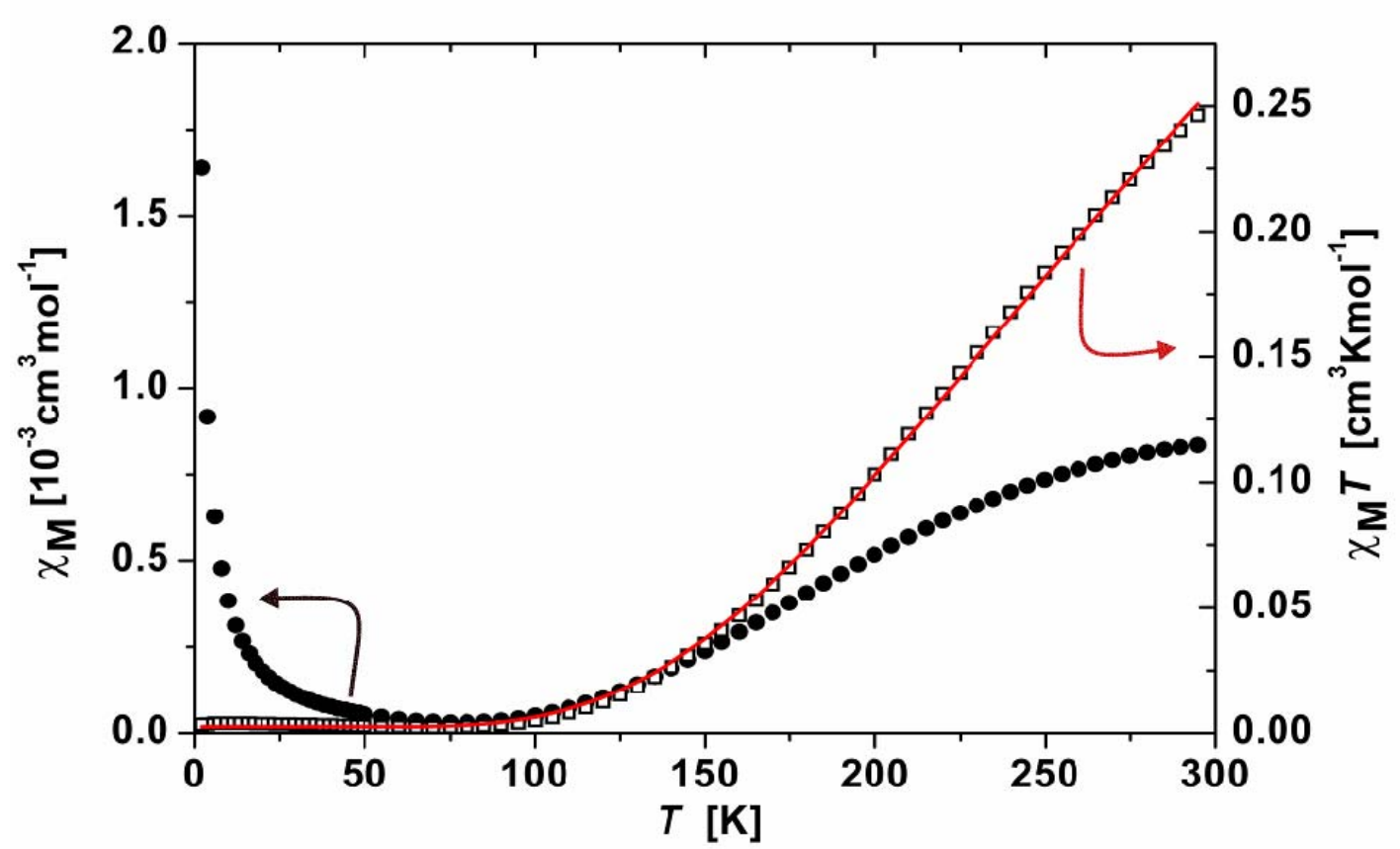

Abbildung 78: Magnetische Suszeptibilität $\chi_{\mathrm{M}}$ vs. $T(\bullet), \chi_{\mathrm{M}} T$ vs. $T(\square)$ und Simulation (-) für den Kupferkomplex $\left[\mathbf{L}^{3} \mathbf{C u}_{2}(\mathbf{O H})\right]$. Der Komplex $\left[\mathbf{L}^{3} \mathbf{C u}_{2}(\mathbf{O H})\right]$ zeigt eine starke antiferromagnetische Spinkopplung (mit $J=-232 \mathrm{~cm}^{-1}$ aus $\hat{\mathrm{H}}=-2 J \hat{S}_{\mathrm{A}} \hat{S}_{\mathrm{B}}$ ).

$$
\begin{gathered}
\mu_{e f f}=g \sqrt{S_{A}\left(S_{A}+1\right)+S_{B}\left(S_{B}+1\right)} \mu_{\beta} \\
\mu_{e f f}=\sqrt{\frac{3 k}{N \mu_{B}^{2}}} \sqrt{\chi_{M} T}=2.83 \sqrt{\chi_{M} T}
\end{gathered}
$$

Ein solches Verhalten ist typisch für zweikernige Komplexe mit intramolekularer antiferromagnetischer Kopplung ( $S=0$ Grundzustand). Der Anstieg von $\chi_{\mathrm{M}}$ bei sehr tiefen Temperaturen wird vermutlich durch einen geringen Anteil paramagnetischer Verunreinigungen verursacht.

Die Simulation der experimentellen Daten von $\left[\mathbf{L}^{3} \mathbf{C u}_{2}(\mathbf{O H})\right]$ erfolgte unter Verwendung des Programms julX, ${ }^{92}$ dass auf einem Heisenberg-Dirac-van-Vleck-Hamiltonoperator mit zusätzlichem Term für die Zeeman-Aufspaltung basiert (Gleichung 3).

$$
\hat{H}=-2 J \hat{S}_{A} \hat{S}_{B}+g \mu_{B} B\left(\hat{S}_{A}+\hat{S}_{B}\right)
$$

Als Ergebnisse werden dabei der Austauschparameter $J$, der $g$-Faktor sowie der Molanteil der Verunreinigungen $\rho$ ermittelt. Unter Verunreinigung - berechnet nach der Gleichung $\chi_{\text {calcd. }}=$ $(1-\rho) \chi+\rho \chi$ mono - versteht man in diesem Zusammenhang einen sehr kleinen Anteil 
paramagnetischer Spezies in der Probe. Es wird vorausgesetzt, dass die Verunreinigung ein Curie-Verhalten aufweist, dieselbe molare Masse und denselben $g$-Faktor wie die untersuchte Verbindung hat. Eine gute Übereinstimmung wurde mit den Parametern $g=2.05$, $J=-232 \mathrm{~cm}^{-1}$ und $\rho=0.6 \%$ erhalten. Eine große negative Kopplungskonstante bestätigt den starken antiferromagnetischen Austausch.

Die Kopplungskonstante $J=-232 \mathrm{~cm}^{-1}$ hängt von dem $\mathrm{Cu}{ }^{\cdots} \mathrm{Cu}$ Abstand und hauptsächlich vom Winkel der $\mathrm{Cu}-\mathrm{O}-\mathrm{Cu}-\mathrm{Brücke} \mathrm{ab}$. Bei $\left[\mathbf{L}^{3} \mathbf{C u}_{\mathbf{2}}(\mathbf{O H})\right]$ beträgt der $\mathrm{Cu} \cdots \mathrm{Cu}$ Abstand $3.31 \AA$ und der $\mathrm{Cu}-\mathrm{O}-\mathrm{Cu}$ Winkel $120.39^{\circ}$. In der Literatur sind die magnetischen Eigenschaften einer Reihe von Pyrazolat-verbrückten bimetallischen $\mathrm{Cu}(\mathrm{II})-(\mu-\mathrm{OH})-\mathrm{Cu}(\mathrm{II})$ Komplexen bekannt. Bei diesen Pyrazolat-verbrückten Cu(II)-Komplexen mit nahezu planarer Geometrie, wie $\left[\mathbf{L}^{3} \mathbf{C u}_{\mathbf{2}}(\mathbf{O H})\right]$, beträgt der $\mathrm{Cu}-\mathrm{O}-\mathrm{Cu}$ Winkel $123-127^{\circ}$ bei einem $\mathrm{Cu} \cdots \mathrm{Cu}$ Abstand von 3.303.50 Å. Die Bestimmung der Kopplungskostanten dieser bimetallischen Kupfer(II)-Komplexe ergab die Werte von $J=-200$ bis $-240 \mathrm{~cm}^{-1} .{ }^{93}$ Somit liegt $J$ für $\left[\mathbf{L}^{3} \mathbf{C} \mathbf{u}_{\mathbf{2}}(\mathbf{O H})\right]$ mit $-232 \mathrm{~cm}^{-1}$ in Bereich der literaturbekannten Werte. ${ }^{93}$

Bei einer antiferromagnetischen Verbindung, wie $\left[\mathbf{L}^{3} \mathbf{C u}_{\mathbf{2}}(\mathbf{O H})\right]$, sind die Spins im Grundzustand $\left(S_{\mathrm{t}}=0\right)$ antiparallel angeordnet. Durch thermische Energie kann der angeregte Zustand mit populiert werden (Abbildung 79). Dieser Zustand kann mittels der ESRSpektroskopie detektiert werden.

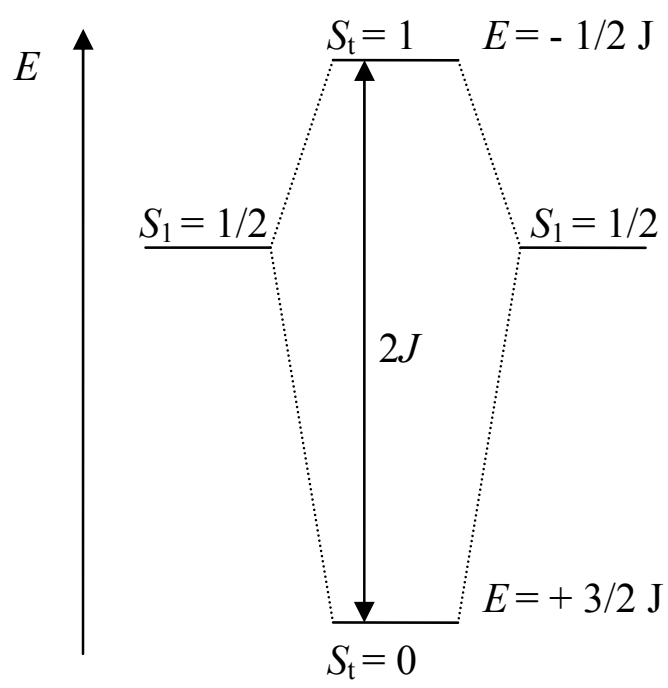

Abbildung 79: Schematische Darstellung des Energiezustands einer antiferromagnetischen Verbindung mit $\mathrm{J}<0$.

Zur Aufnahme von ESR-Spektren wurde der Komplex $\left[\mathbf{L}^{3} \mathbf{C u}_{\mathbf{2}}(\mathbf{O H})\right]$ auf $204 \mathrm{~K}$ gekühlt. Beim Aufwärmen der Probe bis $333 \mathrm{~K}$ wurde bei unterschiedlichen Temperaturen ein ESR- 
Spektrum im X-Band gemessen. In Abbildung 80 ist das ESR-Spektrum von $\left[\mathbf{L}^{3} \mathbf{C} \mathbf{u}_{2}(\mathbf{O H})\right]$ aufgenommen bei $333 \mathrm{~K}$ dargestellt.

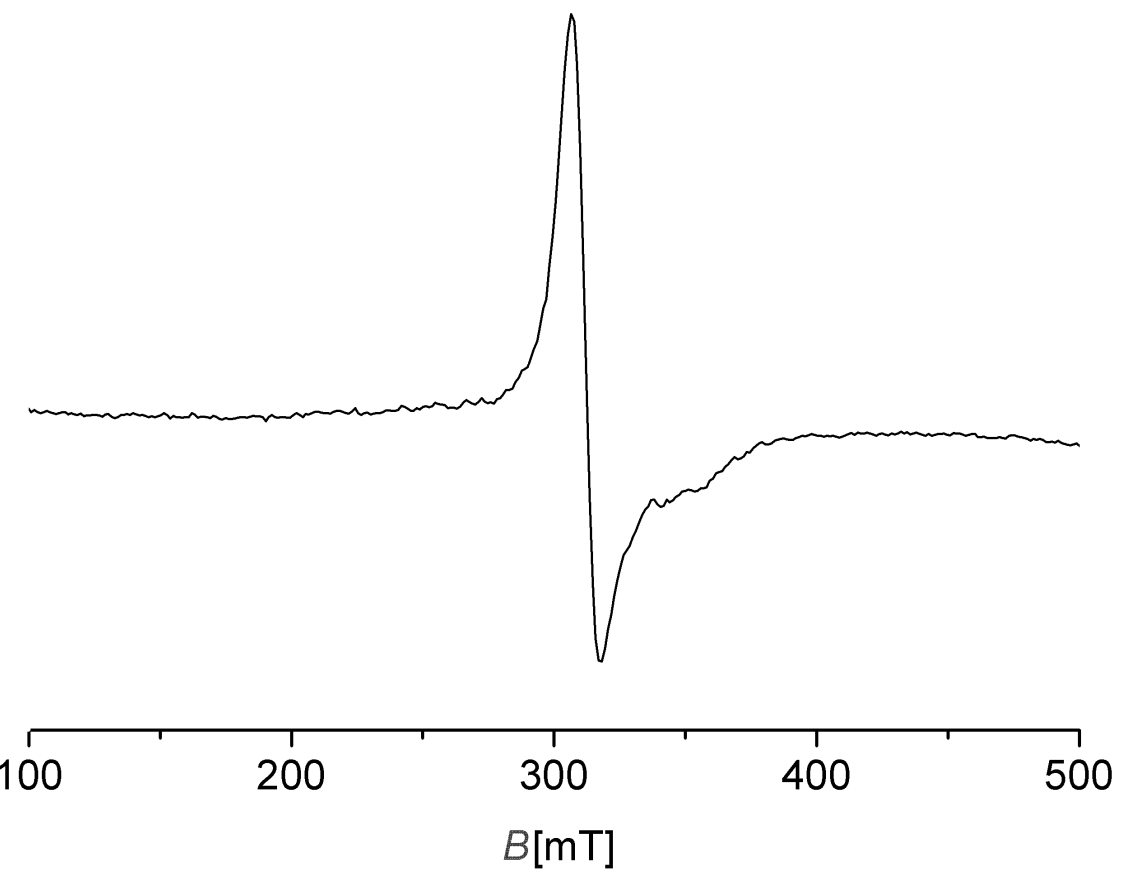

Abbildung 80: ESR-Spektrum (X-Band) des bimetallischen Kupferkomplexes $\left[\mathbf{L}^{3} \mathbf{C} \mathbf{u}_{\mathbf{2}}(\mathbf{O H})\right]$ gemessen im Festkörper bei $333 \mathrm{~K}$.

$\left[\mathbf{L}^{3} \mathbf{C u}_{\mathbf{2}}(\mathbf{O H})\right]$ zeigt das typische breite Signal für den $\Delta m_{\mathrm{s}}= \pm 1$ Übergang eines austauschgekoppelten Systems. Das Halbfeldsignal für den verbotenen $\Delta m_{\mathrm{s}}= \pm 2$ Übergang ist nicht zu sehen.

Aus dem Übergang, der in den ESR-Spektren beobachtet wird, kann über $B_{\text {res }}$ der LandéFaktor $g$ rechnerisch nach Gleichung 4 bestimmt werden:

$$
g=\frac{71.44775 \times v}{B_{\text {res. }}} .
$$

In Tabelle 15 sind die aufgenommenen $B_{\text {res }}$-Werten und die daraus berechneten $g$-Werte aufgelistet. Als Mittelwert für $g$ wurde ein Wert von 2.18 ermittelt. Dieser Wert stimmt nicht genau mit dem Wert $g=2.05$ überein, der aus den SQUID-Messungen abgeleitet wurde. Dies kann unter anderem auf die geringen $\chi_{\mathrm{M}} T$-Werte und die damit verbundene Ungenauigkeiten bei der Simulation zurückzuführen. 
Tabelle 15: Aufgenommene $B_{\text {res }}$ bei unterschiedlichen Temperaturen und resultierender Landé-Faktor $g$.

\begin{tabular}{cccc}
$\mathrm{T}[\mathrm{K}]$ & $v[\mathrm{GHz}]$ & $B_{\text {res }}[\mathrm{mT}]$ & $g$ \\
\hline 293 & 9.445737 & 307.437827 & 2.20 \\
330 & 9.446447 & 310.435371 & 2.17 \\
333 & 9.445491 & 312.477137 & 2.16 \\
& & Mittelwert: & 2.18 \\
\hline
\end{tabular}

Die Messung der magnetischen Eigenschaften ergab für die antiferromagnetische Kopplung der zwei Spins in $\left[\mathbf{L}^{3} \mathbf{C} \mathbf{u}_{2}(\mathbf{O H})\right]$ eine Kopplungskonstante $J$ von $-232 \mathrm{~cm}^{-1}$. Aus $\Delta E=-2 J\left(S_{\mathrm{t}}+1\right)$ kann nach Gleichung 5 und mit Hilfe von $J$ die molare Energie in $[\mathrm{J} / \mathrm{mol}]$ für die Anregung $S_{\mathrm{t}}=0 \rightarrow S_{\mathrm{t}}=1$ berechnet werden (Abbildung 79).

Es gilt:

$$
\begin{aligned}
& \Delta E_{J} \approx h \cdot c \cdot J \cdot N_{A} \\
& \Rightarrow \Delta E_{J}=2775.33 \mathrm{~J} / \mathrm{mol} \\
& \Rightarrow \Delta E_{J}=2775.33 / R=333 \mathrm{~K} \\
& \begin{array}{cc}
\hline N_{A} & 6.02214 \cdot 10^{23} \\
c[\mathrm{~m} / \mathrm{s}] & 299792458 \\
h & 6.62607 \cdot 10^{-34} \\
R[\mathrm{~J} / \mathrm{Kmol}] & 8.314 \\
\hline
\end{array}
\end{aligned}
$$

Demnach werden $2775.33 \mathrm{~J} / \mathrm{mol}$ benötigt um das System aus $S_{\mathrm{t}}=0$ in den Zustand $S_{\mathrm{t}}=1 \mathrm{zu}$ versetzen. Wird der berechnete Energiewert durch die allgemeinen Gaskonstante R dividiert, so kann die Energie in Form einer Temperatur angegeben werden. Der berechnete Wert entspricht $333 \mathrm{~K}$.

Bei Temperaturerhöhung wird nach Boltzmann das $S_{\mathrm{t}}=1$ Niveau populiert, allerdings liegt das System aufgrund der großen Kopplungskonstante auch bei Raumtemperatur noch weitgehend $\operatorname{im} S_{\mathrm{t}}=0$ Zustand vor, das magnetische Moment ist also klein.

$\mathbf{H}_{3} \mathbf{L}^{4}$ unterscheidet sich von den bislang diskutierten Liganden durch die Einführung einer Phenyl-Gruppe am Rückgrat der Pyrazoleinheit in der 4-Position. Durch Umsetzung von $\mathbf{H}_{3} \mathbf{L}^{4}$ mit zwei Äquivalenten Kupfer(II)chlorid entsteht der Komplex $\left[\mathbf{L}^{4} \mathbf{C u}_{2}(\mathbf{O H})\right]$, der mit Hilfe spektroskopischer Untersuchungen eindeutig nachgewiesen werden konnte.

Nach der Metallkomplexierung kommt es im IR-Spektrum zu einer signifikanten Verschiebung der Imin-Bande des Liganden $\left(\mathbf{H}_{3} \mathbf{L}^{4}\right)$ von $1616 \mathrm{~cm}^{-1} \mathrm{zu} 1563 \mathrm{~cm}^{-1}$ (Kapitel 
7.4.1). Diese Verschiebung der Imin-Bande zu kleineren Wellenzahlen konnte auch bei den anderen Liganden nach der Bildung eines bimetallischen Komplexes beobachtet werden.

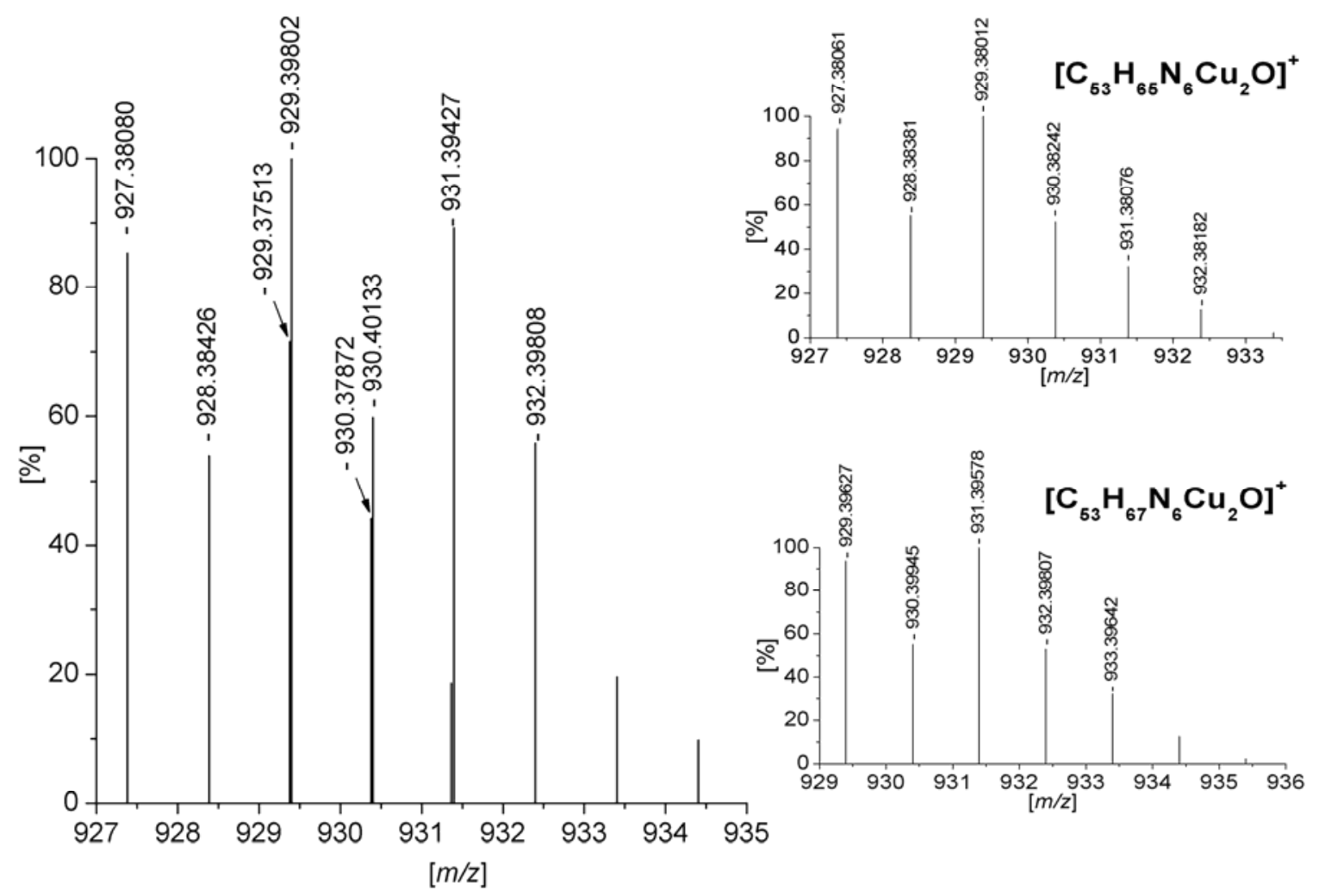

Abbildung 81: Gemessenes HR-MS(ESI+)-Spektrum von $\left[\mathbf{L}^{4} \mathbf{C u}_{2}(\mathbf{O H})\right]$ (links) und Simulation von $\left[\mathrm{C}_{53} \mathrm{H}_{65} \mathrm{~N}_{6} \mathrm{Cu}_{2} \mathrm{O}\right]^{+}[(\mathrm{M}-2 \mathrm{H})+\mathrm{H}]^{+}$(oben -rechts) bzw. $\left[\mathrm{C}_{53} \mathrm{H}_{67} \mathrm{~N}_{6} \mathrm{Cu}_{2} \mathrm{O}\right]^{+}[\mathrm{M}+\mathrm{H}]^{+}$(unten - rechts).

Obwohl Kupferchlorid als Ausgangssubstanz verwendet wurde, entsteht laut der ESI-Massenspektrometrie der Komplex $\left[\mathbf{L}^{4} \mathbf{C u}_{2}(\mathbf{O H})\right]$ mit $\mu$-OH als Brückenligand und nicht $\left[\mathbf{L}^{4} \mathbf{C u}_{2} \mathbf{C l}\right]$. Der Komplex $\left[\mathbf{L}^{4} \mathbf{C u}_{2}(\mathbf{O H})\right]$ wurde in einer MeCN-Lösung mit Hilfe der HRMS(ESI+)-Spektrometrie untersucht. Das gemessene Spektrum ist in Abbildung 81 (links) abgebildet. Im HR-MS-Spektrum wurde das intensivste Signal bei 929.39802 m/z (100 \%) detektiert. Bei genauer Betrachtung des HR-Massenspektrums werden noch drei weitere Signale (bei $929.37513,930.37872$ und $931.37634 \mathrm{~m} / \mathrm{z}$ ) beobachtet. Bei der Ionisierung von $\left[\mathbf{L}^{4} \mathbf{C u}_{2}(\mathbf{O H})\right]$ sollte bei einfacher Protonierung $\left[\left(\mathrm{C}_{53} \mathrm{H}_{65} \mathrm{~N}_{6} \mathrm{Cu}_{2} \mathrm{OH}\right)+\mathrm{H}\right]^{+}$ein Signal bei 931.39578 m/z (100 \%) bzw. mit einem monoisotopischen Signal bei $929.39627 \mathrm{~m} / \mathrm{z}$ zu beobachten sein (simuliertes Spektrum Abbildung 81 rechts unten). Im HR-Massenspektrum liegt der gemessene Wert für diese Spezies bei $929.39802 \mathrm{~m} / \mathrm{z}$ und stimmt somit gut mit dem berechneten Wert überein. Im Rahmen der Messung verliert die Verbindung $\left[\mathbf{L}^{4} \mathbf{C u}_{2}(\mathbf{O H})\right]$ jedoch zwei H-Atome und wird dadurch in Form von $[(\mathrm{M}-2 \mathrm{H})+\mathrm{H}]^{+}$detektiert. Der berechnete 
Wert von $927.38061 \mathrm{~m} / \mathrm{z}$ für das Basissignal dieser oxidierten Verbindung (Simulation Abbildung 81 rechts oben) stimmt mit dem gemessenen Wert bei $927.38081 \mathrm{~m} / \mathrm{z}$ gut überein. Oxidationsvorgänge während den massenspektrometrischen Untersuchungen konnten ebenso bei den bimetallischen Kupferkomplexen ( $\left[\mathbf{L}^{1} \mathbf{C u}_{2}(\mathbf{O H})\right]$ und $\left[\mathbf{L}^{3} \mathbf{C u}_{2}(\mathbf{O H})\right]$ ) beobachtet werden. Auch durch Anwendung anderer Ionisierungsmethoden, in der ESI- oder FABMassenspektrometrie, wurde der gleiche Effekt beobachtet. Dies bestätigt erneut das Vorliegen von „,non-innocent“ Liganden in den synthetisierten Kupferkomplexen $\left[\mathbf{L}^{1} \mathrm{Cu}_{2}(\mathrm{OH})\right],\left[\mathrm{L}^{3} \mathrm{Cu}_{2}(\mathrm{OH})\right]$ und $\left[\mathrm{L}^{4} \mathrm{Cu} \mathbf{u}_{2}(\mathrm{OH})\right]$.

Ein weiterer Kupferkomplex wurde mit dem Liganden $\mathbf{H}_{\mathbf{3}} \mathbf{L}^{\mathbf{5}}$ synthetisiert. Dieser Ligand wurde durch eine Kondensationsreaktion zwischen 1a und dem Methyl-(1-methylimidazol-2ylmethyl)amin ${ }^{94,95}$ dargestellt (Abbildung 82). Trotz mehrfacher säulenchromatographischer Aufreinigung konnte der Ligand nicht in hoher Reinheit gewonnen werden. Das ESI(+)Spektrum zeigt, dass neben dem Produkt $\left(\mathbf{H}_{\mathbf{3}} \mathbf{L}^{\mathbf{5}}\right)$ auch das nur an einem Seitenarm monosubstituiete Nebenprodukt $\mathbf{H}_{\mathbf{3}} \mathbf{L}_{\text {mono }}^{\mathbf{5}}\left[\left(\mathrm{C}_{28} \mathrm{H}_{37} \mathrm{~N}_{7} \mathrm{O}\right)+\mathrm{H}^{+}\right]$mit $488 \mathrm{~m} / \mathrm{z}$ entsteht. Zur Komplexbildung wurde $\mathbf{H}_{\mathbf{3}} \mathbf{L}^{\mathbf{5}}$ in THF gelöst, mit Triethylamin umgesetzt und mit einem leichten Überschuss Kupferacetat (2.1 eq) versetzt. Nach einem Tag wurde das Lösungsmittel entfernt und der Rückstand in Dichlormethan aufgenommen.

Durch langsames Verdampfen des Lösungsmittels konnten grüne Einkristalle für die Röntgenstrukturanalyse gewonnen werden. Die Molekülstruktur des isolierten Kristalls von $\left[\mathbf{L}_{2}^{6} \mathbf{C u}_{2}(\mathbf{O A c})_{4}\right]$ ist in Abbildung 83 dargestellt. Durch Reaktion des Eduktes Methyl-(1methylimidazol-2-ylmethyl)amin mit Trifluoressigsäure innerhalb der säurekatalytischen Darstellung des Liganden $\mathbf{H}_{\mathbf{3}} \mathbf{L}^{\mathbf{5}}$, entsteht in einer Nebenreaktion 2,2,2-Trifluoro- $N$-(1-methyl$1 H$-imidazol-2-ylmethyl)-acetamid. In der nachfolgenden Reaktion mit Kupferacetat wurde daher der bimetallische Kupferkomplex $\left[\mathbf{L}^{6}{ }_{2} \mathbf{C u}_{2}(\mathbf{O A c})_{4}\right]$, der in kristalliner Form gewonnen werden konnte, gebildet (Abbildung 83). 
<smiles>CCc1c(C=O)[nH]c(Cc2cc(Cc3[nH]c(C=O)c(CC)c3CC)[nH]n2)c1CC</smiles>

1a

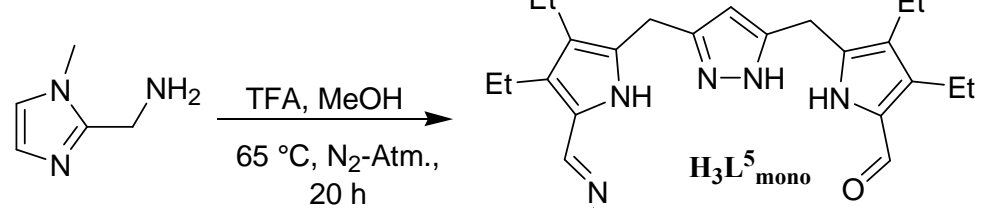

$\mathrm{C}_{28} \mathrm{H}_{37} \mathrm{~N}_{7} \mathrm{O}$

Exakte Masse: $487 \mathrm{gmol}^{-1}$

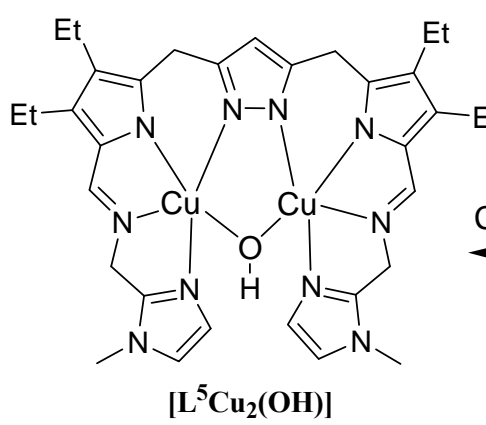<smiles>CCCc1c(Cc2cc(Cc3[nH]c(/C=N/Cc4nccn4C)c(CC)c3CC)[nH]n2)[nH]c(C=NCc2nccn2C)c1CC</smiles>

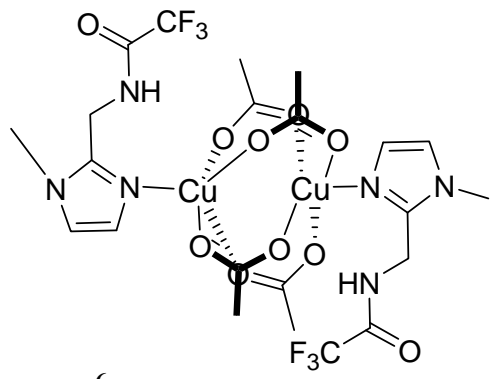

$\left[\mathrm{L}^{6}{ }_{2} \mathrm{Cu}_{2}(\mathrm{OAc})_{4}\right]$

Abbildung 82: Ligand- und Metallkomplexsynthese von $\mathbf{H}_{3} \mathbf{L}^{5}$ bzw. $\left[\mathbf{L}^{5} \mathbf{C u}_{\mathbf{2}}(\mathbf{O H})\right]$ und dem Nebenprodukt $\left[\mathrm{L}^{6}{ }_{2} \mathrm{Cu}_{2}(\mathrm{OAc})_{4}\right]$.

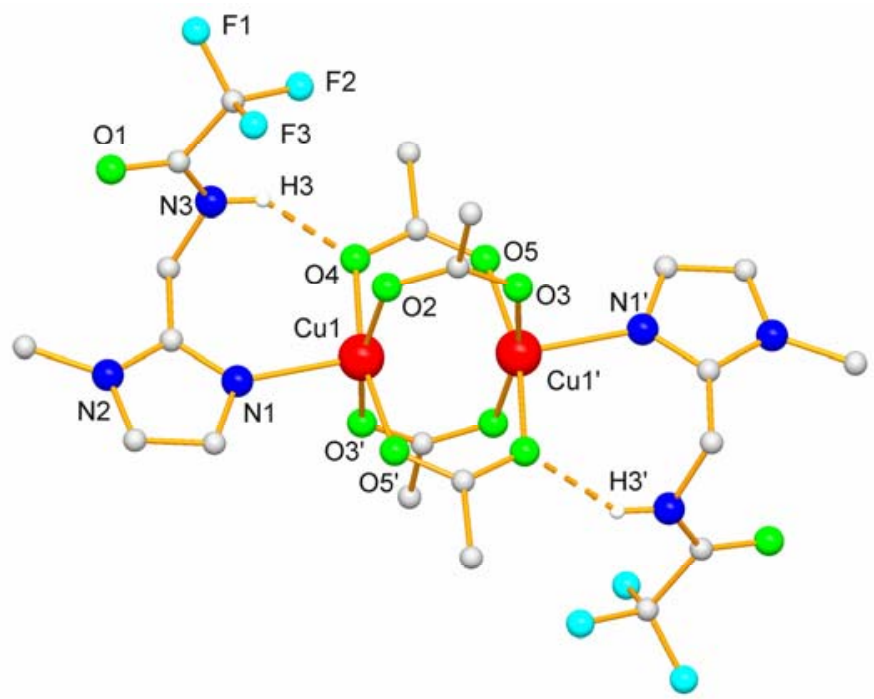

Abbildung 83: Molekülstruktur von $\left[\mathrm{L}_{2}^{6} \mathrm{Cu}_{2}(\mathrm{OAc})_{4}\right]$. 
Die N-Atome (N1 bzw. N1') des Imidazolringes koordinieren an die Kupferatome Cu1 und $\mathrm{Cu} 1^{\prime}$, die eine quadratisch pyramidale Koordinationssphäre und einen $\mathrm{Cu}{ }^{\cdots} \mathrm{Cu}$ Abstand von 2.69(8) $\AA$ aufweisen. Die Struktur von $\left[\mathbf{L}^{6}{ }_{2} \mathbf{C u}_{2}(\mathbf{O A c})_{4}\right]$ zeigt Parallelen zu der bekannten Dimerstruktur des Kupferacetat-Dihydrats $\left[\mathrm{Cu}_{2}(\mathrm{OAc})_{4}\left(\mathrm{H}_{2} \mathrm{O}\right)_{2}\right]^{96}$. Statt der zwei Wassermoleküle koordinieren in $\left[\mathbf{L}^{\mathbf{6}}{ }_{\mathbf{C}} \mathbf{C u}_{\mathbf{2}}(\mathbf{O A c})_{4}\right]$ die zwei N-Atome des Imidazolliganden $\mathbf{L}^{\mathbf{6}}$ an die Kupferatome.

Der Komplex $\left[\mathbf{L}^{\mathbf{5}} \mathbf{C u}_{\mathbf{2}}(\mathbf{O H})\right]$ konnte nicht synthetisiert werden, da der Ligand $\mathbf{H}_{3} \mathbf{L}^{\mathbf{5}}$ nicht in ausreichender reiner Form erhalten werden konnte. Die geometrische Einheit des zweikernigen Komplexes $\left[\mathbf{L}^{5} \mathbf{C u}_{2}(\mathbf{O H})\right]$ bietet keine günstige Koordination an. Die röntgenographische Charakterisierung der anderen Kupferkomplexe hat gezeigt, dass diese Liganden bevorzugt quadratisch planare Komplexe bilden. Die N-Atome der Imidazoleinheiten von $\mathbf{H}_{3} \mathbf{L}^{\mathbf{5}}$ würden somit nicht an der Koordination beteiligen können.

\subsection{Bimetallische Nickel(II)komplexe der Liganden $\mathrm{H}_{3} \mathrm{~L}^{1}, \mathrm{H}_{3} \mathrm{~L}^{3}$ und $\mathrm{H}_{3} \mathrm{~L}^{4}-$ Strukturelle Charakterisierung und NMR-spektroskopische Untersuchungen}

Durch den generell starren Aufbau der vorgestellten Liganden $\left(\mathbf{H}_{3} \mathbf{L}^{\mathbf{x}}\right)$ werden bevorzugt quadratisch planare Komplexe gebildet. Da Nickel(II)ionen bei Komplexreaktionen neben einer sechsfachen Koordination bevorzugt auch eine vierfache Koordination unter Ausbildung quadratisch planarer low-spin Komplexe eingehen, wurde ebenfalls die Nickel(II)komplexe der dargestellten Liganden synthetisiert.

Der jeweilige Ligand $\mathbf{H}_{3} \mathbf{L}^{\mathbf{x}}$ wurde in THF bzw. Chloroform gelöst, mit Triethylamin umgesetzt und mit Nickel(II)acetat-Tetrahydrat versetzt (Abbildung 84). Nach einigen Stunden Rühren wurde das Lösungsmittel entfernt und das rot-braune Rohprodukt in Dichlormethan/Ethanol aufgenommen. Unter langsamem Abdampfen der Lösungsmittel wurden nach mehreren Tagen rot-braune Einkristalle erhalten, die röntgenographisch charakterisiert werden konnten. Die Molekülstruktur von $\left[\mathbf{L}^{1} \mathbf{N i}_{\mathbf{2}}(\mathbf{O H})\right]$ ist in Abbildung 85 gezeigt. Wie erwartet handelt es sich bei dem untersuchten Kristall um einen bimetallischen Nickel(II)komplex mit quadratisch planarer Koordination. Der Komplex kristallisiert in der Raumgruppe $P 2 / c$ mit vier Molekülen in der Einheitszelle. 

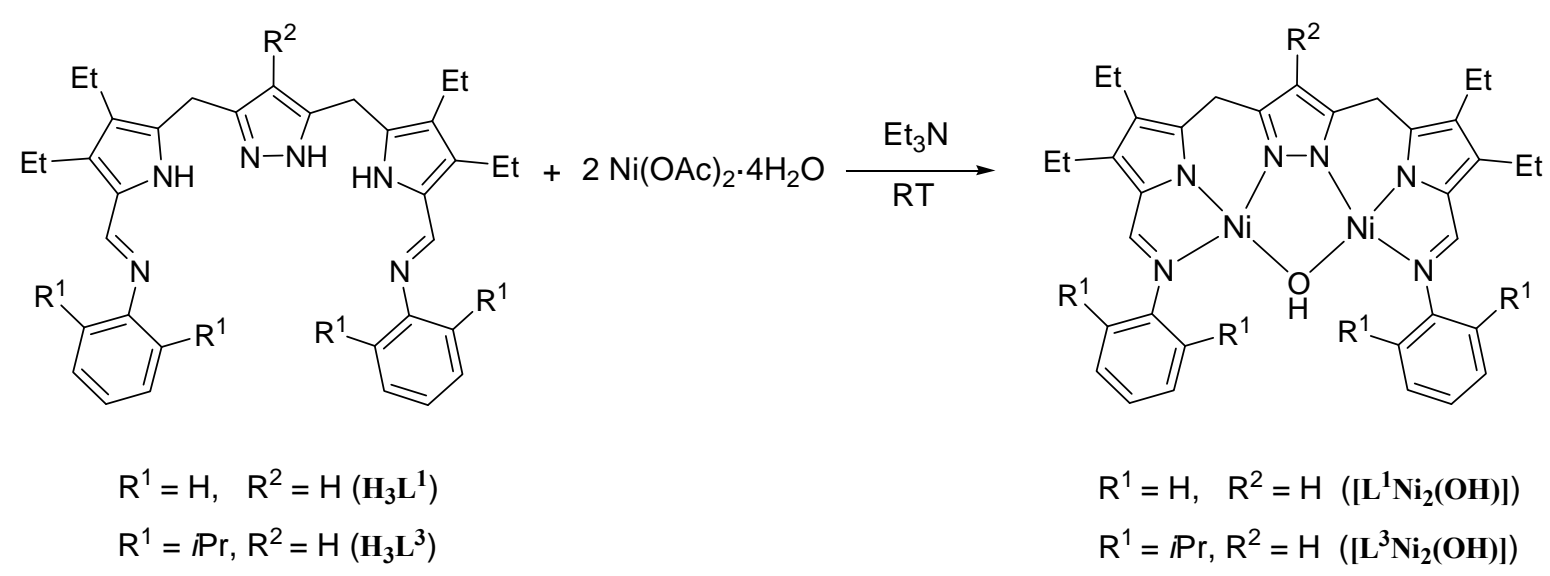

Abbildung 84: Komplexsynthese der bimetallischen Nickel(II)komplexe $\left[\mathbf{L}^{1} \mathbf{N i}_{2}(\mathbf{O H})\right]$ und $\left[\mathbf{L}^{3} \mathbf{N i}_{2}(\mathbf{O H})\right]$.

In Tabelle 16 sind einige ausgewählte Abstände und Winkel von $\left[\mathbf{L}^{\mathbf{1}} \mathbf{N i}_{\mathbf{2}}(\mathbf{O H})\right]$ aufgeführt. Analog zu den diskutierten Kupfer(II)komplexen $\left[\mathbf{L}^{\mathbf{1}}{ }_{\text {ox }} \mathbf{C u}_{\mathbf{2}}(\mathbf{O H})\right]$ und $\left[\mathbf{L}^{3} \mathbf{C u}_{\mathbf{2}}(\mathbf{O H})\right]$ liegt auch hier eine $\mu$-OH Gruppe zwischen den zwei Ni(II)-Ionen als Brückenligand vor. Die OHGruppe entsteht durch das Wasser in der Reaktionsmischung, wobei Acetat bzw. Triethylamin als Base wirken könnten. Beide Nickelatome (Ni2 bzw. Ni1) liegen in der gleichen Ebene wie das $\mu-\mathrm{OH}$. Der $\mathrm{Ni} \cdots \mathrm{Ni}$ Abstand ist mit $3.24 \AA$ relativ klein für Nickel(II)komplexe mit multifunktionellen Pyrazolat-Liganden. ${ }^{97}$ Bei dem Nickel(II)komplexe [ $\left.\mathbf{L}^{1} \mathbf{N i}_{\mathbf{2}}(\mathbf{O H})\right]$ entsteht ein Sechsring-Chelat, welches die zwei Nickelionen in unmittelbar Nähe bringt. Bei den restlich bekannten Nickel(II)komplexe mit multifunktionellen Pyrazolat-Liganden werden hingegen Fünfring-Chelate gebildet. Durch die Ausbildung der Fünfring-Chelate werden die zwei Nickelionen auseinander gezogen und ein größerer $\mathrm{Ni} \cdots \mathrm{Ni}$ Abstand resultiert daraus. ${ }^{97}$

Neben $\left[\mathbf{L}^{\mathbf{1}} \mathbf{N i}_{\mathbf{2}}(\mathbf{O H})\right]$ konnte der bimetallische Nickel(II)komplex $\left[\mathbf{L}^{3} \mathbf{N i}_{\mathbf{2}}(\mathbf{O H})\right]$ erfolgreich synthetisiert werden. Kristalle von $\left[\mathbf{L}^{3} \mathbf{N i}_{2}(\mathbf{O H})\right]$ konnten aus einer Lösung von $\mathrm{CH}_{2} \mathrm{Cl}_{2}$ bzw. $\mathrm{CHCl}_{3}$, die mit Ethanol, Methanol oder Acetonitril überschichtet wurde, isoliert werden. Da es sich bei diesen Komplexen um neutrale Verbindungen handelt, sind sie überwiegend in unpolaren organischen Lösungsmitteln wie Chloroform und Dichlormethan löslich. Der Komplex $\left[\mathbf{L}^{3} \mathbf{N i}_{\mathbf{2}}(\mathbf{O H})\right]$ kristallisiert in der Raumgruppe $P 2 / n$ mit acht Molekülen in der Einheitszelle. Die wichtigsten Bindungslängen und Winkel des Komplexes sind in Tabelle 17 aufgeführt. Die Struktur von $\left[\mathbf{L}^{3} \mathbf{N i}_{\mathbf{2}}(\mathbf{O H})\right]$ ist analog zu $\left[\mathbf{L}^{\mathbf{1}} \mathbf{N i}_{\mathbf{2}}(\mathbf{O H})\right]$. 


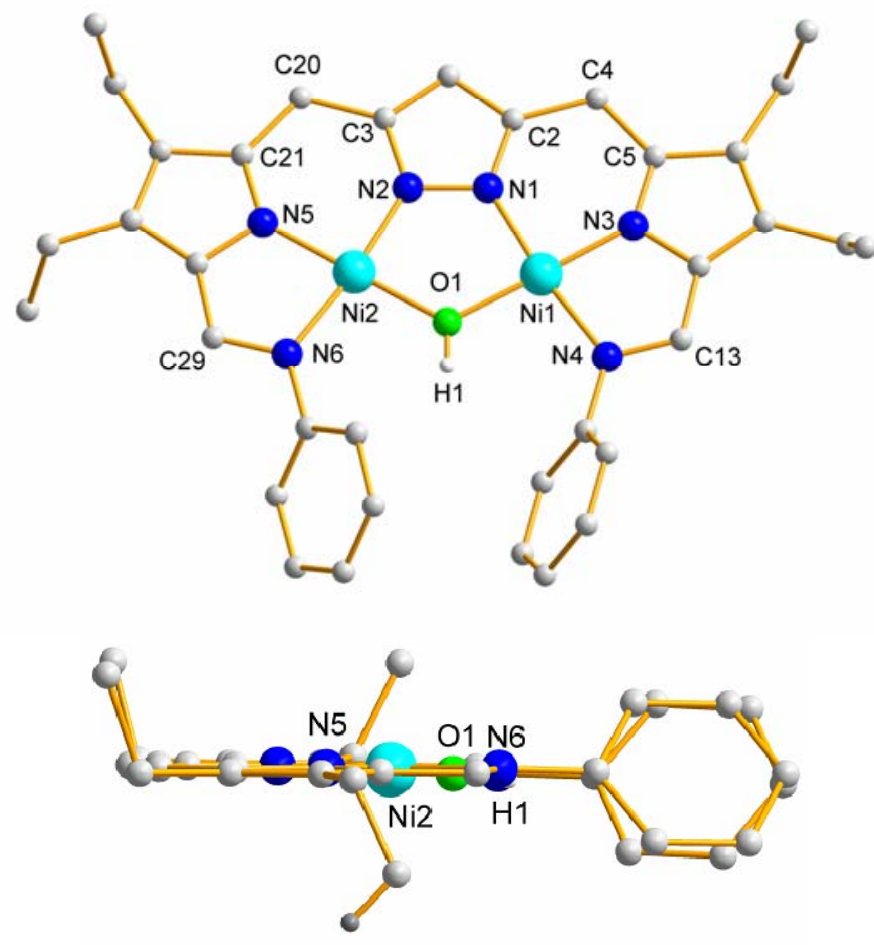

Abbildung 85: Molekülstruktur von $\left[\mathbf{L}^{1} \mathbf{N i}_{2}(\mathbf{O H})\right]$ (oben) und Seitenansicht (unten).

Tabelle 16: Ausgewählte Abstände und Winkel von $\left[\mathbf{L}^{1} \mathbf{N i}_{\mathbf{2}}(\mathbf{O H})\right]$.

\begin{tabular}{|c|c|c|c|}
\hline \multicolumn{4}{|c|}{ Abstände $[\AA]$} \\
\hline $\mathrm{Ni}(1)-\mathrm{Ni}(2)$ & $3.2364(7)$ & $\mathrm{Ni}(2)-\mathrm{N}(5)$ & $1.838(3)$ \\
\hline $\mathrm{Ni}(1)-\mathrm{N}(1)$ & $1.857(3)$ & $\mathrm{Ni}(2)-\mathrm{N}(6)$ & $1.932(3)$ \\
\hline Ni(1)-N(3) & $1.831(3)$ & $\mathrm{Ni}(1)-\mathrm{O}(1)$ & $1.853(3)$ \\
\hline $\mathrm{Ni}(1)-\mathrm{N}(4)$ & $1.918(3)$ & $\mathrm{Ni}(2)-\mathrm{O}(1)$ & $1.846(3)$ \\
\hline $\mathrm{Ni}(2)-\mathrm{N}(2)$ & $1.858(3)$ & & \\
\hline \multicolumn{4}{|c|}{ Winkel $\left[{ }^{\circ}\right]$} \\
\hline $\mathrm{N}(1)-\mathrm{Ni}(1)-\mathrm{N}(4)$ & $173.09(14)$ & $\mathrm{O}(1)-\mathrm{Ni}(2)-\mathrm{N}(2)$ & $89.06(13)$ \\
\hline $\mathrm{N}(3)-\mathrm{Ni}(1)-\mathrm{N}(1)$ & $89.99(14)$ & $\mathrm{O}(1)-\mathrm{Ni}(2)-\mathrm{N}(6)$ & $97.79(12)$ \\
\hline $\mathrm{N}(3)-\mathrm{Ni}(1)-\mathrm{N}(4)$ & $83.21(14)$ & $\mathrm{N}(2)-\mathrm{Ni}(2)-\mathrm{N}(6)$ & $173.14(14)$ \\
\hline $\mathrm{N}(3)-\mathrm{Ni}(1)-\mathrm{O}(1)$ & 178.98(14) & $\mathrm{Ni}(2)-\mathrm{O}(1)-\mathrm{Ni}(1)$ & $122.09(13)$ \\
\hline $\mathrm{N}(5)-\mathrm{Ni}(2)-\mathrm{O}(1)$ & $177.37(15)$ & $\mathrm{O}(1)-\mathrm{Ni}(1)-\mathrm{N}(1)$ & $89.00(13)$ \\
\hline $\mathrm{N}(5)-\mathrm{Ni}(2)-\mathrm{N}(2)$ & $89.58(14)$ & $\mathrm{C}(2)-\mathrm{N}(1)-\mathrm{N}(2)$ & 108.2(3) \\
\hline $\mathrm{N}(5)-\mathrm{Ni}(2)-\mathrm{N}(6)$ & $83.58(13)$ & $\mathrm{O}(1)-\mathrm{Ni}(1)-\mathrm{N}(4)$ & $97.80(12)$ \\
\hline
\end{tabular}


Die zwei Nickelatome sind durch ein $\mu-\mathrm{OH}$ verbrückt und zeigen somit eine quadratisch planare Koordination. Sowohl der Ni*Ni Abstand mit $3.25 \AA$ als auch der Ni2-O1-Ni1 Winkel mit $121.2^{\circ}$ sind nahezu identisch mit den Werten für $\left.\left[\mathbf{L}^{\mathbf{1}} \mathbf{N i}_{\mathbf{2}} \mathbf{( O H}\right)\right]$ mit $(\mathrm{Ni} \cdots \mathrm{Ni}$ Abstand mit $3.24 \AA$ und Ni2-O1-Ni1 Winkel mit $122.1^{\circ}$ ). Wie man aus der Seitenansicht des Komplexes in Abbildung 86 entnehmen kann, liegt der $\mu$-OH Ligand in derselben Ebene wie die zwei Nickelatome.
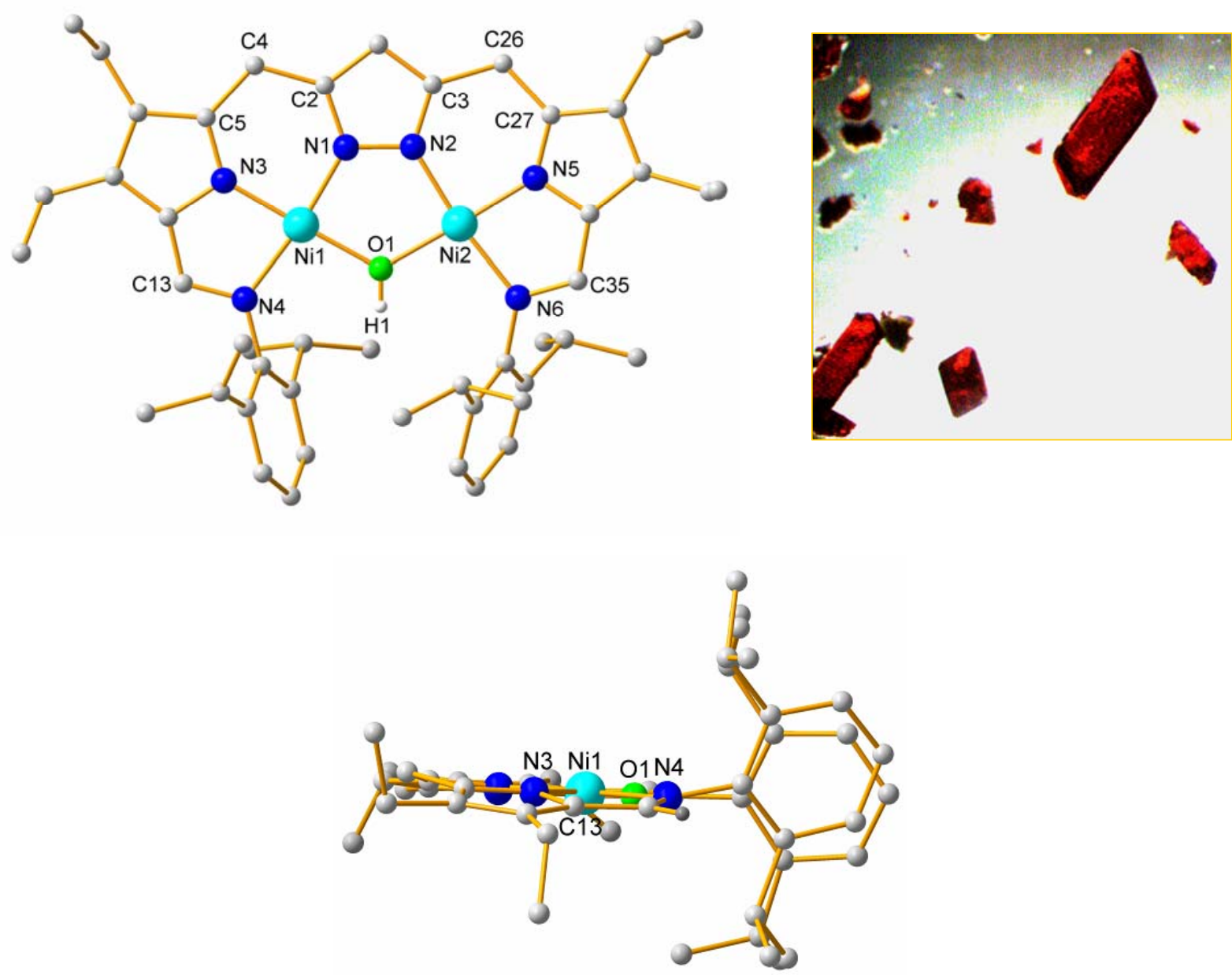

Abbildung 86: Molekülstruktur von $\left[\mathbf{L}^{3} \mathbf{N i}_{2}(\mathbf{O H})\right]$ (oben links) und Seitenansicht (unten). Fotographie der roten $\left[\mathbf{L}^{3} \mathbf{N i}_{\mathbf{2}}(\mathbf{O H})\right]$ Kristalle (oben rechts).

Ein analoger Komplex wurde unter Verwendung eines weiteren Nickelsalzes, mit größerem Anion synthetisiert. Statt des Nickelacetats wurde $\mathrm{NiBr}_{2} \cdot \mathrm{dme}$ verwendet, um den Komplex $\left[\mathbf{L}^{3} \mathbf{N i}_{2} \mathbf{B r}\right]$ zu synthetisieren. Massenspektrometrisch (ESI-MS) konnte ermittelt werden, dass der Ligand zum größten Teil unverbraucht in der Reaktionslösung vorlag und dass erneut der Komplex $\left[\mathbf{L}^{3} \mathbf{N i}_{\mathbf{2}}(\mathbf{O H})\right]$ dargestellt wurde. Diese Ergebnisse und die Daten der 
Molekülstruktur von $\left[\mathbf{L}^{3} \mathbf{N i}_{2}(\mathbf{O H})\right]$ demonstrieren, dass bei der Ausbildung des Komplexes kein Raum für größere Brückenliganden, wie das Bromidion zur Verfügung steht.

Tabelle 17: Ausgewählte Abstände und Winkel von $\left[\mathbf{L}^{3} \mathbf{N i}_{2}(\mathbf{O H})\right]$.

\begin{tabular}{lr|ll}
\multicolumn{5}{c}{ Abstände $[\AA]$} \\
\hline $\mathrm{Ni}(1)-\mathrm{Ni}(2)$ & $3.2472(3)$ & $\mathrm{Ni}(2)-\mathrm{N}(6)$ & $1.9364(16)$ \\
$\mathrm{Ni}(1)-\mathrm{N}(1)$ & $1.8544(16)$ & $\mathrm{Ni}(1)-\mathrm{O}(1)$ & $1.8699(14)$ \\
$\mathrm{Ni}(1)-\mathrm{N}(3)$ & $1.8379(16)$ & $\mathrm{Ni}(2)-\mathrm{O}(1)$ & $1.8680(13)$ \\
$\mathrm{Ni}(1)-\mathrm{N}(4)$ & $1.9275(16)$ & $\mathrm{N}(6)-\mathrm{C}(35)$ & $1.313(2)$ \\
$\mathrm{Ni}(2)-\mathrm{N}(2)$ & $1.8614(16)$ & $\mathrm{C}(3)-\mathrm{C}(26)$ & $1.508(3)$ \\
$\mathrm{Ni}(2)-\mathrm{N}(5)$ & $1.8347(15)$ & $\mathrm{C}(26)-\mathrm{C}(27)$ & $1.489(3)$ \\
& & & \\
& & Winkel [ $\left.{ }^{\circ}\right]$ & $89.54(7)$ \\
$\mathrm{N}(1)-\mathrm{Ni}(1)-\mathrm{N}(4)$ & $172.54(7)$ & $\mathrm{N}(5)-\mathrm{Ni}(2)-\mathrm{N}(2)$ & $82.96(7)$ \\
$\mathrm{N}(1)-\mathrm{Ni}(1)-\mathrm{O}(1)$ & $88.72(6)$ & $\mathrm{N}(5)-\mathrm{Ni}(2)-\mathrm{N}(6)$ & $172.44(6)$ \\
$\mathrm{N}(2)-\mathrm{Ni}(2)-\mathrm{O}(1)$ & $88.57(6)$ & $\mathrm{N}(2)-\mathrm{Ni}(2)-\mathrm{N}(6)$ & $98.55(6)$ \\
$\mathrm{N}(3)-\mathrm{Ni}(1)-\mathrm{N}(1)$ & $89.88(7)$ & $\mathrm{O}(1)-\mathrm{Ni}(1)-\mathrm{N}(4)$ & $98.88(6)$ \\
$\mathrm{N}(3)-\mathrm{Ni}(1)-\mathrm{O}(1)$ & $178.55(7)$ & $\mathrm{O}(1)-\mathrm{Ni}(2)-\mathrm{N}(6)$ & $115.42(16)$ \\
$\mathrm{N}(3)-\mathrm{Ni}(1)-\mathrm{N}(4)$ & $82.84(7)$ & $\mathrm{C}(27)-\mathrm{C}(26)-\mathrm{C}(3)$ & $121.19(8)$ \\
$\mathrm{N}(5)-\mathrm{Ni}(2)-\mathrm{O}(1)$ & $176.80(7)$ & $\mathrm{Ni}(2)-\mathrm{O}(11)-\mathrm{Ni}(1)$ & \\
\hline
\end{tabular}

Der Ligand $\mathbf{H}_{3} \mathbf{L}^{4}$ trägt eine Phenylgruppe am Rückgrat des Pyrazols an der 4-Position (Abbildung 87). Somit wird eine erhöhte Sperrigkeit der Liganden-Peripherie verursacht. Die strukturelle Charakterisierung der Komplexe mit $\mathbf{H}_{3} \mathbf{L}^{4}$ sollte zeigen, ob durch die Sperrigkeit des Ligandrückrats und der Imin-Arylreste das Metallzentrum besser abgeschirmt wird. Der Ligand $\mathbf{H}_{3} \mathbf{L}^{4}$ wurde mit Triethylamin umgesetzt, anschließend wurden zwei Äquivalente $\mathrm{Ni}\left(\mathrm{PPh}_{3}\right)_{2} \mathrm{PhCl}$ zugegeben und das Reaktionsgemisch mehrere Tage bei Raumtemperatur gerührt. Der synthetisierte Komplex wurde durch unterschiedliche spektroskopische Methoden untersucht.

Nach der Reaktion ist im IR-Spektrum eine charakteristische Verschiebung der Imin-Bande des Liganden $\mathbf{H}_{3} \mathbf{L}^{4}$ von $1616 \mathrm{~cm}^{-1}$ zu $1569 \mathrm{~cm}^{-1} \mathrm{zu}$ beobachten. Wie bei den restlichen Komplexen (Kapitel 7.4.1) mit $\mu$-OH Brückenliganden findet sich im IR-Spektrum eine entsprechende Bande bei $3604 \mathrm{~cm}^{-1}$. Diese OH-Gruppe entsteht vermutlich aus Spuren von Wasser. 


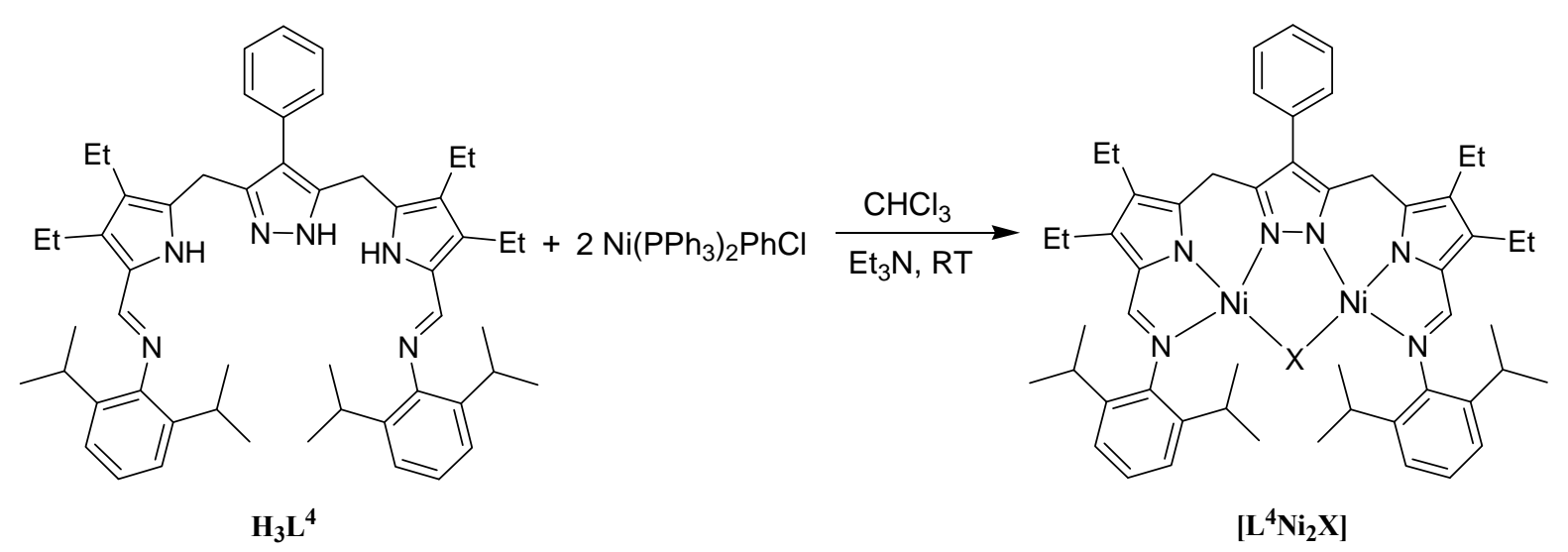

Abbildung 87: Synthese des Nickel(II)komplexes $\left[\mathbf{L}^{4} \mathbf{N i}_{2} \mathbf{X}\right](\mathrm{X}=\mathrm{Cl}, \mathrm{OH})$.

Der Komplex wurde ebenfalls massenspektrometrisch untersucht. Die FAB-Massenspektren zeigen wesentlich drei Signale. Das erste Signal wird bei 790 m/z $\left([\mathrm{M}+\mathrm{H}]^{+}, 20 \%\right)$ detektiert und entspricht dem freien Liganden. Das zweite Signal bei $917 \mathrm{~m} / \mathrm{z}(100 \%)$ ist der Verbindung $\left(\left[\mathbf{L}^{4} \mathbf{N i}_{\mathbf{2}}(\mathbf{O H})\right]+\mathrm{H}\right)^{+}$zuzuordnen. Die Entstehung dieses Komplexes wird durch die Ergebnisse der IR-Spektren gestützt. Das dritte Signal ist bei $937 \mathrm{~m} / \mathrm{z}$ zu beobachten und entspricht $\left(\left[\mathbf{L}^{4} \mathbf{N i}_{2} \mathbf{C l}\right]+\mathrm{H}\right)^{+}$. Als Brückenligand koordiniert hier statt eines $\mu$-OH ein ChloridIon zwischen den zwei Metallionen.

ESI-MS und HR-MS(ESI+) Spektren zeigen ebenfalls ein Signal bei 917 m/z (100\%) für $\left(\left[\mathbf{L}^{4} \mathbf{N i}_{\mathbf{2}}(\mathbf{O H})\right]+\mathrm{H}\right)^{+}$und ein weiteres Signal bei $933 \mathrm{~m} / \mathbf{z}(73 \%)$. Die Bildung von $\left[\mathbf{L}^{4} \mathbf{N i}_{\mathbf{2}} \mathbf{C l}\right]$ konnte hier nicht eindeutig bestätigt werden. Das HR(ESI+)-Massenspektrum der Reaktionslösung und die jeweiligen Berechungen $\mathrm{zu}$ den detektierten Signalen sind in Abbildung 88 (Spektrum A) dargestellt. Das Signal bei $917.39069 \mathrm{~m} / \mathbf{z}$ für $\left(\left[\mathbf{L}^{4} \mathbf{N i}_{2}(\mathbf{O H})\right]^{+H}\right)^{+}$ stimmt gut mit dem berechneten Wert von $917.39216 \mathrm{~m} / \mathrm{z}$ (Spektrum B) überein. Bei dem zweiten Signal bei 933.38542 m/z kann es sich um eine oxygenierte Spezies handeln.

Wie die Berechnungen zeigen (Spektrum C), könnte es sich um den Komplex $\left[\mathbf{C}_{\mathbf{5 3}} \mathbf{H}_{\mathbf{6 5}} \mathbf{N}_{\mathbf{6}} \mathbf{N i}_{\mathbf{2}} \mathbf{O}_{2}\right]^{+}$handeln, bei dem noch ein weiteres Sauerstoffatom in die Struktur eingebunden ist. Es ist daher nicht auszuschließen, dass der Ligand im Komplex - ähnlich dem Kupferkomplex $\left[\mathbf{L}^{\mathbf{1}}{ }_{\text {ox }} \mathbf{C} \mathbf{u}_{\mathbf{2}}(\mathbf{O H})\right]$ an den 3,5-Positionen des Pyrazols ein- bzw. zweifach oxidiert wurde. 

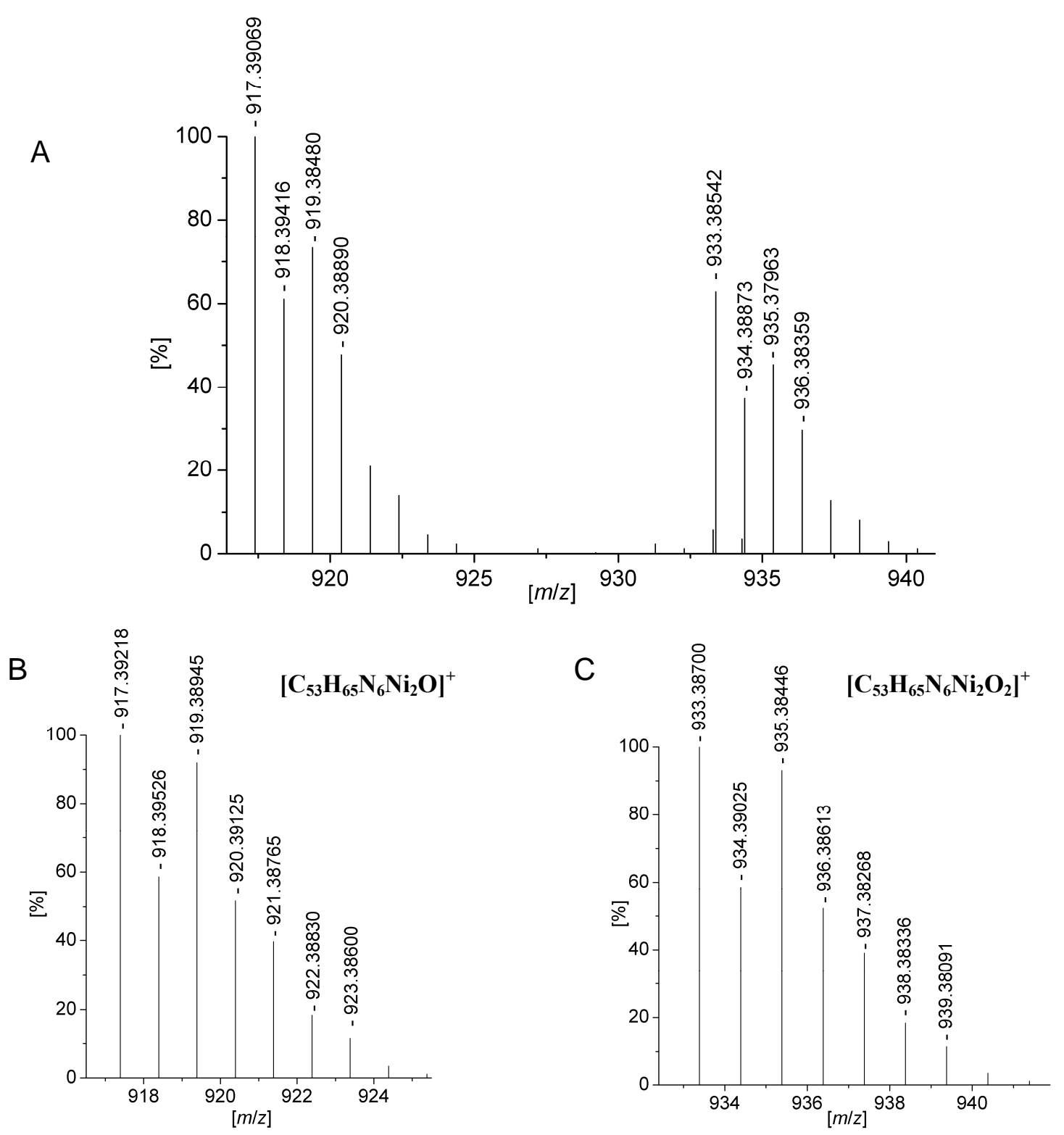

Abbildung 88: HR-MS Spektrum von $\left[\mathbf{L}^{4} \mathbf{N i}_{2} \mathbf{X}\right]$ in Acetonitril (A, oben). Berechnete Werte für die Zusammensetzung $\left[\mathrm{C}_{53} \mathbf{H}_{65} \mathbf{N}_{6} \mathbf{N i}_{2} \mathbf{O}\right]^{+}$(B, unten), und für $\left[\mathrm{C}_{53} \mathbf{H}_{65} \mathbf{N}_{6} \mathbf{N i}_{2} \mathbf{O}_{2}\right]^{+}$(C, unten). 


\subsection{Bimetallischer Palladium(II)komplex mit den Liganden $\mathbf{H}_{3} \mathbf{L}^{3}$ - Strukturelle Charakterisierung und NMR-Untersuchungen}

Durch die Umsetzung des Liganden $\mathbf{H}_{3} \mathbf{L}^{3}$ mit $\mathrm{PdCl}_{2}$ (COD) konnte der Palladium(II)komplex $\left[\mathbf{L}^{\mathbf{3}} \mathbf{P} \mathbf{d}_{\mathbf{2}} \mathbf{C l}\right]$ gemäß des in Abbildung 89 dargestellten Reaktionsschemas synthetisiert werden. Nach mehrtägiger Reaktion bei $50{ }^{\circ} \mathrm{C}$ wurde das Lösungsmittel entfernt, der Rückstand in Dichlormethan aufgenommen und mit Acetonitril überschichtet. Nach wenigen Tagen konnten dunkelgelb-orange Kristalle isoliert werden mit ausreichender Qualität für die Röntgenstrukturanalyse (Abbildung 90). In Tabelle 18 sind einige ausgewählte Bindungslängen und Winkel von Verbindung $\left[\mathbf{L}^{3} \mathbf{P} \mathbf{d}_{2} \mathbf{C l}\right]$ aufgelistet.
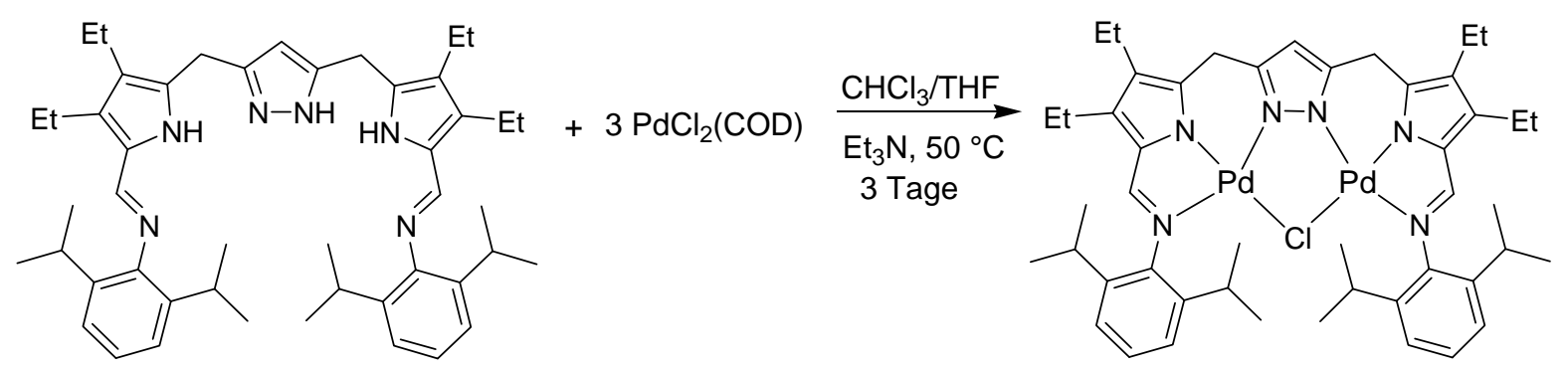

Abbildung 89: Synthese von $\left[\mathbf{L}^{3} \mathbf{P d}_{2} \mathbf{C l}\right]$.

Der Komplex kristallisiert in der Raumgruppe $P^{\overline{1}}$ mit zwei Molekülen in der triklinen Zelle. Beide Palladium(II)ionen nehmen eine quadratisch planare Koordination ein mit einem

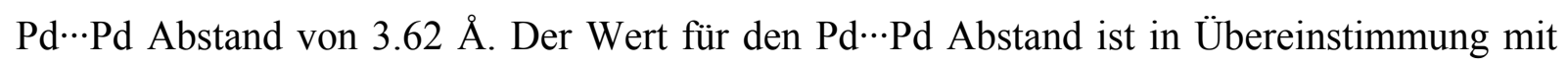
literaturbekannten Werten für Komplexe mit multifunktionellen Pyrazolat-Liganden. ${ }^{84}$ Von den in dieser Arbeit präsentierten Komplexen zeigt der Pd-Komplex den längsten MetallMetall-Abstand. Der Abstand der Metallionen der Kupfer(II)- bzw. Nickel(II)komplexe in $\left[\mathbf{L}^{1} \mathbf{M}_{\mathbf{2}}(\mathbf{O H})\right]$ oder $\left[\mathbf{L}^{3} \mathbf{M}_{\mathbf{2}}(\mathbf{O H})\right]$ beträgt 3.30-3.40 A. Der große Metall-Metall-Abstand kann auf den sperrigeren $\mu$-Cl Brückenliganden und die Flexibilität des Liganden $\mathbf{H}_{3} \mathbf{L}^{3}$ zurückgeführt werden. Der Pd1-Cl1-Pd2 Winkel ist mit 102.3 im Vergleich zum M-O(H)-M Winkel in den jenen Komplexen, die eine $\mu-\mathrm{OH}$ Brücke beinhalten, kleiner. Die Pyrroleinheiten zeigen eine leichte Wölbung und bilden einen Winkel von $7.0^{\circ}$ zueinander. 


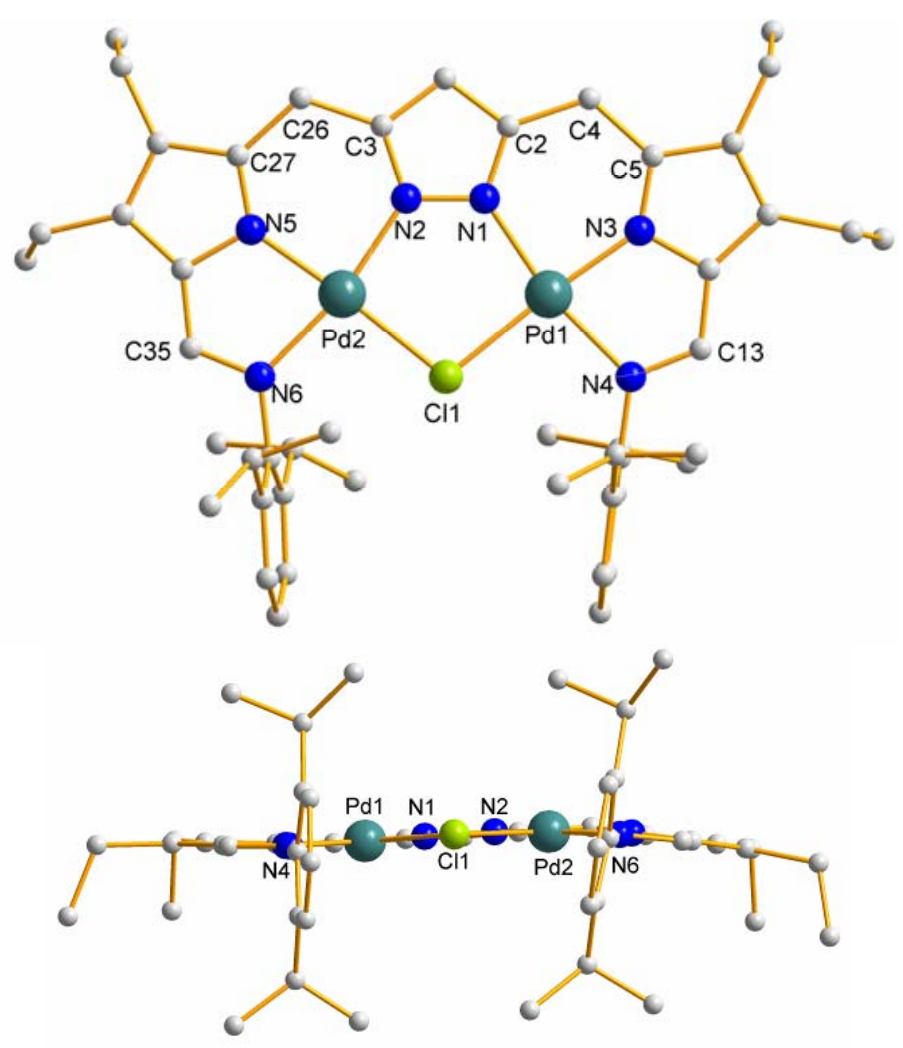

Abbildung 90: Molekülstruktur des bimetallischen Palladium(II)komplexes $\left[\mathbf{L}^{3} \mathbf{P} \mathbf{d}_{2} \mathbf{C l}\right]$ (oben) und Seitenansicht entlang des $\mu$-Cl-Liganden (unten).

Tabelle 18: Ausgewählte Abstände und Winkel von $\left[\mathbf{L}^{3} \mathbf{P d}_{2} \mathbf{C l}\right]$.

\begin{tabular}{ll|ll}
\multicolumn{5}{c}{ Abstände $[\AA]$} \\
\hline $\operatorname{Pd}(1)-\operatorname{Pd}(2)$ & $3.6178(2)$ & $\operatorname{Pd}(2)-\mathrm{N}(6)$ & $2.0449(17)$ \\
$\operatorname{Pd}(1)-\mathrm{N}(1)$ & $2.0077(16)$ & $\operatorname{Pd}(1)-\mathrm{Cl}(1)$ & $2.3194(5)$ \\
$\operatorname{Pd}(1)-\mathrm{N}(3)$ & $1.9444(17)$ & $\mathrm{Pd}(2)-\mathrm{Cl}(1)$ & $2.3261(5)$ \\
$\operatorname{Pd}(1)-\mathrm{N}(4)$ & $2.0423(17)$ & $\mathrm{N}(4)-\mathrm{C}(13)$ & $1.313(3)$ \\
$\operatorname{Pd}(2)-\mathrm{N}(2)$ & $2.0073(16)$ & $\mathrm{C}(2)-\mathrm{C}(4)$ & $1.505(3)$ \\
$\operatorname{Pd}(2)-\mathrm{N}(5)$ & $1.9474(17)$ & $\mathrm{C}(4)-\mathrm{C}(5)$ & $1.492(3)$ \\
\hline
\end{tabular}

Winkel [ $\left.{ }^{\circ}\right]$

\begin{tabular}{lc|cc}
\hline $\mathrm{Pd}(1)-\mathrm{Cl}(1)-\mathrm{Pd}(2)$ & $102.294(18)$ & $\mathrm{N}(3)-\mathrm{Pd}(1)-\mathrm{Cl}(1)$ & $175.89(5)$ \\
$\mathrm{N}(1)-\operatorname{Pd}(1)-\mathrm{N}(4)$ & $169.28(7)$ & $\mathrm{N}(4)-\mathrm{Pd}(1)-\mathrm{Cl}(1)$ & $95.84(5)$ \\
$\mathrm{N}(1)-\operatorname{Pd}(1)-\mathrm{Cl}(1)$ & $94.87(5)$ & $\mathrm{N}(5)-\mathrm{Pd}(2)-\mathrm{N}(2)$ & $88.76(7)$ \\
$\mathrm{N}(2)-\mathrm{Pd}(2)-\mathrm{N}(6)$ & $169.14(7)$ & $\mathrm{N}(5)-\mathrm{Pd}(2)-\mathrm{N}(6)$ & $80.49(7)$ \\
$\mathrm{N}(2)-\operatorname{Pd}(2)-\mathrm{Cl}(1)$ & $94.83(5)$ & $\mathrm{N}(5)-\mathrm{Pd}(2)-\mathrm{Cl}(1)$ & $175.80(5)$ \\
$\mathrm{N}(3)-\operatorname{Pd}(1)-\mathrm{N}(1)$ & $89.18(7)$ & $\mathrm{N}(6)-\mathrm{Pd}(2)-\mathrm{Cl}(1)$ & $95.86(5)$ \\
$\mathrm{N}(3)-\operatorname{Pd}(1)-\mathrm{N}(4)$ & $80.11(7)$ & $\mathrm{C}(5)-\mathrm{C}(4)-\mathrm{C}(2)$ & $118.68(16)$ \\
\hline
\end{tabular}


Abschließend werden die röntgenographisch charakterisierten Pyrrol/Pyrazol-basierten Metallkomplexe in einer Übersichtstabelle (Tabelle 19) miteinander verglichen. Der Tabelle kann entnommen werden, dass der Metall-Metall-Abstand in diesen Pyrazolat-verbrückten Komplexen relativ klein ist.

Tabelle 19: Übersicht wichtiger Winkel und Abstände der röntgenographisch charakterisierten Pyrrol/Pyrazolbasierten Komplexe.

\begin{tabular}{|c|c|c|c|c|c|}
\hline Komplex: & {$\left[\mathrm{L}^{1}{ }_{\mathrm{ox}} \mathrm{Cu}_{2}(\mathrm{OH})\right]$} & {$\left[\mathbf{L}^{3} \mathbf{C u}_{2}(\mathrm{OH})\right]$} & {$\left[\mathrm{L}^{1} \mathrm{Ni}_{2}(\mathrm{OH})\right]$} & {$\left[\mathbf{L}^{3} \mathbf{N i}_{2}(\mathrm{OH})\right]$} & {$\left[\mathbf{L}^{3} \mathbf{P} \mathbf{d}_{2} \mathbf{C l}\right]$} \\
\hline Metall-Metall & $\mathrm{Cu}(1) \cdots \mathrm{Cu}\left(1^{\prime}\right)$ & $\mathrm{Cu}(1) \cdots \mathrm{Cu}(2)$ & $\mathrm{Ni}(1) \cdots \mathrm{Ni}(2)$ & $\mathrm{Ni}(1) \cdots \mathrm{Ni}(2)$ & $\operatorname{Pd}(1) \cdots \operatorname{Pd}(2)$ \\
\hline Abstand $[\AA]$ & $3.3483(7)$ & $3.3108(3)$ & $3.2364(7)$ & $3.2472(3)$ & $3.6178(2)$ \\
\hline $\begin{array}{c}\text { Winkel } \\
\text { M-X-M }\left[^{\circ}\right]\end{array}$ & $122.85(16)$ & $120.38(7)$ & $122.09(13)$ & $121.19(8)$ & $102.294(18)$ \\
\hline $\begin{array}{c}\text { Imin-Bindung } \\
{[\AA]}\end{array}$ & $\begin{array}{c}\mathrm{N}(3)-\mathrm{C}(12) \\
1.306(4)\end{array}$ & $\begin{array}{c}\mathrm{N}(6)-\mathrm{C}(35) \\
1.312(2)\end{array}$ & $\begin{array}{c}\mathrm{N}(4)-\mathrm{C}(13) \\
1.316(5)\end{array}$ & $\begin{array}{c}\mathrm{N}(6)-\mathrm{C}(35) \\
1.313(2)\end{array}$ & $\begin{array}{c}\mathrm{N}(4)-\mathrm{C}(13) \\
1.313(3)\end{array}$ \\
\hline $\begin{array}{c}\text { C-C-Abstand } \\
\text { an der 3,5- } \\
\text { Position } \\
{[\AA]}\end{array}$ & $\begin{array}{c}\mathrm{C}(2)-\mathrm{C}(3) \\
1.486(4) \\
\mathrm{C}(3)-\mathrm{C}(4) \\
1.437(5)\end{array}$ & $\begin{array}{c}\mathrm{C}(3)-\mathrm{C}(26) \\
1.510(2) \\
\mathrm{C}(26)-\mathrm{C}(27) \\
1.496(3)\end{array}$ & $\begin{array}{c}\mathrm{C}(2)-\mathrm{C}(4) \\
1.509(6) \\
\mathrm{C}(4)-\mathrm{C}(5) \\
1.491(6)\end{array}$ & $\begin{array}{c}\mathrm{C}(3)-\mathrm{C}(26) \\
1.508(3) \\
\mathrm{C}(26)-\mathrm{C}(27) \\
1.489(3)\end{array}$ & $\begin{array}{l}\mathrm{C}(2)-\mathrm{C}(4) \\
1.505(3) \\
\mathrm{C}(4)-\mathrm{C}(5) \\
1.492(3)\end{array}$ \\
\hline $\begin{array}{c}\text { Winkel an der } \\
\text { 3,5-Position } \\
\left.{ }^{\circ}\right]\end{array}$ & $\begin{array}{c}C(4)-C(3)-C(2) \\
120.2(3)\end{array}$ & $\begin{array}{c}C(3)-C(26)-C(27) \\
117.16(15)\end{array}$ & $\begin{array}{c}\mathrm{C}(2)-\mathrm{C}(4)-\mathrm{C}(5) \\
116.5(3)\end{array}$ & $\begin{array}{c}\mathrm{C}(27)-\mathrm{C}(26)-\mathrm{C}(3) \\
115.42(16)\end{array}$ & $\begin{array}{c}C(5)-C(4)-C(5) \\
118.68(16)\end{array}$ \\
\hline
\end{tabular}

Die Imin-Bindung ist bei allen Komplexen gleich groß und es gibt keinen Hinweis auf eine mögliche Protonierung dieser Stellen. Entsprechende Protonierungsreaktionen wurden bereits bei den Schiff-Base Makrozyklen beobachtet (Kapitel 5.2.2.3). Die C-C-Abstände der 3,5Position in $\left[\mathbf{L}^{\mathbf{1}}{ }_{\text {ox }} \mathbf{C u}_{\mathbf{2}}(\mathbf{O H})\right]$ sind mit 1.486(4) bzw. 1.437(5) Å die kürzesten Abstände von allen fünf Komplexen. Dies liegt an den Carbonylgruppen und folglich an der $\mathrm{sp}^{2}$ Hybridisierung des C3-Atoms. Entsprechend ist der C4-C3-C2 Winkel an dieser Position geringfügig größer als bei den restlichen bimetallischen Komplexen.

Die Bildung des Komplexes $\left[\mathbf{L}^{\mathbf{3}} \mathbf{P d}_{\mathbf{2}} \mathbf{C l}\right]$ konnte mit Hilfe der ${ }^{1} \mathrm{H}-\mathrm{NMR}$-Spektroskopie verfolgt werden. In Abbildung 91 ist das ${ }^{1} \mathrm{H}-\mathrm{NMR}-$ Spektrum von $\mathbf{H}_{\mathbf{3}} \mathbf{L}^{\mathbf{3}}$ (A) und des 
Palladium(II)komplexes $\left[\mathbf{L}^{3} \mathbf{P d}_{2} \mathbf{C l}\right]$ (B) dargestellt. Es zeigt sich, dass sich durch die Komplexierung die meisten Signale im Spektrum verschieben oder an Schärfe gewinnen.
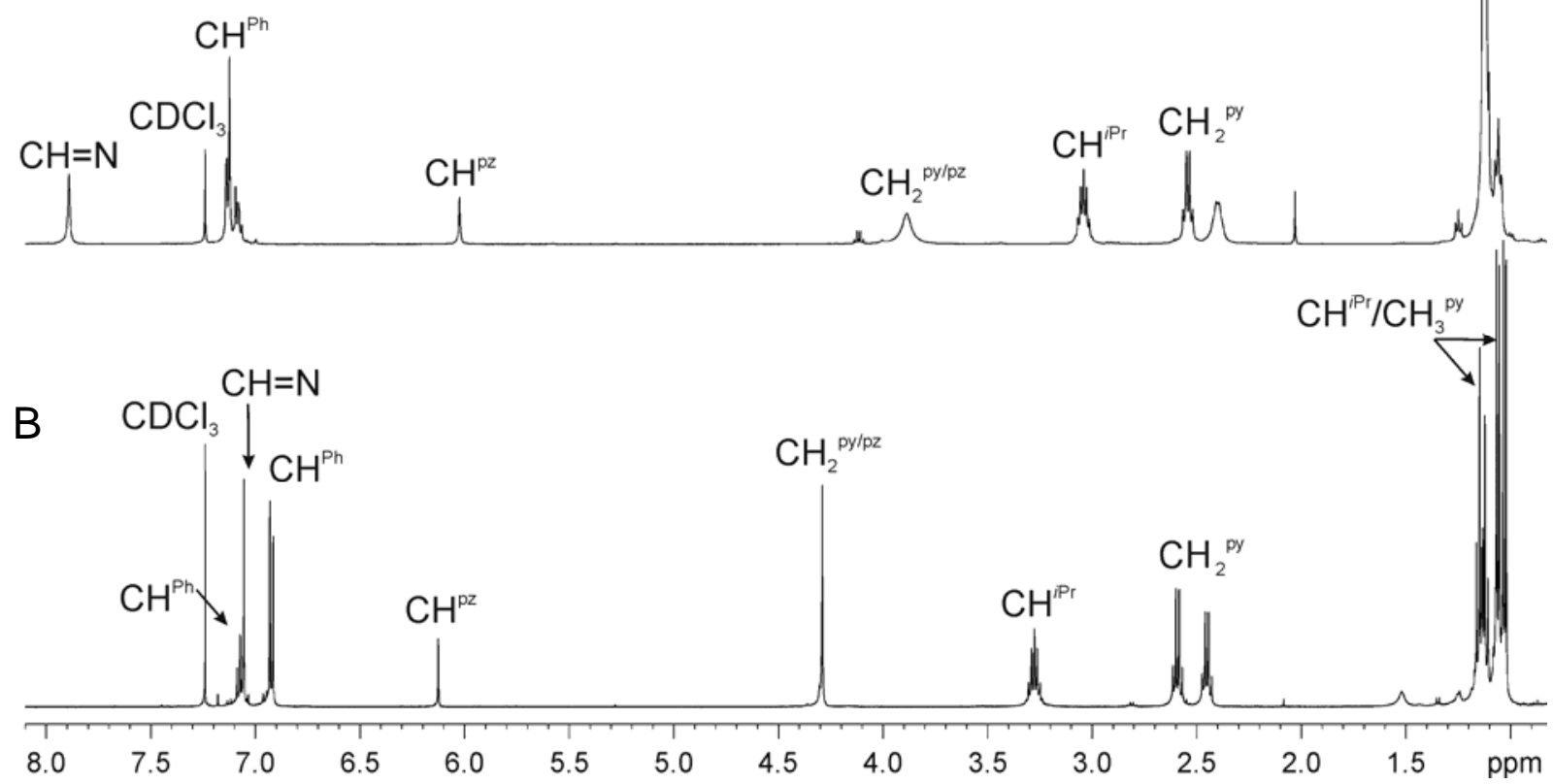

Abbildung 91: ${ }^{1} \mathrm{H}-\mathrm{NMR}-$ Spektrum von $\mathbf{H}_{3} \mathbf{L}^{3}$ (A) und dem bimetallischen Palladium(II)komplexe [ $\left.\mathbf{L}^{3} \mathbf{P d}_{2} \mathbf{C l}\right]$ (B) gemessen in $\mathrm{CDCl}_{3}$.

Das Methinproton der Isopropylreste $\left(\mathrm{CH}^{i \mathrm{Pr}}\right)$ wird durch die Nähe zum Metall von 3.03 ppm auf 3.27 ppm zum Tieffeld verschoben. Das $\mathrm{HC}=\mathrm{N}$ Proton der Imingruppen verschiebt sich nach der Komplexierung mit einer Differenz von $\Delta \delta=0.84$ ppm ins Hochfeld. Diese Änderungen des ${ }^{1} \mathrm{H}-\mathrm{NMR}-$ Spektrums können auf Konformationsänderungen, durch die mäßige Flexibilität und weitere Effekte, die bei der Komplexbildung eintreten, zurückgeführt werden.

Das $\mathrm{CH}^{\mathrm{pz}}$-Signal des Pyrazols an der 4-Position verschiebt sich von $6.02 \mathrm{ppm}$ ins Tieffeld auf $6.13 \mathrm{ppm}$. Vermutlich durch die Komplexreaktion werden die $\mathrm{CH}^{\mathrm{Ph}}$-Protonen an der metaPosition elektronenreicher und so wird eine Hochfeld-Verschiebung des Multipletts von 7.12- 
7.14 ppm auf 6.92-6.93 ppm beobachtet. Das $\mathrm{CH}^{\mathrm{Ph}}$-Proton an der para-Position bleibt dabei unverändert.

Analog zu den ${ }^{1}$ H-NMR-Spektren des Liganden und des Palladiumkomplexes $\left[\mathbf{L}^{3} \mathbf{P d} \mathbf{2} \mathbf{C l}\right.$ werden auch in den ${ }^{13} \mathrm{C}$-NMR-Spektren signifikante Verschiebungen der Imin- und $\mathrm{CH}^{\mathrm{Ph}}$ Kohlenstoffatome beobachtet (Abbildung 92).

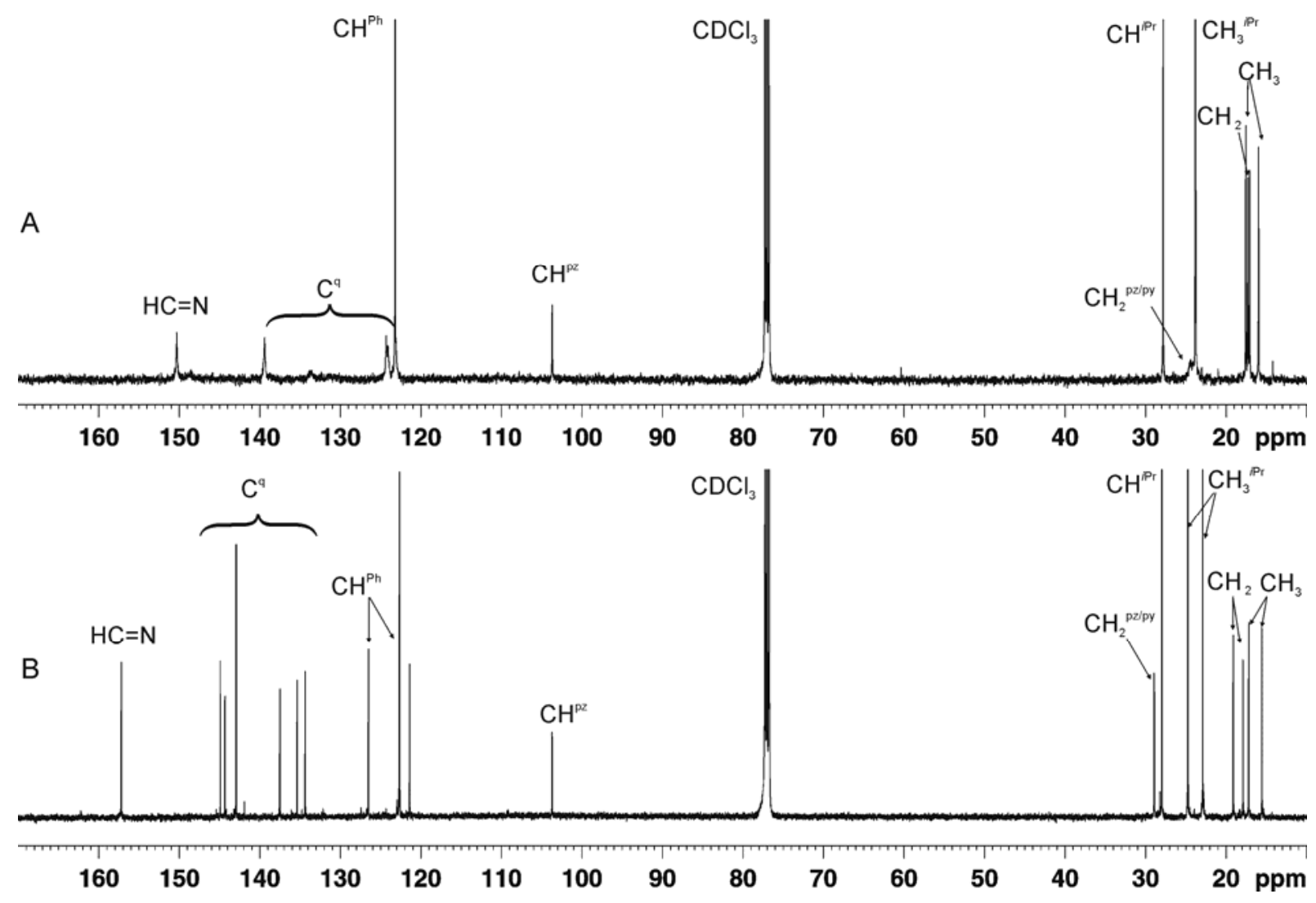

Abbildung 92: ${ }^{13} \mathrm{C}-\mathrm{NMR}$-Spektrum des Liganden $\mathbf{H}_{3} \mathbf{L}^{3}$ (A) und des bimetallischen Palladium(II)komplexes $\left[\mathbf{L}^{3} \mathbf{P d}_{2} \mathbf{C l}\right]$ (B) gemessen in $\mathrm{CDCl}_{3}$.

Die Kohlenstoffatome der $\mathrm{C}=\mathrm{N}$ - und der meta $-\mathrm{CH}^{\mathrm{Ph}}$-Gruppe werden ins Tieffeld verschoben. Im Spektrum des Komplexes wird das Imin-C-Atom bei $157.2 \mathrm{ppm}$ und das der meta- $\mathrm{CH}^{\mathrm{Ph}}$ Gruppe bei 126.5 ppm detektiert. Das ursprüngliche Signal der $\mathrm{CH}^{\mathrm{Ph}}$-Gruppen wird in zwei separate Signale aufgespalten, so dass die Kohlenstoffatome an der meta- bzw. para-Position der $\mathrm{CH}^{\mathrm{Ph}}$-Gruppe unterschieden werden können. Durch die starre Konformation des Liganden im bimetallischen Palladiumkomplex wird das Signal der $\mathrm{CH}_{2}{ }^{\mathrm{py} / \mathrm{pz}}$-Gruppe, das im ${ }^{13} \mathrm{C}-\mathrm{NMR}$ des freien Liganden breit ist und bei 24.2 ppm liegt, in ein scharfes Signal (28.9 ppm) umgewandelt. Besonders deutlich werden ebenfalls die $\mathrm{C}^{\mathrm{q}}$-Kohlenstoffatome (bei 121.4 und zwischen 134.4 bis 144.9 ppm) des Komplexes detektiert. 


\subsection{Spektroskopische Untersuchungen der Metallkomplexe}

\subsubsection{IR-Spektren ausgewählter Metallkomplexe}

Die Liganden bzw. bimetallischen Komplexe wurden mittels der IR-Spektroskopie untersucht, um weitere Informationen über strukturelle Unterschiede zu gewinnen. In diesem Kapitel werden ausgewählte Liganden und ihre entsprechenden Metallkomplexe gezeigt und miteinander verglichen. In Abbildung 93 sind die charakteristischen Imin- (links) und $\mathrm{OH}-$ Valenzschwingungen (rechts) des Liganden $\mathbf{H}_{\mathbf{3}} \mathbf{L}^{1}$ und ausgewählter Metallkomplexe dargestellt.

Die Imin-Valenzschwingung des Liganden wird bei $1612 \mathrm{~cm}^{-1}$ beobachtet. Je größer die Bindungsstärke zwischen zwei Atomen ist, desto höher liegt die Schwingungsfrequenz. Entsprechend wird durch die Bildung eines Metallkomplexes die Imin-Bindung leicht geschwächt und die Bande bei niedrigeren Wellenzahlen beobachtet.
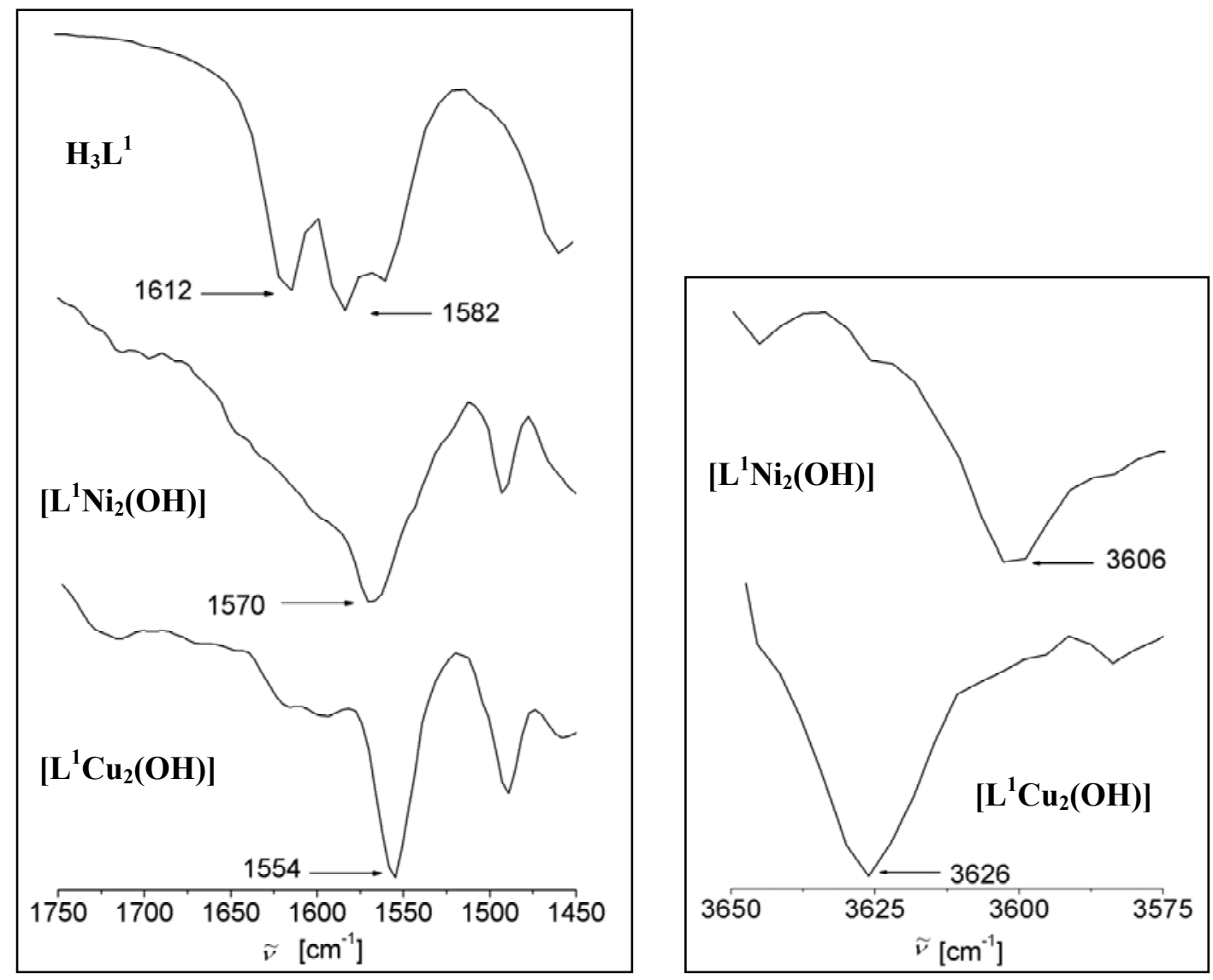

Abbildung 93: IR-Spektrum des Liganden $\mathbf{H}_{3} \mathbf{L}^{1}$ und ausgewählter Komplexe. In der linken Abbildung sind die charakteristischen $\mathrm{C}=\mathrm{N}-\mathrm{Imin}$ Banden und rechts die charakteristische Valenzschwingungen des $\mathrm{OH}$ Brückenliganden zu sehen. 
Die Streckschwingung des OH-Brückenliganden in $\left[\mathbf{L}^{\mathbf{1}} \mathbf{C u}_{\mathbf{2}}(\mathbf{O H})\right]$ bzw. $\left[\mathbf{L}^{\mathbf{1}} \mathbf{N i}_{\mathbf{2}}(\mathbf{O H})\right]$ ist, wie zu erwarten, bei 3626 bzw. $3603 \mathrm{~cm}^{-1}$ zu finden.

Bei dem Ligand $\mathbf{H}_{3} \mathbf{L}^{3}$ und den zugehörigen Metallkomplexen $\left[\mathbf{L}^{3} \mathbf{P d}_{2} \mathbf{C l}\right],\left[\mathbf{L}^{3} \mathbf{N i}_{2}(\mathbf{O H})\right]$, und $\left[\mathbf{L}^{3} \mathbf{C u}_{\mathbf{2}}(\mathbf{O H})\right]$ werden analog Verschiebungen der charakteristischen Banden beobachtet (Abbildung 94). Die Imin-Bande bei $1620 \mathrm{~cm}^{-1}$ verschiebt sich nach Koordination des Metalls zu kleineren Wellenzahlen. Die scharfe Bande bei 3607 bzw. $3615 \mathrm{~cm}^{-1}$ demonstriert wiederholt die Existenz der $\mu$-OH-Gruppe zwischen den Metallionen in $\left[\mathbf{L}^{3} \mathbf{N i}_{2}(\mathbf{O H})\right]$ und $\left[\mathbf{L}^{3} \mathrm{Cu}_{2}(\mathbf{O H})\right]$.
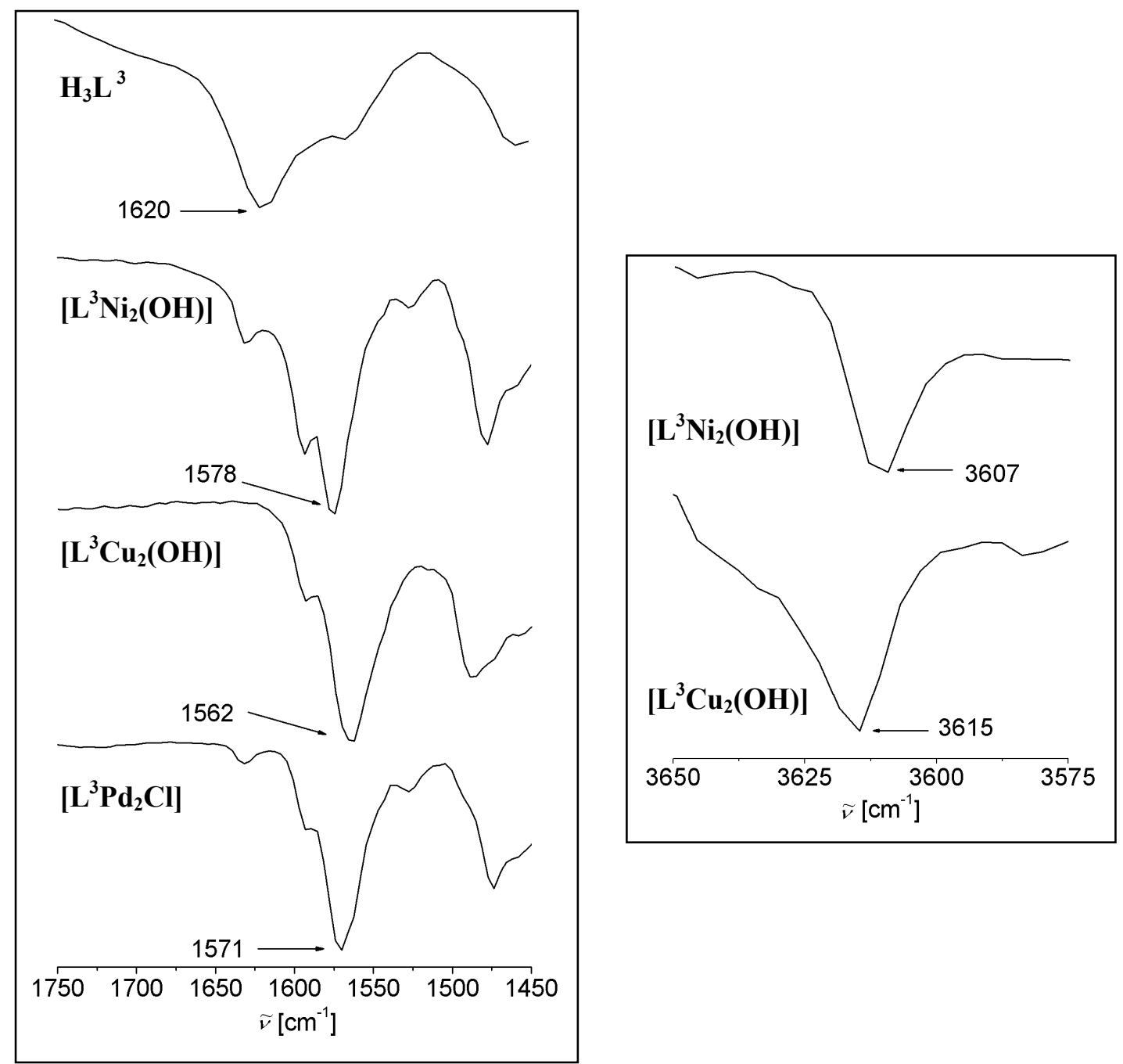

Abbildung 94: Imin-Bande (links) und $\mathrm{OH}-$ Valenzschwingung (rechts) des Liganden $\mathbf{H}_{3} \mathbf{L}^{3}$ und ausgewählter Metallkomplexe im IR-Spektrum.

In Abbildung 95 sind die Imin-Banden von $\mathbf{H}_{\mathbf{3}} \mathbf{L}^{\mathbf{4}}$, des Nickelkomplexes $\left[\mathbf{L}^{\mathbf{4}} \mathbf{N i}_{\mathbf{2}}(\mathbf{O H})\right]$ und des Kupferkomplexes $\left[\mathbf{L}^{4} \mathbf{C u}_{\mathbf{2}}(\mathbf{O H})\right]$ gezeigt. Die Verschiebung der Banden aufgrund der Komplexierungsreaktion ist nahezu identisch $\mathrm{zu}$ den bereits diskutierten Liganden bzw. 
Metallkomplexen. Die OH-Bande von $\left[\mathbf{L}^{4} \mathbf{N i}_{2}(\mathbf{O H})\right]$ wird bei $3601 \mathrm{~cm}^{-1}$ beobachtet. Das Signal für den $\mathrm{OH}-\mathrm{Brückenliganden} \mathrm{in}\left[\mathbf{L}^{4} \mathbf{C} \mathbf{u}_{2}(\mathbf{O H})\right]$ ist nur sehr schwach bei ca. $3600 \mathrm{~cm}^{-1}$ zu beobachten. Der Grund für die schwache $\mathrm{OH}-$ Bande liegt in Verunreinigungen bzw. Wasserspuren in der Probe.

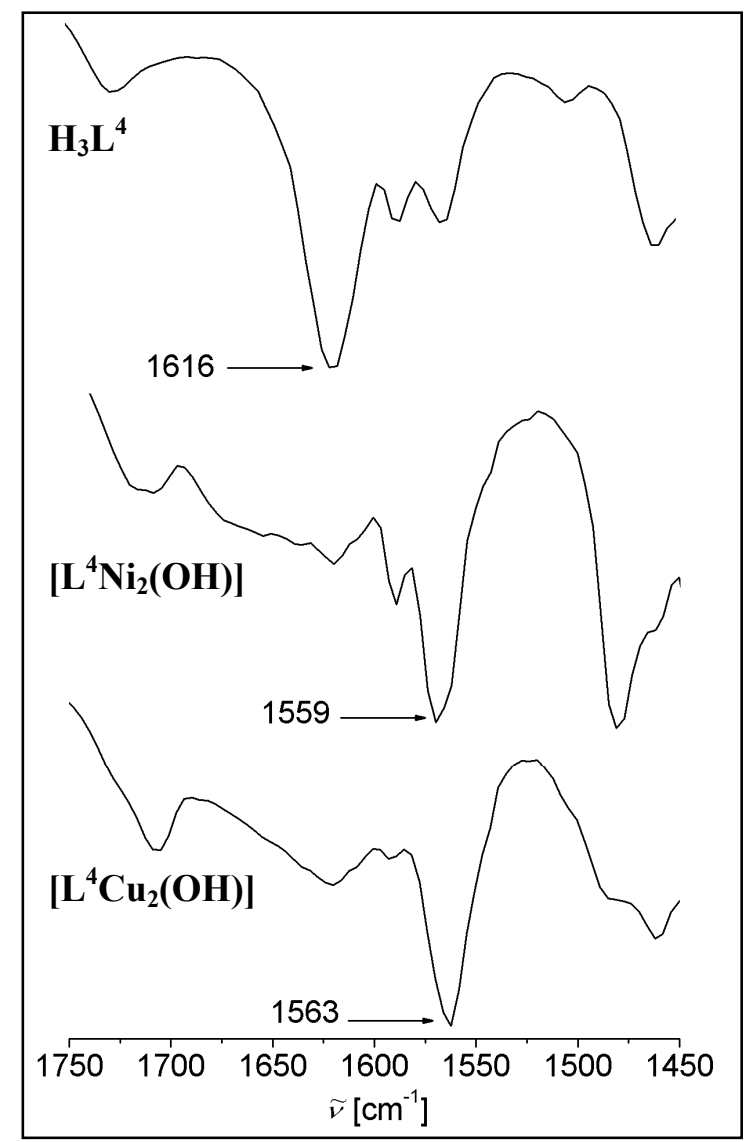

Abbildung 95: Imin-Bande des Liganden $\mathbf{H}_{3} \mathbf{L}^{4}$ und ausgewählter Metallkomplexe im IR-Spektrum. 


\subsubsection{Elektrochemische Untersuchungen ausgewählter bimetallischer Komplexe}

Der Kupfer(II)komplex $\left[\mathbf{L}^{3} \mathbf{C u}_{\mathbf{2}}(\mathbf{O H})\right]$ und der Nickel(II)komplex $\left[\mathbf{L}^{3} \mathbf{N i}_{\mathbf{2}}(\mathbf{O H})\right]$ wurden mittels cyclovoltammetrischer Messungen untersucht. Hierbei wurde das Oxidations- bzw. Reduktionsverhalten der neuartigen Metallkomplexe näher definiert.

In Abbildung 96 ist das Cyclovoltammogramm von $\left[\mathbf{L}^{3} \mathbf{C u}_{\mathbf{2}}(\mathbf{O H})\right]$ in DMF dargestellt. Das Spektrum wurde gegen einen internen Ferrocen-Standard bei Raumtemperatur gemessen und die Potentiale realativ zu SCE (Satured Calomel Electrode) umgerechnet. $^{98}$ Die cyclovoltammetrische Untersuchung des Kupfer(II)komplexes zeigt einen irreversiblen Reduktionsprozeß. Es werden irreversible Reduktionen im Bereich von $-1.91--1.50 \mathrm{~V}$ beobachtet. Dies ist wahrscheinlich auf labile $\mathrm{Cu}(\mathrm{I}) \mathrm{Cu}(\mathrm{II})-$ bzw. $\mathrm{Cu}(\mathrm{I}) \mathrm{Cu}(\mathrm{I})-\mathrm{Spezies}$ zurückzuführen, die in Lösung nach der Reduktion zerfallen. Des Weiteren ist eine irreversible Oxidation bei $E_{p}^{o x d}=0.93 \mathrm{~V}$ zu erkennen.

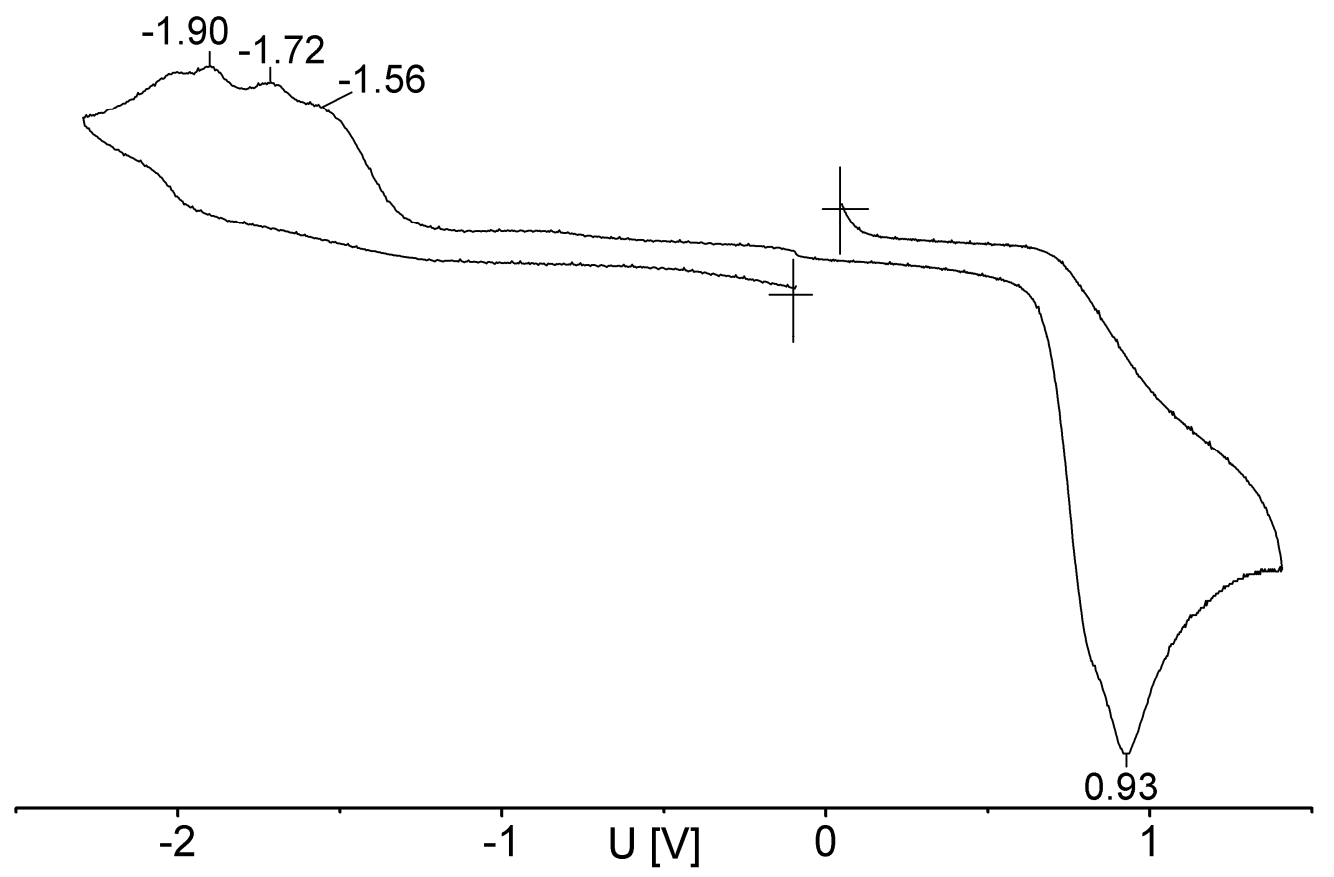

Abbildung 96: Cyclovoltammogramm von $\left[\mathbf{L}^{3} \mathrm{Cu}_{\mathbf{2}}(\mathbf{O H})\right]$ in DMF. Die Probe wurde gegen Ferrocen gemessen und vs. SCE (Satured Calomel Electrode) umgerechnet aufgetragen.

In früheren Arbeiten über Pyrazolat-verbrückte bimetallische Kupfer(II)komplexe mit einer $\mu$ OH-Gruppe zwischen den Kupferionen konnten, analog zu den hier erhaltenen Eigenbissen, zwei irreversible Reduktions- und ein irreversibler Oxidationsschritt beobachtet werden. ${ }^{87}$ 
Der bimetallische Nickel(II)komplex wurde in DMF gegen Decamethylferrocen gemessen. In Abbildung 97 sind die Cyclovoltammogramme des Nickelkomplexes relativ zu SCE ${ }^{98}$ dargestellt. Der Nickel(II)komplex $\left[\mathbf{L}^{3} \mathbf{N i}_{\mathbf{2}}(\mathbf{O H})\right]$ zeigt, ähnlich dem Kupfer(II)komplex $\left[\mathbf{L}^{3} \mathbf{C u}_{2}(\mathbf{O H})\right]$, bei gleicher Vorschubgeschwindigkeit eine irreversible Oxidation bei $E_{p}^{o x d}=$ 0.81 V. Diese irreversible Oxidation wurde bei beiden gemessenen Komplexen beobachtet und findet höchstwahrscheinlich am Liganden statt. Der Komplex $\left[\mathbf{L}^{3} \mathbf{N i}_{\mathbf{2}}(\mathbf{O H})\right]$ zeigt eine quasireversible Reduktion bei $E_{p}^{\text {red }}=-1.97 \mathrm{~V}$ mit der entsprechenden Reoxidation bei $E_{p}^{o x d}=-1.86 \mathrm{~V}$. Die Reduktion führt vermutlich $\mathrm{zu}$ dem gemischtvalenten Komplex $\left[\mathbf{L}^{3} \mathbf{N i}^{\mathbf{I}} \mathbf{N i}^{\mathbf{I I}}(\mathbf{O H})\right]^{-}$. Wird der kathodische Messbereich ausgeweitet, so ist ein zweiter irreversibler Reduktionsschritt bei einem Potential von $E_{p}^{r e d}=-2.65 \mathrm{~V}$ zu beobachten. Dies führt zu einer weiteren Reduktion des Metallkomplexes, entsprechend der möglichen Bildung von $\left[\mathbf{L}^{3} \mathbf{N i}^{\mathbf{I}} \mathbf{N i}^{\mathbf{I}}(\mathbf{O H})\right]^{2-}$. Eine Änderung der Vorschubgeschwindigkeit $\left(>100 \mathrm{mVs}^{-1}\right)$ resultiert auch hier nicht in der Reversibilität der Reduktionsschritte (Abbildung 97 unten).

Es ist bekannt, dass Pyrazolat-basierte bimetallische Nickel(II)- bzw. Palladium(II)komplexe bei einer Reduktion zwei Redoxstufen aufweisen. Von Meyer et al. wurde der reversible Reduktionsschritt jedoch bei niedrigeren Potentialen (zwischen $E_{p}^{\text {red }}=-0.81$ bzw. $-0.98 \mathrm{~V}$ ) beschrieben, als im Rahmen dieser Arbeit gefunden. ${ }^{99}$ Dennoch ist ein direkter Vergleich aufgrund des unterschiedlichen Aufbaus und Beschaffenheit der Metallkomplexe nicht möglich.

Wie in Kapitel (Kapitel 7.1-7.2) bereits diskutiert, konnte in den Massenspektren der bimetallischen Komplexe die Oxidation des Liganden $\mathbf{H}_{\mathbf{3}} \mathbf{L}^{\mathbf{3}}$ mit einem anschließenden „noninnocent" Effekt korreliert werden. Die Ergebnisse der Cyclovoltametrie von $\left[\mathbf{L}^{3} \mathbf{C} \mathbf{u}_{2}(\mathbf{O H})\right]$ und $\left[\mathbf{L}^{3} \mathbf{N i}_{2}(\mathbf{O H})\right]$ stehen somit in Bezug zu der in der Massenspektrometrie beobachtete Oxidation des Liganden. Die irreversible Oxidation der zwei gemessenen Komplexes im Bereich 0.80-0.93 V könnte daher aus der Oxidation des Liganden resultieren. 


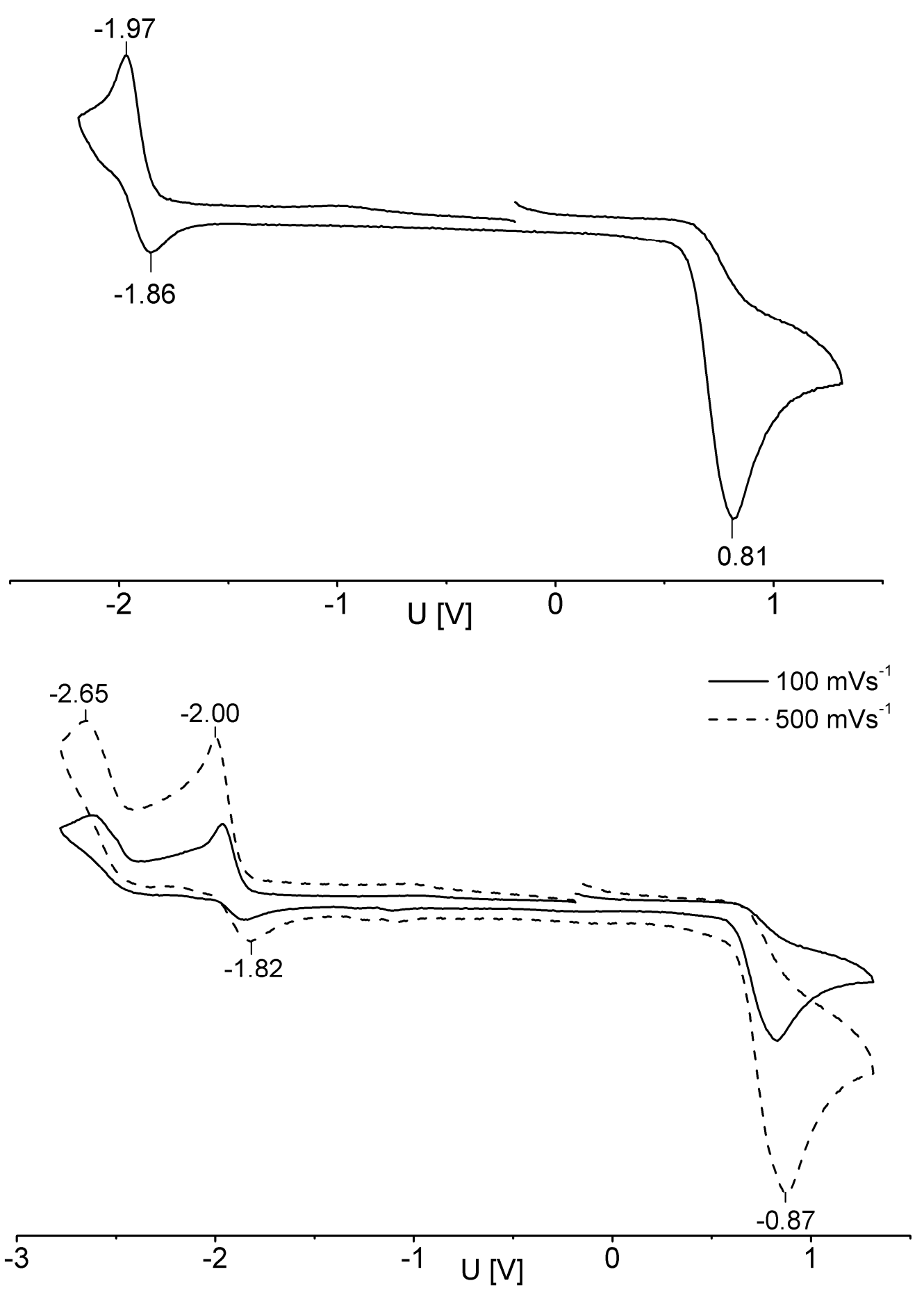

Abbildung 97: Cyclovoltammogramm von $\left.\left[\mathbf{L}^{3} \mathbf{N i}_{\mathbf{2}} \mathbf{( O H}\right)\right]$ in DMF gemessen von 1.4 bis $-2.3 \mathrm{~V}$ bei $100 \mathrm{mVs}^{-1}$ (oben). Messung bei zwei Vorschubgeschwindigkeiten (500 bzw. $100 \mathrm{mVs}^{-1}$ ) und einem Bereich von 2.0 bis $3.0 \mathrm{~V}$ (unten). Die Potentiale sind vs. SCE aufgetragen. 


\subsubsection{UV/Vis-Spektren ausgewählter Metallkomplexe mit azyklischen Pyrrol/Pyrazol-basierten Diimin-Liganden}

In diesem Kapitel werden die Ergebnisse UV/Vis-spektroskopischer Untersuchungen ausgewählter Metallkomplexe des Liganden $\mathbf{H}_{3} \mathbf{L}^{3}$ präsentiert. Untersucht wurden der bimetallische Nickel(II)komplex $\left[\mathbf{L}^{3} \mathbf{N i}_{2}(\mathbf{O H})\right]$, der Kupfer(II)komplex $\left[\mathbf{L}^{3} \mathbf{C} \mathbf{u}_{2}(\mathbf{O H})\right]$ und der Palladium(II)komplex $\left[\mathbf{L}^{3} \mathbf{P d}_{\mathbf{2}} \mathbf{C l}\right]$ und die resultierenden Spektren miteinander verglichen. In Abbildung 98 sind die in Chloroform gemessenen UV/Vis-Spektren des Liganden $\mathbf{H}_{\mathbf{3}} \mathbf{L}^{\mathbf{3}}$ und der jeweiligen bimetallischen Komplexe aufgeführt. Alle drei Komplexe haben quadratischeplanar koordinierte Metallzentren und unterscheiden sich lediglich in ihrem Coliganden $\left(\mathrm{OH}^{-}\right.$ bzw. $\left.\mathrm{Cl}^{-}\right)$.

Die Lösung des freien Liganden weist in Chloroform eine intensive Bande bei $317 \mathrm{~nm}$ $\left[\varepsilon=4.25 \times 10^{4} \mathrm{Lmol}^{-1} \mathrm{~cm}^{-1}\right]$ auf, welche $\pi \rightarrow \pi^{*}$-Übergängen des konjugierten Systems im Ligand zugeordnet werden kann.

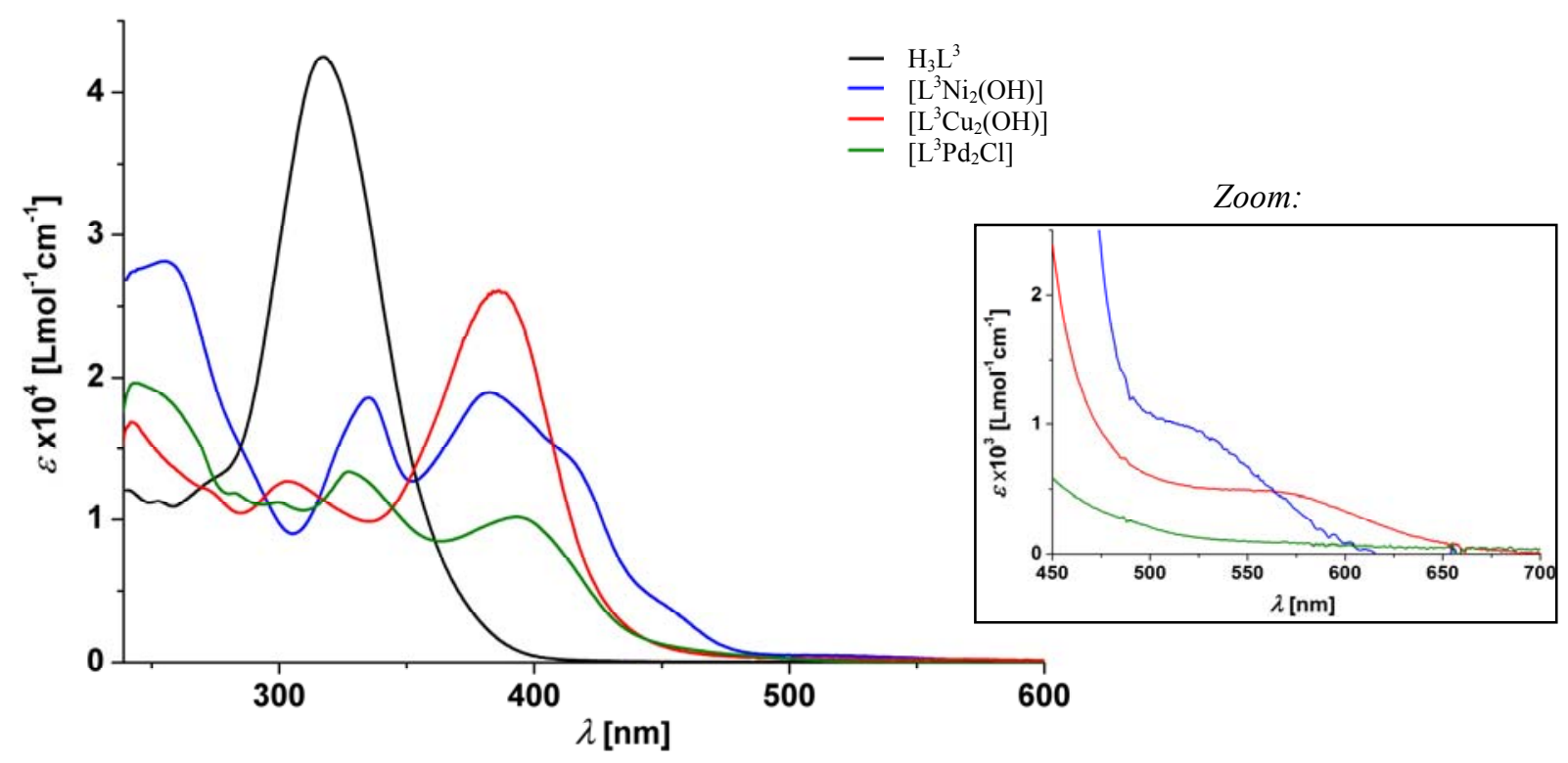

Abbildung 98: UV/Vis-Spektrum des Liganden $\left(\mathbf{H}_{3} \mathbf{L}^{3}[-]\right)$, des Nickel(II)komplexes ( $\left.\left[\mathbf{L}^{3} \mathbf{N i}_{2}(\mathbf{O H})\right][-]\right)$, des Kupfer(II)komplexes ( $\left[\mathbf{L}^{3} \mathbf{C u}_{2}(\mathbf{O H})\right]$ [-]) und des Palladium(II)komplexes ([ $\left.\mathbf{L}^{3} \mathbf{P d}_{2} \mathbf{C l}\right]$ [-]) in Chloroform (links). Vergrößerung der UV/Vis-Spektren der Komplexe von 450-700 nm (rechts).

Das UV/Vis-Spektrum des Kupferkomplexes $\left[\mathbf{L}^{3} \mathbf{C u}_{\mathbf{2}}(\mathbf{O H})\right]$ (Abbildung 98, rote Linie) zeigt mehrere Banden. Die erste Bande wird bei $304 \mathrm{~nm}\left[\varepsilon=1.32 \times 10^{4} \mathrm{Lmol}^{-1} \mathrm{~cm}^{-1}\right]$ beobachtet und resultiert vermutlich ebenfalls aus $\pi \rightarrow \pi^{*}$-Übergängen. Bei $386 \mathrm{~nm}\left[\varepsilon=2.67 \times 10^{4} \mathrm{Lmol}^{-1} \mathrm{~cm}^{-1}\right]$ wird eine intensivere Charge-Transferbande des Kupferkomplexes detektiert. Bei genauerer 
Betrachtung des Spektrums zeigen sich ebenfalls schwache d-d-Übergänge bei $560 \mathrm{~nm}$ $\left[\varepsilon=0.48 \times 10^{3} \mathrm{Lmol}^{-1} \mathrm{~cm}^{-1}\right.$ ] (Abbildung 98, Zoom rechts).

Bei dem Nickel(II)komplex [ $\left.\mathbf{L}^{\mathbf{3}} \mathbf{N i}_{\mathbf{2}}(\mathbf{O H})\right]$ (Abbildung 98, blaue Linie) können analog mehrere Übergänge im UV/Vis-Spektrum beobachtet werden. Die $\pi \rightarrow \pi^{*}$-Übergänge des Liganden werden bei $335 \mathrm{~nm}$ detektiert, weisen jedoch mit $\varepsilon=1.86 \times 10^{4} \mathrm{Lmol}^{-1} \mathrm{~cm}^{-1}$ eine viel schwächere Intensität auf als die $\pi \rightarrow \pi^{*}$-Übergänge innerhalb des Kupferkomplexes. Die Charge-Transfer-Banden erscheinen beim Nickel(II)komplex bei $382 \mathrm{~nm}$ $\left[\varepsilon=1.90 \times 10^{4} \mathrm{Lmol}^{-1} \mathrm{~cm}^{-1}\right]$. Weitere Schultern werden bei 414 und $450 \mathrm{~nm}$ erfasst. Die beobachtete Bande bei $520 \mathrm{~nm}\left[\varepsilon=0.98 \times 10^{3} \mathrm{Lmol}^{-1} \mathrm{~cm}^{-1}\right]$ resultiert aufgrund der d-dÜbergänge der quadratisch planaren Nickel(II)ionen.

Der bimetallische Palladium(II)komplex $\left[\mathbf{L}^{3} \mathbf{P} \mathbf{d}_{2} \mathbf{C l}\right]$ (Abbildung 98, grüne Linie) weist mehrere $\mathrm{n} \rightarrow \pi^{*}$ bzw. $\pi \rightarrow \pi^{*}$ Banden bei $283 \mathrm{~nm}\left[\varepsilon=1.18 \times 10^{4} \mathrm{Lmol}^{-1} \mathrm{~cm}^{-1}\right], 300 \mathrm{~nm}$ $\left[\varepsilon=1.13 \times 10^{4} \mathrm{Lmol}^{-1} \mathrm{~cm}^{-1}\right]$ und $327 \mathrm{~nm}\left[\varepsilon=1.34 \times 10^{4} \mathrm{Lmol}^{-1} \mathrm{~cm}^{-1}\right]$ auf. Die Bande bei $393 \mathrm{~nm}$ $\left[\varepsilon=1.02 \times 10^{4} \mathrm{~mol}^{-1} \mathrm{~cm}^{-1}\right]$ resultiert vermutlich aus einem Charge-Transfer-Übergang. 


\subsection{Experimente zum Austausch des OH-Brückenliganden der Nickel(II)komplexe}

Wie bereits in den Kapiteln 7.1 - 7.2 diskutiert, beinhalten die bimetallischen Kupfer(II)- bzw. Nickel(II)komplexe eine OH-Gruppe als Brückenligand. Die Existenz der OH-Gruppe konnte mit Hilfe der ${ }^{1} \mathrm{H}-\mathrm{NMR}-$ Spektroskopie bewiesen werden. Das ${ }^{1} \mathrm{H}-\mathrm{NMR}-$ Spektrum des Nickel(II)komplexes $\left[\mathbf{L}^{3} \mathbf{N i}_{\mathbf{2}}(\mathbf{O H})\right]$ ist in Abbildung 99 gezeigt. Interessanterweise konnte ein Signal bei -6.70 ppm detektiert werden, welches der $\mu$-OH-Gruppe zugeordnet wird. Analog dazu ist die $\mu$-OH-Gruppe des Nickel(II)komplexes $\left[\mathbf{L}^{\mathbf{1}} \mathbf{N i}_{\mathbf{2}}(\mathbf{O H})\right]$ ebenfalls stark ins Hochfeld verschoben und bei $-5.65 \mathrm{ppm}$ zu finden.

Die starke chemische Hochfeld-Verschiebung der OH-Gruppe lässt sich durch die chemische Umgebung des Protons erklären. Ein zweidimensionales ${ }^{1} \mathrm{H}-\mathrm{NMR}-\mathrm{Spektrum}$ (2D-NOESY) veranschaulicht die unmittelbare Umgebung des OH-Brückenliganden im Komplexmolekül (Abbildung 100).

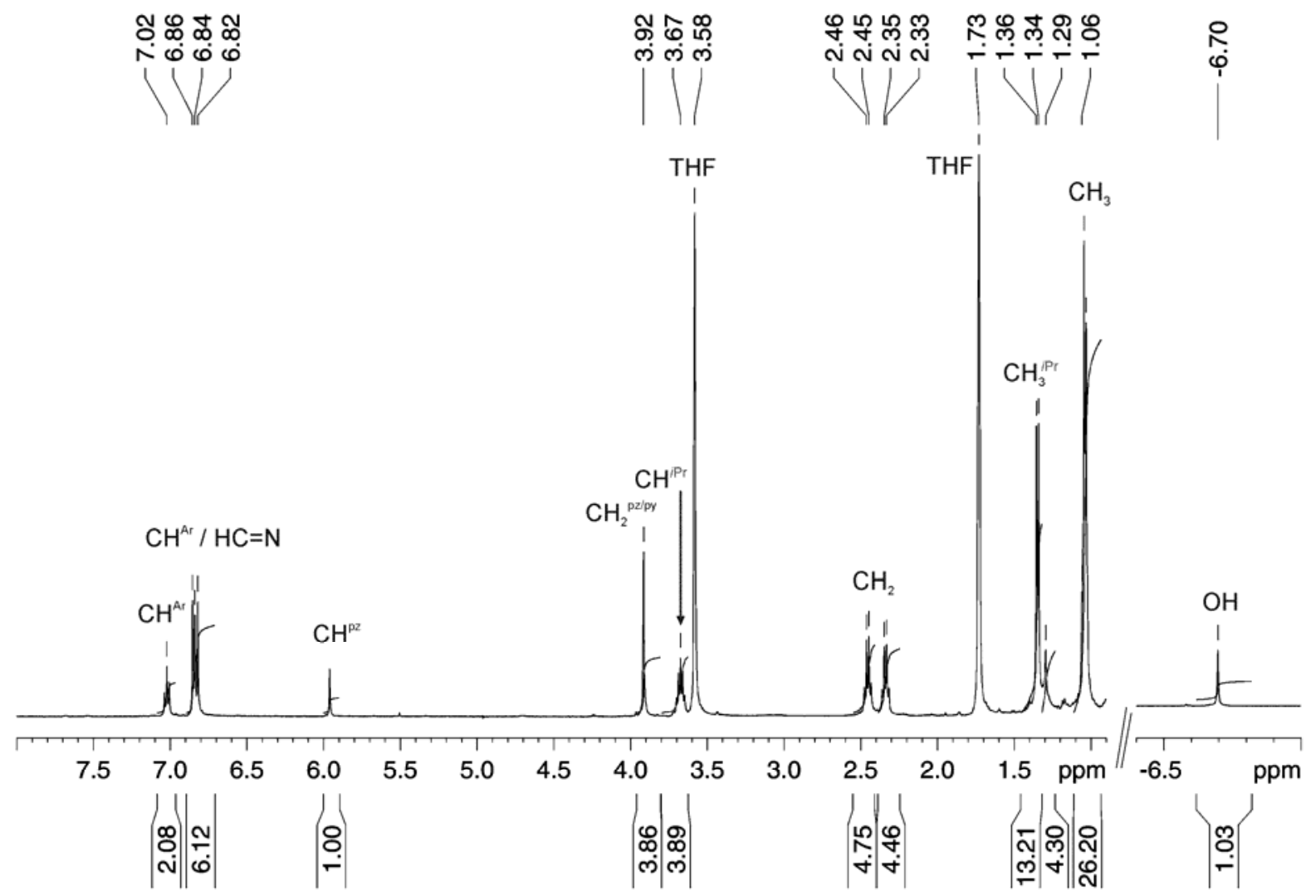

Abbildung 99: ${ }^{1} \mathrm{H}-\mathrm{NMR}-$ Spektrum von $\left[\mathbf{L}^{3} \mathbf{N i}_{2}(\mathbf{O H})\right]$ in THF-d $\mathrm{d}_{8}$.

Die Kreuzsignale des 2D-Spektrums zeigen die räumliche Nachbarschaft der detektierten Kerne an. Dem NOESY-Spektrum kann entnommen werden, dass die OH-Gruppe, welche in 
THF- $\mathrm{d}_{8}$ bei -6.70 ppm erscheint, mit mehreren Protonen der Verbindung in räumlicher Nähe steht. Um die Kopplung des OH-Protons mit den umgebenden Kernen besser zu erkennen, kann eine Spur bei -6.70 ppm gelegt und in ein 1D-Spektrum aufgelöst werden. Dieses 1DSpektrum ist im Vergleich zum ${ }^{1} \mathrm{H}-\mathrm{NMR}$-Spektrum von $\left[\mathbf{L}^{3} \mathbf{N i}_{\mathbf{2}}(\mathbf{O H})\right]$ gemessen in THF- $\mathrm{d}_{8}$, in Abbildung 101 dargestellt.

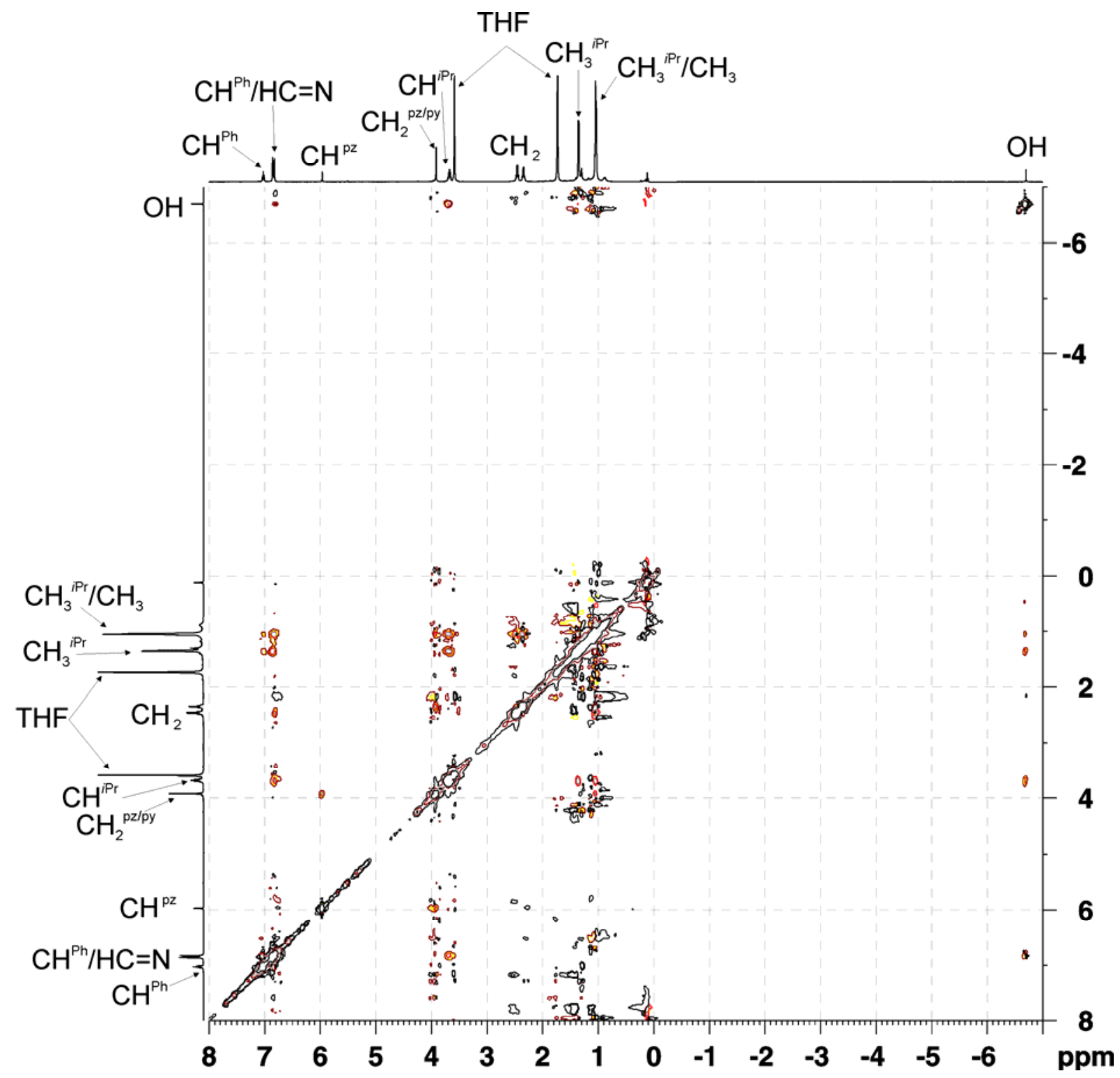

Abbildung 100: H,H-NOESY Spektrum von $\left[\mathbf{L}^{3} \mathbf{N i}_{2}(\mathbf{O H})\right]$ in THF-d $\mathrm{d}_{8}$.

Wie bereits die Molekülstruktur von $\left[\mathbf{L}^{3} \mathbf{N i}_{\mathbf{2}}(\mathbf{O H})\right]$ (Abbildung 86) zeigt, stehen die $\mathrm{CH}_{3}{ }^{i \mathrm{Pr}}$ und die $\mathrm{CH}^{i \mathrm{Pr}}$ Protonen in unmittelbarer Nachbarschaft zur OH-Gruppe. Beobachtet wird zudem eine Wechselwirkung des OH-Protons mit den Phenyl-Protonen in den meta-Positionen. Die $\mathrm{OH}-\mathrm{Gruppe}$ ist dem Ringstromeffekt der zwei aromatischen Phenylgruppen ausgesetzt und erscheinen infolgedessen stark ins Hochfeld verschoben. 


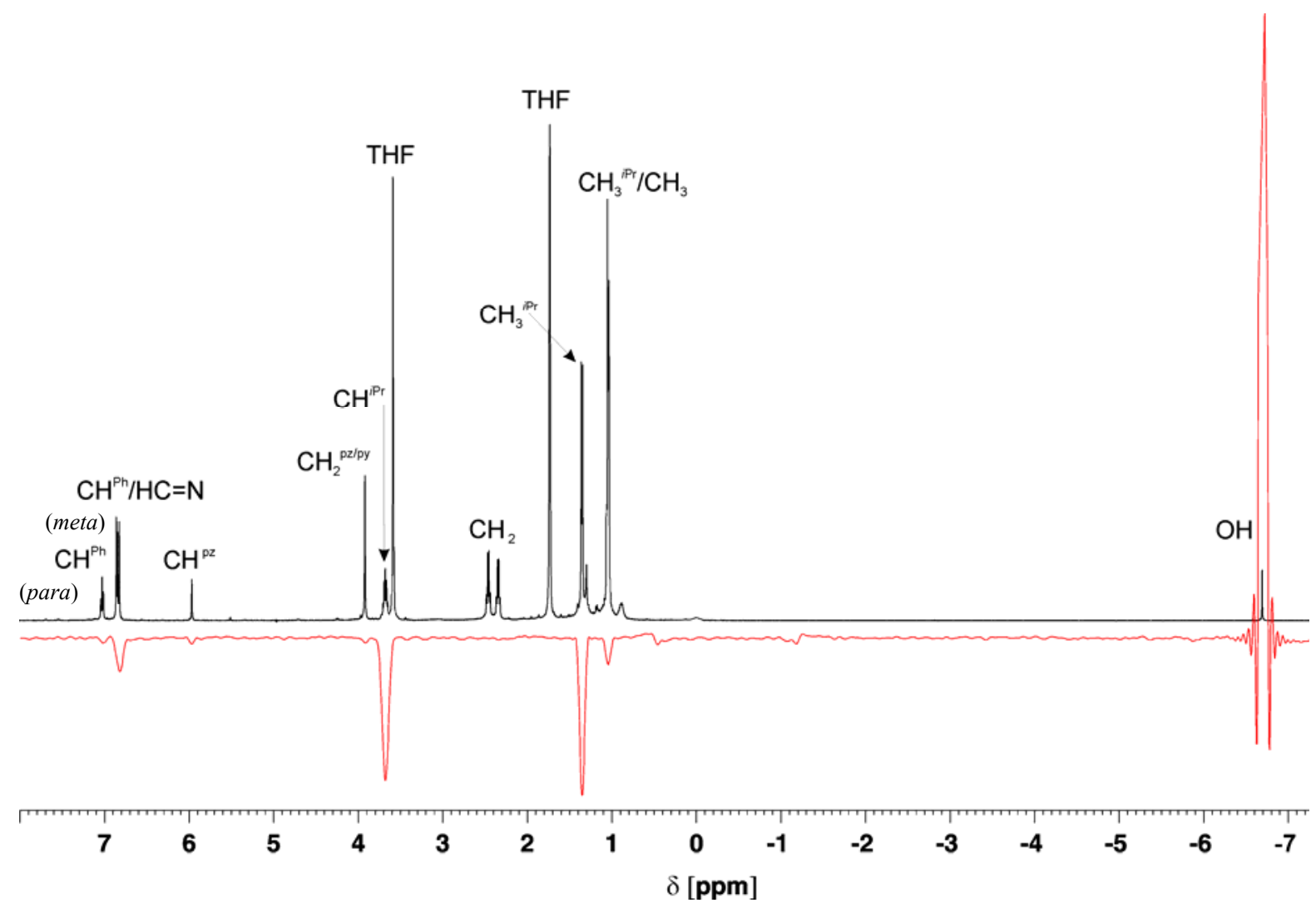

Abbildung 101: ${ }^{1} \mathrm{H}-\mathrm{NMR}-$ Spektrum von $\left[\mathbf{L}^{3} \mathbf{N i}_{2}(\mathbf{O H})\right]$ in THF-d $\mathrm{d}_{8}$ (schwarze Linie) und 1D-Spur des NOESYSpektrums bei -6.70 ppm (rote Linie).

Wird der Komplex $\left[\mathbf{L}^{3} \mathbf{N i}_{2}(\mathbf{O H})\right]$ statt in THF-d $\mathrm{d}_{8}$ in $\mathrm{CDCl}_{3}$ gelöst so sind im ${ }^{1} \mathrm{H}-\mathrm{NMR}-$ Spektrums noch weitere signifikante Änderungen zu erkennen (Abbildung 102, A und B). Wie aus dem ${ }^{1}$ H-NMR-Spektrum A in Abbildung 102 entnommen werden kann liegt der Ligand $\mathbf{H}_{3} \mathbf{L}^{3}$ in hoher Reinheit vor, so dass eventuelle Verunreinigungen durch den Ligand ausgeschlossen werden können.

Wird das $\mathrm{OH}-$ Signal auf eins normiert, so würde für die Integration des $\mathrm{CH}^{\mathrm{pz}}$-Protons an der 4-Position ebenfalls ein Wert von 1 erwartet (Abbildung 102, B). Die Integration des $\mathrm{CH}^{\mathrm{pz}}$ Signals ist jedoch mit 1.77 um 0.77 Einheiten größer. Auch die restlichen Signale des Spektrums haben eine höhere Intensität als erwartet. Manche Signale, wie das Septett für die $\mathrm{CH}^{i \mathrm{Pr}}$ - oder die $\mathrm{CH}_{2}{ }^{\mathrm{py} / \mathrm{pz}}$-Gruppe, erscheinen sogar doppelt im Spektrum. Die Beobachtung könnte von zwei unterschiedlichen Effekten herrühren. Zum einen könnten unter Umständen unterschiedliche Isomere des Komplexes in Lösung existieren, die durch die unmittelbare Nähe zum Metallzentrum unterschiedlich entschirmt werden. Zum anderen ist es möglich, dass sich verschiedene Verbindungen in Lösung befinden. 

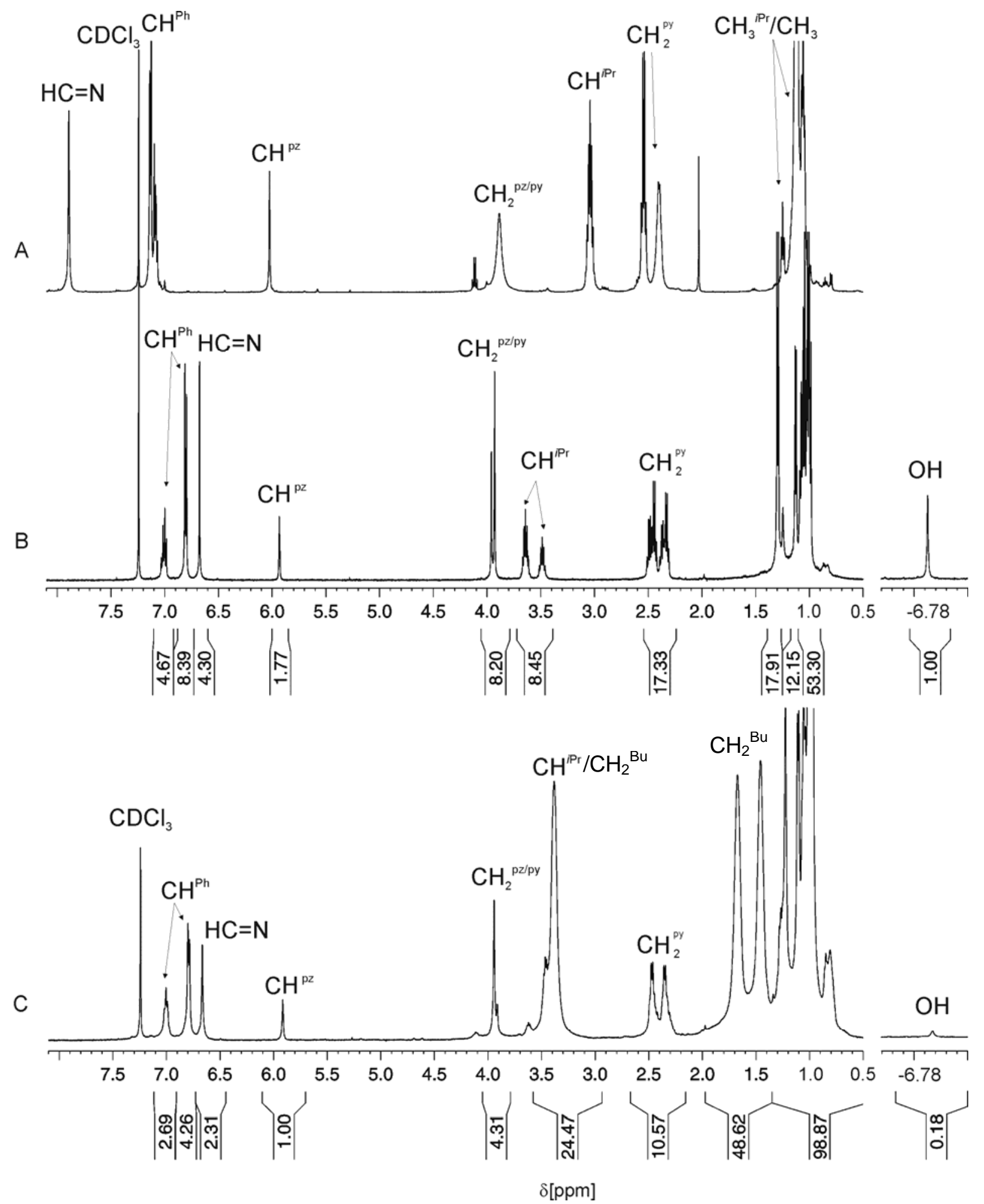

Abbildung 102: ${ }^{1} \mathrm{H}-\mathrm{NMR}$-Spektrum von $\mathbf{H}_{3} \mathbf{L}^{3}$ (A), $\left[\mathbf{L}^{3} \mathbf{N i}_{\mathbf{2}}(\mathbf{O H})\right]$ (B) und $\left[\mathbf{L}^{3} \mathbf{N i}_{2}(\mathbf{O H})\right]$ umgesetzt mit einem Überschuss an Tetrabutylammoniumchlorid zur Darstellung von $\left[\mathbf{L}^{3} \mathbf{N i}_{2} \mathbf{C l}\right](\mathbf{C})$ in $\mathrm{CDCl}_{3}$.

Temperaturabhängige ${ }^{1} \mathrm{H}-\mathrm{NMR}-M e s s u n g e n$ von $\left[\mathbf{L}^{3} \mathbf{N i}_{\mathbf{2}}(\mathbf{O H})\right]$ in $\mathrm{CDCl}_{3}$ können hierbei Aufschluss über die Verbindungen in Lösung geben. In Abbildung 103 ist das Ergebnis der temperaturabhängigen ${ }^{1} \mathrm{H}-\mathrm{NMR}$-Experimente von $\left[\mathbf{L}^{3} \mathbf{N i}_{\mathbf{2}}(\mathbf{O H})\right]$ gezeigt. Die Spektren wurden 
im Bereich von 223 bis $323 \mathrm{~K}$ in $\mathrm{CDCl}_{3}$ gemessen. Es zeigt sich dass das Spektrum in diesem Temperaturbereich temperaturunabhängig ist. Somit ist die Existenz unterschiedlicher Isomere in Lösung unwahrscheinlich. Anscheinend existieren in der Lösung zwei unterschiedliche Komplexe, die die hohe Zahl an Signale im ${ }^{1} \mathrm{H}-\mathrm{NMR}-$ Spektrum verursachen. Da sich die chemische Verschiebung der Imin-Protonen im Vergleich zum Ligand $\left(\mathbf{H}_{3} \mathbf{L}^{\mathbf{3}}\right)$ um $\Delta \delta=1.23$ ppm ändert, kann es sich bei den doppelt vorkommenden Signalen im ${ }^{1} \mathrm{H}-\mathrm{NMR}$ Spektrum von $\left[\mathbf{L}^{3} \mathbf{N i}_{\mathbf{2}}(\mathbf{O H})\right]$ um den Komplex $\left[\mathbf{L}^{3} \mathbf{N i}_{2} \mathbf{C l}\right]$ handeln.

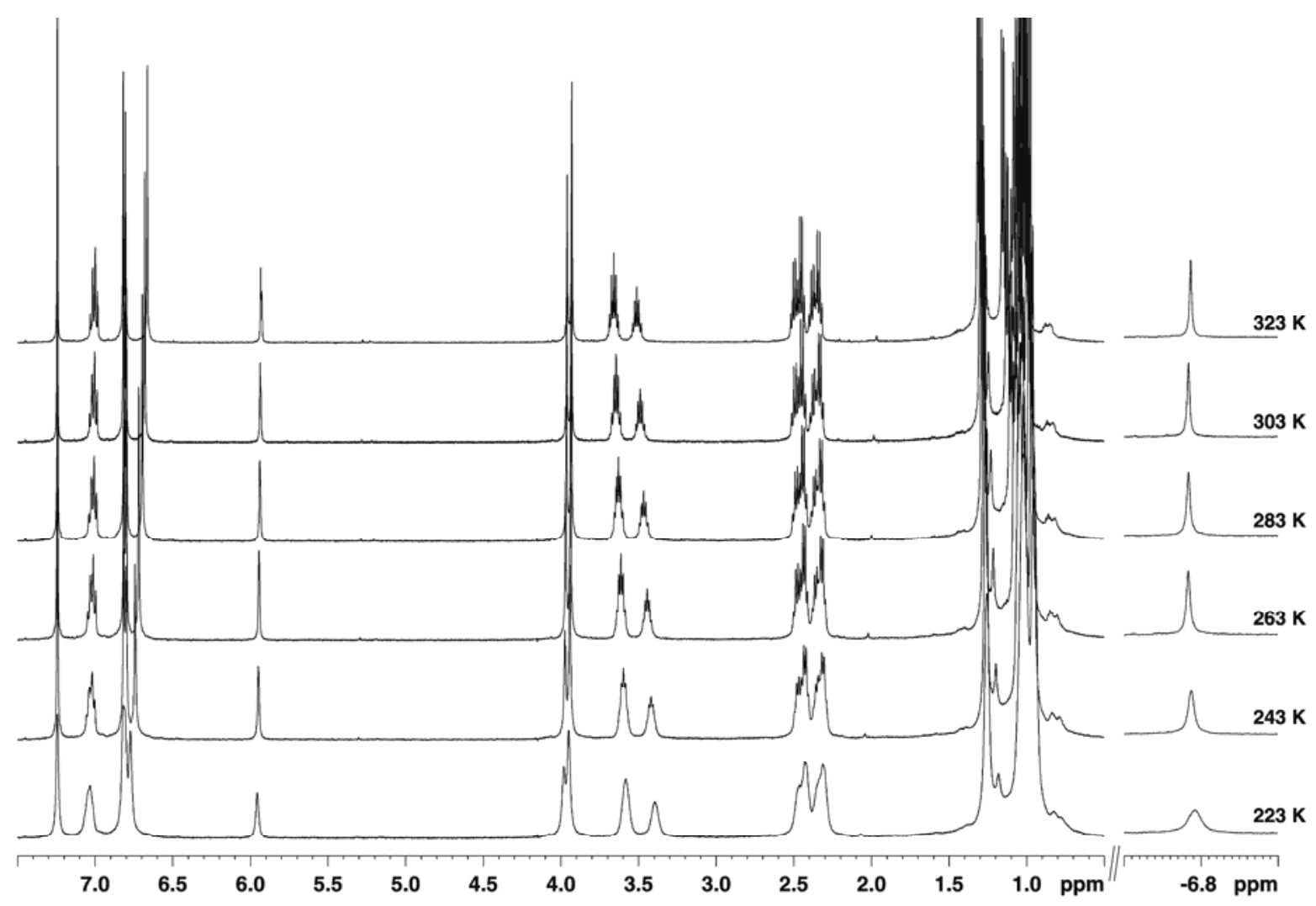

Abbildung 103: ${ }^{1} \mathrm{H}-\mathrm{NMR}$ Hoch- und Tieftemperaturmessungen des Komplexes $\left[\mathbf{L}^{\mathbf{3}} \mathbf{N i}_{\mathbf{2}}(\mathbf{O H})\right]$ in $\mathrm{CDCl}_{3}$ (gemessen bei $500 \mathrm{MHz}$ ).

Diese Beobachtungen legen weiterhin den Ablauf einer Austauschreaktion des Brückenliganden durch Chlorid, welches offenbar in Spuren im Lösungsmittel $\mathrm{CDCl}_{3}$ vorhanden ist.

Um die Annahme einer Austauschreaktion des Brückenliganden zu bestätigen, wurde eine in $\mathrm{CDCl}_{3}$ gelöste NMR-Probe des Nickel(II)komplexes $\left[\mathbf{L}^{3} \mathbf{N i}_{2}(\mathbf{O H})\right]$ mit einem Überschuss an Tetrabutylammoniumchlorid versetzt (Abbildung 104). Die ${ }^{1} \mathrm{H}-\mathrm{NMR}-\mathrm{Spektren}$ vor und nach Zugabe $\mathrm{NBu}_{4} \mathrm{Cl}$ sind in Abbildung 102 (B und C) dargestellt. Nach Zugabe von $\mathrm{NBu}_{4} \mathrm{Cl}$ wird ein schneller Austausch der OH-Gruppe durch Chlorid beobachtet. 

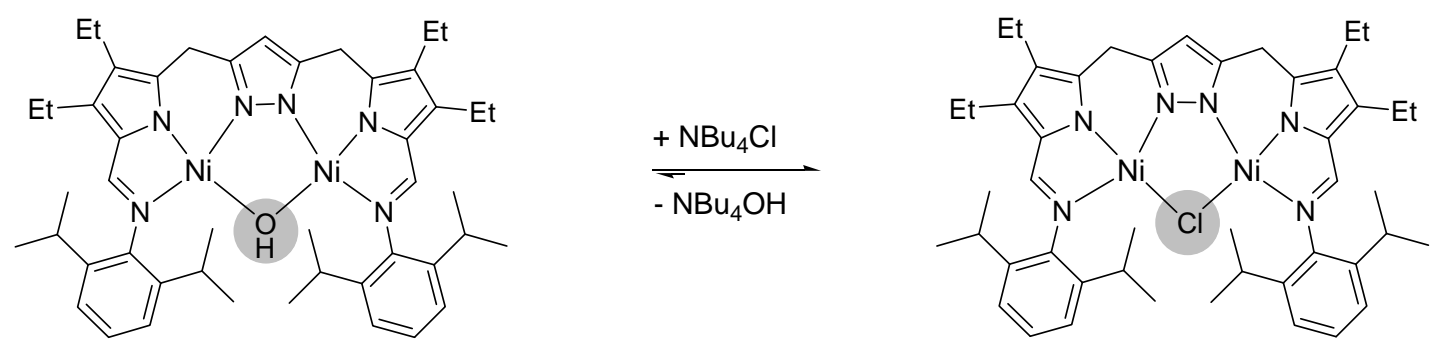

Abbildung 104: Austausch des Brückenliganden $-\mathrm{OH}$ im $\left[\mathbf{L}^{3} \mathbf{N i}_{\mathbf{2}}(\mathbf{O H})\right]$ mit Tetrabutylammoniumchlrorid zu $\left[\mathbf{L}^{3} \mathrm{Ni}_{2} \mathbf{C l}\right]$.

Die Intensität des $\mathrm{OH}-$ Signals bei $-6.78 \mathrm{ppm}$ nimmt rasch ab und die $\mathrm{CH}^{i \mathrm{Pr}}$-Gruppen bzw. $\mathrm{CH}_{2}{ }^{\mathrm{py} / \mathrm{pz}}$-Signale vereinen sich zu einem Signal. Das Gleichgewicht verschiebt sich zur Seite des neu entstandenen $\left[\mathbf{L}^{3} \mathbf{N i}_{\mathbf{2}} \mathbf{C l}\right]$. Es lässt sich daher folgern, dass die doppelte Anzahl der Signale in dem ${ }^{1} \mathrm{H}-\mathrm{NMR}-\mathrm{Spektrum}$ von $\left.\left[\mathbf{L}^{\mathbf{3}} \mathbf{N i}_{\mathbf{2}} \mathbf{( O H}\right)\right]$ in $\mathrm{CDCl}_{3}$ höchstwahrscheinlich aus Spuren von Chlorid im Lösungsmittel resultieren.

Die Existenz von $\left[\mathbf{L}^{3} \mathbf{N i}_{2} \mathbf{C l}\right]$ in Lösung konnte ebenfalls massenspektrometrisch (ESI +/-) bestätigt werden. Außer dem Signal für $\left[\mathbf{L}^{3} \mathbf{N i}_{\mathbf{2}}(\mathbf{O H})\right]$ wird ein weiteres Signal bei $861 \mathrm{~m} / \mathrm{z}$ $[\mathrm{M}+\mathrm{H}]^{+}$für $\left[\left(\mathrm{C}_{47} \mathrm{H}_{61} \mathrm{~N}_{6} \mathrm{Ni}_{12} \mathrm{Cl}\right)+\mathrm{H}\right]^{+}$detektiert, welches dem Komplex $\left[\mathbf{L}^{3} \mathbf{N i}_{2} \mathbf{C l}\right]$ zugeordnet werden kann.

Die Austauschreaktion des Brückenliganden wurde zusätzlich mit deuteriertem Wasser $\left(\mathrm{D}_{2} \mathrm{O}\right)$ untersucht. Findet ein Austausch zwischen der $\mu$-OH-Gruppe und dem $\mathrm{D}_{2} \mathrm{O}$ zu -OD statt, so kann diese Reaktion in der ${ }^{1} \mathrm{H}-\mathrm{NMR}-$ Spektroskopie verfolgt werden.

$\mathrm{Zu}$ diesem Zweck wurden $10 \mathrm{mg}$ des Nickel(II)komplexes $\left[\mathbf{L}^{1} \mathbf{N i}_{\mathbf{2}}(\mathbf{O H})\right]$ in $0.6 \mathrm{~mL} \mathrm{CDCl}_{3}$ gelöst, mit zwei Tropfen $\mathrm{D}_{2} \mathrm{O}(99.9 \%$ ) versetzt und das Reaktionsgemisch anschließend mit Hilfe der ${ }^{1}$ H-NMR-Spektroskopie zeitabhängig untersucht. In den ersten 10-30 Minuten konnte keine signifikante Änderung im NMR-Spektrum beobachtet werden. Nach drei Tagen war allerdings aufgrund sehr langsamen Austausches des Protons gegen Deuterium das $\mathrm{OH}-$ Signal im ${ }^{1}$ H-NMR-Spektrum nicht mehr zu detektieren (Abbildung 105).

Aufgrund des ungewöhnlich langsamen Verlaufes wurde die Kinetik des Proton- bzw. Deuterium-Austausches anhand des Nickel(II)komplexes $\left[\mathbf{L}^{3} \mathbf{N i}_{\mathbf{2}}(\mathbf{O H})\right]$ genauer untersucht (Abbildung 106). Im Vergleich zu $\left[\mathbf{L}^{\mathbf{1}} \mathbf{N i}_{\mathbf{2}}(\mathbf{O H})\right]$ besitzt der Diimin-Ligand in $\left[\mathbf{L}^{3} \mathbf{N i}_{\mathbf{2}}(\mathbf{O H})\right]$ je zwei sterisch anspruchsvolle Isopropylreste an den Phenylgruppen, welche die Zugänglichkeit des Brückenliganden bei einer Austauschreaktion weiter erschweren. 


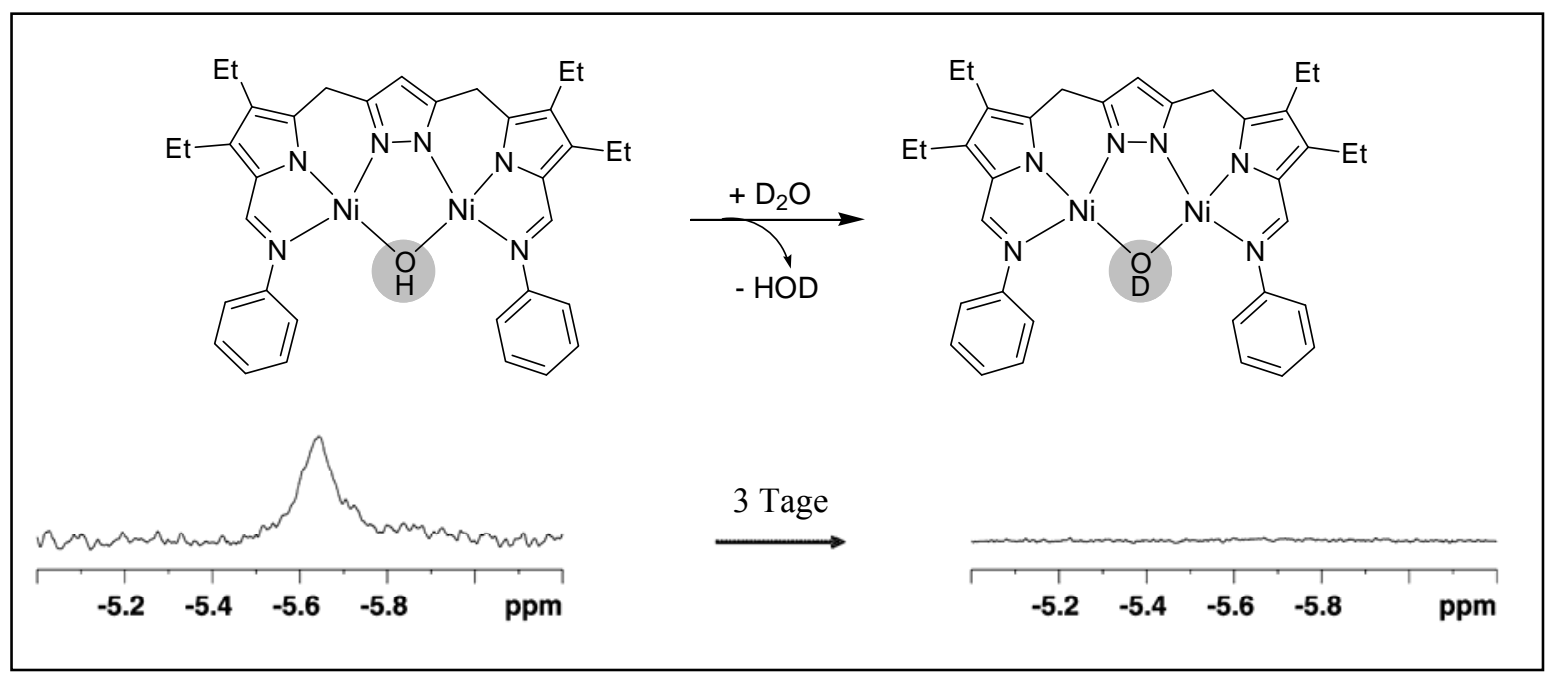

Abbildung 105: Austausch des Brückenliganden -OH mit $\mathrm{D}_{2} \mathrm{O}$ im Nickel(II)komplex $\left[\mathbf{L}^{\mathbf{1}} \mathbf{N i}_{\mathbf{2}}(\mathbf{O H})\right]$. Der OHBrückenligand des Komplexes erscheint im ${ }^{1} \mathrm{H}-\mathrm{NMR}$-Spektrum bei -5.65 ppm (unten links). Nach Zugabe eines Überschusses an $\mathrm{D}_{2} \mathrm{O}$ findet nach drei Tagen der komplette Austausch des Protons zu einem Deuterium statt (unten rechts).

Der Nickel(II)komplex $\left[\mathbf{L}^{3} \mathbf{N i}_{\mathbf{2}}(\mathbf{O H})\right]$ wurde unter wasserfreien Bedingungen in trockenem THF- $\mathrm{d}_{8}$ gelöst und mit 10, 50 und 100 eq. $\mathrm{D}_{2} \mathrm{O}$ versetzt. Der Verlauf des OH/OD-Austausches wurde mittels der ${ }^{1} \mathrm{H}-\mathrm{NMR}-\mathrm{Spektroskopie} \mathrm{mehrere} \mathrm{Tage} \mathrm{lang} \mathrm{verfolgt} \mathrm{(Abbildung} \mathrm{107).}$
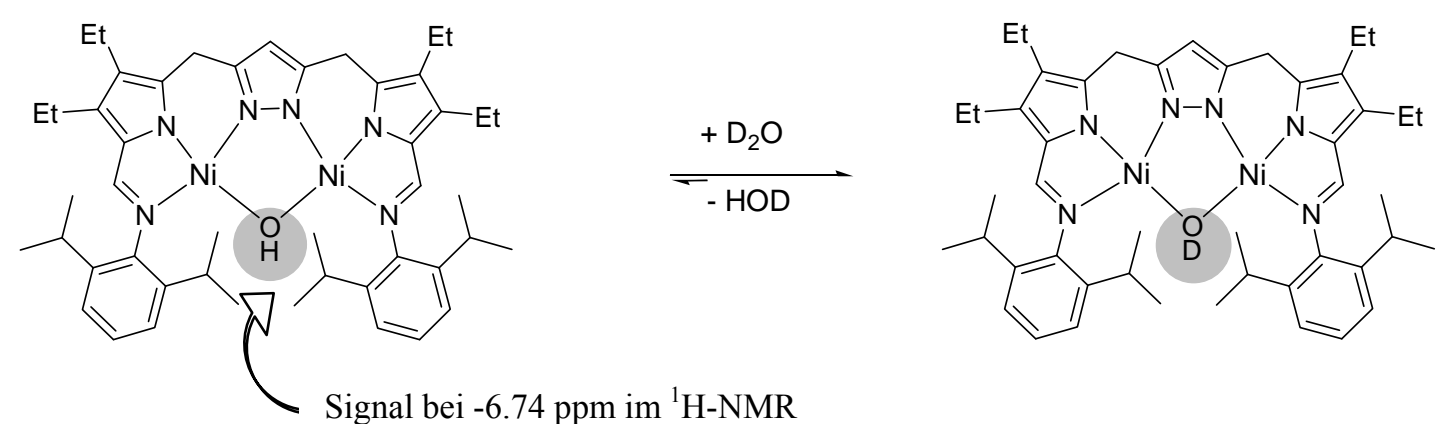

Abbildung 106: Austauschreaktion von $\left[\mathbf{L}^{3} \mathbf{N i}_{2}(\mathbf{O H})\right]$ mit $\mathrm{D}_{2} \mathrm{O}$ zu $\left[\mathbf{L}^{3} \mathbf{N i}_{2}(\mathbf{O D})\right]$ in THF-d .

$\mathrm{Zu}$ Beginn der Reaktion ist das Integral des $\mathrm{CH}^{\mathrm{pz}-4}$-Protons genauso groß wie das der $\mathrm{OH}-$ Gruppe im Komplex entsprechend des erwarteten 1:1 Intensitätsverhältnisses. Während der langsamen Austauschreaktion nimmt die Intensität des Signals der OH-Gruppe bei -6.74 ppm ab. Die Änderung des Peak-Integrals im Vergleich zum $\mathrm{CH}^{\mathrm{pz}-4}$-Signal bei 5.90 ppm ist in Abbildung 107 gegen die Zeit aufgetragen. Hierbei wird ein exponentieller Abfall beobachtet. 


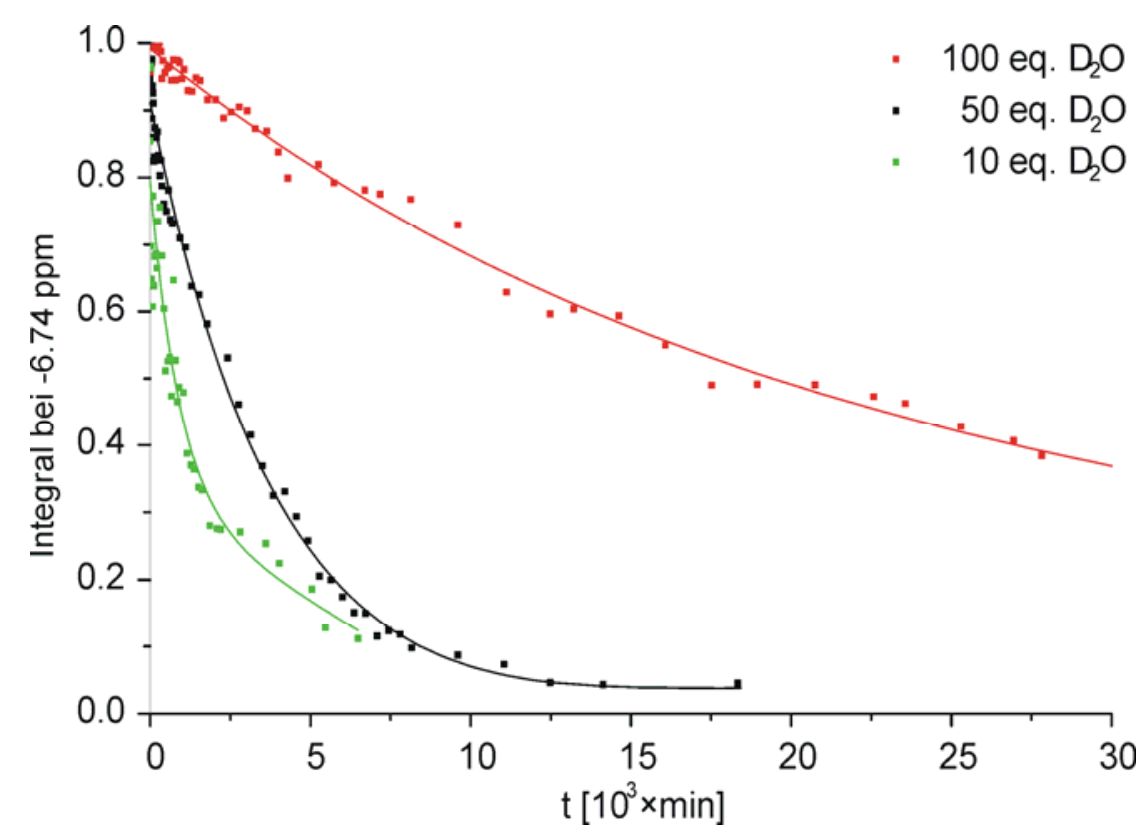

Abbildung 107: Auftragung der Intensität des OH-Signals von $\left[\mathbf{L}^{3} \mathbf{N i}_{2}(\mathbf{O H})\right]$ im ${ }^{1} \mathrm{H}-\mathrm{NMR}-$ Spektrum bei -6.74 ppm nach Zugabe von 10, 50 und 100 eq. $\mathrm{D}_{2} \mathrm{O}$ gegen die Zeit.

Die halblogarithmische Auftragung von $\ln \left(\mathrm{c}\left[\mathbf{L}^{3} \mathbf{N i}_{\mathbf{2}}(\mathbf{O H})\right]\right)$ gegen die Zeit liefert eine Gerade (Abbildung 108). Der lineare Verlauf der Graphen spricht für eine Reaktion pseudoerster Ordnung. Das $\mathrm{D}_{2} \mathrm{O}$ ist in starkem Überschuss vorhanden, so dass die Konzentration als konstant angenommen wird. Die Steigung der Geraden (B) entspricht der Geschwindigkeitskonstanten $k_{\text {obs }}$ der Reaktion (Tabelle 20).

Tabelle 20: Berechnete Werte für die Geschwindigkeitskonstante $k_{\text {obs. }}$.

\begin{tabular}{cc}
\hline eq. $\mathrm{D}_{2} \mathrm{O}$ & $k_{\text {obs }}\left[\mathrm{s}^{-1}\right]$ \\
\hline 10 & $5.09 \pm 0.31 \cdot 10^{-6}$ \\
50 & $4.30 \pm 0.07 \cdot 10^{-6}$ \\
100 & $5.59 \pm 0.08 \cdot 10^{-7}$
\end{tabular}



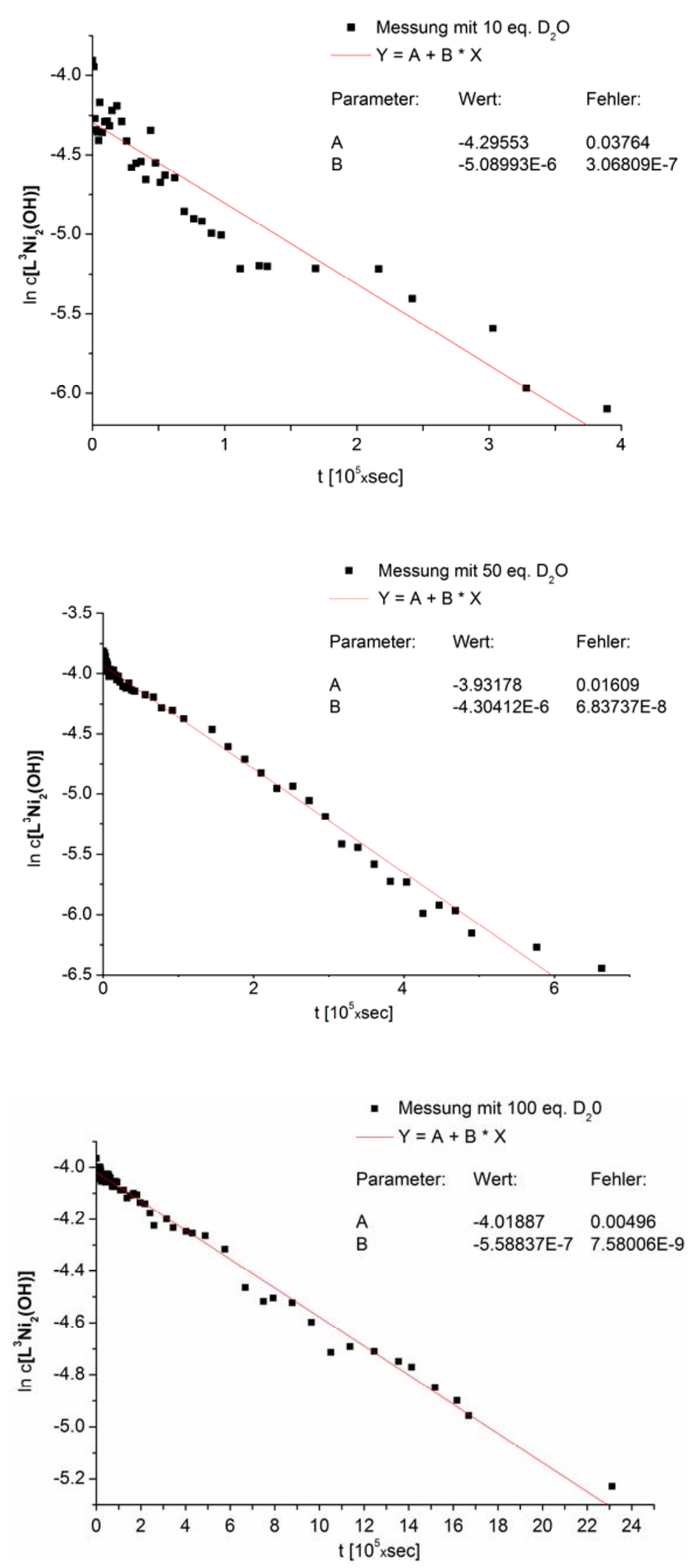

Abbildung 108: Halblogarithmische Auftragung von $\ln c\left[\mathbf{L}_{3} \mathbf{N i}_{2}(\mathbf{O H})\right]$ aufgetragen gegen die Zeit bei der Austauschreaktion mit $\mathrm{D}_{2} \mathrm{O}$. 
Die Austauschreaktion ist außergewöhnlich langsam, die Halbwertszeit beträgt im Fall von 50 eq. $\mathrm{D}_{2} \mathrm{O}$ ca. 2 Tage. Überraschenderweise zeigt der Kurvenverlauf, dass je höher die $\mathrm{D}_{2} \mathrm{O}$ Konzentration in der Lösung ist, desto langsamer läuft die Austauschreaktion ab. Zur Erklärung dieser Beobachtung muss der kinetische H/D-Isotopeneffekt berücksichtigt werden. Zudem muß berücksichtigt werden, dass das verwendete $\mathrm{D}_{2} \mathrm{O}$ einen gewissen Anteil HDO enthält.

Eine weitergehende kinetische Untersuchung und Analyse dieses ungewöhnlich langsamen H/D-Austausches ist in zukünftigen Arbeiten geplant. Dabei ist durch weitere Makierungsexperimente $\left(\mathrm{H}_{2}{ }^{17} \mathrm{O}\right)$ Untersuchung mit ${ }^{17} \mathrm{O}-\mathrm{NMR}-$ Spektroskopie vorab $\mathrm{zu}$ bestätigen, dass die Reaktion mechanistisch - wie angenommen - als Protonierungsaustausch (6) und nicht als Hydroxid-Austausch (7) abläuft.

$$
\begin{aligned}
& {\left[\mathbf{L}^{3} \mathbf{N i}_{\mathbf{2}}(\mathbf{O H})\right]+\mathrm{D}_{2}{ }^{*} \mathrm{O} \rightleftharpoons\left[\mathbf{L}^{\mathbf{3}} \mathbf{N i}_{\mathbf{2}}(\mathbf{O D})\right]+\mathrm{HD} * \mathrm{O}} \\
& {\left[\mathbf{L}^{\mathbf{3}} \mathbf{N i}_{\mathbf{2}}(\mathbf{O H})\right]+\mathrm{D}_{2}{ }^{*} \mathrm{O} \rightleftharpoons\left[\mathbf{L}^{\mathbf{3}} \mathbf{N i}_{\mathbf{2}}\left({ }^{*} \mathbf{O D}\right)\right]+\mathrm{HDO}}
\end{aligned}
$$

Durch die Massenveränderung innerhalb des $\mathrm{H} \rightarrow \mathrm{D}$ Austausches kann die Umsatzrate einer Reaktion beeinflusst werden. Ist der Protonentransfer geschwindigkeitsbestimmend, so wird bei hohen $\mathrm{D}_{2} \mathrm{O}$-Konzentrationen eine deutliche Verlangsamung der Reaktionsgeschwindigkeit beobachtet. ${ }^{100}$ Hierbei müssten auch die Gleichgewichtskonstanten des Wassers $\left(K_{\mathrm{H}}\right)$ und des deuterierten Wassers $\left(K_{\mathrm{D}}\right)$ berücksichtigt werden. Das Verhältnis dieser zwei Konstanten $K_{\mathrm{H}} / K_{\mathrm{D}}$ entspricht $7.47 .^{101}$

Dies bedeutet, dass die Dissoziation des Wassers $\mathrm{zu} \mathrm{H}_{3} \mathrm{O}^{+}$und $\mathrm{OH}^{-}$etwa siebenmal stärker ist, als die entsprechende Dissoziation des deuterierten Wassers. Bei der Umsetzung von $\left[\mathbf{L}^{3} \mathbf{N i}_{2}(\mathbf{O H})\right]$ mit 100 eq. $\mathrm{D}_{2} \mathrm{O}$ kann neben dem abnehmenden $\mathrm{OH}-$ Signal auch das entstehende Signal des $\mathrm{H}_{2} \mathrm{O}$ bzw. HDO beobachtet werden (Abbildung 109).

Der Austausch von einem Proton und einem Deuterium ist ebenfalls in der IR-Spektroskopie $\mathrm{zu}$ sehen. Hierbei können die Wellenzahlen der OH- bzw. OD-Gruppen sowohl spektroskopisch bestimmt, als auch mit Hilfe der reduzierten Massen $(\mu)$ von OH- bzw. ODGruppe berechnet werden. Aus der Gültigkeit der Schwingungsgleichungen (8 und 9) kann durch dividieren der zwei Gleichungen das Verhältnis von $\widetilde{v}_{O H} / \widetilde{v}_{O D}$ ermittelt werden (Gleichung 10). 


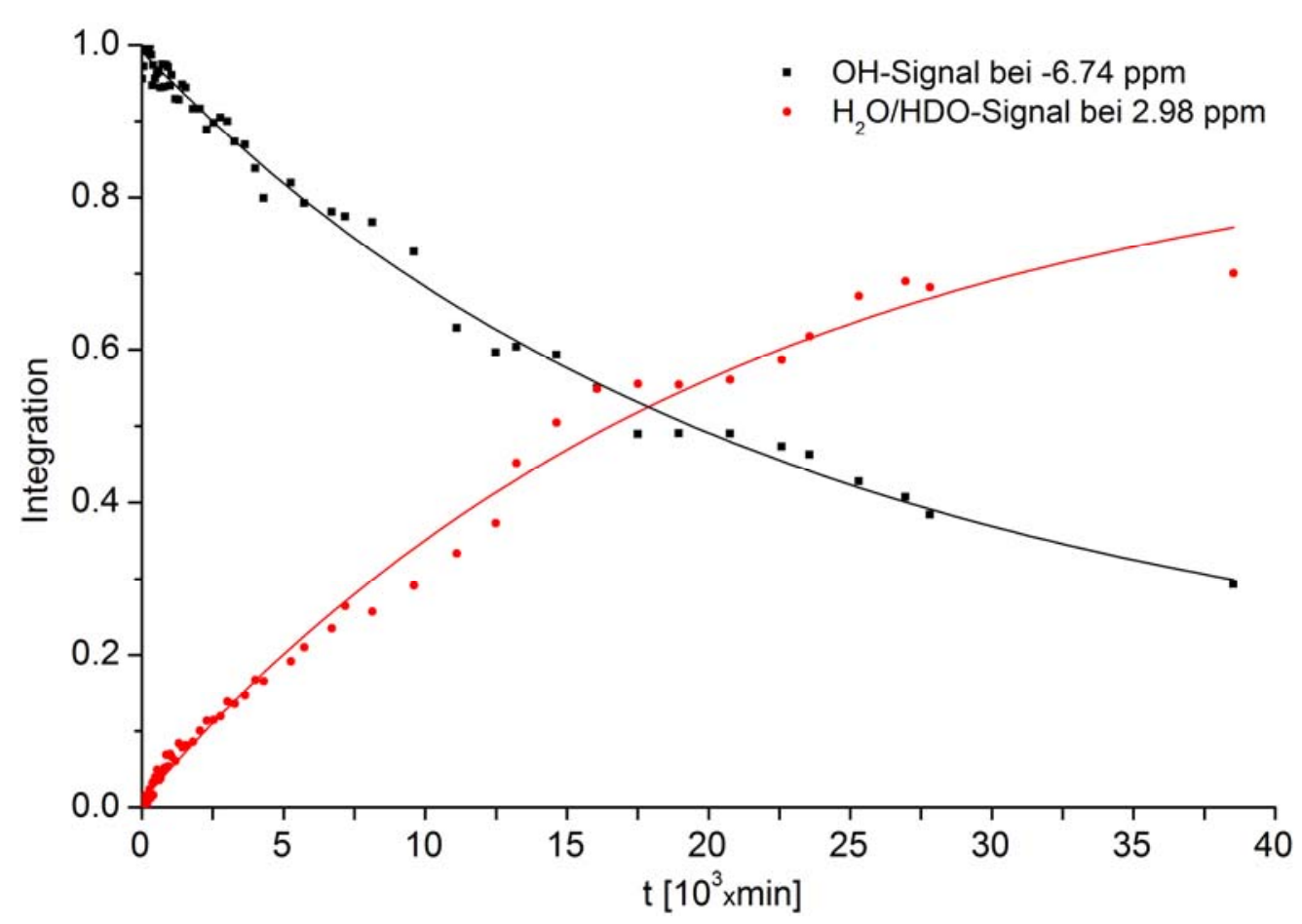

Abbildung 109: Integration der $\mathrm{OH}-$ bzw. $\mathrm{H}_{2} \mathrm{O}$ - Signale während der Austauschreaktion von $\left[\mathbf{L}^{3} \mathbf{N i}_{\mathbf{2}}(\mathbf{O H})\right]$ mit 100 eq. $\mathrm{D}_{2} \mathrm{O}$.

Mit größer werdender Atommasse wird die Absorptionsbande zu kleineren Wellenzahlen hinverschoben. ${ }^{102}$ Die reduzierte Masse $\mu_{\mathrm{OH}}$ beträgt 0.948 und $\mu_{\mathrm{OD}}=1.789$. Daraus ergibt sich für Gleichung 10 ein Verhältnis der Schwingungsfrequenzen $\widetilde{v}_{O H} / \widetilde{v}_{O D}$ von 1.374.

$$
\begin{aligned}
& \widetilde{v}_{O H}=\frac{1}{2 \pi} \sqrt{\frac{k_{O H}}{\mu_{O H}}} \\
& \widetilde{v}_{O D}=\frac{1}{2 \pi} \sqrt{\frac{k_{O D}}{\mu_{O D}}} \\
& \Rightarrow \frac{\widetilde{v}_{O H}}{\widetilde{v}_{O D}} \approx \sqrt{\frac{\mu_{O D}}{\mu_{O H}}}
\end{aligned}
$$

Die OH-Schwingung des Nickel(II)komplexes $\left[\mathbf{L}^{3} \mathbf{N i}_{\mathbf{2}}(\mathbf{O H})\right]$ erscheint im gemessenen Spektrum bei $3607 \mathrm{~cm}^{-1}$ (Abbildung 110, A). Theoretisch wird die OD-Schwingung nach Gleichung 10 bei $2625 \mathrm{~cm}^{-1}$ erwartet. In Abbildung 110 (B) ist das IR-Spektrum nach der Reaktion von $\left[\mathbf{L}^{3} \mathbf{N i}_{2}(\mathbf{O H})\right]$ mit $\mathrm{D}_{2} \mathrm{O}$ aufgeführt. 


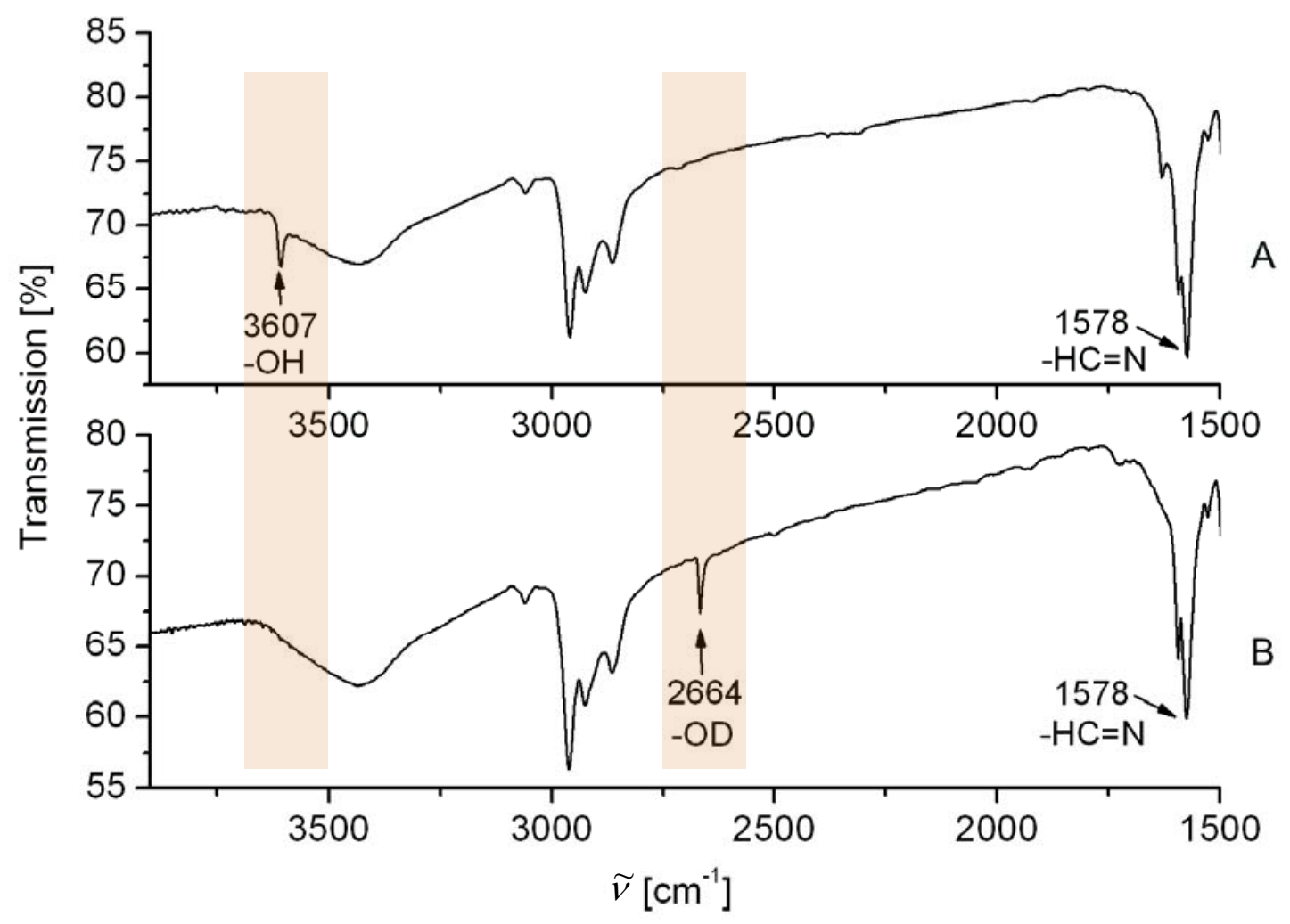

Abbildung 110: IR-Spektrum von $\left[\mathbf{L}^{3} \mathbf{N i}_{\mathbf{2}}(\mathbf{O H})\right]$ gemessen in $\mathrm{KBr}(\mathrm{A})$. $\left[\mathbf{L}^{3} \mathbf{N i}_{\mathbf{2}}(\mathbf{O H})\right]$ nach Austauschreaktion mit $\mathrm{D}_{2} \mathrm{O}$ zu [ $\mathbf{L}^{3} \mathbf{N i}_{2}$ (OD)] (nach 3 Wochen Reaktionszeit; gemessen in $\mathrm{KBr}$ ) (B). Die OH-Bande bei $3607 \mathrm{~cm}^{-1}$ verschwindet und eine neue Bande für den $\mu$-OD Brückenliganden bei $2664 \mathrm{~cm}^{-1}$ entsteht.

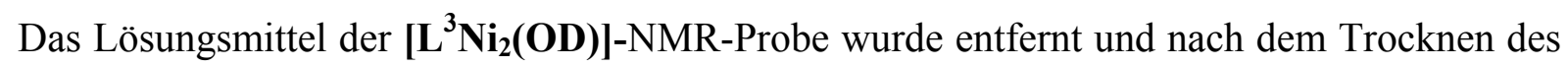
Rückstandes im Hochvakuum erneut IR-spektroskopisch gemessen. In diesem Spektrum ist die OH-Bande bei $3607 \mathrm{~cm}^{-1}$ nicht mehr zu detektieren, während eine neue Bande bei $2664 \mathrm{~cm}^{-1}$ beobachtet wird.

Der berechnete Wert für $\widetilde{v}_{O D}\left(2625 \mathrm{~cm}^{-1}\right)$ stimmt recht gut mit dem gemessenen Wert von $2664 \mathrm{~cm}^{-1}$ überein. Die geringe Abweichung zwischen dem berechneten und dem gemessenen Wert kommt durch die Nährung innerhalb der Berechnungen zustande, da die Kraftkonstanten $\mathrm{k}_{\mathrm{OD}}$ bzw. $\mathrm{k}_{\mathrm{OH}}$ als gleich groß angenommen wurden. Außerdem wird bei der Nährung angenommen, dass die OH-Gruppe völlig isoliert von anderen Schwingungen im System ist.

Mit Hilfe der sogenannten Streckfrequenznäherung kann der primäre kinetische Isotopeneffekt für das Deuterium berechnet werden. ${ }^{101}$ Durch die gemessenen Valenzschwingungen (Abbildung 110) innerhalb der IR-spekroskopischen Untersuchungen von der Geschwindigkeitskonstante von $\left[\mathbf{L}^{3} \mathbf{N i}_{\mathbf{2}}(\mathbf{O H})\right]$ und $\left[\mathbf{L}^{3} \mathbf{N i}_{\mathbf{2}}(\mathbf{O D})\right]$ lässt sich durch 
Gleichung 11 das Verhältnis von $k_{\mathrm{H}} / k_{\mathrm{D}}$ abschätzen. Bei Raumtemperatur (295 K) wird unter Berücksichtigung des kinetischen Isotopeneffekts ein Verhältnis $k_{\mathrm{H}} / k_{\mathrm{D}}$ von 10 berechnet.

$$
k_{H} / k_{D}=\exp \left[\frac{h c}{2 k T}\left(\widetilde{v}_{H}-\widetilde{v}_{D}\right)\right]
$$

Der entstandene deuterierte Komplex $\left[\mathbf{L}^{3} \mathbf{N i}_{\mathbf{2}}(\mathbf{O D})\right]$ wurde zusätzlich mittels der Massenspektrometrie (ESI und HR-ESI) untersucht. Das Signal für $\left.\left[\mathbf{L}^{\mathbf{3}} \mathbf{N i}_{\mathbf{2}} \mathbf{( O H}\right)\right]$ mit $843 \mathrm{~m} / \mathbf{z}$ für $[\mathrm{M}+\mathrm{H}]^{+}$verschiebt sich nach der Reaktion mit $\mathrm{D}_{2} \mathrm{O}$ zu $844 \mathrm{~m} / \mathrm{z}$ (ESI-MS Experiment). Sowohl im $\left[\mathbf{L}^{3} \mathbf{N i}_{\mathbf{2}}(\mathbf{O H})\right]$ als auch in dem deuterierten Komplex $\left[\mathbf{L}^{3} \mathbf{N i}_{2}(\mathbf{O D})\right]$ findet bei der Ionisierung eine Oxidation statt, so dass ein weiteres Signal (841 bzw. $842 \mathrm{~m} / \mathrm{z})$ für [(M$2 \mathrm{H})+\mathrm{H}]^{+}$detektiert wird. Dieser Effekt konnte bei den anderen bimetallischen Komplexen ebenfalls beobachtet werden.

Die Komplexe $\left[\mathbf{L}^{3} \mathbf{N i}_{\mathbf{2}}(\mathbf{O H})\right]$ und $\left[\mathbf{L}^{3} \mathbf{N i}_{\mathbf{2}}(\mathbf{O D})\right]$ wurden in Lösung auch mittels der hochauflösenden Massenspektrometrie untersucht. Die HR-MS ermöglicht eine höhere Auflösung der Massenspektren und somit eine genauere Charakterisierung der Zusammensetzung der zu untersuchenden Substanz. In Abbildung 111 (links) ist das HRMassenspektrum von $\left[\mathbf{L}^{\mathbf{3}} \mathbf{N i}_{\mathbf{2}}\right.$ (OD)] und in Abbildung 112 (links) das Spektrum der Ausgangsverbindung $\left[\mathbf{L}^{3} \mathbf{N i}_{\mathbf{2}}(\mathbf{O H})\right]$ dargestellt. Auch hier werden aufgrund der Ionisierung zwei Spezies beobachtet und zwar $[\mathrm{M}+\mathrm{H}]^{+}$bei $844.38460 \mathrm{~m} / \mathrm{z}$ und die oxidierte Spezies [(M$2 \mathrm{H})+\mathrm{H}]^{+}$bei $842.36674 \mathrm{~m} / \mathrm{z}$. Die berechneten Isotopenmuster (Abbildung 111 rechts oben und unten) stimmen sehr gut mit der gemessenen Verteilung überein. Das Signal bei $844.38460 \mathrm{~m} / \mathrm{z}$ für $[\mathrm{M}+\mathrm{H}]^{+}$weicht unwesentlich von dem berechneten Wert $(844.38276 \mathrm{~m} / \mathrm{z})$ ab. Diese Abweichung kommt hier durch die Überlappung zweier Signale bei 844.36079 und $844.38460 \mathrm{~m} / \mathrm{z} \mathrm{zu}$ Stande. Ein Signal bei $841.36006 \mathrm{~m} / \mathrm{z}$ weist darauf hin, dass neben $\left[\mathbf{L}^{3} \mathbf{N i}_{2}(\mathbf{O D})\right]$ durch geringe Spuren von Wasser auch der Komplex $\left[\mathbf{L}^{3} \mathbf{N i}_{2}(\mathbf{O H})\right]$ (aus [(M$2 \mathrm{H})+\mathrm{H}]^{+}$mit einer molekularen Masse von $841.36801 \mathrm{~m} / \mathrm{z}$ ) vertreten ist. Das HRMassenspektrum von $\left[\mathbf{L}^{3} \mathbf{N i}_{\mathbf{2}}(\mathbf{O H})\right]$ entspricht dem des $\left[\mathbf{L}^{3} \mathbf{N i}_{\mathbf{2}}(\mathbf{O D})\right]$ und zeigt wie erwartet eine Molmassenverschiebung von einer Einheit (Abbildung 112). 


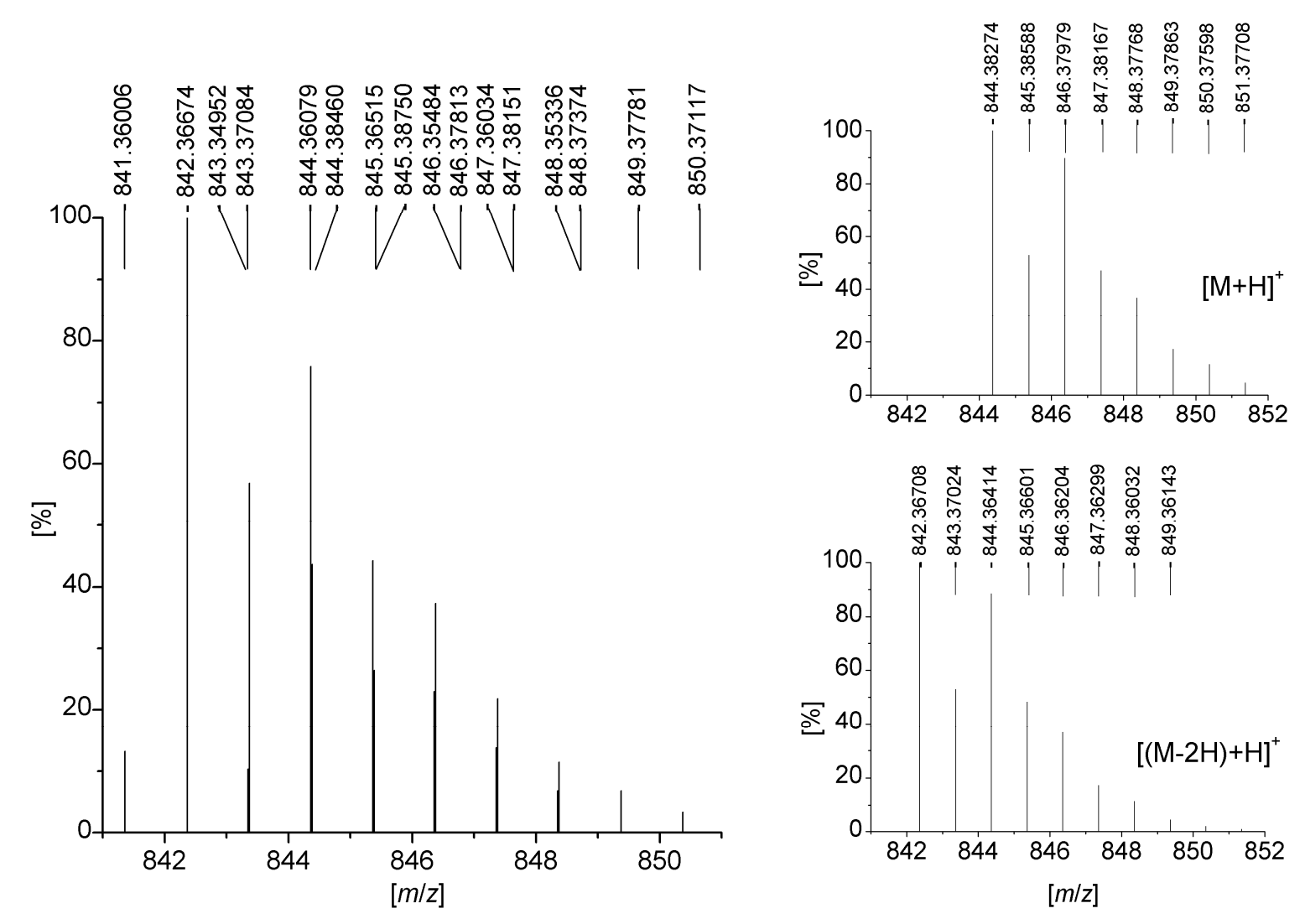

Abbildung 111: HR-Massenspektrum von $\left[\mathbf{L}^{3} \mathbf{N i}_{2}(\mathbf{O D})\right]$ in Acetonitril (links). Berechnete Isotopenmuster für $[\mathrm{M}+\mathrm{H}]^{+}$(rechts oben) und für die oxidierte Spezies $[(\mathrm{M}-2 \mathrm{H})+\mathrm{H}]^{+}$(rechts unten).

In Abbildung 113 sind die Spacefilling-Modelle von $\left[\mathbf{L}^{1} \mathbf{N i}_{2}(\mathbf{O H})\right]$ und $\left[\mathbf{L}^{3} \mathbf{N i}_{2}(\mathbf{O H})\right]$ gezeigt. Von außen ist der $\mu$-OH-Brückenligand zwischen den beiden Phenylresten kaum zugänglich. In $\left[\mathbf{L}^{3} \mathbf{N i}_{2}(\mathbf{O H})\right]$ sorgen die Isopropylreste an den Phenyleinheiten für eine zusätzliche sterische Abschirmung. An den Seiten der Komplexe erhöhen die Ethylgruppen die Sperrigkeit des Komplexes. So bestehen bei einem Austausch nur begrenzte Möglichkeiten für die Annäherung an den Brückenliganden. Ein möglicher Austausch kann am leichtesten direkt von oben bzw. unten erfolgen. 

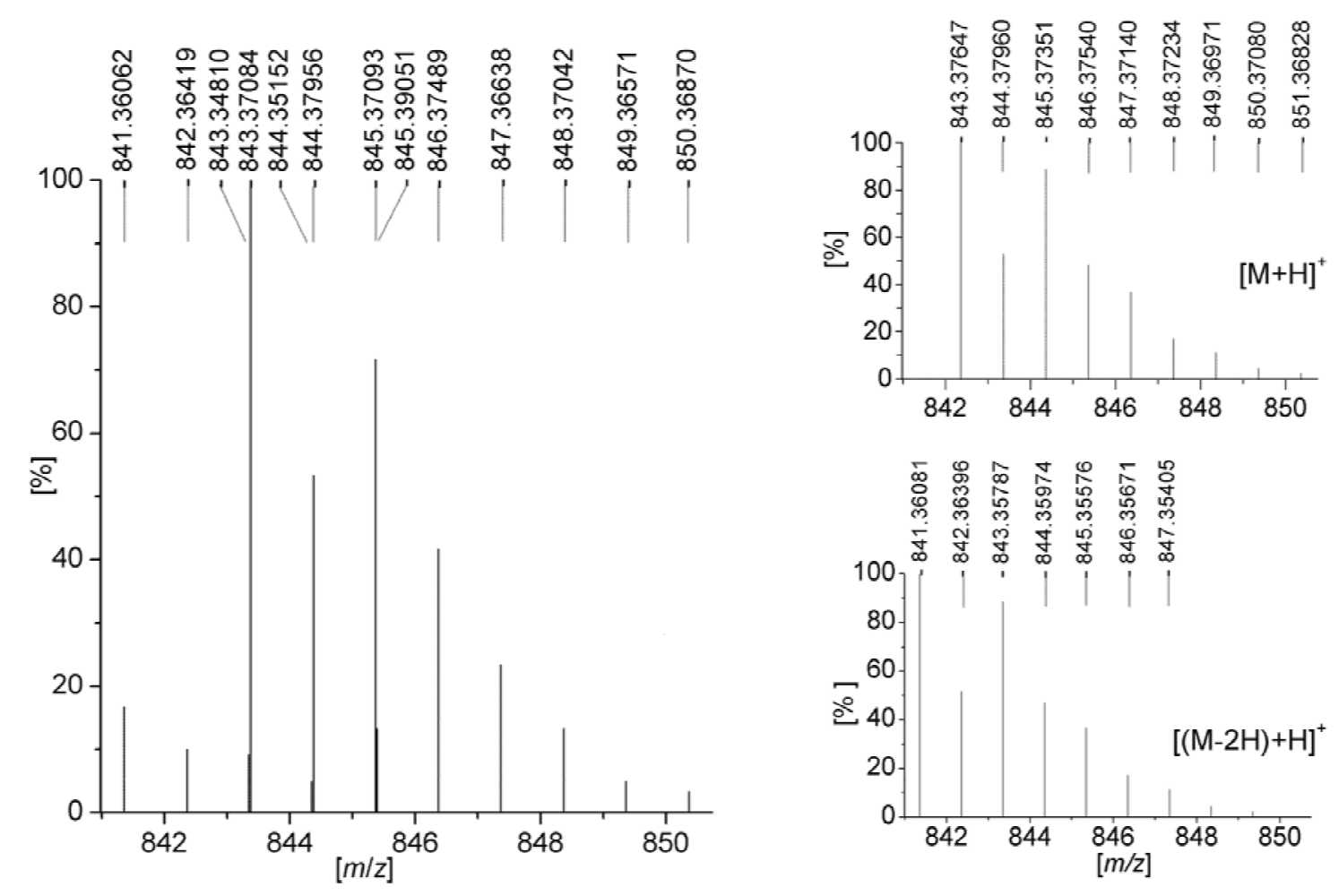

Abbildung 112: HR-Massenspektrum von $\left[\mathbf{L}^{3} \mathbf{N i}_{2}(\mathbf{O H})\right]$ in Methanol (links). Berechnete Isotopenmuster für $[\mathrm{M}+\mathrm{H}]^{+}$(rechts oben) und für die oxidierte Spezies $[(\mathrm{M}-2 \mathrm{H})+\mathrm{H}]^{+}$(rechts unten).

Die Spacefilling-Modelle erklären somit den relativ langsamen Austauschprozess nach Zugabe von $\mathrm{D}_{2} \mathrm{O}$ (Abbildung 113). Die OH-Gruppe im Komplexzentrum ist in das sperrige Ligandengerüst eingebettet und für Konkurrenz-Verbindungen wie das $\mathrm{D}_{2} \mathrm{O}$ schlecht zugänglich. Dies erklärt zudem, warum zwar ein OH/OD-Austausch mit deuteriertem Wasser beobachtet werden kann, aber nicht mit größeren Molekülen wie Methanol bzw. Methanolat. 

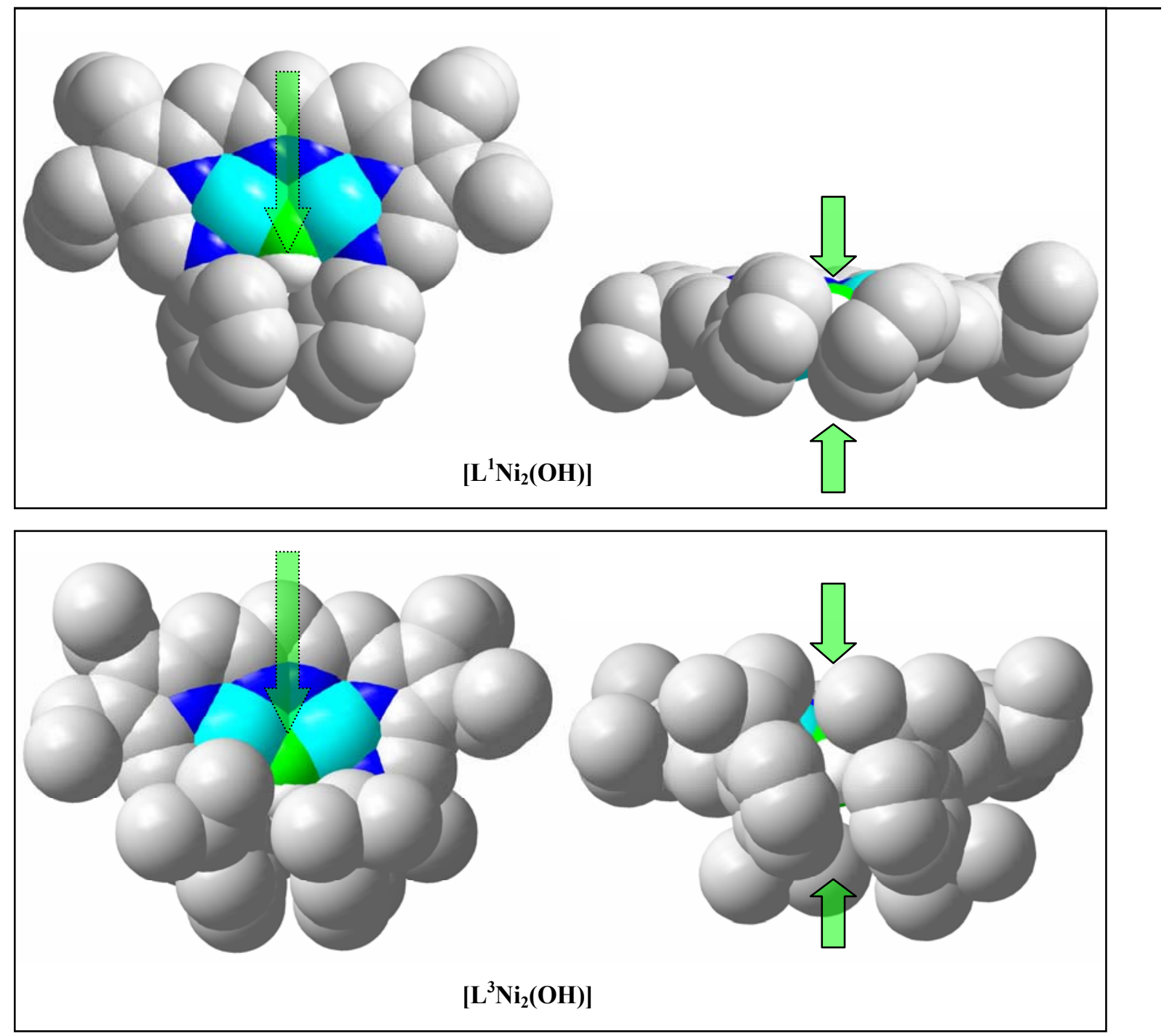

Abbildung 113: Spacefilling-Modelle von $\left[\mathbf{L}^{1} \mathbf{N i}_{2}(\mathbf{O H})\right]$ (oben) und $\left[\mathbf{L}^{3} \mathbf{N i}_{2}(\mathbf{O H})\right]$ (unten), jeweils Aufsicht und Frontansicht. 


\section{Zukunftsperspektiven}

Im Rahmen dieser Arbeit konnte gezeigt werden, dass mit den neuartigen BispyrrolpyrazolBausteinen (1a, 1b) eine Reihe von Hybrid-Makrozyklen und auch azyklischen Systemen erfolgreich synthetisiert werden konnten.

Zukünftige Forschungsarbeiten dürften sich außerdem darauf konzentrieren, Pyrazol-basierte Grundbausteine zu synthetisieren, welche eine Arylgruppe an den Substituenten in den 3,5Positionen des Pyrazols tragen (LVIIa und LVIIb). Durch gute Abgangsgruppen (X = Halogene oder Tosyl) können die Grundbausteine LVIIa bzw. LVIIb in einer Substitutionsreaktion mit unterschiedlichen $\alpha$-unsubstituierten Pyrrolderivaten (z.B. 3,4Diethylpyrrol) den Bispyrrolpyrazol-Baustein (LVI) ergeben. Bei nachfolgender Synthese von LVI könnte dann eventuell das „Doppelporphyrin“ 3j synthetisiert werden (Abbildung 114).
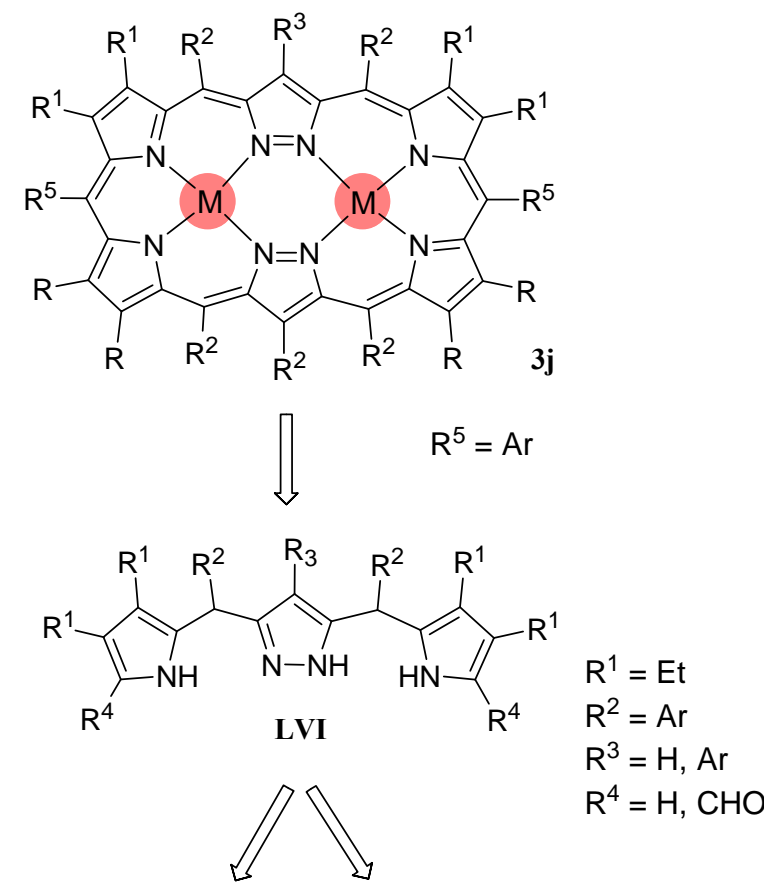

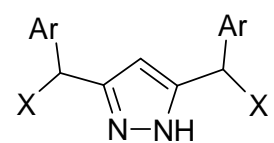

LVIIa

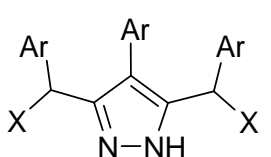

LVIIb

Abbildung 114: Retrosynthese eines „Doppelporphyrins“ 3j aus neuen Pyrazol-basierten Grundbausteinen LVIIa und LVIIb mit arylischen Substituenten an der 3,5-Bismethyl-Position des Pyrazols. 
Die Arylsubstituenten an den 3,5-Substituenten der Pyrazoleinheit in LVI würden die Oxidation des entstandenen Makrozyklus bei einer Zyklisierungsreaktion erleichtern. Somit könnte ein neuartiges Pyrazol-basiertes aromatisches Doppelporphyrin 3j mit $26 \pi$ Elektronen geschaffen werden.

Das Doppelporphyrin 3j besitzt zwei „Bindungstaschen“, in denen zwei Metallionen koordinativ gebunden werden können. Hierbei bietet es sich an, bimetallische Komplexe zu synthetisieren und die kooperativen Eigenschaften der Metallatome im System zu untersuchen.

Seit den 90ern ist das sog. Anthraphyrin (LVIII) bekannt, welches bei einer Schiff-BasenKondensation zwischen dem 1,8-Diaminoanthracen und einem Diformyldipyrrolderivat synthetisiert werden kann. ${ }^{103}$ Analog zum nicht-aromatischen Makrozyklus LVIII könnten Pyrrol/Pyrazol-basierte Schiff-Base Makrozyklen des Typs 2k dargestellt werden (Abbildung 115). 2k bietet einen viel größeren Hohlraum als die bisher synthetisierten Makrozyklen (z. B.

2a bis $\mathbf{2 f}$ ) und könnte somit selektiv an unterschiedliche Metallionen koordinieren.

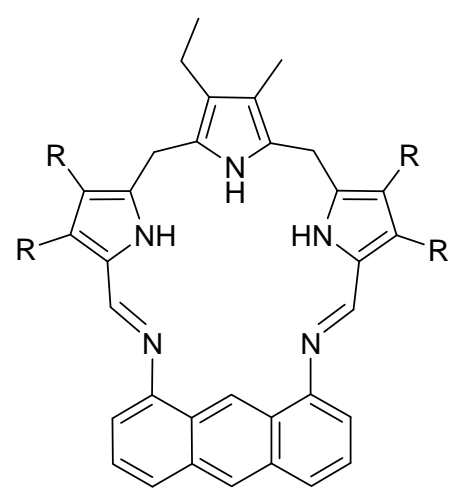

LVIII

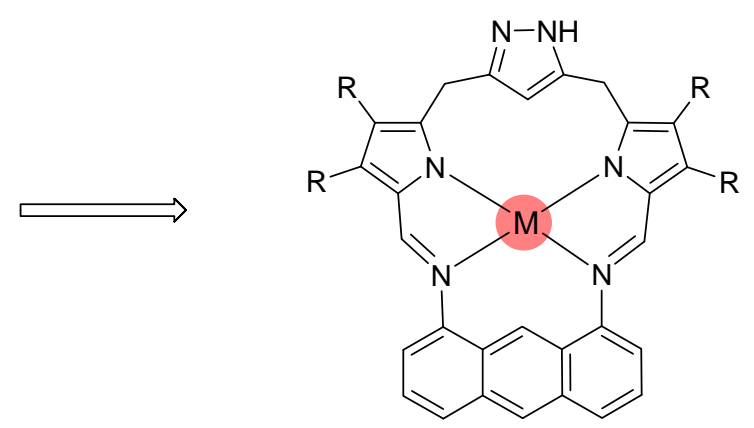

$2 k$

Abbildung 115: Anthraphyrin ${ }^{103}$ (LVIII) und Pyrazol-basiertes Anthraphyrin-Analogon (2k).

Expandierte Porphyrine und Porphyrinogenanaloga können als Anionenrezeptoren wirken. ${ }^{104}$ Damit können sie in zahlreichen Anwendungen wie Anionendetektion bzw. - transport und in der chromatographischen Anionenreinigung interessant sein.

Eine weitere interessante Zukunftsperspektive ist die Synthese eines Pyrrol/Pyrazol-basierten Hybrid-Makrozyklus 2m. Gale et al. berichteten kürzlich über die neuartigen Hybrid Harnstoff/Amid-Makrozyklen (LVIV), die selektiv Anionen binden können. ${ }^{105}$ Der HybridMakrozyklus 2m bietet eine Kavität mit mehreren H-Brücken und könnte als vielversprechender Anionenrezeptor eingesetzt werden (Abbildung 116). 


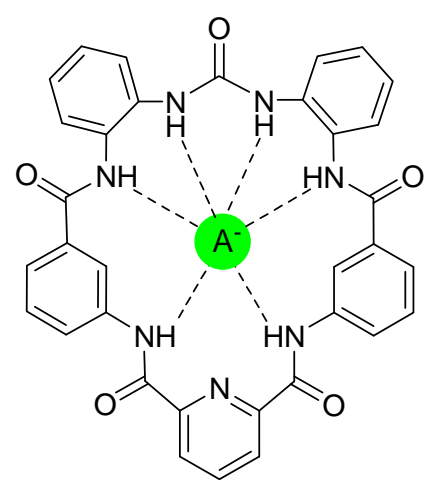

LVIV

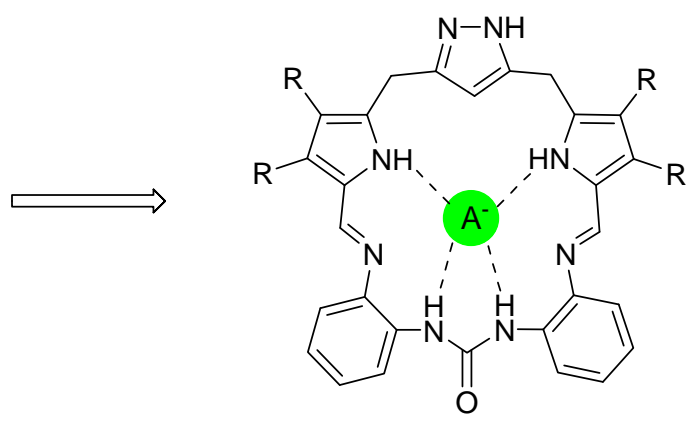

$2 \mathrm{~m}$

Abbildung 116: Hybrid Harnstoff/Amid Makrozyklus (LVIV) ${ }^{105}$ und Pyrrol/Pyrazol-basierter Hybrid Makrozyklus (2m) als Anionenrezeptoren.

Wie in dieser Arbeit diskutiert wurde zeigen die bimetallischen Komplexe $\left[\mathbf{L}^{\mathbf{x}} \mathbf{M}_{2} \mathbf{X}\right]$ interessante spektroskopische Eigenschaften. Zukünftige Forschungsarbeiten dürften sich darauf konzentrieren, den Mechanismus des ungewöhnlich langsam verlaufenden $\mu$-XAustausches zu untersuchen. Statt aromatischer Amine an den Imin-Seitenarmen der Liganden könnten weniger sperrige aliphatische Amine verwendet werden. Hierbei wäre die Änderung der chemischen Verschiebung des $\mu$-OH-Brückenliganden in der ${ }^{1} \mathrm{H}-\mathrm{NMR}-$ Spektroskopie und die Austauschgeschindigkeit der $\mathrm{OH}-\mathrm{Gruppe}$ von Interesse. 


\section{Zusammenfassung}

Die vorliegende Arbeit befasst sich mit der Synthese neuartiger Pyrrol/Pyrazol-basierter zyklischer und azyklischer Verbindungen. $\mathrm{Zu}$ den zyklischen Verbindungen gehören das expandierte Porphyrin, genannt „Doppelporphyrin“ und die nicht-aromatischen Schiff-Basen Makrozyklen (Abbildung 117, A und B). Expandierte Porphyrine sind synthetische Analoga der Porphyrine, die sich von den „klassischen“ Porphyrinen (Tetrapyrrolen) durch einen größeren zentralen Ring mit mindestens 17 Atomen unterscheiden. Durch Erweiterung des Makrozyklus entstehen neuartige Verbindungen mit besonderen spektralen und elektronischen Eigenschaften. ${ }^{21}$ Ziel dieser Arbeit war die Synthese und Charakterisierung der in Abbildung 117 schematisch gezeigten neuen Verbindungen. Hierbei sollten auch die spektrophotochemischen Eigenschaften bzw. Komplexbildungseigenschaften und in einigen Fällen die Fähigkeit der nicht-aromatischen Schiff-Basen Makrozyklen, in bestimmten Protonierungszuständen Anionen zu binden, untersucht werden.

"Doppelporphyrine"

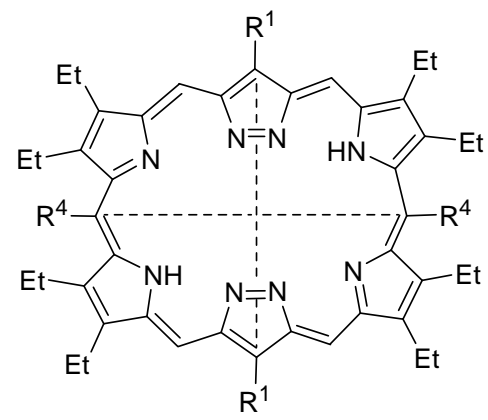

(3)

$$
\begin{aligned}
& \mathrm{R}^{1}=\mathrm{H} \\
& \mathrm{R}^{4}=\mathrm{H}, \mathrm{Ar}
\end{aligned}
$$<smiles>CCCCC</smiles><smiles>CCc1[nH]c(C=O)c(CC)c1CC</smiles><smiles>[R]c1c(C)n[nH]c1Cc1[nH]c(C=O)c(CC)c1CC</smiles>
$\mathrm{R}^{1}=\mathrm{H}(\mathbf{1} \mathbf{a}), \mathrm{Ph}(\mathbf{1 b})$

C

Azyklische Diimin-Liganden

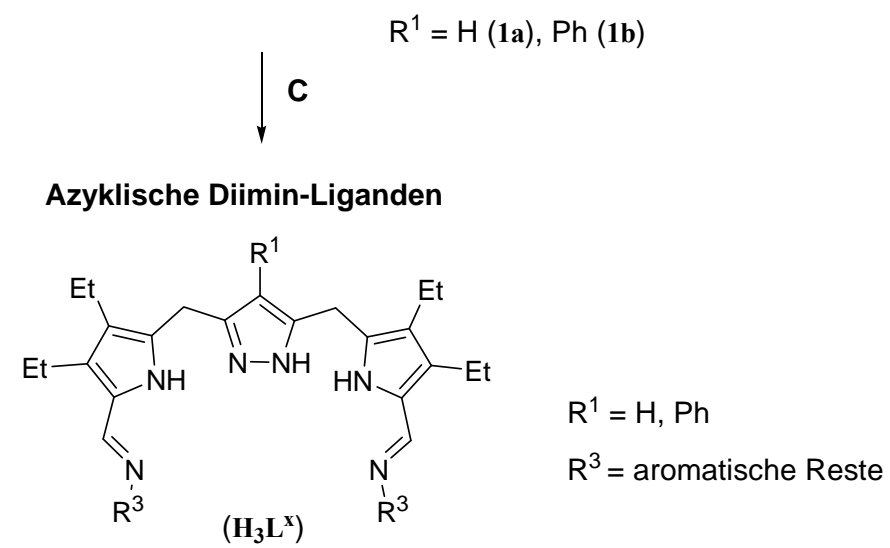

Schiff-Base Makrozyklen

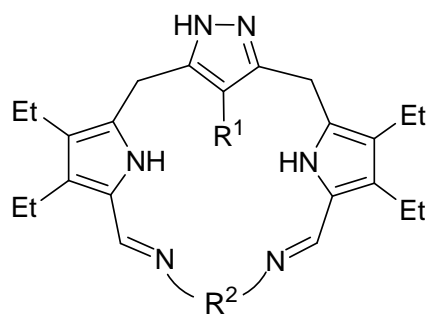

(2)

$$
\begin{aligned}
\mathrm{R}^{1} & =\mathrm{H} \\
\mathrm{R}^{2}= & \text { aliphatische oder } \\
& \quad \text { aromatische Reste }
\end{aligned}
$$

Abbildung 117: Übersicht der in dieser Arbeit neu synthetisierten Verbindungen. 
Als azyklische Systeme wurden die Pyrrol/Pyrazol-basierten Diimin-Liganden synthetisiert (C, Abbildung 117), die erfolgreich in der Komplexsynthese von bimetallischen Systemen eingesetzt wurden. Die Synthese der Bausteine, der Liganden und der bimetallischen Komplexe werden im Rahmen dieser Arbeit erstmals vorgestellt. ${ }^{106}$

Die Grundbausteine (1a und 1b) wurden in einer mehrstufigen Synthese aus dem Literatur bekannten 3,5-Bis-(chlormethyl)pyrazol Hydrochlorid (XXIX) $)^{33}$ bzw. aus in dieser Arbeit neu synthetisiertem 3,5-Bis-(chlormethyl)-4-phenyl-pyrazol Hydrochlorid (4) ${ }^{45,46}$ hergestellt (Abbildung 118).
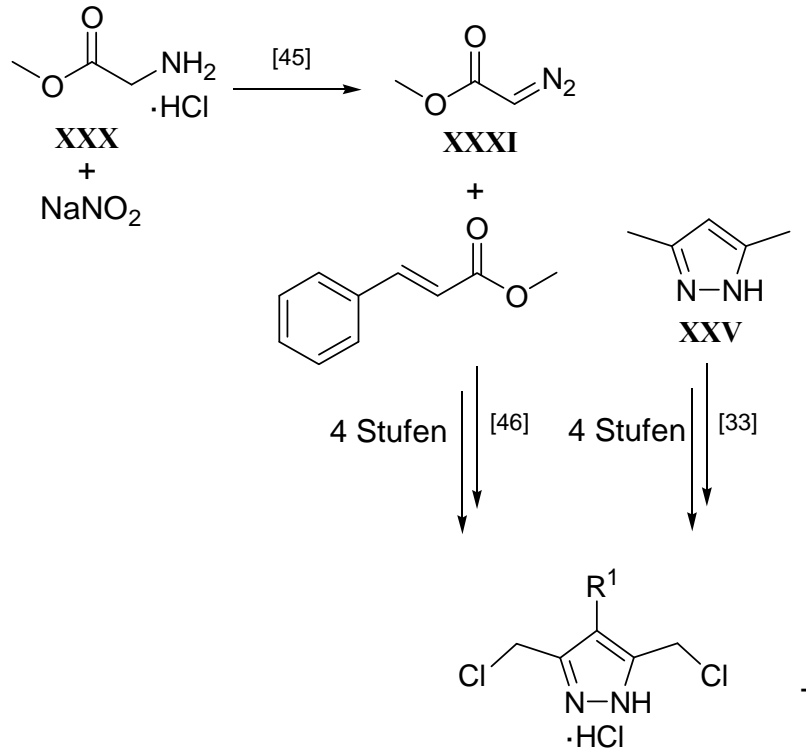

$\mathrm{R}^{1}=\mathrm{H}(\mathbf{X X I X )}, \mathrm{Ph}(4)$
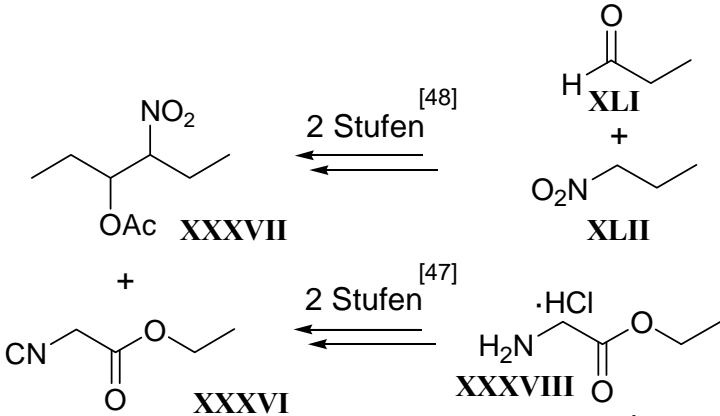<smiles>COC(=O)OC</smiles>

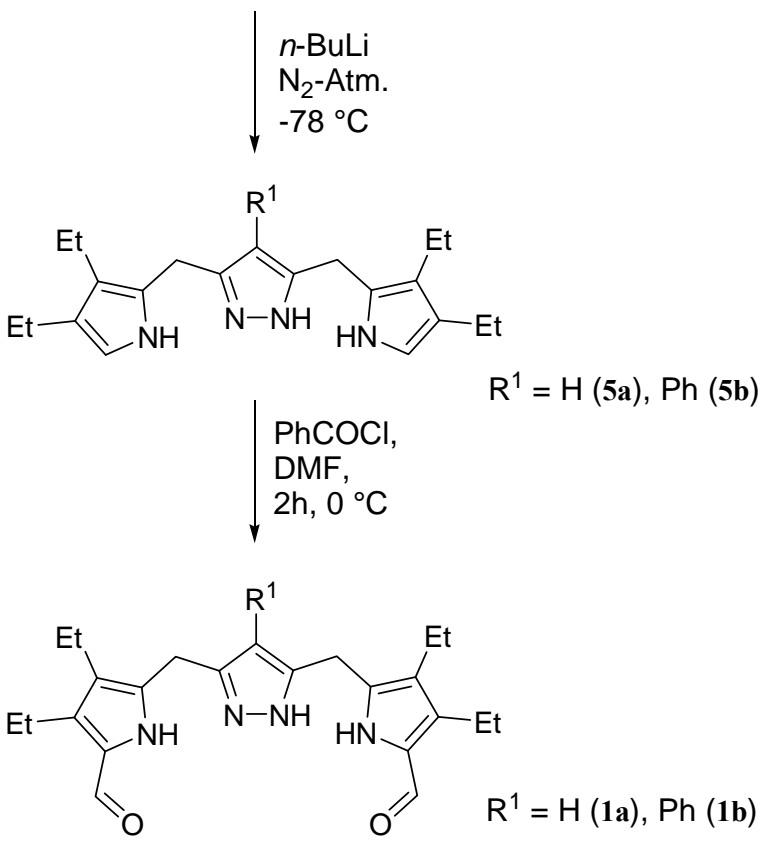

Abbildung 118: Syntheseweg zur Darstellung der Grundbausteine (1a bzw. 1b). 
Pyrrolderivate sind dafür bekannt, dass sie unter Luftzutritt schnell verharzen. Infolgedessen war die Synthese und anschließende Isolierung der Pyrrol/Pyrazol-Derivate (1a, 1b, 5a, und 5b) beschwerlich. Dennoch konnten die erwähnten Verbindungen in relativ hohen Ausbeuten erhalten werden, nachdem die Synthesebedingungen in einer Reihe von Experimenten optimiert wurden.

Im Rahmen dieser Arbeit wurde eine enorme Zahl an unterschiedlichen Zyklisierungsreaktionen zur Darstellung eines Doppelporphyrins mit dem Grundbaustein (1a) durchgeführt. Das Doppelporphyrin kann am erfolgreichsten nach Reduktion des Bisaldahys (1a) zum Bisalkohol (6) in einer [2+2]-Zyklokondensation mit dem $\alpha$-unsubstituierten Dipyrropyrazolderivat (5a) synthetisiert werden. Massenspektroskopisch (HR-MS) konnte der

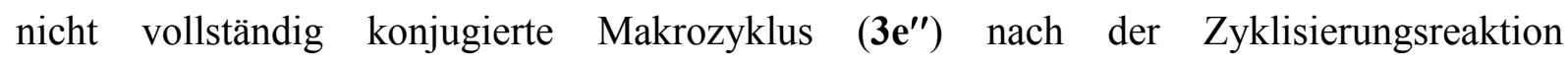
nachgewiesen werden (Abbildung 25, Kapitel 5.1). Der Makrozyklus 3e" ist zwar an den meso-Positionen der Dipyrrolmethan-Einheit oxidiert, aber nicht an den $\mathrm{CH}_{2}{ }^{\mathrm{py} / \mathrm{pz}-}$ Brücken. Unter anderem stellte sich heraus, dass die in der Porphyrinchemie häufig verwendeten Oxidationsmittel DDQ und $p$-Chloranil zur Aromatisierung von Pyrazolderivaten nicht geeignet sind. Auch durch die Anwendung von weiteren Oxidationsmitteln, wie Brom, $\mathrm{Pd} / \mathrm{C}$, $\mathrm{Ag}_{2} \mathrm{O}$ oder $o$-Iodylbenzoesäure $\left(\mathrm{IBX}^{53}\right)$ konnte die Pyrrol/Pyrazol- $\mathrm{CH}_{2}$-Bindung nicht oxidiert werden. Um die Oxidation der $\mathrm{CH}_{2}{ }^{\mathrm{py} / \mathrm{pz}}$-Brücken zu erleichtern, dürften sich zukünftige Forschungsarbeiten auf die Synthese von neuartigen Bausteinen konzentrieren, die Arylsubstituenten an den Armen in den 3,5-Positionen der Pyrazoleinheit tragen. Dies würde die Oxidation des entstandenen Makrozyklus nach einer Zyklisierungsreaktion möglicherweise erleichtern.

Ziel des zweiten Projektes war die Synthese von neuartigen Pyrrol/Pyrazol-basierten SchiffBase Makrozyklen (2). Diese Hybrid-Makrozyklen wurden aus einer Kondensationsreaktion von 1a mit einer Reihe aromatischer bzw. aliphatischer Diamine synthetisiert. Durch Einsatz von Ausgangsverbindungen wie 1,2,4,5-Tetraaminobenzol Tetrahydrochlorid (d) oder 3,3'Diaminobenzidin (e) konnten außerdem biszyklische Hybrid-Makrozyklen (2d und 2e) dargestellt werden.

Die Schiff-Base Makrozyklen wurden auf ihre spektroskopischen Eigenschaften mittels UV/Vis-Spektroskopie untersucht. Hierbei konnte beobachtet werden, dass durch Protonierung der Chromophore der elektronische Zustand der nicht aromatischen Makrozyklen verändert wird. Die Makrozyklen werden durch Säuren an einer der IminEinheiten protoniert und können aufgrund dessen als Protonen-Sonden fungieren. Der Makrozyklus 2a zeigt im UV/Vis-Spektrum eine Bande bei $331 \mathrm{~nm}$ mit einer Schulter bei 
$374 \mathrm{~nm}$. Bei der Titration von 2a mit einer Säure nimmt die Intensität dieser Bande ab und eine Bande bei $362 \mathrm{~nm}$ wächst an (Abbildung 119). Durch Titration von 2a mit unterschiedlichen starken Säuren konnte festgestellt werden, dass nicht nur eine bathochrome Verschiebung $\mathrm{zu}$ beobachten ist, sondern je nach Säurestärke auch das Ausmaß des hyperchromen Effektes bei $362 \mathrm{~nm}$ beeinflusst wird. Der Protonierungsprozeß läuft hierbei reversibel ab.

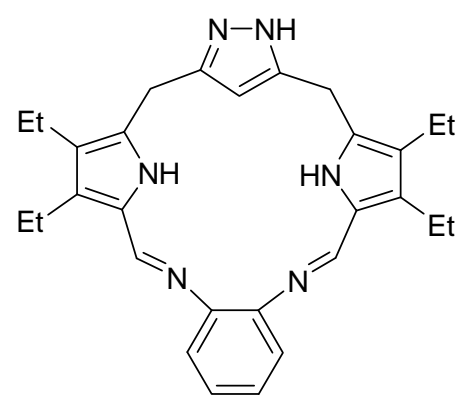

$\mathbf{2 a}$
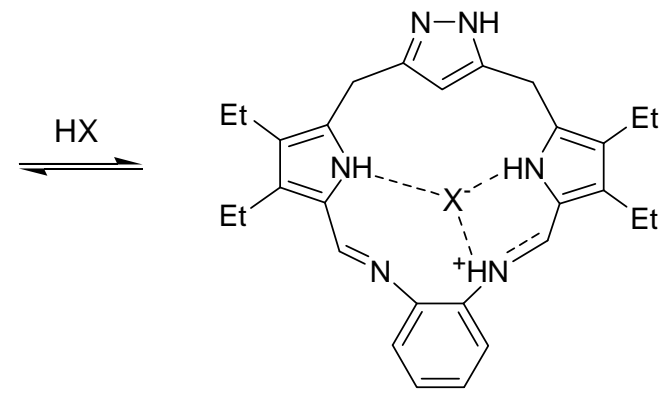

$[2 \mathrm{aH}]^{+}$

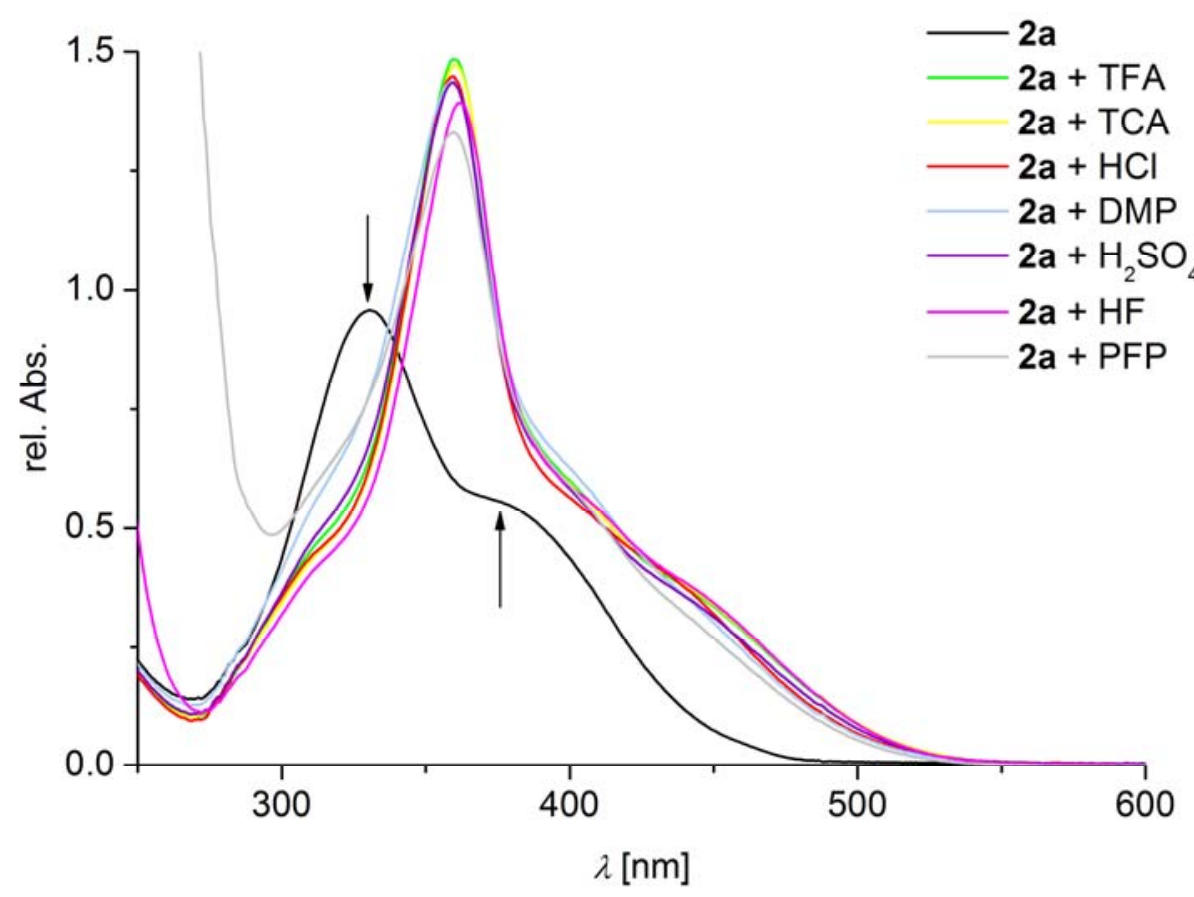

Abbildung 119: UV/Vis-Spektrum von 2a (schwarze Linie) in freier sowie protonierter Form. Die Protonierung erfolgt mit unterschiedlichen Säuren in THF und bei Raumtemperatur.

Der Makrozyklus wurde mit unterschiedlich starken Säuren titriert. Bei allen durchgeführten UV/Vis-spektrophotometrischen Titrationen von 2a konnte ein isosbestischer Punkt bei $344 \mathrm{~nm}$ beobachtet werden. Dies ist ein Hinweis auf den Ablauf einer einheitlichen Protonierungsreaktion des Makrozyklus. Je stärker die ausgewählte Säure, desto intensiver ist 
der hyperchrome Effekt bei ca. $362 \mathrm{~nm}$. In Tabelle 21 sind die unterschiedlichen Säuren, die bei den Titrationen mit 2a verwendet wurden und die zugehörigen $\mathrm{pK}_{\mathrm{a}}$-Werte aufgelistet. Die angegebenen $\mathrm{pK}_{\mathrm{a}}$-Werte wurden in den meisten Fällen in Wasser bestimmt und können nicht direkt mit den hier durchgeführten Messungen in THF verglichen werden. Es besteht jedoch eine gute Übereinstimmung zwischen den Ergebnissen der UV/Vis-Messungen von 2a (Abbildung 119) und dem Trend der $\mathrm{pK}_{\mathrm{a}}$-Werte.

Wie die UV/Vis-Untersuchungen der Schiff-Base Makrozyklen zeigen, eignen sie sich hervorragend als Protonen-Sonde. Schon geringe Spuren saurer Verbindungen können detektiert werden. Anschießend kann die Säurestärke dieser Verbindungen relativ zu bekannten Säuren abgeschätzt werden.

Tabelle 21: Übersichtstabelle der UV/Vis-Banden von 2a nach Protonierung mit unterschiedlichen Säuren in Chloroform $\left(* \mathrm{pK}_{\mathrm{a}}\right.$ Werte wurden in den meisten Fällen in Wasser bestimmt).

\begin{tabular}{|c|c|c|}
\hline Säure & $\mathbf{p K}_{\mathbf{a}}{ }^{*}$ & Banden $[\mathbf{n m}]$ \\
\hline $\mathrm{TFA}$ & $0.52^{69}$ & $362,411(\mathrm{sh}), 444(\mathrm{sh})$ \\
\hline $\mathrm{TCA}$ & $0.77^{107}$ & $364,406(\mathrm{sh}), 447(\mathrm{sh})$ \\
\hline $\mathrm{DMP}$ & $0.47 / 1.88^{70}$ & $363,410(\mathrm{sh}), 449(\mathrm{sh})$ \\
\hline $\mathrm{HCl}$ & $1.92^{71}$ & $366,410(\mathrm{sh}), 445(\mathrm{sh})$ \\
\hline $\mathrm{H}_{2} \mathrm{SO}_{4}$ & $-3.0 / 1.99^{108}$ & $365,408(\mathrm{sh}), 447(\mathrm{sh})$ \\
\hline $\mathrm{HF}$ & $3.25^{71}$ & $363,409(\mathrm{sh}), 450(\mathrm{sh})$ \\
\hline $\mathrm{PFP}$ & $4.10^{72}$ & $362,406(\mathrm{sh}), 449(\mathrm{sh})$ \\
\hline
\end{tabular}

Einige der neu synthetisierten Pyrrol/Pyrazol-basierten Schiff-Base Makrozyklen konnten röntgenographisch charakterisiert werden. Im Fall von 2a ist es gelungen, sowohl die freie Base, als auch die mit TFA protonierte Spezies [2a·TFA] röntgenographisch zu charakterisieren (Abbildung 120). Die Molekülstrukturen zeigen in allen Fällen, dass der Pyrazolring nicht in die Kavität des Makrozyklus gerichtet ist, sondern mit den N-Atomen nach ,außen“ gedreht ist. Über dem Makrozyklus-Hohlraum kann sich ein neutrales oder auch ein geladenes Teilchen platzieren und mit den vorhanden Imin-N- bzw. NH-Atomen der Pyrroleinheiten Wasserstoffbrückbindungen ausbilden. 


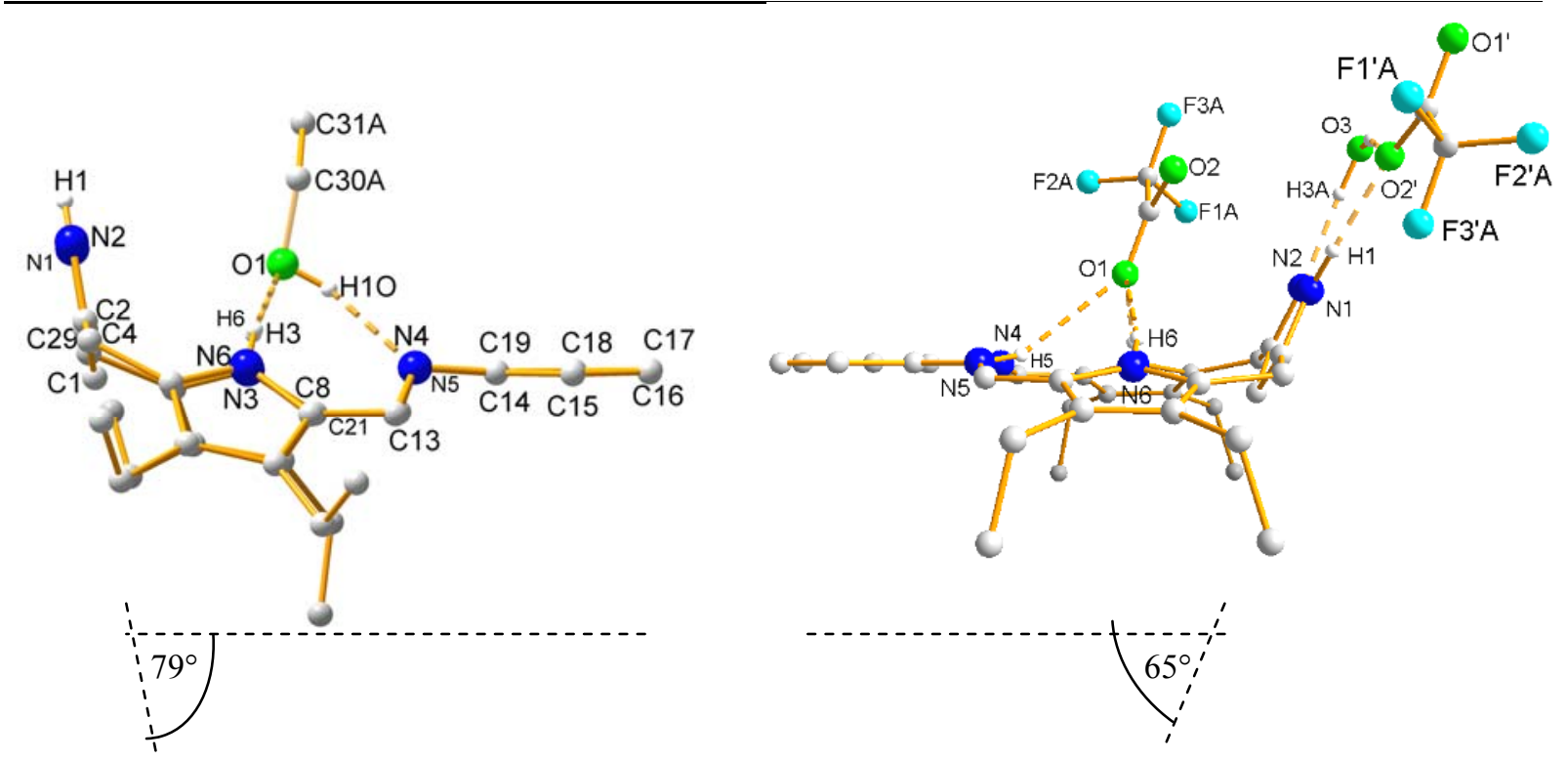

Abbildung 120: Molekülstruktur von [2a·EtOH] (links) und [2a·TFA] (rechts). Mit der gestrichelten Linie wird der im Text angegebene Winkel zwischen Pyrazolring und der planaren Imin-Phenyleinheit illustriert.

Der Makrozyklus hat die besondere Funktion als Protonenakzeptor und -donator dienen zu können. Wie die Studien der UV/Vis-Titration gezeigt haben, untermauern die Festkörperstrukturen der Schiff-Base Makrozyklen die Annahme der Monoprotonierung einer der Imin-Positionen. In Tabelle 22 sind die Abstände der C=N-Imin-Bindung und der Winkel zwischen Pyrazolring und der planaren Imin-Phenyleinheit wiedergegeben.

Aus den aufgelisteten Abständen in Tabelle 22 kann entnommen werden, dass die protonierte Imin-Position in [2a·TFA] (N5-C20), [2b·TFA] (N4-C13) und [2c·TFA] (N4-C13) einen längeren Bindungsabstand besitzt, als die unprotonierte Position. Wie die Molekülstruktur von [2a·TFA] zeigt, gewinnt der Makrozyklus durch die Protonierung des Imins an Planarität. Die Konformationsumwandlung von 2a während der Titration wurde in einer Reihe von NMR-Untersuchungen studiert.

Wie bereits bei den Doppelporphyrinen gefunden wurde, konnten auch die Schiff-Base Makrozyklen (2) in einer Reihe unterschiedlicher Oxidationsreaktion nicht an der $\mathrm{CH}_{2}{ }^{\mathrm{py} / \mathrm{pz}}-$ Verknüpfungsstelle oxidiert werden. 
Tabelle 22: Bindungslängen der Imin-Position bzw. Winkel zwischen dem Pyrazolring und der planaren IminPhenyleinheit der röntgenographisch charakterisierten Schiff-Base Makrozyklen.

\begin{tabular}{|c|c|c|c|}
\hline Verbindung & $\begin{array}{c}\text { Imin } 1 \\
{[\AA ̊]}\end{array}$ & $\begin{array}{c}\text { Imin } 2 \\
{[\AA ̊]}\end{array}$ & $\begin{array}{c}\text { Winkel zwischen } \\
\text { Pyrazolring und der } \\
\text { planaren Imin- } \\
\text { Phenyleinheit }\left[{ }^{\circ}\right] \\
\end{array}$ \\
\hline 2a·EtOH & $\begin{array}{c}\mathrm{N}(4)-\mathrm{C}(13) \\
1.283(3)\end{array}$ & $\begin{array}{c}\mathrm{N}(5)-\mathrm{C}(20) \\
1.278(3)\end{array}$ & 79 \\
\hline 2a.TFA & $\begin{array}{c}\mathrm{N}(4)-\mathrm{C}(13) \\
1.290(3)\end{array}$ & $\begin{array}{c}\mathrm{N}(5)-\mathrm{C}(20) \\
1.319(3)\end{array}$ & 65 \\
\hline 2b-TFA & $\begin{array}{c}\mathrm{N}(4)-\mathrm{C}(13) \\
1.320(4)\end{array}$ & $\begin{array}{c}\mathrm{N}(5)-\mathrm{C}(22) \\
1.284(4)\end{array}$ & 68 \\
\hline $2 c \cdot T F A$ & $\begin{array}{c}\mathrm{N}(4)-\mathrm{C}(13) \\
1.321(6)\end{array}$ & $\begin{array}{c}\mathrm{N}(5)-\mathrm{C}(24) \\
1.307(6)\end{array}$ & 88 \\
\hline
\end{tabular}

Die Pyrrol/Pyrazol-basierten Makrozyklen wurden auch als Chelatliganden zur Darstellung von Metallkomplexen eingesetzt. Die Ergebnisse der Forschungsarbeiten konnten demonstrieren, dass durch die relativ hohe Flexibilität des Pyrazolrings die Koordination eines Metalls verhindert wird. Durch die Rotation des Pyrazolrings um die $\mathrm{CH}_{2}{ }^{\mathrm{py} / \mathrm{pz}}-$ Bindungen wird das Metall aus der Kavität des Makrozyklus herausgedrängt. Zusätzlich bietet der relativ kleine Hohlraum des Makrozyklus nur begrenzt Platz für ein koordiniertes Metall.

Neben den Makrozyklen wurden in einem weiteren Projekt eine Reihe von Pyrrol/Pyrazolbasierten azyklischen Diiminliganden hergestellt (C, Abbildung 117). Analog zu den Pyrrol/Pyrazol-Bausteinen (1a, 1b, 5a und 5b) hat sich die Isolierung der azyklischen Liganden $\left(\mathbf{H}_{\mathbf{3}} \mathbf{L}^{\mathbf{1}}\right.$ bis $\left.\mathbf{H}_{\mathbf{3}} \mathbf{L}^{\mathbf{5}}\right)$ ebenfalls als anspruchsvoll erwiesen.

Die neuartigen Liganden $\left(\mathbf{H}_{3} \mathbf{L}^{\mathbf{1}}\right.$ bis $\left.\mathbf{H}_{\mathbf{3}} \mathbf{L}^{\mathbf{5}}\right)$ wurden in der Komplexsynthese zur Darstellung von zweikernigen Metallkomplexen eingesetzt. Nur Metalle (Cu(II), Ni(II) und Pd(II)), die eine quadratisch planare Koordination eingehen können bzw. aufgrund ihres elektronischen Zustands bevorzugen, können bimetallische Komplexe mit den Liganden $\mathbf{H}_{\mathbf{3}} \mathbf{L}^{\mathbf{1}}$ bis $\mathbf{H}_{\mathbf{3}} \mathbf{L}^{\mathbf{5}}$ eingehen. In Abbildung 121 sind in dieser Arbeit röntgenographisch charakterisierte Metallkomplexe dargestellt.

Die zwei Metallionen (Pd(II), Ni(II) bzw. Cu(II)) im bimetallischen Komplex nehmen eine quadratisch planare Koordination ein. Die röntgenographische Charakterisierung der Metallkomplexe konnte in den meisten Fällen zeigen, dass zwischen den zwei Metallatomen 
ein $\mu$-OH-Molekül koordiniert; die einzige Ausnahme stellt der zweikernige Palladium(II)komplex $\left[\mathbf{L}^{3} \mathbf{P d}_{2} \mathbf{C l}\right]$ dar, der einen $\mu$-Cl-Brückenliganden aufweist. Der MetallMetall-Abstand der Komplexe ist für Pyrazolat-verbrückte bimetallische Komplexe mit 3.31$3.62 \AA$ relativ klein. ${ }^{87,88}$ In einem Fall wurde bei langsamer Kristallisation des Komplexes ([ $\left.\mathbf{L}{ }^{1} \mathbf{C u}_{2}(\mathbf{O H})\right]$ ) an der Luft die $\mathrm{CH}_{2}$-Gruppen an den 3,5-Positionen des Pyrazols $\left(\left[\mathbf{L}^{1}{ }_{\mathbf{o x}} \mathbf{C} \mathbf{u}_{\mathbf{2}}(\boldsymbol{\mu}\right.\right.$ $\mathrm{OH})]$ ) oxidiert.

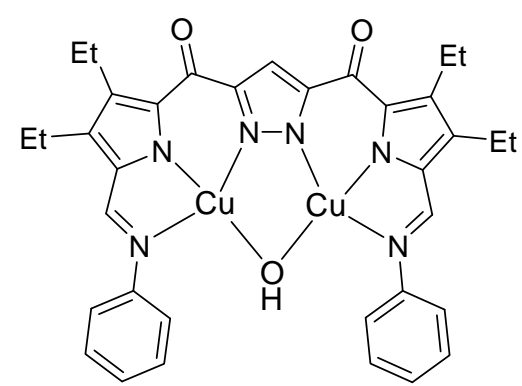

$\left[\mathbf{L}^{1}{ }_{\text {ox }} \mathrm{Cu}_{2}(\mathbf{O H})\right]$

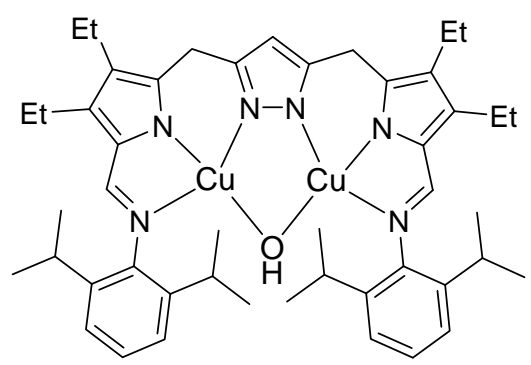

$\left[\mathbf{L}^{3} \mathrm{Cu}_{2}(\mathrm{OH})\right]$

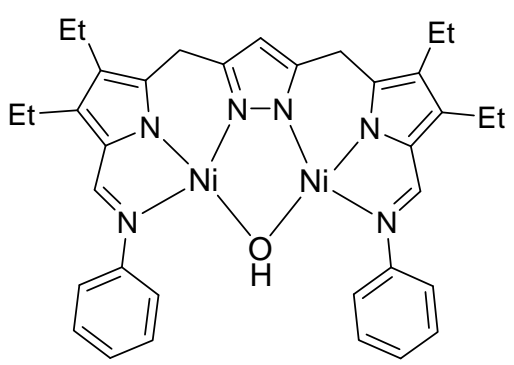

$\left[\mathbf{L}^{1} \mathbf{N i}_{2}(\mathbf{O H})\right]$

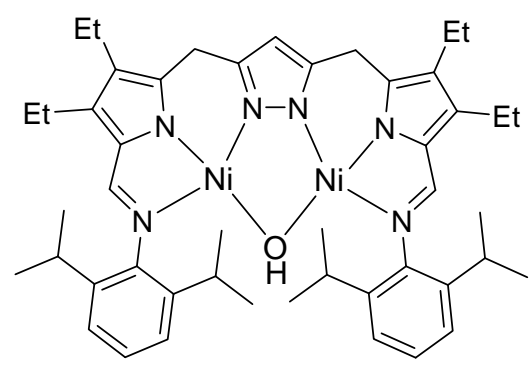

$\left[\mathbf{L}^{3} \mathrm{Ni}_{2}(\mathbf{O H})\right]$

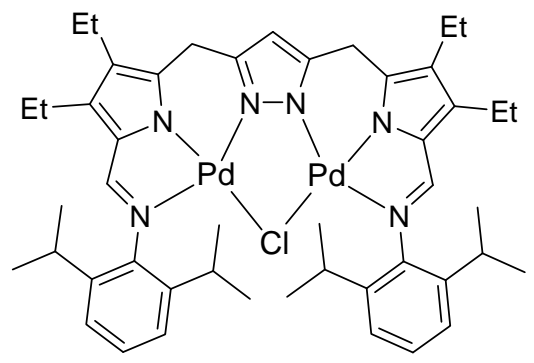

$\left[\mathbf{L}^{3} \mathbf{P d}_{2} \mathbf{C l}\right]$

Abbildung 121: Röntgenographisch charakterisierte bimetallische Komplexe.

Ungewöhnlich ist die chemische Verschiebung der $\mu$-OH-Gruppe der Nickel(II)komplexe in der ${ }^{1}$ H-NMR-Spektroskopie. Die OH-Signale der Nickel(II)komplexe ([ $\left.\mathbf{L}^{1} \mathbf{N i}_{\mathbf{2}}(\mathbf{O H})\right]$ und $\left[\mathbf{L}^{3} \mathbf{N i}_{\mathbf{2}}(\mathbf{O H})\right]$ ) erscheinen im ${ }^{1} \mathrm{H}$-NMR-Spektrum aufgrund ihrer Lage zwischen den Phenylringen stark im Hochfeld verschoben zwischen $\delta=-5.7$ bis $-6.7 \mathrm{ppm}$. 
Hierbei war es von großem Interesse den Austausch der $\mu$-OH-Gruppe der Nickelkomplexe mit weiteren Anionen zu untersuchen (Abbildung 122). Während der Austausch von $\mathrm{OH}^{-}-$ Ionen gegen $\mathrm{Cl}^{-}$-Ionen sofort stattfand, konnte über ${ }^{1} \mathrm{H}-\mathrm{NMR}-$ Spektroskopie ein relativ langsamer Austausch der OH- zur OD-Gruppe ermittelt werden. Die Existenz der $\mu$-ODGruppe in den Nickelkomplexen nach der Reaktion wurde auch mittels Massenspektrometrie und IR-Spektroskopie nachgewiesen.

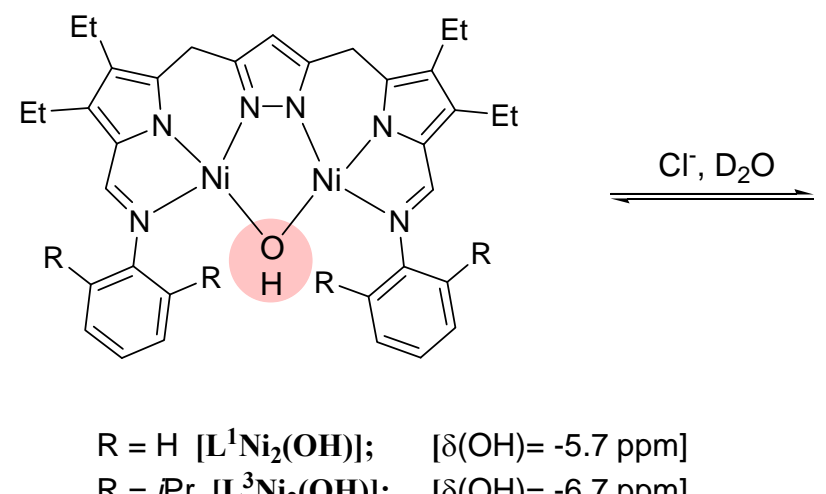

$\mathrm{R}=i \operatorname{Pr}\left[\mathbf{L}^{\mathbf{3}} \mathbf{N i}_{\mathbf{2}}(\mathbf{O H})\right] ; \quad[\delta(\mathrm{OH})=-6.7 \mathrm{ppm}]$

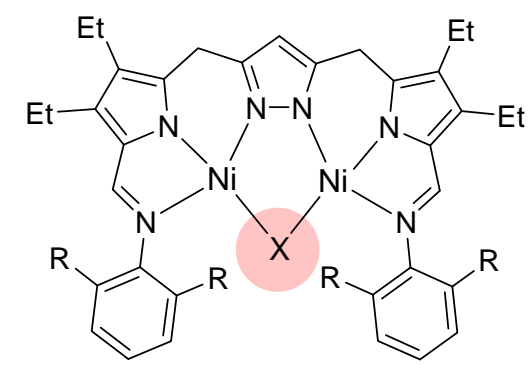

$\mathrm{X}=\mathrm{Cl}, \mathrm{OD}^{*}$

(* aus $\mathrm{D}_{2} \mathrm{O}$ )

Abbildung 122: Austauschreaktionen des OH-Brückenliganden gegen $\mathrm{Cl}^{-}$bzw. $\mathrm{OD}^{-}$in den Nickel(II)komplexen $\left[\mathbf{L}^{1} \mathrm{Ni}_{2}(\mathrm{OH})\right]$ und $\left[\mathbf{L}^{3} \mathrm{Ni}_{2}(\mathrm{OH})\right]$.

Die der $\mu$-OH-Gruppe in $\left[\mathbf{L}^{3} \mathbf{N i}_{\mathbf{2}}(\mathbf{O H})\right]$ zuordnende Bande wurde bei $3607 \mathrm{~cm}^{-1}$ gefunden. Nach dem Austausch mit $\mathrm{D}_{2} \mathrm{O}$ ist die Bande bei $3607 \mathrm{~cm}^{-1}$ nicht mehr zu finden, stattdessen entsteht eine neue Bande bei $2664 \mathrm{~cm}^{-1}$, die der $\mu$-OD-Gruppe in $\left[\mathbf{L}^{3} \mathbf{N i}_{2}\right.$ (OD)] zugeordnet wird (Kapitel 7.5).

In dieser Arbeit wurden neuartige Pyrrol/Pyrazol-basierte Bausteine dargestellt, aus denen sowohl zyklische als auch azyklische Ligandsysteme synthetisiert werden konnten. Über zahlreiche Syntheserouten konnten neuartige Makrozyklen dargestellt werden, die als Protonen-Sonden fungieren können. Die azyklischen Pyrrol/Pyrazol-basierten Bausteine konnten erfolgreich als Ligandsysteme zur Darstellung von bimetallischen Komplexen eingesetzt werden. Die vielversprechende Folgechemie dieser Komplexe und die ungewöhnlich langsam verlaufenden Austauschprozesse des $\mu$-OH-Brückenliganden gegen eine $\mu$-OD-Gruppe in den bimetallischen Metallkomplexen regen zu weiteren Arbeiten an. 


\section{Experimenteller Teil}

\subsection{Allgemeines}

Apparaturen: Es wurden konventionelle Glasapparaturen oder Schlenkkolben verwendet. Sofern nötig, wurden die Apparaturen unter Verwendung eines Heißluftföns ausgeheizt, mehrmals im Ölpumpenvakuum evakuiert und mit Stickstoff gespült. Das Stickstoffgas wurde durch Sicapent der Firma Merck getrocknet.

Absolute Lösungsmittel: Alle Reaktionen wurden in frisch absolutierten, desoxygenierten und destillierten Lösungsmitteln durchgeführt. Die Lösungsmittel wurden anschließend unter Stickstoffatmosphäre und in Molsieb aufbewahrt.

Folgende Trocknungsmittel fanden Verwendung:

Pentan: $\quad$ Natrium/Kalium

Acetonitril: $\quad$ Phosphorpentoxid

Petrolether 40/60: Calciumhydrid

Dichlormethan: Calciumhydrid oder Phosphorpentoxid

Diethylether: Natrium/Kalium

Tetrahydrofuran: Natrium/Kalium

Ethanol: Natrium

Methanol: $\quad$ Magnesiumspäne oder Phosphorpentoxid

Reagentien: Die nicht aufgeführten Verbindungen waren durch die Chemikalienausgabe des Chemischen Institutes erhältlich. Folgende Reagentien wurden über die angegebenen Firmen bezogen:

Reagenz

Chloranil

1,2-Diaminocyclohexan

1,3-Diaminopropan

$\alpha, \alpha$ '-Diamino- $p$-xylen

1,8-Diaminonaphthalin (99\%)

4,5-Dimethyl-1,2-phenylendiamin
Firma

Fluka

Alfa Aesa

Acros

Fluka

Aldrich

Aldrich 
Dimethylphosphat

Nickelacetat Tetrahydrat

Pyrrol-2-carboxaldehyd

Triethylamin Trihydrofluorid (98\%)

Trifluoressigsäure

Dodecafluorodiessigsäure (96 \%)

Pentafluorobenzaldehyd

Pentafluorophenol

$n$-Butyllithium
Aldrich

ABCR

Acros

Aldrich

Spende von Solvay

Aldrich

Acros

Acros

Chemetall

Die folgenden Reagenzien wurden nach literaturbekannten Vorschriften dargestellt:

\section{3,5-Bis-chloromethyl-1H-pyrazol (XXIXa) ${ }^{33,51}$}

3,4-Diethylpyrrol (XXXV) $)^{47,48}$

3,5-Bis-(aminomethyl)pyrazol Dihydrochlorid (L) ${ }^{58}$

2,6-Bis-(aminomethyl)pyridin Hydrochlorid (j) $)^{109,110}$

$\mathrm{PdCl}_{2}(\mathrm{COD})^{111,112,113,114}$

$1 H$-Pyrazol-3,5-dicarbaldehyd ${ }^{59,60}$

Methyl-(1-methylimidazol-2-ylmethyl)amin ${ }^{94,95}$

$1 H$-Pyrazol-3,5-dicarbonyldichlorid Monohydrochlorid (LVI) ${ }^{88,89}$

\subsection{Analytik}

\section{Kernresonanzspektroskopie (NMR)}

Zur Aufnahme der NMR-Spektren wurde ein Spektrometer der Firma Bruker verwendet: Bruker Avance $500\left({ }^{1} \mathrm{H}: 500.13 \mathrm{MHz},{ }^{13} \mathrm{C}\right.$ : $\left.125.77 \mathrm{MHz}\right), 300\left({ }^{1} \mathrm{H}\right.$ : $300.13 \mathrm{MHz},{ }^{13} \mathrm{C}$ : $75.47 \mathrm{MHz}$ ) und $200\left({ }^{1} \mathrm{H}: 200.13 \mathrm{MHz},{ }^{13} \mathrm{C}: 50.32 \mathrm{MHz}\right.$ ). Alle NMR-Spektren wurden (wenn nicht anders angegeben) im Institut für Anorganische Chemie in Göttingen bei $298 \mathrm{~K}$ aufgenommen. In der University of Connecticut in Storrs (USA) erfolgten die NMRMessungen an einem Bruker DRX $400\left({ }^{1} \mathrm{H}: \quad 400.01 \mathrm{MHz},{ }^{13} \mathrm{C}: \quad 100.63 \mathrm{MHz}\right)$. Die angegebenen Werte der Verschiebung $\delta$ beziehen sich auf den internen Standard durch die Lösungsmittel $\mathrm{CDCl}_{3}\left(\delta=7.27\right.$ ppm für ${ }^{1} \mathrm{H}-\mathrm{NMR}$ bzw. $\delta=77.0 \mathrm{ppm}$ für $\left.{ }^{13} \mathrm{C}-\mathrm{NMR}\right)$, THF-d 8 $\left(\delta=1.70\right.$ ppm für ${ }^{1} \mathrm{H}-\mathrm{NMR}$ bzw. $\delta=25.3 \mathrm{ppm}$ für $\left.{ }^{13} \mathrm{C}-\mathrm{NMR}\right)$ und DMSO-d $\mathrm{d}_{6}(\delta=2.50 \mathrm{ppm}$ für ${ }^{1} \mathrm{H}-\mathrm{NMR}$ bzw. $\delta=40.2 \mathrm{ppm}$ für ${ }^{13} \mathrm{C}-\mathrm{NMR}$ ) relativ zu externem Tetramethylsilan (jeweils $\delta=0$ ppm). Die ${ }^{13} \mathrm{C}$-Spektren wurden ${ }^{1} \mathrm{H}$-entkoppelt aufgenommen. Bei den ${ }^{19} \mathrm{~F}-\mathrm{NMR}$ 
Messungen wurde $\mathrm{C}_{6} \mathrm{~F}_{6}$ als interner Standart verwendet. Abkürzungen der Signalmultiplizitäten: $\mathrm{s}=$ Singulett, $\mathrm{d}=$ Dublett, $\mathrm{dt}=$ Dublett vom Triplett, $\mathrm{t}=$ Triplett, $\mathrm{q}=$ Quartett, $\mathrm{m}=$ Multiplett.

\section{Massenspektrometrie (MS)}

Die Massenspektren wurden in Göttingen auf den Geräten Finnigan MAT 8200 für EISpektren und Finnigan MAT 95 für FAB-Spektren aufgenommen. ESI-Spektren wurden mit einem Finnigan MAT LCQ oder mit einem ESI “API $2000^{\mathrm{TM}}-\mathrm{LC} / \mathrm{MS} / \mathrm{MS}$ ” der Firma Applied Biosystems aufgenommen. Dabei wurden die untersuchten Ionen entweder durch Elektronenstoßionisation (EI, 70 eV) oder durch Fast Atom Bombardment (FAB, Xenon als Stoßgas) der Substanz in einer 4-Nitrobenyzlalkohol-Matrix (Nibeol) erzeugt. Hochauflösende Massenspektren wurden mit dem Massenspektrometer „APEX IV 7 TeslaFourier Transform Ion Cyclotron Resonance“ (FTICR) der Firma Bruker aufgenommen. Die Aufnahmen der ESI-Spektren in der University of Connecticut erfolgten mit einem Micromass Quattro II.

\section{Elementaranalyse}

Die Elementaranalyse in Göttingen erfolgte durch das Mikroanalytische Labor des Instituts für Anorganische Chemie der Universität Göttingen mit einem CHN-O-Rapid Elementar bzw. mit einem CHNOS Elementaranalysator vario EL III der Firma Elementar Analysensysteme $\mathrm{GmbH}$. Sofern nicht anders vermerkt, sind die Werte für die angegebene Summenformel berechnet.

\section{Infrarotspektroskopie (IR)}

Die Infrarotspektren wurden in Göttingen auf einem Digilab Excalibur Spektrometer aufgenommen. Die Feststoffe wurden als KBr-Preßling gemessen. Die Lage der Banden ist in Wellenzahlen angegeben $\left(\widetilde{v}\right.$ in $\left.\mathrm{cm}^{-1}\right)$. Die Intensitäten sind wie folgt abgekürzt: $\mathrm{m}=$ mittel, $\mathrm{s}=$ stark, $\mathrm{vs}=$ sehr stark, $\mathrm{w}=$ schwach. Proben wurden mit Hilfe des MIRacle ${ }^{\mathrm{TM}}$ ATR (MIRacle Base Optics Assembly for FTIR Spectrometers) der Firma PIKE Technologies in Lösung gemessen.

\section{UV/VIS-Spektroskopie}

Die Messungen in Göttingen erfolgten auf einem Analytik Jena Specord S 100 Spektrometer und in der University of Connecticut in Storrs auf einem Gerät der Firma Varian GmbH (Cary 
50 Spectrophotometer). Die Spektren wurden in Lösung mit einer Quarzküvette der Dicke $1.0 \mathrm{~cm}$ gemessen. Die Wellenlängen $\lambda$ sind in $\mathrm{nm}$, die Extinktionskoeffizienten $\varepsilon$ sind in $\mathrm{L} \cdot \mathrm{mol}^{-1} \cdot \mathrm{cm}^{-1}$ bzw. in $\mathrm{M}^{-1} \cdot \mathrm{cm}^{-1}$ angegeben. Die Abkürzung „sh“ steht für die beobachtete Schulter in einem UV/Vis-Spektrum.

\section{Dünnschichtchromatographie (DC)}

Für die dünnschichtchromatographische Analytik wurden Fertigfolien „Polygram Alox N/UV 254 “ der Firma Macherey, Nagel \& Co. und „DC-Alufolien Aluminiumoxid“ der Firma Fluka verwendet. Detektion erfolgte unter UV-Licht $(254 \mathrm{~nm})$ und durch Anfärbung mit Brom.

\section{Säulenchromatographie}

Zur Säulenchromatographie wurde basisches aktives Aluminiumoxid (50-200 micron, 70-290 mesh ASTM) der Firma Janssen Chimica oder der Firma Macherey, Nagel GmbH \& Co. KG verwendet.

\section{Schmelzpunktbestimmung}

Die Schmelzpunkte wurden an einem Gerät der Firma Büchi (Glasapparatefabrik FLAWIL, +Pat. 320388) in offenen Glaskapillaren bestimmt oder mit „Optimelt MPA 100“ SRS der Firma Stanford Research Systems Scientific Instruments GmbH. Die Temperatur ist in Grad Celcius $\left({ }^{\circ} \mathrm{C}\right)$ angegeben. Die Werte sind unkorrigiert angegeben.

\section{Magnetische Untersuchungen}

Für die Untersuchung der magnetischen Eigenschaften wurde ein MPMS-5S-SQUIDMagnetometer (Superconducting Quantum Interference Device) der Firma Quantum-Design verwendet. SQUID sind die empfindlichsten heute verfügbaren Detektoren für magnetische Felder. Ihre Funktion beruht auf dem physikalischen Effekt der Supraleitung und der Verdrängung von Magnetfeldern aus einem Supraleiter. Dabei wird der magnetische Fluß in eine Spannung umgewandelt. ${ }^{115}$ Das im Rahmen dieser Arbeit verwendete Gerät erlaubt Temperaturen zwischen $2 \mathrm{~K}$ und $400 \mathrm{~K}$ und homogene Magnetfelder bis zu $5 \mathrm{~T}$. Die Probe wurde in Gelatinekapseln präpariert und in einem nicht magnetischen Probenhalter für die Messung fixiert. Für die diamagnetischen Korrekturen wurden nach Korrektur des Probenhalters (incl. Gelatinekapsel) die aus den Inkrementen ermittelten Werte verwendet. ${ }^{116,117}$ Die Anpassung der Messkurven erfolgte mit dem Programm julX. ${ }^{92}$ 


\section{Cyclovoltammetrie (CV)}

Die cyclovoltammetrischen Messungen wurden an einem Potentiostat/Galvanostat Model 263A der Firma Perkin-Elmer durchgeführt. Als Lösungsmittel wurde DMF mit Tetrabutylammonium-hexafluorophosphat $(0.1 \mathrm{M})$ als Leitsalz verwendet, als Arbeitselektrode eine Glassy Carbon Elektrode, als Gegenelektrode eine Platinelektrode und als Referenzelektrode eine Ag-Elektrode. Die Spektren wurden gegen einen internen Ferrocen-Standard bzw. Decamethylferrocen bei Raumtemperatur gemessen und die Potentiale relativ zu SCE (Satured Calomel Electode) umgerechnet. ${ }^{98}$

\section{ESR-Spektroskopie}

Die ESR-Messungen wurden in einem ESR-Spektrometer ELEXSYS 500 (CW-Spektrometre, X-Band) der Firma Bruker aufgenommen. Sofern nicht vermerkt ist, wurde die Messung im Festkörper durchgeführt. Die Temperatur wurde mit dem ER 4131 VT digitaler Temperatur Controler überprüft. Zum kühlen wurde hierbei flüssiger Stickstoff benutzt.

Warnung!: Das 3,5-Bis-chloromethyl-1H-pyrazol Hydrochlorid (XXIX) und das 3,5-Bis(chloromethyl-4-phenyl)-1H-pyrazol Hydrochlorid (4) sind stark reizend und können bei Kontakt mit der Haut schwere allergische Hautrötungen verursachen!

\subsection{Synthese der Bausteine}

\subsubsection{3,5-Chlormethyl-1H-pyrazol (XXIXa) $)^{33,51}$}

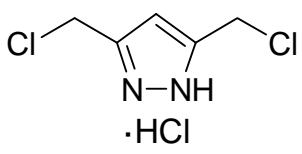

XXIX

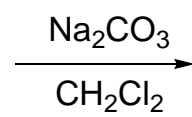

$\mathrm{CH}_{2} \mathrm{Cl}_{2}$

XXIXa

Das 3,5-Bis-chloromethyl-1H-pyrazol Hydrochlorid (XXIX) wurde in einer Dichlormethan Suspension mit einer wässrigen $\mathrm{Na}_{2} \mathrm{CO}_{3}$-Lösung $(0.1 \mathrm{M})$ neutralisiert (dabei ist zu beachten, dass die basische Lösung nur sehr langsam zur Suspension getropft werden darf, da ansonsten das Edukt hydrolysiert wird! Um diese Nebenreaktion zu vermeiden, sollte bei einer größeren Umsetzung das Hydrochlorid in einer THF Suspension mit festem $\mathrm{Na}_{2} \mathrm{CO}_{3}$ und 1-2 Tropfen 
dest. $\mathrm{H}_{2} \mathrm{O}$ über Nacht gerührt werden). Nach einem Tag Rühren bei Raumtemperatur wurde die organische Phase von der wässrigen Phase getrennt und die wässrige Phase mit $\mathrm{CH}_{2} \mathrm{Cl}_{2}$ extrahiert. Die gesammelten organischen Phasen wurden mit $\mathrm{Na}_{2} \mathrm{SO}_{4}$ oder $\mathrm{MgSO}_{4}$ getrocknet und das Lösungsmittel im Vakuum entfernt. Das entstandene Produkt wurde anschließend direkt zur nächsten Stufe umgesetzt.

\section{Auswertung:}

Summenformel:

$\mathrm{C}_{5} \mathrm{H}_{6} \mathrm{Cl}_{2} \mathrm{~N}_{2}$

Molekulargewicht (g/mol): 165.0

${ }^{1} \mathrm{H}-\mathrm{NMR}\left(200 \mathrm{MHz}, \mathrm{CDCl}_{3}\right)$ : $\delta(\mathrm{ppm})=4.62\left(\mathrm{~s}, 4 \mathrm{H}, 2 \times \mathrm{CH}_{2}\right), 6.38\left(\mathrm{~s}, 1 \mathrm{H}, \mathrm{C} H^{\mathrm{pz}-4}\right)$, $10.14(\mathrm{~s}, 1 \mathrm{H}, \mathrm{N} H)$.

${ }^{13} \mathrm{C}-\mathrm{NMR}\left(101 \mathrm{MHz}, \mathrm{CDCl}_{3}\right)$ : $\delta(\mathrm{ppm})=36.5\left(\mathrm{CH}_{2}\right), 105.3\left(\mathrm{CH}^{\mathrm{pz}-4}\right), 145.0\left(C^{\mathrm{pz}-3,5}\right)$.

MS (EI) $m / z(\%)$ : $164(20)[\mathrm{M}]^{+}, 129$ (100) $[\mathrm{M}-\mathrm{Cl}]^{+}, 94$ (16) $[\mathrm{M}-2 \times \mathrm{Cl}]^{+}$, $65(22)\left[\left(\mathrm{C}_{3} \mathrm{HN}_{2}\right)\right]^{+}$.

Schmelzpunkt $\left({ }^{\circ} \mathrm{C}\right)$ : $71-75$.

Elementaranalyse (\%): berechnet: C: 36.39, H: 3.66, N: 16.98, Cl: 42.97; gefunden: C: $36.74, \mathrm{H}: 3.43, \mathrm{~N}: 16.98$.

IR $(\mathrm{KBr})$ : $\widetilde{v}=3192$ (s), 3108 (s), 2994 (s), 2869 (s), 1458 (w), $1315(\mathrm{w}), 1264(\mathrm{~m}), 1142(\mathrm{w}), 1006(\mathrm{w}), 730(\mathrm{~m}) \mathrm{cm}^{-1}$.

\subsubsection{Synthese von 3,5-Bis-(chloromethyl-4-phenyl)-1H-pyrazol Hydrochlorid (4)}<smiles>OCc1n[nH]c(CO)c1-c1ccccc1</smiles>

XXXIV

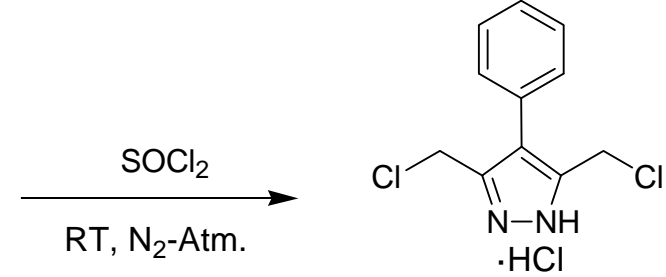

4

Das 3,5-Bis-(hydroxymethyl-4-phenyl)-1H-pyrazol (XXXIV) (3.0 g, 14.7 mmol) wurde unter $\mathrm{N}_{2}$-Atm. langsam und tropfenweise mit Thionylchlorid (10.6 mL, $147 \mathrm{mmol}, 10$ eq.) versetzt*. Die gelbliche Suspension wurde 3 Tage bei Raumtemperatur gerührt. Anschließend wurde das überschüssige $\mathrm{SOCl}_{2}$ bei 50 mbar und $50{ }^{\circ} \mathrm{C}$ destillativ entfernt. Der Rückstand wurde in $\mathrm{Et}_{2} \mathrm{O} / \mathrm{Hexan}(4 \mathrm{~mL} ; 4: 1)$ aufgenommen und die Suspension wurde zwei Tage bei 
$5{ }^{\circ} \mathrm{C}$ zur Kristallisation angesetzt. Der weiße Feststoff (4) wurde kalt abfiltriert und im Hochvakuum getrocknet (3.36 g, $83 \%)$.

(*Bei der Zugabe des $\mathrm{SOCl}_{2}$ kann es zu einer sehr heftigen exothermen Reaktion kommen. Hierbei ist zu empfehlen, einen etwas größeren Rundkolben mit Rückflußkühler und Überdruckventil zu verwenden.)

\section{Auswertung:}

Summenformel:

$\mathrm{C}_{11} \mathrm{H}_{10} \mathrm{~N}_{6} \mathrm{Cl}_{2}(\cdot \times \mathrm{HCl})$

Molekulargewicht $(\mathrm{g} / \mathrm{mol})$ :

240.0

${ }^{1} \mathrm{H}-\mathrm{NMR}\left(200 \mathrm{MHz}, \mathrm{DMSO}-\mathrm{d}_{6}\right)$ : $\delta(\mathrm{ppm})=4.70\left(\mathrm{~s}, 4 \mathrm{H}, 2 \times \mathrm{CH}_{2}\right), 7.39(\mathrm{~m}, 1 \mathrm{H}, \mathrm{Ar}-H)$, 7.46-7.49 (m, 4H, Ar- $H), 8.90$ (br, s, 1H, NH).

${ }^{13} \mathrm{C}-\mathrm{NMR}\left(50 \mathrm{MHz}, \mathrm{DMSO}-\mathrm{d}_{6}\right)$ : $\delta(\mathrm{ppm})=36.7\left(\mathrm{CH}_{2}\right), 117.6\left(C^{\mathrm{q}}\right), 118.1\left(C^{\mathrm{q}}\right), 119.6$ $\left(C^{\mathrm{q}}\right), 126.6\left(C \mathrm{H}^{\mathrm{Ph}}\right), 127.2\left(C \mathrm{H}^{\mathrm{Ph}}\right), 127.4\left(C \mathrm{H}^{\mathrm{Ph}}\right), 128.6$ $\left(C \mathrm{H}^{\mathrm{Ph}}\right), 128.9\left(C \mathrm{H}^{\mathrm{Ph}}\right), 131.4\left(C^{\mathrm{q}}\right), 141.1\left(C^{\mathrm{t}, \mathrm{pz}}\right), 142.8$ $\left(C^{\mathrm{q}}\right)$.

MS (EI) $m / z(\%)$ :

240 (79) $[\mathrm{M}]^{+}, 205(82)[\mathrm{M}-\mathrm{Cl}]^{+}, 169$ (100) [M$(2 \times \mathrm{Cl})]^{+}, 156(23)\left[\mathrm{C}_{10} \mathrm{H}_{8} \mathrm{~N}_{2}\right]^{+}, 139(10)\left[\mathrm{C}_{9} \mathrm{H}_{3} \mathrm{~N}_{2}\right], 115$ (28) $\left[\mathrm{C}_{7} \mathrm{H}_{3} \mathrm{~N}_{2}\right]$.

Schmelzpunkt $\left({ }^{\circ} \mathrm{C}\right)$ : 104-110.

Elementaranalyse (\%): berechnet für $\mathrm{C}_{11} \mathrm{H}_{10} \mathrm{Cl}_{2}(\mathrm{HCl})_{0.75}$ :

$$
\text { C: 49.21; H: 4.04; N: 10.43, Cl: 36.31; }
$$

gemessen: C: 49.29, H: 4.10; N: 10.16; Cl: 35.34.

IR ( $\mathrm{KBr})$ : $\widetilde{v}=3429(\mathrm{w}), 3181(\mathrm{~m}), 3078(\mathrm{w}), 3023(\mathrm{w}), 2962$ (w), 1628 (w), 1520 (m), 1442 (m), 1320 (m), 1258 (s), 1111 (m), 1034 (s), 927 (w), 801 (s), 735 (s), 701 (s), $658(\mathrm{~s}), 525(\mathrm{w}) \mathrm{cm}^{-1}$. 


\subsubsection{Synthese von 3,5-Bis-(3,4-dimethyl-1H-pyrrol-2-yl-methyl)-1H-pyrazol (5a)}

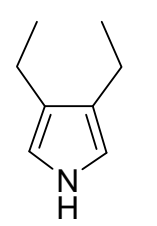

XXXV

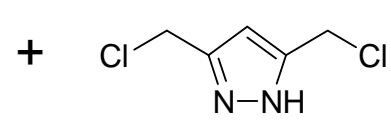

XXIXa

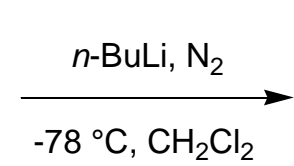

$-78^{\circ} \mathrm{C}, \mathrm{CH}_{2} \mathrm{Cl}_{2}$

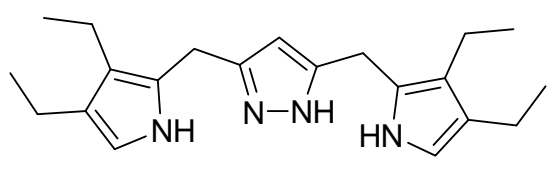

$5 \mathbf{a}$

$\mathrm{Zu}$ einem Gemisch aus 3,4-Diethylpyrrol (XXXV) ${ }^{47,48}$ (3.5 g, $28.4 \mathrm{mmol}, 3.5$ eq.) und trockenem $\mathrm{CH}_{2} \mathrm{Cl}_{2}(200 \mathrm{~mL})$ wurde unter $\mathrm{N}_{2}$-Atmosphäre und bei $-78{ }^{\circ} \mathrm{C} n$-BuLi $(2.5 \mathrm{M}$ in Hexan; $11.4 \mathrm{~mL}, 28.4$ mmol, 3.5 eq.) langsam zugegeben. Nach 1.5 h Rühren wurde das neutrale XXIXa $(1.33 \mathrm{~g}, 8.10 \mathrm{mmol})$ in trockenem $\mathrm{CH}_{2} \mathrm{Cl}_{2}(50 \mathrm{~mL})$ gelöst und bei $-78{ }^{\circ} \mathrm{C}$ vorsichtig portionsweise zum Reaktionsgemisch gegeben. Das Reaktionsgemisch wurde $4 \mathrm{~h}$ bei gleicher Temperatur gerührt und über Nacht auf Raumtemperatur erwärmt. Am nächsten Tag wurde das Reaktionsgemisch mit einer gesättigten $\mathrm{NH}_{4} \mathrm{Cl}$-Lösung (150 mL) neutralisiert und mit $3 \times 100 \mathrm{~mL} \mathrm{CH}_{2} \mathrm{Cl}_{2}$ extrahiert. Nach dem Trocknen mit $\mathrm{MgSO}_{4}$ wurde das Lösungsmittel im Vakuum entfernt und das so erhaltene orange-braune Rohprodukt säulenchromatographisch (Aluminiumoxid [basisch, Aktivität I]), Dichlormethan/Methanol 45:1, $\left(\mathrm{R}_{\mathrm{f}}=0.50\right.$ bei $\left.\mathrm{CH}_{2} \mathrm{Cl}_{2}: \mathrm{MeOH} 1: 20\right)$ gereinigt. Das Produkt 5a wurde in Form eines bräunlichen leicht hygroskopischen Feststoffes (2.49 g, 91 \%) erhalten, der in Lösung nicht lange stabil bleibt. Das Produkt sollte unter tiefen Temperaturen und in $\mathrm{N}_{2}$-Atm. gelagert werden.

\section{Auswertung:}

Summenformel:

$\mathrm{C}_{21} \mathrm{H}_{30} \mathrm{~N}_{4}$

Molekulargewicht (g/mol):

338.5

${ }^{1} \mathrm{H}-\mathrm{NMR}\left(500 \mathrm{MHz}, \mathrm{CDCl}_{3}\right)$ :

$\delta(\mathrm{ppm})=1.11-1.14\left(\mathrm{t},{ }^{3} J(\mathrm{H}, \mathrm{H})=7.5 \mathrm{~Hz}, 6 \mathrm{H}, \mathrm{CH}\right)$, 1.21-1.24(t, $\left.{ }^{3} J(\mathrm{H}, \mathrm{H})=7.51 \mathrm{~Hz}, 6 \mathrm{H}, \mathrm{CH}_{3}\right), 2.45-2.51$ $\left(\mathrm{m},{ }^{3} J(\mathrm{H}, \mathrm{H})=7.5 \mathrm{~Hz}, 8 \mathrm{H}, \mathrm{CH}_{2} \mathrm{CH}_{3}\right), 3.83(\mathrm{~s}, 4 \mathrm{H}$, $\left.\mathrm{CH}_{2}{ }^{\mathrm{py} / \mathrm{pz}}\right), 5.88\left(\mathrm{~s}, 1 \mathrm{H}, \mathrm{CH}^{\mathrm{pz}}\right), 6.33-6.34\left(\mathrm{~m}, 2 \mathrm{H}, \mathrm{C} H^{\mathrm{py}}\right)$, 8.19 (br s, 2H, NH), 8.69-10.19 (br, s, NH).

${ }^{13} \mathrm{C}-\mathrm{NMR}\left(126 \mathrm{MHz}, \mathrm{CDCl}_{3}\right): \quad \delta(\mathrm{ppm})=14.6\left(\mathrm{CH}_{3}\right), 16.1\left(\mathrm{CH}_{3}\right), 17.4\left(\mathrm{CH}_{2}\right), 18.5$ $\left(\mathrm{CH}_{2}\right), 23.8$ (py- $\left.\mathrm{CH}_{2}-\mathrm{pz}\right), 103.5\left(\mathrm{CH}^{\mathrm{pz}}\right), 112.8\left(\mathrm{CH}^{\mathrm{py}}\right)$, $120.3\left(C^{q}\right), 123.8\left(C^{q}\right), 124.8\left(C^{q}\right), 147.1\left(C^{\mathrm{pz}}\right)$. 
MS (EI) $m / z(\%)$ :

338 (62) $[\mathrm{M}]^{+}, 323(10)\left[\mathrm{M}-\left(\mathrm{CH}_{3}\right)\right]^{+}, 309$ (7) [M-

$\left.\left(\mathrm{C}_{2} \mathrm{H}_{5}\right)\right]^{+}, 217(20) \quad\left[\mathrm{M}-\left(\mathrm{C}_{8} \mathrm{H}_{11} \mathrm{~N}\right)\right]^{+}, 200$ (22) $[\mathrm{M}-$

$\left.\left(\mathrm{C}_{9} \mathrm{H}_{16} \mathrm{~N}\right)\right]^{+}, \quad 186 \quad(5) \quad\left[\mathrm{M}-\left(\mathrm{C}_{10} \mathrm{H}_{18} \mathrm{~N}\right)\right]^{+}, \quad 154 \quad$ (12)

$\left[\left(\mathrm{C}_{9} \mathrm{H}_{4} \mathrm{~N}_{3}\right)\right]^{+}, 122(100)\left[\left(\mathrm{C}_{8} \mathrm{H}_{12} \mathrm{~N}\right)\right]^{+}$.

HRMS-(+)ESI $m / z(\%)$ : $\quad$ berechnet $[\mathrm{M}+\mathrm{H}]^{+}: 339.25432$;

(Methanol/Wasser)

gefunden: $[\mathrm{M}+\mathrm{H}]^{+}: 339.25433$.

Elementaranalyse (\%):

berechnet für $\mathrm{C}_{21} \mathrm{H}_{30} \mathrm{~N}_{4} \cdot\left(\mathrm{H}_{2} \mathrm{O}\right)_{0.25}$ :

C: 73.52, H: 8.97, N: 16.34;

gefunden: $\mathrm{C}: 73.59, \mathrm{H}: 8.67, \mathrm{~N}: 16.45$.

IR ( $\mathrm{KBr})$ :

$\widetilde{v}=3356(\mathrm{~s}), 2926(\mathrm{~m}), 1674(\mathrm{~m}), 1558(\mathrm{~m}), 1443(\mathrm{~s})$, 298 (w), 1083 (w), 1002 (w), $779(\mathrm{~m}), 532(\mathrm{~m}) \mathrm{cm}^{-1}$.

\subsubsection{Synthese von 3,5-Bis-(3,4-diethyl-1-formylpyrrol-2-ylmethyl)-1H-pyrazol (1a)}

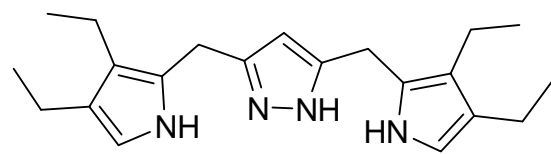

5a

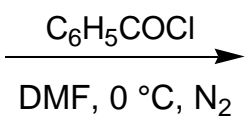

DMF, $0{ }^{\circ} \mathrm{C}, \mathrm{N}_{2}$

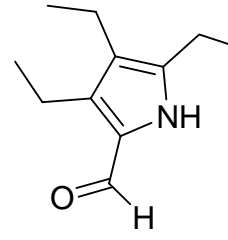

1a

Zu einer im Eisbad gekühlten Lösung aus 5a (282 mg, 0.833 mmol, 1 eq.) in trockenem DMF (608 mg, $8.33 \mathrm{mmol}, 10$ eq.) wurde frisch destilliertes Benzoylchlorid (0.76 mL, $6.7 \mathrm{mmol}$, 8.0 eq.) unter Rühren tropfenweise zugegeben. Das Reaktionsgemisch wurde $2 \mathrm{~h}$ bei $0{ }^{\circ} \mathrm{C}$ und anschließend weitere $2 \mathrm{~h}$ bei Raumtemperatur gerührt. Zur Aufarbeitung wurde eine 50\%ige wässrige Ethanol/ $\mathrm{Na}_{2} \mathrm{CO}_{3}$-Lösung $(1.0 \mathrm{~g}$ in $80 \mathrm{ml})$ unter Kühlung zugegeben. Dazu wurde etwas Dichlormethan gegeben und die wässrige Phase mit $3 \times 100 \mathrm{ml} \mathrm{CH}_{2} \mathrm{Cl}_{2}$ extrahiert. Nach dem Trocknen mit $\mathrm{Na}_{2} \mathrm{SO}_{4}$ wurde das Lösungsmittel im Vakuum entfernt und das so erhaltene schwarz-braune Rohprodukt säulenchromatographisch (Aluminiumoxid [basisch, Aktivität I], Dichlormethan/Methanol 15:1, $\left.\mathrm{R}_{\mathrm{f}}=0.44\right)$ gereinigt. Durch anschließende Umkristallisation des isolierten Produktes in Ethanol wurde 1a in Form eines leicht gelblichen Pulvers (288 mg, 88 \%) erhalten. Bei größeren Umsetzungen beträgt die Ausbeute 40-70 \%. 


\section{Auswertung:}

Summenformel:

$\mathrm{C}_{23} \mathrm{H}_{30} \mathrm{~N}_{4} \mathrm{O}_{2}$

Molekulargewicht (g/mol):

394.5

${ }^{1} \mathrm{H}-\mathrm{NMR}$ (500 MHz, DMSO-d 6 ):

$\delta(\mathrm{ppm})=0.89-0.92\left(\mathrm{t},{ }^{3} J(\mathrm{H}, \mathrm{H})=7.5 \mathrm{~Hz}, 6 \mathrm{H}, \mathrm{CH}_{3}\right)$, 1.09-1.12 (t, $\left.{ }^{3} J(\mathrm{H}, \mathrm{H})=7.5 \mathrm{~Hz}, 6 \mathrm{H}, \mathrm{CH}_{3}\right), 2.29-2.33$ $\left(\mathrm{q},{ }^{3} \mathrm{~J}(\mathrm{H}, \mathrm{H})=7.5 \mathrm{~Hz}, 4 \mathrm{H}, \mathrm{CH}_{2} \mathrm{CH}_{3}\right), 2.60-2.65$ (q, $\left.{ }^{3} J(\mathrm{H}, \mathrm{H})=7.5 \mathrm{~Hz}, \quad 4 \mathrm{H}, \quad \mathrm{CH}_{2} \mathrm{CH}_{3}\right), \quad 3.80 \quad(\mathrm{~s}, \quad 4 \mathrm{H}$; $\left.\mathrm{CH}_{2}^{\mathrm{py} / \mathrm{pz}}\right), 5.74\left(\mathrm{~s}, 1 \mathrm{H}, \mathrm{CH}^{\mathrm{pz}}\right), 9.45$ (s, 2H, CHO), 11.40 (br s, $\mathrm{N} H$ ), 12.28 (br s, NH).

${ }^{13} \mathrm{C}-\mathrm{NMR}\left(126 \mathrm{MHz}, \mathrm{DMSO}-\mathrm{d}_{6}\right): \quad \delta(\mathrm{ppm})=15.8 \quad\left(\mathrm{CH}_{3}\right), \quad 16.2 \quad\left(\mathrm{CH}_{2} \mathrm{CH}_{3}\right), \quad 16.6$ $\left(\mathrm{CH}_{2} \mathrm{CH}_{3}\right), 17.4\left(\mathrm{CH}_{3}\right), 23.4$ (br, py- $\left.\mathrm{CH}_{2}-\mathrm{pz}\right), 102.7$ $\left(C \mathrm{H}^{\mathrm{pz}}\right), 122.9\left(C^{\mathrm{q}}\right), 127.0\left(C^{\mathrm{py}} \mathrm{CHO}\right), 135.5\left(C^{\mathrm{q}}\right), 136.4$ $\left(C^{\mathrm{q}}\right), 144.6\left(\mathrm{br}, C^{\mathrm{q}}\right), 176.3(C \mathrm{HO})$.

$\operatorname{MS}(\mathrm{EI}) m / z(\%)$ :

$394(100)[\mathrm{M}]^{+}, 365(63)\left[\mathrm{M}-\left(\mathrm{C}_{2} \mathrm{H}_{5}\right)\right]^{+}, 351$ (5) [M$\left.\left(\mathrm{C}_{3} \mathrm{H}_{7}\right)\right]^{+}, 337(13)\left[\mathrm{M}-2 \times\left(\mathrm{C}_{2} \mathrm{H}_{5}\right)+\mathrm{H}\right]^{+}, 323(5)[\mathrm{M}-$ $\left.2 \times\left(\mathrm{C}_{2} \mathrm{H}_{5}\right)-\left(\mathrm{CH}_{3}\right)+2 \mathrm{H}\right]^{+}, 243(28)\left[\mathrm{M}-\left(\mathrm{C}_{9} \mathrm{H}_{12} \mathrm{NO}\right)\right]^{+}, 228$ (16) $\left[\mathrm{C}_{13} \mathrm{H}_{16} \mathrm{~N}_{4}\right]^{+}, 214$ (18) $\left[\mathrm{C}_{12} \mathrm{H}_{14} \mathrm{~N}_{4}\right]^{+}, 200$ (12) $\left[\mathrm{C}_{11} \mathrm{H}_{12} \mathrm{~N}_{4}\right]^{+}, \quad 150 \quad$ (30) $\quad\left[\mathrm{C}_{9} \mathrm{H}_{12} \mathrm{NO}\right]^{+}, \quad 122 \quad$ (50) $\left[\mathrm{C}_{8} \mathrm{H}_{12} \mathrm{~N}\right]^{+}, 94(7)\left[\mathrm{C}_{5} \mathrm{~N}_{2} \mathrm{H}_{6}\right]^{+}$.

HRMS-(+)ESI $m / z$ :

(Methanol/Wasser)

Schmelzpunkt $\left({ }^{\circ} \mathrm{C}\right)$ :

Elementaranalyse (\%):

$\operatorname{IR}(\mathrm{KBr})$ : berechnet: $[\mathrm{M}+\mathrm{H}]^{+}:$395.24415;

gemessen: $[\mathrm{M}+\mathrm{H}]^{+}: 395.24413$.

191-195.

berechnet für $\mathrm{C}_{23} \mathrm{H}_{30} \mathrm{~N}_{4} \mathrm{O}_{2} \cdot\left(\mathrm{H}_{2} \mathrm{O}\right)_{0.5}$ :

C: $68.46, \mathrm{H}: 7.74, \mathrm{~N}: 13.88$, O: 9.912 ;

gefunden: C: 68.44, H: 7.74, N: 14.29.

$\widetilde{v}=3243(\mathrm{~s}), 2929(\mathrm{~m}), 1608$ (vs), 1448 (m), 1350 (m), 1280 (w), 1134 (w), 1010 (m), 856 (w), $772(\mathrm{~s}) \mathrm{cm}^{-1}$. 


\subsubsection{Synthese von 3,5-Bis-(3,4-diethyl-1-hydroxy-2-yl-methyl)-1H-pyrazol (6)}

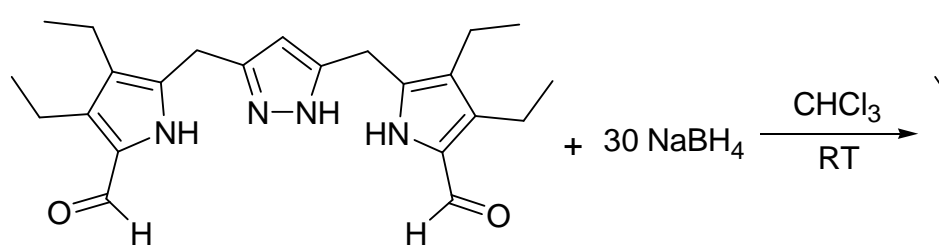

$1 \mathbf{a}$

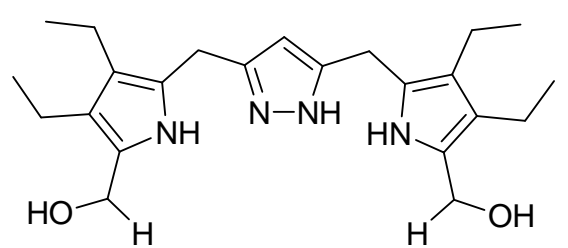

6

Der Dialdehyd 1a (30 mg, $0.076 \mathrm{mmol}, 1$ eq.) wurde in einer Lösung aus trockenem Chloroform/Methanol (50:5 mL) gelöst und mit Natriumborhydrid (86 mg, $2.28 \mathrm{mmol}$, 30 eq.) portionsweise versetzt. Dabei ließ sich das Reaktionsgemisch leicht entfernen. Das Reaktionsgemisch wurde $3 \mathrm{~h}$ bei RT gerührt und die Reaktion wurde per DC-Kontrolle verfolgt. In einem Laufmittel von 1:10 Methanol/ $\mathrm{CH}_{2} \mathrm{Cl}_{2}$ verschwand der einizige Spot bei $\mathrm{R}_{\mathrm{f}}=0.65$ (aufgetragen auf Aluminiumoxidplatten) und ein einzelner Spot bei $\mathrm{R}_{\mathrm{f}}=0.32$ entstand. Beim Anfärben dieser DC-Platte erschein das entstandene Produkt gelb-braun. Das Reaktionsgemisch wurde mit dest. Wasser $(40 \mathrm{~mL})$ gequentcht und die wäßrige Phase wurde mit $3 \times 10 \mathrm{~mL} \mathrm{CH} \mathrm{Cl}_{2}$ extrahiert. Die gesammelten organischen Phasen wurden mit $\mathrm{Na}_{2} \mathrm{SO}_{4}$ getrocknet. Nach dem Trocknen wurde das Lösungsmittel im Vakuum entfernt und das gelbe Ö1 (6) (30 mg, 99 \%) direkt ohne Aufreinigung für die Folgereaktion eingesetzt.

\section{Auswertung:}

Summenformel:

Molekulargewicht (g/mol):

${ }^{1} \mathrm{H}-\mathrm{NMR}\left(400 \mathrm{MHz}, \mathrm{CDCl}_{3}\right)$ :
$\mathrm{C}_{23} \mathrm{H}_{34} \mathrm{~N}_{4} \mathrm{O}_{2}$

398.5

$\delta(\mathrm{ppm})=0.99-1.03\left(\mathrm{~m}, 12 \mathrm{H}, \mathrm{CH}_{3}\right), 2.32-2.42(\mathrm{~m}, 8 \mathrm{H}$, $\left.\mathrm{CH}_{2}\right), 3.66\left(\mathrm{~s}, 4 \mathrm{H}, \mathrm{CH}_{2}{ }^{\mathrm{py} / \mathrm{pz}}\right), 4.29\left(\mathrm{~s}, 4 \mathrm{H}, \mathrm{CH}_{2} \mathrm{OH}\right)$, $5.75\left(\mathrm{~s}, 1 \mathrm{H}, \mathrm{CH}^{\mathrm{pz}}\right), 9.03$ (s, br, $\left.\mathrm{OH}\right)$. 


\subsubsection{Synthese von 3,5-Bis-(3,4-dimethyl-1H-pyrrol-2-yl-methyl)-4-phenyl-1H- pyrazol (5b)}

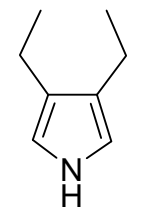

XXXV

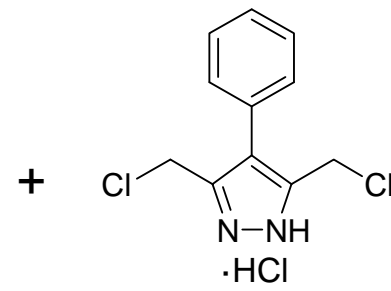

4

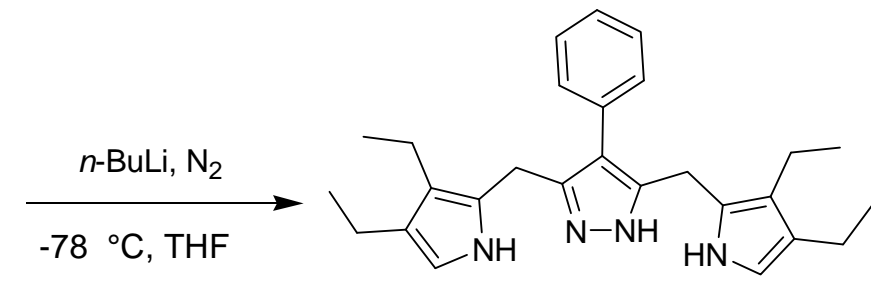

$5 \mathbf{b}$

$\mathrm{Zu}$ einem Gemisch aus 3,4-Diethylpyrrol (XXXV) (0.75 g, $6.0 \mathrm{mmol}, 3.5$ eq.) und $150 \mathrm{~mL}$ trockenem THF wurde unter $\mathrm{N}_{2}$-Atmosphäre und bei $-78{ }^{\circ} \mathrm{C} n$-BuLi (1.6 M in Hexan; $3.8 \mathrm{~mL}$ 6.0 mmol, 3.5 eq.) langsam zugegeben. Parallel wurde in einem weiteren Schlenkkolben das 3,5-Bis-(chloromethyl-4-phenyl)-1H-pyrazol Hydrochlorid (4) (477 mg, 1.7 mmol, 1 eq.) in trockenem THF (25 mL) gelöst. Unter $\mathrm{N}_{2}$-Atm. und bei $-78^{\circ} \mathrm{C}$ wurde das Hydrochlorid 3 mit $n$-BuLi (1.0 mL, 1.7 mmol, 1 eq.) neutralisiert. Nach 45 Min. Rühren wurde das neutralisierte Edukt (4) langsam zum Reaktionsgemisch getropft. Das Reaktionsgemisch wurde $2 \mathrm{~h}$ bei $-78^{\circ} \mathrm{C}$ gerührt und anschließend über Nacht auf Raumtemperatur erwärmt. Am nächsten Tag wurde eine gesättigte (wässrige) $\mathrm{NH}_{4} \mathrm{Cl}$-Lösung $(40 \mathrm{~mL})$ zugegeben und die wässrige Phase mit $3 \times 100 \mathrm{ml} \mathrm{CH}_{2} \mathrm{Cl}_{2}$ extrahiert. Die gesammelten organischen Phasen wurden mit $\mathrm{MgSO}_{4}$ getrocknet und anschließend wurde das Lösungsmittel im Vakuum entfernt. Das erhaltene orange-braune Rohprodukt wurde säulenchromatographisch (Aluminiumoxid [basisch, Aktivität I], Dichlormethan/Methanol 40:1, $\mathrm{R}_{\mathrm{f}}=0.57$ ) gereinigt. Das Produkt 5b wurde in Form eines bräunlichen, leicht hygroskopischen Feststoffes (392 mg, 55 \%) erhalten, der in Lösung nicht lange stabil bleibt. Das Produkt sollte bei tiefen Temperaturen und unter Stickstoffatmosphäre gelagert werden - und, falls möglich, gleich für den weiteren Syntheseschritt eingesetzt werden.

\section{Auswertung:}

Summenformel:

Molekulargewicht (g/mol):

${ }^{1} \mathrm{H}-\mathrm{NMR}\left(500 \mathrm{MHz}, \mathrm{CDCl}_{3}\right.$ ):

$$
\begin{aligned}
& \mathrm{C}_{27} \mathrm{H}_{34} \mathrm{~N}_{4} \\
& 414.6 \\
& \delta(\mathrm{ppm})=0.97-0.99\left(\mathrm{t},{ }^{3} J(\mathrm{H}, \mathrm{H})=7.50 \mathrm{~Hz}, 6 \mathrm{H}, \mathrm{CH}_{3}\right), \\
& 1.15-1.18\left(\mathrm{t},{ }^{3} J(\mathrm{H}, \mathrm{H})=7.50 \mathrm{~Hz}, 6 \mathrm{H}, \mathrm{CH}_{3}\right), 2.30-2.35 \\
& \left(\mathrm{q},{ }^{3} J(\mathrm{H}, \mathrm{H})=7.50 \mathrm{~Hz}, 4 \mathrm{H}, \mathrm{CH}_{2} \mathrm{CH}_{3}\right), 2.39-2.41\left(\mathrm{q},{ }^{3} J\right. \\
& \left.(\mathrm{H}, \mathrm{H})=7.50 \mathrm{~Hz}, 4 \mathrm{H}, \mathrm{CH}_{2} \mathrm{CH}_{3}\right), 3.87\left(\mathrm{~s}, 4 \mathrm{H}, \mathrm{CH}_{2}\right),
\end{aligned}
$$




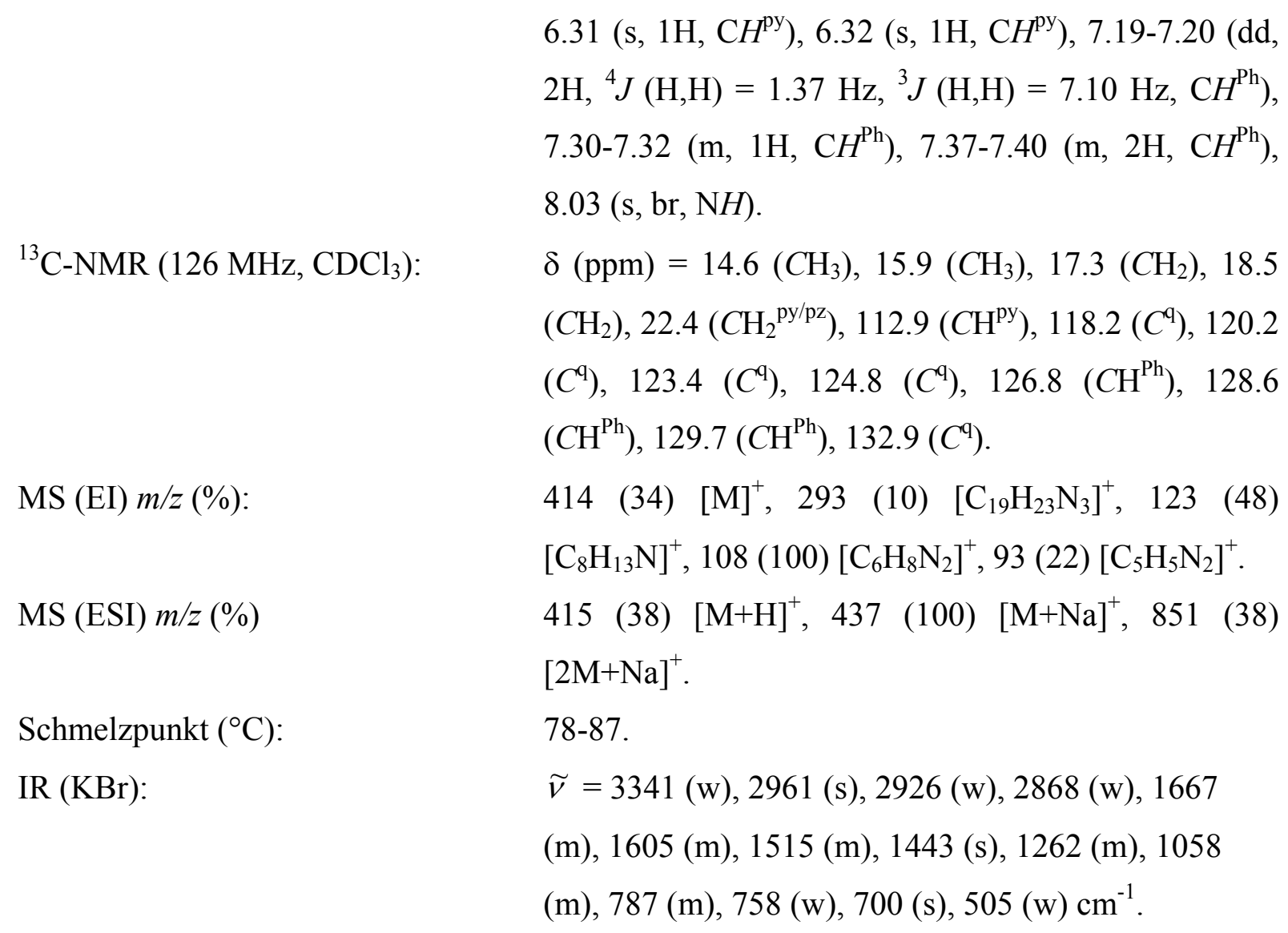

\subsubsection{Synthese von 3,5-Bis-(3,4-diethyl-1-formylpyrrol-2-yl-methyl)-4-phenyl-1H- pyrazol (1b)}

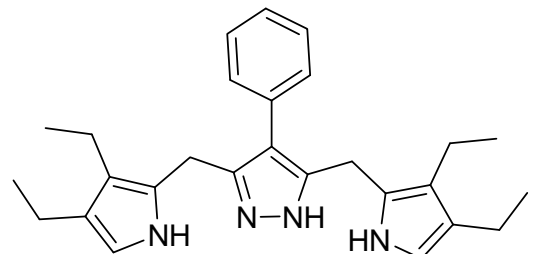

$\mathbf{5 b}$

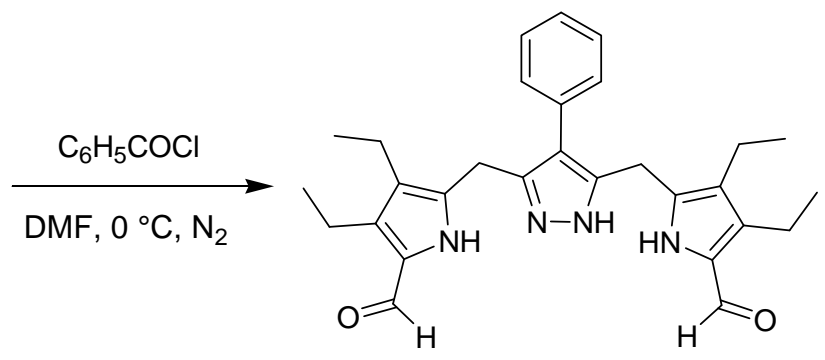

1b

Benzoylchlorid (4.78 g, 3.90 ml, 34.1 mmol, 10 eq.) wurde unter $\mathrm{N}_{2}$-Atmosphäre zu einem im Eisbad gekühlten Reaktionsgemisch aus 5b (1.42 g, $3.4 \mathrm{mmol})$, gelöst in trockenem DMF (2.49 g, $2.65 \mathrm{~mL}, 10$ eq.), unter Rühren zugegeben. Das Reaktionsgemisch wurde anschließend $2 \mathrm{~h}$ bei $0{ }^{\circ} \mathrm{C}$ und noch weitere $3 \mathrm{~h}$ bei $40{ }^{\circ} \mathrm{C}$ gerührt. Zur Aufarbeitung wurde eine $\mathrm{Na}_{2} \mathrm{CO}_{3}$-Ethanol/Wasser-Mischung $(1: 1 ; 150 \mathrm{ml})$ unter Kühlung langsam zugegeben. Dazu wurde etwas Dichlormethan gegeben und die wässrige Phase mit $3 \times 100 \mathrm{ml} \mathrm{CH}_{2} \mathrm{Cl}_{2}$ 
extrahiert. Die gesammelten organischen Phasen wurden mit $\mathrm{Na}_{2} \mathrm{SO}_{4}$ getrocknet und anschließend das Lösungsmittel im Vakuum entfernt. Das erhaltene schwarz-braune Rohprodukt wurde säulenchromatographisch (Aluminiumoxid [basisch, Aktivität I]), Dichlormethan/Methanol/Essigsäureethylester 20:1:1, $\left.\mathrm{R}_{\mathrm{f}}=0.50\right)$ gereinigt. Das isolierte Produkt wurde anschließend in Essigsäureethylester/Methanol umkristallisiert und 1b in Form eines leicht gelb-braunen Feststoffes ( $>122 \mathrm{mg},>8 \%$ ) erhalten.

\section{Auswertung:}

Summenformel:

$\mathrm{C}_{29} \mathrm{H}_{34} \mathrm{~N}_{4} \mathrm{O}_{2}$

Molekulargewicht (g/mol):

470.6

${ }^{1} \mathrm{H}-\mathrm{NMR}\left(500 \mathrm{MHz}, \mathrm{DMSO}-\mathrm{d}_{6}\right)$ :

$\delta(\mathrm{ppm})=0.80-0.83\left(\mathrm{t},{ }^{3} \mathrm{~J}(\mathrm{H}, \mathrm{H})=7.5 \mathrm{~Hz}, 6 \mathrm{H}, \mathrm{CH}\right)$, 1.14-1.17 (t, $\left.{ }^{3} \mathrm{~J}(\mathrm{H}, \mathrm{H})=7.5 \mathrm{~Hz}, 6 \mathrm{H}, \mathrm{CH}_{3}\right), 2.07-2.11$ $\left(\mathrm{q}, J^{3}(\mathrm{H}, \mathrm{H})=7.5 \mathrm{~Hz}, 4 \mathrm{H}, \mathrm{CH}_{2} \mathrm{CH}_{3}\right), 2.60-2.65\left(\mathrm{q},{ }^{3} J\right.$ $\left.(\mathrm{H}, \mathrm{H})=7.5 \mathrm{~Hz}, 4 \mathrm{H}, \mathrm{CH}_{2} \mathrm{CH}_{3}\right), 3.95\left(\mathrm{~s}, 4 \mathrm{H}, \mathrm{CH}_{2}\right)$, 7.11-7.12 (d, $\left.{ }^{3} J(\mathrm{H}, \mathrm{H})=7.2 \mathrm{~Hz}, 2 \mathrm{H}, \mathrm{C} H^{\mathrm{Ph}}\right), 7.29-7.32$ $\left(\mathrm{t},{ }^{3} J(\mathrm{H}, \mathrm{H})=7.2 \mathrm{~Hz}, 1 \mathrm{H}, \mathrm{C} H^{\mathrm{Ph}}\right), 7.35-7.38\left(\mathrm{t},{ }^{3} J\right.$ $\left.(\mathrm{H}, \mathrm{H})=7.2 \mathrm{~Hz}, 2 \mathrm{H}, \mathrm{CH}{ }^{\mathrm{Ph}}\right), 9.42(\mathrm{~s}, \mathrm{br}, 2 \mathrm{H}, \mathrm{CHO})$, $10.30(\mathrm{~s}, \mathrm{br}, \mathrm{N} H)$.

${ }^{13} \mathrm{C}-\mathrm{NMR}\left(126 \mathrm{MHz}, \mathrm{DMSO}-\mathrm{d}_{6}\right): \quad \delta(\mathrm{ppm})=15.6\left(\mathrm{CH}_{3}\right), \quad 16.5 \quad\left(\mathrm{CH}_{2} \mathrm{CH}_{3}\right), \quad 17.2$ $\left(\mathrm{CH}_{2} \mathrm{CH}_{3}\right), 17.6\left(\mathrm{CH}_{3}\right), 22.2\left(\mathrm{~s}, \mathrm{CH}_{2}{ }^{\mathrm{py} / \mathrm{pz}}\right), 119.0\left(\mathrm{C}^{\mathrm{q}}\right)$, $124.6\left(C^{\mathrm{q}}\right), 127.3\left(\mathrm{CH}^{\mathrm{Ph}}\right), 127.6\left(C^{\mathrm{q}}\right), 128.7\left(\mathrm{CH}^{\mathrm{Ph}}\right)$, $129.9\left(C \mathrm{H}^{\mathrm{Ph}}\right), 132.2\left(C^{\mathrm{q}}\right), 135.9\left(C^{\mathrm{q}}\right), 139.4\left(C^{\mathrm{q}}\right), 144.0$ $\left(C^{\mathrm{q}}\right), 176.4(\mathrm{CHO})$.

MS (EI) $m / z(\%)$ : 469 (100) $[\mathrm{M}]^{+}, 441$ (20) $[\mathrm{M}-(\mathrm{COH})]^{+}, 105 \quad(58)$ $\left[\mathrm{C}_{2} \mathrm{~N}_{2} \mathrm{H}_{5}\right]^{+}, 77(20)\left[\left(\mathrm{C}_{4} \mathrm{H}_{5}\right)\right]^{+}$.

MS (ESI in $\mathrm{MeOH}) m / z(\%)$ :

$493 \quad(85) \quad[\mathrm{M}+\mathrm{Na}]^{+}, \quad 963 \quad[2 \mathrm{M}+\mathrm{Na}]^{+}, \quad 1434 \quad(40)$ $[3 \mathrm{M}+\mathrm{Na}]^{+}$.

Schmelzpunkt $\left({ }^{\circ} \mathrm{C}\right)$ : 164-174.

IR (KBr): $\widetilde{v}=3238$ (br, w) 2963 (m), 2929 (w), 2870 (w), 1626 (vs), 1498 (w), 1443 (s), 1381 (w), 1342 (w), 1312 (w), $1258(\mathrm{w}), 1203(\mathrm{w}), 1145(\mathrm{w}), 1107(\mathrm{w}), 1064$ (w), $1010(\mathrm{~m}), 969$ (w), $864(\mathrm{w}), 791$ (w), $754(\mathrm{w})$, $694(\mathrm{~m}) \mathrm{cm}^{-1}$. 


\subsection{Synthese der Makrozyklen}

\subsubsection{Synthese von $2 a$}

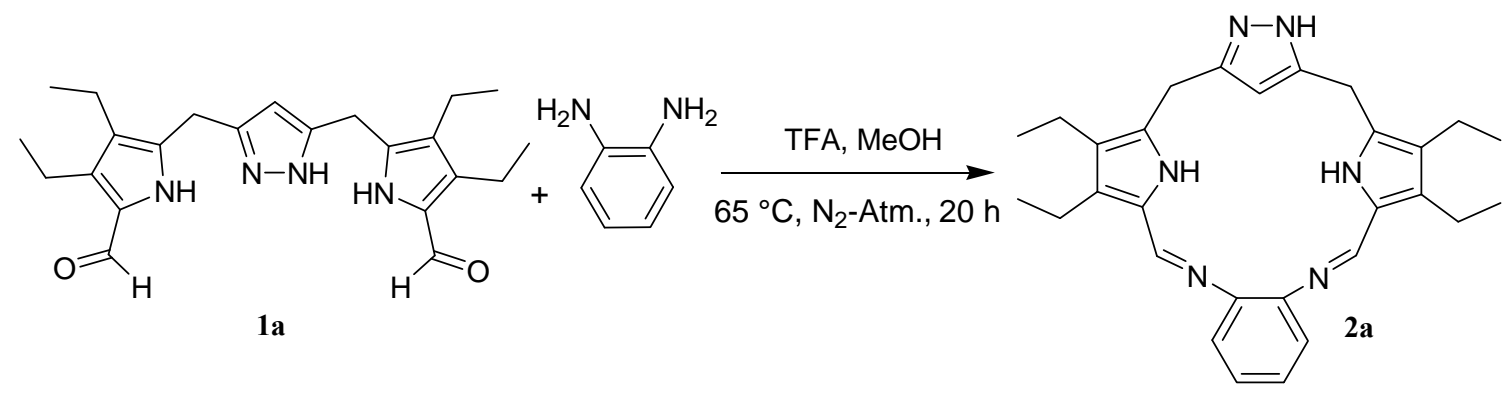

Der Dialdehyd 1a (200 mg, $0.51 \mathrm{mmol}, 1$ eq.) wurde unter $\mathrm{N}_{2}$-Atmosphäre in trockenem Methanol (200 mL) bei $50{ }^{\circ} \mathrm{C}$ gelöst. Das 1,2-Diaminobenzol (54.0 mg, $0.51 \mathrm{mmol}, 1$ eq.) wurde in trockenem Methanol $(10 \mathrm{~mL})$ gelöst und bei $50{ }^{\circ} \mathrm{C}$ langsam zum Dialdehyd zugetropft. Nach einigen Minuten Rühren wurde unter $\mathrm{N}_{2}$-Atm. Trifluoressigsäure $(1.54 \mathrm{~mL}$, 20.0 mmol, 40 eq.) in einem Zeitraum von 10 Minuten langsam zum Reaktionsgemisch dazugegeben. Das Reaktionsgemisch wurde anschließend für 20 Stunden unter Rückfluss erhitzt. Nach Abkühlen auf Raumtemperatur wurde das Lösungsmittel im Vakuum entfernt und das so erhaltene braune Rohprodukt säulenchromatographisch (Aluminiumoxid [basisch, Brockmann Aktivität I], Dichlormethan/Methanol 30:1, $\mathrm{R}_{\mathrm{f}}=0.79$ ) gereinigt. Der isolierte Zyklus 2a wurde in Form eines gelb-orangen Feststoffes (213 mg, 90 \%) erhalten. Einkristalle von $2 \mathrm{a}$ wuchsen bei $5{ }^{\circ} \mathrm{C}$ aus einer gesättigten ethanolischen Lösung, die röntgenographisch analysiert werden konnten.

\section{Auswertung:}

Summenformel:

Molekulargewicht (g/mol):

${ }^{1} \mathrm{H}-\mathrm{NMR}\left(500 \mathrm{MHz}, \mathrm{CDCl}_{3}\right)$ :
$\mathrm{C}_{29} \mathrm{H}_{34} \mathrm{~N}_{6}$

466.6

$\delta(\mathrm{ppm})=1.01-1.04\left(\mathrm{t},{ }^{3} J(\mathrm{H}, \mathrm{H})=7.5 \mathrm{~Hz}, 6 \mathrm{H}, \mathrm{CH}{ }_{3}\right)$,

1.16-1.19 (t, $\left.{ }^{3} J(\mathrm{H}, \mathrm{H})=7.5 \mathrm{~Hz}, 6 \mathrm{H}, \mathrm{CH}_{3}\right), 2.30-2.35$

$\left(\mathrm{m}, 4 \mathrm{H}, \mathrm{CH}_{2} \mathrm{CH}_{3}\right), 2.53-2.57\left(\mathrm{q},{ }^{3} J(\mathrm{H}, \mathrm{H})=7.4 \mathrm{~Hz}\right.$,

$\left.4 \mathrm{H}, \mathrm{CH}_{2} \mathrm{CH}_{3}\right), 3.88\left(\mathrm{~s}, 4 \mathrm{H}, \mathrm{CH}_{2}{ }^{\mathrm{py} / \mathrm{pz}}\right), 5.67(\mathrm{~s}, 1 \mathrm{H}$, $\left.\mathrm{C}^{\mathrm{pz}}\right), 7.17\left(\mathrm{~s}, 4 \mathrm{H}, \mathrm{C} H^{\mathrm{Ph}}\right), 8.16(\mathrm{~s}, 2 \times H \mathrm{C}=\mathrm{N}), 10.50$ (br, s, NH).

$\delta(\mathrm{ppm})=16.0 \quad\left(\mathrm{CH}_{3}\right), \quad 17.0 \quad\left(\mathrm{CH}_{2} \mathrm{CH}_{3}\right), \quad 17.3$

$\left(\mathrm{CH}_{2} \mathrm{CH}_{3}\right), 17.4\left(\mathrm{CH}_{3}\right), 24.0$ (br, $\left.\mathrm{CH}_{2}{ }^{\mathrm{py} / \mathrm{pz}}\right), 103.9$ 


\begin{tabular}{|c|c|}
\hline & $\begin{array}{l}\left(C \mathrm{H}^{\mathrm{pz}}\right), 117.2\left(\mathrm{br}, C^{\mathrm{Ph}}\right), 122.6\left(C^{\mathrm{tert}}\right), 125.7\left(C^{\mathrm{Ph}}\right) \\
132.5\left(\mathrm{br}, C^{\text {tert }}\right), 146.1(\mathrm{br}, \mathrm{HC}=\mathrm{N}) .\end{array}$ \\
\hline MS (ESI in $\mathrm{MeOH}) m / z(\%)$ : & $467(100)[\mathrm{M}+\mathrm{H}]^{+}$. \\
\hline MS (FAB in $4-N B A) ~ m / z(\%)$ : & $467(100)[\mathrm{M}+\mathrm{H}]^{+}$. \\
\hline HRMS-(+)ESI $m / z(\%)$ : & berechnet: $[\mathrm{M}+\mathrm{H}]^{+}: 467.29177$ \\
\hline$\left(\mathrm{MeOH} / \mathrm{H}_{2} \mathrm{O}\right)$ & gefunden: $[\mathrm{M}+\mathrm{H}]^{+}: 467.29177$. \\
\hline Schmelzpunkt $\left({ }^{\circ} \mathrm{C}\right)$ : & $241-244$ \\
\hline \multirow[t]{3}{*}{ Elementaranalyse $(\%)$ : } & berechnet: für $\mathrm{C}_{29} \mathrm{H}_{34} \mathrm{~N}_{6}\left(\mathrm{CH}_{3} \mathrm{OH}\right)_{1.5}(514.7)$ : \\
\hline & $\mathrm{C}: 71.18, \mathrm{H}: 7.83, \mathrm{~N}: 16.33$ \\
\hline & gefunden: C: $71.19, \mathrm{H}: 7.49, \mathrm{~N}: 16.04$. \\
\hline \multirow[t]{4}{*}{ IR (KBr): } & $\widetilde{v}=3428(\mathrm{~m}), 3268(\mathrm{w}), 3061(\mathrm{w}), 2961(\mathrm{~m}), 2926$ \\
\hline & $(\mathrm{w}), 2868(\mathrm{w}), 1612(\mathrm{vs}), 1569(\mathrm{~s}), 1443(\mathrm{~s}), 1383(\mathrm{w})$, \\
\hline & $1335(\mathrm{w}), 1264(\mathrm{~m}), 1210(\mathrm{~m}), 1101(\mathrm{w}), 1057(\mathrm{w})$ \\
\hline & $1010(w), 963(w), 894(w), 807(w), 745(w) \mathrm{cm}^{-1}$. \\
\hline $\mathrm{UV} / \mathrm{Vis}\left(\mathrm{CHCl}_{3} / 0.01 \% \mathrm{Et}_{3} \mathrm{~N}\right)$ : & $\lambda_{\max }[\mathrm{nm}]\left(\varepsilon \times 10^{4} \mathrm{M}^{-1} \mathrm{~cm}^{-1}\right)=332(2.37), 361(\mathrm{sh})$. \\
\hline
\end{tabular}

\subsubsection{Synthese von $2 \mathrm{a} \cdot \mathrm{TFA}$}

2a·TFA wurde über den gleichen Syntheseweg wie in 2a beschrieben dargestellt. Das Lösungsmittel wurde im Vakuum entfernt und das Rohprodukt in $\mathrm{MeOH} / \mathrm{CH}_{2} \mathrm{Cl}_{2}$ für einen Tag bei $5{ }^{\circ} \mathrm{C}$ umkristallisiert. Rote Einkristalle von 2a als TFA-Salz wuchsen durch langsames Abdampfen einer gesättigten Methanol/Dichlormethanlösung und konnten röntgenographisch analysiert werden.

\section{Auswertung:}

Summenformel:

$\mathrm{C}_{29} \mathrm{H}_{34} \mathrm{~N}_{6}\left(\mathrm{CF}_{3} \mathrm{COOH}\right)$

Molekulargewicht (g/mol): 580.6

${ }^{1} \mathrm{H}-\mathrm{NMR}\left(500 \mathrm{MHz}, \mathrm{CDCl}_{3}\right)$ :

$\delta(\mathrm{ppm})=1.13-1.16\left(\mathrm{t},{ }^{3} J(\mathrm{H}, \mathrm{H})=7.5 \mathrm{~Hz}, 6 \mathrm{H}, \mathrm{C} H_{3}\right)$, 1.17-1.20(t, $\left.{ }^{3} J(\mathrm{H}, \mathrm{H})=7.5 \mathrm{~Hz}, 6 \mathrm{H}, \mathrm{CH}_{3}\right), 2.50-2.54$ (m, 4H, $\left.\mathrm{CH}_{2} \mathrm{CH}_{3}, \mathrm{DMSO}_{6}\right), 2.73-2.78\left(\mathrm{q},{ }^{3} J(\mathrm{H}, \mathrm{H})=\right.$ $7.5 \mathrm{~Hz}, 4 \mathrm{H}, \mathrm{CH}_{2} \mathrm{CH}_{3}$ ), 3.42 (br, $\left.\mathrm{NH}\right), 4.13$ (s, 4H, $\left.\mathrm{CH}_{2}{ }^{\mathrm{py} / \mathrm{pz}}\right), \quad 6.21 \quad\left(\mathrm{~s}, \quad 1 \mathrm{H}, \quad \mathrm{CH}^{\mathrm{pz}}\right), \quad 7.30-7.34 \quad(\mathrm{dd}$, $\left.{ }^{3} J(\mathrm{H}, \mathrm{H})=3.3 \mathrm{~Hz}, \quad 2 \mathrm{H}, \quad \mathrm{C} H^{\mathrm{Ph}}\right), \quad 7.80-7.82 \quad(\mathrm{dd}$, 


\begin{tabular}{|c|c|}
\hline & $\begin{array}{l}\left.{ }^{3} J(\mathrm{H}, \mathrm{H})=3.3 \mathrm{~Hz}, 2 \mathrm{H}, \mathrm{C} H^{\mathrm{Ph}}\right), 8.62(\mathrm{~s}, 2 \mathrm{H}, 2 \times \\
H \mathrm{C}=\mathrm{N}), 11.15(\mathrm{br}, \mathrm{s}, \mathrm{N} H) .\end{array}$ \\
\hline${ }^{13} \mathrm{C}-\mathrm{NMR}\left(126 \mathrm{MHz}, \mathrm{DMSO}-\mathrm{d}_{6}\right)$ & $\begin{array}{l}\delta(\mathrm{ppm})=15.1\left(\mathrm{CH}_{3}\right), 16.2 \quad\left(\mathrm{CH}_{2} \mathrm{CH}_{3}\right), \\
\left(C \mathrm{H}_{2} \mathrm{CH}_{3}\right), 17.0\left(C_{3}\right), 24.2\left(\mathrm{br},-C_{2}{ }^{\mathrm{py} / \mathrm{pz}}\right), 104.4 \\
\left(C \mathrm{H}^{\mathrm{pz}}\right), 116.7\left(C^{\mathrm{Ph}}\right), 123.1\left(C^{\mathrm{q}}\right), 125.2\left(C^{\mathrm{q}}\right), 126.6 \\
\left(C \mathrm{H}^{\mathrm{Ph}}\right), 136.0\left(C^{\mathrm{q}}\right), 141.1\left(\mathrm{br}, C^{\mathrm{q}}\right), 142.3(\mathrm{br}, \mathrm{HC}=\mathrm{N}), \\
157.5\left(C^{\mathrm{q}, \mathrm{pz}}\right) .\end{array}$ \\
\hline${ }^{19}$ F-NMR (188 MHz, DMSO-d 6 ): & $\delta(\mathrm{ppm})=89.0\left(\mathrm{~s}, \mathrm{C} F_{3} \mathrm{COO}\right)$ \\
\hline MS (ESI in $\mathrm{MeOH}) m / z(\%)$ : & $\begin{array}{l}467(58)[\mathrm{M}+\mathrm{H}]^{+}, 933(78)[2 \times \mathrm{M}+\mathrm{H}]^{+}, 1047(100) \\
{\left[2 \times \mathrm{M}+\left(\mathrm{CF}_{3} \mathrm{COOH}\right)\right]^{+} .}\end{array}$ \\
\hline Schmelzpunkt $\left({ }^{\circ} \mathrm{C}\right)$ : & $222-235$ \\
\hline $\mathrm{IR}(\mathrm{KBr})$ : & $\begin{array}{l}\widetilde{v}=3430(\mathrm{~m}), 3210(\mathrm{w}), 3178(\mathrm{w}), 2957(\mathrm{~m}), 2947 \\
(\mathrm{w}), 1643(\mathrm{vs}), 1570(\mathrm{~s}), 1552(\mathrm{w}), 1435(\mathrm{w}), 1304 \\
(\mathrm{w}), 1190(\mathrm{~m}), 1180(\mathrm{~m}), 1010(\mathrm{w}), 958(\mathrm{w}), 801(\mathrm{~m}), \\
756(\mathrm{w}) \mathrm{cm}^{-1} .\end{array}$ \\
\hline $\mathrm{UV} / \mathrm{V}$ is $\left(\mathrm{CHCl}_{3} / \mathrm{TFA}\right)$ : & $\lambda_{\max }[\mathrm{nm}]\left(\varepsilon \times 10^{4} \mathrm{M}^{-1} \mathrm{~cm}^{-1}\right)=361(3.58), 441(\mathrm{sh}), 444$ \\
\hline
\end{tabular}
(sh).

\subsubsection{Synthese von $2 \mathrm{~b} \cdot \mathrm{TFA}$}

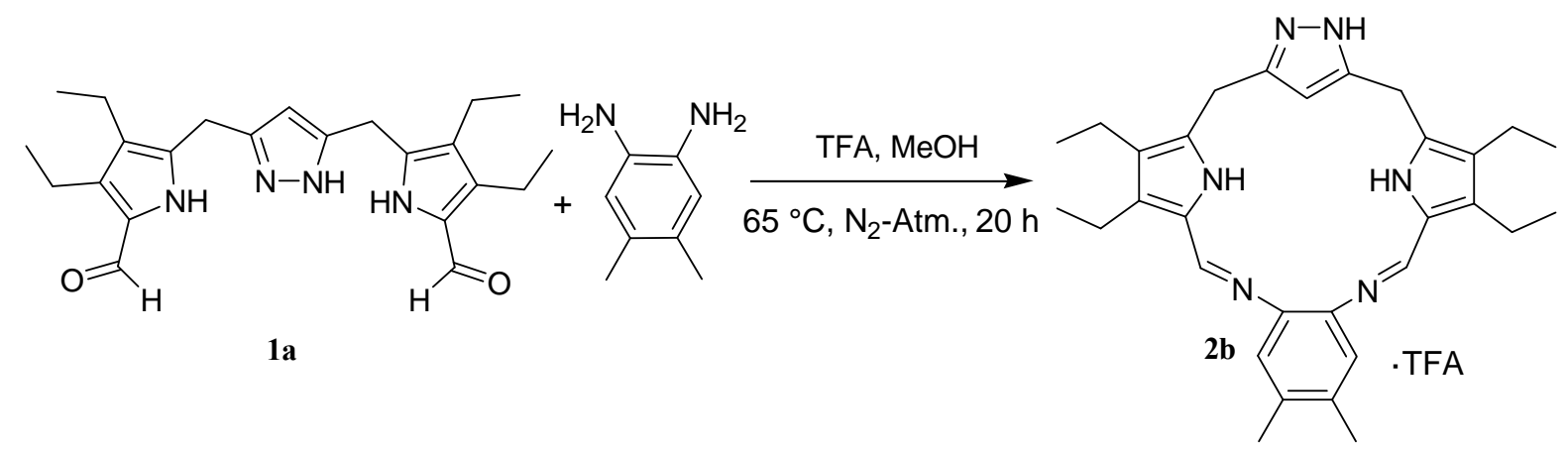

Der Dialdehyd 1a (90 mg, $0.23 \mathrm{mmol})$ wurde unter $\mathrm{N}_{2}$-Atmosphäre in Methanol $(80 \mathrm{~mL}$, getrocknet über Magnesium) bei $50{ }^{\circ} \mathrm{C}$ gelöst. 4,5-Dimethyl-1,2-phenylendiamin (99 \%; $31.0 \mathrm{mg}, 0.23 \mathrm{mmol}, 1.0$ eq.) wurde in Methanol (6 mL) gelöst und langsam zum Dialdehyd zugetropft. Nach einigen Minuten Rühren wurde unter $\mathrm{N}_{2}$-Atm. Trifluoressigsäure $(0.70 \mathrm{~mL}$, 9.12 mmol, 40 eq.) in einem Zeitraum von ca. 5 Minuten langsam zum Reaktionsgemisch dazugegeben. Das dabei braun gewordene Reaktionsgemisch wurde anschließend für 
20 Stunden unter Rückfluss erhitzt. Nach Abkühlen auf Raumtemperatur wurde das

Lösungsmittel im Vakuum entfernt und der Rückstand in $\mathrm{MeOH} / \mathrm{Et}_{2} \mathrm{O}$ bei $5{ }^{\circ} \mathrm{C}$ umkristallisiert. Das Produkt (2b·TFA) erhält man als kupfer roten Feststoff (102 mg, 90 \%).

\section{Auswertung:}

Summenformel:

Molekulargewicht (g/mol):

$\mathrm{R}_{\mathrm{f}}\left(\mathrm{MeOH} / \mathrm{CH}_{2} \mathrm{Cl}_{2}\right.$ 1:30):

${ }^{1} \mathrm{H}-\mathrm{NMR}\left(500 \mathrm{MHz}, \mathrm{DMSO}-\mathrm{d}_{6}\right)$ :

${ }^{13} \mathrm{C}-\mathrm{NMR}\left(126 \mathrm{MHz}, \mathrm{DMSO}-\mathrm{d}_{6}\right)$ :

${ }^{19}$ F-NMR (188 MHz, DMSO-d 6 ):

MS (ESI in $\mathrm{MeOH}) m / z(\%)$ :

MS (FAB, 4-NBA) $m / z(\%)$ :

HRMS-(+)ESI $\left(\mathrm{MeOH} / \mathrm{H}_{2} \mathrm{O}\right) m / z$ :

Elementaranalyse (\%):

Schmelzpunkt $\left({ }^{\circ} \mathrm{C}\right)$ :

IR $(\mathrm{KBr})$ :
$\mathrm{C}_{31} \mathrm{H}_{38} \mathrm{~N}_{6}\left(\mathrm{CF}_{3} \mathrm{COOH}\right)$

608.7

0.45

$\delta(\mathrm{ppm})=1.12-1.15\left(\mathrm{t},{ }^{3} J(\mathrm{H}, \mathrm{H})=7.5 \mathrm{~Hz}, 6 \mathrm{H}, \mathrm{CH}_{3}\right)$, 1.16-1.19(t, $\left.{ }^{3} J(\mathrm{H}, \mathrm{H})=7.5 \mathrm{~Hz}, 6 \mathrm{H}, \mathrm{CH}_{3}\right), 2.29(\mathrm{~s}, 6 \mathrm{H}$, $\left.2 \times \mathrm{CH}_{3}{ }^{\mathrm{Ar}}\right), 2.49-2.53\left(\mathrm{q},{ }^{3} J(\mathrm{H}, \mathrm{H})=7.5 \mathrm{~Hz}, 4 \mathrm{H}\right.$, $\left.\mathrm{CH}_{2} \mathrm{CH}_{3}\right), 2.72-2.77\left(\mathrm{q},{ }^{3} \mathrm{~J}(\mathrm{H}, \mathrm{H})=7.5 \mathrm{~Hz}, 4 \mathrm{H}\right.$, $\left.\mathrm{CH}_{2} \mathrm{CH}_{3}\right), 4.12\left(\mathrm{~s}, 4 \mathrm{H}, 2 \times \mathrm{CH}_{2}\right), 6.20\left(\mathrm{~s}, 1 \mathrm{H}, \mathrm{CH}^{\mathrm{pz}}\right)$, $7.63\left(\mathrm{~s}, 2 \mathrm{H}, \mathrm{C} H^{\mathrm{Ph}}\right), 8.58(\mathrm{~s}, 2 \mathrm{H}, H \mathrm{C}=\mathrm{N}), 11.08-11.54$ (br, $\mathrm{N} H$ ).

$\delta(\mathrm{ppm})=15.3\left(\mathrm{CH}_{3}\right), \quad 16.4 \quad\left(\mathrm{CH}_{2} \mathrm{CH}_{3}\right), \quad 16.7$ $\left(\mathrm{CH}_{2} \mathrm{CH}_{3}\right), \quad 17.2\left(\mathrm{CH}_{3}\right), \quad 19.2\left(\mathrm{CH}_{3}{ }^{\mathrm{Ph}}\right), 24.3$ (br, $\left.\mathrm{CH}_{2}{ }^{\mathrm{py} / \mathrm{pz}}\right), 104.5\left(C \mathrm{H}^{\mathrm{pz} 4}\right), 116.1\left(C^{\mathrm{q}}\right), 117.5\left(C \mathrm{H}^{\mathrm{Ph}}\right)$, $118.4\left(C^{\mathrm{q}}\right), 123.1\left(C^{\mathrm{q}}\right), 125.0\left(C^{\mathrm{q}}\right), 133.7\left(C^{\mathrm{q}}\right), 135.5$ $\left(C^{\mathrm{q}}\right), 140.6\left(C^{\mathrm{q}}\right), 141.6(C \mathrm{H}=\mathrm{N}), 157.8,158.0,158.1$, $158.3\left(\mathrm{q},{ }^{3} J(C, \mathrm{~F})=30.8 \mathrm{~Hz}, \mathrm{TFA}\right)$.

$\delta(\mathrm{ppm})=88.6\left(\mathrm{~s}, \mathrm{C} F_{3} \mathrm{COO}\right)$.

$495(100)[\mathrm{M}+\mathrm{H}]^{+}, 989(65)[2 \times \mathrm{M}+\mathrm{H}]^{+}, 1102(61)$

$\left[2 \times \mathrm{M}+\mathrm{CF}_{3} \mathrm{COOH}\right]^{+}$.

$495(100)[\mathrm{M}]^{+}$.

berechnet $[\mathrm{M}+\mathrm{H}]^{+}:$495.32307;

gemessen $[\mathrm{M}+\mathrm{H}]^{+}: 495.32312$.

berechnet für $\mathrm{C}_{31} \mathrm{H}_{38} \mathrm{~N}_{6}(\mathrm{TFA})_{1}\left(\mathrm{H}_{2} \mathrm{O}\right)_{1.5}$ :

C: 62.35, H: 6.66, N: 13.22;

gemessen: C: 62.15, H: 6.63, N: 12.44.

216-218

$\widetilde{v}=3436(\mathrm{~m}), 3265(\mathrm{w}), 3177(\mathrm{w}), 2965(\mathrm{~m})$, 2932 (w), 2874 (w), 2359 (w), 1679 (s), 1641 (vs), 1590 (s), 1447 (m), 1385 (m), 1335 (w), 1312 (w), 
$1264(\mathrm{~s}), 1195$ (w), 1099 (m), 1017 (m), 866 (w), 802

$\mathrm{UV} / \mathrm{V}$ is $(\mathrm{MeCN} / \mathrm{TFA})$ : (vs), $717(\mathrm{w}) \mathrm{cm}^{-1}$.

$\lambda_{\max }[\mathrm{nm}]\left(\varepsilon \times 10^{4} \mathrm{M}^{-1} \mathrm{~cm}^{-1}\right)=362(2.88), 413(\mathrm{sh}), 467$

(sh).

\subsubsection{Synthese von $2 b$}

Der neutrale Makrozyklus 2b wurde durch den gleichen Syntheseweg wie bei bereits in 2b-TFA beschrieben dargestellt. 2b-TFA kann durch Überschuss einer Base $\left(\mathrm{Et}_{3} \mathrm{~N}\right.$ oder $\mathrm{Na}_{2} \mathrm{CO}_{3}$-wäß.) oder durch Filtration über basischem Aluminiumoxid (Brockmann Aktivität I, $\mathrm{CH}_{2} \mathrm{Cl}_{2} / \mathrm{MeOH}$ 15:1) neutralisiert werden. Einkristalle von 2 b wuchsen unter langsamem Abdampfen einer gesättigten $\mathrm{MeOH} / \mathrm{CH}_{2} \mathrm{Cl}_{2} / \mathrm{Et}_{3} \mathrm{~N}$-Lösung.

\section{Auswertung:}

Summenformel:

$\mathrm{C}_{31} \mathrm{H}_{38} \mathrm{~N}_{6}$

Molekulargewicht (g/mol): 494.7

${ }^{1} \mathrm{H}-\mathrm{NMR}\left(300 \mathrm{MHz}, \mathrm{CDCl}_{3}\right)$ :

$\delta(\mathrm{ppm})=1.00-1.05\left(\mathrm{t},{ }^{3} J(\mathrm{H}, \mathrm{H})=7.5 \mathrm{~Hz}, 6 \mathrm{H}, \mathrm{CH}\right)$, 1.13-1.18(t, $\left.{ }^{3} J(\mathrm{H}, \mathrm{H})=7.5 \mathrm{~Hz}, 6 \mathrm{H}, \mathrm{CH}_{3}\right), 2.27(\mathrm{~s}, 6 \mathrm{H}$, $\left.2 \times \mathrm{CH}_{3}{ }^{\mathrm{Ph}}\right), 2.31-2.38\left(\mathrm{q},{ }^{3} J(\mathrm{H}, \mathrm{H})=7.5 \mathrm{~Hz}, 4 \mathrm{H}\right.$, $\left.\mathrm{CH}_{2} \mathrm{CH}_{3}\right), 2.51-2.58\left(\mathrm{q},{ }^{3} \mathrm{~J}(\mathrm{H}, \mathrm{H})=7.5 \mathrm{~Hz}, 4 \mathrm{H}\right.$, $\left.\mathrm{CH}_{2} \mathrm{CH}_{3}\right), 3.88\left(\mathrm{~s}, 4 \mathrm{H}, 2 \times \mathrm{CH}_{2}\right), 5.75\left(\mathrm{~s}, \mathrm{br}, 1 \mathrm{H}, \mathrm{CH}^{\mathrm{pz}}\right)$, $6.94\left(\mathrm{~s}, 2 \mathrm{H}, \mathrm{C} H^{\mathrm{Ph}}\right), 8.07(\mathrm{~s}, 2 \mathrm{H}, 2 \times H \mathrm{C}=\mathrm{N}),(\mathrm{NH})$ nicht beobachtet.

MS (ESI in $\left.\mathrm{MeCN} / \mathrm{CH}_{2} \mathrm{Cl}_{2}\right) \mathrm{m} / z(\%)$ : $495(100)[\mathrm{M}+\mathrm{H}]^{+}, 989(65)[2 \times \mathrm{M}+\mathrm{H}]^{+}$.

$\mathrm{UV} / \mathrm{V}$ is $\left(\mathrm{MeCN} / 0.01 \% \mathrm{Et}_{3} \mathrm{~N}\right)$ : $\lambda_{\text {max }}[\mathrm{nm}]\left(\varepsilon \times 10^{4} \mathrm{M}^{-1} \mathrm{~cm}^{-1}\right)=334$ (2.06), 388 (1.24). 


\subsubsection{Synthese von 2c-TFA}

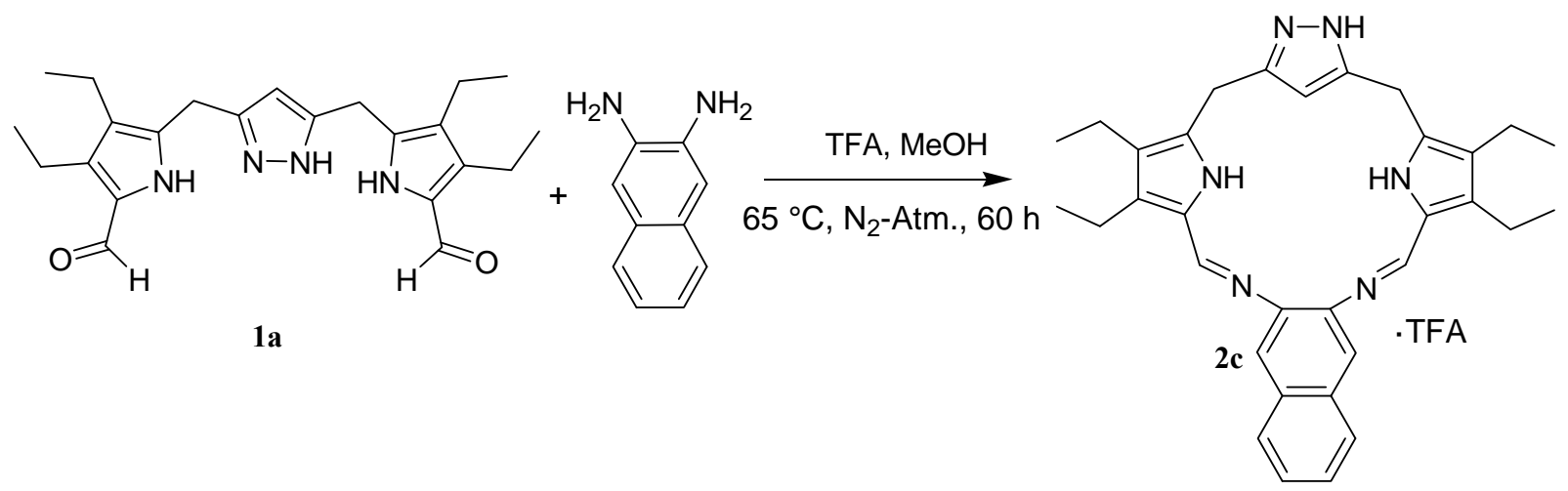

Der Dialdehyd 1a $(150 \mathrm{mg}, 0.38 \mathrm{mmol})$ wurde unter $\mathrm{N}_{2}$-Atmosphäre in Methanol $(90 \mathrm{~mL}$, getrocknet über $\mathrm{Mg}$ ) bei $50{ }^{\circ} \mathrm{C}$ gelöst. $\mathrm{Zu}$ dieser Lösung wurde Diaminonaphtnaline $(60.0 \mathrm{mg}$, $0.38 \mathrm{mmol}$, 1 eq.), in Methanol (8 mL) gelöst, langsam zugetropft. Nach 10 Min. Rühren wurde unter $\mathrm{N}_{2}$-Atm. Trifluoressigsäure (1.20 mL, $15.2 \mathrm{mmol}, 40$ eq.) in einem Zeitraum von 3 Min. langsam zum Reaktionsgemisch dazugegeben. Das dabei braun gewordene Reaktionsgemisch wurde anschließend für 20 Stunden unter Rückfluss erhitzt. Nach Abkühlen auf Raumtemperatur wurde das Lösungsmittel im Vakuum entfernt und der Rückstand in $\mathrm{MeOH} / \mathrm{Et}_{2} \mathrm{O} / \mathrm{CH}_{2} \mathrm{Cl}_{2}$ bei $5{ }^{\circ} \mathrm{C}$ umkristallisiert. Das Produkt wurde als rotbrauner Feststoff (173 mg, $88 \%$ ) erhalten. Unter langsamen Abdampfen einer gesättigten $\mathrm{MeOH} / \mathrm{CH}_{2} \mathrm{Cl}_{2}$ Lösung von 2c-TFA wuchsen nach einigen Tagen Einkristalle, die röngenographisch analysiert wurden.

\section{Auswertung:}

Summenformel:

$\mathrm{C}_{33} \mathrm{H}_{36} \mathrm{~N}_{6}$ (TFA)

Molekulargewicht (g/mol):

630.7

$\mathrm{R}_{\mathrm{f}}\left(\mathrm{MeOH} / \mathrm{CH}_{2} \mathrm{Cl}_{2} 1: 30\right)$ :

0.57

${ }^{1} \mathrm{H}-\mathrm{NMR}\left(300 \mathrm{MHz}, \mathrm{CDCl}_{3}\right.$ ):

$\delta(\mathrm{ppm})=1.03-1.08\left(\mathrm{t},{ }^{3} J(\mathrm{H}, \mathrm{H})=7.5 \mathrm{~Hz}, 6 \mathrm{H}, \mathrm{CH}_{3}\right)$, $1.13-1.18\left(\mathrm{t},{ }^{3} J(\mathrm{H}, \mathrm{H})=7.5 \mathrm{~Hz}, 6 \mathrm{H}, \mathrm{CH}_{3}\right), 2.36-2.43$ (q, $\left.{ }^{3} J(\mathrm{H}, \mathrm{H})=7.5 \mathrm{~Hz}, \quad 4 \mathrm{H}, \quad \mathrm{CH}_{2} \mathrm{CH}_{3}\right), \quad 2.57-2.65 \quad(\mathrm{q}$, $\left.{ }^{3} J(\mathrm{H}, \mathrm{H})=7.5 \mathrm{~Hz}, 4 \mathrm{H}, \mathrm{CH}_{2} \mathrm{CH}_{3}\right), 4.10\left(\mathrm{~s}, 4 \mathrm{H}, 2 \times \mathrm{CH}_{2}\right)$, $6.30\left(\mathrm{~s}, 1 \mathrm{H}, \mathrm{C} H^{\mathrm{pz}}\right), 7.39-7.43\left(\mathrm{~m},{ }^{3} J(\mathrm{H}, \mathrm{H})=3.1 \mathrm{~Hz}\right.$, $\left.2 \mathrm{H}, \mathrm{C} H^{\text {naphtyl }}\right), 7.70-7.74\left(\mathrm{~m},{ }^{3} J(\mathrm{H}, \mathrm{H})=3.1 \mathrm{~Hz}, 4 \mathrm{H}\right.$, $\left.\mathrm{C} H^{\text {naphtyl }}\right), 8.16(\mathrm{~s}, 2 \mathrm{H}, 2 \times H \mathrm{C}=\mathrm{N}), 12.23(\mathrm{~s}, \mathrm{br}, \mathrm{N} H)$.

${ }^{13} \mathrm{C}-\mathrm{NMR}\left(126 \mathrm{MHz}, \mathrm{DMSO}-\mathrm{d}_{6}\right): \quad \delta(\mathrm{ppm})=15.3\left(\mathrm{CH}_{2} \mathrm{CH}_{3}\right), 16.4\left(\mathrm{CH}_{2} \mathrm{CH}_{3}\right), 16.7$ $\left(\mathrm{CH}_{2} \mathrm{CH}_{3}\right), 17.3\left(\mathrm{CH}_{2} \mathrm{CH}_{3}\right), 24.4\left(\mathrm{br}, \mathrm{CH}_{2}{ }^{\mathrm{py} / \mathrm{pz}}\right), 104.5$ 


\begin{tabular}{|c|c|}
\hline & $\begin{array}{l}\left(C \mathrm{H}^{\mathrm{pz}-4}\right), 113.8\left(C \mathrm{H}^{\mathrm{naphtl}}\right), 123.3\left(C^{\mathrm{q}}\right), 125.4\left(C \mathrm{H}^{\mathrm{naphtl}}\right), \\
126.2\left(C^{\mathrm{q}}\right), 127.5\left(C \mathrm{H}^{\mathrm{naphtl}}\right), 131.6\left(C^{\mathrm{q}}\right), 135.5\left(C^{\mathrm{q}}\right), \\
141.3(\mathrm{br}, \mathrm{N}=C \mathrm{H}), 142.9\left(C^{\mathrm{q}}\right), 158.0\left(C^{\mathrm{q}}\right) .\end{array}$ \\
\hline MS (ESI in $\mathrm{MeOH}) m / z(\%)$ : & $\begin{array}{l}517(100)\left[\mathrm{M}^{+}+\mathrm{H}\right], 1033(24)[2 \mathrm{M}+\mathrm{H}]^{+}, 1069(40) \\
{\left[2 \times\left(\mathrm{M}+\mathrm{H}_{2} \mathrm{O}\right)+\mathrm{H}\right]^{+}, 1146(42)[2 \mathrm{M}+\mathrm{TFA}]^{+}}\end{array}$ \\
\hline HRMS- $(+)$ ESI $\left(\mathrm{MeOH} / \mathrm{H}_{2} \mathrm{O}\right)$ : & $\begin{array}{l}\text { berechnet für } \mathrm{C}_{33} \mathrm{H}_{36} \mathrm{~N}_{6}:[\mathrm{M}+\mathrm{H}]^{+}: 517.30742 \text {; } \\
\text { gemessen für } \mathrm{C}_{33} \mathrm{H}_{36} \mathrm{~N}_{6}:[\mathrm{M}+\mathrm{H}]^{+}: 517.30720 \text {. }\end{array}$ \\
\hline MS (FAB, 4-NBA) $m / z(\%):$ & $517(100)[\mathrm{M}+\mathrm{H}]^{+}, 1033(1)[2 \times \mathrm{M}+\mathrm{H}]^{+}$. \\
\hline Elemenmtaranalyse (\%): & $\begin{array}{l}\text { berechnet für } \mathrm{C}_{33} \mathrm{H}_{36} \mathrm{~N}_{6}\left(\mathrm{CF}_{3} \mathrm{COOH}\right)\left(\mathrm{H}_{2} \mathrm{O}\right) \text { : } \\
\text { C: } 64.80, \mathrm{H}: 6.06, \mathrm{~N}: 12.96 \\
\text { gemessen: C: } 64.20, \mathrm{H}: 6.40, \mathrm{~N}: 12.74 \text {. }\end{array}$ \\
\hline Schmelzpunkt $\left({ }^{\circ} \mathrm{C}\right)$ & $270-279$ \\
\hline $\mathrm{IR}(\mathrm{KBr})$ : & $\begin{array}{l}\tilde{v}=3422(\mathrm{w}), 3230(\mathrm{~m}), 3050(\mathrm{w}), 2960(\mathrm{~m}), \\
2925(\mathrm{w}), 2875(\mathrm{w}), 2354(\mathrm{w}), 1604(\mathrm{vs}), 1433(\mathrm{~s}), \\
1328(\mathrm{~m}), 1265(\mathrm{~m}), 1222(\mathrm{~m}), 1156(\mathrm{w}), 1009(\mathrm{w}), \\
861(\mathrm{w}), 801(\mathrm{w}), 746(\mathrm{w}), 620(\mathrm{w}) \mathrm{cm}^{-1} .\end{array}$ \\
\hline $\mathrm{UV} / \mathrm{Vis}\left(\mathrm{CHCl}_{3} / \mathrm{TFA}\right)$ : & $\lambda_{\max }[\mathrm{nm}]\left(\varepsilon \times 10^{4} \mathrm{M}^{-1} \mathrm{~cm}^{-1}\right)=342(2.44), 381(\mathrm{sh})$ \\
\hline $\mathrm{UV} / \mathrm{V}$ is $\left(\mathrm{CHCl}_{3} / 0.01 \% \mathrm{Et}_{3} \mathrm{~N}\right)$ : & $\lambda_{\max }[\mathrm{nm}]\left(\varepsilon \times 10^{4} \mathrm{M}^{-1} \mathrm{~cm}^{-1}\right)=369(2.87), 414(\mathrm{sh})$ \\
\hline
\end{tabular}

\subsubsection{Synthese von $2 d \cdot T F A$}

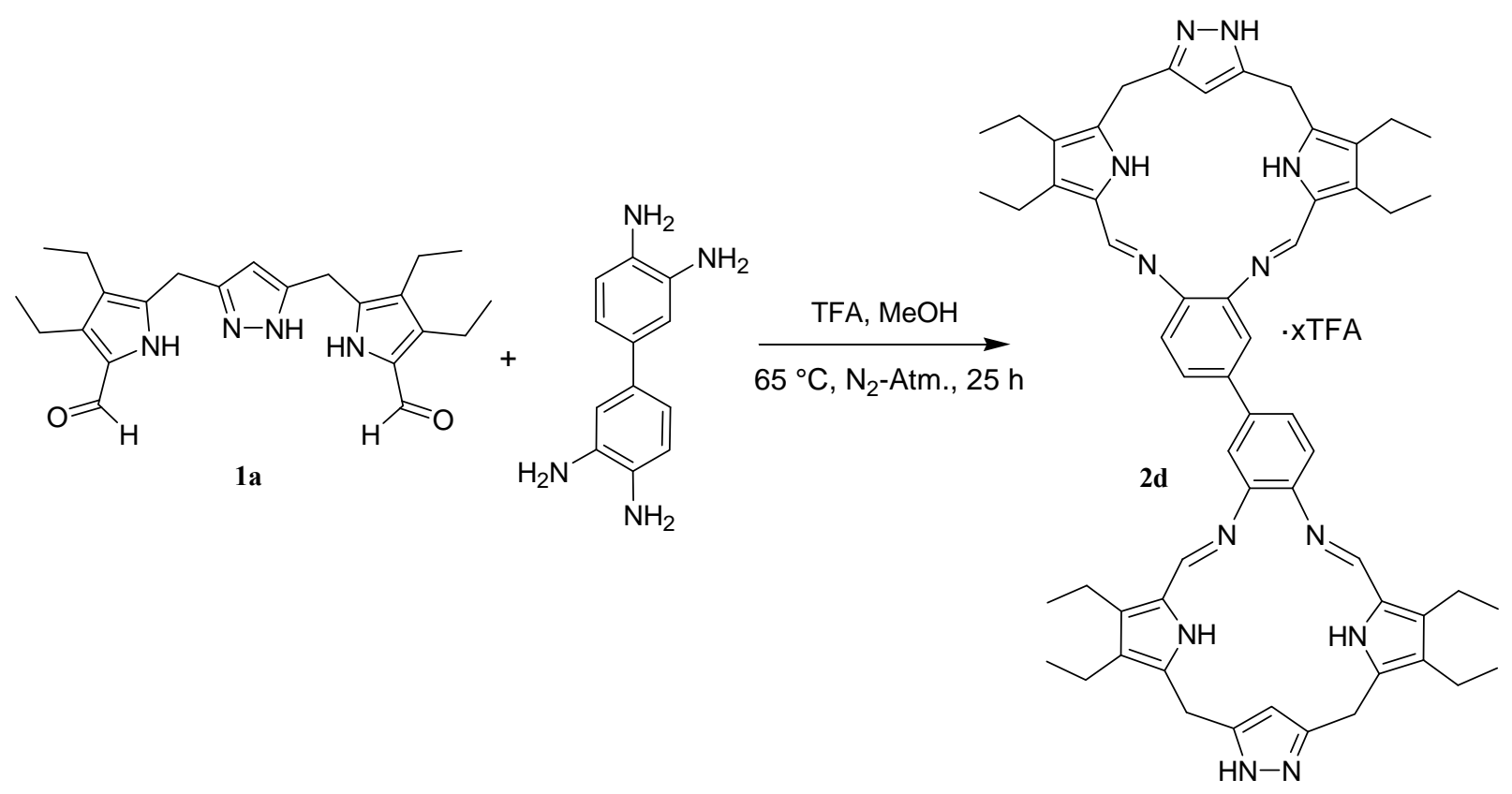


1 a (108 mg, 0.20 mmol, 2.0 eq,) wurde unter $\mathrm{N}_{2}$-Atmosphäre in Methanol (90 mL, getrocknet über $\mathrm{Mg}$ ) bei $60{ }^{\circ} \mathrm{C}$ gelöst. $\mathrm{Zu}$ dieser Lösung wurde 3,3'-Diaminobenzidin (21.8 $\mathrm{mg}$, $0.10 \mathrm{mmol}$, 1.0 eq.), in einer Suspension aus Methanol/Dichlormethan (2/5 mL) gelöst langsam dazu getropft. Nach 10 Minuten Rühren wurde unter $\mathrm{N}_{2}$-Atm. Trifluoressigsäure (156 $\mu 1, \quad 10.9$ mmol, 40 eq.) in einem Zeitraum von 5 Minuten langsam zum Reaktionsgemisch dazugegeben. Das dabei violett gewordene Reaktionsgemisch wurde anschließend unter Lichtausschluss für 26 Stunden unter Rückfluss erhitzt. Nach Abkühlen auf Raumtemperatur wurde das Lösungsmittel im Vakuum entfernt und das so erhaltene braune Rohprodukt in Methanol/Dichlormethan bei $5{ }^{\circ} \mathrm{C}$ zur Kristallisation angesetzt. Der rote Feststoff (2d·TFA) wurde abfiltriert und mit $\mathrm{Et}_{2} \mathrm{O}$ gewaschen (94 mg, 99 \%).

\section{Auswertung:}

Summenformel:

$\mathrm{C}_{58} \mathrm{H}_{66} \mathrm{~N}_{12}(\cdot \mathrm{xTFA})$

Molekulargewicht (g/mol):

931.2

$\mathrm{R}_{\mathrm{f}}\left(\mathrm{MeOH} / \mathrm{CH}_{2} \mathrm{Cl}_{2}\right.$ 1:15):

0.56

${ }^{1} \mathrm{H}-\mathrm{NMR}\left(500 \mathrm{MHz}, \mathrm{DMSO}-\mathrm{d}_{6}\right): \quad \delta(\mathrm{ppm})=1.11-1.19\left(\mathrm{~m},{ }^{3} \mathrm{~J}=7.5 \mathrm{~Hz}, 27 \mathrm{H}, \mathrm{CH}_{3}\right), 2.50$ $\left(\mathrm{m},{ }^{3} J(\mathrm{H}, \mathrm{H})=7.5 \mathrm{~Hz}, 8 \mathrm{H}, \mathrm{CH}_{2} \mathrm{CH}_{3}, \mathrm{DMSO}\right), 2.79$ (q, $\left.{ }^{3} J(\mathrm{H}, \mathrm{H})=7.5 \mathrm{~Hz}, 8 \mathrm{H}, \mathrm{CH}_{2} \mathrm{CH}_{3}\right), 4.15\left(\mathrm{~s}, 8 \mathrm{H}, 2 \times \mathrm{CH}_{2}\right)$, $6.22\left(\mathrm{~s}, 2 \mathrm{H}, \mathrm{C} H^{\mathrm{pz}}\right), 7.75-7.83\left(\mathrm{t}, 2 \mathrm{H}, \mathrm{C} H^{\mathrm{Ar}}\right), 7.93-7.98$ $\left(\mathrm{d}, 2 \mathrm{H}, \mathrm{C} H^{\mathrm{Ar}}\right), 8.20\left(\mathrm{~s}, 2 \mathrm{H}, \mathrm{C} H^{\mathrm{Ar}}\right), 8.69(\mathrm{~s}, 2 \mathrm{H}$, $2 \times H \mathrm{C}=\mathrm{N}), 8.82(\mathrm{~s}, 2 \mathrm{H}, 2 \times H \mathrm{C}=\mathrm{N}), 11.20(\mathrm{~s}, 1 \mathrm{H}, \mathrm{NH})$, $11.33(\mathrm{~s}, 1 \mathrm{H}, \mathrm{N} H)$.

${ }^{13} \mathrm{C}-\mathrm{NMR}\left(126 \mathrm{MHz}, \mathrm{DMSO}-\mathrm{d}_{6}\right): \quad \delta(\mathrm{ppm})=15.1\left(\mathrm{CH}_{3}\right), \quad 15.15\left(\mathrm{CH}_{2} \mathrm{CH}_{3}\right), \quad 16.8$ $\left(\mathrm{CH}_{2} \mathrm{CH}_{3}\right), 16.6\left(\mathrm{CH}_{3}\right), 16.9,17.1,24.2\left(\mathrm{br}, \mathrm{CH}_{2}{ }^{\mathrm{py} / \mathrm{pz}}\right)$, $24.4\left(\mathrm{CH}_{2}{ }^{\mathrm{py} / \mathrm{pz}}\right), 104.6\left(\mathrm{CH}^{\mathrm{pz}-4}\right), 115.7\left(\mathrm{CH}^{\mathrm{Ar}}\right), 117.3$ $\left(C \mathrm{H}^{\mathrm{Ar}}\right), 123.0\left(C^{\mathrm{q}}\right), 123.5\left(C^{\mathrm{q}}\right), 125.0,125.2,125.6$ $\left(C \mathrm{H}^{\mathrm{Ar}}\right), 135.8\left(C^{\mathrm{q}}\right), 136.2\left(C^{\mathrm{q}}\right), 137.5\left(C^{\mathrm{q}}\right), 140.9\left(C^{\mathrm{q}}\right)$, $142.2(C \mathrm{H}=\mathrm{N}), 142.6(C \mathrm{H}=\mathrm{N}), 143.3\left(C^{\mathrm{q}}\right),[157.5$, $157.7,157.9,158.2]$ (q, $\left.\mathrm{CF}_{3} \mathrm{COOH}\right)$.

${ }^{19}$ F-NMR $\left(188 \mathrm{MHz}, \mathrm{DMSO}-\mathrm{d}_{6}\right): \quad \delta(\mathrm{ppm})=88.05\left(\mathrm{~s}, \mathrm{C} F_{3} \mathrm{COO}\right)$.

MS (ESI in $\mathrm{MeOH}) m / z(\%)$ :

467 (48) $[\mathrm{M}+2 \mathrm{H}]^{2+}, 931(100) \quad[\mathrm{M}+\mathrm{H}]^{+}, 954$ (7)

$[\mathrm{M}+\mathrm{Na}]^{+}, \quad 1862 \quad(20) \quad[2 \times \mathrm{M}+\mathrm{H}]^{+}, \quad 1884 \quad(7)$ $[2 \times \mathrm{M}+\mathrm{Na}]^{+}$.

HRMS-(+)ESI $m / z$ : berechnet für $\mathrm{C}_{58} \mathrm{H}_{66} \mathrm{~N}_{12}$ : $[\mathrm{M}+2 \mathrm{H}]^{2+}: 466.28395$; 
$\left(\mathrm{MeOH} / \mathrm{H}_{2} \mathrm{O}\right)$

MS (FAB, 4-NBA) $m / z(\%)$ :

Elementaranalyse (\%):

$\mathrm{UV} / \mathrm{V}$ is $(\mathrm{MeOH} / \mathrm{TFA})$ :

$\mathrm{UV} / \mathrm{V}$ is $\left(\mathrm{MeOH} / \mathrm{Et}_{3} \mathrm{~N}\right)$ :

Schmelzpunkt $\left({ }^{\circ} \mathrm{C}\right)$ :

IR ( $\mathrm{KBr})$ : gemessen für $\mathrm{C}_{58} \mathrm{H}_{66} \mathrm{~N}_{12}$ : $[\mathrm{M}+2 \mathrm{H}]^{2+}$ : 466.28388 .

$932(100)[\mathrm{M}+\mathrm{H}]^{+}$.

berechnet für $\mathrm{C}_{58} \mathrm{H}_{66} \mathrm{~N}_{12}\left(\mathrm{CF}_{3} \mathrm{COOH}\right)_{3}\left(\mathrm{H}_{2} \mathrm{O}\right)_{2}$ :

C: 58.71; H: 5.62, N: 12.84;

gemessen: C: 58.39, H: 5.50; N: 12.55 .

237-239.

$\widetilde{v}=3429$ (br), 3229 (br), 2955 (w), 2361 (w), 1640

(vs), 1577 (m), 1436 (m), 1322 (w), 1335 (w), 1260

(w), $1188(\mathrm{w}), 1145(\mathrm{~m}), 1012(\mathrm{~m}), 866(\mathrm{~m}) \mathrm{cm}^{-1}$.

$\lambda_{\max }[\mathrm{nm}]\left(\varepsilon \times 10^{4} \mathrm{M}^{-1} \mathrm{~cm}^{-1}\right)=376(3.79), 422(\mathrm{sh})$.

$\lambda_{\max }[\mathrm{nm}]\left(\varepsilon \times 10^{4} \mathrm{M}^{-1} \mathrm{~cm}^{-1}\right)=348(2.39), 393(\mathrm{sh})$.

\subsubsection{Synthese von 2d·DFA}

2d·DFA wurde nach der gleichen Vorschrift wie bereits in 2d beschrieben synthetisiert. Als Säure wurde hierbei 1.0 eq. Dodekafluorodiessigsäure (96 \%) statt TFA verwendet. Das Reaktionsgemisch wurde unter $\mathrm{N}_{2}$-Atmosphäre für $31 \mathrm{~h}$ unter Rückfluss erhitzt. Das Lösungsmittel wurde anschließend im Vakuum entfernt und der rot-braune Rückstand in $\mathrm{MeOH} / \mathrm{Et}_{2} \mathrm{O}$ für einige Tage bei $5{ }^{\circ} \mathrm{C}$ zur Kristallisation angesetzt (152 mg, $94 \%$ ).

\section{Ausbeute:}

Summenformel:

$\mathrm{C}_{58} \mathrm{H}_{66} \mathrm{~N}_{12}(\cdot x D F A)$

Molekulargewicht (g/mol):

931.2

${ }^{1} \mathrm{H}-\mathrm{NMR}$ (500 MHz, DMSO-d 6 ):

$\delta(\mathrm{ppm})=1.13-1.20\left(\mathrm{~m},{ }^{3} J(\mathrm{H}, \mathrm{H})=7.5 \mathrm{~Hz}, 27 \mathrm{H}\right.$, $\left.\mathrm{CH}_{3}\right), 2.50\left(\mathrm{~m},{ }^{3} \mathrm{~J}(\mathrm{H}, \mathrm{H})=7.5 \mathrm{~Hz}, 8 \mathrm{H}, \mathrm{CH}_{2} \mathrm{CH}_{3}\right.$, DMSO), $2.79\left(\mathrm{q},{ }^{3} J(\mathrm{H}, \mathrm{H})=7.5 \mathrm{~Hz}, 8 \mathrm{H}, \mathrm{CH}_{2} \mathrm{CH}_{3}\right.$ ), 3.40 (br, s, $\mathrm{H}_{2} \mathrm{O}$ ), 4.14 (s, $4 \mathrm{H}, 2 \times \mathrm{CH}_{2}$ ), 4.15 (s, $4 \mathrm{H}$, $\left.2 \times \mathrm{CH}_{2}\right), 6.22\left(\mathrm{~s}, 2 \mathrm{H}, \mathrm{C} H^{\mathrm{pz}}\right), 7.73-7.75\left(\mathrm{~d}, 2 \mathrm{H}, \mathrm{C} H^{\mathrm{Ar}}\right)$, 7.92-7.93 (d, 2H, CH $\left.H^{\mathrm{Ar}}\right), 8.19\left(\mathrm{~s}, 2 \mathrm{H}, \mathrm{C} H^{\mathrm{Ar}}\right), 8.67$ (s, $2 \mathrm{H}, 2 \times H \mathrm{C}=\mathrm{N}), 8.79(\mathrm{~s}, 2 \mathrm{H}, 2 \times H \mathrm{C}=\mathrm{N}), 11.13(\mathrm{~s}, 1 \mathrm{H}$, $\mathrm{NH}) 11.27$ (s, 1H, NH).

${ }^{13} \mathrm{C}-\mathrm{NMR}\left(126 \mathrm{MHz}, \mathrm{DMSO}-\mathrm{d}_{6}\right): \quad \delta(\mathrm{ppm})=15.1\left(\mathrm{CH}_{3}\right), 15.2\left(\mathrm{CH}_{3}\right), 16.1\left(\mathrm{CH}_{2} \mathrm{CH}_{3}\right)$, $16.4\left(\mathrm{CH}_{2} \mathrm{CH}_{3}\right), 16.7\left(\mathrm{CH}_{2} \mathrm{CH}_{3}\right), 17.0\left(\mathrm{CH}_{3}\right), 17.1$ 


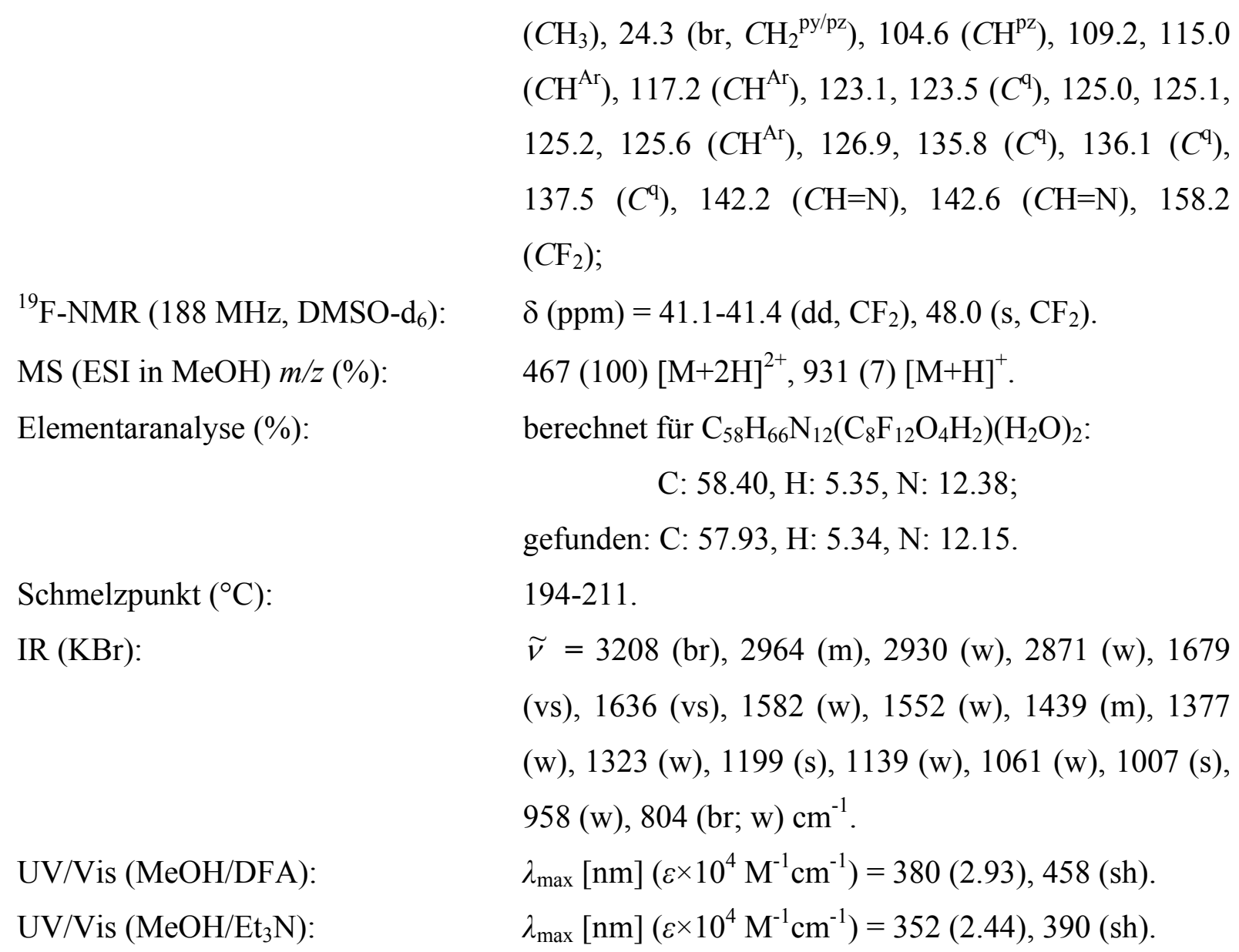

10.4.8. Synthese von $2 \mathrm{e} \cdot$ TFA
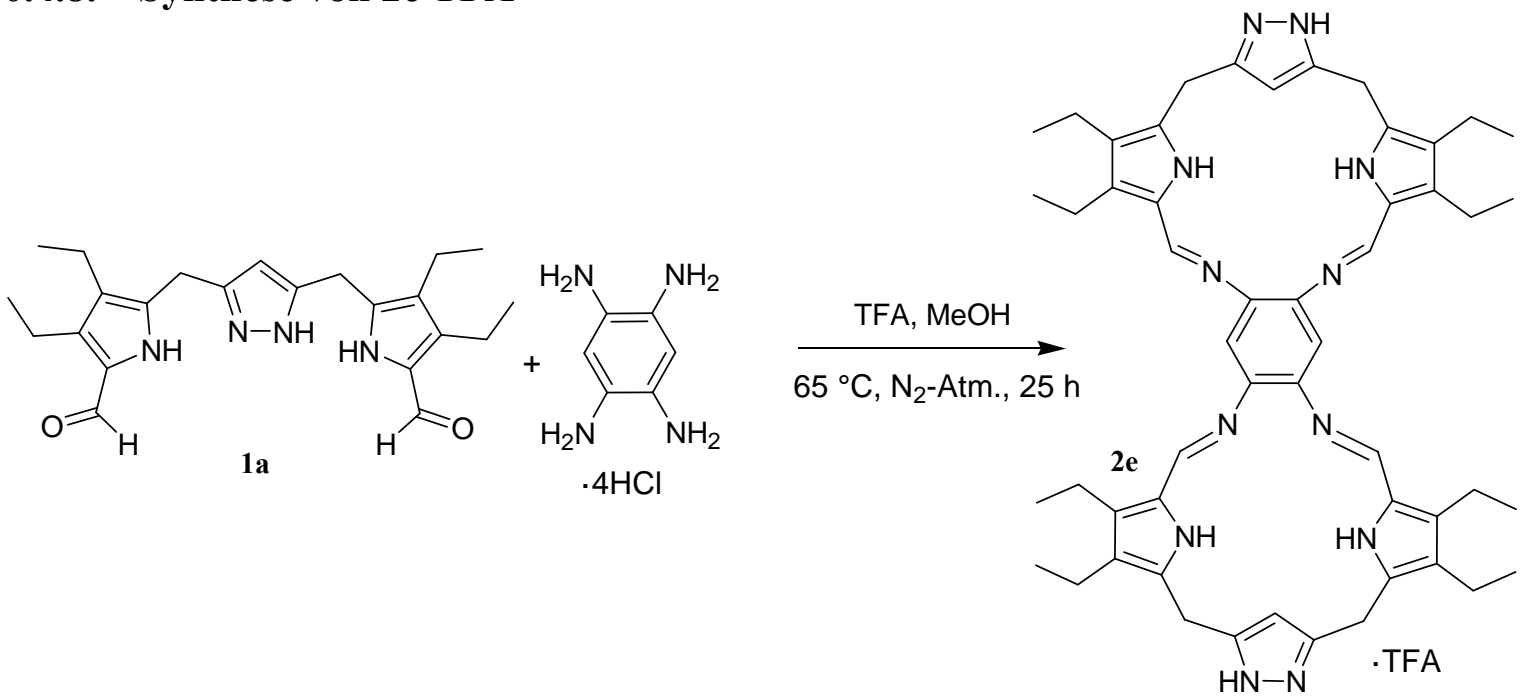

$1 \mathrm{a}\left(108 \mathrm{mg}, 0.27 \mathrm{mmol}, 2.0\right.$ eq.) wurde unter $\mathrm{N}_{2}$-Atmosphäre in trockenem $\mathrm{MeOH}(90 \mathrm{~mL})$ bei $60{ }^{\circ} \mathrm{C}$ gelöst. $\mathrm{Zu}$ dieser Lösung wurde 1,2,4,5-Tetraaminobenzol Tetrahydrochlorid (38.9 mg, $0.14 \mathrm{mmol}, 1.0$ eq.) in einer Suspension aus $\mathrm{MeOH}(8 \mathrm{~mL})$ dazugetropft. Nach 10 Min. wurde in einem Zeitraum von 5 Minuten TFA $(53 \mu \mathrm{L}, 0.69 \mathrm{mmol}, 5.0$ eq.) 
tropfenweise zum Reaktionsgemisch gegeben. Das dabei violett gewordene Reaktionsgemisch wurde anschließend für 30 Stunden unter Rückfluss erhitzt. Nach Abkühlen auf Raumtemperatur wurde das Lösungsmittel im Vakuum entfernt und das so erhaltene braune Rohprodukt säulenchromatographisch (Aluminiumoxid [basisch, Brockmann Aktivität I], Dichlormethan/Methanol 20:1, $\mathrm{R}_{\mathrm{f}}=0.65$ in Dichlormethan/Methanol 15:1) gereinigt. Das Produkt (2e-TFA) wurde als violett-roter Feststoff (107 mg, $91 \%)$ erhalten.

\section{Ausbeute:}

Summenformel:

$\mathrm{C}_{52} \mathrm{H}_{62} \mathrm{~N}_{12}(\cdot x T F A)$

Molekulargewicht (g/mol):

855.1

MS (ESI in $\mathrm{MeOH}) m / z(\%)$ :

$429(63)[\mathrm{M}+2 \mathrm{H}]^{2+}, 856(100)[\mathrm{M}+\mathrm{H}]^{+}, 1710(8)$ $[2 \times \mathrm{M}]^{+}$.

MS (FAB, in 4-NBA) $m / z(\%)$ :

$855(100)[\mathrm{M}+\mathrm{H}]^{+}$.

HRMS-(+)ESI $\left(\mathrm{MeOH} / \mathrm{H}_{2} \mathrm{O}\right) \mathrm{m} / z$ : berechnet für $\mathrm{C}_{52} \mathrm{H}_{62} \mathrm{~N}_{12}:[\mathrm{M}+\mathrm{H}]^{+}$: 855.52932;

gemessen für $\mathrm{C}_{52} \mathrm{H}_{62} \mathrm{~N}_{12}$ : $[\mathrm{M}+\mathrm{H}]^{+}:$: 855.52896.

berechnet für $\mathrm{C}_{52} \mathrm{H}_{62} \mathrm{~N}_{12}$ : $[\mathrm{M}+2 \mathrm{H}]^{2+}$ : 428.26830 ;

gemessen für $\mathrm{C}_{52} \mathrm{H}_{62} \mathrm{~N}_{12}$ : $[\mathrm{M}+2 \mathrm{H}]^{2+}: 482.26810$.

IR ( $\mathrm{KBr})$ : $\widetilde{v}=3375(\mathrm{w}), 3171(\mathrm{w}), 2932(\mathrm{~m}), 1631(\mathrm{vs}), 1546$ (w), 1443 (m), 1269 (m), 1165 (w), 1010 (s), 957 (w), $796(\mathrm{~m}) \mathrm{cm}^{-1}$.

Schmelzpunkt $\left({ }^{\circ} \mathrm{C}\right)$ : $<290$.

$\mathrm{UV} / \mathrm{Vis}\left(\mathrm{CHCl}_{3} / \mathrm{MeOH} / \mathrm{TFA}\right)$ :

$\lambda_{\max }[\mathrm{nm}]\left(\varepsilon \times 10^{4} \mathrm{M}^{-1} \mathrm{~cm}^{-1}\right)=396(5.02), 452(\mathrm{sh})$.

$\mathrm{UV} / \mathrm{Vis}\left(\mathrm{CHCl}_{3} / \mathrm{MeOH} / \mathrm{Et}_{3} \mathrm{~N}\right)$ : $\lambda_{\text {max }}[\mathrm{nm}]\left(\varepsilon \times 10^{4} \mathrm{M}^{-1} \mathrm{~cm}^{-1}\right)=369$ (3.06), 434 (2.79).

\subsubsection{Synthese von $2 \mathrm{f}$}

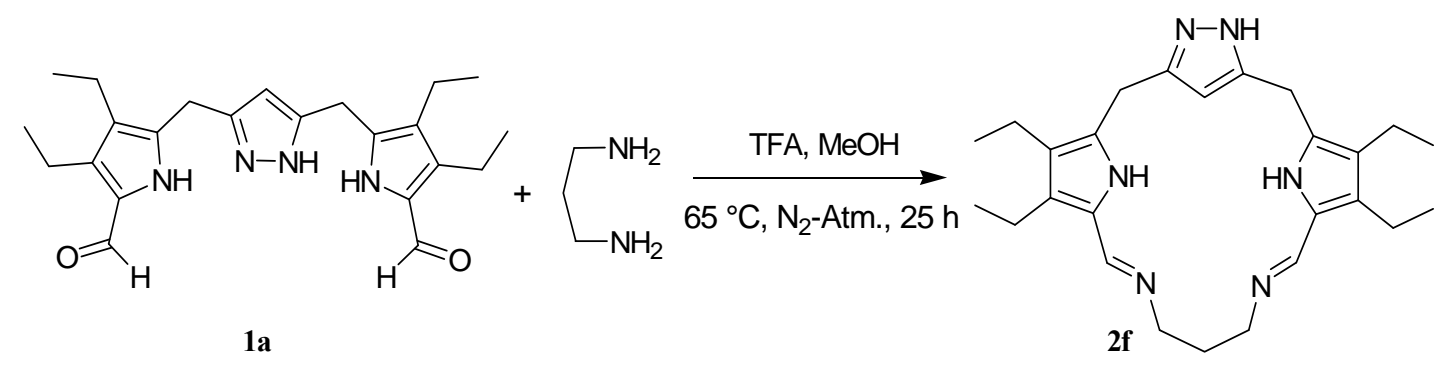


$1 \mathrm{a}\left(104 \mathrm{mg}, 0.26 \mathrm{mmol}, 1.0\right.$ eq.) wurde unter $\mathrm{N}_{2}$-Atmosphäre in trockenem $\mathrm{MeOH}(80 \mathrm{~mL})$ bei $60{ }^{\circ} \mathrm{C}$ gelöst. Hierzu wurde erst 1,3-Diaminopropan $(22.0 \mu \mathrm{L}, 0.26 \mathrm{mmol}, 1.0$ eq.) getropft und nach 10 Min. anschließend TFA ( $2 \mu \mathrm{L}, 0.69 \mathrm{mmol}, 0.1$ eq.) verdünnt in $\mathrm{MeOH}(1 \mathrm{~mL})$ zum Reaktionsgemisch gegeben. Das Reaktionsgemisch wurde für 25 Stunden unter Rückfluss erhitzt. Nach Abkühlen auf Raumtemperatur wurde das Lösungsmittel im Vakuum entfernt und das so erhaltene rot-braune Rohprodukt säulenchromatographisch (Aluminiumoxid [basisch, Brockmann Aktivität I], Dichlormethan/Methanol 20:1, $\mathrm{R}_{\mathrm{f}}=0.38$ ) gereinigt. Das Produkt (2f) wurde als gelb-oranger Feststoff (107 mg, 94 \%) erhalten.

\section{Auswertung:}

Summenformel:

$\mathrm{C}_{26} \mathrm{H}_{36} \mathrm{~N}_{6}$

Molekulargewicht (g/mol):

432.6

${ }^{1} \mathrm{H}-\mathrm{NMR}\left(300 \mathrm{MHz}, \mathrm{CD}_{2} \mathrm{Cl}_{2}\right)$ :

$\delta(\mathrm{ppm})=0.99-1.05\left(\mathrm{t},{ }^{3} J(\mathrm{H}, \mathrm{H})=7.5 \mathrm{~Hz}, 6 \mathrm{H}, \mathrm{CH}\right)$, 1.07-1.12(t, $\left.{ }^{3} J(\mathrm{H}, \mathrm{H})=7.5 \mathrm{~Hz}, 6 \mathrm{H}, \mathrm{CH}_{3}\right), 1.91(\mathrm{~m}, 2 \mathrm{H}$, $\left.\mathrm{CH}_{2}{ }^{\text {prop }}\right), \quad 2.31-2.38\left(\mathrm{q},{ }^{3} J(\mathrm{H}, \mathrm{H})=7.5 \mathrm{~Hz}, 4 \mathrm{H}\right.$, $\left.\mathrm{CH}_{2} \mathrm{CH}_{3}\right), 2.46-2.54\left(\mathrm{q},{ }^{3} \mathrm{~J}(\mathrm{H}, \mathrm{H})=7.5 \mathrm{~Hz}, 4 \mathrm{H}\right.$, $\left.\mathrm{CH}_{2} \mathrm{CH}_{3}\right), 3.66-3.71\left(\mathrm{~m}, 4 \mathrm{H}, \mathrm{CH}_{2}{ }^{\text {prop }}\right), 3.84(\mathrm{~s}, 4 \mathrm{H}$, $\left.2 \times \mathrm{CH}_{2}\right), 5.53\left(\mathrm{~s}, 1 \mathrm{H}, \mathrm{CH}^{\mathrm{pz}}\right), 7.99(\mathrm{~s}, 2 \mathrm{H}, 2 \times H \mathrm{C}=\mathrm{N})$, $9.46(\mathrm{~N} H)$.

${ }^{13} \mathrm{C}-\mathrm{NMR}\left(126 \mathrm{MHz}, \mathrm{CD}_{2} \mathrm{Cl}_{2}\right): \quad \delta(\mathrm{ppm})=16.48\left(\mathrm{CH}_{3}\right), \quad 17.4 \quad\left(\mathrm{CH}_{2} \mathrm{CH}_{3}\right), \quad 17.7$ $\left(\mathrm{CH}_{2} \mathrm{CH}_{3}\right), \quad 18.6\left(\mathrm{CH}_{3}\right), 24.1$ (br, $\left.\mathrm{CH}_{2}{ }^{\mathrm{py} / \mathrm{pz}}\right), 30.1$ $\left(\mathrm{CH}_{2} \mathrm{CH}_{2} \mathrm{CH}_{2}\right), \quad 34.7, \quad 62.3 \quad\left(\mathrm{CH}_{2} \mathrm{CH}_{2} \mathrm{CH}_{2}\right), \quad 104.3$ $\left(C \mathrm{H}^{\mathrm{pz}-4}\right), 122.4,124.4,131.1,143.5,150.0(\mathrm{HC}=\mathrm{N})$.

MS (ESI in $\mathrm{MeOH}) m / z(\%)$ : $433(100)[\mathrm{M}+\mathrm{H}]^{+}, 865(69)[2 \times \mathrm{M}+\mathrm{H}]^{+}$.

MS (FAB in 4-NBA) $m / z(\%)$ : $433(100)[\mathrm{M}+\mathrm{H}]^{+}$.

HRMS-(+)ESI $\left(\mathrm{MeOH} / \mathrm{H}_{2} \mathrm{O}\right) \mathrm{m} / z$ : $\quad$ berechnet: $[\mathrm{M}+\mathrm{H}]^{+}:$433.30742; gemessen: $[\mathrm{M}+\mathrm{H}]^{+}: 433.30731$.

Schmelzpunkt $\left({ }^{\circ} \mathrm{C}\right)$ : $>180$.

IR ( $\mathrm{KBr})$ : $\widetilde{v}=3245(\mathrm{w}), 2947$ (s), 1628 (vs), 1443 (s), 1261 (s), $1080(\mathrm{~m}), 1020(\mathrm{~m}), 801(\mathrm{~s}) \mathrm{cm}^{-1}$.

$\mathrm{UV} / \mathrm{V}$ is $\left(\mathrm{CHCl}_{3} / \mathrm{TFA}\right)$ : $\lambda_{\text {max }}[\mathrm{nm}]\left(\varepsilon \times 10^{4} \mathrm{M}^{-1} \mathrm{~cm}^{-1}\right)=325(1.70)$.

$\mathrm{UV} / \mathrm{V}$ is $\left(\mathrm{CHCl}_{3} / 0.01 \% \mathrm{Et}_{3} \mathrm{~N}\right)$ : $\lambda_{\text {max }}[\mathrm{nm}]\left(\varepsilon \times 10^{4} \mathrm{M}^{-1} \mathrm{~cm}^{-1}\right)=312(1.56), 431(\mathrm{sh})$. 


\subsubsection{Synthese von $2 \mathrm{~g}$}

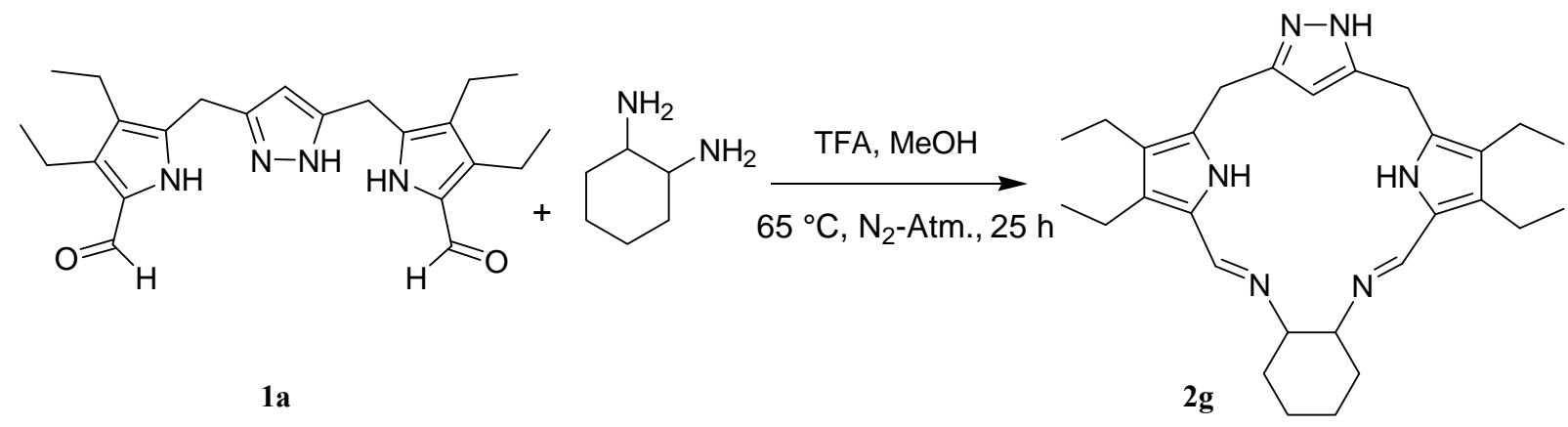

1a (104mg, $0.26 \mathrm{mmol}, 1.0 \mathrm{eq})$ wurde unter $\mathrm{N}_{2}$-Atmosphäre in trockenem Methanol $(50 \mathrm{~mL})$ bei $50{ }^{\circ} \mathrm{C}$ gelöst. 1,2-Diaminocyclohexan (30 mg, $0.26 \mathrm{mmol}, 1.0$ eq.) wurde unter Rühren dazugefügt und anschließend wurde TFA (200 $\mu \mathrm{L}, 2.64 \mathrm{mmol}, 10.0$ eq.) langsam dazugetropft. Das Reaktionsgemisch wurde unter $\mathrm{N}_{2}$-Atmosphäre 3 Tage unter Rückfluss erhitzt. Das entstandene Produkt konnte sowohl durch Säulenchromatographie (Aluminiumoxid [basisch, Brockmann Aktivität I], Dichlormethan/Methanol 30:1) als auch durch Umkristallisation nicht isoliert werden.

\section{Auswertung:}

Summenformel:

$\mathrm{C}_{29} \mathrm{H}_{40} \mathrm{~N}_{6}$

Molekulargewicht (g/mol):

472.7

MS (ESI in $\mathrm{MeOH}) m / z(\%)$ :

$474(36)[\mathrm{M}+\mathrm{H}]^{+}, 945(11)[2 \times \mathrm{M}+\mathrm{H}]^{+}, 1059(100)$

$[2 \times \mathrm{M}+\mathrm{H}]^{+}$.

\subsection{Ligandsynthese}

\subsubsection{Synthese von 3,5-Bis-(2,3-dihydro-1H-perimidin)-1H-pyrazol (10)}<smiles>O=Cc1cc(C=O)[nH]n1</smiles>

LI<smiles>Nc1cccc2cccc(N)c12</smiles>

LII

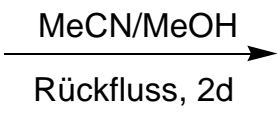

Rückfluss, 2d

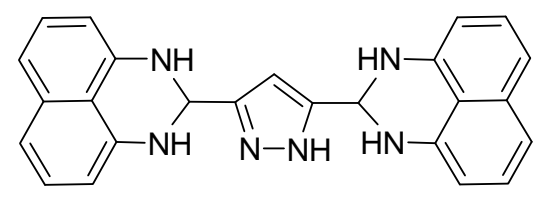

10 
1H-Pyrazol-3,5-dicarbaldehyd (LI) $)^{59,60}(0.392 \mathrm{~g}, 3.2 \mathrm{mmol})$ wurde in einem Gemisch aus Acetonitril $(150 \mathrm{~mL})$ und warmes Methanol $(10 \mathrm{~mL})$ gelöst. Nachdem das Reaktionsgemisch auf $50{ }^{\circ} \mathrm{C}$ erwärmt wurde, wurde eine Lösung aus 1,8-Diaminonaphthalin (LII) (1.0 g, $6.3 \mathrm{mmol}, 2$ eq.) in Methanol (5 mL) dazugegeben. Die Suspension wurde zwei Tage unter Rückfluss erhitzt. Das Lösungsmittel wurde entfernt und der Rückstand in Dichlormethan aufgenommen. Der dabei leicht gelbliche schwerlösliche Feststoff (9) konnte mit Hilfe einer Zentrifuge isoliert und im Hochvakuum getrocknet werden (Ausbeute 57 \%).

Die Reaktion kann durch katalytische Mengen von Säure (TFA, $p$-TSA oder HCOOH) beschleunigt werden. Der Reaktionsablauf wurde mittels Dünnschichtchromatographie verfolgt.

\section{Auswertung:}

Summenformel:

Molekulargewicht (g/mol):

${ }^{1} \mathrm{H}-\mathrm{NMR}(500 \mathrm{MHz}$, DMSO-d 6 ):
$\mathrm{C}_{25} \mathrm{H}_{20} \mathrm{~N}_{6}$

404.5

$\delta(\mathrm{ppm})=5.50(\mathrm{~s}, 2 \mathrm{H}, 2 \times \mathrm{CH}), 6.35\left(\mathrm{~s}, 1 \mathrm{H}, \mathrm{C} H^{\mathrm{pz}}\right)$, 6.52-6.53 (d, $\left.{ }^{3} J(\mathrm{H}, \mathrm{H})=7.3 \mathrm{~Hz}, 4 \mathrm{H}, \mathrm{C} H^{\mathrm{Ar}-5 / 5^{\prime}}\right), 6.68$ (br, s, 4H, NH), 6.97-6.99 (d, ${ }^{3} J(\mathrm{H}, \mathrm{H})=8.1 \mathrm{~Hz}, 4 \mathrm{H}$, $\left.\mathrm{C} H^{\mathrm{Ar}-6 / 6^{\prime}}\right), 7.13-7.16\left(\mathrm{t}, 4 \mathrm{H}, \mathrm{C} H^{\mathrm{Ar}-7 / 7^{\prime}}\right), 12.90(\mathrm{br}, \mathrm{s}, 1 \mathrm{H}$, $\left.\mathrm{N} H^{\mathrm{pz}}\right)$.

${ }^{13} \mathrm{C}-\mathrm{NMR}\left(126 \mathrm{MHz}, \mathrm{DMSO}-\mathrm{d}_{6}\right): \quad \delta(\mathrm{ppm})=60.3(\mathrm{br}, \mathrm{CH}), 101.7\left(\mathrm{CH}^{\mathrm{pz}-4}\right), 104.6$ $\left(\mathrm{C} H^{\mathrm{Ar}-5 / 5^{\prime}}\right), 112.5\left(C^{\mathrm{Ar}-\mathrm{q}}\right), 115.3\left(\mathrm{CH}^{\mathrm{Ar}-6 / 6^{\prime}}\right), 126.7$ $\left(C \mathrm{H}^{\mathrm{Ar}-7 / 7^{\prime}}\right), 134.2\left(C^{\mathrm{Ar}-\mathrm{q}}\right), 142.1\left(C^{\mathrm{pz}}\right), 145.0\left(\mathrm{br}, C^{\mathrm{q}}\right)$.

MS (ESI+; DMSO/MeOH) m/z (\%): 909 (15) $[2 \times \mathrm{M}+\mathrm{Na}+\mathrm{DMSO}]^{+}, 831(25)[2 \times \mathrm{M}+\mathrm{Na}]^{+}$, 505 (65) $[\mathrm{M}+\mathrm{Na}+\mathrm{DMSO}]^{+}, 427(8)[\mathrm{M}+\mathrm{Na}]^{+}$.

MS (ESI-; DMSO/MeOH) $m / z(\%)$ :

844 (8) $[2 \times \mathrm{M}+\mathrm{Cl}]^{-}, 807$ (16) $[2 \times \mathrm{M}-\mathrm{H}]^{-}, 439$ (75) $[\mathrm{M}+\mathrm{Cl}]^{-}, 403$ (100) [M-H]'.

MS (FAB+; 4-NBA) $m / z(\%)$ : $404(10)[\mathrm{M}]^{+}$.

MS (FAB-; 4-NBA) $m / z(\%)$ :

MS (EI) $m / z(\%)$ :

Schmelzpunkt:

IR ( $\mathrm{KBr})$ :
403 (15) [M-H].

404 (1) $[\mathrm{M}]^{+}, 402$ (14) $[\mathrm{M}-2 \mathrm{H}]^{+}, 400$ (24) $[\mathrm{M}-4 \mathrm{H}]^{+}$, 248 (14) $\left[\mathrm{M}-\left(\mathrm{C}_{10} \mathrm{H}_{8} \mathrm{~N}_{2}\right)\right]^{+}, 234$ (26) $\left[\mathrm{M}-\left(\mathrm{C}_{11} \mathrm{H}_{10} \mathrm{~N}_{2}\right)\right]^{+}$, $158(100)\left[\mathrm{C}_{10} \mathrm{H}_{10} \mathrm{~N}_{2}\right]^{+}$.

$<260{ }^{\circ} \mathrm{C}$ $\widetilde{v}=3364(\mathrm{~m}), 3193(\mathrm{w}), 2353(\mathrm{w}), 1600(\mathrm{~s}), 1404(\mathrm{~s})$, $1242(\mathrm{~m}), 1090(\mathrm{w}), 761(\mathrm{~s}) \mathrm{cm}^{-1}$. 


\subsubsection{Synthese von [5-(Morpholin-4-carbonyl)-2H-pyrazol-3-yl]-morpholin-4-yl- methanon (11)}<smiles>O=C(Cl)c1cc(C(=O)Cl)[nH]n1</smiles>

LVI

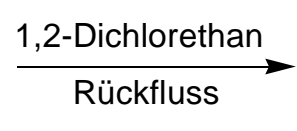<smiles>[C]1CNCCO1</smiles>
Rückfluss<smiles>O=C(c1cc(C(=O)N2CCOCC2)[nH]n1)N1CCOCC1</smiles>

11

Das 1H-Pyrazol-3,5-dicarbonyldichlorid Monohydrochlorid (LVI) (2.0 g, $10.5 \mathrm{mmol})$ wurde in trockenem 1,2-Dichlorethan $(100 \mathrm{~mL})$ bei $80^{\circ} \mathrm{C}$ gelöst. Nach 15 Min. wurde zur entstandene Suspension Morpholin $(10 \mathrm{~mL},>10$ eq.) portionsweise gegeben und das inzwischen klar gewordene Reaktionsgemisch über Nacht unter Rückfluss erhitzt. Das Reaktionsgemisch wurde auf Raumtemperatur gebracht und das Nebenprodukt Morpholin Monohydrochlorid durch Filtration entfernt. Das Filtrat wurde eingeengt und der gelbliche Rückstand in $\mathrm{MeOH}$ aufgenommen und in $\mathrm{Et}_{2} \mathrm{O}$ bei $5{ }^{\circ} \mathrm{C}$ einige Stunden gefällt. Das Produkt (11) wurde in Form eines weißen Feststoffes (3.00 g, $97 \%$ ) erhalten.

\section{Auswertung:}

Summenformel:

Molekulargewicht (g/mol):

${ }^{1} \mathrm{H}-\mathrm{NMR}\left(200 \mathrm{MHz}, \mathrm{CDCl}_{3}\right)$ :

${ }^{13} \mathrm{C}-\mathrm{NMR}\left(126 \mathrm{MHz}, \mathrm{CDCl}_{3}\right)$ :

MS (EI) $m / z(\%)$ :

Schmelzpunkt $\left({ }^{\circ} \mathrm{C}\right)$ :

Elementaranalyse (\%):

IR ( $\mathrm{KBr})$ :
$\mathrm{C}_{13} \mathrm{H}_{18} \mathrm{~N}_{4} \mathrm{O}_{4} \cdot(\mathrm{xHCl})$

294.3

$\delta(\mathrm{ppm})=3.62\left(\mathrm{br}, \mathrm{s}, 16 \mathrm{H}, \mathrm{CH}_{2}\right), 6.88\left(\mathrm{~s}, 1 \mathrm{H}, \mathrm{C} H^{\mathrm{pz}}\right)$, 13.95 (s, br, $\mathrm{N} H)$.

$\delta(\mathrm{ppm})=54.0\left(\mathrm{CH}_{2}\right), 65.9\left(\mathrm{CH}_{2}\right), 66.2\left(\mathrm{CH}_{2}\right), 108.4$ $\left(C \mathrm{H}^{\mathrm{pz}}\right), 160.5(C=\mathrm{O})$.

$294(30)[\mathrm{M}]^{+}, 208(35)\left[\mathrm{M}-\left(\mathrm{C}_{4} \mathrm{H}_{8} \mathrm{NO}\right)\right]^{+}, 181$ (48)

$\left[\mathrm{C}_{8} \mathrm{H}_{11} \mathrm{~N}_{3} \mathrm{O}_{2}\right]^{+}, 86(100)\left[\mathrm{C}_{4} \mathrm{H}_{8} \mathrm{NO}\right]^{+}$.

176-185.

berechnet für $\mathrm{C}_{13} \mathrm{H}_{18} \mathrm{~N}_{4} \mathrm{O}_{4}(\mathrm{HCl})_{0.25}$ :

C: 51.46, H: 6.06, N: 18.47;

gemessen: C: 51.31, H: 6.48, N: 16.86.

$\widetilde{v}=3441(\mathrm{w}), 3171(\mathrm{~m}), 2863(\mathrm{w}), 2418(\mathrm{~m}), 1625$

(s), 1446 (s), 1388 (m), 1235 (s), 1110 (s), 990 (s), 844

(s), $759(\mathrm{~m}), 596(\mathrm{~m}), 505(\mathrm{~m}) \mathrm{cm}^{-1}$. 


\subsubsection{Synthese von 3,5-Bis-(1H-pyrrol-2-yl-methylen-amin)-1H-pyrazol (7)}

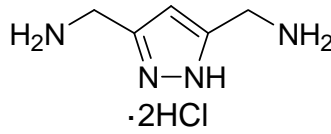

$\mathbf{L}$

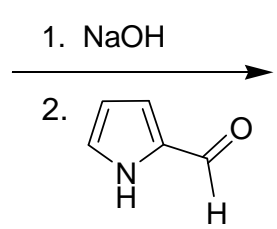

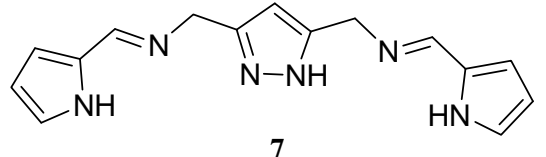

7

L (650 mg, $3.28 \mathrm{mmol})$ wurde in dest. Wasser $(10 \mathrm{~mL})$ gelöst. $\mathrm{Zu}$ dieser Lösung wurde Natriumhydroxid (263 mg, $6.57 \mathrm{mmol}, 2$ eq.) gelöst in $\mathrm{H}_{2} \mathrm{O}(2 \mathrm{~mL})$, tropfenweise unter Rühren zugegeben und einige Minuten bei Raumtemperatur weiter gerührt. Anschließend wurde das Pyrrol-2-carboxaldehyd (1.25 g, 13.12 mmol, 4 eq.) und Dichlormethan (4 mL) zum besseren Lösen des Pyrrolderivates zum Reaktionsgemisch gegeben. Die Suspension wurde $1 \mathrm{~d}$ bei RT gerührt. Am nächsten Tag wurde das Reaktionsgemisch destillativ auf die Hälfte des Reaktionsvolumens eingeengt und der entstandene weiße Niederschlag abfiltriert und mit $\mathrm{MeOH} / \mathrm{Et}_{2} \mathrm{O}$ umkristallisiert $\left(5^{\circ} \mathrm{C}, 1 \mathrm{~d}\right)$. Das Produkt 7 wurde in Form eines weißen Feststoffes (692 mg, $75 \%$ ) isoliert.

\section{Auswertung:}

Summenformel:

$\mathrm{C}_{15} \mathrm{H}_{16} \mathrm{~N}_{6}$

Molekulargewicht (g/mol):

280.3

${ }^{1} \mathrm{H}-\mathrm{NMR}\left(500 \mathrm{MHz}, \mathrm{DMSO}-\mathrm{d}_{6}\right)$ :

$\delta(\mathrm{ppm})=4.59\left(\mathrm{~s}, 4 \mathrm{H}, 2 \times \mathrm{CH}_{2}\right), 6.02\left(\mathrm{~s}, 1 \mathrm{H}, \mathrm{C} H^{\mathrm{pz}}\right)$, 6.09-6.11 (m, 2H, $\left.\mathrm{CH}^{\mathrm{py}-3}\right), 6.45-6.46\left(\mathrm{~d},{ }^{3} J(\mathrm{H}, \mathrm{H})=\right.$ $\left.2.2 \mathrm{~Hz}, 2 \mathrm{H}, \mathrm{C} H^{\mathrm{py}-4}\right), 6.86\left(\mathrm{~s}, 2 \mathrm{H}, \mathrm{CH}^{\mathrm{py}-2}\right), 8.14(\mathrm{~s}, 2 \mathrm{H}$, $\mathrm{C} H=\mathrm{N}), 11.4$ (br, $\mathrm{N} H), 12.4$ (br, $\mathrm{N} H$ ).

${ }^{13} \mathrm{C}-\mathrm{NMR}\left(126 \mathrm{MHz}, \mathrm{DMSO}-\mathrm{d}_{6}\right): \quad \delta(\mathrm{ppm})=55.3\left(\mathrm{CH}_{2}\right), 102.8\left(\mathrm{CH}^{\mathrm{pz}}\right), 108.8\left(C \mathrm{H}^{\mathrm{py}-3}\right)$, $113.6\left(C \mathrm{H}^{\mathrm{py}-4}\right), 122.1 \quad\left(C \mathrm{H}^{\mathrm{py}-2}\right), 129.9\left(C^{\mathrm{q}}\right), 152.7$ $(C \mathrm{H}=\mathrm{N})$.

MS (EI) $m / z(\%)$ : $280(100)[\mathrm{M}]^{+}, 188(58)\left[\mathrm{M}-\left(\mathrm{C}_{5} \mathrm{H}_{5} \mathrm{~N}_{2}\right)\right]^{+}, 157$ (38) $\left[\left(\mathrm{C}_{8} \mathrm{H}_{5} \mathrm{~N}_{4}\right)\right]^{+}, 94(39)\left[\left(\mathrm{C}_{5} \mathrm{H}_{6} \mathrm{~N}_{2}\right)\right]^{+}, 65(13)\left[\left(\mathrm{C}_{4} \mathrm{H}_{3} \mathrm{~N}\right)\right]^{+}$.

Schmelzpunkt $\left({ }^{\circ} \mathrm{C}\right)$ : 169-178.

Elementaranalyse (\%): berechnet: C: 64.27, H: 5.75, N: 29.98; gemessen: C: 63.96, H: 5.77, N: 28.32.

IR ( $\mathrm{KBr})$ : $\widetilde{v}=3171(\mathrm{~m}), 3092(\mathrm{w}), 2870(\mathrm{~m}), 2353(\mathrm{w}), 1636$ (vs), 1570 (w), 1422 (m), 1362 (w), 1265 (m), 1137 
(w), $1096(\mathrm{w}), 1039(\mathrm{~m}), 1000(\mathrm{w}), 828(\mathrm{w}), 741(\mathrm{~m})$, $606(\mathrm{w}) \mathrm{cm}^{-1}$.

\subsubsection{Synthese von 3,5-Bis-(methyl-pyridin-2-yl-methylen-amin)-1H-pyrazol (8)}<smiles>NCc1cc(CN)[nH]n1</smiles>

$\mathbf{L}$

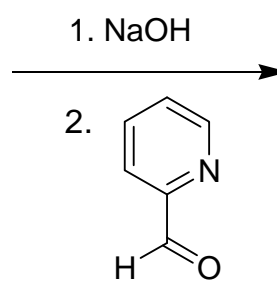

L (200 mg, $1.00 \mathrm{mmol})$ wurde in dest. Wasser $(5 \mathrm{~mL})$ gelöst. Zu dieser Lösung wurde Natriumhydroxid ( $80 \mathrm{mg}, 2.00 \mathrm{mmol}, 2$ eq.) gelöst in $\mathrm{H}_{2} \mathrm{O}(2 \mathrm{~mL})$ tropfenweise unter Rühren dazu gegeben und einige Minuten bei Raumtemperatur weiter gerührt. Anschließend wurde 2Pyridin-carboxaldehyd (191 $\mu \mathrm{L}, 2.00 \mathrm{mmol}, 2$ eq.) zum Reaktionsgemisch zugegeben. Nach einiger Zeit entsteht ein trüber Niederschlag. $\mathrm{Zu}$ diesem wurde $\mathrm{CH}_{2} \mathrm{Cl}_{2}(6 \mathrm{~mL})$ gegeben und die Suspension $1 \mathrm{~d}$ bei RT gerührt. Am nächsten Tag wurde die organische Phase abgetrennt

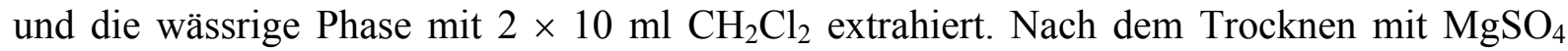
wurde das Lösungsmittel im Vakuum entfernt. Das erhaltene gelbe Öl (8) wurde in $\mathrm{CHCl}_{3} / \mathrm{MeOH} / \mathrm{Et}_{2} \mathrm{O}$ bei $5{ }^{\circ} \mathrm{C}$ umkristallisiert (279 mg, $92 \%$ ).

\section{Auswertung:}

Summenformel:

Molekulargewicht (g/mol):

${ }^{1} \mathrm{H}-\mathrm{NMR}\left(500 \mathrm{MHz}, \mathrm{CDCl}_{3}\right)$ :

${ }^{13} \mathrm{C}-\mathrm{NMR}\left(126 \mathrm{MHz}, \mathrm{CDCl}_{3}\right)$ :
$\mathrm{C}_{17} \mathrm{H}_{16} \mathrm{~N}_{6}$

304.4

$\delta(\mathrm{ppm})=4.84\left(\mathrm{~s}, 4 \mathrm{H}, \mathrm{CH}_{2}\right), 6.18\left(\mathrm{~s}, 1 \mathrm{H}, \mathrm{C} H^{\mathrm{pz}}\right), 7.21-$

$7.22\left(\mathrm{~d},{ }^{3} J(\mathrm{H}, \mathrm{H})=1.2 \mathrm{~Hz}, 1 \mathrm{H}, \mathrm{C} H^{\mathrm{Ph}}\right), 7.22-7.23(\mathrm{~d}$, $\left.1 \mathrm{H}, \mathrm{C} H^{\mathrm{Ph}}\right), 7.62-7.65\left(\mathrm{dt},{ }^{3} J(\mathrm{H}, \mathrm{H})=7.9 \mathrm{~Hz},{ }^{4} J(\mathrm{H}, \mathrm{H})\right.$

$\left.1.2 \mathrm{~Hz}, 2 \mathrm{H}, \mathrm{CH}^{\mathrm{Ph}}\right), 7.92-7.94\left(\mathrm{~d},{ }^{3} J(\mathrm{H}, \mathrm{H})=7.9 \mathrm{~Hz}\right.$, $\left.2 \mathrm{H}, \mathrm{C} H^{\mathrm{Ph}}\right), 8.44(\mathrm{~s}, 2 \mathrm{H}, \mathrm{C} H=\mathrm{N}), 8.56-8.57\left(\mathrm{qd},{ }^{3} J\right.$ $\left.(\mathrm{H}, \mathrm{H})=4.8 \mathrm{~Hz},{ }^{4} J(\mathrm{H}, \mathrm{H})=1.62 \mathrm{~Hz}, 2 \mathrm{H}, \mathrm{CH}^{\mathrm{Ph}}\right)$.

$\delta(\mathrm{ppm})=56.8\left(\mathrm{CH}_{2}\right), 103.1\left(C \mathrm{H}^{\mathrm{pz}}\right), 121.5\left(\mathrm{CH}^{\mathrm{pyr}}\right)$, $124.7\left(C \mathrm{H}^{\mathrm{pyr}}\right), 136.5\left(C \mathrm{H}^{\mathrm{pyr}}\right), 149.3\left(C \mathrm{H}^{\mathrm{pyr}}\right), 154.2$ $\left(C^{\mathrm{q}}\right), 163.1(\mathrm{H} C=\mathrm{N})$. 


\begin{tabular}{|c|c|}
\hline MS (ESI) $m / z(\%)$ & 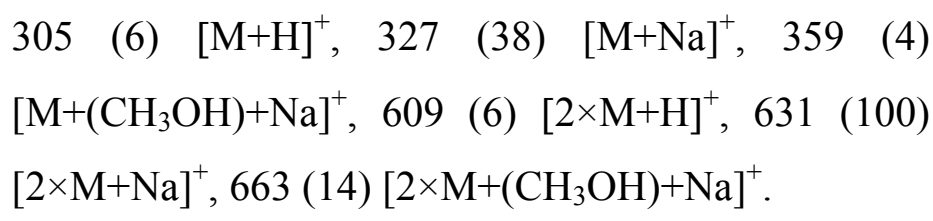 \\
\hline MS (EI) $m / z(\%)$ & 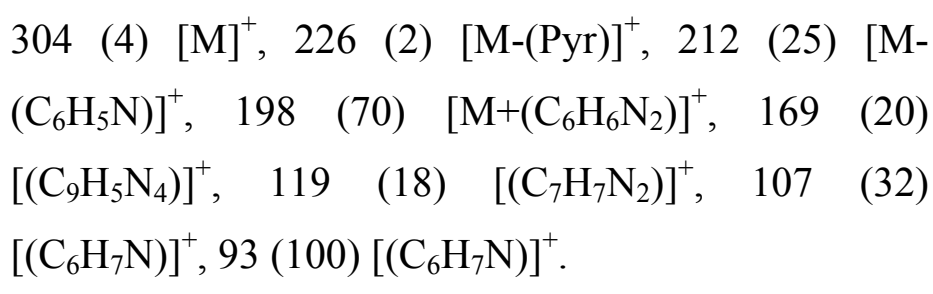 \\
\hline Schmelzpunkt $\left({ }^{\circ} \mathrm{C}\right)$ : & $71-75$ \\
\hline IR (KBr): & $\begin{array}{l}\tilde{v}=3195(\mathrm{w}), 3011(\mathrm{w}), 2965(\mathrm{w}), 2874(\mathrm{~m}), 1643 \\
(\mathrm{vs}), 1588(\mathrm{vs}), 1568(\mathrm{~m}), 1468(\mathrm{~s}), 1434(\mathrm{~s}), 1360 \\
(\mathrm{~m}), 1312(\mathrm{~m}), 1262(\mathrm{w}), 1215(\mathrm{w}), 1138(\mathrm{~m}), 1094 \\
(\mathrm{w}), 1019(\mathrm{~m}), 993(\mathrm{~m}), 781(\mathrm{~s}) \mathrm{cm}^{-1} .\end{array}$ \\
\hline
\end{tabular}

10.5.5. Synthese von 3,5-Bis-(benzyliden-2-yl-methylenamin)-1H-pyrazol (9)<smiles>NCc1cc(CN)[nH]n1</smiles>

$\mathbf{L}$
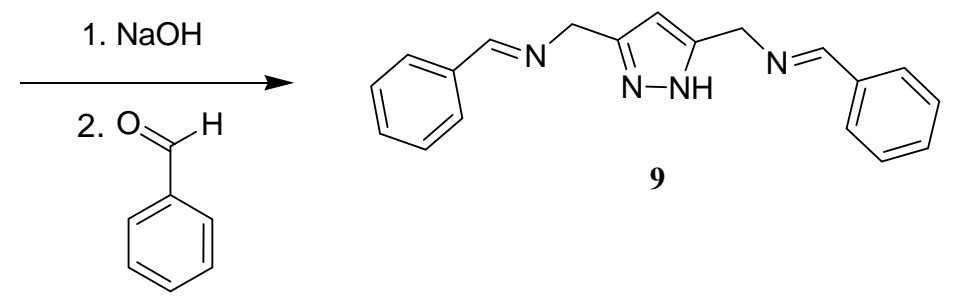

L (805 mg, $4.07 \mathrm{mmol}$ ) wurde in $20 \mathrm{ml}$ dest. Wasser gelöst. Zu dieser Lösung wurde Natriumhydroxid (325 mg, $8.14 \mathrm{mmol}, 2$ eq.), gelöst in $\mathrm{H}_{2} \mathrm{O}(2 \mathrm{~mL})$, tropfenweise unter Rühren dazu gegeben und einige Minuten bei Raumtemperatur gerührt. Anschließend wurde Benzaldehyd ( $845 \mu \mathrm{L}, 8.14$ mmol, 2 eq.) zum Reaktionsgemisch gegeben. Nach einiger Zeit entsteht ein trüber Niederschlag im Kolben. Hierzu wurden $\mathrm{CH}_{2} \mathrm{Cl}_{2}(5 \mathrm{~mL})$ gegeben und die Suspension 1 d bei RT gerührt. Am nächsten Tag wurde die organische Phase abgetrennt und die wässrige Phase mit $3 \times 10 \mathrm{~mL} \mathrm{CH}_{2} \mathrm{Cl}_{2}$ extrahiert. Nach dem Trocknen mit $\mathrm{MgSO}_{4}$ wurde das Lösungsmittel im Vakuum entfernt und das so erhaltene gelbe Öl im Hochvakuum bei $80^{\circ} \mathrm{C}$ mehrere Stunden getrocknet. Das Produkt 9 wurde in Form eines gelben Feststoffes (877 mg, $71 \%$ ) erhalten. 


\section{Auswertung:}

Summenformel:

$\mathrm{C}_{19} \mathrm{H}_{18} \mathrm{~N}_{4}$

Molekulargewicht (g/mol):

302.4

${ }^{1} \mathrm{H}-\mathrm{NMR}\left(400 \mathrm{MHz}, \mathrm{CDCl}_{3}\right.$ ):

$\delta(\mathrm{ppm})=4.81\left(\mathrm{~s}, 2 \mathrm{H}, \mathrm{CH}_{2}\right), 4.82\left(\mathrm{~s}, 2 \mathrm{H}, \mathrm{CH}_{2}\right), 6.20$

$\left(\mathrm{s}, 1 \mathrm{H}, \mathrm{C} H^{\mathrm{pz}}\right), 7.38-7.41\left(\mathrm{~m}, 6 \mathrm{H}, \mathrm{C} H^{\mathrm{Ph}}\right), 7.72(\mathrm{~m}, 4 \mathrm{H}$, $\left.\mathrm{C} H^{\mathrm{Ph}}\right), 8.34(\mathrm{~s}, 2 \mathrm{H}, H \mathrm{C}=\mathrm{N})$.

${ }^{13} \mathrm{C}-\mathrm{NMR}\left(126 \mathrm{MHz}, \mathrm{CDCl}_{3}\right)$ :

$\delta(\mathrm{ppm})=57.0\left(\mathrm{CH}_{2}\right), 102.8\left(\mathrm{CH}^{\mathrm{pz}}\right), 128.2\left(\mathrm{CH}^{\mathrm{Ph}}\right)$, $128.5\left(C \mathrm{H}^{\mathrm{Ph}}\right), 130.8\left(C \mathrm{H}^{\mathrm{Ph}}\right), 135.8\left(C^{\mathrm{q}-\mathrm{Ph}}\right), 146.3(\mathrm{br}$, $\left.C^{\mathrm{q}-\mathrm{pz}}\right), 162.9(C \mathrm{H}=\mathrm{N})$.

MS (ESI) $m / z(\%)$ :

$303(63)[\mathrm{M}+\mathrm{H}]^{+}, 605(45)[2 \times \mathrm{M}+\mathrm{H}]^{+}, 627(100)$

$[2 \times \mathrm{M}+\mathrm{Na}]^{+}$.

Schmelzpunkt $\left({ }^{\circ} \mathrm{C}\right)$ :

70-82.

Elementaranalyse (\%):

berechnet: C: 75.47; H: 6.00; N: 18.53;

gemessen: C: 75.05, H: 6.14; N: 18.21.

IR ( $\mathrm{KBr})$ :

$\widetilde{v}=3412(\mathrm{w}), 3192(\mathrm{w}), 3026(\mathrm{w}), 2862(\mathrm{w}), 1628$

(vs), 1585 (w), 1449 (m), 1376 (w), 1310 (m), 1214

(w), 1142 (w), 1011 (s), 754 (m), 692 (s), 486

(m) $\mathrm{cm}^{-1}$.

\subsubsection{Synthese von $\mathrm{H}_{3} \mathrm{~L}^{1}$}

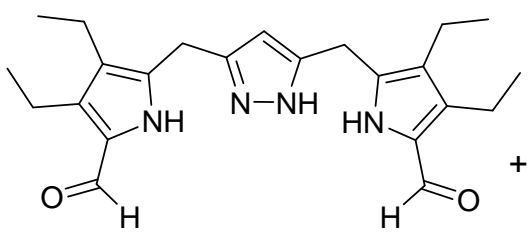

$1 \mathbf{a}$<smiles>Nc1ccccc1</smiles>

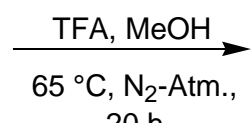
$20 \mathrm{~h}$

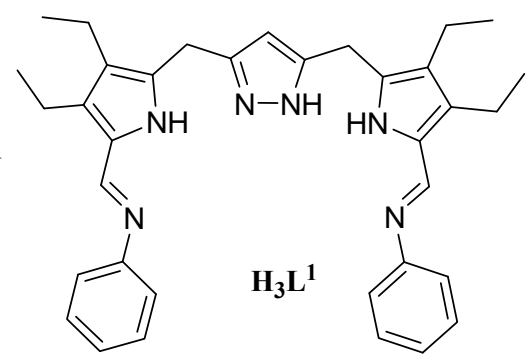

1 a (115 mg, $0.29 \mathrm{mmol}, 1$ eq.) wurde unter $\mathrm{N}_{2}$-Atmosphäre in trockenem Methanol $(80 \mathrm{~mL}$, über $\mathrm{Mg}$ getrocknet) bei $50{ }^{\circ} \mathrm{C}$ gelöst. $\mathrm{Zu}$ dieser Lösung wurde frisch dest. Anilin $(53 \mu 1,0.58$ mmol, 2 eq.) langsam zugetropft. Nach einigen Minuten Rühren wurde unter $\mathrm{N}_{2}$-Atm. Trifluoressigsäure ( $56 \mu 1,0.73 \mathrm{mmol}, 2.5$ eq.) langsam zum Reaktionsgemisch dazugegeben. Das Reaktionsgemisch wurde anschließend für 20 Stunden unter Rückfluss erhitzt. Nach Abkühlen auf Raumtemperatur wurde das Lösungsmittel im Vakuum entfernt und das so 
erhaltene braune Rohprodukt säulenchromatographisch (Aluminiumoxid [basisch, Brockmann Aktivität I], Dichlormethan/Methanol 30:1, $\mathrm{R}_{\mathrm{f}}=0.51$ ) gereinigt. Die noch zurückgebliebenen Reste an Anilin konnten mit Hilfe einer Kugelrohdestille (Hochvakuum, $80{ }^{\circ} \mathrm{C}$ ) entfernt werden. $\mathbf{H}_{3} \mathbf{L}^{1}$ erhält man in Form eines orange-braunen Feststoffes (132 mg, $83 \%)$.

\section{Auswertung:}

Summenformel:

$\mathrm{C}_{35} \mathrm{H}_{40} \mathrm{~N}_{6}$

Molekulargewicht (g/mol):

544.7

${ }^{1} \mathrm{H}-\mathrm{NMR}\left(500 \mathrm{MHz}, \mathrm{CDCl}_{3}\right)$ :

$\delta(\mathrm{ppm})=1.01-1.04\left(\mathrm{t},{ }^{3} J(\mathrm{H}, \mathrm{H})=7.5 \mathrm{~Hz}, 6 \mathrm{H}, \mathrm{CH}\right)$, 1.14-1.19 (t, $\left.{ }^{3} J(\mathrm{H}, \mathrm{H})=7.5 \mathrm{~Hz}, 6 \mathrm{H}, \mathrm{CH}_{3}\right), 2.36-2.41$ $\left(\mathrm{q},{ }^{3} J(\mathrm{H}, \mathrm{H})=7.5 \mathrm{~Hz}, 4 \mathrm{H}, \mathrm{CH}_{2} \mathrm{CH}_{3}\right), 2.57-2.62$ (q, $\left.{ }^{3} J(\mathrm{H}, \mathrm{H})=7.5 \mathrm{~Hz}, 4 \mathrm{H}, \mathrm{CH}_{2} \mathrm{CH}_{3}\right), 3.88\left(\mathrm{~s}, 4 \mathrm{H}, \mathrm{CH}_{2}\right)$, $5.91\left(\mathrm{~s}, 1 \mathrm{H}, \mathrm{CH}^{\mathrm{pz}}\right), 7.09-7.15\left(\mathrm{~m}, 6 \mathrm{H}, \mathrm{C} H^{\mathrm{Ph}}\right), 7.29-7.34$ $\left(\mathrm{m}, 4 \mathrm{H}, \mathrm{C} H^{\mathrm{Ph}}\right), 8.16(\mathrm{~s}, 2 \mathrm{H}, 2 \times H \mathrm{C}=\mathrm{N}), 9.4(\mathrm{~s}, \mathrm{~N} H)$.

${ }^{13} \mathrm{C}-\mathrm{NMR}\left(126 \mathrm{MHz}, \mathrm{CDCl}_{3}\right)$ :

$\delta(\mathrm{ppm})=16.0 \quad\left(\mathrm{CH}_{3}\right), \quad 17.0 \quad\left(\mathrm{CH}_{2} \mathrm{CH}_{3}\right), \quad 17.4$ $\left(\mathrm{CH}_{2} \mathrm{CH}_{3}\right), 17.5\left(\mathrm{CH}_{3}\right), 24.3\left(\mathrm{CH}_{2}\right), 103.8\left(\mathrm{CH}^{\mathrm{pz}}\right)$, $120.8\left(C \mathrm{H}^{\mathrm{Ph}}\right), 124.9\left(C \mathrm{H}^{\mathrm{Ph}}\right), 129.3\left(C \mathrm{H}^{\mathrm{Ph}}\right), 147.7(\mathrm{br}$, $\mathrm{H} C=\mathrm{N}), 151.9\left(C^{\mathrm{Ph}, \mathrm{q}}\right)$.

MS (EI) $m / z(\%)$ :

544 (23) $[\mathrm{M}]^{+}, 469$ (31) $\left[\mathrm{M}-\left(\mathrm{C}_{6} \mathrm{H}_{5}\right)\right]^{+}, 394$ (37) [M$\left.2 \times\left(\mathrm{C}_{6} \mathrm{H}_{5}\right)\right]^{+}, \quad 364$ (23) $\left[\mathrm{M}-2 \times\left(\mathrm{N}-\mathrm{C}_{6} \mathrm{H}_{5}\right)\right]^{+}, \quad 243$ (12) $\left[\left(\mathrm{C}_{15} \mathrm{H}_{7} \mathrm{~N}_{4}\right)\right]^{+}, 122(22)\left[\mathrm{C}_{8} \mathrm{H}_{12} \mathrm{~N}\right]^{+}, 93(100)\left[\mathrm{C}_{5} \mathrm{H}_{5} \mathrm{~N}_{2}\right]^{+}$, $66(22)\left[\mathrm{C}_{3} \mathrm{H}_{2} \mathrm{~N}_{2}\right]$.

MS (ESI in $\mathrm{MeOH}) \mathrm{m} / \mathrm{z}(\%)$ : $545(100)[\mathrm{M}+\mathrm{H}]^{+}$.

HRMS-(+)ESI m/z: berechnet: $[\mathrm{M}+\mathrm{H}]^{+}$545.33872;

(MeOH/Ammoniumacetat): gemessen: $[\mathrm{M}+\mathrm{H}]^{+} 545.33852$.

Schmelzpunkt $\left({ }^{\circ} \mathrm{C}\right)$ : 93-104.

IR $(\mathrm{KBr})$ :

$\widetilde{v}=3414(\mathrm{w}), 3194(\mathrm{w}), 2951(\mathrm{~m}), 2925(\mathrm{~m})$, 2872 (w), 2339 (w), 1612 (s), 1582 (s), 1450 (s), 1312 (m), 1188 (m), 1107 (w), 1008 (w), 763 (m), 693 (m), 540 (w) $\mathrm{cm}^{-1}$. 


\subsubsection{Synthese von $\mathrm{H}_{3} \mathrm{~L}^{3}$}

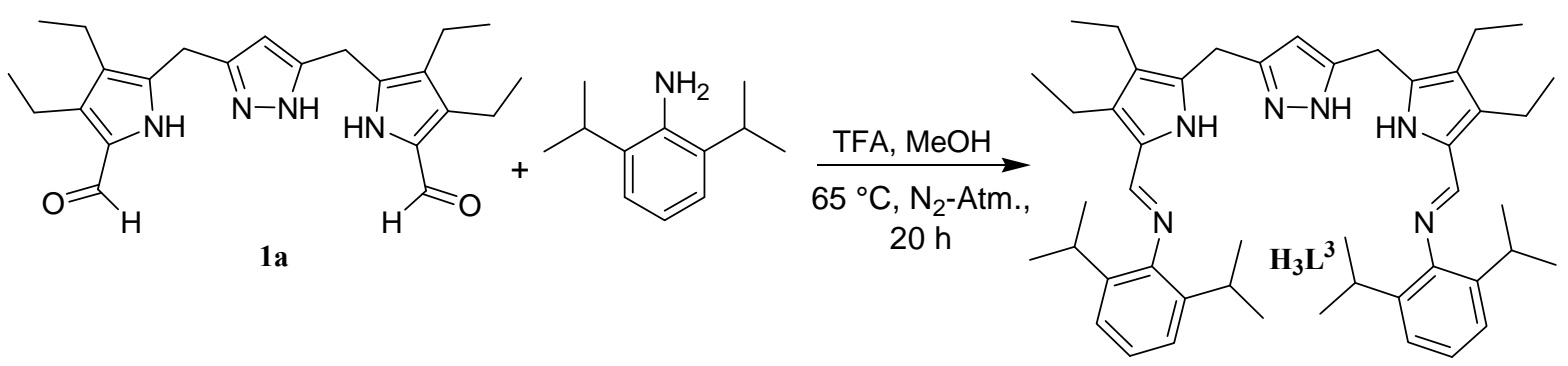

1 a (100 mg, $0.25 \mathrm{mmol}, 1$ eq.) wurde unter $\mathrm{N}_{2}$-Atmosphäre in trockenem Methanol $(60 \mathrm{~mL}$, getrocknet über $\mathrm{Mg}$ ) bei $50{ }^{\circ} \mathrm{C}$ gelöst. $\mathrm{Zu}$ dieser Lösung wurde frisch dest. 1,2Diisopropylanilin (176 $\mu \mathrm{L}, 1.52 \mathrm{mmol}, 6$ eq.) getropft und nach einigen Minuten Rühren wurde unter $\mathrm{N}_{2}$-Atm. Trifluoressigsäure (195 $\mu \mathrm{L}, 2.54 \mathrm{mmol}, 10$ eq.) langsam zum Reaktionsgemisch gegeben. Das Reaktionsgemisch wurde anschließend für 3 Tage unter Rückfluss erhitzt. Nach Abkühlen auf Raumtemperatur wurde das Lösungsmittel im Vakuum entfernt und das so erhaltene braune Rohprodukt säulenchromatographisch (Aluminiumoxid [basisch, Brockmann Aktivität I], Dichlormethan/Essigsäureethylester 10:1, $\mathrm{R}_{\mathrm{f}}=0.85$ ) gereinigt. Durch anschließende Umkristallisation in Essigsäureethylester bei tiefen Temperaturen erhält man $\mathbf{H}_{3} \mathbf{L}^{3}$ in Form eines weißen Feststoffes (92 mg, $51 \%$ ).

\section{Auswertung:}

Summenformel:

$\mathrm{C}_{47} \mathrm{H}_{64} \mathrm{~N}_{6}$

Molekulargewicht (g/mol):

713.1

${ }^{1} \mathrm{H}-\mathrm{NMR}\left(500 \mathrm{MHz}, \mathrm{CDCl}_{3}\right)$ :

$\delta(\mathrm{ppm})=1.05-1.10\left(\mathrm{t},{ }^{3} J(\mathrm{H}, \mathrm{H})=7.5 \mathrm{~Hz}, 6 \mathrm{H}, 2 \times\right.$ $\left.\mathrm{CH}_{3}\right), 1.11-1.12\left(\mathrm{~m}, 30 \mathrm{H}, 10 \times \mathrm{CH}_{3}\right), 2.39-2.41(\mathrm{q}$, $\left.{ }^{3} J(\mathrm{H}, \mathrm{H})=7.5 \mathrm{~Hz}, \quad 4 \mathrm{H}, \quad \mathrm{CH}_{2} \mathrm{CH}_{3}\right), 2.51-2.56 \quad(\mathrm{q}$, $\left.{ }^{3} J(\mathrm{H}, \mathrm{H})=7.5 \mathrm{~Hz}, 4 \mathrm{H}, \mathrm{CH}_{2} \mathrm{CH}_{3}\right), 3.03-3.05(\mathrm{~m}, 4 \mathrm{H}$, $\left.\mathrm{CH}^{\mathrm{iPr}}\right), 4.11(\mathrm{~s}, \mathrm{br}, 4 \mathrm{H}, \mathrm{CH}), 6.03\left(\mathrm{~s}, 1 \mathrm{H}, \mathrm{C} H^{\mathrm{pz}}\right), 7.09-$ $7.11\left(\mathrm{~m}, 2 \mathrm{H}, \mathrm{C} H^{\mathrm{Ph}}\right), 7.13-7.15\left(\mathrm{~m}, 4 \mathrm{H}, \mathrm{C} H^{\mathrm{Ph}}\right), 7.89(\mathrm{~s}$, $2 \mathrm{H}, 2 \times H \mathrm{C}=\mathrm{N})$.

\footnotetext{
${ }^{13} \mathrm{C}-\mathrm{NMR}\left(126 \mathrm{MHz}, \mathrm{CDCl}_{3}\right): \quad \delta(\mathrm{ppm})=16.0 \quad\left(\mathrm{CH}_{3}\right), \quad 17.1 \quad\left(\mathrm{CH}_{2} \mathrm{CH}_{3}\right), \quad 17.3$ $\left(\mathrm{CH}_{2} \mathrm{CH}_{3}\right), 17.5\left(\mathrm{CH}_{3}\right), 23.8\left(\mathrm{CH}^{i \mathrm{Pr}}\right), 24.2\left(\mathrm{CH}_{2}\right), 103.8$ $\left(C \mathrm{H}^{\mathrm{pz}}\right), 123.2\left(C \mathrm{H}^{\mathrm{Ph}}\right), 124.0\left(C^{\mathrm{q}}\right), 124.4\left(C \mathrm{H}^{\mathrm{Ph}}\right), 139.5$ $\left(\mathrm{N} C^{\mathrm{ph}}\right), 150.4(\mathrm{br}, \mathrm{HC}=\mathrm{N})$.
} 
MS (EI) $m / z(\%)$ :

$$
\begin{aligned}
& \text { MS (ESI(+) in } \mathrm{MeOH}) m / z(\%) \text { : } \\
& \text { MS (ESI(-) in } \left.\mathrm{CHCl}_{3}\right) m / z(\%) \text { : } \\
& \text { HRMS-(+)ESI } m / z \text { : } \\
& \text { (MeOH/Ammoniumacetat) } \\
& \text { Elementaranalyse (\%): }
\end{aligned}
$$

Schmelzpunkt $\left({ }^{\circ} \mathrm{C}\right)$ :

$\mathrm{IR}(\mathrm{KBr})$ :

$\mathrm{UV} / \mathrm{Vis}\left(\mathrm{CHCl}_{3}\right)$ :
$713(100)[\mathrm{M}]^{+}, 697(20)\left[\mathrm{M}-\left(\mathrm{CH}_{3}\right)\right]^{+}, 669$ (42) [M-

$$
\begin{aligned}
& \left.\left(\mathrm{C}_{3} \mathrm{H}_{7}\right)\right]^{+}, \quad 524 \quad(40) \quad\left[\left(\mathrm{C}_{33} \mathrm{H}_{44} \mathrm{~N}_{6}\right)\right]^{+}, \quad 309 \\
& {\left[\left(\mathrm{C}_{19} \mathrm{H}_{25} \mathrm{~N}_{4}\right)\right]^{+} \text {. }}
\end{aligned}
$$$$
714(100)[\mathrm{M}+\mathrm{H}]^{+} \text {. }
$$$$
711(100)[\mathrm{M}-\mathrm{H}]^{-} \text {. }
$$

berechnet: $[\mathrm{M}+\mathrm{H}]^{+}$713.52652,

gemessen: $[\mathrm{M}+\mathrm{H}]^{+} 713.52623$.

berechnet für $\mathrm{C}_{47} \mathrm{H}_{64} \mathrm{~N}_{6}\left(\left(\left(\mathrm{CH}_{3} \mathrm{H}_{2}\right)_{2} \mathrm{CO}_{2}\right)_{0.25}\right)$

C: 79.17, H: 9.05, N: 11.79;

gemessen: $\quad$ C: $78.07, \mathrm{H}: 8.94, \mathrm{~N}: 11.47$.

182-187.

$\widetilde{v}=3401(\mathrm{w}), 3219(\mathrm{w}), 2961(\mathrm{~s}), 2927(\mathrm{~m}), 2867$

(w), 1620 (vs), 1436 (s), 1102 (m), 1004 (m), 1008

(w), $787(\mathrm{w}) \mathrm{cm}^{-1}$.

$\lambda_{\max }[\mathrm{nm}]\left(\varepsilon \times 10^{4} \mathrm{M}^{-1} \mathrm{~cm}^{-1}\right)=317[4.25]$.

\subsubsection{Synthese von $\mathrm{H}_{3} \mathrm{~L}^{4}$}

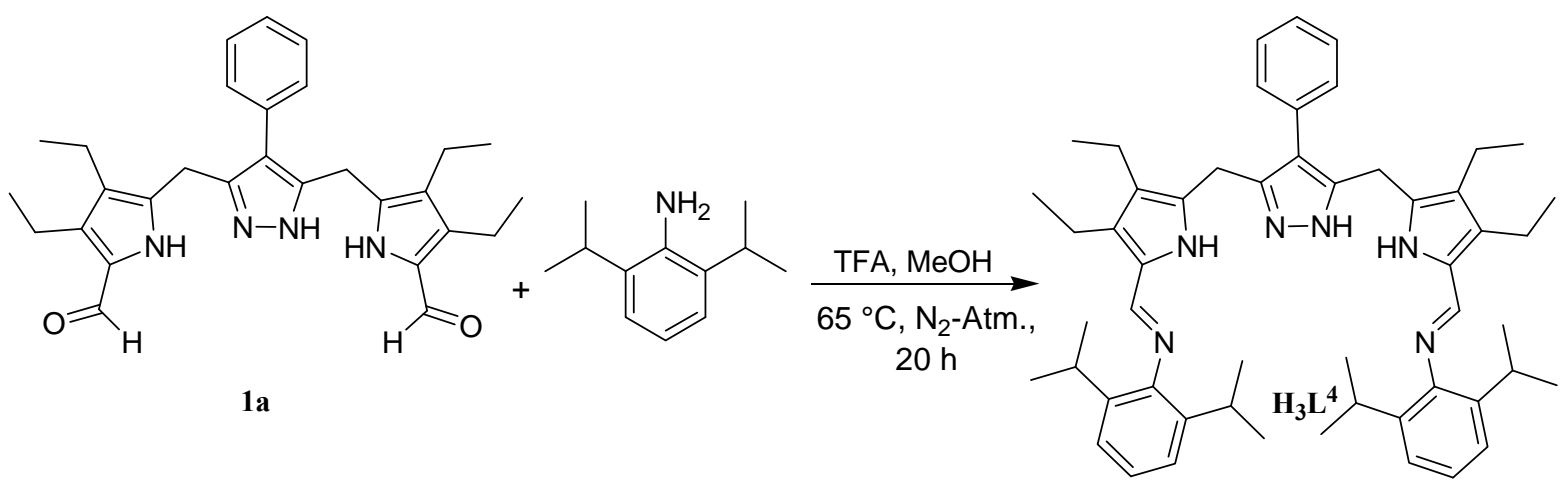

Der Dialdehyd (1a) (80 mg, $0.17 \mathrm{mmol}, 1$ eq.) wurde unter $\mathrm{N}_{2}$-Atmosphäre in Methanol $\left(80 \mathrm{~mL}\right.$, getrocknet über $\mathrm{Mg}$ ) bei $50{ }^{\circ} \mathrm{C}$ gelöst. $\mathrm{Zu}$ dieser Lösung wurde frisch destilliertes 1,2-Diisopropylanilin ( $79 \mu \mathrm{L}, 0.68 \mathrm{mmol}, 4$ eq.) langsam getropft und nach einigen Minuten Rühren wurde unter $\mathrm{N}_{2}$-Atm. Trifluoressigsäure (131 $\mu \mathrm{L}, 4.80 \mathrm{mmol}, 10$ eq.) dazugegeben. Das Reaktionsgemisch wurde anschließend für 3 Tage unter Rückfluss erhitzt. Nach Abkühlen auf Raumtemperatur wurde das Lösungsmittel im Vakuum entfernt und das so erhaltene braune Rohprodukt säulenchromatographisch (Aluminiumoxid [basisch, 
Brockmann Aktivität I], Dichlormethan/Essigsäureethylester 20:1, $\mathrm{R}_{\mathrm{f}}=0.30$ ) gereinigt. $\mathbf{H}_{\mathbf{3}} \mathbf{L}^{\mathbf{4}}$ erhält man in Form eines gelben, leicht bräunlichen Feststoffes (60 mg, 45 \%).

\section{Auswertung:}

Summenformel:

$\mathrm{C}_{53} \mathrm{H}_{68} \mathrm{~N}_{6}$

Molekulargewicht (g/mol):

789.2

${ }^{1} \mathrm{H}-\mathrm{NMR}\left(500 \mathrm{MHz}, \mathrm{CDCl}_{3}\right)$ :

$\delta(\mathrm{ppm})=0.93-0.96\left(\mathrm{t},{ }^{3} J(\mathrm{H}, \mathrm{H})=7.6 \mathrm{~Hz}, 6 \mathrm{H}, 2 \times\right.$ $\left.\mathrm{CH}_{3}\right), 1.07-1.15\left(\mathrm{~m}, 30 \mathrm{H}, 10 \times \mathrm{CH}_{3}\right), 2.23-2.28(\mathrm{q}$, $\left.{ }^{3} J(\mathrm{H}, \mathrm{H})=7.6 \mathrm{~Hz}, 4 \mathrm{H}, \quad \mathrm{CH}_{2} \mathrm{CH}_{3}\right), 2.48-2.53$ (q, ${ }^{3} J(\mathrm{H}, \mathrm{H})=7.6 \mathrm{~Hz}, 4 \mathrm{H}, \mathrm{CH}_{2} \mathrm{CH}_{3}$ ), 2.96-3.04 (sept, $\left.{ }^{3} J(\mathrm{H}, \mathrm{H})=6.9 \mathrm{~Hz}, 4 \mathrm{H}, \mathrm{C} H^{\mathrm{iPr}}\right), 3.93\left(\mathrm{~s}, 4 \mathrm{H}, \mathrm{CH}_{2}^{\mathrm{pz} / \mathrm{py}}\right)$, 7.08-7.08 $\left(\mathrm{m}, 2 \mathrm{H}, \mathrm{C} H^{\mathrm{Ph} / \mathrm{DIPA}}\right), 7.10-7.12(\mathrm{~m}, 4 \mathrm{H}$, $\left.\mathrm{CH}^{\mathrm{Ph} / \mathrm{DIPA}}\right), 7.21-7.22\left(\mathrm{~m}, 2 \mathrm{H}, \mathrm{C} H^{\mathrm{Ph} / \mathrm{pz}}\right), 7.26-7.29(\mathrm{tt}$, $\left.{ }^{3} J(\mathrm{H}, \mathrm{H})=7.3 \mathrm{~Hz},{ }^{4} J(\mathrm{H}, \mathrm{H})=2.0 \mathrm{~Hz}, 1 \mathrm{H}, \mathrm{C} H^{\mathrm{Ph} / \mathrm{pz}}\right)$, 7.34-7.37 (m, $\left.2 \mathrm{H}, \mathrm{C} H^{\mathrm{Ph} / \mathrm{pz}}\right), 7.85(\mathrm{~s}, 2 \mathrm{H}, 2 \times H \mathrm{C}=\mathrm{N})$.

${ }^{13} \mathrm{C}-\mathrm{NMR}\left(126 \mathrm{MHz}, \mathrm{CDCl}_{3}\right): \quad \delta(\mathrm{ppm})=15.8\left(\mathrm{CH}_{3}\right), \quad 16.9 \quad\left(\mathrm{CH}_{2} \mathrm{CH}_{3}\right), \quad 17.2$ $\left(\mathrm{CH}_{2} \mathrm{CH}_{3}\right), 17.5\left(\mathrm{CH}_{3}\right), 23.7\left(\mathrm{CH}_{3}{ }^{i \mathrm{Pr}}\right), 27.7\left(\mathrm{CH}^{i \mathrm{Pr}}\right)$, $119.1\left(C^{\mathrm{q}}\right), 123.0\left(C \mathrm{H}^{\mathrm{Ph} / \mathrm{DIPA}}\right), 124.0\left(C \mathrm{H}^{\mathrm{Ph} / \mathrm{DIPA}}\right), 127.0$ $\left(C \mathrm{H}^{\mathrm{Ph} / \mathrm{pz}}\right), 128.6\left(C \mathrm{H}^{\mathrm{Ph} / \mathrm{pz}}\right), 129.8\left(C \mathrm{H}^{\mathrm{Ph} / \mathrm{pz}}\right), 132.5\left(C^{\mathrm{q}}\right)$, $139.1\left(C^{\mathrm{q}}\right), 150.0(\mathrm{H} C=\mathrm{N})$.

$\mathrm{MS}(\mathrm{ESI}(+)$ in $\mathrm{MeOH}) \mathrm{m} / \mathrm{z}(\%)$ : $395(91)[\mathrm{M}+2 \mathrm{H}]^{2+}, 789(100)[\mathrm{M}+\mathrm{H}]^{+}$.

HRMS-(+)ESI: berechnet: $[\mathrm{M}+\mathrm{H}]^{+}$789.55782,

(MeOH/Ammoniumacetat) $\mathrm{m} / \mathrm{z}$ : gemessen: $[\mathrm{M}+\mathrm{H}]^{+} 789.55764$.

Schmelzpunkt $\left({ }^{\circ} \mathrm{C}\right)$ : 105-110.

IR ( $\mathrm{KBr})$ : $\widetilde{v}=3429(\mathrm{w}), 3210(\mathrm{br}, \mathrm{w}), 3060(\mathrm{w}), 2961$ (s), 2928 (m), $2869(\mathrm{w}), 1720$ (w), 1616 (vs), 1565 (m), 1417 (s), 1342 (w), 1261 (w), 1177 (m), 1096 (m), $1010(\mathrm{w}), 887(\mathrm{w}), 800(\mathrm{w}), 757(\mathrm{~m}), 694(\mathrm{w}) \mathrm{cm}^{-1}$. 


\subsubsection{Synthese von $H_{3} L^{5}$}

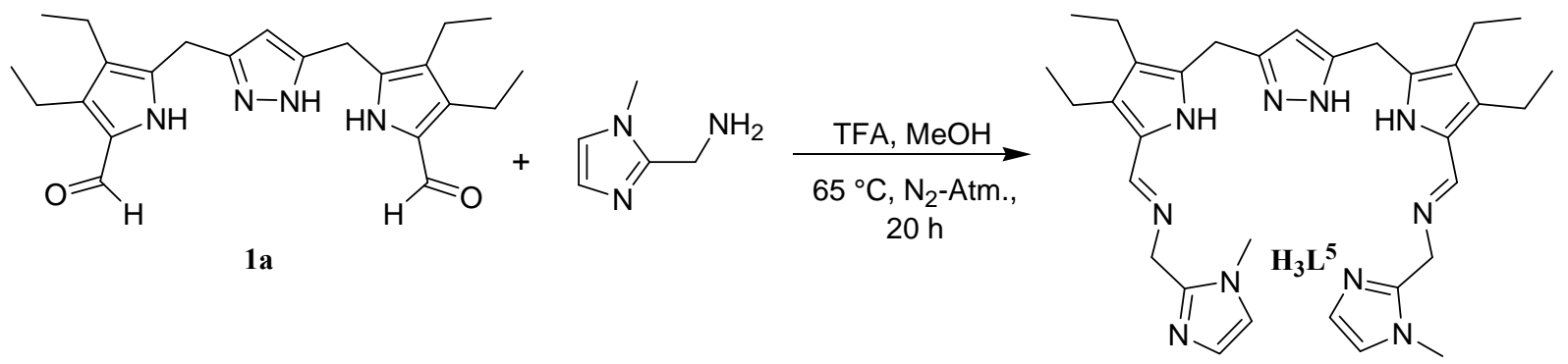

$1 \mathrm{a}\left(200 \mathrm{mg}, 0.51 \mathrm{mmol}, 1\right.$ eq.) wurde unter $\mathrm{N}_{2}$-Atmosphäre in trockenem Methanol $(80 \mathrm{~mL}$; getrocknet über $\mathrm{Mg}$ ) bei $50{ }^{\circ} \mathrm{C}$ gelöst. $\mathrm{Zu}$ dieser Lösung wurden das Methyl-(1methylimidazol-2-ylmethyl)amin (109.6 mg, 1.02 mmol, 2 eq.) zugetropft. Nach einigen Minuten Rühren wurde unter $\mathrm{N}_{2}$-Atm. Trifluoressigsäure (78 $\mu \mathrm{L}, 1.02 \mathrm{mmol}, 2.0$ eq.) langsam zum Reaktionsgemisch zugegeben. Das Reaktionsgemisch wurde anschließend 24 Stunden unter Rückfluss erhitzt. Nach Abkühlen auf Raumtemperatur wurde das Lösungsmittel im Vakuum entfernt und das so erhaltene braune Rohprodukt säulenchromatographisch (Aluminiumoxid [basisch, Brockmann Aktivität I], Dichlormethan/Methanol 30:1, $\mathrm{R}_{\mathrm{f}}=0.55$ ) gereinigt. Als Nebenprodukt entstand das monosubstituierte Produkt (Produkt/Nebenprodukt 1:0.5), welches eine Trennung des gewünschten Produktes $\left(\mathbf{H}_{3} \mathbf{L}^{3}\right)$ nicht möglich machte. Das Produkt erhält man als leicht bräunlicher Feststoff (133 mg, 45 \%) erhalten.

\section{Auswertung:}

Summenformel:

$\mathrm{C}_{33} \mathrm{H}_{44} \mathrm{~N}_{10}$

Molekulargewicht (g/mol): 580.8

${ }^{1} \mathrm{H}-\mathrm{NMR}\left(200 \mathrm{MHz}, \mathrm{CDCl}_{3}\right)$ :

$\delta(\mathrm{ppm})=0.098-1.09\left(\mathrm{~m}, \mathrm{CH}_{3}\right), 2.36-2.51\left(\mathrm{~m}, \mathrm{CH}_{2}\right)$, $3.65\left(\mathrm{~s}, \mathrm{CH}_{3}{ }^{\mathrm{imid}}\right), 3.68\left(\mathrm{~s}, \mathrm{CH}_{3}{ }^{\mathrm{imid}}\right), 3.89(\mathrm{~s}, \mathrm{br}$, $\left.\mathrm{CH}_{2} \mathrm{~N}^{\mathrm{imid}}\right), 4.53\left(\mathrm{~s}, \mathrm{CH}_{2}{ }^{\mathrm{py} / \mathrm{pz}}\right), 4.70\left(\mathrm{~s}, \mathrm{CH}_{2}{ }^{\mathrm{py} / \mathrm{pz}}\right), 5.95$ (s, $\left.\mathrm{CH}^{\mathrm{pz}}\right), 5.99\left(\mathrm{~s}, \mathrm{CH}^{\mathrm{pz}}\right), 6.84-6.92\left(\mathrm{~m}, \mathrm{CH}_{2}{ }^{\mathrm{imid}}\right), 8.10(\mathrm{~s}$, $H \mathrm{C}=\mathrm{N})$.

MS (ESI in $\mathrm{MeOH}) m / z(\%)$ :

$488(100)\left[\left(\mathrm{C}_{28} \mathrm{H}_{37} \mathrm{~N}_{7} \mathrm{O}\right)+\mathrm{H}\right]^{+}, 581(80)[\mathrm{M}+\mathrm{H}]^{+}, 975$

$$
\left[2 \times\left(\mathrm{C}_{28} \mathrm{H}_{37} \mathrm{~N}_{7} \mathrm{O}\right)+\mathrm{H}\right]^{+}, \quad 1068
$$

$\left[\left(\mathrm{C}_{28} \mathrm{H}_{37} \mathrm{~N}_{7} \mathrm{O}\right)+\mathrm{M}+\mathrm{H}\right]^{+}$. 


\subsection{Komplexsynthese}

\subsubsection{Synthese von $\left[\mathrm{L}^{1}{ }_{\text {ox }} \mathrm{Cu}_{2}(\mathrm{OH})\right]$}
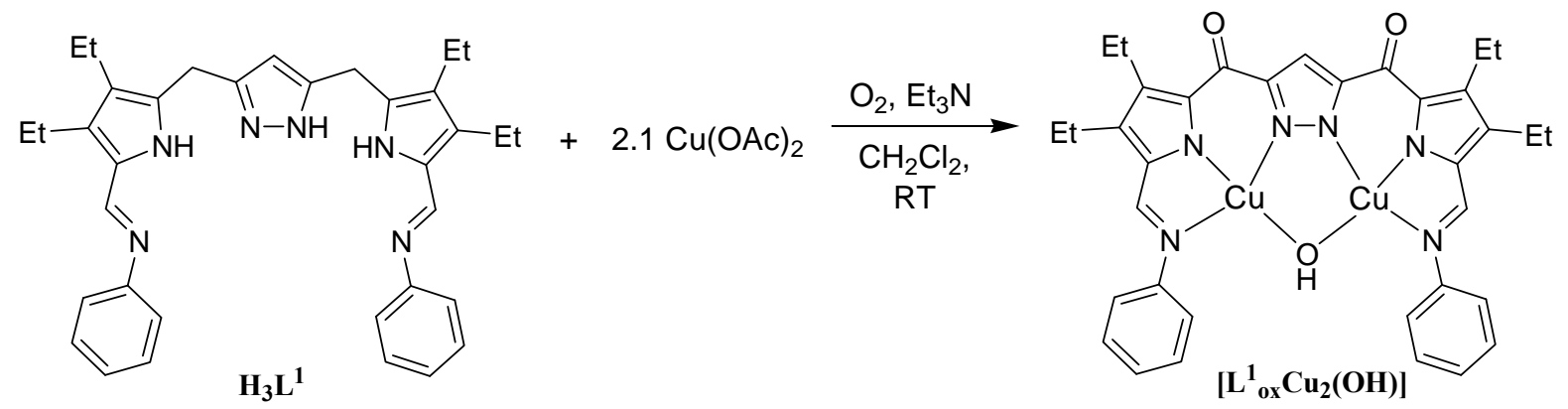

Der Ligand $\left(\mathbf{H}_{\mathbf{3}} \mathbf{L}^{\mathbf{1}}\right)$ (47 $\mathrm{mg}, 0.086 \mathrm{mmol}, 1$ eq.) wurde in THF (10 mL) gelöst und mit Triethylamin $(37 \mu \mathrm{L}, 0.26 \mathrm{mmol}, 3.0$ eq.) umgesetzt. Nach 10 Min. Rühren wurde Kupferacetat (33 mg, $0.18 \mathrm{mmol}, 2.1$ eq.) gegeben und das Reaktionsgemisch über Nacht bei RT gerührt. Das Lösungsmittel wurde entfernt und der grün-brauner Rückstand in Dichlormethan gelöst und Hexan/Et ${ }_{2} \mathrm{O}$ überschichtet. Da nach einigen Tagen keine Kristalle wuchsen, wurde die Lösung durch erneutes Lösen und langsames Abdampfen zum Kristallisieren gebracht. Nach mehreren Wochen konnte ein Einkristall isoliert und röntgenographisch analysiert werden.

\section{Auswertung:}

Summenformel:

$\mathrm{C}_{35} \mathrm{H}_{34} \mathrm{~N}_{6} \mathrm{Cu}_{2} \mathrm{O}_{3}$

Molekulargewicht (g/mol):

713.8

MS (ESI) $m / z(\%)$ :

$683(100)\left[\mathrm{C}_{35} \mathrm{H}_{37} \mathrm{~N}_{6} \mathrm{Cu}_{2} \mathrm{O}\right]^{+}, 788(50)$

$\left(\mathrm{MeOH} / \mathrm{CH}_{2} \mathrm{Cl}_{2}\right)$

$\left[\mathrm{C}_{35} \mathrm{H}_{33} \mathrm{~N}_{6} \mathrm{Cu}_{2} \mathrm{O}_{2}\left(\mathrm{CH}_{3} \mathrm{COO}\right)\left(\mathrm{CH}_{3} \mathrm{OH}\right) \mathrm{H}\right]^{+}$.

IR ( $\mathrm{KBr})$ :

$\widetilde{v}=3627(\mathrm{w}), 3434$ (br, w), 2963 (s), 2926 (w), 2868

(w), 1718 (w), 1608 (w), 1560 (m), 1481 (m), 1365

(w) $\mathrm{cm}^{-1}$. 


\subsubsection{Synthese von $\left[\mathrm{L}^{1} \mathrm{Cu}_{2}(\mathrm{OH})\right]$}
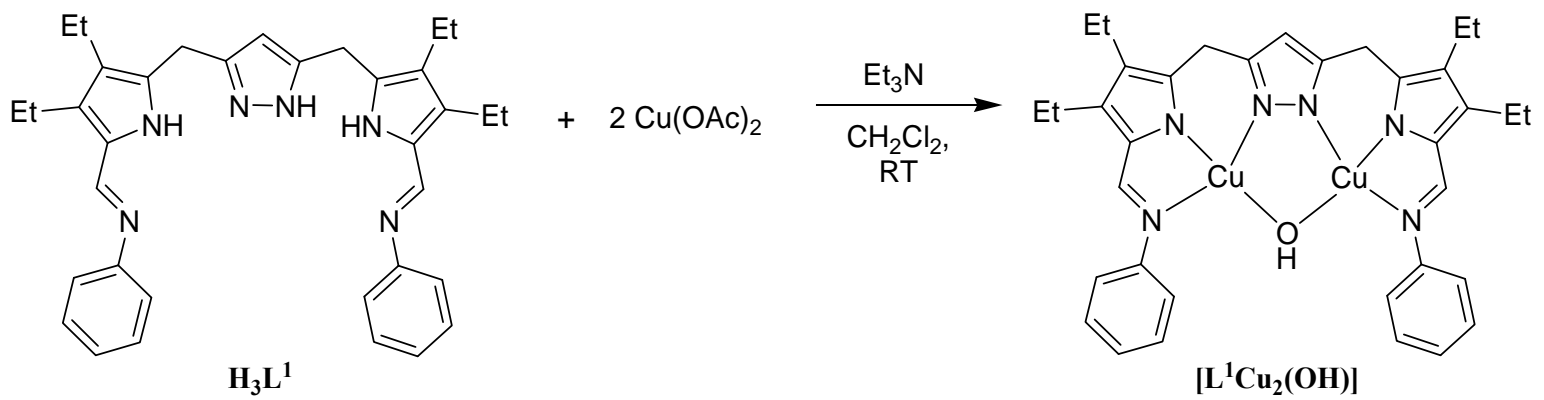

Der Ligand $\mathbf{H}_{3} \mathbf{L}^{1}$ (27 mg, $0.050 \mathrm{mmol}, 1$ eq.) wurde in $\mathrm{CH}_{2} \mathrm{Cl}_{2}$ (2 mL) gelöst und mit Triethylamin (3 Tropfen) deprotoniert. Nach 10 Min. Rühren wurde Kupferacetat (18 mg, $0.099 \mathrm{mmol}, 2$ eq.) gelöst in Ethanol (3 mL) dazugegeben und das Reaktionsgemisch wurde über Nacht bei RT gerührt. Das Lösungsmittel wurde entfernt und das Rohprodukt in $\mathrm{CH}_{2} \mathrm{Cl}_{2}$ aufgenommen, mit etwas Ethanol versetzt und durch langsames Abdampfen der Lösungsmittel kristallisiert. Der Komplex entstand in Form eines grün-braunen Feststoffes (18.3 mg, $54 \%)$.

\section{Auswertung:}

Summenformel:

$\mathrm{C}_{35} \mathrm{H}_{38} \mathrm{~N}_{6} \mathrm{Cu}_{2} \mathrm{O}$

Molekulargewicht (g/mol): 685.8

MS (ESI) $m / z(\%)$ : $685(70)\left[\mathrm{C}_{35} \mathrm{H}_{38} \mathrm{Cu}_{2} \mathrm{~N}_{6} \mathrm{O}\right]^{+}, 725$ (45)

$\left(\mathrm{MeCN} / \mathrm{CH}_{2} \mathrm{Cl}_{2}\right)$

$\left[\mathrm{C}_{35} \mathrm{H}_{37} \mathrm{Cu}_{2} \mathrm{~N}_{6}\left(\mathrm{C}_{2} \mathrm{O}_{2} \mathrm{H}_{3}\right)+\mathrm{H}\right]^{+}, 748(100)$

IR ( $\mathrm{KBr})$ :

$\left[\mathrm{C}_{35} \mathrm{H}_{37} \mathrm{Cu}_{2} \mathrm{~N}_{6}\left(\mathrm{C}_{2} \mathrm{O}_{2} \mathrm{H}_{3}\right)+\mathrm{Na}\right]^{+}$.

$\widetilde{v}=3626$ (m), 3262 (br, w), 2959 (m), 2911 (m), 2866 (w), 1703 (w), 1597 (w), 1554 (vs), 1481 (m), 1457 (m), 1363 (s), 1296 (w), 1260 (m), 1211 (m), $1018(\mathrm{~s}), 893(\mathrm{~m}) \mathrm{cm}^{-1}$. 


\subsubsection{Synthese von $\left[\mathrm{L}^{11} \mathrm{Co}_{2} \mathrm{Cl}\left(\mathrm{CH}_{3} \mathrm{OH}\right)_{2}\right]$}
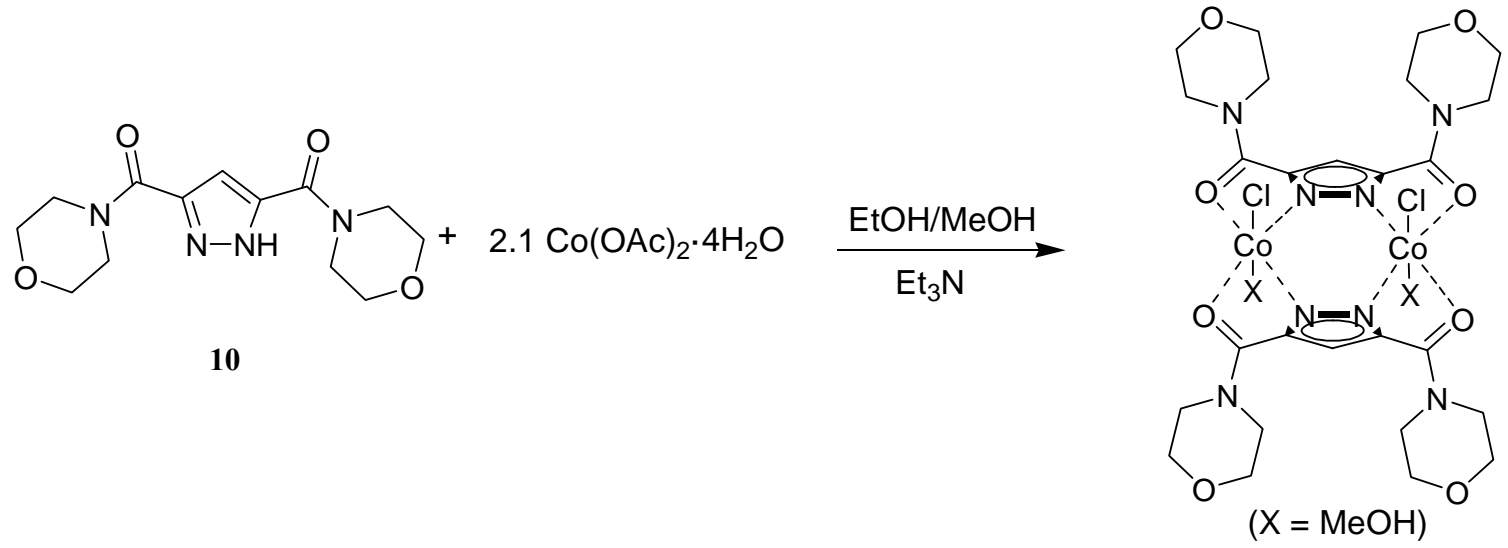

$\left[\mathrm{L}^{11} \mathrm{Co}_{2} \mathrm{Cl}\left(\mathrm{CH}_{3} \mathrm{OH}\right)_{2}\right]$

Der Ligand (11) (144 mg, $0.49 \mathrm{mmol}$ ) wurde in einer Mischung aus Ethanol/Methanol gelöst und mit zehn Tropfen Triethylamin deprotoniert. $\mathrm{Zu}$ dieser Lösung wurde KobaltacetatTetrahydrat (256 mg, $1.0 \mathrm{mmol}, 2.1$ eq.) gegeben und über Nacht bei Raumtemperatur gerührt. Am nächsten Tag wurde das Reaktionsgemisch eingeengt und mit Diethylether durch langsame Diffusion zum Kristallisieren gebracht. Nach einigen Tagen wuchsen kleine kubische lila-rote Einkristalle.

\section{Auswertung:}

Summenformel:

$\mathrm{C}_{26} \mathrm{H}_{34} \mathrm{~N}_{8} \mathrm{O}_{10} \mathrm{Co}_{2} \mathrm{Cl}_{2}$

Molekulargewicht (g/mol):

839.45

MS (ESI) $m / z(\%)($ in $\mathrm{MeOH})$ :

$740(77)\left[\left(\mathrm{C}_{13} \mathrm{H}_{17} \mathrm{~N}_{4} \mathrm{O}_{4}\right)_{2} \mathrm{Co}_{2} \mathrm{Cl}\right]^{+}, 763(100)$

$\left[\left(\mathrm{C}_{13} \mathrm{H}_{17} \mathrm{~N}_{4} \mathrm{O}_{4}\right)_{2} \mathrm{Co}_{2}\left(\mathrm{CH}_{3} \mathrm{COO}\right)\right]^{+}, 997$ (41)

$\left[\left(\mathrm{C}_{13} \mathrm{H}_{17} \mathrm{~N}_{4} \mathrm{O}_{4}\right)_{3} \mathrm{Co}_{2}\right]^{+}$.

MS (FAB(+) in 4-NBA) $m / z(\%)$ :

997 (100) $\left[\left(\mathrm{C}_{13} \mathrm{H}_{17} \mathrm{~N}_{4} \mathrm{O}_{4}\right)_{3} \mathrm{Co}_{2}\right]^{+}$.

$\operatorname{IR~}(\mathrm{KBr})$ :

$\widetilde{v}=3441(\mathrm{br}, \mathrm{m}), 2963(\mathrm{w}), 2353(\mathrm{~m}), 1600(\mathrm{w}), 1571$

(s), 1427 (m), 1289 (m), 1088 (m), 1021 (s), 804 (m), $664(\mathrm{~m}), 609(\mathrm{w}) \mathrm{cm}^{-1}$. 


\subsubsection{Synthese von $\left[\mathrm{L}^{3} \mathrm{Cu}_{2}(\mathrm{OH})\right]$}

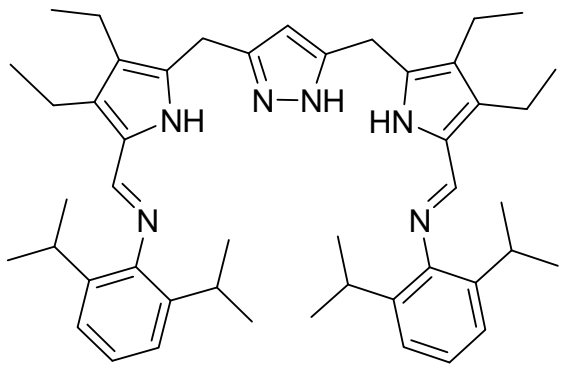

$\mathrm{H}_{3} \mathrm{~L}^{3}$
1. $\mathrm{Et}_{3} \mathrm{~N}$

2. 2 eq. $\mathrm{Cu}(\mathrm{OAc})_{2}$

$\mathrm{CH}_{2} \mathrm{Cl}_{2} / \mathrm{EtOH}$

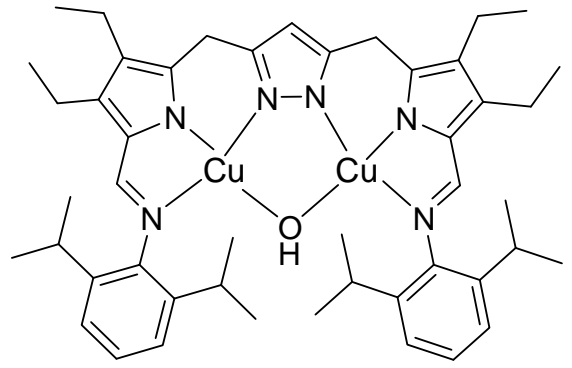

$\left[\mathrm{L}^{3} \mathrm{Cu}_{2}(\mathrm{OH})\right]$

Der Ligand $\mathbf{H}_{\mathbf{3}} \mathbf{L}^{\mathbf{3}}$ (60 mg, $0.08 \mathrm{mmol}, 1$ eq.) wurde in Dichlormethan (5 mL) gelöst und mit Triethylamin (3 Tropfen) deprotoniert. Nach 10 Min. Rühren wurde Kupferacetat (30.6 mg, $0.16 \mathrm{mmol}, 2.0$ eq.), gelöst in Ethanol (2 mL), dazugegeben und das Reaktionsgemisch wurde 5 Tage bei RT gerührt. Das Lösungsmittel wurde entfernt und der Rückstand in Dichlormethan gelöst und mit Ethanol (1:2) überschichtet. Nach wenigen Tagen wuchsen in der Lösung rot-braune Kristall des Komplexes $\left[\mathbf{L}^{3} \mathbf{C u}_{\mathbf{2}}(\mathbf{O H})\right](50.1 \mathrm{mg}, 70 \%$ ), die röntgenographisch analysiert werden konnten.

\section{Auswertung:}

Summenformel:

$\mathrm{C}_{47} \mathrm{H}_{62} \mathrm{~N}_{6} \mathrm{Cu}_{2} \mathrm{O}$

Molekulargewicht (g/mol):

854.1

MS (ESI in $\mathrm{MeOH} / \mathrm{CH}_{2} \mathrm{Cl}_{2}$ ) m/z (\%): 714 (100) $\left[\mathrm{C}_{47} \mathrm{H}_{64} \mathrm{~N}_{6}+\mathrm{H}\right]^{+}, 774$ (47) $\left[\mathrm{C}_{47} \mathrm{H}_{63} \mathrm{~N}_{6} \mathrm{Cu}_{2}\right]^{+}$, $853 \quad(72) \quad\left[\left(\left(\mathrm{C}_{47} \mathrm{H}_{62} \mathrm{~N}_{6} \mathrm{Cu}_{2}\right)-2 \mathrm{H}\right)+\mathrm{H}\right]^{+}, \quad 1488$ $\left[\left(\mathrm{C}_{47} \mathrm{H}_{62} \mathrm{~N}_{6}\right)_{2} \mathrm{Cu}+\mathrm{H}\right]^{+}, 1550(3)\left[\left(\mathrm{C}_{47} \mathrm{H}_{62} \mathrm{~N}_{6}\right)_{2} \mathrm{Cu}_{2}+\mathrm{H}\right]^{+}$.

MS (FAB in 4-NBA) $m / z(\%): \quad 853 \quad(58) \quad\left[\left(\left(\mathrm{C}_{47} \mathrm{H}_{62} \mathrm{~N}_{6} \mathrm{Cu}_{2}\right)-2 \mathrm{H}\right)+\mathrm{H}\right]^{+}, \quad 853 \quad(100)$ $\left[\left(\mathrm{C}_{47} \mathrm{H}_{62} \mathrm{~N}_{6} \mathrm{Cu}_{2} \mathrm{OH}\right)-3 \mathrm{H}\right]^{+}, 871(96)$ $\left[\left(\mathrm{C}_{47} \mathrm{H}_{62} \mathrm{~N}_{6} \mathrm{Cu}_{2}(\mathrm{OH})_{2}\right)-3 \mathrm{H}\right]^{+}$.

HRMS-(+)ESI $(\mathrm{MeOH}) \mathrm{m} / \mathrm{z}: \quad$ berechnet: $[\mathrm{M}+\mathrm{H}]^{+}:$853.36499, gemessen: $[\mathrm{M}+\mathrm{H}]^{+}:$853.36490; berechnet: $[(\mathrm{M}-2 \mathrm{H})+\mathrm{H}]^{+}: 851.34934$, gemessen: $[(\mathrm{M}-2 \mathrm{H})+\mathrm{H}]^{+}: 851.34889$.

IR ( $\mathrm{KBr})$ : $\widetilde{v}=3615$ (m), 3453 (br, w), 2955 (s), 2924 (m), 2867 (w), 1562 (vs), 1477 (s), 1381 (m), 1277 (m), 1215 (s), 
1192 (m), 1099 (w), 1018 (m), 976 (w), 922 (m), 801

(w), $756(\mathrm{~m}), 727(\mathrm{w}) \mathrm{cm}^{-1}$.

$\mathrm{UV} / \mathrm{Vis}(\mathrm{nm})\left(\mathrm{CHCl}_{3}\right)$

$\lambda_{\max }\left[\varepsilon^{\times} \times 10^{4} \mathrm{M}^{-1} \mathrm{~cm}^{-1}\right]=304[1.32], 386[2.67], 560$

$[0.05]$.

\subsubsection{Synthese von $\left[\mathrm{L}^{4} \mathrm{Cu}_{2}(\mathrm{OH})\right]$}
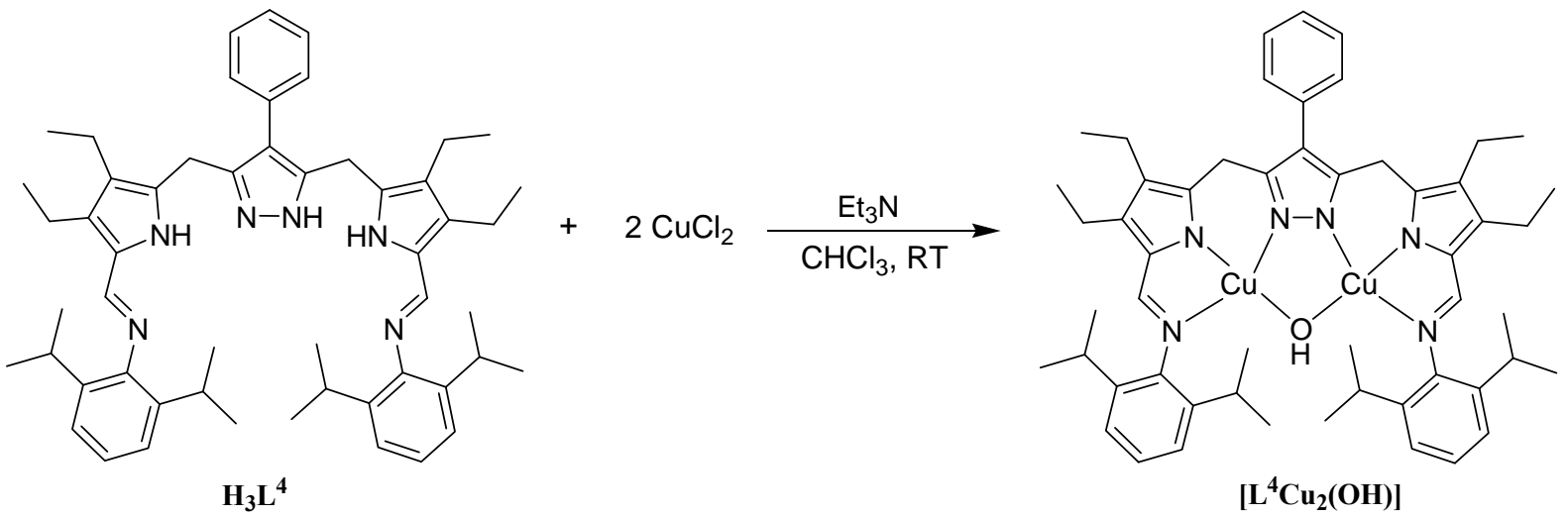

Der Ligand $\mathbf{H}_{3} \mathbf{L}^{4}$ (27 mg, $0.034 \mathrm{mmol}, 1$ eq.) wurde in $\mathrm{CHCl}_{3}$ (3 mL) gelöst und mit Triethylamin (4 Tropfen) deprotoniert. Nach 10 Min. Rühren wurde Kupferchlorid (9.2 mg, 0.068 mmol, 2 eq.) dazugegeben und das Reaktionsgemisch 5 Tage bei RT gerührt. Das Lösungsmittel wurde entfernt und das Rohprodukt in $\mathrm{CH}_{2} \mathrm{Cl}_{2}$ aufgenommen und mit $\mathrm{MeCN}$ überschichtet. Der Komplex entstand in Form eines grün-braunen Feststoffes.

\section{Auswertung:}

Summenformel:

Molekulargewicht (g/mol):

HRMS-(+) (ESI) $m / z(\%)$ :

$(\mathrm{MeCN})$
$\mathrm{C}_{53} \mathrm{H}_{66} \mathrm{~N}_{6} \mathrm{Cu}_{2} \mathrm{O}$

930.2

$[(\mathrm{M}-2 \mathrm{H})+\mathrm{H}]^{+}$berechnet: 929.38012,

$[(\mathrm{M}-2 \mathrm{H})+\mathrm{H}]^{+}$gemessen: 929.37513 ;

$[\mathrm{M}+\mathrm{H}]^{+}$berechnet: 931.39578,

$[\mathrm{M}+\mathrm{H}]^{+}$gemessen: 931.39427 .

MS (ESI $(+)$; MeCN) $m / z(\%)$ :

MS (ESI(-); MeCN) $m / z(\%)$ :

MS (FAB(+); 4-NBA) $m / z(\%)$ :
929 (100) $\left[\mathrm{C}_{53} \mathrm{H}_{65} \mathrm{~N}_{6} \mathrm{Cu}_{2}(\mathrm{OH})+\mathrm{H}\right]^{+}$.

$947 \quad$ (73) $\quad\left[\mathrm{C}_{53} \mathrm{H}_{64} \mathrm{~N}_{6} \mathrm{Cu}_{2} \mathrm{Cl}\right]^{-}, \quad 965$

$\left[\mathrm{C}_{53} \mathrm{H}_{64} \mathrm{~N}_{6} \mathrm{Cu}_{2} \mathrm{Cl}+\mathrm{F}\right]^{-}$.

948 (100) $\left[\mathrm{C}_{35} \mathrm{H}_{65} \mathrm{Cu}_{2} \mathrm{~N}_{6} \mathrm{Cl}\right]^{+} ; 930$ (25) $\left[\mathrm{C}_{35} \mathrm{H}_{65} \mathrm{Cu}_{2} \mathrm{~N}_{6}\right.$ $(\mathrm{OH})+\mathrm{H}]^{+}, 912(22)\left[\mathrm{C}_{35} \mathrm{H}_{35} \mathrm{Cu}_{2} \mathrm{~N}_{6}\right]^{+}$. 
IR (KBr):

$\widetilde{v}=3441$ (br, m), $3066(\mathrm{w}), 2959$ (s), 2928 (m), 2878

(w), $2678(\mathrm{w}), 2493(\mathrm{w}), 1707(\mathrm{w}), 1621(\mathrm{w}), 1563$

(vs), $1461(\mathrm{~m}), 1381(\mathrm{~m}), 1326(\mathrm{w}), 1270(\mathrm{w}), 1184$

(w), $1096(\mathrm{~m}), 1031(\mathrm{~m}), 922(\mathrm{w}), 804(\mathrm{~m}), 760(\mathrm{w})$, $694(\mathrm{w}) \mathrm{cm}^{-1}$.

\subsubsection{Synthese von $\left[\mathrm{L}^{1} \mathrm{Ni}_{2}(\mathrm{OH})\right]$}
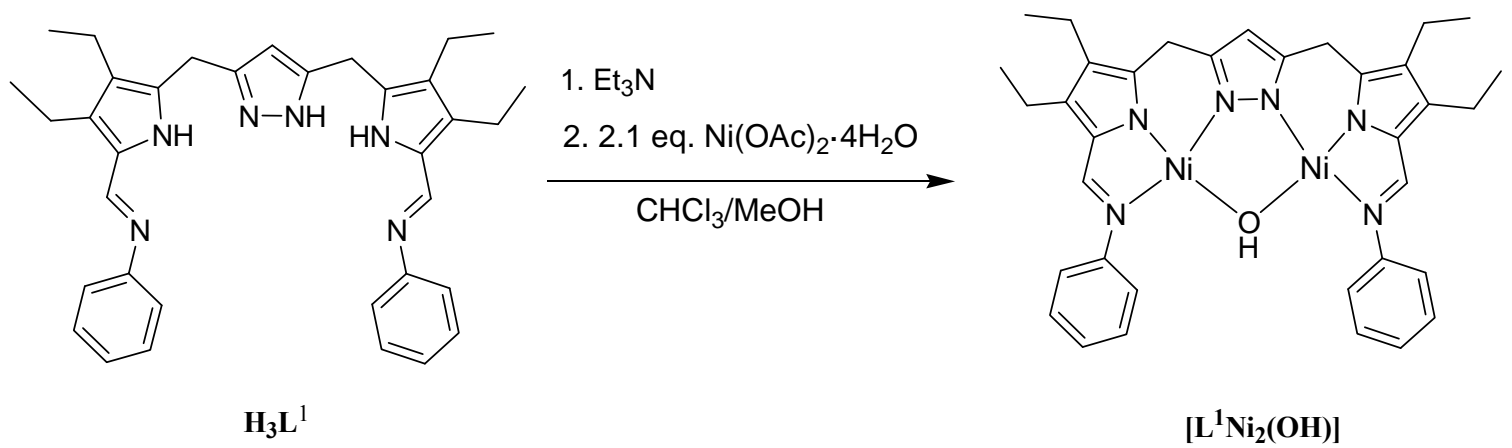

Der Ligand $\mathbf{H}_{\mathbf{3}} \mathbf{L}^{\mathbf{1}}$ (47 mg, $0.09 \mathrm{mmol}, 1$ eq.) wurde in THF (2-3 mL) gelöst und mit Triethylamin (5 Tropfen) deprotoniert. Anschließend wurde Nickelacetat-Tetrahydrat (45 mg, $0.18 \mathrm{mmol}, 2.1$ eq.), gelöst in Methanol ( $2 \mathrm{~mL})$ zum Reaktionsgemisch gegeben und $1 \mathrm{~d}$ gerührt. Das Lösungsmittel wurde destillativ entfernt und der Rückstand in einer Lösung aus Dichlormethan/Ethanol (1:2) gelöst. Beim langsamen Abdampfen des Lösungsmittels wuchsen nach einigen Tagen kleine orange-rote Kristalle, die röntgenographisch charakterisiert werden konnten (14 mg, $24 \%$ ).

\section{Auswertung:}

Summenformel:

$\mathrm{C}_{35} \mathrm{H}_{38} \mathrm{~N}_{6} \mathrm{Ni}_{2} \mathrm{O}$

Molekulargewicht (g/mol):

674.18

${ }^{1} \mathrm{H}-\mathrm{NMR}\left(500 \mathrm{MHz}, \mathrm{CDCl}_{3}\right)$ :

$\delta(\mathrm{ppm})=-5.65(\mathrm{~s}, 1 \mathrm{H}, \mathrm{OH}), 0.82-0.86\left(\mathrm{t},{ }^{3} J(\mathrm{H}, \mathrm{H})=\right.$ $\left.7.5 \mathrm{~Hz}, 6 \mathrm{H}, 2 \times \mathrm{CH}_{3}\right), 1.01-1.04\left(\mathrm{t},{ }^{3} J(\mathrm{H}, \mathrm{H})=7.5 \mathrm{~Hz}\right.$, $\left.6 \mathrm{H}, 2 \times \mathrm{CH}_{3}\right), 2.30-2.35\left(\mathrm{q},{ }^{3} J(\mathrm{H}, \mathrm{H})=7.5 \mathrm{~Hz}, 4 \mathrm{H}\right.$, $\left.\mathrm{CH}_{2} \mathrm{CH}_{3}\right), \quad 2.46-2.50\left(\mathrm{q},{ }^{3} J(\mathrm{H}, \mathrm{H})=7.5 \mathrm{~Hz}, 4 \mathrm{H}\right.$, $\left.\mathrm{CH}_{2} \mathrm{CH}_{3}\right), 3.93\left(\mathrm{~s}, 4 \mathrm{H}, 2 \times \mathrm{CH}_{2}\right), 5.93\left(\mathrm{~s}, 1 \mathrm{H}, \mathrm{CH}^{\mathrm{pz}}\right)$, 6.81-6.90 (m, 10H, $\left.\mathrm{CH}^{\mathrm{Ph}}\right), 7.00(\mathrm{~s}, 2 \mathrm{H}, 2 \times H \mathrm{C}=\mathrm{N})$.

${ }^{13} \mathrm{C}-\mathrm{NMR}\left(126 \mathrm{MHz}, \mathrm{CDCl}_{3}\right): \quad \delta(\mathrm{ppm})=15.5\left(\mathrm{CH}_{3}\right), 17.0\left(\mathrm{CH}_{3}\right), 17.7\left(\mathrm{CH}_{2} \mathrm{CH}_{3}\right)$, $18.9\left(\mathrm{CH}_{2} \mathrm{CH}_{3}\right), 27.0\left(\mathrm{CH}_{2}{ }^{\mathrm{py} / \mathrm{pz}}\right), 102.7\left(\mathrm{CH}^{\mathrm{pz}}\right), 122.4$ 


$\begin{array}{ll} & \left(C \mathrm{H}^{\mathrm{Ph}}\right), 124.4\left(C^{\mathrm{q}}\right), 125.7\left(C \mathrm{H}^{\mathrm{Ph}}\right), 129.1\left(\mathrm{CH}^{\mathrm{Ph}}\right), 135.3 \\ & \left(C^{\mathrm{q}}\right), 141.9\left(C^{\mathrm{q}}\right), 145.5\left(C^{\mathrm{q}}\right), 148.0\left(C^{\mathrm{q}}\right), 154.6 \\ & (\mathrm{HC}=\mathrm{N}) . \\ & 673(100)\left[\mathrm{C}_{35} \mathrm{H}_{37} \mathrm{~N}_{6} \mathrm{Ni}_{2} \mathrm{O}\right]^{+}, 697(13) \\ \mathrm{MS}(\mathrm{ESI}) \mathrm{m} / z(\%): & {\left[\left(\mathrm{C}_{35} \mathrm{H}_{37} \mathrm{~N}_{6} \mathrm{Ni}_{2} \mathrm{OH}\right)+\mathrm{Na}\right]^{+} .} \\ \left(\mathrm{MeOH} / \mathrm{CH}_{2} \mathrm{Cl}_{2}\right) & 675(100)[\mathrm{M}+\mathrm{H}]^{+}, 657(38)[\mathrm{M}-\mathrm{OH}]^{+} . \\ \mathrm{MS}(\mathrm{FAB}(+) \text { in 4-NBA}) m / z(\%): & \text { berechnet: }[\mathrm{M}+\mathrm{H}]^{+}: 675.18868, \\ \mathrm{HRMS}-(+) \mathrm{ESI} m / z: & \text { gemessen: }[\mathrm{M}+\mathrm{H}]^{+}: 675.18884 ; \\ \left(\mathrm{MeOH} / \mathrm{CH}_{2} \mathrm{Cl}_{2}\right) & \text { berechnet: }[(\mathrm{M}-2 \mathrm{H})+\mathrm{H}]^{+}: 673.17303, \\ & \text { gemessen: }[(\mathrm{M}-2 \mathrm{H})+\mathrm{H}]^{+}: 673.17354 . \\ & >350 \\ \text { Schmelzpunkt }\left({ }^{\circ} \mathrm{C}\right): & \widetilde{v}=3603(\mathrm{~m}), 3426(\mathrm{br}, \mathrm{w}), 2963(\mathrm{~s}), 2925(\mathrm{w}), 2862 \\ \mathrm{IR}(\mathrm{KBr}): & (\mathrm{w}), 1570(\mathrm{~s}), 1491(\mathrm{w}), 1408(\mathrm{~m}), 1262(\mathrm{~s}), 1215(\mathrm{~m}), \\ & 1092(\mathrm{~m}), 1021(\mathrm{~m}), 982(\mathrm{~m}), 867(\mathrm{w}), 800(\mathrm{vs}), 683 \\ & (\mathrm{~m}), 615(\mathrm{w}) \mathrm{cm}{ }^{-1} .\end{array}$

\subsubsection{Synthese von $\left[\mathrm{L}^{3} \mathrm{Ni}_{2}(\mathrm{OH})\right]$}
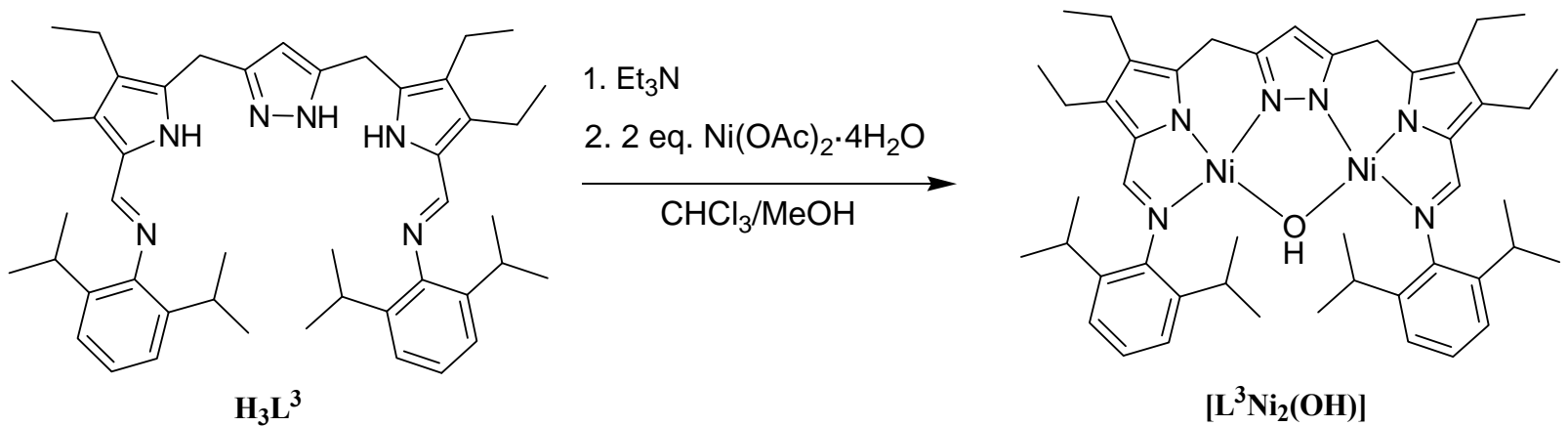

$\left[\mathbf{L}^{3} \mathrm{Ni}_{2}(\mathrm{OH})\right]$

$\mathbf{H}_{3} \mathbf{L}^{3}$ (60 mg, $0.084 \mathrm{mmol}, 1$ eq.) wurde in THF (5 mL) gelöst und mit Triethylamin (4 Tropfen) deprotoniert. Nach 10 Min. wurde Nickelacetat Tetrahydrat (41.9 mg, $0.168 \mathrm{mmol}$, 2 eq.) gelöst in Methanol ( $2 \mathrm{~mL}$ ) dazu gegeben und das Reaktionsgemisch 3 Tage bei RT gerührt. Das Lösungsmittel wurde entfernt und der Rückstand in einer Mischung aus $\mathrm{THF} / \mathrm{CH}_{2} \mathrm{Cl}_{2} / \mathrm{MeCN}$ (2:1:1) gelöst. Beim langsamen Abdampfen der Lösungsmittel wuchsen nach einigen Tagen lange rote Nadeln des Komplexes $\left[\mathbf{L}^{3} \mathbf{N i}_{\mathbf{2}}(\mathbf{O H})\right](48.2 \mathrm{mg}, 68 \%$ ), die röntgenographisch analysiert wurden. 


\section{Auswertung:}

Summenformel:

Molekulargewicht (g/mol):

${ }^{1} \mathrm{H}-\mathrm{NMR}$ (500 MHz, THF-d 8 ):

${ }^{13} \mathrm{C}-\mathrm{NMR}\left(126 \mathrm{MHz}, \mathrm{THF}-\mathrm{d}_{8}\right)$ :

$\operatorname{MS}(\operatorname{ESI}(+)) m / z(\%)$ :

(in $\mathrm{MeOH} / \mathrm{CH}_{2} \mathrm{Cl}_{2}$ )

$\mathrm{MS}(\mathrm{FAB}(+)$ in $4-\mathrm{NBA}) \mathrm{m} / z(\%)$ :

HRMS-(+) (ESI in MeOH) $m / z$ :

Schmelzpunkt (\%):

IR $(\mathrm{KBr})$ :

$\mathrm{UV} / \mathrm{V}$ is $(\mathrm{nm})\left(\mathrm{CHCl}_{3}\right)$ :
$\mathrm{C}_{47} \mathrm{H}_{62} \mathrm{~N}_{6} \mathrm{Ni}_{2} \mathrm{O}$

844.4

$\delta(\mathrm{ppm})=-6.70(\mathrm{~s}, 1 \mathrm{H}, \mathrm{OH}), 1.03-1.06\left(\mathrm{~m},{ }^{3} J(\mathrm{H}, \mathrm{H})=\right.$ $\left.7.5 \mathrm{~Hz}, \quad 24 \mathrm{H}, \quad \mathrm{CH}_{3} / \mathrm{CH}_{3}{ }^{i \mathrm{Pr}}\right), \quad 1.29-1.36 \quad(\mathrm{~d}, \quad 12 \mathrm{H}$, $\left.\mathrm{CH}_{3} / \mathrm{CH}_{3}{ }^{i \mathrm{Pr}}\right), 2.32-2.36\left(\mathrm{q},{ }^{3} \mathrm{~J}(\mathrm{H}, \mathrm{H})=7.5 \mathrm{~Hz}, 4 \mathrm{H}\right.$, $\left.\mathrm{CH}_{2} \mathrm{CH}_{3}\right), \quad 2.43-2.48\left(\mathrm{q},{ }^{3} J(\mathrm{H}, \mathrm{H})=7.5 \mathrm{~Hz}, 4 \mathrm{H}\right.$, $\mathrm{CH}_{2} \mathrm{CH}_{3}$ ), 3.63-3.72 (sept, ${ }^{3} J(\mathrm{H}, \mathrm{H})=6.80 \mathrm{~Hz}, 4 \mathrm{H}$, $\left.\mathrm{C}^{\mathrm{iPr}}\right), 3.92\left(\mathrm{~s}, 4 \mathrm{H}, 2 \times \mathrm{CH}_{2}\right), 5.96\left(\mathrm{~s}, \mathrm{br}, 1 \mathrm{H}, \mathrm{CH}^{\mathrm{pz}}\right)$, $6.82(\mathrm{~s}, 2 \mathrm{H}, 2 \times H \mathrm{C}=\mathrm{N}), 6.84-6.86\left(\mathrm{~d},{ }^{3} J(\mathrm{H}, \mathrm{H})=\right.$ $\left.7.90 \mathrm{~Hz}, 4 \mathrm{H}, \mathrm{C} H^{\mathrm{Ph}}\right), 7.01-7.04\left(\mathrm{t},{ }^{3} J(\mathrm{H}, \mathrm{H})=7.90 \mathrm{~Hz}\right.$, $\left.2 \mathrm{H}, \mathrm{C} H^{\mathrm{Ph}}\right)$.

$\delta(\mathrm{ppm})=16.0\left(\mathrm{CH}_{3}\right), 17.8\left(\mathrm{CH}_{3}\right), 18.4\left(\mathrm{CH}_{2} \mathrm{CH}_{3}\right)$, $19.4\left(\mathrm{CH}_{2} \mathrm{CH}_{3}\right), 23.8\left(\mathrm{CH}_{3}{ }^{i \mathrm{Pr}}\right), 27.5\left(\mathrm{CH}_{2}{ }^{\mathrm{py} / \mathrm{pz}}\right), 29.0$ $\left(\mathrm{CH}_{3}{ }^{{ }^{\mathrm{Pr}}}\right), 103.6\left(\mathrm{CH}^{\mathrm{pz}}\right), 124.0\left(C^{\mathrm{q}}\right), 124.4\left(\mathrm{CH}^{\mathrm{Ph}}\right), 127.0$ $\left(C \mathrm{H}^{\mathrm{Ph}}\right), 135.5\left(C^{\mathrm{q}}\right), 135.8\left(C^{\mathrm{q}}\right), 142.3\left(C^{\mathrm{q}}\right), 143.7\left(C^{\mathrm{q}}\right)$, $144.4\left(C^{\mathrm{q}}\right), 146.7\left(C^{\mathrm{q}}\right), 158.2(\mathrm{H} C=\mathrm{N})$.

$843(20)\left[\mathrm{C}_{47} \mathrm{H}_{62} \mathrm{~N}_{6} \mathrm{Ni}_{2} \mathrm{OH}+\mathrm{H}\right]^{+}, 413$ (100)

$\left[\left(\mathrm{C}_{47} \mathrm{H}_{62} \mathrm{~N}_{6} \mathrm{Ni}_{2}\right)^{+}+\mathrm{H}^{+}\right]^{2+}$.

$843(100)[\mathrm{M}+\mathrm{H}]^{+}$.

$[\mathrm{M}+\mathrm{H}]^{+}$berechnet: 843.37648 ,

$[\mathrm{M}+\mathrm{H}]^{+}$gemessen: 843.37609;

$[\mathrm{M}+\mathrm{Na}]^{+}$berechnet: 865.35843,

$[\mathrm{M}+\mathrm{Na}]^{+}$gemessen: 865.35798.

128-132.

$\widetilde{v}=3607$ (m), 3433 (br, w), 3059 (w), 2955 (s), 2924 (s), $2864(w), 2353(w), 2340(w), 1578$ (vs), 1470 (s), 1396 (m), 1320 (w), 1266 (m), 1194 (w), 1097 (w), 1024 (m), 982 (m), 931 (w), 799 (m), 763 (m), 731 (m), $586(\mathrm{w}) \mathrm{cm}^{-1}$.

$\lambda_{\max }\left[\varepsilon^{\times} \times 10^{4} \mathrm{M}^{-1} \mathrm{~cm}^{-1}\right]=335$ [1.86], 382 [1.90], $414(\mathrm{sh})$ [1.42], 450 (sh) [0.42], 520 [0.10]. 


\subsubsection{Synthese von $\left[\mathrm{L}^{4} \mathrm{Ni}_{2}(\mathrm{OH})\right]$}

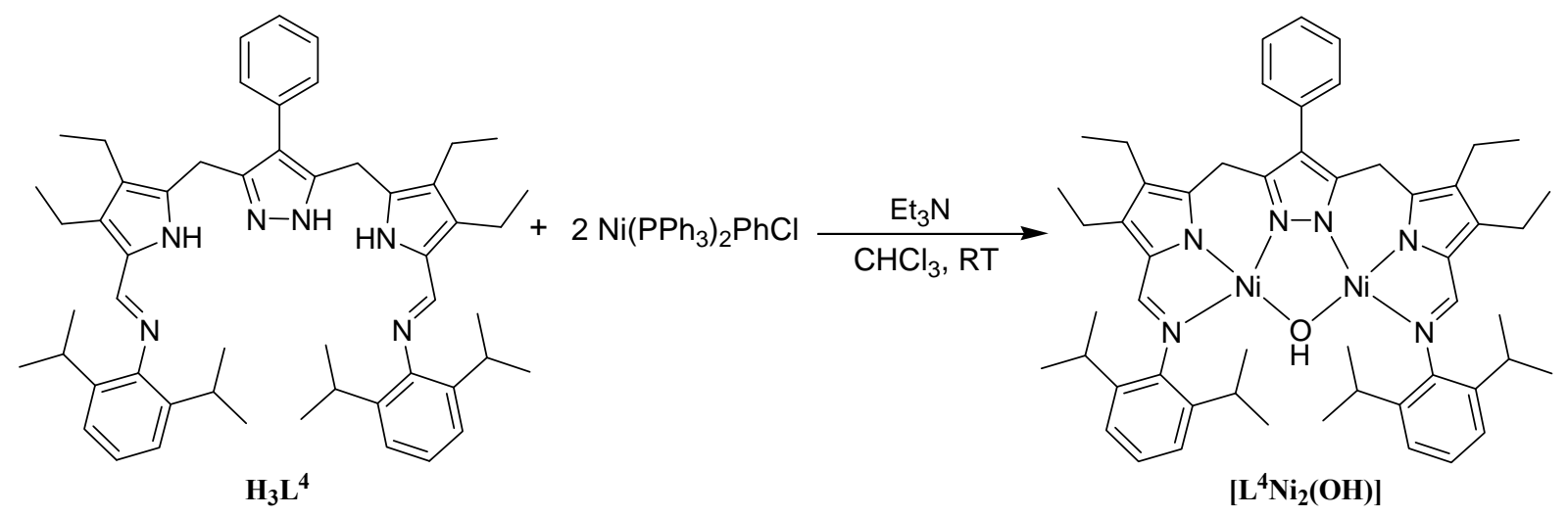

Der Ligand $\left(\mathbf{H}_{3} \mathbf{L}^{4}\right)\left(27 \mathrm{mg}, 0.034 \mathrm{mmol}, 1\right.$ eq.) wurde in $\mathrm{CHCl}_{3}(3 \mathrm{~mL})$ gelöst und mit Triethylamin (4 Tropfen) deprotoniert. Nach $10 \mathrm{Min}$. Rühren wurde $\mathrm{Ni}\left(\mathrm{PPh}_{3}\right)_{2} \mathrm{PhCl}$ (47.5 mg, 0.068 mmol, 2 eq.) dazugegeben und das Reaktionsgemisch 5 Tage bei RT gerührt. Das Lösungsmittel wurde entfernt und das Rohprodukt in $\mathrm{CH}_{2} \mathrm{Cl}_{2}$ aufgenommen und mit $\mathrm{MeCN}$ überschichtet. Der Komplex entstand in Form eines rot-braunen Feststoffes.

\section{Auswertung:}

Summenformel:

$\mathrm{C}_{53} \mathrm{H}_{66} \mathrm{~N}_{6} \mathrm{Ni}_{2} \mathrm{O}$

Molekulargewicht (g/mol):

920.5

HR-MS (ESI(+)) $m / z(\%)$ :

$\left[\left(\left(\mathrm{C}_{53} \mathrm{H}_{65} \mathrm{~N}_{6} \mathrm{Ni}_{2}\right)(\mathrm{OH})-2 \mathrm{H}\right)+\mathrm{H}\right]^{+}$berechnet: 917.39218 , $\left[\left(\left(\mathrm{C}_{53} \mathrm{H}_{65} \mathrm{~N}_{6} \mathrm{Ni}_{2}\right)(\mathrm{OH})-2 \mathrm{H}\right)+\mathrm{H}\right]^{+}$gemessen: 917.39069 ;

$\left[\left(\mathrm{C}_{53} \mathrm{H}_{65} \mathrm{~N}_{6} \mathrm{Ni}_{2}\right)\left(\mathrm{O}_{2}\right)\right]^{+}$berechnet: 933.38542,

$\left[\left(\mathrm{C}_{53} \mathrm{H}_{65} \mathrm{~N}_{6} \mathrm{Ni}_{2}\right)\left(\mathrm{O}_{2}\right)\right]^{+}$gemessen: 933.38700 .

MS (ESI $(+)$; MeCN) $m / z(\%)$ :

919 (100) $\left[\mathrm{C}_{35} \mathrm{H}_{65} \mathrm{Ni}_{2} \mathrm{~N}_{6}(\mathrm{OH})+\mathrm{H}\right]^{+}$.

MS (ESI(-); MeCN) $m / z(\%)$ :

979

$\left[\mathrm{C}_{35} \mathrm{H}_{64} \mathrm{Ni}_{2} \mathrm{~N}_{6}(\mathrm{Ph})\right]^{-}$,

997

$\left[\mathrm{C}_{35} \mathrm{H}_{64} \mathrm{Ni}_{2} \mathrm{~N}_{6}(\mathrm{Ph})+\mathrm{F}\right]^{-}$.

MS (FAB(+); 4-NBA) $m / z(\%)$ :

$919(100) \quad\left[\mathrm{C}_{35} \mathrm{H}_{65} \mathrm{Ni}_{2} \mathrm{~N}_{6}(\mathrm{OH})+\mathrm{H}\right]^{+}, \quad 937$

$\left[\mathrm{C}_{35} \mathrm{H}_{65} \mathrm{Ni}_{2} \mathrm{~N}_{6}(\mathrm{Cl})\right]^{+}$.

IR (KBr):

$\widetilde{v}=3601(\mathrm{w}), 3423$ (br, m), 3054 (w), 2951 (m) 2930

(m), 2869 (w), 2740 (w), 2677 (m), 2492 (w), 1709

(w), 1620 (m), 1569 (s), 1474 (s), 1437 (vs), 1385 (m), $1273(\mathrm{~m}), 1025$ (w), 805 (w) $\mathrm{cm}^{-1}$. 


\subsubsection{Synthese von $\left[\mathrm{L}^{3} \mathrm{Pd}_{2} \mathrm{Cl}\right]$}

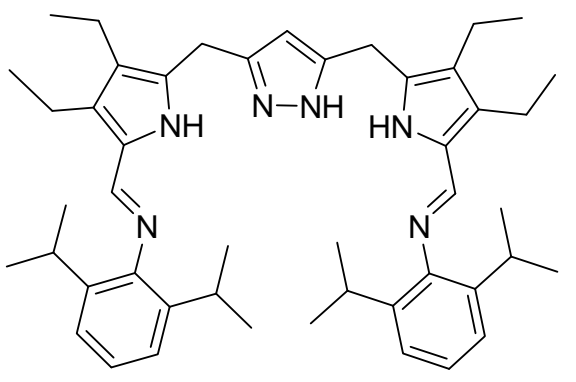

$\mathbf{H}_{3} L^{3}$
1. $\mathrm{Et}_{3} \mathrm{~N}$

2. 2 eq. $\mathrm{PdCl}_{2}(\mathrm{COD})$

$\mathrm{CHCl}_{3} / \mathrm{THF}$

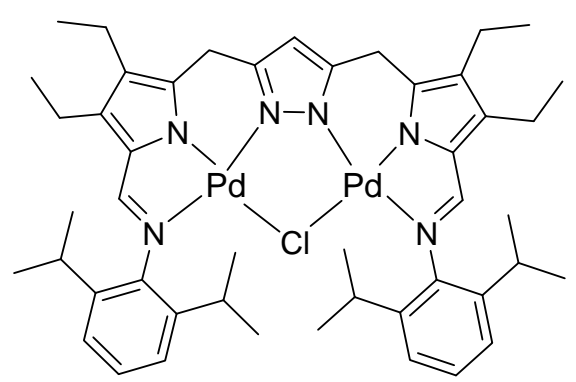

$\left[\mathbf{L}^{3} \mathbf{P d}_{2} \mathbf{C l}\right]$

Der Ligand $\left(\mathbf{H}_{3} \mathbf{L}^{\mathbf{3}}\right)$ (21 mg, $0.03 \mathrm{mmol}, 1$ eq.) wurden in Chloroform (2 mL) gelöst und mit Triethylamin (5 Tropfen) deprotoniert. Anschließend wurde $\mathrm{PdCl}_{2}(\mathrm{COD})$ (16.7 mg, $0.06 \mathrm{mmol}, 2.0$ eq.) in einer Suspension mit THF ( $2 \mathrm{~mL})$ zum Reaktionsgemisch gegeben und 3 d unter Rückfluss bei $50{ }^{\circ} \mathrm{C}$ (Ölbadtemperatur) erwärmt. Das Lösungsmittel wurde entfernt und der Rückstand in Chloroform gelöst und mit $\mathrm{MeCN}$ überschichtet. Nach einigen Tagen wuchsen kleine gelb-orange kubische Kristalle, die röntgenographisch charakterisiert werden konnten $(24.5 \mathrm{mg}, 87 \%)$.

\section{Auswertung:}

Summenformel:

$\mathrm{C}_{47} \mathrm{H}_{61} \mathrm{~N}_{6} \mathrm{ClPd}_{2}$

Molekulargewicht (g/mol):

958.32

${ }^{1} \mathrm{H}-\mathrm{NMR}\left(500 \mathrm{MHz}, \mathrm{CDCl}_{3}\right)$ :

$\delta(\mathrm{ppm})=1.02-1.08\left(\mathrm{~m},{ }^{3} J(\mathrm{H}, \mathrm{H})=7.5 \mathrm{~Hz}, 18 \mathrm{H}, 4 \times\right.$ $\left.\mathrm{CH}_{3}{ }^{i \mathrm{Pr}}, 2 \times \mathrm{CH}_{2} \mathrm{CH}_{3}\right), 1.12-1.16\left(\mathrm{q},{ }^{3} J(\mathrm{H}, \mathrm{H})=7.5 \mathrm{~Hz}\right.$, $\left.18 \mathrm{H}, 4 \times \mathrm{CH}_{3}{ }^{i \mathrm{Pr}}, 2 \times \mathrm{CH}_{2} \mathrm{CH}_{3}\right), 2.44-2.46\left(\mathrm{q},{ }^{3} \mathrm{~J}(\mathrm{H}, \mathrm{H})=\right.$ $\left.7.5 \mathrm{~Hz}, \quad 4 \mathrm{H}, \quad \mathrm{CH}_{2} \mathrm{CH}_{3}\right), \quad 2.58-2.60\left(\mathrm{~m},{ }^{3} \mathrm{~J}(\mathrm{H}, \mathrm{H})=\right.$ $7.5 \mathrm{~Hz}, 4 \mathrm{H}, \mathrm{CH}_{2} \mathrm{CH}_{3}$ ), 3.26-3.29 (sept, ${ }^{3} J(\mathrm{H}, \mathrm{H})=$ $\left.6.80 \mathrm{~Hz}, 4 \mathrm{H},\left(\mathrm{CH}_{3}\right)_{2} \mathrm{CH}\right), 4.29\left(\mathrm{~s}, 4 \mathrm{H}, 2 \times \mathrm{CH}_{2}\right), 6.13$ $\left(\mathrm{s}, 1 \mathrm{H}, \mathrm{C} H^{\mathrm{pz}}\right), 6.92-6.93\left(\mathrm{~d},{ }^{3} J(\mathrm{H}, \mathrm{H})=7.90 \mathrm{~Hz}, 4 \mathrm{H}\right.$, $\left.\mathrm{CH}^{\mathrm{Ph}}\right), 7.05(\mathrm{~s}, 2 \mathrm{H}, 2 \times H \mathrm{C}=\mathrm{N}), 7.07-7.09(\mathrm{~m}$, $\left.{ }^{3} J(\mathrm{H}, \mathrm{H})=7.90 \mathrm{~Hz}, 2 \mathrm{H}, \mathrm{CH} H^{\mathrm{Ph}}\right)$.

${ }^{13} \mathrm{C}-\mathrm{NMR}\left(126 \mathrm{MHz}, \mathrm{CDCl}_{3}\right)$ : $\delta(\mathrm{ppm})=15.5\left(\mathrm{CH}_{3}\right), 17.2\left(\mathrm{CH}_{3}\right), 17.9\left(\mathrm{CH}_{2} \mathrm{CH}_{3}\right)$, $19.1\left(\mathrm{CH}_{2} \mathrm{CH}_{3}\right), 22.9 / 24.8\left(\mathrm{CH}_{3}{ }^{i \mathrm{Pr}}\right), 27.9\left(\mathrm{CH}^{i \mathrm{Pr}}\right), 28.9$ $\left(\mathrm{CH}_{2}{ }^{\mathrm{py} / \mathrm{pz}}\right), 103.7\left(C \mathrm{H}^{\mathrm{pz}}\right), 121.4\left(C^{\mathrm{q}}\right), 122.7\left(C^{\mathrm{Ph}}\right)$, $126.5\left(C \mathrm{H}^{\mathrm{Ph}}\right), 134.4\left(C^{\mathrm{q}}\right), 135.4\left(C^{\mathrm{q}}\right), 137.5\left(C^{\mathrm{q}}\right), 142.9$ $\left(C^{\mathrm{q}}\right), 144.4\left(C^{\mathrm{q}}\right), 144.9\left(C^{\mathrm{q}}\right), 157.2(\mathrm{H} C=\mathrm{N})$. 
$\mathrm{MS}(\mathrm{ESI}) \mathrm{m} / \mathrm{z}(\%)\left(\mathrm{MeOH} / \mathrm{CHCl}_{3}\right): \quad 958(100)[\mathrm{M}+\mathrm{H}]^{+}$.

$\mathrm{MS}\left(\mathrm{FAB}(+)\right.$ in 4-NBA) $m / z(\%): \quad 957(100)[\mathrm{M}+\mathrm{H}]^{+}$.

HRMS-(+)ESI:

berechnet: $[\mathrm{M}+\mathrm{H}]^{+}$958.28136,

(MeOH/Ammoniumacetat) $m / z$ : gemessen: $[\mathrm{M}+\mathrm{H}]^{+}$958.28055.

IR $(\mathrm{KBr})$ : $\widetilde{v}=3446$ (br, w), 3057 (w), 2956 (s), 2925 (w), 2864 (w), 1571 (vs), 1466 (s), 1398 (m), 1319 (w), 1289 (m), 1262 (m), 1230 (s), 1184 (m), 1092 (br; m), 1022 (s), $992(\mathrm{w}), 931(\mathrm{w}), 803(\mathrm{~s}), 766(\mathrm{w}), 728(\mathrm{w}) \mathrm{cm}^{-1}$.

$\mathrm{UV} / \mathrm{Vis}\left(\mathrm{CHCl}_{3}\right)(\mathrm{nm})$ : $\lambda_{\max }\left[\varepsilon \times 10^{4} \mathrm{M}^{-1} \mathrm{~cm}^{-1}\right]=283$ [1.18], 300 [1.13], 327 [1.34], 393 [1.02]. 


\section{Röntgenstrukturdaten}

Die Bestimmung der Elementarzelle und die Sammlung der Meßdaten erfolgte an der Universität Göttingen an einem STOE AED-2 Gerät, einem Vierkreisdiffraktometer mit Offset-Eulerwiege der Firma Huber mit einem Bruker SMART 4K CCD-Zähler mit graphitmonochromatisierter Mo-K $\alpha$-Strahlung $(\lambda=0.711 \AA)$ sowie an einem SMART 6000 CCD Diffraktometer mit $\mathrm{Cu}-\mathrm{K} \alpha$-Strahlung $(\lambda=1.542 \AA)$. Die Meßwerte wurden bezüglich Lorentz- und Polarisationseffekten korrigiert. Zusätzlich erfolgten Absorptionskorrekturen. Die Lösung und Verfeinerung wurde mit den Programmen SHELXS-97 ${ }^{118}$ und SHELXL$97^{119}$ durchgeführt. Bei den Kristalldaten in Kapitel 3.2 und den folgenden Gitterkonstanten sind in Klammern hinter den jeweiligen Werten die Standardabweichungen in Einheiten der letzten Dezimale angegeben. Alle Messungen wurden bei 133(2) K durchgeführt. Kristalloder Röntgenstrukturanalysen wurden von Herrn Dr. Sebastian Dechert vorgenommen. 
Tabelle 23

\begin{tabular}{|c|c|c|c|}
\hline Verbindung & $2 a$ & 2a.TFA & 2b-TFA \\
\hline Interner Name & sk4 & sk8 & sk5 \\
\hline Summenformel & $\mathrm{C}_{33} \mathrm{H}_{46} \mathrm{~N}_{6} \mathrm{O}_{2}$ & $\mathrm{C}_{31} \mathrm{H}_{37} \mathrm{~F}_{3} \mathrm{~N}_{6} \mathrm{O}_{3}$ & $\mathrm{C}_{33} \mathrm{H}_{41} \mathrm{~F}_{3} \mathrm{~N}_{6} \mathrm{O}_{3}$ \\
\hline Molmassse $\left[\mathrm{gmol}^{-1}\right]$ & 558.76 & 598.67 & 626.72 \\
\hline Kristallsystem & Monoklin & Triklin & Triklin \\
\hline Raumgruppe & $P 2 / c$ & $P \overline{1}$ & $P \overline{1}$ \\
\hline $\mathrm{a}[\AA]$ & $19.8688(8)$ & $8.8877(7)$ & $9.2223(9)$ \\
\hline $\mathrm{b}[\AA]$ & $7.9486(5)$ & $11.7586(9)$ & $12.0717(12)$ \\
\hline $\mathrm{c}[\AA]$ & $21.8773(8)$ & $14.9846(12)$ & $15.0175(15)$ \\
\hline$\alpha\left[^{\circ}\right]$ & 90 & $111.537(6)$ & $66.062(7)$ \\
\hline$\beta\left[^{\circ}\right]$ & 111.26 & $91.417(6)$ & $87.286(8)$ \\
\hline$\gamma\left[{ }^{\circ}\right]$ & 90 & $94.439(6)$ & $83.853(8)$ \\
\hline Volumen $\left[\AA^{3}\right]$ & $3219.8(3)$ & $1449.9(2)$ & $1519.3(3)$ \\
\hline Z & 4 & 2 & 2 \\
\hline$\rho_{\text {ber. }}\left[\mathrm{Mg} / \mathrm{m}^{3}\right]$ & 1.153 & 1.371 & 1.370 \\
\hline$\mu(\mathrm{Mo}-\mathrm{K} \alpha)\left[\mathrm{mm}^{-1}\right]$ & 0.073 & 0.103 & 0.102 \\
\hline$F(000)$ & 1208 & 632 & 664 \\
\hline$\theta$ Bereich $\left[{ }^{\circ}\right]$ & $1.90-24.88$ & $1.46-24.85$ & $1.48-24.84$ \\
\hline$h k l$ Berech & $\pm 23, \pm 9, \pm 25$ & $\pm 10, \pm 13,-15-17$ & $\pm 10, \pm 14,-17-16$ \\
\hline Gemessene Reflexe & 62808 & 27128 & 15739 \\
\hline Unabhängige Reflexe $\left(R_{\text {int }}\right)$ & $5588(0.0979)$ & $4999(0.0914)$ & $5210(0.0754)$ \\
\hline $\mathrm{T}_{\max } / \mathrm{T}_{\min }$ & - & $0.9717 / 0.7983$ & $0.9797 / 0.8444$ \\
\hline Daten / Restraints / Parameter & $5588 / 0 / 416$ & 4999 / 0 / 426 & $5210 / 3 / 436$ \\
\hline Goodness-of-fit & 1.006 & 1.033 & 1.017 \\
\hline \multirow[t]{2}{*}{$R_{1}[I>2 \sigma(I)]$} & $R_{1}=0.0506$ & $R_{1}=0.0533$ & $R_{1}=0.0617$ \\
\hline & $\mathrm{w} R_{2}=0.1142$ & $\mathrm{w} R_{2}=0.1194$ & $\mathrm{w} R_{2}=0.1324$ \\
\hline \multirow[t]{2}{*}{$R_{\text {ind. (alle Daten) }}$} & $R_{1}=0.0825$ & $R_{1}=0.0894$ & $R_{1}=0.1121$ \\
\hline & $\mathrm{w} R_{2}=0.1263$ & $\mathrm{w} R_{2}=0.1339$ & $\mathrm{w} R_{2}=0.1531$ \\
\hline Restelektrondichte $\left[\mathrm{e} \AA^{-3}\right]$ & $0.267 /-0.265$ & $0.276 /-0.318$ & $0.399 /-0.335$ \\
\hline
\end{tabular}


Tabelle 24

\begin{tabular}{|c|c|c|c|}
\hline Verbindung & $2 c \cdot T F A$ & {$\left[\mathbf{L}_{2}^{6} \mathrm{Cu}_{2}(\mathrm{OAc})_{4}\right]$} & {$\left[\mathrm{L}^{1}{ }_{0 \mathrm{x}} \mathrm{Cu}_{2}(\mathrm{OH})\right]$} \\
\hline Interner Name & sk6 & sk10 & sk11 \\
\hline Summenformel & $\mathrm{C}_{35} \mathrm{H}_{39} \mathrm{~F}_{3} \mathrm{~N}_{6} \mathrm{O}_{3}$ & $\mathrm{C}_{26} \mathrm{H}_{36} \mathrm{Cu}_{2} \mathrm{~F}_{6} \mathrm{~N}_{6} \mathrm{O}_{11}$ & $\mathrm{C}_{35} \mathrm{H}_{34} \mathrm{Cu}_{2} \mathrm{~N}_{6} \mathrm{O}_{3}$ \\
\hline Molmassse $\left[\mathrm{gmol}^{-1}\right]$ & 648.72 & 849.69 & 713.76 \\
\hline Kristallsystem & Triklin & Triklin & Monoklin \\
\hline Raumgruppe & $P \overline{1}$ & $P \overline{1}$ & $C 2 / c$ \\
\hline $\mathrm{a}[\AA]$ & $9.2295(16)$ & $7.9772(9)$ & $27.0441(16)$ \\
\hline $\mathrm{b}[\AA]$ & $11.894(2)$ & $10.1534(12)$ & $13.3370(12)$ \\
\hline $\mathrm{c}[\AA]$ & $14.983(3)$ & $11.8106(16)$ & $8.9087(6)$ \\
\hline$\alpha\left[^{\circ}\right]$ & $104.052(13)$ & $67.885(9)$ & 90 \\
\hline$\beta\left[^{\circ}\right]$ & $98.876(13)$ & $70.561(10)$ & $106.492(5)$ \\
\hline$\gamma\left[{ }^{\circ}\right]$ & $91.087(14)$ & $79.389(9)$ & 90 \\
\hline Volumen $\left[\AA^{3}\right]$ & $1573.8(5)$ & $833.81(18)$ & $3081.1(4)$ \\
\hline $\mathrm{Z}$ & 2 & 1 & 4 \\
\hline$\rho_{\text {ber. }}\left[\mathrm{Mg} / \mathrm{m}^{3}\right]$ & 1.369 & 1.692 & 1.539 \\
\hline$\mu(\mathrm{Mo}-\mathrm{K} \alpha)\left[\mathrm{mm}^{-1}\right]$ & 0.101 & 0.1375 & 1.428 \\
\hline$F(000)$ & 684 & 434 & 1472 \\
\hline$\theta$ Bereich $\left[{ }^{\circ}\right]$ & $1.77-24.83$ & $2.17-24.84$ & $1.57-24.70$ \\
\hline$h k l$ Bereich & $\pm 10,-14-13,-17-14$ & $\pm 9,-11-10, \pm 13$ & $\pm 31, \pm 15, \pm 10$ \\
\hline Gemessene Reflexe & 11129 & 7555 & 22786 \\
\hline Unabhängige Reflexe $\left(R_{\text {int }}\right)$ & $5082(0.1100)$ & $2701(0.0581)$ & $2632(0.0620)$ \\
\hline $\mathrm{T}_{\max } / \mathrm{T}_{\min }$ & $0.9797 / 0.8444$ & $0.8042 / 0.6456$ & $0.7685 / 0.6431$ \\
\hline Daten / Restraints / Parameter & $5082 / 38 / 462$ & $2701 / 0 / 235$ & $2632 / 4 / 264$ \\
\hline Goodness-of-fit & 1.006 & 1.013 & 1.005 \\
\hline \multirow[t]{2}{*}{$R_{1}[I>2 \sigma(I)]$} & $R_{1}=0.0716$ & $R_{1}=0.0382$ & $R_{1}=0.0331$ \\
\hline & $\mathrm{w} R_{2}=0.1102$ & $\mathrm{w} R_{2}=0.0868$ & $\mathrm{w} R_{2}=0.0784$ \\
\hline \multirow[t]{2}{*}{$R_{\text {ind. }}$ (alle Daten) } & $R_{1}=0.1642$ & $R_{1}=0.0528$ & $R_{1}=0.0497$ \\
\hline & $\mathrm{w} R_{2}=0.1348$ & $\mathrm{w} R_{2}=0.0911$ & $\mathrm{w} R_{2}=0.0830$ \\
\hline Restelektrondichte $\left[\mathrm{e} \AA^{-3}\right]$ & $0.249 /-0.221$ & $0.413 /-0.385$ & $0.382 /-0.272$ \\
\hline
\end{tabular}


Tabelle 25

\begin{tabular}{|c|c|c|c|}
\hline Verbindung & {$\left[\mathrm{L}^{11} \mathrm{Co}_{2} \mathrm{Cl}\left(\mathrm{CH}_{3} \mathrm{OH}\right)_{2}\right]$} & {$\left[\mathbf{L}_{3} \mathrm{Cu}_{2}(\mathrm{OH})\right]$} & {$\left[\mathbf{L}^{1} \mathbf{N i}_{2}(\mathrm{OH})\right]$} \\
\hline Interner Name & sk13 & sk18 & sk15 \\
\hline Summenformel & $\mathrm{C}_{29} \mathrm{H}_{46} \mathrm{C}_{12} \mathrm{Co}_{2} \mathrm{~N}_{8} \mathrm{O}_{11}$ & $\mathrm{C}_{48} \mathrm{H}_{64} \mathrm{Cl}_{2} \mathrm{Cu}_{2} \mathrm{~N}_{6} \mathrm{O}$ & $\mathrm{C}_{35} \mathrm{H}_{38} \mathrm{~N}_{6} \mathrm{Ni}_{2} \mathrm{O}$ \\
\hline Molmassse $\left[\mathrm{gmol}^{-1}\right]$ & 871.50 & 939.03 & 676.13 \\
\hline Kristallsystem & Monoklin & Triklin & Monoklin \\
\hline Raumgruppe & $C 2 / c$ & $P \overline{1}$ & $P 2 / c$ \\
\hline $\mathrm{a}[\AA]$ & $28.480(3)$ & $11.0255(5)$ & $11.3637(9)$ \\
\hline $\mathrm{b}[\AA]$ & $8.7919(5)$ & $15.1061(6)$ & $18.2132(10)$ \\
\hline $\mathrm{c}[\AA \AA]$ & $16.2926(15)$ & $15.5401(6)$ & $15.2535(11)$ \\
\hline$\alpha\left[^{\circ}\right]$ & 90 & $65.519(3)$ & 90 \\
\hline$\beta\left[^{\circ}\right]$ & $120.115(7)$ & $78.161(3)$ & $99.357(6)$ \\
\hline$\gamma\left[{ }^{\circ}\right]$ & 90 & $80.000(3)$ & 90 \\
\hline Volumen $\left[\AA^{3}\right]$ & $3528.9(5)$ & $2294.01(16)$ & $3115.0(4)$ \\
\hline Z & 4 & 2 & 4 \\
\hline$\rho_{\text {ber. }}\left[\mathrm{Mg} / \mathrm{m}^{3}\right]$ & 1.640 & 1.359 & 1.442 \\
\hline$\mu(\mathrm{Mo}-\mathrm{K} \alpha)\left[\mathrm{mm}^{-1}\right]$ & 1.162 & 1.086 & 1.248 \\
\hline$F(000)$ & 1808 & 988 & 1416 \\
\hline$\theta$ Bereich $\left[{ }^{\circ}\right]$ & $1.65-24.72$ & $1.62-27.32$ & $1.76-24.67$ \\
\hline$h k l$ Bereich & $-31-32, \pm 10,-18-19$ & $\pm 14, \pm 19,(-19)-20$ & $\pm 13, \pm 21, \pm 17$ \\
\hline Gemessene Reflexe & 10034 & 62615 & 31983 \\
\hline Unabhängige Reflexe $\left(R_{\text {int }}\right)$ & $2843(0.0495)$ & $10269(0.0366)$ & $5248(0.0884)$ \\
\hline $\mathrm{T}_{\max } / \mathrm{T}_{\min }$ & $0.9276 / 0.8227$ & $0.6476 / 0.5744$ & $0.8607 / 0.7866$ \\
\hline Daten / Restraints / Parameter & $2843 / 13 / 267$ & $10269 / 8 / 576$ & $5248 / 1 / 405$ \\
\hline Goodness-of-fit & 1.060 & 1.070 & 1.060 \\
\hline$R_{1}[I>2 \sigma(I)]$ & $R_{1}=0.0423$ & $R_{1}=0.0330$ & $R_{1}=0.0475$ \\
\hline \multirow{3}{*}{$R_{\text {ind. }}($ alle Daten $)$} & $\mathrm{w} R_{2}=0.0967$ & $\mathrm{w} R_{2}=0.0880$ & $\mathrm{w} R_{2}=0.0801$ \\
\hline & $R_{1}=0.0607$ & $R_{1}=0.0376$ & $R_{1}=0.0855$ \\
\hline & $\mathrm{w} R_{2}=0.1047$ & $\mathrm{w} R_{2}=0.0902$ & $\mathrm{w} R_{2}=0.0894$ \\
\hline Restelektrondichte $\left[\mathrm{e} \AA^{-3}\right]$ & $0.926 /-0.542$ & $0.509 /-0.702$ & $0.273 /-0.245$ \\
\hline
\end{tabular}


Tabelle 26

\begin{tabular}{|c|c|c|}
\hline Verbindung & {$\left[\mathbf{L}^{3} \mathbf{N i}_{2}(\mathbf{O H})\right]$} & {$\left[\mathbf{L}^{3} \mathbf{P d}_{2} \mathbf{C l}\right]$} \\
\hline Interner Name & sk14 & sk17 \\
\hline Summenformel & $\mathrm{C}_{47} \mathrm{H}_{62} \mathrm{~N}_{6} \mathrm{Ni}_{2} \mathrm{O}$ & $\mathrm{C}_{48} \mathrm{H}_{63} \mathrm{Cl}_{3} \mathrm{~N}_{6} \mathrm{Pd}_{2}$ \\
\hline Molmassse $\left[\mathrm{gmol}^{-1}\right]$ & 844.45 & 1043.19 \\
\hline Kristallsystem & Monoklin & Triklin \\
\hline Raumgruppe & $P 2 / n$ & $P \overline{1}$ \\
\hline $\mathrm{a}[\AA]$ & $14.7609(4)$ & $11.9896(5)$ \\
\hline $\mathrm{b}[\AA]$ & $16.2459(3)$ & $14.8987(6)$ \\
\hline $\mathrm{c}[\AA]$ & $35.8641(10)$ & $15.7262(7)$ \\
\hline$\alpha\left[^{\circ}\right]$ & 90 & $113.058(3)$ \\
\hline$\beta\left[^{\circ}\right]$ & $92.938(2)$ & $96.510(3)$ \\
\hline$\gamma\left[^{\circ}\right]$ & 90 & $107.441(3)$ \\
\hline Volumen $\left[\AA^{3}\right]$ & $8589.1(4)$ & $2379.17(17)$ \\
\hline $\mathrm{Z}$ & 8 & 2 \\
\hline$\rho_{\text {ber. }}\left[\mathrm{Mg} / \mathrm{m}^{3}\right]$ & 1.306 & 1.456 \\
\hline$\mu(\mathrm{Mo}-\mathrm{K} \alpha)\left[\mathrm{mm}^{-1}\right]$ & 0.919 & 0.964 \\
\hline$F(000)$ & 3600 & 1072 \\
\hline$\theta$ Bereich $\left[{ }^{\circ}\right]$ & $1.14-24.86$ & $1.60-27.34$ \\
\hline$h k l$ Bereich & $\pm 17,-19-17, \pm 42$ & $\pm 15, \pm 19,-19-20$ \\
\hline Gemessene Reflexe & 128210 & 52565 \\
\hline Unabhängige Reflexe $\left(R_{\text {int }}\right)$ & $14813(0.0551)$ & $10698(0.0341)$ \\
\hline $\mathrm{T}_{\max } / \mathrm{T}_{\min }$ & $0.9046 / 0.6439$ & $0.8556 / 0.7495$ \\
\hline Daten / Restraints / Parameter & $14813 / 18$ / 1095 & $10698 / 13 / 589$ \\
\hline Goodness-of-fit & 1.013 & 1.040 \\
\hline$R_{1}[I>2 \sigma(I)]$ & $\begin{array}{l}R_{1}=0.0292 \\
\mathrm{w} R_{2}=0.0705\end{array}$ & $\begin{array}{l}R_{1}=0.0250, \\
\mathrm{w} R_{2}=0.0627\end{array}$ \\
\hline$R_{\text {ind. }}$ (alle Daten) & $\begin{array}{l}R_{1}=0.0427 \\
\mathrm{w} R_{2}=0.0737\end{array}$ & $\begin{array}{l}R_{1}=0.0329 \\
\mathrm{w} R_{2}=0.0645\end{array}$ \\
\hline Restelektrondichte $\left[\mathrm{e}^{-3}\right]$ & $0.402 /-0.325$ & $0.607 /-0.574$ \\
\hline
\end{tabular}




\section{Verzeichnis der verwendeten Abkürzungen}

$\AA$

Abb.

Abs.

Ar

B

$n$-BuLi

${ }^{\circ} \mathrm{C}$

COD

$\mathrm{CV}$

$\mathrm{CH}_{2} \mathrm{Cl}_{2}$

$\delta$

DC

DFA

DMAP

DME

DMSO

DHP

DBU

E

EI

Et

EtOH

ESI

ESR bzw. EPR

$\mathrm{Et}_{2} \mathrm{O}$

$\varepsilon$

eq.

FAB

G

$\mathrm{h}$

HRMS

$\mathrm{Hz}$
Ångstrøm $\left(10^{-10} \mathrm{~m}\right)$

Abbildung

Absorption

Aryl

Feld

n-Butyllithium

Grad Celsius

Zyklooktadien

Cyklovoltametrie

Dichlormethan

Chemische Verschiebung

Dünnschichtchromatographie

Dodekafluorodicarbonsäure

Dimethylaminopyridin

Dimethoxyethan

Dimethylsulfoxid

3,4-Dihydro-2H-pyran

1,8-Diazabicyclo-[5.4.0]undec-7-en

Energie

Elektronenionisation

Ethyl

Ethanol

Elektronensprayionisation

Elektronen-Spin-Resonanz bzw.

Electron Paramagnetic Resonance

Diethylether

Extinktionskoeffizient $\left[\mathrm{mol} \cdot \mathrm{l}^{-1} \cdot \mathrm{cm}^{-1}\right]$

Äquivalente

Fast Atom Bombardment

Gauß

Stunde

High Resolution Mass Spectroscopy

(Hochauflösende Massenspektroskopie)

Hertz 


\begin{tabular}{|c|c|}
\hline$i \operatorname{Pr}$ & Isopropyl \\
\hline IR & Infrarot \\
\hline$J$ & Kopplungskonstante \\
\hline Kat. & Katalysator \\
\hline $\mathrm{KO}^{t} \mathrm{Bu}$ & Kalium-tert-butylat \\
\hline $\mathrm{L}$ & Ligand \\
\hline$\lambda$ & Wellenlänge [nm] \\
\hline $\mathrm{M}^{+}$ & Molekülkation \\
\hline $\mathrm{Me}$ & Methyl \\
\hline $\mathrm{MeCN}$ & Acetonitril \\
\hline $\mathrm{MeOH}$ & Methanol \\
\hline Min & Minute \\
\hline MS & Massenspektrometrie \\
\hline $\mathrm{m} / \mathrm{z}$ & Masse/Ladungs-Verhältnis \\
\hline $\mathrm{NiBr}_{2} \cdot \mathrm{dme}$ & Nickelbromidethylenglycoldimethylether \\
\hline $\mathrm{nm}$ & Nano Meter \\
\hline \multirow[t]{2}{*}{ NMR } & Nuclear Magnetic Resonance \\
\hline & (Kernresonanzspektoskopie) \\
\hline NOESY & Nuclear Overhauser Enhancement Spectroscopy \\
\hline$\tilde{v}$ & Wellenzahl \\
\hline$v$ & Frequenz \\
\hline Nibeol oder 4-NBA & 4-Nitrobenzylalkohol \\
\hline PFP & Pentafluorophenol \\
\hline $\mathrm{Ph}$ & Phenyl \\
\hline ppm & parts per million \\
\hline py & Pyrrolyl \\
\hline $\mathrm{pz}$ & Pyrazolyl \\
\hline$p$-TSA & para-Toluolsulfonsäure \\
\hline $\mathrm{R}$ & Rest \\
\hline$S$ & Spin \\
\hline SCE & Satured Calomel Electrode \\
\hline $\sec$ & Sekunde \\
\hline $\operatorname{sh}$ & Schulter \\
\hline TCA & Trichloressigsäure \\
\hline TFA & Trifluoressigsäure \\
\hline THF & Tetrahydrofuran \\
\hline $\mathrm{UV} / \mathrm{Vis}$ & Ultraviolett/Visible \\
\hline
\end{tabular}




\section{Literaturverzeichnis}

\section{Literaturverzeichnis}

${ }^{1}$ L. R. Milgrom, The Colors of Life, Oxford, Oxford University Press, 1997.

${ }^{2}$ I. Bertini, H. B. Gray, E. I. Stiefel, J. S. Valentine, Biological Inorganic Chemistry, Structure \& Reactivity, University Science Books, Sausalito, California, 2007.

${ }^{3}$ W. Kaim, B. Schwederski, Bioarganische Chemie, Teubner Studienbücher, Stuttgart, 1991.

4 a) Y.-H. Liang, X.-Z. Liu, S.-H. Liu, G.-Y. Lu, Acta. Cryst. 2001, D57, 1850-1856.

b) www.pcsb.org/pdp/explore.do?structureld=1FAW.

5 a) D. Horigome, H. Satoh, N. Itoh, K. Mitsunaga, I. Oonishi, A. Nakagawa, A. Uchida, J. Bio. Chem. 2007, 9 , 6525-6531. b) www.rcsb.org/pdp/explore.do?structureld=2DRE.

${ }^{6} \mathrm{http}: / /$ www.img.bio.uni-goettingen.de/mswww/teaching/Oli/Biochemie\%20der\%20Metalloproteine/BdM2006\%20-\%2005\%20Reaktionszentren\%20$\% 20$ Handout.pdf.

${ }^{7}$ P. Marek, T. S. Balaban, Nachrichten aus der Chemie, Biomimetische Solarzellen, 11/2006, 1072-1077.

${ }^{8}$ M. Linke-Schaetzel, A. D. Bhise, H. Gliemann, T. Koch, T. Schimmel, T. S. Balaban, Thin Solid Films, 2004, 451-452, 16-21.

${ }^{9}$ H. Bresser; www.drbresser.de/cms/PDT.461.0.html.

${ }^{10}$ J. L. Sessler, E. Tomat, Acc. Chem. Res. 2007, 40, 371-379.

${ }^{11}$ A. Gorski, T. Köhler, D. Seidel, J. Tae Lee, G. Orzanowska, J. L. Sessler, J. Waluk, Chem. Eur. J. 2005, 11, 4179-4184.

${ }^{12}$ Y. Kamimura, S. Shimizu, A. Osuka, Chem. Eur. J. 2007, 13, 1620-1628.

${ }^{13}$ M. Suzuki, A. Osuka, Chem. Eur. J. 2007, 13, 196-202.

${ }^{14}$ S. Mori, A. Osuka, J. Am. Chem. Soc. 2005, 127, 8030-8031.

15 J. L. Sessler, S. Camiolo, P. A. Gale, Coord. Chem. Rev. 2003, 240, 17-55.

${ }^{16}$ G. Cafeo, F. H. Kohnke, A. J. P. White, D. Garozzo, A. Messina, Chem. Eur. J. 2007, 13, 649-656.

${ }^{17}$ M. M. Linn, D. C. Poncio, V. G. Machado, Tetrahedron Lett. 2007, 48, 4547-4551.

${ }^{18}$ V. J. Bauer, D. L. J. Clive, D. Dolphin, J. B. Paine, F. L. Harris, M. M. King, J. Loder, S.-W. C. Wang, R. B. Woodward, J. Am. Chem. Soc. 1983, 105, 6429-6436.

${ }^{19}$ a) J. L. Sessler, G. Hemmi, T. D. Mody, T. Murai, A. Burrell, S. W. Young, Acc. Chem. Res. 1994, 27, 43. b)

S. Hannah, V. M. Lynch, N. Gerasimchuk, D. Magda, J. L. Sessler, Org. Lett. 2001, 3, 3911-3914.

${ }^{20}$ J. L. Sessler, R. A. Miller, Biochem. Pharmacol. 2000, 59, 733-739.

${ }^{21}$ J. L. Sessler, D. Seidel, Angew. Chem. 2003, 115, 5292-5333.

22 J. L. Sessler, T. Morishima, V. Lynch, Angew. Chem. 1991, 103,1018-1020; Angew. Chem. Ed. Engl. 1991, 30, 977-980.

${ }^{23}$ R. Charrière, T. A. Jenny, H. Rexhausen, A. Gossauer, Heterocycles 1993, 36, 1561-1575.

${ }^{24}$ A. Srinivasan, T. Ishizuka, A. Osuka, H. Furuta, J. Am. Chem. Soc. 2003, 125, 878-879.

${ }^{25}$ A. Srinivasan, S. Mahajan, S. K. Pushpan, M. Ravikumar, T. K. Chandrashekar, Tetrahedron Lett. 1998, 39, 1961-1964.

${ }^{26}$ T. D. Mody, J. L. Sessler, J. Porphyrins Phthalocyanines, 2001, 5, 134-142. 


\section{Literaturverzeichnis}

${ }^{27}$ A) F. V. Acholla, K. B. Mertes, Tetrahedron Lett. 1984, 25, 3269-3270. b) F. V. Acholla, F. Takusagawa, K. B. Mertes, J. Am. Chem. Soc. 1985, 24, 6902-6908.

${ }^{28}$ a) N. N. Gerasimchuk, A. Gerges, T. Clifford, A. Danby, K. Bowman-James, Inorg. Chem. 1999, 38, 56335636; b) W. A. Reiter, A. Gerges, S. Lee, T. Deffo, T. Clifford, A. Danby, K. Bowman-James, Coord. Chem. Rev. 1998, 174, 343-359.

${ }^{29}$ G. Givaja, A. J. Blake, C. Wilson, M. Schröder, J. B. Love, Chem. Commun. 2003, 2508-2509.

${ }^{30}$ G. Givaja, M. Volpe, J. W. Leeland, M. A. Edwards, T. K. Young, S. B. Darby, S. D. Reid, A. J. Blake, C. Wilson, J. Wolowska, E. J. L. McInnes, M. Schröder, J. B. Love, Chem. Eur. J. 2007, 12, 3707-3723.

${ }^{31}$ C.-H. Lee, K. T. Oh, Tetrahedron Lett. 1999, 40, 1921-1924.

${ }^{32}$ D.-H. Won, C.-H. Lee, Tetrahedron Lett. 2001, 42, 1969-1972.

${ }^{33}$ T. G. Schenck, J. M. Downes, C. R. C. Milne, P. B. MacKenzie, H. Boucher, J. Whelan, B. Bosnich, Inorg. Chem. 1985, 24, 2334-2337.

${ }^{34}$ T. G. Schenck, C. R. Milne, J. F. Sawyer, B. Bosnich, Inorg. Chem. 1985, 24, 2338-2344.

35 a) B. Bauer-Siebenlist, Dissertation, Georg-August-Universität Göttingen, 2004. b) J. Ackermann, Dissertation, Georg-August-Universität Göttingen, 2003.

${ }^{36}$ J. Ackermann, F. Meyer, E. Kaifer, H. Pritzkow, Chem. Eur. J. 2002, 8, 247-258.

${ }^{37}$ F. Meyer, Eur. J. Inorg. Chem. 2006, 3789-3802.

${ }^{38}$ E. Lind, MSc.Thesis, Michigan State University, East Lansing, MI, USA, 1987.

39 a) M. Kumar, V. J. Arán, P. Navarro, Tetrahedron Lett. 1993, 19, 3159-3162. b) M. Kumar, V. J. Arán, P. Navarro, Tetrahedron Lett. 1995, 12, 2161-2164.

${ }^{40}$ C. Miranda, F. Escartí, L. Lamarque, E. Garcia-España, P. Navarro, J. Latorre, F. Lloret, H. R. Jiménez, M. J. R. Yunta, Eur. J. Inorg. Chem. 2005, 189-208.

${ }^{41}$ V. J. Arán, M. Kumar, J. Molina, L. Lamarque, P. Navarro, E. García-España, J. A. Ramírez, S. V. Luis, B. Escuder, J. Org. Chem. 1999, 64, 6135-6145.

${ }^{42}$ C. Acerete, J. M. Bueno, L. Campayo, P. Navarro, M. I. Rodriguez-Franco, Tetrahedron 1994, 50, 4765-4774.

${ }^{43}$ D. J. de Geest, A. Noble, B. Moubaraki, K. S. Murray, D. S. Larsen, S. Brooker, Dalton Trans. 2007, 467-475.

${ }^{44}$ N. E. Borisova, M. D. Reshetova, Y. A. Ustynyuk, Chem. Rev. 2007, 107, 46-79.

${ }^{45}$ E. Buchner, C. Heide, Ber. Dtsch. Chem. Ges. 1902, 35, 31-34.

46 A. Sachse, Institut für Anorganische Chemie, Georg-August-Universität, Göttingen, unveröffentlichte Arbeiten.

${ }^{47}$ G. D. Hartman, L. M. Weinstock, Org. Synth., Coll. Vol VI 1988, 620.

${ }^{48}$ J. L. Sessler, A. Mozaffari, M. R. Johnson, Org. Synth. 1992, 70, 68-78.

49 a) A. D. Adler, F. R. Longo, J. D. Finarelli, J. Goldmacher, J. Assour, L. Korsakoff, J. Org. Chem. 1967, 2 , 476-476. b) K. S. Chamberlain, E. LeGoff, Heterocycles, 1979, 12, 1567-1570.

${ }^{50}$ G.P. Arsenault, E. Bullock, S.F. MacDaonald, J. Am. Chem. Soc. 1960, 82, 4384-4389. b) R. B. Woodward, Angew. Chem. 1960, 72, 651-662.

${ }^{51}$ S. Katsiaouni, Diplomarbeit, Georg-August-Universität Göttingen, Germany, 2004.

${ }^{52}$ P. D. Rao, S. Dhanalekshmi, B. J. Littler, J. S. Lindsey, J. Org. Chem. 2000, 65, 7323-7344.

${ }^{53}$ K. C. Nicolau, J. N. C. Mathison, T. Montagnon, J. Am. Chem Soc. 2004, 126, 5192-5201. 


\section{Literaturverzeichnis}

${ }^{54}$ M. Suzuki, M.C. Yoon, D. Y. Kim, J. H. Kwon, H. Furuta, D. Kim, A. Osuka, Chem. Eur. J. 2006, 12, 17541759.

${ }^{55}$ P. Piątek, V. M. Lynch, J. L. Sessler, J. Am. Chem. Soc. 2004, 126, 16073-16076.

${ }^{56}$ C. Brückner, R.W. Boyle, J. Posakony, B. James, D. Dolphin, Org. Synth. 1999, 76, 287-293.

${ }^{57}$ C. Brückner, V. Karunaratne, S. J. Retting, D. Dolphin, Can. J. Chem. 1996, 74, 2182-2193.

${ }^{58}$ T. Kamiusuki, H. Okawa, H. Inoue, N. Matsumoto, M. Kodera, S. Kida, J. Coord. Chem. 1991, 23, $201-211$.

${ }^{59}$ M. Kumar, V. J. Arán, P. Navarro, Tetrahedron Lett. 1993, 34, 3159-3164.

${ }^{60}$ V. J. Arán, M. Kumar, J. Molina, L. Lamarque, P. Navarro, E. García-España, J. A. Ramírez, S. V. Luis, B. Escuder, J. Org. Chem. 1999, 64, 6135-6145.

${ }^{61}$ a) G. Jung, S. U. Son, K. H. Park, K.-C. Chung, J. W. Lee, Y. K. Chung, Organometallics 2003, 22, 47154720. b) S. U. Son, K.-C. Chung, H.-Y. Jang, I. S. Lee, Y. K. Chung, Organometallics 1998, 17, 3236-3239.

${ }^{62}$ E. J.T. Chrystal, L. Couper, D. J. Robins, Tetrahedron, Vol. 51, 1995, 37, 10241-10252.

${ }^{63}$ C. Reichert, A. Blum, K. Harms, G. Schäfer, Liebigs Ann./Recueil 1997, 707-720.

${ }^{64}$ V. Chiroli, F. Benedini, E. Ongini, P. Del Soldato, Eur. J. Med. Chem. 2003, 38, 441-446.

${ }^{65}$ G. Lee, M. Oka, H. Takemura, Y. Miyahara, N. Shimizu, T. Inazu, J. Org. Chem. Vol. 61, 1996, 23, 83048306.

${ }^{66}$ P. M. Dominiak, A. Makal, P. R. Mallinson, K. Trzcinska, J. Eilmes, E. Grech, M. Chruszcz, W. Minor, K. Wozniak, Chem. Eur. J 2006, 12, 1941-1949.

${ }^{67}$ J. L. Sessler, M. R. Johnson, V. Lynch, J. Org. Chem. 1987, 52, 4394-4397.

${ }^{68}$ Y. Tian, J. Tong, G. Frenzen, J.-Y. Sun, J. Org. Chem. 1999, 64, 1442-1446.

${ }^{69}$ E. P. Serjeant, B. Dempsey, Ionisation constants of organic acids in aqueous solution, Pergamon Press, Oxford, 1979.

${ }^{70}$ V. I. Galkin, R. D. Sayakov, A. R. Garifzyanov, R. A. Cherkasov, A. N. Pudovik, Dokl. Akad. Nauk SSSR 1991, 318, 114-116.

${ }^{71}$ P. W. Atkins, Physikalische Chemie, VCH Verlangsgesellschaft mbH, Weinheim, 1990, 869.

${ }^{72}$ K. Bernhard, J. Geimer, M. Canle-Lopez, J. Reynisson, D. Beckert, R. Gleiter, S. Steenken, Chem. Eur. J. 2001, 21, 4640-4650.

${ }^{73}$ P. A. Gale, J. L. Sessler, V. Kra'1, V. Lynch, J. Am. Chem. Soc. 1996, 118, 5140-5141.

${ }^{74}$ P. D. Beer, P. A. Gale, Angew. Chem. Int. Ed. 2001, 40, 486-516.

${ }^{75}$ M. Shionoya, H. Furuta, V. Lynch, A. Harriman, J. L. Sessler, J. Am. Chem. Soc. 1992, 114, 5714-5722.

${ }^{76}$ Calculated using Advanced Chemistry Development (ACD/Labs) Software V8.14 for Solaris (C) 1994-2007 $\mathrm{ACD} / \mathrm{Labs})$.

77 a) J. L. Hughey IV, S. Knapp, H. Schugar, Synthesis, 1980, 489-490. b) H. Firouzababi, E. Ghaderi, Tetrahedron Lett. 1978, 839-840. c) H. I. Schlesinger, H. B. Siems, J. Am. Chem. Soc. 1924, 46, 1965-1978.

${ }^{78}$ J. Elguero, in Comprehensive Heterocyclic Chemistry, ed. K. T. Potts, Pergamon Press, Oxford, 1st edn, 1984, Vol. 5, 167-303.

${ }^{79}$ T. Ueno, Y. Urano, H. Kojima, T. Nagano, J. Am. Chem. Soc. 2006, 128, 10640-10641.

${ }^{80}$ H. Maeda, Y. Ito, Y. Kusunose, T. Nakanishi, Chem. Commun. 2007, 1136-1138.

${ }^{81}$ P. Plitt, D. E. Gross, V. M. Lynch, J. L. Sessler, Chem. Eur. J. 2007, 13, 1374-1381. 


\section{Literaturverzeichnis}

${ }^{82}$ a) J. H. Weber, Inorg. Chem. 1967, 6, 258-262. b) L. Yang, Q. Chen, Y. Li, S. Xiong, G. Li, J. S. Ma, Eur. J. Inorg. Chem. 2004, 1478-1487. c) A. Bacchi, M. Carcelli, L. Gabba, S. Ianelli, P. Pelagatti, G. Pelizzi, D. Rogolino, Inorg. Chim. Acta 2003, 342, 229-235.

${ }^{83}$ Y. Wang, H. Fu, F. Shen, X. Sheng, A. Peng, Z. Gu, H. Ma, J. S. Ma, J. Yao, Inorg. Chem. 2007, 46, 35483556.

${ }^{84}$ a) G. Noël, J. C. Röder, S. Dechert, H. Pritzkow, L. Bolk, S. Mecking, F. Meyer, Adv. Synth. Catal. 2006, 348, 887-897. b) G. Noël, Dissertation, Georg-August-Universität Göttingen, 2005.

${ }^{85}$ J. C. Röder, Dissertation, Ruprecht-Karls-Universität Heidelberg, 2001.

${ }^{86}$ E. A. Katayev, K. Severin, R. Scopelliti, Y. A. Ustynyuk, Inorg. Chem. 2007, 46, 5465-5467.

${ }^{87}$ J. Ackermann, F. Meyer, E. Kaifer, H. Pritzkow, Chem. Eur. J. 2002, 8, 247-258.

${ }^{88}$ F. Meyer, K. Heinze, B. Nuber, L. Zsolnai, J. Chem. Soc. Dalton, Trans. 1998, 207-213.

${ }^{89}$ F. Meyer, U. Ruschewitz, P. Schober, B. Antelmann, L. Zsolnai, J. Org. Soc., Dalton Trans., 1998, $1181-$ 1186.

${ }^{90}$ P. J. Zinn, D. R. Powell, V. W. Day, M. P. Hendrich, T. N. Sorell, A. S: Borovik, Inorg. Chem. 2006, 45, 3484-3486.

${ }^{91}$ F. Meyer, S. Beyreuther, K. Heinze, L. Zsolnai, Chem. Ber/Recueil 1997, 130, 605-613.

${ }^{92}$ Das hier verwendete Programm julX wurde von Dr. E. Bill, Max-Planck-Institut für Bioanorganische Chemie, Mülheim an der Ruhr, zur Verfügung gestellt.

93 a) E. Escrivà, J. García-Lozano, J. Martínez-Lillo, H. Nuñez, J. Server-Carrió, L. Soto, R. Carrasco, J. Cano, Inorg. Chem. 2003, 42, 8328-8336. b) W. Mazurek, B. J. Kennedy, K. S. Murray, M. J. O’Connor, J. R. Rodgers, M. R. Snow, A. G. Wedd, P. R. Zwack, Inorg. Chem. 1985, 24, 3258-3264. c) Y. Elerman, H. Kara, A. Elmali, Z. Naturforsch. 2003, 58a, 363-372. d) S.-F. Huang, Y.-C. Chou, P. Misra, C.-J. Lee, S. Mohanta, H.-H. Wei, Inorg. Chim. Acta 2004, 357, 1627-1631.

94 a) M. C. Rodríguez, I. Morgenstern-Badarau, M. Cesario, J. Guilhem, B. Keita, L. Nadjo, Inorg. Chem. 1996, 35, 7804-7810. b) K. J. Oberhausen, J. F. Richardson, R. M. Buchanan, Polyhedron 1989, 8, 659-668.

${ }^{95}$ M. Gil-Alvariño, Dissertation, Georg-August-Universität Göttingen, 2006.

${ }^{96}$ P. de Meester, S. R. Fletcher, A. C. Skapski, J. Chem. Soc., Dalton Trans. 1973, 2575-2578.

${ }^{97}$ S. Buchler, F. Meyer, E. Kaifer, H. Pritzkow, Inorg. Chim. Acta. 2002, 337, 371-386.

98 J. R. Aranzaes, M.-C. Daniel, D. Astruc, Can. J. Chem. 2006, 84, 288-299.

${ }^{99}$ J. C. Röder, F. Meyer, E. Käfer, H. Pritzkow, Eur. J. Chem. 2004, 1646-1660.

${ }^{100}$ H. Bisswanger, Enzymkinetik, 3. neu bearbeitete Auflage, Wiley-VCH Weinheim, 2000.

${ }^{101}$ A. V. Willi, Isotopeneffekte bei chemischen Reaktionen, Georg Thieme Verlag, Stuttgart, 1983.

${ }^{102}$ M. Hesse, H. Meier, B. Zeeh, Spektroskopische Methoden in der organischen Chemie, 5. überarbeitete Auflage, Georg Thieme Verlag Stuttgart, 1995, $29 \mathrm{ff}$.

103 J. L. Sessler, T. D. Mody, D. A. Ford, V. Lynch, Angew. Chem. 1992, 104, 461-464.

${ }^{104}$ J. L. Sessler, A. Andrievsky, P. A. Gale, V. Lynch, Angew. Chem. 1996, 108, 2954-2957.

${ }^{105}$ S. J. Brooks, S. E. García-Garrido, M. E. Light, P. A. Cole, P. A. Gale, Chem. Eur. J. 2007, 13, 3320-3329.

${ }^{106}$ S. Katsiaouni, S. Dechert, C. Brückner, F. Meyer, Chem. Commun. 2007, 951-953.

${ }^{107}$ www.cem.msu.edu/ reusch/VirtualText/acidity2.htm 


\section{Literaturverzeichnis}

108 http://daecr1.harvard.edu/pdf/evans_pKa_table.pdf

${ }^{109}$ E. J. T. Chrystal, L. Couper, D. J. Robins, Tetrahedron 1995, 51, 10241-10252.

${ }^{110}$ C. Reichert, A. Blum, K. Harms, G. Schäfer, Liebigs Ann./Recueil 1997, 707-720.

${ }^{111}$ J. Chatt, L. M. Vallarino, L. M. Venanzi, J. Chem. Soc. 1957, 3413-3416.

${ }^{112}$ R. E. Rülke, J. M. Ernsting, A. L. Spek, C. J. Elsevier, P. W. N. M. VanLeeuwen, K. Vrieze, Inorg. Chem. 1993, 32, 5769-5778.

${ }^{113}$ V. Chiroli, F. Benedini, E. Ongini, P. Del Soldato, Eur. J. Med. Chem. 2003, 38, 441-446.

${ }^{114}$ G. Lee, M. Oka, H. Takemura, Y. Miyahara, N. Shimizu, T. Inazu, J. Org. Chem. 1996, 61, 8304-8306.

115 S. J. Swithenby, Contemp. Phys. 1974, 15, 249-267.

116 a) W. Haberditzl, Angew. Chem. 1966, 78, 277-288; b) Angew. Chem., Int. Ed. Engl. 1966, 5, 288-298.

${ }^{117}$ W. Haberditzl, Magnetochemie, Akademie-Verlag, Berlin, 1968.

${ }^{118}$ G. M. Sheldrick, SHELXS-97, Program for Crystal Structure Solution, Universität Göttingen, 1997.

${ }^{119}$ G. M. Sheldrick, SHELXL-97, Program for Crystal Structure Refinement, Universität Göttingen, 1997. 


\section{Formelübersicht}<smiles>CCc1c(C=O)[nH]c(Cc2cc(Cc3[nH]c(C=O)c(CC)c3CC)[nH]n2)c1CC</smiles><smiles>CCc1c(C=O)[nH]c(Cc2n[nH]c(Cc3[nH]c(C=O)c(CC)c3CC)c2-c2ccccc2)c1CC</smiles>

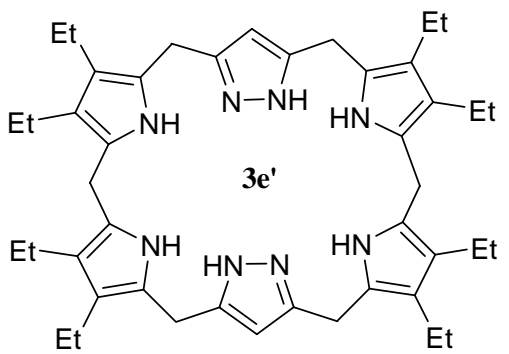

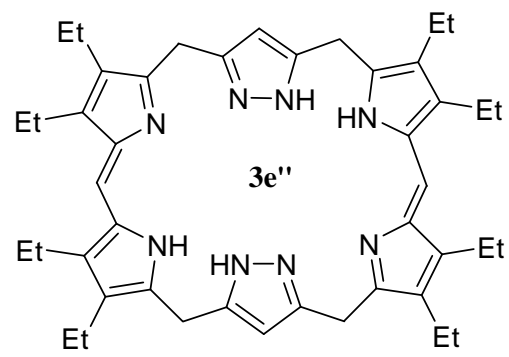<smiles>ClCc1n[nH]c(CCl)c1-c1ccccc1</smiles>

$\cdot \mathrm{HCl}$

4<smiles>CCc1c[nH]c(Cc2cc(Cc3[nH]cc(CC)c3CC)[nH]n2)c1CC</smiles>

$5 a$<smiles>CCc1c[nH]c(Cc2n[nH]c(Cc3[nH]cc(CC)c3CC)c2-c2ccccc2)c1CC</smiles>

$5 b$<smiles>CCc1c(CO)[nH]c(Cc2cc(Cc3[nH]c(CO)c(CC)c3CC)[nH]n2)c1CC</smiles><smiles>C(=NCc1cc(CN=Cc2ccc[nH]2)[nH]n1)c1ccc[nH]1</smiles>

7<smiles>C(=N/Cc1cc(C/N=C/c2ccccn2)[nH]n1)\c1ccccn1</smiles><smiles>C(=NCc1cc(CN=Cc2ccccn2)[nH]n1)c1ccccn1</smiles>

9

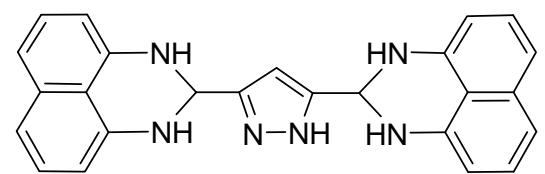

10<smiles>O=C(c1cc(C(=O)N2CCOCC2)[nH]n1)N1CCOCC1</smiles> 


\section{Formelübersicht}
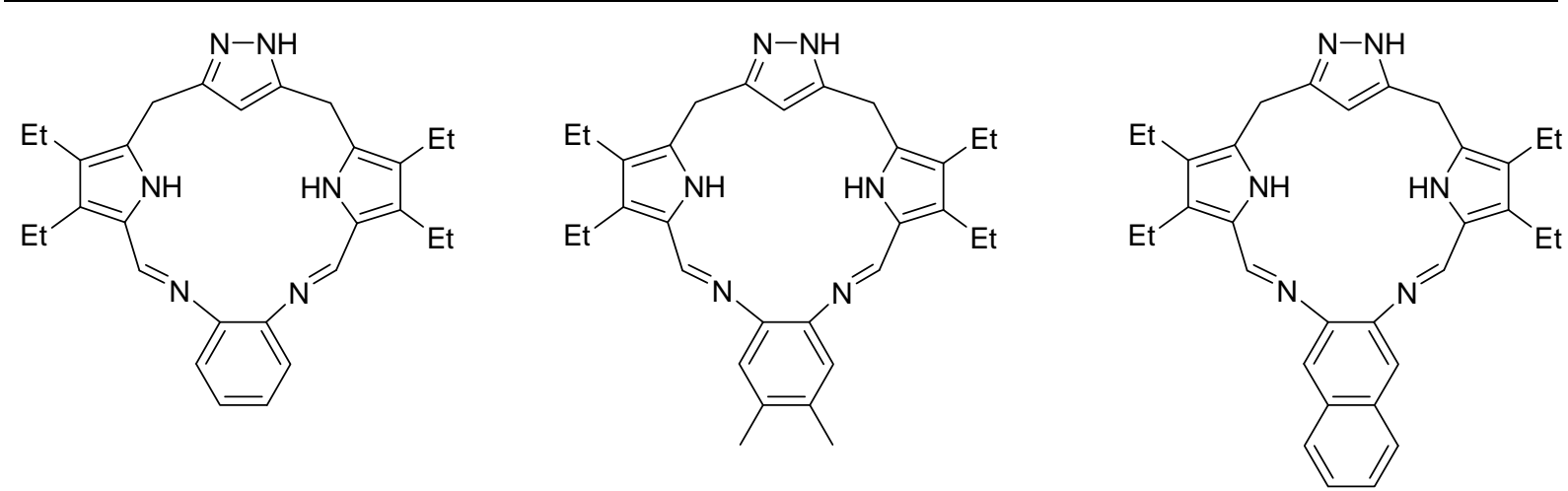

2a

$2 b$

2c
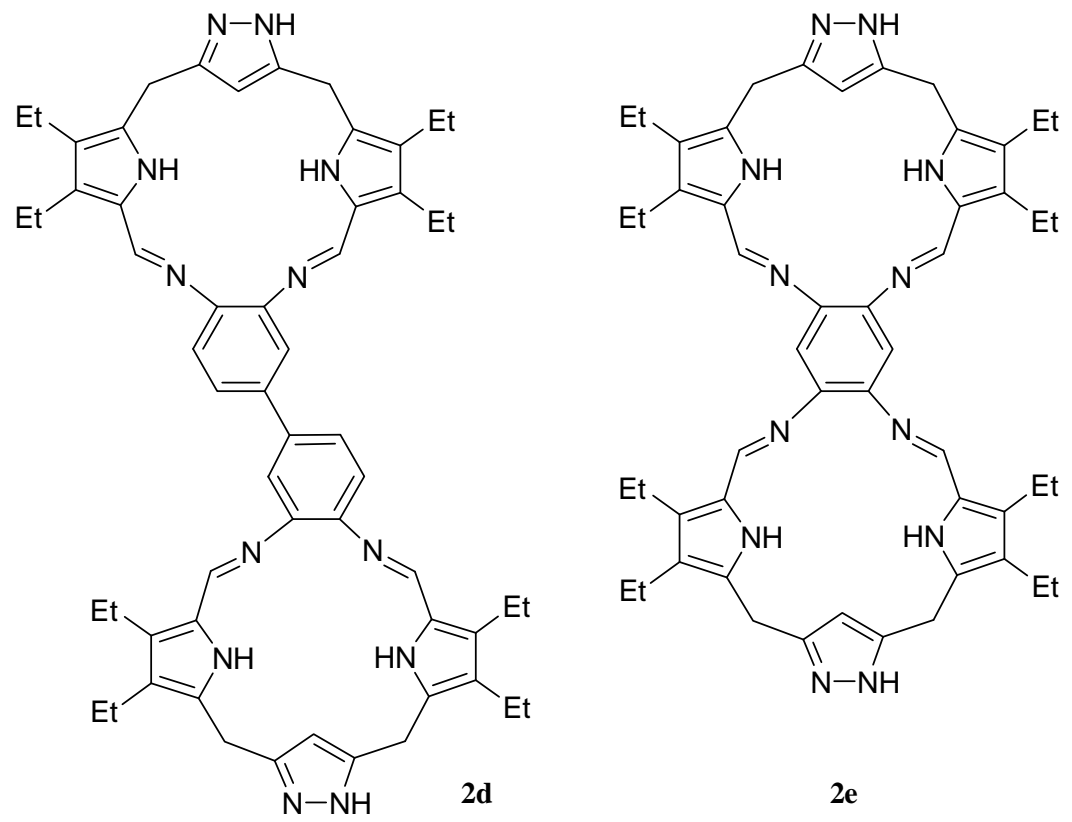

2e

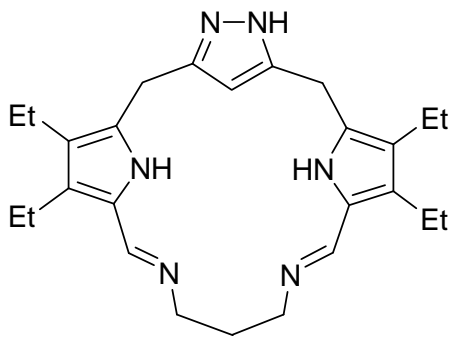

2f

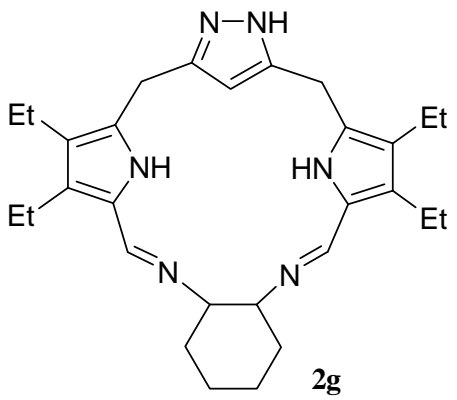

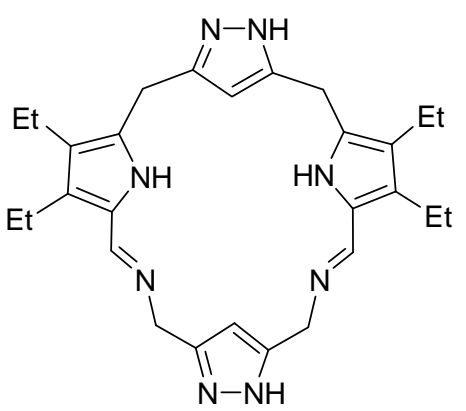

$2 \mathrm{~L}$

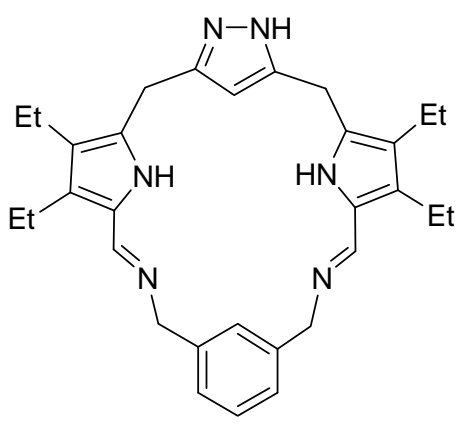

$2 \mathbf{i}$

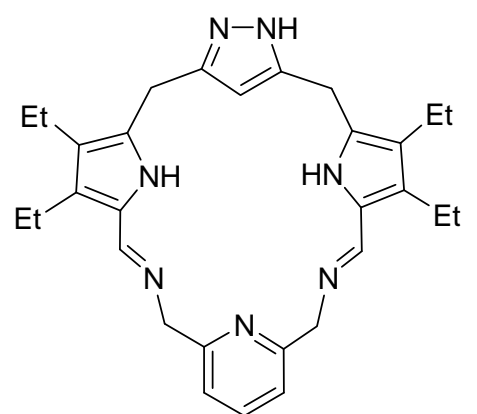

2j 


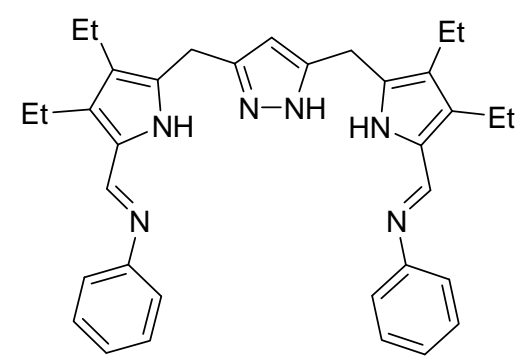

$\mathrm{H}_{3} \mathbf{L}^{1}$

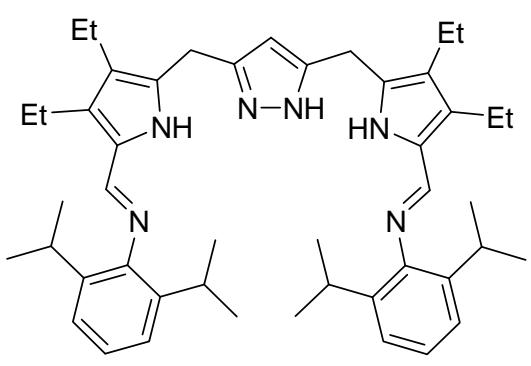

$\mathbf{H}_{3} \mathbf{L}^{3}$

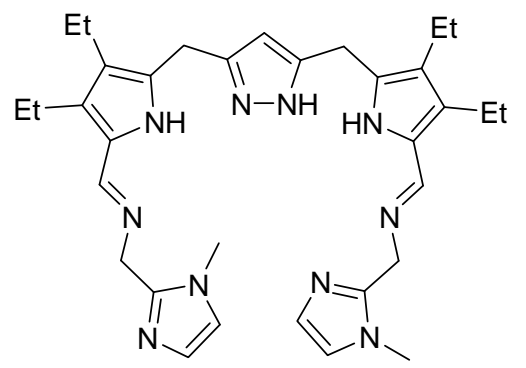

$\mathbf{H}_{3} \mathbf{L}^{5}$

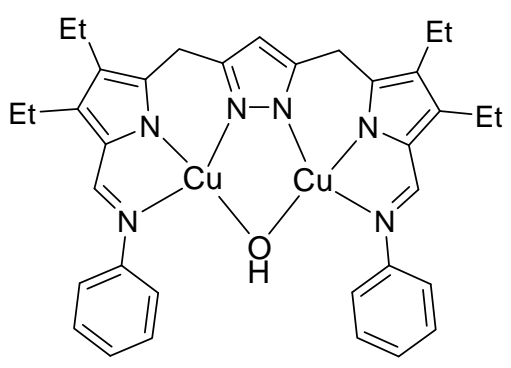

$\left[\mathrm{L}^{1} \mathrm{Cu}_{2}(\mathrm{OH})\right]$

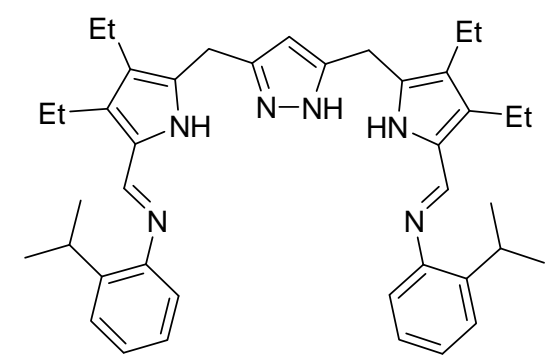

$\mathbf{H}_{3} \mathrm{~L}^{2}$

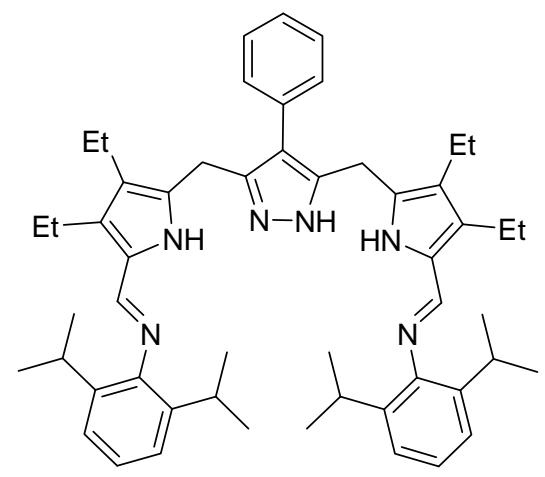

$\mathrm{H}_{3} \mathrm{~L}^{4}$

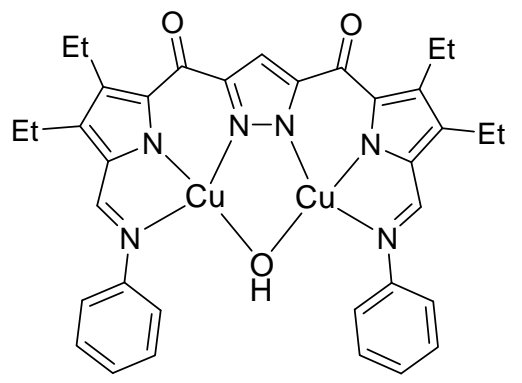

$\left[\mathrm{L}^{1}{ }_{\text {ox }} \mathrm{Cu}_{2}(\mathrm{OH})\right]$

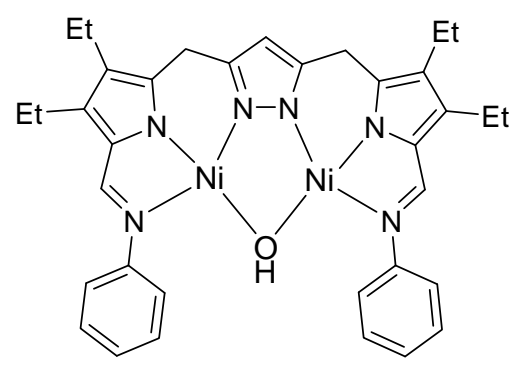

[L $\left.{ }^{1} \mathrm{Ni}_{2}(\mathrm{OH})\right]$ 


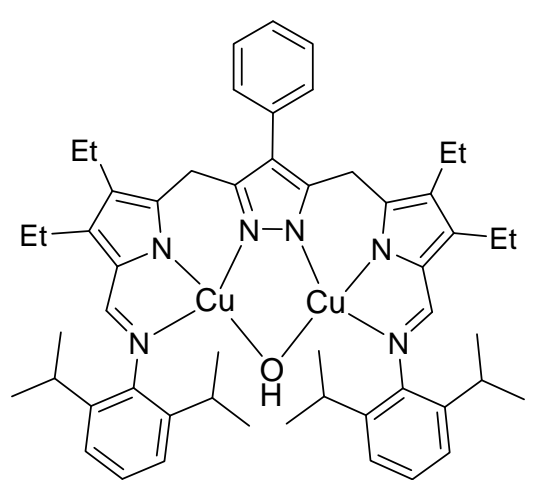

$\left[\mathrm{L}^{4} \mathrm{Cu}_{2}(\mathrm{OH})\right]$

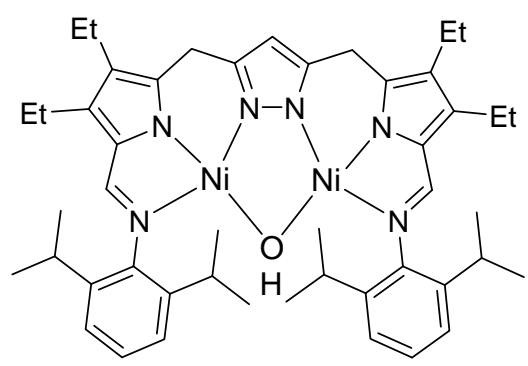

$\left[\mathrm{L}^{3} \mathrm{Ni}_{2}(\mathrm{OH})\right]$

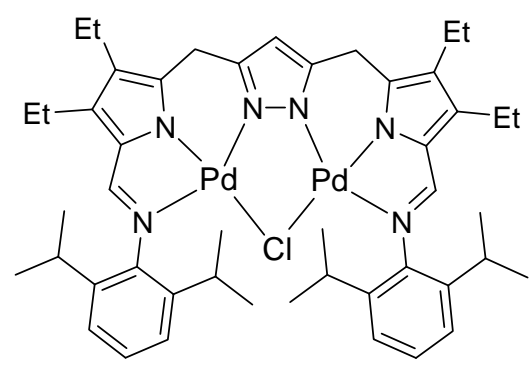

[ $\left.\mathbf{L}^{3} \mathbf{P d}_{2} \mathbf{C l}\right]$

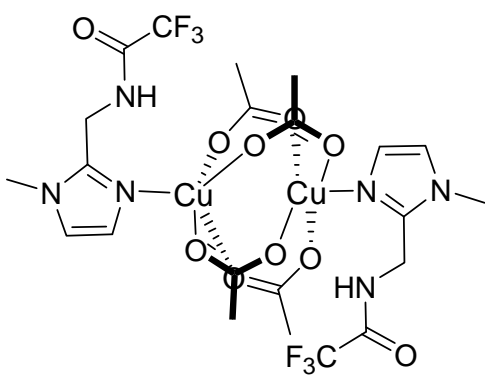

$\left[\mathbf{L}_{2}^{6} \mathrm{Cu}_{2}(\mathbf{O A c})_{4}\right]$

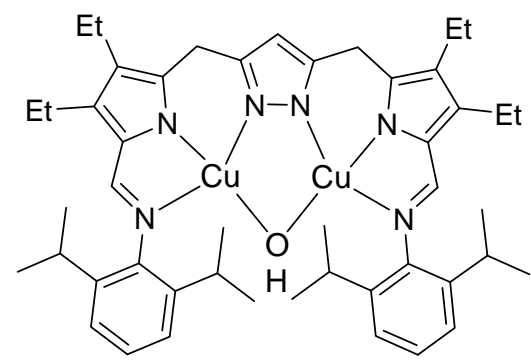

$\left[\mathrm{L}^{3} \mathrm{Cu}_{2}(\mathrm{OH})\right]$

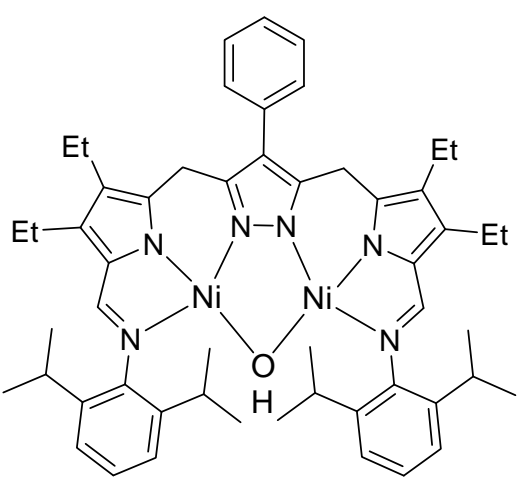

$\left[\mathrm{L}^{4} \mathrm{Ni}_{2}(\mathrm{OH})\right]$

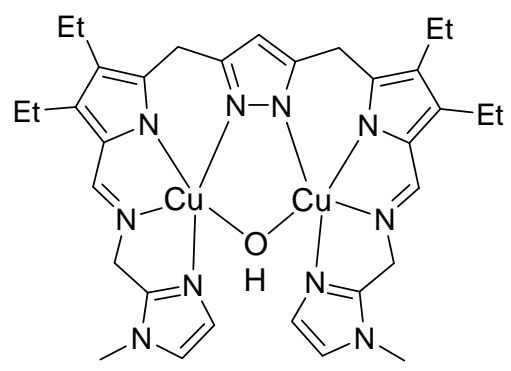

[L $\left.{ }^{5} \mathrm{Cu}_{2}(\mathrm{OH})\right]$

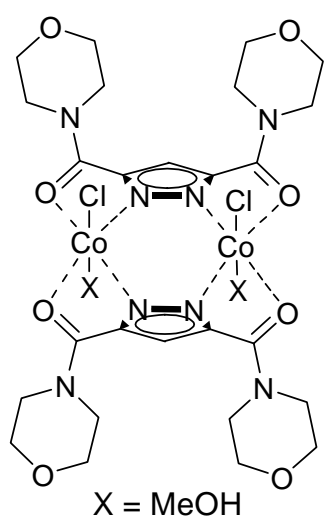

$\left[\mathrm{L}^{11} \mathrm{Co}_{2} \mathrm{Cl}_{2}(\mathrm{MeOH})_{2}\right]$ 


\section{Publikationen}

S. Katsiaouni, S. Dechert, C. Brückner, F. Meyer, Chem. Commun. 2007, 951-953.

"A versatile building block for pyrazole-pyrrole hybrid macrocycles".

S. Katsiaouni, S. Dechert, R. P. Briñas, C. Brückner, F. Meyer, in Vorbereitung.

„Schiff base macrocycles containting pyrroles and pyrazoles“.

\section{Poster}

International Conference on Porphyrin and Phtalocyanin (ICPP 4), July 2006, Rom, Italien "Pyrrole-Pyrazole Hybrid Macrocycles: “Tropopiophyrin” - Synthesis and binding studies of a novel macrocycle".

3. Koordinationschemie-Treffen, Berlin 2007

"A versatile class of pyrazole/pyrrole building blocks: new scaffolds for bimetallic complexes".

Erster Fakultätstag der Göttingen Chemie, Göttingen 2007

“Neuartige Pyrrol/Pyrazol Hybrid-Makrozyklen”. 



\section{Danksagung}

An erster Stelle möchte ich mich bei Herrn Prof. Dr. Franc Meyer für die freundliche Aufnahme in den Arbeitskreis, für die Betreuung meiner Arbeit und die Gewährung der wissenschaftlichen Freiheit für meine Forschungsarbeiten bedanken.

Besonders möchte ich mich außerdem bei Herrn Prof. Dr. Christian Brückner von der University of Connecticut für die freundliche Aufnahme in Storrs und den netten lehrreichen Aufenthalt in den USA bedanken. Christian und Heike, ihr habt großartig für mich/uns gesorgt und meinen Aufenthalt unvergesslich gemacht. Thank you so much!

Danken möchte ich ebenso allen, die zum Gelingen der Arbeit beigetragen haben: Dem Kristallographen Dr. Sebastian Dechert, der aus den winzigen Kristallen immer die besten Ergebnisse erhalten konnte; dem Mikroanalytischen Labor des Instituts für Anorganische Chemie und der MS-Abteilung; den Werkstätten und Glasbläsern des Instituts, der NMRAbteilung - speziell Herrn Zolke, Herrn Schöne und Herrn Dr. M. John für die „wochenlangen“ Messungen; Dr. Walter Maringgele, Andreas Schwarz und Jörg Teichgräber danke ich für die Synthese einiger wichtiger Präparate, für die Ausgabe von Laborzubehör und für die CV-Messungen; Frau Dr. Claudia Stückl danke ich für die ESR-Messungen und die administrative Arbeit; unserer Sekretärin Frau Petra Unger möchte ich mich für die administrative Hilfe und die netten Gespräche danken; Herrn Dr. Serhiy Demeshko danke ich für die SQUID-Messung; Holger Müller und Ulrich Scheele danke ich für die Hilfe bei den Arbeiten mit der Glovebox; bei Frau Dr. Rosana Ferarri möchte ich mich für die Hilfe in Sachen ,pH- und Titration“ bedanken; Katharina Fejfar und Matthias Bischoff danke ich für die engagierte Arbeit während ihrer Forschungspraktika; and I thank Michelle Dean for her great committed work in the lab during her stay in Göttingen with the demanding pyrrol/pyrazol-building blocks.

Prof. Dr. Martin Bröring von der Phillips-Universität in Marburg möchte ich für die Ideen, die Anregungen und für den Versand von Chemikalien danken.

Mein besonderer Dank gilt Frau Kathrin Meindl, Frau Dr. Eftichia Alexopoulos, Frau Dr. Sarah Fakih, Herrn Dr. Marcus Hensch, Herrn Dr. Gilles Noël, Herrn Ulrich Scheele, Herrn Prof. Dr. Christian Brückner und Prof. Dr. Franc Meyer für das schnelle Korrekturlesen dieser Arbeit.

Allen Mitgliedern und Ex-Mitgliedern der Gruppe danke ich für die Anregungen, Ideen, das angenehme Arbeitsklima und vor allem für die vielen gemeinsamen Unternehmungen. 
Insbesondere möchte ich mich bei Dr. Gilles Noël, Dr. Rosana Ferrari, Dr. Jens Ackermann, Dr. Bernhard Bauer-Siebenlist, Dr. Julia Klingele, Dr. Marco Klingele, Dr. Miguel Alvariño, Dr. Anupama Ranganathan, Michelle Dean, Ulrich Scheele und Holger Müller für die lustigen Gespräche und die langen amüsanten Abende im Sozialraum bedanken.

Meiner „griechisch-französischen“ Familie danke für die großartige Unterstützung in allem!

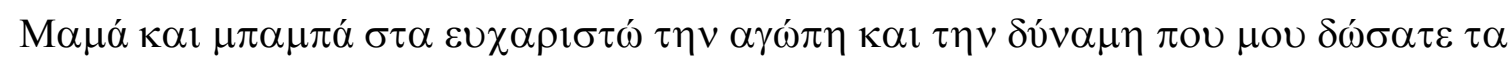
$\tau \varepsilon \lambda \varepsilon \cup \tau \alpha i ́ \alpha \chi \chi \rho o ́ v i \alpha !$

Meinen Schwestern Panagiota und Ekaterini Katsiaouni danke ich für ihre Liebe, Hilfe und die lustigen Momente mit ihnen. Besonders in der Endphase dieser Arbeit habt ihr mich durch eure zahlreichen Telefonate bei guter Laune gehalten.

Mein besonderer Dank gilt meinem großartigen Ehemann Dr. Gilles Noël für die Hilfe (besonders bei Soft- und Hardware Problemen), Geduld, Liebe und für das Verständnis während dieser Arbeit. Merci pour tout mon cœur! 


\section{Lebenslauf}

Name

Geburtsdatum

Geburtsort

Familienstand

Staatsangehörigkeit

Schulausbildung

06.1999

\section{Hochschulstudium}

10.1999

10.2001

07.2003-03.2004

05.2004

\section{Promotion}

06.2004-11.2007

\section{Beschäftigungsverhältnisse}

05.2003-08.2004

seit 09.2004

\section{Praktika/Auslandsaufenthalt}

10. -12.2002
Stamatia Katsiaouni

26. März 1980

Serres (Griechenland)

verheiratet

griechisch

Abitur, Wilhelm Raabe Gymnasium, Lüneburg.

Diplomstudiengang Chemie an der Georg-August-Universität Göttingen.

Vordiplom Chemie, Georg-August-Universität Göttingen.

Diplomarbeit am Lehrstuhl für Anorganische Chemie der Georg-August-Universität unter der Leitung von Prof. Dr. Franc Meyer; Thema: "Synthesebausteine zum Aufbau von Doppelporphyrinen".

Abschluss als Diplom-Chemikerin

Dissertation an der Georg-August-Universität Göttingen mit dem Thema: „Neuartige Pyrrol/Pyrazol-Bausteine für die Synthese von Hybrid-Makrozyklen, azyklischen Ligandsystemen und bimetallischen Komplexen“.

Wissenschaftliche Hilfskraft am Institut für Anorganische Chemie der Georg-August-Universität Göttingen.

Wissenschaftliche Angestellte am Institut für Anorganische Chemie der Georg-August-Universität Göttingen.

Organisch-chemisches Praktikum in der University of Manchester (UK), bei Dr. Andrew C. Regan (ERASMUS Austauschprogramm). 
10.-12.2003 und

09.-11.2004

\section{Stipendien}

10. -12.2002

10.-12.2003 und

09.- -11.2004

\section{Symposien/Konferenzen}

29.08.-02.09.2004

26.06.-01.07.2005

19.02.-21.02.2006

02.07.-07.07.2006

18.02.-20.02.2007

29.06.2007
Forschungsaufenthalt bei Prof. Dr. Christian Brückner im Department of Chemistry der University of Connecticut, Storrs (USA) im Rahmen einer DAAD/NSF Kooperation.

ERASMUS - University of Manchester (UK).

DAAD-Stipendium für den Aufenthalt im Department of Chemistry der University of Connecticut in Storrs (USA).

$7^{\text {th }}$ European Biological Inorganic Chemistry Conference, Garmisch-Partenkirchen.

$2^{\text {nd }}$ Blue Sky Conference, Sorrento, Italien.

2. Koordinationschemikertreffen, Institut für Anorganische Chemie, Göttingen.

$4^{\text {th }}$ International Conference on Porphyrins and Phthalocyanines, Rom, Italien.

3. Koordinationschemie-Treffen, TU-Berlin.

Erster Fakultätstag der Göttinger Chemie, Göttingen. 